\title{
Schulleiterinnen und Schulleiter im Spannungsverhältnis zwischen programmatischen Zielvorgaben und alltäglicher Praxis
}

Eine empirische Studie zur Berufsauffassung von Schulleiterinnen und Schulleitern

\footnotetext{
Dissertation

Zur Erlangung des sozialwissenschaftlichen Doktorgrades des Sozialwissenschaftlichen Fakultät der GeorgAugust-Universität Göttingen
}

\author{
vorgelegt von \\ Maike Languth \\ aus Kiel
}

Göttingen 2006 
1,Gutachterin: Frau Prof. Dr. Doris Lemmermöhle

2. Gutachter: Herr Prof. Dr. Wulf Hopf

Tag der mündlichen Prüfung: 31.10.06 
Inhalt

Seite

Vorwort

1. Einleitung 6

1.1 Forschungsinteresse 9

1.1.1 Fragestellung 9

1.2 Struktur und Aufbau der Arbeit 10

2. Schulleitungsforschung 10

2.1 Funktion und Rolle der Schulleiterinnen und 11

Schulleiter

2.2 Empirische Befunde 16

2.2.1 Ansätze aus der Organisationsperspektive 16

2.2.2 Profession als Fokus der Forschung 24

2.2.3 Berufswechsel als Sozialisationsprozess 26

2.2.4 Handlungsdimensionen von Schulleiterinnen 29 und Schulleitern

2.2.5 Geschlechtsspezifische Forschungsansätzen 30

2.3 Zusammenfassung 32

3. Theoretische Grundlagen 33

3.1 Führungstheorie $\quad 35$

3.1.1 Personenorientierte Ansätze $\quad 36$

3.1.2 Verhaltensorientierte Ansätze 36

3.1.3 Positionsorientierte Führungstheorien 38

3.1.4 Situationstheorien der Führung 39

3.1.5 Ausgewählte Führungskonzepte und
ihre Bedeutung für die Schule

3.1.5.1 Konsultative Führung 40

3.1.5.2 Kooperative Führung 41

3.1.5.3 Delegative Führung 43

3.1.5.4 Transaktionale Führung $\quad 45$

3.1.5.5 Transformationale Führung 45

3.1.6 Zusammenfassung $\quad 47$

3.2 Organisationstheorie 48

3.2.1 Entwicklung der Organisationstheorie $\quad 49$

3.2.2 Schule als Organisation 51

3.2.3 Schulentwicklung 53

3.2.3.1 Stationen der Schulentwicklungsdiskussion 54

3.2.4 Schulentwicklung und Schulleitung 56

3.2.5 Zusammenfassung $\quad 57$

3.3 Professionstheorie 58

3.3.1 Professionstheoretische Vorüberlegungen 59 
3.3.2 Pädagogische Professionsdiskussion

3.3.2.1 Werner Helsper: Konzept der konstitutiven 65 Antinomien der professionellen Praxis

3.3.3 Pädagogisches Professionswissen 69

3.3.4 Professionalität von Schulleiterinnen und $\quad 71$ Schulleitern

3.3.5 Berufsauffassungen $\quad 73$

3.3.6 Zusammenfassung 76

4. Eigene Untersuchung 77

4.1 Zielsetzung und methodisches Vorgehen $\quad 77$

$\begin{array}{ll}\text { 4.1.1 Fragestellung } & 79\end{array}$

4.2 Triangulation 79

4.2.1 Methodologische-Triangulation 80

4.3 Quantitative Erhebung 80

4.3.1 Fragebogen 81

4.3.2 Auswahl des Samples 82

4.3.2.1 Status der Schulleiter und Schulleiterinnen 83 in Niedersachsen

4.3.2.2 Rechtliche Stellung 83

4.3.2.3 Berufsbild in Niedersachsen 84

4.3.2.4 Auswahl und Qualifizierung 86

4.3.3 Beschreibung der untersuchten Stichprobe 86

4.3.4 Antworthäufigkeiten zu einzelnen Items $\quad 87$

4.3.4.1 Umgang mit Lehrerinnen und Lehrern $\quad 88$

4.3.4.2 Berufszufriedenheit und Belastungsempfinden 90

4.3.4.3 Ergebnisse Schulentwicklung 92

4.3.4.4 Führungsverhalten 94

4.3.4.5 Entscheidungsfindung 96

4.3.5 Dimensionen schulischen Führungshandelns 97

4.3.5.1 Korrelationen zwischen individuellen $\quad 99$ Merkmalen und den vier Dimensionen

4.3.5.2 Zusammenhänge zwischen den vier Dimensionen 101

4.3.6 Zusammenfassung 101

4.4 Leitfadengestützte qualitative Interviews mit Schul- 102 leiterinnen und Schulleitern methodisches Vorgehen bei der Datenerhebung

4.4.1 Erhebungsverfahren 102

4.4.2 Expertenstatus 103

4.4.2.1 Wissenssoziologische Definition 104 des Expertenbegriffs

4.4.3 Erhebung der leitfadengestützten 105 problemzentrierten Interviews mit Experten

4.4.3.1 Leitfaden und Durchführung der Interview 108

4.4.3.2 Die qualitative Stichprobe 110

4.4.4 Auswertung der problemzentrierten Interviews 111 
5. Berufsauffassungen von Schulleiterinnen und Schulleitern- 114 das empirische Material

5.1 Darstellung der Ergebnisse anhand ausgewählter 116

Fallbeispiele

5.1.1 Konstruktiver Jongleur

5.1.2 Resignierter Einsamer

5.1.3 Formelle Führungskraft

5.1.4 Resoluter Systemkritiker

5.1.5 Bodenständiger Seelsorger

6. Schule leiten in der beruflichen Praxis

6.1 Muster von Berufsauffassungen

6.2 Schulleitungshandeln- Aushandlungsprozess zwischen Stabilität und Innovation

6.3 Das Spannungsverhältnis zwischen Programmatiken 191 und Alltagshandeln

7. Resümee

7.1 Zusammenfassende Diskussion der Ergebnisse

7.2 Ausblick

8. Literatur

Abbildungen

Anlagen 


\section{Vorwort}

Die vorliegende Untersuchung ist dem Bereich Schulforschung zuzuordnen. Sie versucht, einen Ausschnitt der "Schulwirklichkeit" mit wissenschaftlichen Methoden aufzuklären, ein Teil des Schullebens wird dabei fokussiert, nämlich die Schulleiterinnen und Schulleiter in ihrem komplexen Handlungsfeld. Durch den Einsatz einer quantitativen und einer qualitativen Befragung werden die Berufsauffassungen der Akteure erfasst. Insbesondere die qualitativen Interviews mit den Schulleiterinnen und Schulleitern in Niedersachsen ermöglichen Erkenntnisse über die Sichtweise der Schulpraxisexperten vor Ort.

Ohne die Bereitschaft der Schulleiterinnen und Schulleiter, mir Einblick in ihre berufliche Praxis zu gestatten, wäre diese Studie nicht möglich gewesen. Ich möchte innen allen für ihr Offenheit und die mir zugestandene Zeit sehr danken. Die Ergebnisse zeigen, mit welchem Engagement Schulleiterinnen und Schulleiter ihren eigenen Beruf reflektieren und analysieren.

Diese Arbeit wäre ohne die Hilfe und Unterstützung anderer Menschen nicht zustande gekommen. Mein Dank gilt denjenigen, die mich in diesem Forschungsprozess durch kritische Diskussionen, Anregungen und Hilfestellungen begleitet haben. Ganz besonders möchte ich Frau Prof. Dr. Doris Lemmermöhle für ihre intensive Begleitung und Beratung danken. Des Weiteren danke ich Herrn Prof. Dr. Wulf Hopf für seine Anregungen sowie dem Promotionskolloquium der Universität Göttingen. Für seine Ermutigung und die konstruktive Unterstützung danke ich Herrn Prof. Dr. Waldemar Pallasch. Für die Hilfe bei der Analyse des statistischen Datenmaterials danke ich Felix Brümmer.

Mein Dank gilt vor allem auch den Menschen, die mich tagtäglich ermutigt und die Höhen und Tiefen dieses Prozesses begleitet haben. Ganz besonders Heidrun Wiedemann, Inge Henze, Gabi Schatz, Peter und Ulrike Brammer. Ebenso danke ich meinen Eltern Heidi und Hans-Jürgen Schulz für ihren Zuspruch und ihre Unterstützung.

Iris Bekedorf und Brigitte Bialkowski danke ich sehr für ihre Bereitschaft, den Text auf formale und stilistische Richtigkeit zu prüfen. 


\begin{abstract}
„Das ist schwer. Das ist schwer, es ist ja schwer mit der Führungskompetenz, das muss ich mal vorweg sagen, weil Lehrer ja ein sehr unregierbares Völkchen sind. Ich weiß nicht, ob Ihnen das klar ist?"1
\end{abstract}

\title{
1. Einleitung
}

"Leitstern am Lehrerhimmel“, so lautet die Überschrift eines Artikels aus dem Jahr 2004 in der ZEIT zum Thema Schulleitung. ${ }^{2}$ Sternenklar, so wird der Eindruck vermittelt, sei doch die herausragende Bedeutung der Schulleitung für den Erfolg der Schule, Schulleiterinnen und Schulleiter seien Kristallisationspunkte der Veränderung und neue Schulen bräuchten eine andere Führung. Charismatische Führungspersönlichkeiten für die Schule werden gefordert, die faszinieren, die ein Bündel von Ideen, Überzeugungen besitzen und die durch ihre Ausstrahlung Erfolge herbeiführen. Der alte "Direx" hat ausgedient, gefragt sind jetzt Ideengeber und Motivatoren, Menschensammler, Visionäre, Innovationsmanager - das begriffliche Zuden-Sternen-greifen wird ergänzt durch eine Berufskonzeption des Allgemeinen Schulleitungsverbandes, der in Niedersachsen gemeinsam mit dem Kultusministerium ein komplexes Anforderungsprofil erarbeitet hat.

Welche Sternschnuppe muss zu Boden fallen, um diesem Idealbild entsprechen zu können?

Ganz anders sieht der Blick der Schulleiterinnen und Schulleiter aus, wenn sie von sich meinen, dass sie „zahnlose Tiger" seien, dass sie sich von „der Behörde im Stich gelassen fühlen“ oder gar die Schule insgesamt als "innovationsfeindlich und ineffektiv" einschätzen, dann scheint offensichtlich ein Spannungsverhältnis zwischen den Himmelsbeschreibungen und dem Erdendasein zu bestehen.

Heute müssen sich Schulleiterinnen und Schulleiter nicht mehr über mangelnde Aufmerksamkeit beklagen, im Gegenteil: Die Ansprüche und Erwartungen, die an sie gestellt werden, steigern sich ins Irrationale. Die Bedingungen für die Verwirklichung der Erwartungen im Hinblick auf Auswahl und Qualifizierung der Schulleiterinnen und Schulleiter, bezüglich der Unterstützung, die sie durch die Schulbehörde erhalten und ebenso die ihnen öffentlich entgegengebrachte Wertschätzung entsprechen hingegen nicht unbedingt diesen Ansprüchen.

Für Schulleiterinnen und Schulleiter gilt, dass sie keine homogene Gruppe darstellen, sondern Individuen sind, die mit unterschiedlichem Vorwissen und unterschiedlichen Kompetenzen das Amt der Schulleiterin/ des Schulleiters antreten. Bisher trifft für sie alle, die amtierenden und die neuen Schulleiterinnen und Schulleiter, zu, dass sie keine systematische Berufsausbildung erfahren und insofern ein wichtiges Kriterium der allgemeingültigen Vorstellung von Professionalität nicht erfüllen, obwohl von innen professionelles Führungshandeln erwartet wird.

Die Rolle der Schulleiterin/ des Schulleiters als der praktizierenden Lehrkraft mit einigen zusätzlichen, vornehmlich verwaltungsmäßigen Aufgaben

\footnotetext{
${ }^{1}$ Interviewzitat SI 3, 1.

${ }^{2}$ Vgl. ZEIT 37/2004.
} 
ist längst abgelöst worden von der Position „Allround-Manager“ und Entwickler/-in von personellen, finanziellen und sachlichen Ressourcen. Eine Fülle von unterschiedlichen Tätigkeiten, z.B. Schnellentscheidungen und sofortiger Regelungsbedarf, bestimmen ihren Arbeitsalltag. Ein hohes Maß an Arbeitssouveränität und Umgangskultur von Konfliktlösungskompetenz und Veränderungsmanagement werden von innen erwartet und dabei gilt es oft, Spannungszustände „professionell zu meistern“.

In den letzten Jahren hat sich durch gesellschaftliche Wandlungsprozesse und zunehmende Kritik an den öffentlichen Schulen die Schulentwicklung zum Hauptaufgabenfeld der Schulleiterinnen und Schulleiter ausgebildet. Insbesondere durch das vergleichsweise schlechte Abschneiden deutscher Schülerinnen und Schüler innerhalb internationaler Vergleichsuntersuchungen hat sich eine kritische Haltung gegenüber Schulen, Lehrkräften und auch Schulleiterinnen und Schulleitern entwickelt. Diese zunehmende Kritik und die gleichzeitig durch knappere Ressourcen verursachten schwierigeren Arbeitsbedingungen in den Schulen lösen in den Kollegien zuweilen Abwehr aus und verhindern notwendige Umgestaltungen. Rosenbusch spricht von "Sättigungsgrenzen“ der Schulentwicklung. ${ }^{3}$ In diesem Bereich ist das Aushandeln unterschiedlicher Interessenlagen innerhalb und außerhalb der Schule von Bedeutung für das Gelingen von Veränderungsprozessen. Gerade hier setzt eine Schlüsselanforderung an Schulleiterinnen und Schulleiter an: Ihnen soll es gelingen, mit Repräsentanten unterschiedlicher und zuweilen widerstreitender Auffassungen in wertschätzende Kommunikationsprozesse zu treten, um Veränderungsprojekte zu initiieren und zu implementieren.

Schulleiterinnen und Schulleiter stehen in einem Spannungsverhältnis zwischen der Aufgabenvielfalt und den hohen Erwartungen an die Schulleitungsarbeit auf der einen Seite und den vorgefundenen Bedingungen der konkreten Schulleitungstätigkeit auf der anderen Seite. Neben der im Schulgesetz verankerten Gesamtverantwortung für die Schule gehören zu den neuen Aufgaben vor allen Dingen wirtschaftliche, personelle, organisatorische und curriculare Gestaltungsaufgaben, z.B. Mittelbewirtschaftung, Unterrichtsorganisation (flexiblere Stundentafel), Hinwirkung auf Aus- und Fortbildung der Lehrkräfte, Verbesserung der Unterrichts- und Erziehungsarbeit und des Schullebens, Förderung innerschulischer Selbstorganisation, Erstellung und Weiterentwicklung des Schulprogramms, Förderung und Öffnung der Schule zum Umfeld, um nur einiges zu nennen. ${ }^{4}$ Diesem komplexen Anforderungsprofil stehen Hierarchie innerhalb der Schule mit differenzierten Entscheidungswegen, ein eher traditionelles Rollenverständnis der Lehrkräfte, welches sich in der Scheu vor Veränderungen ausdrückt und ein traditioneller Verwaltungsrahmen der Schulverwaltung sowie unzureichende finanzielle Führungsmittel gegenüber.

Durch die KMK und die Gesetzgebung der Länder hat die Position der Schulleiterinnen und Schulleiter sich verändert. Die Gestaltungs-, Handlungs- und Entscheidungsspielräume der Schulleiterinnen und Schulleiter werden ausgedehnt. Diese Aufgabenvielfalt steht in einem Missverhältnis

\footnotetext{
${ }^{3}$ Vgl. Rosenbusch 2005, $119 \mathrm{ff}$.

${ }^{4}$ Vgl. Brockmeyer 1998,131.
} 
zu dem zugebilligten Maß an eigenverantwortlichen Entscheidungsmöglichkeiten - so bleiben beispielsweise die Entscheidungskompetenzen der Schulleitung im Bereich der Personalführung aufgrund fehlender Ermächtigung durch die Schulaufsicht eingeschränkt.

Schulleiterinnen und Schulleiter befinden sich in der schwierigen Situation, dass sie einerseits den neuen Anforderungen einer Profilierung der Einzelschule gerecht werden sollen, andererseits das stark auf Klassenunterricht ausgerichtete Berufsverständnis von Lehrerinnen und Lehrern im Hinblick auf die Entwicklung der Schule als Ganzes lenken sollen. Daneben steht die Einzelschule im Systemzusammenhang staatlicher Kontrolle des Schulsystems, wodurch sich ein Wechselspiel Innen- und Außenwelt ergibt, an deren Schnittstelle die Schulleiterinnen und Schulleiter stehen.

Neben dem komplexen Tätigkeitsspektrum der Schulleiterinnen und Schulleiter und den sich daraus ergebenden Anforderungen an die Berufspraxis bestehen seitens der Politik, der Wissenschaft, der Kultusbehörden, der Wirtschaft, der Öffentlichkeit und vor allen Dingen seitens aller an Schule beteiligten Interessengruppen hohe Erwartungen an die optimale Erfüllung all dieser Aufgaben.

Ein Beispiel für ein Anforderungsprofil stellt das Ergebnis einer Projektgruppe „Arbeitsplatz Schulleitung"5 des niedersächsischen Kultusministeriums dar. Insgesamt werden dreißig verschiedene Kompetenzbereiche und über zwanzig Fähigkeiten genannt, die Schulleiterinnen und Schulleiter besitzen sollten. Es ist wohl die Eigenheit von Anforderungskatalogen, dass durch sie Vorstellungen eines Idealbildes vermittelt werden, gefordert wird die perfekte Schulleiterin/ der perfekte Schulleiter - ein Allroundgenie. Zwar lässt sich ein Idealbild beschreiben, aber in der realen Welt können wir es wahrscheinlich nicht verwirklicht finden, und das ist der Unterschied zwischen idealen Vorstellungen, gesellschaftlichen Erwartungen und der realen Praxis des Berufes.

Die Frage nach der idealen Schulleiterin/ dem idealen Schulleiter ist möglicherweise daher falsch und nicht zu beantworten, vielmehr sollte hinterfragt werden, welche theoretischen Bezüge Einfluss haben, welche Vorstellungen durch die gestellten Anforderungen herausgebildet werden und wie sich z.B. vorgegebene Berufsbilder auswirken. Mit der vorliegenden Untersuchung soll versucht werden, Aufschluss darüber zu erhalten, welche Berufsauffassung Schulleiterinnen und Schulleiter haben. Als Menschen, als Persönlichkeiten sind Schulleiterinnen und Schulleiter unterschiedlich, sie werden höchstwahrscheinlich keinen Anforderungsprofilen und schon gar nicht Idealtypen entsprechen, aber vermutlich zieht sich durch ihr Handeln und ihre Auffassung über den eigenen Beruf ein roter Faden, ein Muster. Der Suche nach diesen Eigenarten gilt mein Forschungsinteresse, das im Folgenden erläutert werden soll.

\footnotetext{
${ }^{5}$ Vgl. Arbeitsplatz Schulleitung Nds. Kultusministerium 2002.
} 


\subsection{Forschungsinteresse}

Das Thema Schulleitung hat in den letzten Jahren an Aktualität gewonnen. Im Zuge der in den 80er und 90er Jahren international und national geführten Debatte über die Umgestaltung und Weiterentwicklung der Schulen hat sich die Tätigkeit der Schulleiterinnen und Schulleiter zu einem eigenen Forschungsthema ausgebildet, aus dem heraus sich der Appell nach einer Anerkennung als eigenständigem Beruf sowie nach berufsspezifischer Qualifikation ergeben hat. ${ }^{6}$

Die Forderungen nach Veränderungen und Innovation im Sinne einer kontinuierlichen Schulentwicklung, bezogen auf die Einzelschule, stellen eine besondere Herausforderung für das schulische Leitungspersonal dar. Schulleiterinnen und Schulleiter werden dazu aufgefordert, an der „Qualitätsentwicklung" und der Umgestaltung der eigenen Schulorganisation unter der Prämisse der "Qualitätsverbesserung“ mitzuwirken. Das Aufgabenspektrum der Schulleitung hat sich erweitert und verändert, die Schulleiterinnen und Schulleiter müssen sich mit Erwartungen unterschiedlicher an Schule beteiligter Personengruppen auseinandersetzen.

Für die Schule als einer formalen sozialen Organisation kann angenommen werden, dass sie bürokratisch und hierarchisch organisiert ist. An ihre Ablauforganisation und an ihre Weiterentwicklung werden professionelle Anforderungen gestellt. Da ein konstitutives Element in komplexen Arbeitsfeldern - wie noch zu zeigen sein wird - Handlungsparadoxien darstellen, ist davon auszugehen, dass Schulleiterinnen und Schulleiter mit widersprüchlichen Anforderungen umgehen müssen. Diese Widersprüche können teilweise strukturell bedingt sein, sie können aber auch durch Widersprüche bei der Erfüllung des Berufsauftrages in den Personen selbst begründet sein. Eine kritische Reflexion der realen Berufsauffassung der Schulleiterinnen und Schulleiter selbst und dem idealen Berufsverständnis, wie es programmatisch verkündet wird, kann meiner Einschätzung nach einen Beitrag dazu leisten, eine reflektierte Haltung bei allen Akteuren auszubilden und den bewussten Umgang mit Dilemmata zu fördern.

\subsubsection{Fragestellung}

Ziel der vorliegenden Studie ist es, durch eine quantitative Befragung zu prüfen, ob und in welcher Weise sich die durch die Schulleitungsforschung herausgearbeiteten führungsrelevanten Handlungsdimensionen in den Selbsteinschätzungen der Schulleiterinnen und Schulleiter in Niedersachsen wieder finden lassen.

Gefragt wird daher durch eine qualitative Interviewstudie nach der berufsbiographisch erworbenen Berufsauffassung von Schulleiterinnen und Schulleitern und insbesondere nach ihrem Führungsverständnis. Von Bedeutung ist in diesem Kontext auch die Frage, wie Schulleiterinnen und Schulleiter mit den spezifischen Anforderungen, die sich einerseits aus der empirisch fundierten Kritik - siehe z.B. Ergebnisse der Internationalen Leistungsuntersuchungen - und aus der medial veröffentlichten Kritik und andererseits aus gesellschaftlichen Veränderungen - z.B. veränderte Qualifikationsanforderungen der Berufs- und Arbeitswelt, Jugenderwerbs-

\footnotetext{
${ }^{6}$ Vgl. Wissinger 2002 u. ASD 2005
} 
losigkeit, Bedeutungssteigerung und Bedeutungsverlust von Schulabschlüssen- umgehen und durch welche dieser Anforderungen sie sich in besondere Weise belastet fühlen.

Hinter diesen Fragen steht die Annahme, dass Veränderungen in der Schule die Professionalität von Schulleiterinnen und Schulleitern benötigen, und zwar insbesondere im Hinblick auf den Umgang mit Antinomien. Gerade die neuen Tendenzen der Kultusbehörde die Eigenverantwortung der Schulen zu erhöhen, erweitert die Aufgaben und Befugnisse der Akteure, bringt sie jedoch noch stärker in den Zwiespalt Dienstvorgesetzter und gleichzeitig der kooperativer Partner der Schulentwicklung zu sein.

\subsection{Struktur und Aufbau der Arbeit}

Abb. 1 Aufbau der Arbeit

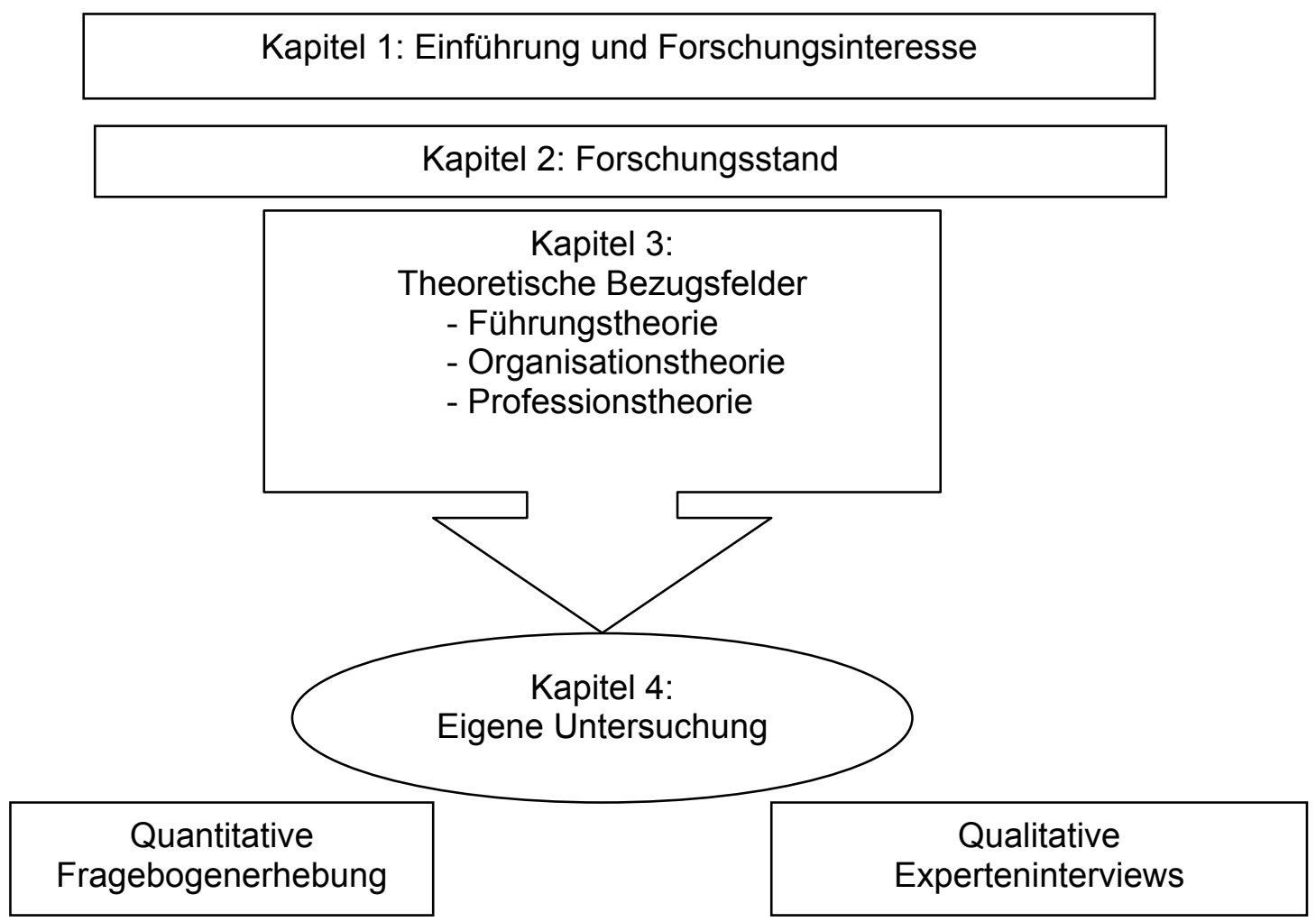

Kapitel 5 :

Berufsauffassungen von Schulleiterinnen und Schulleitern - das empirische Material

Kapitel 6:

Diskussion der Ergebnisse

Kapitel 7:

Resümee

Die vorliegende Arbeit gliedert sich in sieben Kapitel. Kapitel 1 dient der Einführung in das Thema und expliziert das Forschungsinteresse. Kapitel 2 gibt den Forschungsstand wieder. Kapitel 3 hat die Funktion, die für den Forschungsgegenstand relevanten Bezugstheorien darzustellen und sie auf die Schule zu übertragen, die Ansätze dienen als Erklärungsmodell für 
die Auswertung der Untersuchung. In Kapitel 4 wird der eigene Untersuchungsansatz mit seinen beiden Teilbereichen erläutert und das genaue methodische Vorgehen dargelegt. In Kapitel 5 die Durchführung beschrieben und die Ergebnisse ausführlich dargestellt. Das Kapitel 6 diskutiert ausgehend vom Forschungsinteresse die Ergebnisse und gibt Hinweise auf mögliche Konsequenzen. Kapitel 7 dient einem zusammenfassenden Resümee.

\section{Schulleitungsforschung}

Das Interesse an der wissenschaftlichen Erforschung der Funktion und Rolle der Schulleiterin/ des Schulleiters ist in Deutschland in den letzten Jahren deutlich gestiegen. Dies zeigt sich durch eine vermehrte Erforschung der Berufsrolle und Untersuchungen zur Wirksamkeit von Schulleitungshandeln im Hinblick auf die Schulentwicklung. Die Tatsache, dass gerade in den vergangen Jahren einige Untersuchungen zum Schulleiterberuf erschienen sind, steht sicherlich im Zusammenhang mit einer breiteren Diskussion über die notwendige Veränderung von Schule und so ist auch die Rolle der Schulleitung stärker in den Blick gerückt.

Gleichzeitig erstellte die „Arbeitsgemeinschaft der Schulleiterverbände Deutschlands“ (ASD 1994) ein Positionspapier „Schulleitung in Deutschland" und beschreibt darin erstmalig ein Berufsbild von Führungskräften im Schulwesen. ${ }^{7}$ Durch erhebliche Veränderungen und Weiterentwicklungen des Systems Schule haben sich zwangsläufig auch die Anforderungen an das schulische Führungshandeln verändert. In den letzten Jahren wurden Verantwortlichkeiten, die bis daher der Schulbehörde vorbehalten waren, auf die Schulleitung übertragen. Einerseits erleben die im Schulleitungsverband organisierten Schulleiterinnen und Schulleiter die Übertragung von neuen Aufgaben als Stärkung ihrer eigenen Rolle als Dienstvorgesetzte, andererseits fordern sie, dass die Grundlage für die Bestellung von Schulleiterinnen und Schulleitern sowie ihrer Stellvertreter eine fundierte Ausbildung sein müsse. Daher schlägt der ASD in seiner Schrift „Schulleitung in Deutschland 2005" die Einführung der Ausbildung von Schulleiterinnen und Schulleitern an einer Bundesakademie für pädagogische Führungskräfte vor. ${ }^{8}$

In den folgenden Kapiteln möchte ich auf Veränderungen im Anforderungsprofil der Schulleiterinnen und Schulleiter eingehen, den Forschungsstand der Schulleitungsforschung in Deutschland skizzieren und die Situation der Schulleiterinnen und Schulleiter in Niedersachsen darstellen.

\subsection{Funktion und Rolle der Schulleiterinnen und Schulleiter}

So alt wie die Schule selbst ist auch die Diskussion um ihre Reformbedürftigkeit und Veränderungsnotwendigkeit, dies wird damit begründet, dass Schule auf den gesellschaftlichen Wandel reagieren müsse. Die in diesem Zusammenhang neu geführte Diskussion über Autonomie von Schule ist nicht neu, denn bereits Anfang der 70er Jahre wurde vom Deutschen Bildungsrat in seinem Strukturplan eine „vermehrte Autonomie“ von Schulen

\footnotetext{
${ }^{7}$ Vgl. ASD-Bundesvorstand 1994.

${ }^{8} \mathrm{Vgl}$. ASD 2005, $7 \mathrm{f}$.
} 
empfohlen. ${ }^{9}$ Der Begriff der Autonomie ist im Zusammenhang der Diskussion unscharf, denn es geht nicht um das Herauslösen aus der staatlichen Aufsicht, sondern vielmehr um das Gewähren und den Nutzen von Gestaltungsfreiräumen, dem Sachverhalt der Gestaltungsautonomie im Rahmen eines schulrechtlichen und

schulaufsichtlichen Rahmens trägt Dubs (1997) durch den Begriff „Teilautonomie" Rechnung. Es werden Bereiche der Lehrplanautonomie, der Budgetautonomie und Organisationsautonomie unterschieden. ${ }^{10}$ Das Konzept der teilautonomen Schule greift die nordrhein-westfälische Bildungskommission auf und stellt ihren Überlegungen zur Schulreform die Überlegung voran, „dass die Schule nur eine Zukunft hat, wenn sie rechtzeitig versteht, dass sie in der tradierten Form und Struktur keine Zukunft hat." ${ }^{11}$ Die neuere Diskussion um Schulreform, die Mitte der 80er Jahre einsetzt, unterscheidet sich von früheren Forderungen nach einer „äußeren Schulreform" darin, dass die Einzelschule als Ort der Entwicklung angesehen wird. Das Erscheinen der Denkschrift „Zukunft der Bildung Schule der Zukunft" (Bildungskommission NRW) kann als Programmatik für eine neue Gestaltung von Schule eingeordnet werden, denn sie markiert den Einstieg in eine breite Diskussion zur Schulentwicklung, die nun aus den Schulen selbst heraus entstehen sollen. Auf die einzelnen Schulen werden Verantwortungsbereiche übertragen und den Schulleiterinnen und Schulleitern neue Aufgabenbereiche zugesprochen. Die Umgestaltung der Schulleitungsrolle ergibt sich aus den Ansätzen der Schulentwicklung. Dabei sind zwei Richtungen zu nennen, die sich nicht voneinander trennen lassen, aber zu unterschiedlichen Schwerpunktsetzungen führen: Die institutionelle Schulentwicklung, die darauf ausgerichtet ist, die Schule als soziale Organisation im Sinne einer lernenden Organisation zu verändern. Schulentwicklung wird dabei als Organisationsentwicklung verstanden, Schulleiterinnen und Schulleiter erhalten in diesem Sinne mehr Kompetenz, z.B. die Möglichkeit der Budgetierung von Lehrerstunden, ein größeres Mitspracherecht bei Stellenausschreibungen. Im Rahmen der Organisationsentwicklung wird der Managementbegriff im Sinne eines Gestaltens der Organisation verwendet.

Die pädagogische Schulentwicklung konzentriert sich stärker auf die Veränderung des Unterrichts. Die Schulleitung trägt Verantwortung für die Implementierung neuer Unterrichtsformen, Teambildungsprozesse im Kollegium, schulinterne Curricula. ${ }^{12}$ Von der Schulleitung werden Management- und Führungsaufgaben gefordert, wobei Führung den Bereich der pädagogischen Weiterentwicklung und Profilierung sowie Personalentwicklung beinhaltet und Management die oganisationsbezogenen Aufgaben. ${ }^{13}$

Die Orientierung an der Einzelschule macht es notwendig, dass alle Beteiligten innerhalb der eigenen Schule Ziele für ihre Weiterarbeit vereinbaren. „Die auch rechtlich abzusichernden Freiheiten sollen also nicht auf den

\footnotetext{
9 Vgl. Deutscher Bildungsrat 1972, , 262.

${ }^{10}$ Vgl. Dubs 1997, 107.

${ }^{11}$ Vgl. Bildungskommission NRW 1995, 69.

${ }^{12}$ Vgl. Bastian 1997/ Klippert 1997.

${ }^{13}$ Vgl. Dalin, Rolff, Buchen (1995) und Bildungskommission NRW 1995.
} 
Einzelnen bezogen sein, sie sind vielmehr auf eine gemeinsame Gestaltung und Leistung von Schulleitung und Kollegium angewiesen." 14

Das Schulprogramm wird als Festschreibung dieser Zielsetzungen verstanden, es werden darin eigene Entwicklungsschwerpunkte benannt, pädagogische Grundsätze vereinbart und konkrete Arbeitsziele beschlossen. Von der Idee her sollte das Schulprogramm zu einer inneren Auseinandersetzung des Kollegiums und der Schulleitung in der Schule führen und einer gemeinsamen zielgerichteten Weiterentwicklung der Schule dienen. Eine Beteiligung des Kollegiums, z.B. durch die Einrichtung von Steuergruppen, wurde angeregt. Die stärkere Selbstorganisation der einzelnen Schule geht von der Beteiligung aller Schulmitglieder an Entwicklungsprozessen aus, verlangt aber ausdrücklich von Schulleiterinnen und Schulleitern ein "Innovationsmanagement", eine Führungsverantwortung, die sich durch Kompetenzen in den Bereichen Management, Kommunikation und Kooperation ausdrückt. Diese neue Richtung der Führung wird auch als „transformationale Führung“ oder „dialogische Führung“ bezeichnet. ${ }^{15}$

Zunächst wurden in den Bundesländern Pilotprojekte zur Schulprogrammentwicklung durchgeführt, z.B. "Qualitätsverbesserung in Schulen und Schulsystemen“ (QuiSS), und ein Orientierungsrahmen "Schulqualität“ erarbeitet. In Niedersachsen wurde 2003 das Gesetz zur „Verbesserung der Bildungsqualität und zur Sicherung von Schulstandorten" verabschiedet, welches in erster Linie die Abschaffung der Orientierungsstufe forderte. ${ }^{16}$

Hierin wird die Aufgabe und Rolle der Schulleiterinnen und Schulleiter, wie sie sich das Land Niedersachsen vorstellt, benannt. „Schulleiterinnen und Schulleiter haben eine besondere Verantwortung dafür, dass jede Schule, jedes Kollegium sich darum bemüht, den Erfolg ihrer pädagogischen Arbeit regelmäßig zu überprüfen und in wichtigen Bereichen zu verbessern." ${ }^{17}$ An der Entwicklung eines Orientierungsrahmen für Schulqualität arbeitete die vom Niedersächsischen Kultusministerium einberufene Projektgruppe „Arbeitsplatz Schulleitung“, die sich mit den Veränderungen der Anforderungen an die Tätigkeit der Schulleiterinnen und Schulleiter auseinandersetzte. (vgl. Kapitel 2.3).

Neben den traditionellen Tätigkeitsbereichen der Schulleitung, Verwaltungs-, Gestaltungs- und Hierarchieverantwortung (Vorgesetztenfunktion und Weisungsrecht), wie sie bereits Nevermann $(1982)^{18}$ herausgearbeitet hat, kommen nun explizit Aufgaben, die sich auf pädagogische und organisatorische Entwicklungen beziehen, hinzu, z.B. das zielgerichtete gemeinsame Handeln des Lehrerkollegiums zu fördern oder der Schule ein eigenes Profil zu geben. Die Vielschichtigkeit der Aufträge wird durch die allgemeine Beschreibung dieser Aufgaben deutlich, „Sicherung der Schulqualität" kann vieles beinhalten. Den Veränderungen des Aufgabenbereiches, die sich auch aktuell im Programm „Eigenverantwortliche Schule“ zeigen, ist gemein, dass die Rolle der Schulleiterinnen und Schulleiter ge-

\footnotetext{
${ }^{14}$ Bildungskommission NRW 1995, 16.

${ }^{15} \mathrm{Vgl}$. Rolff 1993, 27

${ }^{16}$ Vgl. Orientierungsrahmen Schulqualität in Niedersachsen 2003.

${ }^{17}$ Schulqualität in Niedersachsen 2003, 2.

${ }^{18}$ Vgl. Nevermann 1982, 45.
} 
stärkt wird, sie werden Dienstvorgesetzte und erhalten erweiterte personalrechtliche Befugnisse, z.B. Anordnung von (vergüteter) Mehrarbeit, Beförderung bis A 14, dienstliche Beurteilung der an der Schule tätigen Lehrkräfte. Über die Zuständigkeit und Rolle der Gesamtkonferenz wird momentan diskutiert, im Gespräch ist ein Schulvorstand, der lediglich beratende Funktion übernimmt, dadurch könnten manche Entscheidungen in Zukunft allein von den Schulleiterinnen und Schulleitern getroffen werden. Parallel zu diesen schulinternen Neuerungen soll eine externe Schulinspektion ab 2006 verpflichtend eingeführt werden, die Schulen werden an Qualitätsstandards evaluiert und erhalten eine Gesamtbewertung, die ihnen Anreiz zur Weiterarbeit im Sinne einer Verbesserung dieser Bewertung bietet. ${ }^{19}$

Der Schulleitungsverband greift den Anspruch auf pädagogische und organisatorische Weiterentwicklung auf und spricht vom Schulleiter als Manager und Entwickler. „Der Schulleiter trägt in der Neugestaltung der Schulgesetze der Bundesländer und im öffentlichen Verständnis die gesamte Verantwortung für seine Schule, d.h. er ist für die Erfüllung des staatlichen Erziehungs- und Unterrichtsauftrages verantwortlich. “20

Die Verlagerung des Aufgabenschwerpunktes der Schulleitung könnte das Bild erwecken, als seien die Schulleiterinnen und Schulleiter ausschließlich für Aufgaben der Schul- und Personalentwicklung zuständig. Es darf nicht vergessen werden, dass sie alle administrativen und organisatorischen Aufgaben weiterhin zu erledigen haben. Im Unterschied zu angloamerikanischen und skandinavischen Ländern haben die Schulleiterinnen und Schulleiter in Deutschland ebenso Lehrtätigkeit auszuüben. Die zu erteilenden Unterrichtsstunden werden in Abhängigkeit zur Größe der Schule (Schülerzahlen/ Anzahl der Klassen) berechnet. Die gleichzeitige Tätigkeit als Lehrerin/ als Lehrer und Schulleiterin/ Schulleiter wird unterschiedlich bewertet, so gibt es die Meinung, dass Schulleiterinnen und Schulleiter aufgrund ihrer vielfältigen Leitungstätigkeiten von Unterrichtstätigkeiten befreit werden sollten. Schratz (2002) beschreibt dieses Problem als „primus inter pares Mythos“. Schulleiterinnen und Schulleiter seien nach ihrem Selbstverständnis eher Lehrkraft als Führungskraft und Vorgesetzter. Dies sei ein Anzeichen dafür, dass die „neue“ Rolle einer Führungskraft, eines Managers und Moderators vor Ort noch nicht angenommen wurde. ${ }^{21}$

Andererseits wird der Unterrichtstätigkeit ein hoher Stellenwert eingeräumt, weil sie zu einer Bewahrung der eigenen Identität als Lehrerin/ Lehrer führe. Je nach Schwerpunkt der Schulentwicklungsansätze wird die eigene Unterrichtstätigkeit der Schulleitung beurteilt. Schulentwicklung, die vorrangig auf Organisationsentwicklung ausgerichtet ist, spricht sich für eine Abgrenzung Lehrperson/Schulleiterin/Schulleiter aus und Schulentwicklung, die Unterrichtsreformen voran bringen will, geht davon aus, dass Schulleiterinnen und Schulleiter gerade durch ihre Unterrichtstätigkeit ein Vorbild abgeben müssten.

\footnotetext{
${ }^{19}$ Vgl. Nds. Kultusministerium 2005

${ }^{20}$ ASD 2005, 15.

${ }^{21}$ Vgl. Schratz 2002, 162.
} 
Nicht nur von staatlicher Seite, sondern wie in Kapitel 2.2 gezeigt wird, auch durch die Ergebnisse der Schulleitungsforschung wird ein umfangreiches Tätigkeitsprofil, ein verändertes Rollenverständnis und eine bessere Qualifizierung von Schulleiterinnen und Schulleitern gefordert. Rosenbusch (2000) sieht Schulleiterinnen und Schulleiter als „Instanz, die entscheidenden Einfluss auf Schulreformprozesse haben kann, und zwar im Positiven wie im Negativen. ${ }^{\text {22 }}$

Pfeiffer (2002) fasst die Ergebnisse der Schulleitungsforschung dahingehend zusammen: „Erfolgreichen Schulleitern gelingt es, die verschiedenen schulischen Handlungsebenen bzw. -felder (Unterricht, Kollegiums-, Schulleitungsebene) im Hinblick auf eine gemeinsame „Vision“ zu verknüpfen, ohne dabei die Aufrechterhaltung eines geregelten Schulbetriebes aus den Augen zu verlieren." Hier werden zwei Aspekte angesprochen, nämlich auf der einen Seite die Orientierung an der Weiterentwicklung der Schule und auf der anderen Seite die Organisation eines intakten Schulbetriebes.

Die Anforderungen, die an den Beruf Schulleiterin/Schulleiter gestellt werden, sind höher geworden, denn sie haben eine Vielzahl von Aufgaben zu erfüllen, die in den Bereich des Qualitätsmanagements reichen (vgl. Kapitel 3.1 in dieser Arbeit). Seitens verschiedener Personengruppen der Praxis - Lehrerinnen und Lehrer, Schülerinnen und Schüler, Eltern, Schulbehörde, zukünftige Arbeitgeber - aber auch durch Ergebnisse der Schulleitungsforschung werden unterschiedliche Erwartungshaltungen an die Schulleiterinnen und Schulleiter herangetragen. Die hier beschriebenen Facetten von Schulleitungshandeln machen die Herausforderung deutlich, die darin besteht, die verschiedenen Tätigkeitsbereiche in Einklang zu bringen und in der eigenen Person so zu verwirklichen, dass das Verhalten dem Gelingen von Schule dienen kann.

Baumert (1989) stellt fest, dass das Schulleiterverhalten in hohem Maß an Schulen variiert und es kein einheitliches erfolgreiches Modell von Schulleitungshandeln gibt, „vielmehr wird davon ausgegangen, dass es unterschiedliche Strategien erfolgreicher Schulführung gibt, wobei die Leiter ihrer jeweiligen Persönlichkeitsstruktur entsprechend - für sich einen individuellen Weg finden müssen. Das setzt Selbstkontrolle über ihre jeweiligen Stärken und Schwächen voraus. Und gerade diese selbstreflexive Kompetenz erwies sich als ein Wesenszug erfolgreicher Schulleiter." ${ }^{23}$

In meiner Forschungsarbeit möchte ich diese selbstreflexive Kompetenz der Schulleiterinnen und Schulleiter nutzen und die Person in den Blick nehmen. Dabei interessiert mich, ob Diskrepanzen zwischen einem idealen Berufsverständnis, wie es Ministerien und auch Schulforschungsexperten fordern, und dem eigenen Berufserleben der Schulleiterinnen und Schulleiter bestehen. Es gilt ferner herauszuarbeiten, ob die Berufsauffassung der amtierenden Schulleiterinnen und Schulleiter den aus der Theorie entwickelten modernen Ansprüchen, z.B. hinsichtlich Führung und Professionsgrad, entspricht.

\footnotetext{
${ }^{22}$ Vgl. Rosenbusch 2000, 12.

${ }^{23}$ Baumert 1989, 60.
} 


\subsection{Empirische Befunde}

Der Stand der Schulleitungsforschung wurde lange Zeit als defizitär beschrieben, so verweist Baumert (1989) darauf, dass empirische Forschung zur Schulleitung in Deutschland nur in Ansätzen existiert. Zu diesem Zeitpunkt wird vorwiegend auf Forschungsergebnisse im englischsprachigen Raum hingewiesen. Bis Ende der 80er Jahre finden sich meist deskriptive Untersuchungen, die sich hauptsächlich auf die Tätigkeitsprofile von Schulleitung beziehen. Die neue Akzentuierung der Schulen als Motor der Entwicklung von Schulen führte spätestens seit den 90er Jahren zu einer grundlegenderen Auseinandersetzung mit neuen Leitungsaufgaben der Schulleitung. Die Anerkennung der Schulleitungstätigkeit als eigenständigem Beruf lassen auch in Deutschland eine eigenständige Schulleitungsberufsforschung entstehen.

Die Ergebnisse der Schulleitungsforschung werden insoweit vorgestellt, als sie für den in der vorliegenden Arbeit zu untersuchenden Gegenstandsbereich - Berufsauffassungen und berufliche Orientierung von Schulleiterinnen und Schulleitern - bereits Erkenntnisse beinhalten, bzw. insoweit, als sie Anregungen für Kategorisierungen und Differenzierung der Ergebnisse der vorliegenden Studie liefern können. Entsprechend ihren theoretischen Ansätzen lassen sich die vorliegenden Untersuchungen grob folgendermaßen zuordnen: Zu unterscheiden sind - auch wenn die Unterscheidungen nicht in jedem Fall ganz eindeutig erfolgen können-

1. Ansätze, die die Schulleitungsfunktion und- tätigkeit vorrangig aus der Perspektive von Schule als einer spezifischen Organisation mit besonderen Organisationsmerkmalen betrachten.

2. Ansätze, die von Schulleitung als spezifischer Profession ausgehen und das Kriterium der Professionalität in den Mittelpunkt der empirischen Untersuchung stellen.

3. Ansätze, die den Sozialisationsprozess, d.h. den Wechsel von dem Beruf des Lehrers/der Lehrerin in den des Schulleiters/ der Schulleiterin und die damit verbundenen sozialisatorischen Veränderungen fokussieren.

4. Ansätze, die handlungstheoretisch argumentieren und nach den Handlungsdimensionen und -strategien von Schulleiterinnen und Schulleitern fragen.

5. Geschlechtsspezifische Forschungsansätze, die Frauen in Schulleitungspositionen sichtbar machen und auf stereotype Verhaltenszuschreibungen eingehen.

\subsubsection{Ansätze aus der Organisationsperspektive}

Erste Ansätze der Schulleitungsforschung, die von einem theoretischen Modell der Schule als „loosely coupled organization“ ausgehen, liegen von Baumert/Leschinsky (1986) vor.

Ihr Forschungsinteresse gilt den Einflussmöglichkeiten der Schulleiterinnen und Schulleiter auf die „Kultur" einer Schule. Sie ermitteln fünf faktorielle Dimensionen des Schulleiterbildes: Direktoriale Leitung; Gleichgewicht zwischen Direktorial- und Kollegenrechten; Aufteilung von Zuständigkeiten; Vorbild und Kooperation und Kontrolle. ${ }^{24}$ Baumert/Leschinsky

\footnotetext{
${ }^{24}$ Vgl. Baumert/ Leschinsky 1986/2, 256.
} 
kommen zu dem Ergebnis, „dass nach dem Selbstverständnis von Schulleitern Vorbild und Kooperation zentrale Elemente ihrer Rolle darstellen." Die Ergebnisse der Befragung verweisen auf eine Tendenz zu direktorialen Leitungsformen der Schulleiterinnen und Schulleiter. Diese Leitungsform wird durch eine effektive Geschäftsführung beschrieben, d.h. dass die administrativen Aufgaben möglichst beim Schulleiter/der Schulleiterin bleiben, rechtliche Entscheidungen werden schnell und ohne längere Diskussion allein getroffen, Delegation wird nur wenig geschätzt. Die Prüfung von spezifischen Zusammenhängen von einzelnen Aspekten der Rollendefinition und der Qualität des Schullebens zeigen, dass die Neigung zu einer direktorialen Leitung nicht förderlich für die Gestaltung eines vielgestaltigen Schullebens ist. ${ }^{25}$

Der Einfluss der Schulleiterinnen und Schulleiter auf das Schulleben ist nicht Teil meiner Fragestellung, aber die Aussagen zu den Dimensionen und grundsätzlichen Positionen der Schulleiterinnen und Schulleiter sind bemerkenswert, da die Ansätze der Schulentwicklung und Ergebnisse aus der Führungsforschung immer wieder auf die bedeutende Rolle eines kooperativen Führungsstils hinweisen.

In ihrem Resümee verweisen Baumert/Leschinsky (1986) auf das Problem, dass es die Rolle des Schulleiters/ der Schulleiterin aufgrund der schulischen Struktur erfordert, zwischen zwei teilweise gegensätzlichen Verhaltensweisen zu balancieren, nämlich einerseits im Besitz formeller Befugnisse zu sein und gleichzeitig im Kontext intensiver kollegialer Kontakte zu stehen. ${ }^{26}$

Insbesondere der Aspekt der widersprüchlichen Anforderungen ist interessant, da sich in den letzen Jahren durch die Schulgesetznovellierungen neue Aufgaben für Schulleiterinnen und Schulleiter ergeben haben.

Hasenbank (2000) beschäftigt sich mit der Führung und Leitung an Berufsschulen unter einem organisationstheoretischen Blickwinkel. Dabei betrachtet er Schulführung als Dimension und Rahmenbedingung berufsschulischer Entwicklung. Hasenbank geht der Frage nach, welche Rolle und Funktion Berufsschulleiter im Kontext der Schulentwicklung einnehmen. Er analysiert die Tätigkeit von Schulleiterinnen und Schulleiter vor dem Hintergrund unterschiedlicher Schulentwicklungsmodelle. In seiner in Bayern durchgeführten quantitativen Befragung ermittelt er Daten von 126 Berufsschulleitern/ -leiterinnen, des Weiteren führt er vier problemzentrierte Interviews. Die Interviewauszüge nehmen innerhalb seiner Arbeit illustrierende Funktion ein und untermauern Ergebnisse des Fragebogens.

Der von ihm entwickelte Fragebogen geht auf die Ausbildung bayerischer Schulleiterinnen und Schulleiter ein, überprüft den bereits oben thematisierten Praxisschock und fragt nach dem Führungsstil der Schulleiterinnen und Schulleiter. Seine Ergebnisse verweisen auf die Schlüsselrolle der Schulleiterinnen und Schulleiter für die Schulentwicklung. Seine Annahme, dass Schulleiterinnen und Schulleiter nicht angemessen auf ihre Tätigkeit vorbereitet sind, findet er bestätigt, insbesondere im Bereich Personalent-

\footnotetext{
${ }^{25}$ Baumert/ Leschinsky 1986/2, 256.

${ }^{26}$ Ebd., 263.
} 
wicklung (Teamentwicklung und Konfliktfähigkeit) äußern die Befragten Weiterbildungsbedürfnis.

Die Ergebnisse zum Führungsstil geben Hinweise darauf, dass die Schulleitungstätigkeit stark administrativ geprägt ist und sich Schulleiterinnen und Schulleiter zeitlich von Verwaltungsaufgaben belastet fühlen. Er kommt des Weiteren zu dem Ergebnis, dass Schulleiterinnen und Schulleiter eine persönlich geprägte kommunikative Führung praktizieren und sich dabei an eigenen Erfahrungen mit Schulführungshandeln orientieren.

„Angesichts der als hoch empfundenen zeitlichen Belastung der Schulleiter mit administrativen Aufgaben, einer Vielzahl von Funktionsstellen und den oben skizzierten Vorteilen einer kooperativen Führung ist es erstaunlich, dass „management by delegation“ als Führungsstil „nur“ 41, 2 \% Zustimmung findet. 17, $5 \%$ der Schulleiter lehnen delegative Führung für sich sogar ab." 27 Dieses Ergebnis führt er auf die mangelnde Bereitschaft der Schulleiterinnen und Schulleiter, Einfluss abzugeben, zurück.

Hasenbank fordert aufgrund seiner Ergebnisse ein Schulführungskräftekonzept, welches die Schlüsselrolle der Schulleitung unter dem Gesichtspunkt Schulentwicklungsmaßnahmen berücksichtigt und die Ausbildung der Schulleiterinnen und Schulleiter in Personal-, Unterrichts- und Organisationsentwicklung einbettet.

Obwohl Berufsschulen nicht das Feld meiner Untersuchung bilden, sind die Ergebnisse dieser Untersuchung insofern von Interesse, als dass $\mathrm{Ha}$ senbank für Schulleiterinnen und Schulleiter der Berufsschulen nur eine mittlere Zustimmung zu einer kooperativen-delegativen Führung nachweisen kann, was gerade unter dem Gesichtspunkt der Größe der Organisation Berufsschule verwundert. Da für mich die Führungskonzepte der Schulleiterinnen und Schulleiter wichtig sind, wird zu zeigen sein, welche Bedeutung Delegation und Kooperation in der eigenen Berufsauffassung haben.

Bonsen/Gathen/Iglhaut/Pfeiffer (2002) untersuchen im Rahmen eines Forschungsprojektes des Dortmunder Instituts für Schulentwicklungsforschung in Nordrhein-Westfalen die Wirksamkeit der Schulleitung im Hinblick auf die Unterrichtsentwicklung. Diese Untersuchung kann einerseits dem Ansatz der Wirkungsforschung zugeordnet werden, da sie sich aber im zweiten für mich relevanten Teil auf organisationstheoretische Ansätze bezieht, ordne ich sie diesen zu.

In dem ersten Teil der Untersuchung wird eine standardisierte Lehrerbefragung an 25 Schulen (10 Grundschulen, 10 Gymnasien und 5 Integrierten Gesamtschulen) aus Nordrhein-Westfalen und 5 Gymnasien im Schweizerischen Kanton Basel-Land durchgeführt. Ziel ist es, durch die Einschätzung der Lehrkräfte führungsrelevante Handlungsdimensionen der Schulleitung zu identifizieren, dabei werden aber deutsche und schweizerische Ergebnisse nicht verglichen und auf Unterschiede oder Ähnlichkeiten wird nicht eingegangen. Hier liegt ein völlig anderer Zugang vor als bei der Befragung von Schulleiterinnen und Schulleitern, da die

\footnotetext{
${ }^{27}$ Hasenbank 2000, 256.
} 
Ergebnisse der Standardbefragung der Lehrkräfte von Bonsen et al aber als Basis für die Kriterien erfolgreichen Schulleiterhandelns angesehen werden, nenne ich sie hier zunächst, bevor ich auf die qualitative Untersuchung mit Schulleiterinnen und Schulleitern eingehe.

Unter einem explorativen Anspruch werden die ermittelten Einschätzungen auf der Basis eines Vergleichs "guter“ beziehungsweise „verbesserungsbedürftiger" Schulen abgeglichen. Dieses Vorgehen knüpft an die Forschungsergebnisse über die „gute“ Schule an. ${ }^{28}$ Auf der Grundlage von Skalenwerten wird den befragten Schulen ein Rangplatz zugeordnet und werden die Extremgruppen gebildet. Der Fragebogen enthält eine Reihe von Items, die Aspekte von Führungshandeln aufgreifen, die Items beziehen sich zum Beispiel auf Innovationsförderung, Beziehungsorientierung etc.

Anhand der gewonnenen Daten der Lehrereinschätzungen zu Handlungsdimensionen von Schulleiterinnen und Schulleitern kommen Bonsen/Gathen/Iglhaut/Pfeiffer zu folgendem Ergebnis: „Vor allem die Dimensionen der zielbezogenen Führung, der Innovationsbereitschaft des Schulleiters lassen sich als bedeutsame Merkmale erfolgreicher Schulleiter identifizieren."29 Mit zielorientierter Führung sind im Rahmen der Ausführungen von Bonsen et al gemeinsam formulierte pädagogische Ziele gemeint, die sich an einem Leitbild der Schule orientieren. Dabei sind sowohl schulbezogene Zielsetzungen, z.B. Profilentwicklung, als auch individuelle Arbeitsziele, z.B. Verfassen eines Förderkonzeptes, gemeint. Dieser Zielbezogenheit der Schulleitung wird in den untersuchten Schulen ein großer Stellenwert beigemessen. Dabei fällt auf, dass die befragten Lehrkräfte die Außenrepräsentation des gemeinsamen Leitbildes und die Entwicklung langfristiger Ziele für bedeutsamer halten als schulinterne Führung im Kollegium, z.B. die Sorge um die Umsetzung curricularer Vorgaben. ${ }^{30}$

Es besteht offensichtlich aus Sicht des Kollegiums ein Zusammenhang zwischen der Qualität der Schule und der Qualität der Schulleiterinnen und Schulleiter. Es fällt auf, dass insbesondere die auf Entwicklung zielenden Dimensionen (zielgerichtete Führung, Innovationsbereitschaft) als besonders bedeutsam von den Lehrerinnen und Lehrern eingeschätzt werden.

Die quantitative Untersuchung wird durch die Erhebung qualitativer Daten ergänzt, es werden 25 qualitative Interviews mit Schulleiterinnen und Schulleitern geführt. Inhaltliche Schwerpunkte der Interviews sind Selbsteinschätzung der eigenen Tätigkeit und Wirksamkeit des eigenen Handelns bezogen auf zwei Bereiche: erstens Schulleitung und Unterricht und zweitens Schulleitung und Schulentwicklung, beides unter dem Fokus Qualitätsverbesserung.

Im ersten Bereich wird der Frage nachgegangen, ob Schulleiterinnen und Schulleiter durch ihre Führungspraxis Einfluss auf Unterrichtsentwicklung haben, z.B. durch unterrichtsbezogenes Feedback. Es werden unterrichtsbezogene Tätigkeiten ermittelt, deutlich wird, dass Schulleiterinnen und Schulleiter administrativ bzw. koordinierend auf Unterricht einwirken, jedoch nicht auf die direkte Entwicklung bzw. Veränderung Einfluss neh-

\footnotetext{
${ }^{28}$ Vgl. Steffens/Bargel 1993.

${ }^{29} \mathrm{Vgl}$. Bonsen 2002, 170.

${ }^{30} \mathrm{Vgl}$. Bonsen 2002, 121.
} 
men, so werden Unterrichtsbesuche im Durchschnitt nur ein Mal pro Woche durchgeführt. ${ }^{31}$

Die Frage der eigenen Unterrichtsverpflichtung beantworten die Schulleiterinnen und Schulleiter mehrheitlich in dem Sinne, dass sie unterrichten wollen und ihre Rolle als unterrichtende Pädagogen sehen, andererseits ihr Dilemma darin sehen, dass sie dann weniger Zeit für andere Aufgaben haben. Hier ist besonders auf die Unterschiede der Schulformen hinzuweisen, Grundschulleiterinnen und -leiter unterrichten im Durchschnitt 13,6 Std. und Schulleiterinnen und Schulleiter an weiterführenden Schulen unterrichten durchschnittlich 7,3 Std. Insgesamt weisen die Ergebnisse nach, dass administrative Tätigkeitsbereiche vor entwicklungsbezogenen Aufgaben stehen.

Bonsen et al fordern, dass pädagogische Führung insgesamt stärker auf die Unterrichtsgestaltung ausgerichtet sein muss, so z.B. durch die Förderung der Lehrerkooperation, eine „unterrichtsbezogene Feedbackkultur“. ${ }^{32}$

Die Ergebnisse machen deutlich, dass ein Widerspruch zwischen der Einschätzung der Lehrerinnen und Lehrer, die eine zielgerichtete und innovative Leitung besonders schätzen, und dem tatsächlich ermittelten Aufgabenbereich der Schulleiterinnen und Schulleiter besteht, denn die Schulleiterinnen und Schulleiter richten ihr Führungshandeln vornehmlich auf die direkte und individuell ausgerichtete Einflussnahme auf das Handeln einzelner Lehrkräfte. Bonsen et al fordern unter der Prämisse Schulentwicklung eine stärkere Orientierung an gemeinsamen Entwicklungsbemühungen, z.B. kooperative Arbeitsweisen, Maßnahmen zur Unterrichtsverbesserung. Für meine Untersuchung wird hierdurch ein Hinweis auf den Aspekt Fremd- und Selbsteinschätzung gegeben.

Den zweiten Bereich der Untersuchung „Schulentwicklung“ betrachten Bonsen et al aus dem Fokus der Organisationsentwicklung und gehen davon aus, dass Management und Führung komplementäre Bereiche des Schulleitungshandelns darstellen. Dabei ist mit Management ein aktives Problemlösen, ein Kommunizieren gemeinsamer Handlungsstrategien, gemeinsame Visionen sowie die Inspiration der Organisationsmitglieder gemeint. Diese Leitungskonzeption wird von den Autoren in Anlehnung an angloamerikanische Ergebnisse der Schulforschung übernommen. ${ }^{33}$

Die Interviews werden nun theoriegeleitet ausgewertet, da das Interesse dem organisationstheoretischen Bezugsrahmen der führungsbezogenen Aussagen gilt, werden leitungsrelevanten Textstellen auf der Grundlage des theoretischen Modells „Refraiming Organizations“ nach Bolman/Deal Rahmenkonstrukten zugeordnet. ${ }^{34} \mathrm{Da}$ vor allen Dingen die Übertragung des theoretischen Modells von Bolmann/Deal aus meiner Einschätzung heraus nachvollziehbare und wichtige Impulse für die organisationstheoretische Einordnung von schulischem Führungshandeln bietet, möchte ich an dieser Stelle kurz darauf eingehen.

\footnotetext{
${ }^{31} \mathrm{Vgl}$. Bonsen et al 2002a, 67f.

32 Ebd., 92

${ }^{33}$ Vgl. Bonsen et al 2002a, 99 und Fiedler 1992, 26.

34 Das theoretische Modell wird ausführlich in Kapitel 4. erläutert.
} 
Das Rahmenkonzept von Bolman/Deal 1997 stellt ein integratives organisationstheoretisches Modell dar, denn es verbindet Ansätze aus der Führungstheorie und der Organisationstheorie. Die unterschiedlichen Rahmen dienen dem Individuum zur Interpretation von Gegebenheiten und bieten die Grundlage zur Auswahl von Handlungsvollzügen. Auf der Grundlage unterschiedlicher organisationstheoretischer Zugänge und deren Begrifflichkeiten entwickeln die Autoren vier Rahmen:

Abb. 2: $\quad$ Vier-Rahmenmodell Bolmann/Deal ${ }^{35}$

\begin{tabular}{|l|l|}
\hline Struktureller Rahmen & $\begin{array}{l}\text { Die Struktur einer Organisation wird durch Techno- } \\
\text { logie und Umwelt beeinflusst, die formalen Struktu- } \\
\text { ren von Organisationen stehen im Mittelpunkt. Diese } \\
\text { können genutzt werden, um Effektivität und Produk- } \\
\text { tivität zu fördern. } \\
\text { Mit der Verteilung von Aufgaben werden Rollen bzw. } \\
\text { Positionen und Verfahrensweisen festgelegt. } \\
\text { Die aktive Gestaltung günstiger Strukturen ist ein } \\
\text { wichtiges Ziel für Führungspersonen. }\end{array}$ \\
\hline Personaler Rahmen & $\begin{array}{l}\text { Die Beziehungen und damit einhergehende unter- } \\
\text { schiedliche Bedürnisse der Mitarbeiter einer Organi- } \\
\text { sation sind von Bedeutung für eine Zielerreichung. } \\
\text { Mitarbeitermotivation wird ein hoher Stellenwert } \\
\text { eingeräumt. } \\
\text { Die organisationellen und individuellen Bedürfnisse } \\
\text { in Einklang zu bringen, ist ein Ziel der } \\
\text { Führungspersonen. }\end{array}$ \\
\hline Politischer Rahmen & $\begin{array}{l}\text { Unterschiedliche Interessengruppen verfolgen unter- } \\
\text { schiedliche Ziele, die auf Werthaltungen und Einstel- } \\
\text { lungen beruhen. Organisationsziele und Entschei- } \\
\text { dungen beruhen auf dem Verhandeln solcher Unter- } \\
\text { schiedlichkeiten. Dabei spielen knappere Ressour- } \\
\text { cen eine große Rolle. Die Verteilung von materiellen } \\
\text { und personellen Ressourcen sind strategische wich- } \\
\text { tig. }\end{array}$ \\
\hline Symbolischer Rahmen \\
$\begin{array}{l}\text { Unsicherheiten und Unklarheiten werden unter die- } \\
\text { sem Aspekt berücksichtigt, d.h. nicht nur rationale } \\
\text { Gesichtspunkte spielen eine Rolle in Organisationen, } \\
\text { sondern gerade der Umgang mit Mehrdeutigkeiten } \\
\text { muss beachtet werden, um Richtungen vorzugeben } \\
\text { und Ziele zu setzen. Menschen handeln aufgrund } \\
\text { von Bedeutungszuschreibungen und Sinngebungen. } \\
\text { Ein wichtiges Ziel von Führung ist es demnach, Mit- } \\
\text { arbeitern ein Gefühl von Überzeugungen zu vermit- } \\
\text { teln, die Identifikation mit der Organisation zu fördern } \\
\text { und Werthaltungen zu vermitteln. }\end{array}$ \\
\hline
\end{tabular}

In der Untersuchung von Bonsen et al (2002) und auch von Bonsen (2003) wird dieser Organisationsansatz aufgegriffen und auf die Schule übertragen:

\footnotetext{
${ }^{35}$ Nach Bolmann/Deal 1997.
} 
Struktureller Rahmen: Notwendigkeit formaler Rollen und Beziehungen in Schule, Verteilung von Aufgaben und Verantwortungen sind wichtige Aspekte einer „Organisationspolitik“.

Personaler Rahmen: Fähigkeiten, Bedürfnisse und Gefühle von Lehrerinnen und Lehrern müssen bei der Organisationsgestaltung berücksichtigt werden.

Politischer Rahmen: Schule wird als Organisation mit begrenzten Ressourcen betrachtet. Die Verteilung wird auf der Basis von Macht und Einfluss der Individuen sowie von Gruppen geregelt.

Symbolischer Rahmen: Die Organisation Schule wird durch gemeinsame Werthaltungen und Schulkultur zusammengehalten. Lehrerinnen und Lehrer würden stärker durch Rituale, Zugehörigkeitsgefühl und Tradition zusammengehalten als durch formale Regeln. ${ }^{36}$

Die Ergebnisse der Untersuchung von Bonsen et al 2002 zeigen, dass der strukturelle und personale Rahmen signifikant häufiger von Schulleiterinnen und Schulleitern eingenommen wird. Unter dem Blickwinkel eines personalen Rahmens sind aus Sicht der befragten Lehrkräfte die Handlungsdimensionen „Management der sozialen Beziehungen“ und „Förderung der Partizipation in der Entscheidungsfindung" hervorzuheben. ${ }^{37}$ Dies könnte ein Hinweis darauf sein, dass die eigene Position und damit die Sicherung der Verteilung von Aufgaben und Verantwortlichkeiten von großer Bedeutung für die Schulleiterinnen und Schulleiter sind. Die Betonung des personalen Rahmens stimmt mit der von den Lehrkräften als wichtig eingestufter Dimension überein, was sich aus der Wechselseitigkeit der Kommunikationssituationen ergibt.

Anhand des Vergleichs der Einschätzungen von Lehrerinnen und Lehrern zu Handlungsdimensionen von Schulleiterinnen und Schulleiter zeigt sich, dass dort die Dimensionen zielbezogene Führung, Innovationsbereitschaft des Schuleiters sowie Organisationskompetenz als Merkmale erfolgreicher Schulleiterinnen und Schulleiter angesehen werden. Im Zusammenhang mit zielbezogener Führung wird von Leitungspersonen gefordert, für die Akzeptanz der Ziele zu sorgen und für die Umsetzung der Ziele zu motivieren. Unter diesem Aspekt erhält der symbolische Rahmen (gemeinsame Werthaltung, Leitbild, Schulkultur) eine besondere Bedeutung.

Bonsen et al (2002) kommen in ihrem Resümee zu dem Ergebnis: „(...) erfolgreiches pädagogisches Führungshandeln mit der Zielrichtung besonderer und außergewöhnlicher Schulqualität („leadership towards excellence") vor allem durch die Handlungsdimensionen der zielbezogenen Führung, der Innovationsbereitschaft des Schulleiters sowie die wahrgenommene Organisationskompetenz des Schulleiters beschreiben. “38

Bonsen et al (2002) beziehen in ihrer Untersuchung zur Wirksamkeit von Schulentwicklung sowohl Lehrkräfte als auch Schulleiterinnen und Schulleiter mit ein. Die Autoren weisen die Bedeutung der zielbezogenen Füh-

\footnotetext{
${ }^{36} \mathrm{Vgl}$. Bonsen et al 2002a, 100-102.

37 Bonsen 2002a, 123.

${ }^{38}$ Bonsen et al 2002, 173.
} 
rung empirisch nach, dabei ordnen sie die Dimension der zielbezogenen Führung in der Analyse dem symbolischen Rahmen zu. ${ }^{39}$

Martin Bonsen (2003) ${ }^{40}$ wertet die in der oben dargestellten Untersuchung gewonnenen Daten erneut im Hinblick auf das Führungsverständnis von Schulleiterinnen und Schulleitern aus. Er ermittelt mit der Methode der Clusteranalyse ${ }^{41}$ bezüglich ihrer Aufmerksamkeitsprofile Typen von Schulleitungspersonen, dabei wird das theoretische Modell der Vier-Rahmen von Bolman/Deal (1997) als Folie der Auswertung benutzt (vgl. S. 20 in dieser Arbeit). Ziel der clusteranalytischen Typenbildung ist die Beschreibung empirisch vorfindbarer Führungsaufmerksamkeiten der untersuchten Schulleitungspersonen (25 Schulleiterinnen und Schulleiter). Auf der Grundlage des Rahmenkonzeptes werden die generierten Rahmungstypen durch zentrale Zitate aus den Interviews qualitativ als besonders „typischer Fall" dargestellt und damit eine typenbezogene Verallgemeinerung vorgenommen. Bonsen verweist darauf, dass diese Typen nicht in einem engeren wissenschaftlichen Verständnis benannt werden können, sondern dass die Fallbeschreibung unter dem Fokus des organisationstheoretischen Aufmerksamkeitsrahmens vorgenommen wird. ${ }^{42}$

Bonsen ermittelt vier Gruppen:

Die konventionell-klassische Führungsaufmerksamkeit, die Schulleiterinnen und Schulleiter beziehen sich in ihrem Führungshandeln vornehmlich auf den strukturellen und personalen Rahmen. Die Führungsbereiche beziehen sich auf Mitarbeiterorientierung und den funktionalen Ablauf des $\begin{array}{llll}\text { Organisationsbetriebes. Diesem } & \text { Typus entsprechen }\end{array}$ Schulleitungspersonen von 25.

Konventionell-klassisches Führungsverständnis mit erhöhter politischer Aufmerksamkeit. Dieser Typus stellt sozusagen eine Erweiterung des erstgenannten dar, der personale und strukturelle Rahmen werden ergänzt durch eine erhöhte Aufmerksamkeit im politischen Rahmen. Insbesondere mikropolitische Zusammenhänge werden von den in diesem Cluster angesiedelten Schulleiterinnen und Schulleiter wahrgenommen und angesprochen, es geht um das Aushandeln der Konflikte auch in der Zusammenarbeit mit externen Bereichen der Schule. Bonsen betont, dass in diesem Cluster 7 Schulleiterinnen und Schulleiter an größeren Organisationseinheiten (Gymnasium, Gesamtschule) arbeiten und stellt den Zusammenhang Organisationsgröße und politischer Führungsrahmen heraus, demnach ist der politische Führungsrahmen an größeren Schulen stärker ausgeprägt.

„Multi-Framing“- die Integration aller vier Führungsrahmen. Dieser Typus wird durch 5 Schulleiterinnen und Schulleiter größerer Organisationen rep-

\footnotetext{
${ }^{39}$ Ebd., 121.

${ }^{40} \mathrm{Vgl}$. Bonsen 2003.

${ }^{41}$ Die Clusteranalyse ist ein statistisches Verfahren, welches das Auffinden einer Klassifikation ermöglicht. Auf Grundlage einer Befragung bestimmter Personen (Klassifikationsobjekte) werden diese nach bestimmten Merkmalsausprägungen (in dieser Untersuchung Führungsrahmen) gruppiert. Die Verschmelzung einzelner Cluster wurde nach der "Complete-Linkage - oder Maximummethode" vorgenommen.

${ }^{42}$ Vgl. Bonsen 2003, 251.
} 
räsentiert. Dem symbolischen Rahmen kommt eine besondere Bedeutung zu. Dieser Typus zeichnet sich dadurch aus, dass die Schulleiterinnen und Schulleiter neben der optimalen Gestaltung der organisatorischen Aufgaben den strukturellen Rahmen beachten, ihn aber individuell akzentuieren. Im Bereich des personalen Rahmens wird besonderes Gewicht auf Begleitung und Unterstützung der Lehrkräfte gelegt. Die deutliche Formulierung von "Visionen" und die konkretere Ausformulierung von Zielrichtungen, z.B. „mehr Schülerorientierung“, ordnet Bonsen dem symbolischen Rahmen zu. Schulleiterinnen und Schulleiter dieses Typus sind sich ihrer Modellhaftigkeit bewusst und nehmen zielgerichtet Einfluss auf die Schulkultur.

Klassisch-symbolische Führung in der Schule: Die 4 Schulleiterinnen/ Schulleiter zeigen keine ausgeprägte Aufmerksamkeit im politischen Rahmen, aber zeichnen sich durch hohe Werte in den anderen Bereichen, vor allen Dingen bezüglich des symbolischen Rahmens, aus. Die Schulleiterinnen und Schulleiter setzen sich für mehr Kooperation im Kollegium ein und haben großes Interesse an der Weiterentwicklung und Veränderung ihrer Schulen.

In seiner Zusammenfassung fordert Bonsen (2003), dass Schulleiterinnen und Schulleiter sich stärker mit theoretischen Ansätzen auseinander setzen müssen, um Schule aus unterschiedlichen Blickwinkeln betrachten zu können. Er verweist darauf, dass Schulleiterinnen und Schulleiter im kulturellen und institutionellen Kontext der Schule von einer symbolisch akzentuierten Führung profitieren können.

Nicht ganz nachvollziehbar erscheint die Fokussierung auf einen Rahmen des Modells, unter dem Fokus Schulentwicklung ist die Betonung des symbolischen Rahmens plausibel. Das Rahmenmodell von Bolman/ Deal (1997) zeichnet sich aber gerade durch eine ganzheitliche Betrachtungsweise aus, die sich aus vier Betrachtungsperspektiven zusammensetzt, durch diese Multiperspektivität sollte das Handeln der Schulleiterinnen und Schulleiter bestimmt sein, um den komplexen Anforderungen der Organisation Schule gerecht werden zu können.

Bonsen illustriert am Ende seiner Arbeit ein Bild der guten Schulleiterin/ des guten Schulleiters, welches einer anspruchsvollen Idealvorstellung entspricht. „In einer Person des Schulleiters oder einer Schulleiterin vereinigt sich idealtypisch somit der Pädagoge mit einem "inneren Team" aus Manager und Sozialarchitekt, Berater und Betreuer, Prediger und Prophet und gegebenenfalls auch Anwalt und Politiker. ${ }^{\text {“43 }}$

\subsubsection{Profession als Fokus der Forschung}

Neulinger (1991) geht in seiner quantitativen und qualitativen Untersuchung in Baden-Württemberg der Fragestellung nach, „ob es sich bei den Schulleitern um eine homogen denkende, eingestellte, motivierte und sozial herkunftsgeprägte Lehrer-Elite handelt, die sich aus weitgehend voneinander unterscheidenden schulartspezifisch erkennbaren Schulleitergruppen zusammensetzt. “44 Konkret versucht Neulinger herauszufinden, ob die berufliche Selbsteinschätzung der Schulleiterinnen und Schulleiter sich hinsichtlich der Differenzkategorie Schulform nachweisen lässt. Eine

\footnotetext{
${ }^{43}$ Bonsen 2003, 298.

${ }^{44}$ Neulinger 1991, 22.
} 
seiner Hypothesen besteht darin, dass sich die Einstellungen der Schulleiterinnen und Schulleiter stark nach Schulformen unterscheiden werden. ${ }^{45}$

Der quantitative Teil umfasst 345 Schulleiter und 23 Schulleiterinnen der Sonderschule, Realschule, des Gymnasiums und der Grund- und Hauptschule. Im qualitativen Teil werden narrative Interviews mit 27 Schulleitern und einer Schulleiterin geführt.

Neulinger bezieht sich in seiner Einführung auf professionstheoretische Überlegungen. Er vergleicht die Professionalitätskriterien für den Lehrerberuf mit denen des Schulleiterberufs. Grundlage der Analyse sind 6 Kriterien der Professionalisierung (1. Berufsausübung auf wissenschaftlicher Grundlage; 2. lange Spezialausbildung, 3. altruistisch motivierte klientenbezogene Berufsausübung, 4. sorgfältige Kontrolle der Ausbildung und der Ausübung des Berufes, 5. gut organisierte Berufsverbände, die über die Einhaltung berufsethischer Grundsätze wachen und 6. Autonomie der Berufsausübung). Auf der Basis dieses Analyseinstrumentariums arbeitet Neulinger Unterschiede heraus, die als Beleg für die fehlende Professionalität der Schulleiterinnen und Schulleiter herangezogen werden.

- „Der Schulleiter kennt keine Berufsausübung auf wissenschaftlicher Grundlage.

- Der Schulleiterberuf kennt keine lange wissenschaftliche Ausbildung.

- Der Schwerpunkt der Arbeit eines Schulleiters liegt in den Bereichen, die ein überwiegend altruistisch motiviertes und klientenbezogenes Berufshandeln nicht erforderlich machen oder nicht ermöglichen.

- Da es eine Ausbildung zum Schulleiter nicht gibt, kann dieselbe auch nicht kontrolliert werden.

- Es gibt zwar bundesweit Berufsfachverbände der Schulleiter, die in der Arbeitsgemeinschaft der Schulleiterverbände Deutschlands zusammengeschlossen sind, aber für sie gilt dasselbe wie für die Lehrerverbände: Sie haben das Berufsethos als Thema noch nicht erkannt; ein entsprechender Kodex wird von ihnen auch nicht überwacht.

- Die für Professionen geforderte Autonomie gibt es für den Schulleiter im Rahmen seines Berufshandelns nicht. "46

Dieser professionstheoretische Ansatz erscheint lohnend, vor allen Dingen im Hinblick auf die eigene Berufsauffassung der Schulleiterinnen und Schulleiter. Von Interesse wird im Rahmen meiner Untersuchung sein, ob die Schulleiterinnen und Schulleiter professionstheoretische Überlegungen äußern und ob sie sich selbst als Professionelle verstehen. Leider greift Neulinger seine zu Beginn der Untersuchung ausgeführten theoretischen Überlegungen im weiteren Verlauf nicht wieder auf.

Die von ihm entwickelten Fragenkomplexe beziehen sich nur teilweise auf den erarbeiteten theoretischen Hintergrund der Profession, z.B. die Fragen zum Umgang mit institutionellen Normierungen, die Frage nach einem Praxisschock angesichts fehlender Ausbildung und Fragen zur Motivation

\footnotetext{
45 Neulinger 1991, 23.

${ }^{46}$ Neulinger 1991, 49-50.
} 
der Berufswahl. Die schulformspezifischen Analysen der Daten bestätigen die Annahme Neulingers, wonach sich Schulleiter verschiedener Schulformen in ihren Einstellungen signifikant voneinander unterscheiden.

Neulinger kommt zu dem Ergebnis, dass vor allen Dingen Schulleiterinnen und Schulleiter des Gymnasiums eine ausgeprägte Tendenz zur Beachtung schulgesetzlicher Vorgaben haben und ein starker Wunsch nach Stärkung der eigenen Position in der Schule besteht. Materielle Motive und der Wunsch nach persönlichem Ansehen sind insbesondere bei Grund-/ Hauptschulrektoren nachzuweisen, hingegen ist bei Sonderschulrektoren die größte Bereitschaft zu kollegialer Gestaltung der Schule zu erkennen. Die befragten Schulleiterinnen und Schulleiter schätzen ihre eigene berufliche Qualifikation als sehr hoch ein und zeigen eine allgemeine Berufszufriedenheit.

Neulinger kommt nach seiner schulformspezifischen Analyse zu der Einschätzung: „Die Schulleiter insgesamt wirken in ihren Einstellungen zum bestehenden Schulsystem deutlich systemerhaltend. Sie zeigen in ihren Einstellungen zu schulbezogenen gesellschaftlichen Fragen eine keineswegs zur Veränderung oder Weiterentwicklung drängende Haltung. ${ }^{.47}$ Die Ergebnisse bezüglich der Schulleiterinnen und Schulleiter des Gymnasiums sind für mich von Interesse und bieten die Möglichkeit, die eigene Positionierung der von mir befragten Schulleiterinnen und Schulleiter in den Blick zu nehmen.

Im qualitativen Teil seiner Arbeit wird das Verhältnis Schulleiterin/Schulleiter zu den anderen Mitgliedern der Schule in den Blick genommen. Des Weiteren wird ermittelt, wie der berufliche Wechsel von der Lehrkraft zum Schulleiter/zur Schulleiterin erlebt wird, indem konkret nach einem „Praxisschock“ gefragt wird. Die in den Interviews geäußerten Negativerlebnisse, z.B. Enttäuschungen oder Frustrationen, wertet Neulinger als „Praxisschock“. Im Bereich der innerkollegialen Beziehungen zeigen die Ergebnisse, dass das Berufsleben der Schulleiterinnen und Schulleiter in besonderem Maße vom Umgang mit Lehrkräften bei auftretenden Konflikten geprägt ist. Die subjektiv empfundene Belastung der befragten Schulleiterinnen und Schulleiter wird als hoch, aber zu bewältigen eingeschätzt. Von allen Schulleiterinnen und Schulleitern werden die seelischmenschlichen Anforderungen als am stärksten belastend erlebt. Die hohen Belastungen in beiden Bereichen führt Neulinger vor allen Dingen auf die fehlende Ausbildung und damit auf eine mangelnde Professionalisierung zurück.

Dies gibt Anregungen zu neuen Fragestellungen bezüglich des Belastungsempfindens und deren Erklärungen seitens der Schulleiterinnen und Schulleiter meiner Untersuchungsgruppe.

\subsubsection{Berufswechsel als Sozialisationsprozess}

Den Wechsel vom Lehr- zum Schulleitungsberuf betrachtet Storath (1995). Er führt eine Fragebogenerhebung und zwölf problemzentrierte Interviews mit neu ernannten Schulleiterinnen und Schulleitern der Volks- und Sonderschule in Bayern durch. Storath geht von der Annahme aus, dass sich

\footnotetext{
${ }^{47}$ Neulinger 1991, 354.
} 
insbesondere Schulleiterinnen und Schulleiter in der Anfangsphase ihres Berufes durch fehlende Vorbereitung und unzureichende Qualifizierung einem „Praxisschock“ ausgesetzt fühlen.

Anhand der Fragen zum beruflichen Werdegang kann gezeigt werden, dass Schulleiterinnen und Schulleiter vor ihrem Amtsantritt eine Funktionsstelle inne hatten, zum Teil langjährig in der stellvertretenden Schulleitung tätig waren und sie daher ihre jetzige Tätigkeit in der Rückschau erwartungsgemäß beurteilen. Die erlebten Belastungen hatten die befragten Schulleiterinnen und Schulleiter von vornherein realistisch eingeschätzt. Belastung drückt sich vor allen Dingen in der zeitlichen Dimension aus, $96,3 \%$ geben an, dass sie sich zeitlich stark eingespannt fühlen. ${ }^{48}$

Während seine quantitativen Daten die Annahme eines „Praxisschocks“ nicht unbedingt bestätigen, zeigen die qualitativen Daten, dass die befragten Schulleiterinnen und Schulleiter auf eine Diskrepanz zwischen Erwartung und Realität hinweisen. So berichten einige der Befragten von Schwierigkeiten bei der Umsetzung von Innovationen und geben Beispiele von „frustrierenden Erlebnissen“ mit Kollegen. Insgesamt sieht Storath (1995) seine Hypothese des Praxisschocks bestätigt. „Die Ist-Soll- Diskrepanz muss gerade zu Beginn der Tätigkeit besonders schmerzhaft erlebt werden, wenn idealistisch-euphorische Aufgeschlossenheit, die hohe Innovationsbereitschaft in Kollision gerät mit nicht einkalkulierbaren personalen und /oder sachlich-organisatorischen Widerständen." ${ }^{49}$ Als Konsequenz fordert er eine bessere Qualifizierung, zum Beispiel in Form von Führungsseminaren und kollegialer Praxisberatung.

Die Untersuchungsgruppe ist für mich zwar nicht von Interesse, da er sich auf eine andere Schulform und ausschließlich auf neu ernannte Schulleiterinnen und Schulleiter bezieht, da das Thema "Praxisschock" aber evtl. in meinen Interviews angesprochen wird, werde ich es als Problem im Blick behalten.

Unter sozialisationstheoretischer Perspektive und organisationstheoretischen Überlegungen untersuchte Wissinger (1996) in Bayern mittels eines standardisierten Fragebogens das Selbstverständnis von Schulleiterinnen und Schulleitern unter der Perspektive des Führungshandelns. Die quantitative Stichprobe ist schulformübergreifend ausgerichtet und umfasst Aussagen von 198 Schulleiterinnen und Schulleitern.

Wissinger (1995) setzt sich ausführlich mit der angloamerikanischen Schuleffektivitäts- und Schulqualitätsforschung auseinander und problematisiert den Einfluss von Schulleiterinnen und Schulleitern auf den Erfolg der Schulen. Die hohen Erwartungen, die an Schulleitungen gestellt werden würden, können nach Wissingers Meinung durch die Voraussetzungen des Leitungshandelns nicht erfüllt werden. ${ }^{50}$

Das Schulleitungshandeln wird von Wissinger als ein Wechselspiel zwischen "individuellem Lernprozess" und Prozessen des "OrganisationsLernens" verstanden. Wie bereits in anderen Untersuchungen verweist auch Wissinger unter dem Gesichtspunkt der Schulentwicklung auf die

\footnotetext{
${ }^{48} \mathrm{Vgl}$. Storath 1995, 185.

${ }^{49}$ Storath 1995, 215

${ }^{50}$ Vgl. Wissinger 1996, 11.
} 
strukturell problematische Doppelrolle der Schulleiterinnen und Schulleiter, da sie auf Denk- und Verhaltensmuster zurückgreifen würden, die sie als Lehrerin/ Lehrer erworben hätten und diese Rollenidentität im Widerspruch zu den Anforderungen eines modernen Führungsverständnisses als Schulleiterin/ als Schulleiter stehe. ${ }^{51}$

Wissinger fordert eine Trennung zwischen schulischem Lehrberuf und schulischem Leitungsberuf und verlangt eine unterschiedliche Qualifizierung. Dabei geht er davon aus, dass die Organisationsentwicklung die bestimmende Zielgröße für das Handeln von Schulleiterinnen und Schulleiter sein muss. ${ }^{52}$ Die berufliche Sozialisation im Kontext der Organisation Schule wird unter der Fragestellung des eigenen Rollenverständnisses untersucht. Inhaltlich werden Fragen zum Rollenverständnis, zum Berufswahlmotiv, zu Arbeitsbedingungen, zu Aufgaben und zum Führungsverhalten gestellt.

Wissinger konstatiert innerhalb seiner Ergebnisdarstellung, dass es möglich ist, trotz der Schulformunterschiede von einer relativ homogenen Gruppe der Schulleiterinnen und Schulleiter zu sprechen. ${ }^{53}$ Unter dem Fokus des vollzogenen Rollenwechsels von der Lehrkraft zur Schulleiterin/zum Schulleiter interpretiert er die Ergebnisse als Bestätigung seiner Hypothese und hält fest, dass das Führungsverständnis und -verhalten persönlich, emotional und auf individuelle Zuwendung ausgelegt ist und dass Schulleiterinnen und Schulleitern auf ihre Rollenidentität als Lehrerin/als Lehrer zurückgreifen. Er weist kritisch darauf hin, dass Schulleiterinnen und Schulleiter eher auf der Individualebene handeln würden als auf der Systemebene und fordert einen Perspektivenwechsel, indem das eigene Selbstverständnis reflektiert werde und Schulleiterinnen und Schulleiter bereit sein sollten, ihre Rolle neu zu definieren. Die Schulleiterin/der Schulleiter hätte aufgrund der Gesamtverantwortung für die schulische Handlungseinheit die Aufgabe, sowohl personen- als auch systembezogene Maßnahmen zur Entwicklung ihrer/seiner Schule umzusetzen. ${ }^{54}$ Wissinger bezieht sich vornehmlich auf den strukturell bedingten Rollenkonflikt der Schulleiterin/ des Schulleiters und fasst zusammen: „Die vorliegende Arbeit hat am Beispiel zu illustrieren versucht, dass von der Schulleitungsfunktion und -rolle unter Berücksichtigung bestehender Arbeitsbedingungen, eines spezifischen Rollenverständnisses, spezifischer Führungsvorstellungen sowie einer unzureichenden Qualifikation zum gegenwärtigen Zeitpunkt keine Impulse für die Entwicklung der Schule als Organisation und als je spezifische, kontextgebundene Handlungseinheit erwartet werden können." ${ }^{\text {"55 }}$ Die Entwicklung der Schule hängt seiner Meinung nach davon $a b$, ob es Schulleiterinnen und Schulleitern gelingt, einen Rollenwechsel vorzunehmen. Sein Verständnis von Schulleitungshandeln ist eingebettet in die Ansätze der Organisationsentwicklung, daher betont er die Managementfunktion der Schulleiterinnen und Schulleiter.

\footnotetext{
${ }^{51}$ Ebd., 75.

52 Ebd., 187.

53 Ebd., 155.

${ }^{54}$ Vgl. Wissinger 1996, 182.

${ }^{55}$ Wissinger 1996, 173.
} 
Interessant ist dieses Ergebnis im Hinblick auf meine Untersuchung, da sich in den letzen Jahren in Niedersachsen das Aufgabenspektrum für Schulleiterinnen und Schulleiter vornehmlich im Bereich der aktiven Schulentwicklung erweitert hat.

\subsubsection{Handlungsdimensionen von Schulleiterinnen u. Schulleitern}

Bonsen/Iglhaut/Pfeiffer (1999) ${ }^{56}$ vom Dortmunder Institut für Schulentwicklung untersuchen im Rahmen eines Schulprojektes Handlungsdimensionen von Schulleiterinnen und Schulleitern. In einer qualitativen Interviewstudie in zwei benachbarten Kleinstädten in Niedersachsen wurden mit 20 Schulleiterinnen und Schulleitern (18 Männer/2 Frauen) aller Schulformen Interviews geführt.

Das Forschungsinteresse gilt den Handlungsdimensionen und Handlungsstrategien. Die Leitfaden-Interviews werden nach thematischen Segmenten geordnet, im Vordergrund stehen Tätigkeitsfelder.

Auf der Basis ihres Materials arbeiten die Autoren heraus, dass das Handeln von Schulleiterinnen und Schulleitern stark davon geprägt ist, situationsbezogenen Anforderungen gerecht zu werden. Das Haupttätigkeitsfeld sind Gespräche mit verschiedenen Akteuren der Schule. Auf die Frage nach Kernkompetenzen werden daher auch Kommunikations- und Konfliktfähigkeit von der Mehrheit der Befragten genannt.

Diese eigene Einschätzung der Schulleiterinnen und Schulleiter ist für meine Forschungsfrage bedeutsam, weil gerade die nicht planbaren Kommunikationssituationen meiner Einschätzung nach sehr viel Raum in der täglichen Arbeit einnehmen und zu Belastungen führen können.

Im Bereich Unterrichtstätigkeit sehen sich die Befragten als Unterrichtende und die Mehrheit der Untersuchten geben an, dass sie nach ihrem Selbstverständnis gerne Lehrer und Pädagogen seien. ${ }^{57}$

Die zeitlich höchste Beanspruchung erfahren die Schulleiterinnen und Schulleiter durch die Verwaltung und die Organisation der Schule.

Als wichtiges Element ihrer Tätigkeit schätzen die Untersuchten ihre eigene Präsenz und Erreichbarkeit in der Schule ein. Diese Einschätzung wird ergänzt durch die zurückhaltende Beantwortung der Frage nach Delegation. Einerseits wird es als wichtig eingestuft, Aufgaben an andere Mitglieder der Schulleitung zu delegieren, andererseits wird in dem Zusammenhang aber auf die letztendliche Verantwortung des Schulleiters/ der Schulleiterin hingewiesen.

Widersprüchliche Anforderungen werden von den Befragten als hinderliche Bedingung für das eigene Führungshandeln herausgestellt. „Nicht erstaunlich ist die weitgehend geteilte Meinung, dass eine gute Ausstattung mit Personal und Material eine hilfreiche und günstige Rahmenbedingung darstellt und umgekehrt. Dagegen wird die große Menge und Unübersichtlichkeit (bis zur teilweisen Widersprüchlichkeit) der Anforderungen an

\footnotetext{
${ }^{56}$ Vgl. Bonsen/lglhaut/Pfeiffer 1999. Die Ergebnisse dieses Forschungsprojektes greifen die Autoren in Ihrer Untersuchung von 2002 erneut auf und erweitern dort ihre Forschungsperspektive durch organisationstheoretische Überlegungen.

${ }^{57}$ Bonsen et al 1999, 22.
} 
Schule und Schulleitung als erschwerende Bedingung für erfolgreiches Schulleitungshandeln herausgestellt.“58

Bonsen/lglhaut/Pfeiffer liefern durch ihre Befragung wichtige Hinweise zu den Handlungsdimensionen von Schulleiterinnen und Schulleitern. Die Vielschichtigkeit des Handelns und die situationsabhängige, auch auf den Ablauf des Schuljahres bezogene erfahrene Tätigkeit werden herausgestellt. Insbesondere die zusammenfassende Analyse verweist auf das Spannungsfeld der Akteure zwischen Rahmenbedingungen und eigenen Handlungsdimensionen.

\subsubsection{Geschlechtsspezifische Forschungsansätze}

Es gibt neben den oben dargestellten theoretischen Ansätzen der Schulleitungsforschung auch Untersuchungen, die die Kategorie Geschlecht fokussieren.

Winterhager-Schmids (1997) Evaluationsstudie „Berufsziel Schulleiterin. Professionalität und weibliche Ambition" kann nur in einem weiten Sinne zu den Untersuchungen über Schulleiterinnen gezählt werden, da überwiegend keine im Amt befindlichen Schulleiterinnen befragt wurden, sondern an Funktionsstellen interessierte Lehrerinnen. Im Rahmen eines Modellversuchs 'Schulleitung als Aufgabe für Frauen' in Niedersachsen nehmen interessierte Lehrerinnen an Orientierungskursen teil. Das Ziel dieser Kurse ist es, die Motivation von Frauen zu stärken, sich auf ein Amt als Schulleiterin zu bewerben. Neben einer Fragebogenerhebung werden 17 narrative Interviews mit Teilnehmerinnen geführt, die Fragen zu Veränderungen seit der Kursteilnahme und zum gesamten beruflichen Werdegang enthalten. Gefragt wird nach dem Interesse an Leitungstätigkeiten, dem Reiz, den die Schulleitungstätigkeit ausübt sowie nach persönlichen „Vereinbarkeitsmodellen“ von Familie, Privatleben und Beruf.

Winterhager-Schmid kommt zu dem Ergebnis, dass für Lehrerinnen die Aufstiegsmotive weniger selbstverständlich sind als vermutlich bei Lehrern. Winterhager-Schmid deutet dies als „Abwägungsspielraum“ von Verlust und Gewinn durch eine Leitungsfunktion, wobei sie der Dimension von Macht und Einfluss eine besondere Rolle einräumt. „Eine besondere Rolle spielt dabei für sie die berufsethische Dimension von Macht und Einfluss. Nur wenn sie den Eindruck haben, die Übernahme von mehr Verantwortung öffne innen Gestaltungsräume für die Veränderung von Schule und wenn sie zudem davon ausgehen können, willkommen zu sein als Leiterinnen, zumindest aber nicht offen bekämpft zu werden, wird Leitung für sie zu einer attraktiven Herausforderung. "59

Forberg (1997) befragt in ihrer Studie „Rollen- und Führungsverständnis von Schulleiterinnen beruflicher Schulen“ 125 Schulleiterinnen der alten Bundesländer. Ihre Ausgangsthese orientiert sich an Ansätzen der geschlechtsspezifischen Sozialisation: „Die geschlechtsdifferente Sozialisation hat prägenden Einfluss auf das Individuum und dessen Präferenz für bestimmte Arbeits- und Kommunikationsformen. Mit der Übernahme der Führungsrolle als Schulleiter/ Schulleiterin werden positionsspezifische

\footnotetext{
${ }^{58}$ Bonsen et al 1999, 117.

${ }^{59}$ Vgl. Winterhager-Schmid 1997, 218.
} 
Rollenerwartungen an das Individuum gerichtet, das bereits in seiner Subjektivität geprägt ist." 60

Im qualitativen Teil ihrer Untersuchung führt Forberg (1997) 24 Interviews und entwickelt auf der Grundlage ihrer Daten eine Typologie, in der das Rollen- und Führungsverständnis erfasst wird und folgende Rollen von ihr identifiziert werden: Teamerin und Förderin kollegialer Kooperation, Vermittlerin in Konfliktfällen und Türöffnerin für pädagogische Innovationen und konstituiert in ihrem Resümee, dass Schulleiterinnen eine stärker ausgeprägte 'sozio-emotionale Dimension` in ihrem Führungsstil aufweisen. ${ }^{61}$

Miller (2001) ${ }^{62}$ befragt in ihrer Studie 634 Schulleiterinnen und Schulleiter an Grundschulen in Nordrhein-Westfalen zu biografischen Aspekten sowie dem beruflichen Werdegang. Hinsichtlich der Ausgestaltung der Schulleitungstätigkeit stellt Miller überwiegend eine Geschlechtergleichheit fest, z.B. hinsichtlich der Präferenz zwischen pädagogischen und administrativen Aufgaben. Einen Unterschied zwischen Schulleiterinnen und Schulleitern gibt es bei der Übernahme der Klassenleitung, Schulleiterinnen sind zu 70\% Klassenlehrerin dagegen Schulleiter zu 50\%.

Auf die Frage nach der Rangordnung der Tätigkeitsbereiche unterscheiden sich Männer und Frauen um weniger als 3\% bei sechs der zwölf Tätigkeitsbereiche. Schülerinnen und Schüler erziehen und zu unterrichten sowie die Stundenplan- und Unterrichtsorganisation werden annähernd gleich gerne von Schulleiterinnen und Schulleitern ausgeführt. Signifikante Unterschiede liegen bei den Aufgaben der Haushalts- und Finanzorganisation und bei der Kooperation mit dem Schulträger vor, diese Tätigkeiten werden von Männern lieber ausgeführt als von Frauen. ${ }^{63}$

Die genannten Führungsvorstellungen könnten dazu verleiten, einen 'weiblichen' und 'männlichen' Führungsstil im Sinne eines traditionellen Gegensatzpaares (Kooperation, Beziehungsorientierung vs. Macht, Hierarchie) anzunehmen. Doch die Ergebnisse sind in mehrfacher Hinsicht vorsichtiger zu deuten, denn während Forbergs und Winterhager-Schmids Befunde einen Vergleich Frau - Mann nicht möglich machen, weisen Millers Ergebnisse eine nur geringe Differenz hinsichtlich der Ausgestaltung des Führungshandelns aus.

In der Untersuchung von Kansteiner-Schänzlin (2002) werden schulformübergreifend Lehrkräfte in Baden-Würtemberg über das wahrgenommene Führungsverhalten ihrer Schulleiterinnen und Schulleiter befragt. Mittels eines standardisierten Fragebogens werden aus den Rückmeldungen jeweils von drei Lehrerinnen und drei Lehrern einer Schule Führungsprofile ermittelt.

In ihren theoretischen Überlegungen stellt Kansteiner-Schänzlin zunächst ausführliche Befunde zur Personalführung in Unternehmen im Vergleich zur Personalführung in der Schule dar, sie resümiert, dass der Blick auf

\footnotetext{
${ }^{60}$ Vgl. Forberg 1997, 13.

${ }^{61}$ Ebd., 187.

${ }^{62}$ Miller 2001.

${ }^{63}$ Ebd., 261.
} 
Führungskräfte im Wirtschaftbereich eine Zweigeteiltheit, wie sie oben angedeutet wurde, als nicht zutreffend herausgestellt. ${ }^{64}$

Dieser Befund deckt sich mit den Ergebnissen von Wunderer (2003), seit Beginn der neunziger Jahre wurde in Unternehmen der Frage nach der Existenz eines `typisch weiblichen` Führungsstils nachgegangen. Wunderer (2003) befragt 700 Personen (Personalexperten, Mitarbeiterinnen und Mitarbeiter, Führungskräfte) hinsichtlich dieser Einschätzung. Die weiblichen und männlichen Führungskräfte wurden sehr ähnlich beurteilt. Ein „kooperativer Führungsstil wird entgegen der Erwartung von Frauen nicht häufiger angewandt". ${ }^{65}$

Die Ergebnisse der Untersuchung von Kansteiner-Schänzlin zeigen, dass Lehrerinnen und Lehrer hinsichtlich der meisten Kompetenzen keine Unterschiede zwischen Schulleiterinnen und Schulleitern feststellen. Differenzen bestehen aber im Bereich Kontrolle, Autorität und Leistungsbereitschaft: „Schulleiter zeigen ein Mehr an Vertrauen und an Rückkopplung sowie ein Mehr an Autorität und ein Weniger an Kontrolle. (...)Viele Schulleiter scheinen weniger einzufordern als ihre Kolleginnen und umgekehrt jedoch auch weniger zu unterstützen und zu investieren. (...) Das Mehr an Leistungsbereitschaft und Engagement der Schulleiterinnen und ihr Mehr an Förderung von Teams und Projektgruppen geht einher mit einem Mehr an Kontrolle und einem Weniger an Teilung der Entscheidungsmacht. "66

Gemeinsam ist den hier vorgestellten Studien, dass sie beabsichtigen, Frauen in Führungspositionen von Schule sichtbar zu machen und eventuell stereotype Vorstellungen über Schulleiterinnen durch die Darstellung vielfältiger Berufsbiographien zu erweitern

Der Fokus Geschlecht wird in meiner Untersuchung nicht explizit thematisiert, spielt aber selbstverständlich immer eine Rolle. Da ich sowohl Schulleiterinnen als auch Schulleiter befrage, kann es zu Äußerungen kommen, die diesen Aspekt aufgreifen, insofern dienen die Ergebnisse als Sensibilisierung.

\subsection{Zusammenfassung}

Die hier dargestellten Forschungsergebnisse machen deutlich, dass das Amt der Schulleiterin/ des Schulleiters in den letzten Jahren in Deutschland verstärkt wahrgenommen worden ist und zu einer Reihe von Forschungsbeiträgen geführt hat. Im Hinblick auf den Forschungsgegenstand dieser Studie sind drei Untersuchungen von besonderer Bedeutung. Viele der vorliegenden sind organisationstheoretisch orientiert und versuchen zu klären, inwieweit die Amtsinhaber die Anforderungen der Schulentwicklung in ihre berufliche Handlungspraxis integriert haben. Baumert/ Leschinsky, Storath und Wissinger verweisen auf die Bedeutung einer Rollenidentität als Schulleiterin/ Schulleiter, in der sich die Akteure an einem veränderten Verständnis von Schule als lernender Organisation orientieren.

Im Unterschied zu den drei genannten Studien greift Neulinger (1991) das Problem der Professionalisierung auf. Er ermittelt bildungspolitische und

\footnotetext{
${ }^{64}$ Vgl. Kansteiner-Schänzlin 2002,137.

${ }^{65}$ Wunderer 2003, 249.

${ }^{66}$ Kansteiner-Schänzlin 2002, 242.
} 
pädagogische Einstellungen der Schulleiterinnen und Schulleiter und konstatiert einerseits eine fehlende Professionalität der Akteure, andererseits und dies ist im Rahmen der aktuellen Diskussion um Schulentwicklung hin zur Einzelschule als Gestaltungseinheit von Bedeutung, dass die Einstellungen der Schulleiterinnen und Schulleiter keine Veränderungsbereitschaft im Sinne der Weiterentwicklung der Schule aufweisen. Beide Aspekte sind für meine Untersuchung von Interesse. Zu fragen wird sein, wie heutige Schulleiterinnen und Schulleiter mit dem hohen Veränderungsdruck umgehen. Insbesondere die von ihm erörterten professionstheoretischen Ausführungen werde ich aufgreifen und weiter führen im Hinblick auf die Ambivalenz zwischen strukturellen und individuellen Problemen der Schulleiterinnen und Schulleiter.

Bei all ihrer Unterschiedlichkeit und differenzierten Schwerpunktsetzungen ist festzustellen, dass in der Mehrzahl der Studien das Schulleitungshandeln an den Vorstellungen einer optimalen Organisationsgestaltung, eines wirkungsvollen Führungshandelns und dem Erreichen von Schulentwicklungsprozessen gemessen wird. Diese Inspektion der Schulleiterinnen und Schulleiter an Modellen der Schulentwicklung oder Konzepten der Qualitätsverbesserung ist gewiss wertvoll und hat eine Menge wichtige Ergebnisse geliefert. In allen vorliegenden Untersuchungen wird die bedeutsame Rolle der Schulleiterinnen und Schulleiter betont. Ebenfalls wird in den Forschungsbeiträgen auf die Abhängigkeit des Schulleitungshandelns von veränderten Umwelterwartungen bzw. den daraus erwachsenen Innovationsdruck verwiesen.

Wie genau es den Akteuren in der Schule gelingen kann, diesen Anforderungen gerecht zu werden und welchen Stellenwert dabei neben der Bewältigung des Alltagsgeschäftes die Schulentwicklung haben kann, dies ist eine wichtige Frage in meiner Untersuchung. Es gilt also, das Spannungsverhältnis zwischen programmatischen Zielvorgaben und der alltäglichen Praxis in den Blick zu nehmen und dabei sowohl die Schulleiterinnen und Schulleiter selbst als auch die Rahmenbedingungen der Organisation Schule zu berücksichtigen.

\section{Theoretische Grundlagen}

In diesem Teil der Arbeit werden die theoretischen Grundlagen der Untersuchung beschrieben. Im Zentrum der vorliegenden Arbeit steht die individuelle Führungsperson der Einzelschule, also die Schulleiterin/ der Schulleiter. Aus dieser Blickrichtung soll die Berufsauffassung der Akteure erschlossen werden. Diese Perspektive macht es notwendig, sich mit den theoretischen Hintergründen der Führungsforschung und ihren Bezug zur Schule zu beschäftigen. Im ersten Teil werden führungstheoretische Ansätze behandelt. Es werden unterschiedliche Führungstheorien aus der Literatur genannt und die für die Schule relevanten Führungskonzeptionen beschrieben.

Aus einer zweiten Perspektive wird die Organisation Schule ${ }^{67}$ in den Fokus der Betrachtung gezogen, denn die zunehmende Selbstständigkeit

\footnotetext{
${ }^{67}$ Die Begriffe Institution und Organisation werden häufig gleichermaßen für die Schule verwendet. Institution als staatliche Einrichtung ist die Schule insofern, als dass ein insti tutionelles Rechtsdenken das Zusammenleben der Schulmitglieder ordnet und regelt und somit das Funktionsgerüst der Schule liefert. Soziologisch gilt "Institution“ als Sam-
} 
von Einzelschulen verlangt eine größere organisationale Selbststeuerung, die vornehmlich von Schulleiterinnen und Schulleitern umgesetzt werden muss. Im zweiten Teil werde ich daher auf Ansätze der Organisationstheorie eingehen und Schulentwicklungsansätze beschreiben, die sich aus der Organisationsentwicklung abgeleitet haben.

Die Betrachtung dieser beiden Perspektiven macht deutlich, dass das Verhältnis zwischen Individuum und Organisation von großer Bedeutung ist, so spricht Schütze (1996) von der Balance zwischen professioneller Berufsratio und Organisationsratio. ${ }^{68}$ Diese strukturellen Probleme des Berufes sind Gegenstand der Professionstheorie, die damit als Klammer der oben genannten Theorieansätze verstanden werden kann. In dem dritten Teil werde ich professionstheoretische Überlegungen darstellen und dabei insbesondere die konstitutiven Antinomien des pädagogischen Handelns und das Pädagogische Professionswissen in den Blick nehmen.

Die theoretischen Bezüge werden im Hinblick auf die Rolle der Schulleiterinnen und Schulleiter dargestellt, an einigen Stellen ist es jedoch wichtig, den Kontext der Bezugstheorie ausführlicher darzustellen, um bestimmte Entwicklungen, die sich auch für das heutige Berufsbild ergeben, nachvollziehbar darzulegen.

Abschließend sollen diese Ansätze im Hinblick auf den Forschungsgegenstand zusammengeführt und ein Konstrukt der Berufsauffassung von Schulleiterinnen und Schulleitern entwickelt werden.

Abb. 3: Theoretische Bezüge

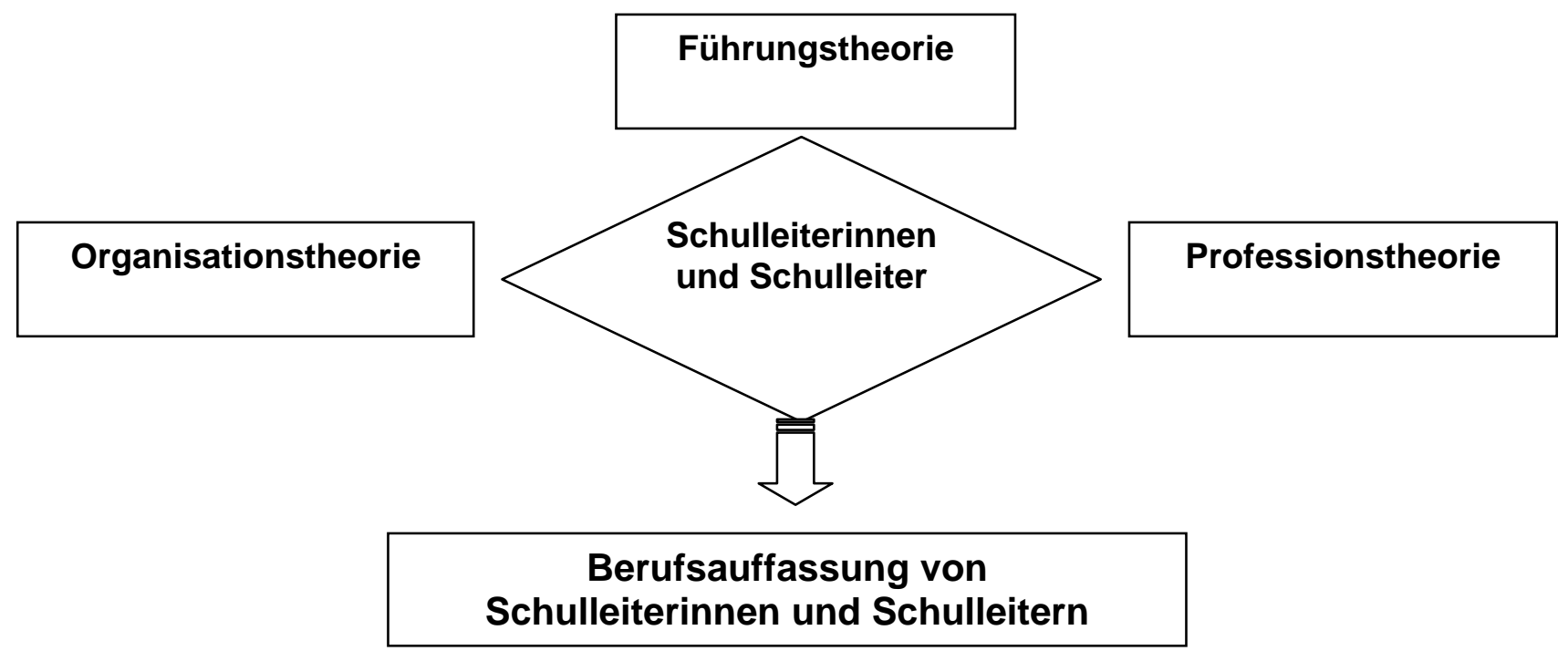

melbezeichnung für soziale Gebilde und Organisationen verschiedenster Art. Unterschieden werden Institutionen als abstrakte niedergelegte, regulative Prinzipien und Institutionen als konkrete gesellschaftliche Gruppen, Organisationen, in denen sich diese Prinzipien repräsentieren. In der vorliegenden Arbeit werde ich den Begriff der Organisation verwenden, Schule wird als soziales System betrachtet, in dem Menschen in einem Strukturzusammenhang stehen (institutionaler Organisationsbegriff), Regeln das Handeln in diesem System festlegen (instrumentaler Organisationsbegriff) und gleichzeitig Tätigkeiten des Gestaltens vollzogen werden (funktionaler Organisationsbegriff). Vgl. Brockhaus 2006.

${ }^{68} \mathrm{Vgl}$. Schütze 1996 


\subsection{Führungstheorie}

Die Führungstheorien beschreiben und erklären Bedingungen, Strukturen, Prozesse, Ursachen und Konsequenzen von Führung. Die Führungsforschung begann Anfang bis Mitte des 20. Jahrhunderts damit, die Eigenschaften einer Person als ausschlaggebendes Moment der Führung in den Mittelpunkt zu stellen und später verschiedene Stile hinsichtlich ihres Erfolges zu vergleichen. Im weiteren Verlauf werden konstituierende Momente einer Situation, in der Führung geschieht, betrachtet. Zunehmend wird ein einseitiger Blick auf die Führungsperson hin zu den Geführten und den Aspekten des Vorgesetztenverhaltens erweitert.

Der Begriff der Führung wird in der Literatur und im praktischen Sprachgebrauch in zahlreichen Varianten benutzt. Insbesondere in der Betriebswirtschaftslehre wird eine definitorische Verknüpfung der Begriffe Führung und Management vorgenommen, dabei wird unterschieden zwischen dem Gestalten, Lenken und Entwickeln von Institutionen (Management) und dem Lenken und Entwickeln von Mitarbeitern (Führung). In der angloamerikanischen Literatur werden die Termini 'Management' (Unternehmensführung), Leadership (Personalführung) und Headship (Leitung) abgegrenzt. Management im Sinne einer zielorientierten Führung bezieht stärker die Gestaltungsfunktion und Steuerungsfunktion in Organisationen mit ein und Führung die Steuerung des Wandels durch Erkennen und Verstehen. ${ }^{69}$

Eine in allen Definitionsversuchen wiederkehrende Übereinstimmung besteht darin, dass Führung die zielorientierte soziale Einflussnahme zur Erfüllung gemeinsamer Aufgaben ist. „Führung wird verstanden als zielund ergebnisorientierte, aktivierende und wechselseitige, soziale Beeinflussung zur Erfüllung gemeinsamer Aufgaben in und mit einer strukturierenden Arbeitssituation." ${ }^{\text {"70 }}$ In diesem Sinne beinhaltet Führung zwei Aspekte, den strukturellen Aspekt, die Verhaltensbeeinflussung indirekt im Sinne optimaler Gestaltung der Arbeitsabläufe und Arbeitssituation, wie zum Beispiel Jahresplan, Gestaltung des Stundenplans etc.), und den personalen Aspekt, die Verhaltensbeeinflussung bezogen auf die Mitarbeiter. Beide Aspekte lassen sich wiederum auf zwei Ebenen betrachten, die Systemebene, also Führung für das Personal als Systemgestaltung und die Prozessebene, Führung des Personals durch Verhaltenssteuerung.

In der Literatur werden folgende führungstheoretische Ansätze unterschieden, die wiederum spezifiziert werden: Der personenorientierte Ansätze, der sich mit den Eigenschaften und Einstellungen der Führungsperson beschäftigt, der verhaltensorientierte Ansätze, der mit den Handlungen der Führungspersonen auseinandersetzt, hierzu gehört die Führungsstilforschung, die positionsorientierte Ansätze, die den strukturellen Rahmen der Führung in den Vordergrund stellt. Die situativen Ansätze und die Kontingenzansätze beschreiben die Faktoren, die erfolgreiches Führungsverhalten bestimmen. Konkretisierung erfahren diese theoretischen Ansät-

\footnotetext{
${ }^{69} \mathrm{Vgl}$. Berthel 2000, 8.

${ }^{70}$ Vgl. Wundere 2003, Stogdill 1974, Staehle 1999, Hentze et al. 1986, Berthel 2000.
} 
ze in den Führungskonzeptionen, die als Modelle für die Führungspraxis entwickelt werden.

\subsubsection{Personenorientierte Ansätze}

Der personenorientierte Ansatz beschäftigt sich mit den Eigenschaften und Einstellungen der Führungsperson, daher wird auch von der Eigenschaftstheorie gesprochen. Die Theorie basiert auf einem individualpsychologischen Ansatz, darin werden Führungserfolge durch individuelle Attribute des Führers erklärt. Als Eigenschaften werden relativ konstante Dispositionen zu bestimmten Verhaltensweisen, die konsistent in verschiedenen Situationen auftreten, angesehen. ${ }^{71}$

Der personale Einflussfaktor ist die Persönlichkeit der Führungskraft, d.h. die Fähigkeiten, Kenntnisse, Persönlichkeitsmerkmale, Charakterzüge, Eigenschaften, Erfahrungen, Qualitäten und Kompetenzen. Einerseits erfährt die Rolle der Führungskraft durch diese Ansätze eine hohe Aufwertung, andererseits werden Erwartungsnormen aufgestellt, die das Bild einer idealtypischen Universalgenies zeichnen. Es lässt sich zwar ein Idealtypus beschreiben, wissenschaftlich abgesicherte Aussagen darüber, dass Führungserfolg auf außergewöhnliche Eigenschaften und Fähigkeiten einzelner Führungspersonen zurückgeführt werden kann, konnte durch die Führungsforschung nicht belegt werden, obwohl zahlreiche Untersuchungen vor allen Dingen in den USA durchgeführt worden sind. ${ }^{72}$

Die traditionelle Führungstheorie, die von der Person des Führenden ausging, gewann in den letzten Jahren wieder zunehmend an Bedeutung, so gibt es einen eigenen Ansatz der „charismatischen“ Führungstheorie. Die charismatische Führungspersönlichkeit zeichnet sich durch eine stark beeinflussende „Ausstrahlung“ aus, wodurch Einfluss auf die Werthaltungen und Emotionen der Geführten genommen werden soll. Charismatische Personen vermitteln demnach Visionen und können Werte und Verhalten in grundsätzlicher Weise verändern. Sie bieten Problemlösungen an und treten für Veränderungen ein. ${ }^{73}$

Die Kritik an diesem Ansatz besteht darin, dass mit der Zuweisung von Eigenschaften an einzelne Führungspersonen andere Einflussfaktoren außer Acht gelassen werden und das komplexe Geschehen der Führung lediglich auf einen Faktor reduziert wird. Die Betonung der Führungspersönlichkeit widerspricht dem aktuellen Bemühen um stärkere Mitarbeiterorientierung und der zunehmend gewünschten Selbststeuerung am Arbeitsplatz. Diese Tendenzen kollidieren mit Konzepten der Schulentwicklung, die auf aktive, selbstständige und eigenverantwortliche Mitwirkung des Kollegiums setzen.

\subsubsection{Verhaltensorientierte Ansätze}

Im Zentrum vieler Führungsdefinitionen steht der Prozess der zielbezogenen Verhaltensbeeinflussung der Geführten bzw. Mitarbeiter. Im Zusammenhang mit dem Verhaltensansatz (Behavioral Approach) ist der Füh-

\footnotetext{
71 Ebd.

72 Vgl. Stogdill 1974,17.

${ }^{73}$ Vgl. Wunderer 2003, 277.
} 
rungsprozess als ein Reiz-Reaktions-Modell zwischen Verhalten von Vorgesetzen gegenüber Mitarbeitern zu verstehen. Das Führungsverhalten bezeichnet alle Verhaltensweisen, die auf eine zielorientierte Einflussnahme zur Erfüllung gemeinsamer Aufgaben ausgerichtet ist. Demgegenüber bezeichnet der Führungsstil eine Kombination verschiedener Verhaltenskomponenten ${ }^{74}$.

Seit den 40er Jahren wurde versucht in zahlreichen Labor- und Feldstudien Führungsverhalten zu erfassen, zu klassifizieren und seine Auswirkungen zu untersuchen. ${ }^{75}$ Es sollten bestimmte Verhaltensmuster zu einem Führungsstil zusammengefasst werden, die als erfolgsbestimmend für Führung in Organisationen angesehen wurden.

Die „Michigan-Studie“ identifizierte zwei grundlegende Orientierungsmuster: Aufgabenorientierung und Mitarbeiterorientierung, die jeweils die Extrempole eines Kontinuums repräsentieren. Das wichtigste Ergebnis dieser Studie ist, dass effektive und effiziente Vorgesetzte eher mitarbeiter- als aufgabenorientiert sind. ${ }^{76}$

In der Literatur werden häufig vier Idealtypen von Führungsstilen unterschieden: Patriarchalischer Führungsstil; Charismatischer Führungsstil;. Autokratischer Führungsstil und Bürokratischer Führungsstil. ${ }^{77}$

Unter den typologischen Ansätzen ist bis heute die Kontinuums-Theorie von Tannenbaum/Schmidt (1958) bedeutsam. Sie soll hier kurz dargestellt werden, weil sich die Führungsstilforschung nach wie vor auf die Begrifflichkeiten dieses Modells bezieht. Nach dem Grad der Mitarbeiterbeteiligung bei Entscheidungen werden sieben Führungsstile unterschieden. Die Begrifflichkeiten dieser Typologie bildeten die Grundlage für die Entwicklung von Führungskonzepten.

Abb. 4: Führungsstilkontinuum nach Tannebaum/Schmidt ${ }^{78}$

\begin{tabular}{|c|c|c|c|c|c|c|}
\hline \multicolumn{5}{|c|}{$\begin{array}{l}\text { Entscheidungsspielraum } \\
\text { des Vorgesetzten }\end{array}$} & \multicolumn{2}{|c|}{$\begin{array}{l}\text { Entscheidungsspielraum } \\
\text { des Mitarbeiters }\end{array}$} \\
\hline 1 & 2 & 3 & 4 & 5 & 6 & 7 \\
\hline $\begin{array}{l}\text { Vorgesetzter } \\
\text { entscheidet } \\
\text { allein }\end{array}$ & $\begin{array}{l}\text { Vorgesetzter ent- } \\
\text { scheidet, versucht } \\
\text { aber Mitarbeiter } \\
\text { von seinen Ent- } \\
\text { scheidungen } \\
\text { zu überzeugen }\end{array}$ & $\begin{array}{l}\text { Vorgesetzter } \\
\text { entscheidet, } \\
\text { fördert aber eine } \\
\text { vorherige Aus- } \\
\text { einander- } \\
\text { setzung, um } \\
\text { Akzeptanz zu } \\
\text { erreichen }\end{array}$ & $\begin{array}{l}\text { Vorgesetzter infor- } \\
\text { miert vor der Ent- } \\
\text { scheidung seine } \\
\text { Mitarbeiter, diese } \\
\text { können Meinungen } \\
\text { äußern, bevor } \\
\text { entschieden wird }\end{array}$ & $\begin{array}{l}\text { Die Mitarbeiter } \\
\text { entwickeln } \\
\text { Vorschläge, } \\
\text { Vorgesetzter } \\
\text { entscheidet } \\
\text { sich nach } \\
\text { Beurteilung } \\
\text { dieser Vor- } \\
\text { schläge }\end{array}$ & $\begin{array}{l}\text { Vorgesetzter } \\
\text { zeigt Proble- } \\
\text { me und Gren- } \\
\text { zen des Ent- } \\
\text { scheidungs- } \\
\text { spielraums } \\
\text { auf, die Mitar- } \\
\text { beiter ent- } \\
\text { scheiden. }\end{array}$ & $\begin{array}{l}\text { Mitarbeiter } \\
\text { entscheiden, } \\
\text { Vorgesetzter } \\
\text { fungiert als } \\
\text { Koordinator } \\
\text { nach innen u. } \\
\text { vor allem nach } \\
\text { außen }\end{array}$ \\
\hline autoritär & patriarchalisch & informierend & beratend & kooperativ & delegativ & autonom \\
\hline
\end{tabular}

Die Kritik an diesem Modell gilt vor allen Dingen der Eindimensionalität des Ansatzes, denn Tannenbaum/ Schmidt (1958) reduzieren den Führungsstil auf das Entscheidungsverhalten der Führungsperson. Dabei

\footnotetext{
${ }^{74}$ Vgl. Wunderer 2003, 204.

${ }^{75}$ Vgl. Wunderer /Grunwald 1980, Bd I.

${ }^{76}$ Vgl. Wunderer 2003, $206 f$.

${ }^{77}$ Vgl. Staehle 1999, $335 \mathrm{ff} /$ Berthel 2000, $63 \mathrm{ff}$.

78 Tannenbaum/ Schmidt 1958.
} 
bleiben soziale Aspekte der Beziehungsgestaltung ausgeblendet. Das Führungsstilkontinuum von Tannenbaum/ Schmidt wird unter anderem von Wunderer (2003) weiterentwickelt, er entfaltet ein zweidimensionales Konzept, welches den Führungsstil durch die Faktoren Partizipation (Teilhabe) und prosoziale Beziehungsgestaltung (Teilnahme) beschreibt. Führung umfasst danach sowohl eine Machtdimension (gewährte Entscheidungsbeteiligung) als auch eine prosoziale Dimension als Charakteristikum zwischenmenschlicher Qualität. ${ }^{79}$

Prosoziale Dimension

Abb. 5: Führungsstiltypologie nach Wunderer ${ }^{80}$

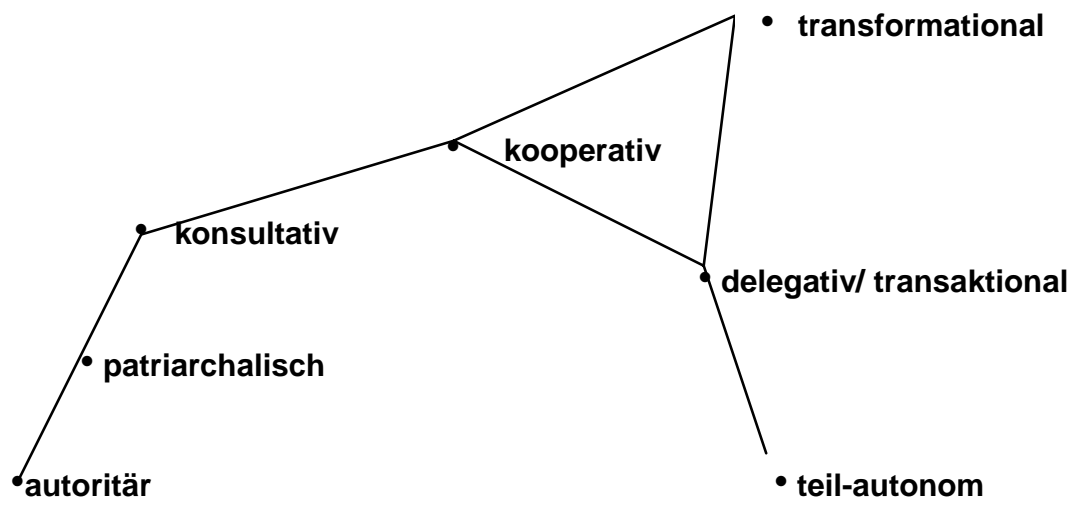

Machtdimension der Führunq

Wunderer (2003) kommt nach seinen Erhebungen in deutschsprachigen Unternehmen zu dem Ergebnis, dass die Mehrzahl der real praktizierten Führungsstile sich zwischen konsultativer und delegativer Führung bewegen. $^{81}$

\subsubsection{Positionsorientierte Führungstheorien}

Innerhalb der positionsorientierten Führungstheorien findet eine Perspektivverschiebung von der Persönlichkeit auf die Position einer Führungsperson innerhalb eines Gefüges statt. Führungserfolg wird daran gemessen, wie sich diese Anforderungen mit der eigenen Definition des Stelleninhabers decken und inwieweit die Erwartungen der Bezugsgruppe erfüllt werden. In der Rollentheorie, die als Hauptansatz gilt, definieren die Erwartungen von anderen sozialen Positionen (z.B. Lehrpersonen, Eltern, Schülerinnen und Schüler) an eine Position (z.B. Schulleiterin/ Schulleiter) die Führungsrolle. Unter Rolle wird ein Bündel normativer Erwartungen von unterschiedlichen Erwartungsträgern, die sich an den Inhaber einer bestimmten sozialen Position richten, verstanden. ${ }^{82}$

In diesem Zusammenhang stehen strukturelle Rollenkonflikte, die sich aus den unterschiedlichen Erwartungen an dieselbe Führungsperson ergeben.

\footnotetext{
${ }^{79}$ Vgl. Wunderer 2003, 210.

${ }^{80}$ Wunderer 2003, 248.

${ }^{81}$ Vgl. Wunderer 1995.

${ }^{82}$ Vgl. Wunderer 2003, 294.
} 
Neuberger (1994) unterscheidet sechs klassische Rollenkonflikte der Führungskraft. ${ }^{83}$

- Intra-Sender

- Inter-Sender-Konflikt

- Inter-Rollen-Konflikt

- Person-Rollen-Konflikt

- Rollen-Ambiguität

- Rollen-Überlastung
Widersprüchliche Erwartungen an sich selbst

verschiedene Positionsinhaber richten sich widersprechende Erwartungen an die Führungskraft

Unterschiedliche Rollenzugehörigkeiten konfrontieren mit Erwartungen

Rollenerwartungen und Selbstbild kollidieren

Erwartungen an die Führungskraft sind unklar

Die Menge der positionsspezifischen

Anforderungen überfordert die Führungskraft

Die Rollentheorie macht auf die widersprüchlichen Anforderungen und Erwartungen aufmerksam, die an die Führungsposition gestellt werden. Zugleich zeigt sie, dass der Führungserfolg nicht nur von der Person des Führers abhängig ist, sondern ebenso von strukturellen Gegebenheiten beeinflusst wird. Die Rollentheorie der Führung ist für Theorie und Praxis von großer Bedeutung und bietet die Möglichkeit einer dynamischen Situationsorientierung.

\subsubsection{Situationstheorien der Führung}

In den situationstheoretischen Führungstheorien wird eine Vielzahl von Einflussfaktoren aus den bereits beschriebenen Theorien einbezogen. Der Führungserfolg wird nicht an die Führungseigenschaften gekoppelt, sondern in Abhängigkeit von einem situativen Kontext, in dem Führungsperson und Geführte interagieren, betrachtet. Das Führungsverhalten wird in Abhängigkeit von der Gruppe - den Geführten-, der Aufgabe und der Führungssituation analysiert.

Das auch heute noch beachtete Kontingenzmodell von Fiedler wurde bereits in den sechziger Jahren entwickelt. Fiedler $(1967)^{84}$ geht davon aus, dass die Effizienz von Führung von den motivationalen Orientierungen des Führers sowie von organisationsinternen situativen Konstellationen abhängt.

Die zugrunde liegende Dimension ist der Führungsstil, dem Fiedler(1967) drei Situationsvariablen gegenüberstellt: Aufgabenstruktur, Positionsmacht des Führers und Führer-Mitarbeiter-Beziehung. Er kommt zu dem Ergebnis, dass aufgabenorientierte Führungspersonen in Situationen mit extremen Ausprägungen erfolgreich sind, z.B. sehr komplexe oder sehr einfache Arbeitsstruktur. Hingegen seien mitarbeiterorientierte Führer in mittleren Günstigkeiten erfolgreich.

Der Ansatz erweitert zwar den Bezugsrahmen und stellt operationalisierbare und systematische Analyseinstrumente vor, Kritik wird jedoch an der einseitigen Ausrichtung auf die Sichtweise der Führungsperson geübt.

\footnotetext{
${ }^{83}$ Vgl. Neuberger 1994 a, $65 f$.

${ }^{84}$ Vgl. Fiedler (1967).
} 
Durch die Situations- und Kontingenzansätze wird belegt, dass die Anforderungen an das Führungsverhalten je nach Situation variieren, dadurch wurde das Verständnis von Führungsprozessen erweitert.

\subsubsection{Ausgewählte Führungskonzepte und ihre Bedeutung für die Schule}

Inwieweit und wie sich in der Praxis bewusst oder unbewusst Führungskräfte ihr Handeln an den theoretischen Ausrichtungen orientieren, ist abhängig davon, welches Führungs- bzw. Organisationsverständnis dominiert und wie die Möglichkeiten der Beeinflussung dieser Unternehmensbzw. Organisationskultur eingeschätzt werden. Idealtypisch werden in der Literatur drei Konzepte unterschieden:

- Autokratisch-zentralistisches Konzept: Führung orientiert sich an einem autoritativen Entwurf, welcher auf Befolgung der Anweisungen abzielt, Respekt und formale Autorität stehen im Vordergrund.

- Konsultativ-kooperatives Teamkonzept: Die Forderung nach Partizipation und die höhere Qualifikation der Mitarbeiter prägte eine neue Vorstellung von kooperativer Führung, in diesem Konzept ist der Vorgesetzte „Primus inter Pares“ und die Unternehmenskultur wird vom Team gestaltet.

- Kooperativ-delegatives Konzept: Ähnlich wie der zuvor genannte Ansatz zielt das kooperativ-delegative Konzept auf den Einbezug des Mitarbeiters, hier wird noch stärker die Mitverantwortung gefordert, die Aufgabe, das gemeinsame Ziel soll im Zentrum der Arbeit stehen. Kooperation mit dem Anspruch einer klarer Ergebnisorientierung und Problemlösekompetenz sind charakteristische Merkmale dieses Ansatzes. ${ }^{85}$

Aus den Erkenntnissen der Führungsforschung und im Zusammenhang mit Anleitungen für die Führungspraxis entstanden unterschiedliche Führungskonzeptionen, die neben dem Führungsverhalten und dem Führungsstil ebenso die Führungsbeziehung berücksichtigen. Im Folgenden werde ich auf einige Führungskonzeptionen eingehen und die für die Schule relevanten beschreiben.

\subsubsection{1}

Konsultative Führung

Konsultative Führung kann als Vorstufe einer kooperativen-delegativen Führung angesehen werden, denn sie bildet den Einstieg in eine wechselseitige Führungsbeziehung. Bei konsultativer Führung werden Mitarbeiter auf Initiative des Vorgesetzten beratend mit einbezogen, eine selbstinitiierte Einflussnahme wird aber nicht erwartet. Die Entscheidungspartizipation ist gering ausgeprägt. Konsultative Führung ist generell auftragsbezogen und die Entscheidungskompetenz liegt allein bei der Führungsperson, es wird von einer lenkenden Führung gesprochen.

Obwohl das diesem Ansatz zugrunde liegende Führungsverständnis nicht den stärker am Mitarbeiter orientierten Ansätzen entspricht, ist es der am häufigsten erlebte Führungsstil, der in Unternehmen durch Führungsanalysen ermittelt wurde. ${ }^{86}$

\footnotetext{
${ }^{85} \mathrm{Vgl}$. Wunderer 2003, 170.

${ }^{86}$ Vgl. Wunderer 1995.
} 
In den Rahmen einer konsultativen Führung lassen sich z.B. direktionale Führungsstile der Schulleiterinnen und Schulleiter einordnen (vgl. Seite 16 in dieser Arbeit). Dort wo Schulleiterinnen und Schulleiter ihren Tätigkeitsbereich stärker im administrativen und verwaltenden Aufgabenspektrum ansiedeln und eher planende, leitende, koordinierende und kontrollierende Tätigkeiten verfolgen kann von einem konsultativen Führungsverständnis ausgegangen werden.

\subsubsection{Kooperative Führung}

Kooperative Führung hat bereits in den 60er Jahren Eingang in die Diskussion um Führungskonzeptionen gefunden, Kooperative Führung gilt als Führungsmodell, das den Veränderungen von Organisationen und Mitarbeitern entgegenkommt.

Führungsfaktoren wie Kommunikation und Teamorientierung stehen im Mittelpunkt. Konstitutive Merkmale kooperativer Führung sind „Partizipation“(Teilhabe) und „prosoziale Dimension“ (Teilnahme). Kooperative Führung basiert auf der Vorstellung, dass die Führungsfunktion dauerhaft im Miteinander mit den Mitarbeitern ausgestaltet wird und eine hohe Entscheidungsbeteiligung aufweist. Wunderer (2003) nennt vier Dimensionen der kooperativen Führung: ${ }^{87}$

- Zielorientierte soziale Einflussnahme zur Erfüllung gemeinsamer Aufgaben - Ziel-Leistungsaspekt

- in/mit einer strukturierten Arbeitssituation - Kontextaspekt

- Unter wechselseitiger, teilweise symmetrischer Einflussausübung partizipativer Aspekt

- und konsensfähige Gestaltung der Arbeits- und Sozialbeziehung prosozialer Aspekt

Das wichtigste Merkmal kooperativer Führung ist die hohe Interaktionsdichte zwischen Führungsperson und Geführten. Der interaktionelle Aspekt bezieht sich auf die direkte Gestaltung der Beziehung zwischen Vorgesetzten und Mitarbeitern, die zwischenmenschliche Beeinflussung des Verhaltens in Form eines Miteinanderarbeitens, das auf Partizipation und Gleichberechtigung beruht, sollen zu einer aufgabenadäquaten Kooperation führen. ${ }^{88}$ Deutlich wird hier, dass sowohl von der Führungsperson als auch von den Geführten ein hohes Maß an Sozialkompetenz erwartet wird. Die gruppendynamischen Beziehungen können zu Konflikten führen, die durch Aushandeln und Verhandeln geregelt werden müssen.

Kooperative Führung ist zeitintensiv und erfordert eine häufige Anwesenheit der Führungskraft. Andererseits kann durch eine hohe Beteiligung an Arbeits- und Entscheidungsprozessen, z.B. durch die Bildung von Arbeitsgruppen bzw. Projektgruppen, ein eigenständiges Arbeiten erreicht werden, welches Erklärungs- und Überzeugungsaufwand reduziert.

Der strukturelle Rahmen einer kooperativen Führung wird durch die Schaffung institutionalisierter Kooperationssituationen und durch die Gestaltung günstiger Organisationsstrukturen erreicht. Daneben spielen Faktoren eine Rolle, die die Qualität der Vorgesetzen - Mitarbeiterbeziehung beeinflus-

\footnotetext{
${ }^{87}$ Wunderer 2002, 220.

${ }^{88} \mathrm{Vgl}$. Münch 1999, 135.
} 
sen sowie eine generelle Führungsphilosophie, die Kooperation, Wechselseitigkeit und Partizipation wirklich befürwortet.

Neue Arbeitsformen wie Gruppen- und Projektarbeit erhöhen die Bedeutung des wechselseitigen Austauschs. Kooperative Führung ist sicherlich sozial erwünscht und birgt viele Vorteile für Geführte und Führungspersonen, sie entspricht den dominanten Werten unserer Gesellschaft und daher erscheint die Favorisierung dieses Ansatzes nachvollziehbar. ${ }^{89}$ Dennoch soll auch auf die Grenzen und Probleme hingewiesen werden.

Bezüglich der Dimensionen Partizipation und prosoziale Dimension können mögliche Einwände erhoben werden: Partizipation muss institutionell durch Gesetze, Verfassungen abgesichert werden und setzt voraus, dass die Teilhabe an Entscheidungen einem gemeinsamen Leitbild folgt. Die prosoziale Dimension erfordert Gruppen- und Interaktionsprozesse, die von wechselseitiger fachlicher und persönlicher Akzeptanz und gegenseitigem Vertrauen und Willen zur Zusammenarbeit getragen sind. Das bedeutet, dass kooperative Führung sowohl von Führungskräften als auch von den Mitarbeitern tatsächlich gewollt sein muss. Dies ist nicht immer der Fall. Es kommt bei der Bewertung der kooperativen Führung zu einer Differenz zwischen einer hohen kognitiven und sozialen Bedeutungszuschreibung durch die offizielle Bewertung kooperativen Führungsverhaltens und einer wesentlich geringeren Zustimmung bei der tatsächlichen Bewertung und Praktizierung durch Vorgesetzte. Gründe hierfür könnten vor allen Dingen das hohe Maß an gleichen Einstellungen und Überzeugungen für die gemeinsame Sache sein. ${ }^{90}$

In der Diskussion um kooperative Führung in der Schule werden Begriffe wie partizipative Führung, kooperativer Führungsstil und situativkooperative Führung häufig synonym verwendet.

Diese Führungskonzeption entspricht einer Grundhaltung, die letztlich dem dezidierten Bildungs- und Erziehungsziel der Schule selbst entspricht. Die Trennung zwischen den Positionen ebenso zwischen Lehrenden und Lernenden wie auch zwischen Schulleiterin/Schulleiter und Lehrkräften wird nicht mehr eng an den hierarchischen Status gebunden werden, das Abgeben von Verantwortung soll dem Ziel der Demokratisierung der Schule dienen. $^{91}$

In dem von Dubs (1994) formulierten Anspruch an Führung, den er an heutige Schulleiterinnen und Schulleiter stellt, findet sich der Gedanke wieder, dass Schulleitung einen kooperativen Führungsstil verpflichtet ist und im Dienste der Weiterentwicklung steht: „Im Vordergrund der kooperativen Führung steht die Idee, der Lehrerschaft und dem Verwaltungspersonal der Schule bei der Erfüllung ihrer Aufgaben möglichst viele Gestaltungs- und Entfaltungsmöglichkeiten zu geben, damit über eine bessere Motivation bessere Leistungen und eine größere Arbeitszufriedenheit entstehen. Deshalb wird nicht mehr ausschließlich von oben her befohlen und angeordnet sowie sinnlos kontrolliert, sondern Vorgesetzte setzen in ihre

\footnotetext{
${ }^{89} \mathrm{Vgl}$. Wiswede/ Wiendieck 1990, 140.

$90 \mathrm{Vgl}$. Wunderer 2003, $226 \mathrm{ff}$.

${ }^{91}$ Vgl. Huber 2003, 62.
} 
Mitarbeiter Vertrauen, indem sie mit ihnen Ziele vereinbaren (Führung durch Zielsetzung) und innen eigene Aufgaben-, Kompetenz- und Verantwortungsbereiche übertragen (Delegation) sowie einen Führungsstil pflegen, bei dem die Mitarbeiter bei guten sozialen Beziehungen Mitwirkungsmöglichkeiten haben (partizipativ-situativer Führungsstil). “92

In diesem Zitat werden mehrere Führungsstile angesprochen, die noch ausgeführt werden. Die Komponenten der kooperativen Führung (Kooperation, Delegation, prosozialer Aspekt, partizipativer Aspekt und Organisationsaspekt) lassen sich im Kontext Schule finden, z.B. in den Bereichen zielorientierte soziale Einflussnahme zu Erfüllung gemeinsamer Aufgaben (Schulprogramm und Schulentwicklungsmaßnahmen, Unterrichtsentwicklung, wechselseitige Einflussnahme und Teilhabe an Entscheidungen, z.B. Gesamtkonferenzbeschlüsse über Grundsatzentscheidungen, konsensfähige Gestaltung der Arbeits- und Sozialbeziehungen, z.B. Schulklima, Zusammenarbeit, Gremienarbeit). Eine strukturierte Arbeitssituation ist durch Unterrichtsverteilung, also den Unterrichtseinsatz, durch Konferenzen, Dienstbesprechungen und Arbeitsgruppen etc. vorgegeben.

Insbesondere die bereits in Kapitel (2.2) erwähnte Untersuchung von Bonsen et al (2002) brachte Ergebnisse, die die Handlungsdimensionen einer kooperativen Führungskonzeption als besonders relevant für eine erfolgreiche Schulleitung nachweisen konnten. Zielbezogene Führung, Innovationsförderung durch die Schulleitung, Partizipation in der Entscheidungsfindung und Organisationskompetenz. Dabei wird unter zielbezogener Führung der Prozess einer Verständigung über die Leitvorstellungen einer Schule verstanden. ${ }^{93}$

\subsubsection{Delegative Führung}

Die delegative Führung geht gewissermaßen noch einen Schritt weiter als die kooperative Führung, denn hier werden Rechte und Pflichten übertragen bzw. zugewiesen. Die für kooperative Führung geltende Gemeinsamkeit bei der Entscheidungsfindung und der Umsetzung im Team ist weniger ausgeprägt. Vorgesetzte und Mitarbeiter arbeiten unabhängiger und müssen Entscheidungsaktivitäten grundsätzlicher, planmäßiger und systematischer durchführen.

Im Bereich der Beziehungsebene ist die wechselseitige Interaktion geringer, erforderlich ist ein hohes Maß an Vertrauen des Delegierenden in Fähigkeiten des Mitarbeiters.

\footnotetext{
${ }^{92}$ Dubs 1994, 17. Der Terminus „pädagogische Führung“ hat seit Mitte der 80er Jahre in der Literatur zur Schulleitung einen eigenen Stellenwert erlangt. ${ }^{92}$

Der Begriff der Pädagogischen Führung verweist auf die Besonderheit der Führung in einer pädagogischen Organisation und bezieht somit den Systemkontext mit ein. Basierend auf den angloamerikanischen Forschungsergebnissen zum „effective principal“ sieht Dubs (1992) die Aufgabe der pädagogischen Führung über die bloß administrative Führung hinausgehend und Ziele der Führung vornehmlich pädagogische Innovationen sind, wie z.B. Unterrichtsentwicklung zu verwirklichen. Münch (1999) verweist darauf, dass der Begriff „Pädagogische Führung" bisher zu unscharf definiert ist und es sich nicht als ein eigenes Führungskonzept der Schule beschreiben lässt, da lediglich Bezug zu anderen Ansätzen genommen wird. Vgl. Münch 1999, 89ff.

${ }^{93} \mathrm{Vgl}$. Bonsen et al 2002a, 28.
} 
Dem delegativen Führungsansatz liegen drei Dimensionen zugrunde:

- Aufgabenorientierte Delegation: eigenständige Handlungsbereiche mit Festlegung der damit verbundenen Entscheidungs- und Weisungsmacht.

- Zielorientierte Delegation: Dies bezieht sich auf das Konzept der zielorientierten Mitarbeiterführung (Management by Objectives) oder auch transaktionale Führung genannt. Grundprinzipien sind Kenntnisse der Ziele, Kenntnis der Leistungsbeurteilung, regelmäßige Zielüberprüfung. Die Vorteile der zielorientierten Führung liegen in einer Entlastung der Führenden und einer höheren Selbstständigkeit der Geführten in der Zielumsetzung. Durch eine Evaluation der Ziele mit entsprechenden Konsequenzen (z.B. materieller Honorierung bei Zielerreichung, Förderung von Fortbildung bei unbefriedigender Zielerreichung) wird eine unternehmerische Ausrichtung der Mitarbeiter gefördert und gefordert.

- Visionsorientierte Delegation: Ansätze der Managementtheorie beziehen verstärkt Visionen in ihre Überlegungen mit ein und erhofften sich dadurch eine stärkere Identifikation und Motivation der Mitarbeiter durch den Einbezug der Sinnfrage des Handelns. Die Entwicklung einer Vision meint, dass ungenutzte Chancen und Mängel einer bestehenden Situation benannt werden sollen und daraus ein Veränderungsziel - eine Vision als eine Art Zukunftsbild entwickelt werden soll. Wunderer (2003) weist aber deutlich darauf hin, dass der Begriff der Visionen oft zur Leerformel und als modische Instrumentalisierung benutzt wurde. Wenn Visionen oder Zukunftsmodelle nicht auf ihre Realisierbarkeit hin überprüft werden, dann wird aus ihnen eine Vision im wahren Wortsinn, nämlich ein Trugbild.

Das Konzept der delegativen Führung ist abhängig von strukturellen und verhaltensbezogenen Aspekten. Wie bei der kooperativen Führung ist auch in diesem Konzept ein gemeinsames Wertsystem wichtig, damit Selbstständigkeit und Kooperation in einem Rahmen gewährt werden können, der Zielen entspricht, die vorgegeben oder gemeinsam erarbeitet wurden.

Innerhalb der Aufgabenverteilung wird eine klare und eindeutige Regelung von Zuständigkeiten und Verantwortlichkeiten getroffen. Das Beziehungssystem ist von Vertrauen, aber auch einem Verpflichtungsbewusstsein geprägt. Da die Interaktionsintensität minimiert wird, muss auf Seiten der Mitarbeiter eine hohe Verantwortungsbereitschaft und auf Seiten des Vorgesetzten der Wunsch wirklich delegieren - also auch Verantwortung abgeben zu wollen - bestehen.

Ein großer Unterschied zur kooperativen Führung besteht im Kontrollsystem der delegativen Führung. Die Kontrolle ist ergebnisorientiert und institutionell ausgerichtet, d.h. es wird offen mit Zielerreichung oder NichtErreichung umgegangen. Neben dem Anreiz einer intrinsischen Motivation wird von einer variablen Vergütung der erbrachten Leistung ausgegangen. 
Der Aspekt der Delegation ist im schulischen Bereich meist der kooperativen Führungskonzeption untergeordnet und beinhaltet die Übertragung wichtiger Aufgaben an einzelne Lehrkräfte oder Teile des Kollegium, z.B. die Fachgruppen, die Steuergruppe im Rahmen der Schulentwicklung oder Arbeitsgruppen zu gezielten Themen (Methodentraining u.a.), hier können durch die Vorbereitung eines Antrages an die Gesamtkonferenz wichtige Entscheidungen angebahnt und durch Mehrheitsbeschlüsse getroffen werden. Die Aufgabe der Führungsperson, also der Schulleiterin/des Schulleiters, besteht darin, diese Prozesse in ein Gesamtprofil zu integrieren und den Erarbeitungsprozess moderierend zu gestalten.

\subsubsection{Transaktionale Führung}

Das transaktionale Führungsmodell betont die dynamische FührungskraftMitarbeiter-Transaktion. Das Modell geht davon aus, dass Führungsbeziehungen auf der Grundlage von Leistung und Gegenleistung beruhen. Die Zielorientierung mit einem verbindlichen Charakter steht im Vordergrund, d.h. dass man sich auf vorgegebene Ziele konzentrieren muss, dass diese Ziele klar und operational definiert sind. Die Zielverträglichkeit von Arbeitszielen und Bedürfnissen der Mitarbeiter muss dabei analysiert werden und die Fähigkeiten der Mitarbeiter im Sinne der Zielerreichung gefördert werden. Die Ziele selbst können nicht auf ihre Verträglichkeit hin untersucht werden, sondern die zielerreichenden Maßnahmen müssen dahingehend überprüft werden, ob sie ihre Wirkung erreichen oder diese behindern. Dabei ist eine Trennung von Zielsetzungen einerseits auf der Ebene der Produktivität von der Institution (Ziele des Betriebs) und andererseits Zufriedenheit von Menschen (Ziele Mitarbeiter) nicht möglich, sondern es geht um die Integration beider Zielbereiche. ${ }^{94}$

Die Grundprinzipien der transaktionalen Führung sind: Die Zielerreichung wird belohnt und es wird eingegriffen, wenn das Ziel verfehlt wird. Zwei Faktoren spielen dabei eine Rolle: Contingent Reward (Weg - Zielklärung sowie leistungsbezogene Belohnungsvergabe) und Management by Exception (die Geführten sind bemüht ihre Arbeit nach den Zielvorstellungen der Führungspersönlichkeit zu erfüllen und erhalten dafür die gewünschte Belohnung). Es wird deutlich, dass dieses Führungskonzept stark auf den rationalen Nutzen der Zielerreichung ausgerichtet ist. ${ }^{95}$ Dieser Führungsansatz kann in klar strukturierten Unternehmen mit Hierarchien sicher zu einer Rollenklarheit und auch zur Arbeitszufriedenheit führen. In vielen Organisationen wird das Handeln aber nicht nur von Kosten-NutzenÜberlegungen geleitet, daher greift hier der transformationale Führungsansatz.

\subsubsection{Transformationale Führung}

Die transformationale Führung zeichnet sich durch eine stärkere Orientierung an den Überzeugungen und Werten der Mitarbeiter aus, die Führungskraft „transformiert“ Werthaltungen und Motive der Mitarbeiter. Die Führungsperson soll visionäre, anregende Inhalte vermitteln und dadurch die emotionale Beteiligung der Organisationsmitglieder im Sinne der gemeinsamen Ziele erreichen. Die Wirkung transformationaler Führung beginnt dort, wo Belohnungs- und Sanktionsmöglichkeiten oder andere instrumentelle Effekte nicht gegeben sind. ${ }^{96}$ Die Bedeutung von ${ }^{94} \mathrm{Vgl}$. Berthel 2000, 66.

${ }^{95}$ Vgl. Bass 1995, 123. 
strumentelle Effekte nicht gegeben sind. ${ }^{96}$ Die Bedeutung von Zielen und Aufgaben soll transparent und nachvollziehbar sein. Die Führungsperson gilt als Identifikationsperson für Leistung und Erfolg. Statt extrinsischer Belohnung soll eine innere Überzeugung für sachbezogene Werte, Ziele und Aufgaben erreicht werden.

Die transformationale Führungskonzeption integriert vor allem Elemente der kooperativen und delegativen Führung.

In der aktuellen Literatur der Führungsforschung wird der transformationalen Führung große Bedeutung beigemessen, da eine werteorientierte Führung unternehmerisches Denken fördert, weil sie Mitarbeiter sowohl kognitiv als auch emotional anspricht. Diese vertrauensbildende und anerkennende Haltung hat Einfluss auf die Eigenverantwortung und fördert die Identifikation mit dem Unternehmen bzw. der Organisation. ${ }^{97}$

Kritik wird an der transformationalen Führungskonzeption insofern geübt, als dass sie nicht allein als Konzeption Bestand haben kann, sie setzt die Ziel- und Aufgabenorientierung voraus und kann nur ein Bestandteil einer übergeordneten Führungskonzeption sein. Im Zusammenhang mit der häufig idealisierten Darstellung dieses Führungsansatzes wird erneut die Führungsperson mit ihrer persönlichen Ausstrahlung, ihrem Charisma in den Mittelpunkt gerückt. Diese Tendenz steht aber den Autonomiebestrebungen der Mitarbeiter, einer zunehmenden Teamorientierung und betrieblichen Organisationsabläufen entgegen. ${ }^{98}$

Dieses ursprünglich von Burns (1978) in den USA entwickelte Führungskonzept wurde von Leithwood (1999) auf die Schule übertragen. ${ }^{99}$ Die Veränderungsansätze, die sich in den oben genannten Zielen andeuten, münden in die für die Schule richtungweisende Konzeption des „transformational leadership“ bzw. transformationale Führungskonzeption. Die transformationale Führung konzentriert vornehmlich auf die Aufgaben der Gestaltung eines kooperativen und engagierten Klimas im Kollegium, so verweisen Ergebnisse einzelner Forschungsbeiträge darauf, dass "gute" Schulen sich durch das Vorherrschen eines Vertrauensklimas auszeichnen würden. Dahinter steht die Überzeugung, dass sich Schulen aus sich selbst heraus weiter entwickeln sollen (vgl. Schulentwicklung). Es werden reale Veränderungen bzw. Verbesserungen innerhalb der Schule angestrebt, die als qualitative Ziele beschrieben werden. Die Führungsverantwortung besteht darin, die Entwicklung von gemeinsamer getragener Zielvorstellung zu ermöglichen und voranzutreiben, eine Verbesserung der schulinternen Kommunikation positiv zu beeinflussen und effiziente Strategien für Entscheidungsfindung und Problemlösung zu entwickeln. Die Führungskraft muss ein hohes Maß an Motivations- und Einbindungskompetenz besitzen.

Parallel zu den theoretischen Erkenntnissen werden Aufgaben für Schulleiterinnen und Schulleiter beschrieben, die den Merkmalen einer trans-

\footnotetext{
${ }^{96}$ Ebd., 124.

${ }^{97}$ Vgl. Wunderer 2003, 538.

${ }^{98}$ Vgl. Bass 1995 u. Wunderer $2003,247$.

${ }^{99}$ Vgl. Burns 1978.
} 
formationalen Führung entsprechen: Schulleiterinnen und Schulleiter sollen das Kollegium bei der Entwicklung einer kooperativen und professionellen Lernkultur unterstützen, aktive Personalentwicklung betreiben und effektive Problemlöse- und Umsetzungsstrategien fördern. ${ }^{100}$

\subsubsection{Zusammenfassung}

Die Darstellung der Führungsforschung verdeutlicht, dass auf der einen Seite versucht wird, die Führungssituationen in ihrer Komplexität zu analysieren und auf der anderen Seite die Anleitungen für die Führungspraxis je nach Standpunkt und Zielsetzung differenzierter werden, so zu Beispiel mit den Konzept der transformationalen Führung, in dem eine Mitarbeiterorientierung zu gemeinsamen Zielsetzungen und letztendlich zur Weiterentwicklung führen soll. Auf der Seite der Führungspraxis gilt es Veränderungen innerhalb der Betriebswirtschaft bzw. des schulischem Kontextes permanent zu berücksichtigen und darauf zu reagieren, dadurch entwickeln sich jeweils neue Akzentuierungen. Die größten Übereinstimmungen zwischen Führungskonzepten für die Betriebswirtschaft und der Schule scheinen sich im Modell der kooperativen Führung zu treffen. Kooperative Führung betont den Aspekt der Zusammenarbeit und Beteiligung und bezieht gleichermaßen die Mitarbeiter/-innen und Führungspersonen mit ein. Zwei Aspekte gilt es dabei zu berücksichtigen, erstens die Schule ist weiterhin ein hierarchisches System und die Schulleiterinnen und Schulleiter sind Dienstvorgesetzte, die bei entstehenden Konflikten nicht immer als symmetrische Kommunikationspartner fungieren können. Zweitens die Theorie der kooperativen Führung geht davon aus, dass gemeinsam Ziele festzulegen sind, die den Organisationszielen entsprechen und alle gemeinsam ihren Beitrag zur Erreichung dieser Ziele leisten. In jedem Unternehmen und in jeder Schule wird es Situationen geben, die Gegensätzlichkeiten von Ansprüchen der Mitarbeiter und der Führungskräfte aufzeigen, wo auf der einen Seite personen- und organisationsbezogene Bedingungen miteinander konkurrieren.

Meiner Einschätzung nach hatte Ende der 90er Jahre in den Schulen die Profilierung der Einzelschule, die in der Formulierung eines Schulprogramms münden sollten, Vorrang vor der Orientierung auf Leistungsmessung der Schülerinnen und Schüler. In den letzten Jahren ab 2000 wird in Niedersachsen durch knappere Lehrerversorgung wieder stark die Schulleitung in ihrem Organisationskompetenz gefordert, um zunächst einmal Unterricht zu sichern. In dem einen Fall müsste Schulleitung kooperative Arbeitsgruppen initiieren und die Implementierung neuer Akzente in der Schule prüfen, in dem anderen Bereich müsste Schulleitung konkret in den Unterricht gehen, Leistungsergebnisse kritisch hinterfragen und Evaluationsinstrumente für Unterricht einsetzen. Im Bereich der Unterrichtsversorgung geht es um Personalentwicklung vor Ort und Ressourcensicherung bei der Schulbehörde.

Wie bereits oben kritisch angemerkt, stellt sich für die Schulleiterinnen und Schulleiter immer die Frage, in welchem Ausmaß eigene Ziele zum Tragen kommen, denn einerseits tritt an schulische Führungskräfte der Anspruch heran, sich in der Zusammenarbeit mit Lehrerinnen zurück zu

${ }^{100}$ Vgl. Buhren /Rolff 2002, 53ff. 
nehmen, damit Entwicklungen von Lehrerteams angeregt werden können, und andererseits setzen Schulentwicklungsprozesse voraus, dass die Führungskräfte eigene Visionen und Ziele entwickeln und diese auch zu vermitteln wissen. Die Besonderheit einer Führungstheorie liegt darin, dass es um das Verhältnis von 'Führenden' und 'Geführten' geht, von Führenden kann zu recht erwartet werden, dass sie sagen, wo es lang geht und/ oder den Prozess organisieren, der die Zielfindung zum Ziel hat. Ein Kernproblem scheint aus meiner Sicht das Spannungsverhältnis zwischen den Rechten der Schulleiterinnen und Schulleiter und den Rechten der Kolleginnen und Kollegen zu sein, denn formal ist die Rechtsposition der Schulleiterinnen und Schuleiter zwar hierarchisch, sie werden bei formal-rechtlichen Fehler zur Rechenschaft gezogen, in ihrem Alltagshandeln fordern aber nicht nur Programmatiken, sondern auch die vorgesetzte Dienstbehörde ein kooperatives Vorgehen und Konfliktlösungsstrategien auf einer symmetrischen Ebene. Was passiert aber, wenn gemeinsame Zielsetzungen nicht umgesetzt werden, wenn Kolleginnen und Kollegen sich nicht an den Zielen der Schule orientieren? Die bisher noch stark traditionell ausgerichteten Strukturen der Organisation Schule lassen Führungskonzeptionen, die eine Verbindlichkeit in der Zielerreichung einfordern, z.B. transaktionale Führung, bisher noch nicht zu. Es wäre meiner Einschätzung nach sehr lohnenswert, ob eine Korrelation zwischen höherem Weisungsrecht der Schulleiterinnen und Schulleiter und der Schulqualität besteht.

\subsection{Organisationstheorie}

Im Hinblick auf das Thema meiner Untersuchung werde ich aus dem Kontext der Organisationstheorie den Bereich Organisationsentwicklung hervorheben und auf die Besonderheiten der Schule als Organisation eingehen. Denn aus den Modellen der Organisationsentwicklung ent-wickelten sich die Konzepte der Schulentwicklung, die wiederum Einfluss auf die Rolle und die Aufgaben der Schulleiterinnen und Schulleiter haben.

Es bestehen grundsätzlich zwei unterschiedliche Organisationsverständnisse: Einerseits das instrumentelle Organisationsverständnis, nach dem Organisationen ein zeitlich überdauerndes, fest gefügtes Gebilde sind. Auf der anderen Seite wird der Blick auf die Prozesse des Organisierens selbst gerichtet. Organisationen werden als dynamische Systeme aufgefasst, die sich per se im Wandel befinden. Aus dieser Perspektive heraus sind die Akteure der Organisation in der Lage die organisatorischen Erfordernisse (Aufbau/ Aufgaben) entsprechend der eigene Anforderungen und der Umwelt zu gestalten. ${ }^{101}$

Charakteristische Merkmale von Organisationen sind:

- Organisationen sind aus Individuen und Gruppen zusammengesetzt,

- streben nach der Erreichung bestimmter Ziele oder Zwecke,

- und diese Ziele werden durch rationale Koordination und Führung erreicht,

- Organisationen sind auf Dauer angelegt. ${ }^{102}$

\footnotetext{
${ }^{101}$ Vgl. Bormann 2002, 27.

102 Staehle 1999, 416.
} 
Für die Schule gilt ebenso wie für andere Arbeitsorganisationen, dass ihre Möglichkeiten der Problemlösung sowohl von übergreifenden Bedingungen als auch von Einstellungen und Verhaltensweisen ihrer Mitglieder abhängig sind.

\subsubsection{Entwicklung der Organisationstheorie}

Die Darstellung der Organisationstheorie orientiert sich auch heute noch an der 1961 von Scott entwickelten historischen Gliederung, die Siedenbiel (2001) aufgreift. Die klassische Organisationstheorie bildet die Grundlage für die Organisationslehre. Die neoklassische Organisationstheorie basiert auf dem Human-Relations-Ansatz, der entscheidenden Einfluss auf die Ausbildung der Organisationsentwicklung hatte, und der betrieblichen Organisationslehre. Als zentrale Kategorien der modernen Organisationstheorie werden Entscheidungen, System und Situation angesehen. ${ }^{103}$

Abb. 6: Entwicklung der Organisationstheorie leicht verändert nach Bormann 2002 und Bonsen 2003. ${ }^{104}$
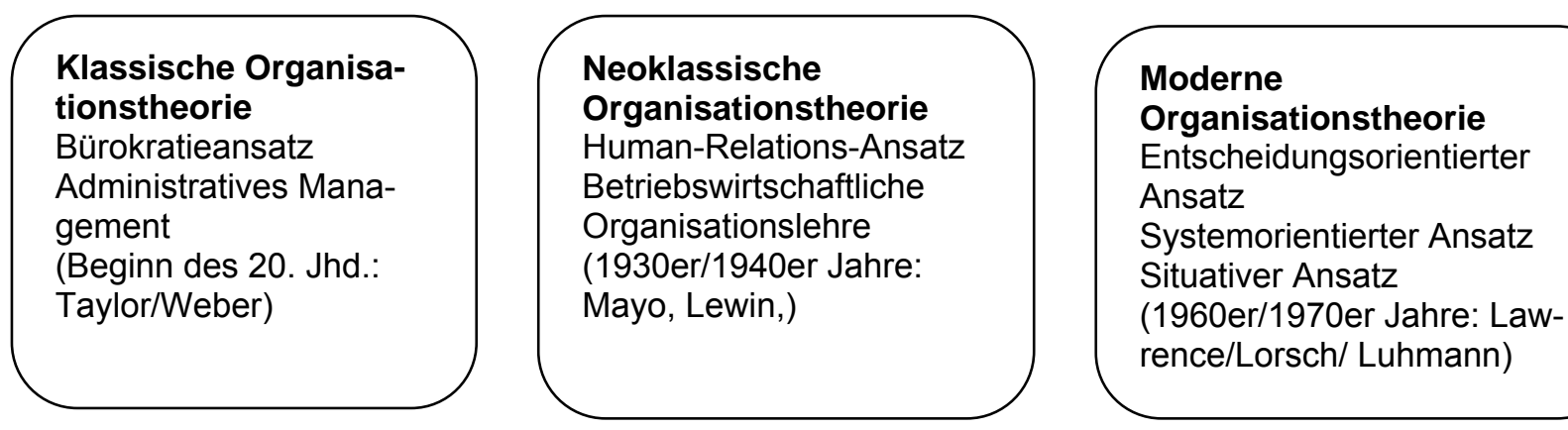

Klassische Organisationstheorien

Insbesondere der Bürokratieansatz mit den konstitutiven Prinzipien einer Bürokratie, z.B. eine geregelten Arbeitsteilung mit einer personenunabhängigen und generell festgelegten Verteilung von Zuständigkeiten, ein System von Über- und Unterordnung von Stellen sowie eindeutige Vorgaben zur Amtsführung, beschreiben eine bürokratisch strukturierte Organisation. Dieser Ansatz kann heute noch als Modell angesehen werden, an dem es möglich ist, zu messen, wie bürokratisch eine Organisation ist. Die Organisation Schule im Rückgriff auf die bürokratietheoretischen Ausführungen als formale Organisation zu beschreiben, wie es Überlegungen der Organisationssoziologie in den 70er und 80er Jahren taten, greifen aber nur den formalen Aspekt der Organisation auf.

\section{Neoklassische Organisationstheorien}

Die Human-Relations-Theorie aus der Neoklassischen Organisationstheorie betrachtet eine Organisation als ein soziotechnisches System, das sich mit den Gegebenheiten der Umwelt auseinandersetzen muss. Neben den formalen Strukturen einer Organisation werden die informellen Aspekte

\footnotetext{
${ }^{103}$ Zur Genese von Organisationstheorien siehe ausführlich Philipps 1999, Siedenbiedel 2001, Bonsen 2003.

${ }^{104}$ Vgl. Siedenbiedel 2001, 36 und Bonsen 2003, 69.
} 
(Machtverteilung, Status, Kommunikation, zwischenmenschliche Beziehungen) berücksichtigt. ${ }^{105}$

Der Human-Relation Ansatz gab entscheidende Impulse für das Konzept der Organisationsentwicklung. Die Organisationsentwicklung gilt zunächst als Praxiskonzept, welches erst rückblickend theoretisch fundiert wurde. Als erste Form der Organisationsentwicklung im deutschsprachigen Raum wird das Ende der sechziger Jahre entwickelte Konzept der Institutionsberatung von Fürstenau ${ }^{106}$ angesehen. Die Konzepte der Organisationsentwicklung zeichnen sich durch ihre ganzheitliche Sicht aus, es werden personen- und aufgabenbezogenen Aspekte berücksichtigt. Daher werden die Ansätze der Organisationsentwicklung als förderlich für die weichen (Organisationsklima, Arbeitszufriedenheit) wie auch die harten (Quantität und Qualität der Arbeitsergebnisse) Erfolgkriterien angesehen ${ }^{107}$

\section{Moderne Organisationstheorien}

Innerhalb der modernen Organisationstheorie sind vor allen Dingen die verhaltenswissenschaftliche Entscheidungstheorie und die systemorientierte Organisationstheorie zu nennen.

Eine Grundfrage der Entscheidungstheorie ist, unter welchen Bedingungen Menschen sich in Organisationen engagieren und warum sie motiviert sind, die zum Erhalt der Organisation notwendigen Beiträge zu leisten. Der theoretische Ansatz dieser Konzeption geht davon aus, dass Organisationen aus Handlungen bestehen. Um wichtige Ziele erreichen zu können, müssen Individuen Beiträge leisten, dabei treffen Organisationsmitglieder nicht immer objektiv rationale Entscheidungen (Prinzip der beschränkten Rationalität). Die Analyse des individuellen Entscheidungsverhaltens steht im Mittelpunkt der gesamten Theorie. ${ }^{108}$

Ausgehend von dieser Theorie hat sich beispielsweise der Ansatz des organisationalen Lernens entwickelt, der durch Peter Senges (1990) die „fünfte Disziplin“ einen Aufschwung erlebt. Der beschränkten Rationalität menschlichen Handelns wird in diesem Ansatz die Lernfähigkeit gegenübergestellt. Aufgrund von Erfahrungen passt der Mensch sein Verhalten durch vielfache Versuchs-Irrtums-Prozesse einer guten Lösung an und revidiert gegebenenfalls seine Handlungsweisen. Das Konzept des organisationalen Lernens geht davon aus, dass derartige Prozesse auch auf der Ebene der Organisation beobachtet werden können.

Der systemorientierte Ansatz der Organisationstheorie resultiert aus unterschiedlichen Forschungsrichtungen, denen gemeinsam ist, dass sie Organisationen als Systeme deuten. ${ }^{109}$ Die Zustandsveränderungen offener Systeme hängen einerseits vom Austausch mit der Umwelt und andererseits auch von systeminternen Transformationsprozessen ab. Systeme können somit Außen- und Inneneinflüssen gegenüber geschlossen oder offen, stabil oder instabil, lern- oder lernunfähig sein. Der Erfolg und Be-

\footnotetext{
${ }^{105}$ Vgl. Kieser 2001, 115.

106 Vgl. Fürstenau 1972, 511ff.

107 Vgl. Kieser 2001, 120f.

${ }^{108}$ Vgl. Siedenbiel 2001, 64.

${ }^{109}$ Vgl. Luhmann1984.
} 
stand einer Organisation hängt davon ab, inwieweit sie Entwicklungen ihrer Umwelt frühzeitig wahrnimmt und notwendige Veränderungen initiiert. Die Aspekte Dynamik, Komplexität und Zielorientierung und die Vorhersehbarkeit von Verhaltensweisen spielen innerhalb der systemischen Betrachtungsweise eine wichtige Rolle. ${ }^{110}$

Die Fokussierung auf die Führungskraft als „Organisator" dieser Ordnung wurde in modernen Führungs- und Organisationstheorien relativiert, denn Ordnung kann nicht als Ergebnis der Maßnahmen einer Person angesehen werden, sondern ist vielmehr Produkt unterschiedlicher relativ autonomer, auf sich selbst bezogener, vielfältiger Strukturen und Verhaltensweisen. Konzepte der Selbstorganisation gehen von einer nur begrenzt zielgerichteten Gestaltbarkeit einer Organisation aus. ${ }^{111}$

In selbstorganisierten Systemen werden Aktivitäten und Gestaltungsmaßnahmen über das ganze System verteilt, wodurch Entscheidungen dezentral getroffen werden und Informationen vom gesamtem System aufgenommen und verarbeitet werden können. Durch diese Ansätze hat sich das Bild von Führung, Organisation und Organisationsgestaltung stark verändert. Mit dem Begriff Selbstorganisation wird die Aufmerksamkeit auf die eigene Flexibilität, die Selbstentwicklung und Erneuerungsprozesse gelenkt. ${ }^{112}$

\subsubsection{Schule als Organisation}

Die Bestimmung der Schule als Organisation erfolgt vor dem Hintergrund des Schulsystems in seiner Gesamtheit, die Schule ist in staatlich gefasste formale Strukturen des Bildungssystems eingebunden und insofern eine formale Organisation. Dennoch hat jede Einzelschule ein eigenes spezifisches Profil (Abläufe, Routinen, eigene Regeln und Absprachen etc.), welches sich durch dynamische Prozesse innerhalb ihrer Organisation herausbildet. ${ }^{113}$

Als Teil des Bildungssystems und durch die Formalisierung ihrer Strukturen stellt Schule zunächst ein geschlossenes System dar, welches auf einer bürokratischen Administration aufbaut. Die Schule als Bildungs- und Erziehungseinrichtung dient dem Zweck, die Erfüllung gesellschaftlicher Aufgaben (Bildungs- und Erziehungsauftrag) möglichst personenunabhängig sicherzustellen. Dabei besteht folgende Grundstruktur:

- Eine arbeitsteilige Funktionsgliederung, die Lern- Lehr- Beratungsund Verwaltungsaufgaben den Inhabern spezifischer Positionen zuweist.

- Formale Lehr- und Verwaltungsrollen mit Amtscharakter mit formalisierter Einberufung auf der Basis von Kompetenz und Bewährung, gesetzliches Anstellungsverhältnis als Landesbeamter.

- Hierarchische Ordnung der Ämter auf der Basis gesetzlich definierter Amtsvollmacht, formellen Entscheidungsmodi und vorgezeichneten Kommunikationslinien (Dienstweg), Weisungsbefugnisse.

\footnotetext{
${ }^{110}$ Vgl. Siedenbiedel 2001, 68.

111 Vgl. Probst 1992, 2259.

112 Vgl. Probst 1992, 2266.

${ }^{113}$ Vgl. Türk 1989, Wissinger 1996, 54ff und Bormann 2002, 31.
} 
- Regelhaftigkeit der Handlungsvollzüge, die durch Ziel- und Durchführungsbestimmungen dienstlicher Maßnahmen der willkürlichen Amtsausübung Grenzen setzt. ${ }^{114}$

Schule stellt gleichzeitig ein offenes System dar, welches sich mit ständig wandelnden, unvorhersehbaren Bedingungen und Aufgaben auseinander setzen muss. Die Schule ist auf den einzelnen Menschen als relativ autonomen Entscheidungsträger angewiesen und zeichnet sich durch die Besonderheit aus, dass der Organisationserfolg vom Kooperationswillen der Mitglieder bestimmt wird. ${ }^{115}$

Rolff (1995) stellt zwei idealtypische Organisationen gegenüber, die professionelle Organisation und die bürokratisch-mechanische Organisation. Die professionelle Organisation (z.B. Universität) zeichnet sich durch transzendierende Inhalte, begrenzte Technologisierbarkeit, Fallverstehen, Selbstkontrolle, teamartige Kooperation und Reflexivität der Ziele aus - die bürokratisch-mechanische (z.B. konventionelle Fabrik) durch Informationsverarbeitung, unbegrenzte Technologisierbarkeit, instrumentelle Tätigkeit, Fremdkontrolle, gefügeartige Kooperation und operative Ziele aus . ${ }^{116}$ Für die Schule gilt, dass sie in ihrer Aufbauorganisation tendenziell bürokratisch organisiert ist, an ihre Ablauforganisation bzw. an das pädagogische Handeln aber professionelle Anforderungen gestellt werden, was nach Rolff (1995) zu Diskrepanzen zwischen pädagogischen Idealvorstellungen und realen Alltagerfordernissen führen kann.

Aus den systemisch orientierten Ansätzen der Organisationstheorie entwickelte sich ein Bild von Schule als selbstlernende und veränderungsfähige Organisation. Die Grundidee ist dabei, dass Schulen nicht mehr nur LernOrganisationen, d.h. die Organisation systematischer Lernprozesse, sondern als Organisation selbst zu Lernprozessen fähig sind. Dieser Ansatz hat sich seit Beginn der neunziger Jahre durchgesetzt, man sprach auch vom Paradigmenwechsel. ${ }^{117}$

Der Terminus „Organisationspädagogik“, der von Rosenbusch (1988) erstmals in die Diskussion gebracht wurde, entwickelt sich als Arbeitsbereich der Pädagogik. Die Organisationspädagogik beschäftigt sich mit dem Einfluss der Organisiertheit von Erziehung und Unterricht im Hinblick auf den Einzelnen und die Schule als System. ${ }^{118}$ Das Schulleitungshandeln wird als pädagogisches Organisationshandeln verstanden und soll einen Beitrag zum Gelingen der pädagogischen Zieltätigkeit auf allen Ebenen der Organisation Schule leisten. Damit ist die Schulkultur, die Unterrichtstätigkeit, die Kooperation der Lehrkräfte und letztendlich der Schülerinnen und Schüler gemeint. Die administrativen und strukturellen Bedingungen sind den pädagogischen Prinzipien unterzuordnen und so zu gestalten, dass sie pädagogisches Handeln optimal ermöglichen, z.B. Aufteilung von Aufgaben und Verantwortlichkeiten, Konferenzstruktur etc. ${ }^{119}$

\footnotetext{
${ }^{114}$ Vgl. Fend 1981, 232

${ }^{115}$ Vgl. Steffens/ Bargel 1993, 23.

116 Vgl. Rolff 1995, 135ff.

117 Vgl. Rolff 1992 a, 290.

${ }^{118}$ Vgl. Rosenbusch 2005, 6.

${ }^{119}$ Vgl. Rosenbusch 1997, 333.
} 
Für Schulleiterinnen und Schulleiter bedeutet dies, dass sie einer Führungskonzeption des "organisationspädagogischen Managements" folgen. Auch in diesem Ansatz, der versucht, die Trennung Administration und Erziehung aufzuheben, steht die Kooperation als Handlungsprinzip im Mittelpunkt.

Diese Beschäftigung mit Fragen der Organisation war der Auslöser für eine neue Richtung in der Schulentwicklung und fand ihren Ausdruck in Begrifflichkeiten wie „Erweiterte schulische Verantwortung“, „Organisationsentwicklung an Schulen“, „Schulisches Management“, Selbstständige Schule oder aktuell in Niedersachsen das Modell der "Eigenverantwortlichen Schulen“. Ausgehend von den Überlegungen der Organisationsentwicklung wurden Modellprojekte in Schulen initiiert, die das Ziel hatten, die Kompetenzen der Einzelschule im Hinblick auf die inhaltliche, personelle und auch finanzielle Gestaltung zu erhöhen.

\subsubsection{Schulentwicklung}

Der Begriff der Schulentwicklung findet verstärkt seit zwei Jahrzehnten Verwendung, davor wurde er aber bereits in den 70er Jahren benutzt. Ähnlich wie die Begriffe Innovation und Schulreform betont auch der Begriff Schulentwicklung das prozesshafte Geschehen, im Gegensatz zu konkreten Einzelmaßnahmen oder umfassenden Reformmodellen liegt die Akzentuierung der Schulentwicklung auf der Veränderung des pädagogischen Handelns und der organisatorischen Strukturen. Die Prozesshaftigkeit verweist darauf, dass Schulentwicklung nicht irgendwann ein Ende hat, sondern als Daueraufgabe angesehen wird. Die Tatsache, dass die Schullandschaft durch ein hohes Maß an Hetreogenität gekennzeichnet ist, führt zu der Erkenntnis, dass gezielte Entwicklungsprozesse nicht verordnet werden können, vielmehr sind die Auswirkungen und Umsetzungen staatlicher Interventionen abhängig vom Handeln der einzelnen Schulleitung bzw. des Lehrerkollegiums. ${ }^{120}$

Schulentwicklung kann durch unterschiedliche Motive und Interessen initiiert sein und verfolgt jeweils spezifische Ziele, dabei spielen sowohl ökonomische Aspekte eine Rolle (finanzielle Kosten für Reformen) als auch das Engagement, die Innovationsfreudigkeit der Beteiligten und Betroffenen der Schulpraxis. Seit Mitte der 90er Jahre stehen die Qualitätsentwicklung und Qualitätssicherung im Vordergrund des Interesses. Innerhalb der Qualitätsverbesserung in schulischen Bereichen wird von der Trias (Personalentwicklung/ Unterrichtsentwicklung/ Organisationsentwicklung) der Schulentwicklung gesprochen. ${ }^{121}$

Das Konzept der Schulentwicklung bezieht sich vornehmlich auf die Ebene der Einzelschule, dennoch müssen bildungspolitische Entscheidungen getroffen werde, die die Akteure dieser Entwicklung mit dem nötigen Wissen und den erforderlichen Ressourcen ausstatten. Die Entwicklung der Schule wird einerseits auf der Ebene des Handelns einzelner Schulleitungen und Lehrkräfte zurückgeführt und andererseits haben die Ebenen Administration, Politik, Wirtschaft, die die Gestaltungsspielräume beschließen und ermöglichen können, einen erheblichen Einfluss. Daher fordern Altrichter und Rolff (1998) die Mikroebene der Einzelschule um die

\footnotetext{
${ }^{120}$ Vgl. Rolff 1993, 175.

${ }^{121} \mathrm{Vgl.}$ Rolff 1998, 16.
} 
Makroebene der politischen Steuerung des Systems Schule zu erweitern. ${ }^{122}$

Im Zuge von mehr Schulautonomie muss mehr Verantwortung für das Management dieser Schulentwicklung von den Schulen selbst übernommen werden. Seit 2000 wird der Autonomiebegriff zum Leitbegriff des qualitätsorientierten Schulentwicklungsdiskurses, aufgrund ihrer staatlichen Verfasstheit kann jedoch der Einzelschule keine vollkommene Autonomie übertragen werden, dennoch werden den einzelnen Schulen in Teilbereichen erweiterte Handlungsspielräume zugestanden. Beispiele hierfür sind die Budgetierung von Lehrerstunden und die so genannten "schulscharfen Stellen" bei denen die Schule größeres Mitspracherecht bei der Neueinstellung hat. ${ }^{123}$

\subsubsection{Stationen der Schulentwicklungsdiskussion}

Der Verlauf und die unterschiedliche Akzentuierung des Schulentwicklungsdiskurses lassen sich für den deutschsprachigen Raum grob in drei Phasen einteilen:

Abb. 7: Schulentwicklungsstationen

\begin{tabular}{|c|c|c|}
\hline $\begin{array}{l}\text { Beschreibung der } \\
\text { Schule als Organisa- } \\
\text { tion } \\
\text { Späte 1970er/frühe } \\
\text { 1980er Jahre }\end{array}$ & $\begin{array}{l}\text { Anpassung und } \\
\text { Transfer von Orga- } \\
\text { nisationsenicklung- } \\
\text { sansätzen auf die } \\
\text { Schule } \\
\text { 1980er Jahre }\end{array}$ & $\begin{array}{l}\text { Eigenständiger Schul- } \\
\text { entwicklungsdiskurs } \\
\text { mit drei Ausrichtun- } \\
\text { gen: } \\
\text { - Institutionelle } \\
\text { Schulentwicklung } \\
\text { - Pädagogische } \\
\text { Schulentwicklung } \\
\text { - Lernende Schule } \\
\text { 1990er Jahre }\end{array}$ \\
\hline
\end{tabular}

Zunächst geht es um die Definition der Schule als Organisation mit ihren für sie konstitutiven Abläufen. Im Zuge der Übertragung von Ansätzen der Organisationsentwicklung auf die Schule werden zunehmend Probleme bei der schulischen Entwicklung reflektiert. Deutlich wird, dass die Initiative für die Entwicklung bei den Lehrkräften liegt, die weitgehend unabhängig voneinander agieren. Die Konsequenz daraus ist, dass die Einzelschule als Ort der Entwicklung in den Mittelpunkt der Debatte gerückt wird. Bulla (1982) entwirft ein Bild von Schulen als "front-line-organizations" und beschreibt Hindernisse in Hinblick auf die Kontrolle der Handlungen. Probleme entstehen durch die starke Individualisierung der Lehrkräfte, dem Mangel an professioneller Kommunikation und Kooperation. Das Konzept der Organisationsentwicklung würde sich zur Überwindung dieser Probleme anbieten, da es zur Veränderungen auf der individuellen, auf der interpersonellen und auf der Ebene des Gesamtsystems beitragen kann. ${ }^{124}$ In den folgenden Jahren wird die Schulentwicklungsdiskussion stärker aus einer lösungsorientierten Perspektive herausgeführt. Schule wird als inno-

\footnotetext{
${ }^{122}$ Ebd.

${ }^{123}$ Vgl. Fullan 1999, 222.

${ }^{124}$ Vgl. Bulla 1982, $35 f$.
} 
vative soziale Organisation betrachtet, die trotz ihrer ambivalenten Struktur, durch Maßnahmen der Organisationsentwicklung gestaltungsfähig sein kann. Ein entscheidendes Moment stellt die Partizipation der von Veränderung Betroffenen dar. Es entwickelt sich das Leitbild einer partizipativen und innovativen Schule. Für Rolff (1986) bedeutet Organisationsentwicklung in Schulen „ein offenes, planmäßiges, zielorientiertes und langfristiges Vorgehen im Umgang mit Veränderungsanforderungen und Veränderungsabsichten in sozialen Systemen“. ${ }^{125}$ In dieser Phase der Diskussion wird versucht, die Ambivalenz zwischen Person und Organisation, Struktur und Verhalten aufzuheben. Die Mitglieder der Organisation Schule werden als Träger von Entwicklungsprozessen in den Mittelpunkt der Auseinandersetzung gerückt.

Zu Beginn der neunziger Jahre sind verschiedene Ansätze der Schulentwicklung zu verzeichnen.

Institutionelle Schulentwicklung

Schule wird im institutionellen Schulentwicklungsansatz in ihrem Gesamtzusammenhang betrachtet, sie wird als interdependentes Subsystem im gesamtgesellschaftlichen Kontext verstanden. Die Schulentwicklungsprozesse sollen sich zunächst auf der Mikroebene der Einzelschule konzentrieren. Die einzelnen Organisationsmitglieder lernen sich zu entwickeln und aus diesen Lernprozessen resultiert dann eine umfassende Organisationsentwicklung der Schule. Dazu ist es notwendig, Vorbehalte gegenüber Veränderungen abzubauen und Betroffene zu Beteiligten zu machen. Schule wird so zu einer Einheit des Wandels. ${ }^{126}$ Die integrativen Ansätze der Organisationsentwicklung favorisieren die Veränderung von innen. Institutionelle Schulentwicklung hat eine allgemeine Schulverbesserung zum Ziel.

\section{Pädagogische Schulentwicklung}

Innerhalb der pädagogischen Schulentwicklung wird dem Unterrichtsgeschehen die größte Beachtung geschenkt. Pädagogische Schulentwicklung basiert auf den Erkenntnissen der „Gute Schule-Studien“127 und rückt methodisch-didaktische Merkmale von als gut wahrgenommen Unterricht in den Vordergrund. Schulentwicklungsprozesse sollen genutzt werden, um veränderte Unterrichtsformen und Unterrichtsmethoden in der Schule zu verankern. Im Hinblick auf Planung und Umsetzung von Entwicklungskonzepten bedeutet das, dass vorhandene Kompetenzen der Lehrerinnen und Lehrer im Sinne eines eigenverantwortlichen Handelns systematisch genutzt werden sollen, um Methoden- Kommunikations- und Teamentwicklungstraining durchzuführen. Pädagogische Schulentwicklung hat die Unterrichtsverbesserung zum Ziel.

Schule als lernende Organisation

Seit Beginn der 90er Jahre spricht man zunehmend von der Schule als lernender Organisation. Aus einer systemischen Perspektive wird ein zent-

\footnotetext{
${ }^{125} \mathrm{Vgl.} \mathrm{Rolff} \mathrm{1986,} 13$.

${ }^{126}$ Vgl. Dalin/Rolff/Buchen 1996, 328.

${ }^{127}$ Vgl. Tillmann 1995.
} 
rales Thema der Auseinandersetzung der Umgang mit Komplexität. ${ }^{128}$ Wichtige Elemente einer lernenden Schule sind Konzentration auf vorhandene Stärken und Prozessorientierung. Das Team und dessen Kooperation erhält eine bedeutende Rolle. Die lernende Schule kann als letztes Ziel der Organisationsentwicklung angesehen werden. Wenn von einer lernenden Schule gesprochen wird, meint dies, dass Lehrerinnen und Lehrer durch praktisches Tun ihren Arbeitsalltag und ihre Schule so verändern, dass sie zufriedener und erfolgreicher unterrichten. Die lernende Schule rückt das Problemlösen in den Vordergrund, Probleme der Schule werden als Aufgabe verstanden, die es gemeinsam miteinander zu lösen gilt. ${ }^{129}$

Die durch die Schulentwicklung hervorgebrachte Überzeugung, dass Schulen lernende Organisationen sind, hat zu einer Neubestimmung der Aufgaben von Schulleitung geführt. Schulleiterinnen und Schulleiter werden die Gestalter des Wandels in Organisationen, die "change agents“, wie Fullan (1999) sie nennt. ${ }^{130}$ Führungskräfte in lernenden Organisationen befähigen ihre Mitarbeiter dazu, produktiv mit den entscheidenden Problemen umgehen zu können. Sie entwickeln eigene Visionen und bemühen sich um die Integration der Zielvorstellungen von Mitarbeitern in ein gemeinsames Ganzes. Von Bedeutung ist es, dass die Mitarbeiter systemische Einsichten in die Organisation entwickeln und selbst Verantwortung für das System übernehmen. ${ }^{131}$ Demnach erfolgt die Steuerung der Schule über drei Aufgabenbereiche:

\section{Administration und Organisation}

Schule ist eine organisatorische bürokratische Einheit, die Verwaltungsabläufe unter den gegeben Erlassen und Verordnungen autonom regeln soll. Führung und Leitung: Neben interner Kooperation und Kommunikation wird der Schulleitung eine große Verantwortung für die Imagepflege der Schule als öffentliche Non-profit-Organisation übertragen.

\section{Pädagogische Aufgaben}

Strategische Maßnahmen müssen ergriffen werden, die zu einer Unterrichtsarbeit beitragen, die den Bildungsauftrag der Schule sichern. Pädagogische Beratung, die dem Erziehungsauftrag der Schule entspricht.

\section{Qualitätskontrolle}

Bestandsaufnahmen Soll-Ist-Vergleich, die der Schulleitung dazu dienen soll, die Auswirkungen der Steuerung zu überprüfen und weitere Maßnahmen zu ergreifen. Diese Bestandaufnahmen können intern oder auch durch externe Berater im Austausch mit den Gremien der Schule erfolgen. $^{132}$

\subsubsection{Schulentwicklung und Schulleitung}

Die Schulentwicklung hat einen großen Einfluss auf die Funktion und Rolle der Schulleiterin/ des Schulleiters. Wichtige Impulse für die Weiterentwicklung der Schule, insbesondere das Konzept der lernenden Schule, wurden

\footnotetext{
${ }^{128}$ Vgl. Rolff 1992/ Fullan 1999.

${ }^{129}$ Vgl. Rolff et al 1998, 37.

${ }^{130}$ Vgl. Fullan, 1999, 127.

${ }^{131}$ Vgl. Senge 1999, 411.

132 Vgl. Ulrich/Probst 1991, 289.
} 
positiv in der Schullandschaft aufgenommnen. Viele Schulentwicklungsprojekte wurden in letzten Jahren initiiert, insbesondere konkrete Maßnahmen der Unterrichtsentwicklung wurden aufgenommen, z.B. Methodencurricula.

Für Schulleiterinnen und Schulleitern hat sich dadurch das Aufgabenfeld stark erweitert und sie haben andere und neue Aufgaben zu bewältigen, ohne dass sie dabei von administrativen Aufgaben befreit werden. Den Schulleiterinnen und Schulleiter wird innerhalb der Schulentwicklung eine zentrale Rolle beigemessen, denn sie sind einerseits in einer Position, die es ermöglicht zu initiieren und zu unterstützen; sie können aber auch Entwicklungen bremsen, wenn sie sich überfordert fühlen oder befürchten durch veränderte Strukturen in ihrer Rolle geschwächt zu werden. Die Begrifflichkeiten, die im Zusammenhang mit der Schulentwicklungsdiskussion für die Schulleiterin/den Schulleiter verwendet werden, bestätigen dieses Bild: „Türöffner“, „change agents“, „Initiatoren“, „Motor der Schulentwicklung" etc. ${ }^{133}$

Innerhalb der pädagogischen Schulentwicklung wird die Wirkung des Führungshandelns auf die Lernleistungen der Schülerinnen und Schüler betrachtet.

Bonsen (2002) kommt in seiner Untersuchung zur Wirksamkeit von Schule und Schulentwicklung zu dem Ergebnis, dass der Beitrag der Schulleitung zur Erklärung von Leistungen der Schülerinnen und Schülern eher gering ausfällt. Schulenwicklung kann nur im Zusammenschluss mit dem Kollegium geschehen und hat dann Einfluss auf Schülerleistungen. Die Aufgabe der Schulleiterin/ des Schulleiters kann daher nicht nur auf der direkten und individuell ausgerichteten Einflussnahme auf das Handeln der einzelnen Lehrkräfte bezogen bleiben, sondern muss stärker in der „Einflussnahme und Führung des Kollegiums in Richtung kooperativer Arbeitsweisen, gemeinsamer Entwicklungsbemühungen mit der Zielrichtung einer Verbesserung der Qualität von Schule und Unterricht sein. “" ${ }^{334}$

\subsubsection{Zusammenfassung}

Die Schule aus dem Blickwinkel Schulleiterin und Schulleiter zeichnet sich einerseits durch hierarchische Entscheidungs- und Führungsstruktur aus. So sind Schulleiterinnen und Schulleiter die Vorgesetzten der Lehrkräfte und haben bestimmte Befugnisse beziehungsweise Verpflichtungen innen gegenüber, z.B. Anordnung von Mehrarbeit etc.. Andererseits haben sie selbst innerhalb einer vertikalen Rangfolge Vorgesetzte, die ihnen Weisungen geben können. Die vielen Willensbildungs- und Entscheidungsprozesse innerhalb der Organisation Schule verlaufen aber selbstverständlich auch auf symmetrischen Ebenen und nicht nur von oben nach unten $a b$, sondern sind ebenso von unten nach oben möglich, so binden Beschlüsse der Gesamtkonferenz ${ }^{135}$ die Schulleiterinnen und Schulleiter. Neben den innerschulischen Strukturen ist die Einzelschule abhängig von

\footnotetext{
133 Vgl. Fullan 1999, 204. Bennis 1998.

134 Bonsen et al 2002a, 193.

${ }^{135}$ Die rechtliche Stellung der Gesamtkonferenz wird zurzeit in Niedersachsen kontrovers diskutiert, wobei sich das MK für mehr Entscheidungsrechte der Schulleitung ausspricht.
} 
einem hierarchischen Überbau - Schulaufsicht, Schulträger. Die Schule ist zudem durch bürokratische Vorgaben - Schulverwaltung - in ihrer Ablauforganisation konstruiert, insofern handelt es sich um eine komplexe Organisation.

Die Handlungsrationalitäten in Schulen orientieren sich dabei an unterschiedlichen Ebenen, die technische Vollzugsebene der Schulverwaltung, die Ebene eines zielorientierten-rationalen Organisationsverständnis und einer kommunikativ-interaktionale Ebene. ${ }^{136}$ Schulleiterinnen und Schulleiter arbeiten an den Schnittstellen dieser Ebenen und können sich in der Konfrontation mit konkreten Ereignissen, Problemen und Vorfällen in der Schule, der einen oder anderen Handlungsebene eher verbunden fühlen.

Hinzu kommt, dass Schulleiterinnen und Schulleiter nicht nur in der Organisationsform der Einzelschule agieren, sondern sich einer linearen Hierarchie der Schulbehörde gegenüber sehen, deren Beschlüsse und Verordnungen sie umzusetzen haben. Ein aktuelles Beispiel hierfür stellt die Schulstrukturreform in Niedersachsen dar, die durch die Abschaffung der Orientierungsstufe und der damit einhergehenden Angliederung der 5. und 6. Klassen an die weiterführenden Schulen zu einer Umbruchsituation führte. Es mussten erhebliche pädagogische, organisatorische und personelle Veränderungen in kürzester Zeit umgesetzt werden.

Ohne die wichtigen Impulse für die Weiterentwicklung von Schulen durch die Ansätze der Organisations- bzw. Schulentwicklung in Abrede stellen zu wollen, muss meines Erachtens darauf hingewiesen werden, dass der Aspekt der formalen Struktur insbesondere in neueren Theorien der Schulentwicklung zuweilen vernachlässigt wird. Die Funktion der Schulleiterin/des Schulleiters bewegt sich in dem Dilemma zweier Handlungswirklichkeiten, nämlich zwischen bürokratischer Administration und pädagogischer Innovation. ${ }^{137}$ Keiner der Schulentwicklungsansätze löst sich von dem externen administrativen System (Kultusministerium, Amt für Schule). Es wurden unzählige Aufgaben an die Schulleitungen übertragen, auch verwaltungsrechtliche, aber die Personalhoheit und die finanzielle Verwaltung der Schule bleiben bei der übergeordneten Behörde. Insofern bewegt sich das Handeln der Schulleiterinnen und Schulleiter immer im Spannungsverhältnis Autonomie und Dependenz. Rosenbusch (2005) spricht in diesem Zusammenhang von einem „strukturell gestörten Verhältnis“, welches durch die Strukturen und Aufgabenzuweisungen der Organisation, in der Tradition des Rollenverständnisses und der Selbstdefinition im erlebten Umfeld begründet ist.

\subsection{Professionstheorie}

Die Besonderheiten der Schule als Organisation wurden im vorherigen Kapitel ausgeführt. Die im Folgenden zu erläuternde Professionstheorie nimmt Bezug auf das Verhältnis zwischen Individuum und Organisation, somit bietet sie die Chance strukturelle berufliche Probleme zu reflektieren. Baumgartner et al (1996) verweisen darauf, dass im Prozess der Organisationsentwicklung die Gruppe der Professionellen vor typischen Problemen stehen würde. So stünden die Kreativität der Professionellen

\footnotetext{
${ }^{136}$ Vgl. Terhart 1997, 14.

${ }^{137}$ Vgl. Rosenbusch/Wissinger 1989, 14.
} 
im Widerspruch zu bürokratischen Regelungen und Formalismen und die Gestaltungskraft des Professionellen vertrage sich nicht mit einer starren Führungshierarchie. ${ }^{138}$ Fritz Schütze (1996) bezieht sich auf diese Position und problematisiert in seinen Abhandlungen Organisationszwänge und deren Auswirkungen auf professionelles Handeln. Die in seinen Ausführungen angesprochenen Strukturprobleme sollen im Folgenden im Hinblick auf die Position von Schulleiterinnen und Schulleitern thematisiert werden.

Ihren Ursprung hat die Professionstheorie in der Berufssoziologie. Ich gehe daher zunächst auf das soziologische Professionskonzept ein, dann sollen Eigenheiten des pädagogischen Handelns erörtert werden.

Im Hinblick auf die pädagogische Professionsdiskussion soll die Besonderheit der Schulleiterinnen und Schulleiter durch ihre Position im Kontext Schule Berücksichtigung finden. Das pädagogische Professionswissen kann als Reflexionsspiegel genutzt werden, in dem der Blickwinkel der Akteure reflektiert wird. In diesem Zusammenhang steht die Frage, auf welche Wissensvorräte Schulleiterinnen und Schulleiter zurückgreifen.

\subsubsection{Professionstheoretische Vorüberlegungen}

In der Literatur wird die Frage, was die Merkmale einer Profession seien, nicht einheitlich beantwortet, es lassen sich grob zwei Phasen der Theoriebildung unterscheiden. In der ersten Phase bis Ende der 80er Jahre stehen Professionskonzepte, die anhand von Merkmalskatalogen die Besonderheit bestimmter Berufe zu beschreiben versuchen. Hier dienen vor allen Dingen die so genannten klassischen Professionen (Ärzte, Juristen) als Folie für die Beschreibung. Von diesem Professionskonzept ausgehend gehören zu den Merkmalen einer Profession systematisches Wissen, ein Berufsethos, welches am Allgemeinwohl orientiert ist, und eine gewisse Unabhängigkeit gegenüber der von außen vorgenommenen Beurteilung. ${ }^{139}$

Gegenüber diesen an den Kriterien des Berufs orientierten Definitionen bezieht sich die neuere Debatte seit Beginn der 90 er Jahre stärker auf die spezifischen Aufgaben und die Bearbeitung gesellschaftlicher Probleme durch die Professionen. Diese aus der interaktionistischen und strukturtheoretischen Theoriebildung entwickelten Ansätze setzen unterschiedliche Schwerpunkte, weisen aber eine Vielzahl von Übereinstimmungen auf. Der Akzent der interaktionistischen Theoriebildung liegt auf konkreten Arbeitsabläufen innerhalb professioneller Handlungsbereiche, also mit den Handlungsleistungen der an der Interaktion beteiligten Personen. Hingegen betont die strukturfunktionalistische Theoriebildung das Expertenwissen des Professionellen und unterstreicht die gemeinschaftliche Funktion der Professionen als Mechanismen der sozialen Kontrolle, so wäre zum Beispiel im Fall des Lehrberufes abzuwägen zwischen Lern- und Entwicklungsprozessen des Individuums und den traditionellen Erwartungsmustern der gesellschaftlichen Anforderungen. ${ }^{140}$

\footnotetext{
${ }^{138}$ Vgl. Baumgartner e.a 1996, 63ff.

${ }^{139}$ Vgl. Tenorth 1989, 537.

140 Vgl. Schütze 1996, 185-186. Bei den folgenden Ausführungen ist zu beachten, dass Schütze seine Überlegungen aus dem Kontext der Sozialarbeit erläutert, daher spricht er
} 
Schütze referiert folgende Bestimmungsmerkmale als typisch für Professionen: ${ }^{141}$

1. Professionen ist von der Gesellschaft ein Mandat zur Bearbeitung eines Problembereichs übertragen worden.

2. Eine Profession hat einen Wissensvorsprung (aus ihrer Fachwissenschaft), der in einer akademischen Ausbildung erlernt und in seiner praktischen Anwendung eingeübt werden muss. Die Profession trifft zur Problembearbeitung Arbeitsvereinbarungen, die gegenseitige Kompetenzzuschreibungen und Vertrauensvorschüsse voraussetzt.

3. Die Profession hat Handlungs- und Problembearbeitungsverfahren, die sie im gesellschaftlichen Auftrag anwendet, auch wenn damit dem Klienten Unannehmlichkeiten zugefügt werden, wenn das Handeln dem Wohle der Gesellschaft dient.

4. Die Profession ist sowohl innerlich als auch äußerlich in eine Organisationsstruktur eingebettet, die sie für die Steuerung komplexer Abläufe nutzt, die sie aber auch kontrolliert. Diese Einbindung in Organisationsstrukturen gewährleistet einerseits zum Beispiel bestimmte Routineleistungen durch Arbeitsteilung, andererseits erfordert dies auch kommunikative Absprachen und eine gewisse Unterordnung unter die spezifischen Bedingungen der Organisation.

5. Die professionelle Interaktion geschieht in einer bestimmten Art und Weise, die vom Professionellen als Verfahrensverwalter in Gang gesetzt und aufrechterhalten wird. Dabei werden Mitteilungen des Klienten unter Rückgriff auf spezielle Wissensbestände gedeutet.

6. Die Angehörigen einer Profession entwickeln durch ihre Berufstätigkeit eine spezielle biographische Identität als Professionelle.

Schütze stellt in den Mittelpunkt seiner Ausführungen den Zusammenhang zwischen institutionellen Strukturkomponenten und Fehlentwicklungspotentialen professionellen Handelns, dabei geht er davon aus, dass allen Professionen Fehlentwicklungspotentiale zu Eigen sind, da sie strukturell begründet sind und daher gewissermaßen unaufhebbar sind. Schütze geht davon aus, dass mögliche Fehler im Handeln nur dann nicht realisiert werden, wenn die Fehlerpotentiale reflektiert werden. Es geht ihm um die Erkenntnis, dass es sich bei den Kernproblemen des Arbeitsfeldes um Konsequenzen professioneller Handlungsparadoxien handelt. Die Identifizierung dieser immanenten Widersprüche und erst das Akzeptieren dieser Paradoxien für das berufliche Handeln macht eine Ausbalancierung widersprüchlicher Anforderungen möglich.

Schütze bezieht sich stark auf die von der interaktionistischen Forschungsrichtung betonten, institutionellen Strukturkomponenten der Profession, die das Fehlerhafte des professionellen Handelns, das Paradoxe in den Vordergrund stellen.

„Die Kernprobleme des professionellen Handelns sind paradox, wie auch die Interaktionsbasis des Alltagslebens angesichts der unaufhebbaren Unvereinbarkeit der interaktionslogische notwendig idealisierenden, wechselseitigen Unterstellungen der Interaktionspartner mit dem jeweiligen fak-

vom Klient bzw. Abnehmer und wählt zur Konkretisierung seiner Paradoxien das Beispiel der Aktenverwendung im Sozialwesen.

${ }^{141}$ Vgl. Schütze 1996, $183 \mathrm{ff}$. 
tischen Zustand der Interaktion- zum Beispiel wechselseitig Sinnübereinstimmung zu Beginn der Interaktion bereits zu unterstellen, bevor Interaktionspartner diese überhaupt „austesten“ konnten." ${ }^{142}$

Deutlich wird hier, dass die Professionellen mit drei wichtigen BezugsgröBen arbeiten, zwei wurden bereits genannt. Die Gesellschaft als Auftraggeber, die Fachwissenschaft als Sinnquelle und die dritte, die unter interaktionistischer Betrachtungsweise an erster Stelle stehen sollte, ist das Individuum als Klient, also die Interaktion zwischen Professionellem und Klient, die sich im Kern durch ein widersprüchliches Verhältnis auszeichnet.

Unter interaktionistischer Sichtweise kommen nun zu den oben bereits genannten Merkmalen der Profession nachstehende hinzu: ${ }^{143}$

7. Professionen bilden eine besondere ethisch und wissenschaftlich begründete Sinnwelt aus, an dem sich das berufliche Handeln orientiert. Einerseits haben die Professionellen das Privileg einen besonderen Zugang zu Wissensbeständen zu erhalten, andererseits können Laien ihnen auch mit Misstrauen begegnen. Das Professionelle wird erst sichtbar durch die Anwendung im Einzelfall, also die Bearbeitung bestimmter Problembereiche.

8. Die Anwendung des Professionswissens durch eine konkrete Handlung ist in den Lebenskontext des Klienten eingebunden und dieser befindet sich in einem dynamischen Wandel nicht zuletzt durch die Intervention des Professionellen. Die Praxis hat also Einfluss auf die Theorie, weil der Professionelle mit Änderungen der Rahmenbedingungen rechnen muss und durch die Entwicklung des Einzelfalls eine Umformulierung abstrakter Kategorien notwendig werden kann.

9. In der Berufssozialisation kommt es zur biographischen Identifizierung des Professionellen mit den Werten seines Berufes. Dieses Expertenwissen der jeweiligen Fachwissenschaft dient zwar als Steuerungsinstrument, Schütze verweist aber auch auf die Gefahr, dass Professionelle durch eine zu starke Bindung an die Berufsratio bei auftretenden Schwierigkeiten eher vom Beruf ausgesaugt werde und in eine Berufsfalle verstrickt wird. Gründe hierfür sieht Schütze vor allen Dingen in einem Mangel an Distanzierungsfähigkeit. So können z.B. bei einer zu starken biographischen Identifizierung Probleme, die im Berufsleben unweigerlich auftreten, als persönliche Niederlagen erlebt werden und ein problemorientiertes Handeln evtl. einschränken.

10. Professionen wehren sich aus ihrer verinnerlichten beruflichen Identität heraus gegen Einschränkungen und Übergriffe der Organisation. Das Widerspruchsverhältnis wird besonders dort deutlich, wo ein soziotechnischer Wandel zur Verknappung der Ressourcen führt und die Spielräume innerhalb der jeweiligen Organisation kleiner werden. Professionen zeichnen sich einerseits durch den gesellschaftlichen Auftrag und andererseits durch eine autonome Problembearbeitung aus. Die Auseinandersetzungen über Einschränkungen finden nicht auf der Ebene der Gesellschaft statt,

\footnotetext{
${ }^{142}$ Schütze 1996, 188.

${ }^{143}$ Vgl. Schütze 1996, 190-196.
} 
sondern werden in der Regel über die Organisation bzw. ihre Mitglieder vermittelt. In diesem Zusammenhang verweist Schütze auf die Probleme, die entstehen, wenn Professionelle aus der verinnerlichten Berufssicht heraus zu einer Abwehr gegen Organisationszwänge neigen, da eine organisationsfeindliche Atmosphäre ein Hindernis dafür darstellen kann, die Systemzusammenhänge zu durchschauen und erfolgreich mit ihnen umzugehen.

11. Jede Profession hat in ihrem Arbeitsablauf mit für sie typischen Störpotentialen zu kämpfen. Die Profession befindet sich mit ihrer Klientel in einem spannungsreichen und paradoxen sozialen Verhältnis. Zum einen ist der Professionelle dem Klienten notwendigerweise an Wissen, Können und Macht überlegen, aber er ist ebenso auf das Vertrauen und die freiwillige Mitarbeit der Klientel als Basis einer Zusammenarbeit angewiesen.

Vor dem Hintergrund dieser Beschreibung professioneller Handlungslogik entwickelt Schütze typische Professionelle Paradoxien. Diese unaufhebbaren Paradoxien professionellen Handelns müssen nach Schütze immer wieder professionsethisch, in der eigenen Selbsterfahrung und persönlicher Handlungsreflexion sowie sozialwissenschaftlich durchdacht werden.

Ich werde im Zusammenhang mit der pädagogischen Professionalität noch auf Paradoxien eingehen (vgl. 4.4.2) und benenne an dieser Stelle nicht die durch die Interaktion zwischen Professionellen und Klienten auftretenden internen Fehlerquellen, sondern gehe auf die von Schütze hergeleiteten widersprüchlichen Wirkungen der staatlichen bzw. organisationalen Steuerungsmechanismen ein.

Die Organisation hat nach Schütze positive und negative Wirkungen, sie bietet Gelegenheit zum Informationsaustausch, zur Vernetzung, sie macht Arbeitsteilung notwendig und möglich. Sie ermöglicht zudem eine gewisse Standardisierung, Nachprüfbarkeit und systematische Kritik und insofern ist die Organisation eine wichtige Bedingung für die professionelle Qualitätskontrolle. ${ }^{144}$ Auf der anderen Seite bilden Organisationen eigene Denkund Handlungsrationalitäten aus, die zum Teil im Widerspruch zu den Werten und Überzeugungen der Profession an sich stehen kann. Die Professionellen können versuchen sich auf unabhängige professionsinterne Kriterien, d.h. Selbstverständnis der Profession, Erkenntnisse der Forschung etc., zurückzuziehen. Schütze verwendet hierfür den Terminus Binnenarena-Debatte und stellt diese der Außenarena-Debatte gegenüber. Der Professionelle übt dann Kritik an organisatorischen Restriktionen oder an Ausbildungs-, Kontroll-, Träger- und Finanzinstanzen.

Es gibt unterschiedliche Möglichkeiten, mit den Vorgaben der Organisation umzugehen, nämlich Verinnerlichung und Annpassung oder aber Ablehnung der Organisation, wobei dann durch das Verschieben der Verantwortlichkeiten nach außen eine aktive und gestaltende Einflussmöglichkeit verhindert wird.

Die Bedeutung eines reflektierten Umganges mit diesem schwierigen Verhältnis einer Profession zu ihren Organisationsstrukturen wurde bereits oben erwähnt, die Frage der Einbindung der Lehrerinnen und Lehrer in die

\footnotetext{
${ }^{144}$ Vgl. Schütze 1996, 221-222.
} 
Organisation wird im Kontext der pädagogischen Professionstheorie z.B. von Helsper thematisiert.

\subsubsection{Pädagogische Professionsdiskussion}

Einer allgemeinen Darstellung des soziologischen Professionskonzepts nach Schütze (1996) schließen sich nun die auf den pädagogischen Kontext spezifizierten Ansätze an. Die professionsbezogene Forschung hat sich mit den Spezifika pädagogischen Handelns und vornehmlich mit dem Professionsstatus des Lehrberufs beschäftigt. Über die zentrale Frage des Professionsstatus gibt es keine einheitliche Antwort, mehrheitlich wird aber davon ausgegangen, dass der Lehrberuf professionalisierbar bzw. professionalisierungsbedürftig ist.

In der ersten Phase der Professionstheorie ging es darum, ein Entsprechung für den angelsächsischen Begriff „professionals“ zu finden und Merkmale zu formulieren, anhand derer Berufe identifiziert werden könnten, die dem entwickelten Konzept des Professionellen entsprächen. Die pädagogische Professionalisierungsdebatte setzte die Professionalisierung des Lehrberufes gleich mit einer Verwissenschaftlichung der Lehrertätigkeit. Die Professionalität liegt zu Recht im Beherrschen des Fachwissens, was vermittelt wird. Noch Ende der 80er Jahre bezeichnete Ulf Schwänke den Lehrberuf als Semi-Profession und bezog diese Einschätzung vor allem auf das Merkmal 'Autonomie`, da Lehrerinnen und Lehrer ihren Beruf nicht selbstständig ausüben, sondern in einem untergeordneten Handlungskontext der Organisation Schule. ${ }^{145}$ Die ausgelöste Auseinandersetzung mit der Professionalisierungsfrage diente einerseits durch den Rekurs auf Wissenschaft den Pädagogen an der Hochschule und andererseits wurde die Defizit-Analyse des Semi-Professionellen als Herausforderung zur eigenen Professionalisierung angenommen, damit wurde der statische Professionsbegriff abgelöst.

In einer zweiten Phase der Professionsdebatte im pädagogischen Kontext wurden stärker personale Faktoren der Lehrerpersönlichkeit und des pädagogischen Verstehens in den Blick genommen. Die Diskussion, die in der allgemeinen Erziehungswissenschaft seit Beginn der neunziger Jahre geführt wird, behandelt zunehmend den Aspekt der pädagogischen Professionalisierung. Dies geschah in Anlehnung an die Merkmale, die die allgemeine berufssoziologische Theorie im Hinblick auf die klassischen Professionen formuliert hatte.

In diesem Zusammenhang erscheinen Arbeiten, in denen die Zuordnung des Lehrberufs im Sinne dieser Merkmale als professionalisiert angesehen wird.

Die Diskussion seit Beginn der neunziger Jahre behandelt nun den Aspekt einer spezifischen pädagogischen Professionalisierung. Die in der interaktionistischen Professionstheorie bevorzugte Bestimmung der Profession über eine spezifische Logik der Interaktion zwischen Professionellem und Klienten wird von der aktuellen pädagogischen Professionstheorie aufgegriffen. Die von der interaktionistischen Theoriebildung angenommene paradoxale oder antinomische Struktur der professionellen Interaktion wird

${ }^{145}$ Vgl. Schwänke 1988. 
dabei weitgehend übernommen, wobei die genaue Bestimmung des eigentlich „Paradoxalen“ jeweils variiert.

Neuere erziehungswissenschaftliche Arbeiten beschäftigen sich mit dem Prozess der Professionalisierung des Lehrberufs oder mit dem Status pädagogischer Professionalität und versuchen der Forderung nach einem eigenen Theorieanspruch nachzukommen. Als Überblick über den aktuellen Stand der Diskussion in der Erziehungswissenschaft kann der von Combe/Helsper (1996) herausgegebene Sammelband „Pädagogische Professionalität" angesehen werden. Die aktuelle Debatte bezieht sich in der Regel theoretisch auf den strukturanalytischen-funktionslogischen Professionsansatz von Oevermann.

Im Zentrum der Überlegungen Oevermanns steht das Konzept der „stellvertretenden Deutung“, ${ }^{146}$ welche er aus dem ursprünglichen Kontext der Therapie auf die Pädagogik überträgt. Unter dem Blickwinkel einer strukturellen Analogie der pädagogischen Praxis zum therapeutischen Handeln weist Oevermann eine therapeutisch-prophylaktische Ebene im Lehrerhandeln nach, die für ihn professionalisierungsbedürftig ist.

Für die Darstellung der pädagogischen Professionalität dient inm das Modell der Therapie, weil nach Oevermann strukturelle Parallelen zwischen Therapie und Pädagogik bestehen. Pädagogisches Handeln unterscheidet sich (wie auch Therapie) von naturwüchsiger Sozialisation, weil es zielgerichtet auf Bildung und Erziehung angelegt ist und einer Rollenspezifizierung der Beteiligten bedarf. Andererseits greifen beide - Pädagogik wie Therapie - auf die Strukturen der naturwüchsigen Sozialisation zurück. Dies ist für Oevermann der Grundwiderspruch des pädagogischen Handelns, er liegt sozusagen im Arbeitsbündnis Lehrkraft-Schülerin/Schüler begründet, denn seiner Ansicht nach, müsse es um das gemeinsame Bemühen um die Autonomie des Schülers/ der Schülerin gehen und diese stehe im Widerspruch (strukturlogisch) zur Institutionalisierung der Schule auf der Basis der Schulpflicht. Deutlich wird, dass sich der Anspruch auf Freiwilligkeit des Arbeitsbündnisses Klient/Therapeut nicht auf den Schulkontext übertragen lässt. Als Hauptgrund für die fehlende Professionalisierung sieht er die gesetzliche Schulpflicht und ihre Folgen. ${ }^{147}$ In diesem Widerspruch ein Professionalisierungsdefizit des Lehrerhandelns zu sehen erscheint wenig hilfreich für die pädagogische Praxis, da Problemursachen externalisiert werden, nämlich die gegebenen Bedingungen von Schule.

Die Widersprüchlichkeit des Lehrerhandelns hat auch Helsper (1996) diskutiert. Er arbeitet mit einem Professionsverständnis, das einige Gemeinsamkeiten mit dem Konzept von Schütze und Oevermann hat. Die Besonderheit seines Ansatzes resultiert daraus, dass er Antinomien in Beziehung setzt zu vier „Modernisierungsparadoxien“, mit denen er sich auf van der Loo und van Reijen (1992/1997) bezieht.

\footnotetext{
${ }^{146}$ Stellvertretende Deutung bezeichnet dort jenen Vorgang, bei dem der Therapeut stellvertretend für den Klienten zu der Lösung von dessen Problemen beiträgt, indem er in der klientenbezogenen Anwendung theoretischen Wissens latente Strukturen des Klientenhandelns offen legt.

${ }^{147}$ Vgl. Oevermann 1996, 181.
} 


\subsubsection{Werner Helsper: Konzept der konstitutiven Antinomien der professionellen Praxis}

Das Typische der Profession sieht Helsper in einer komplexen Form der Interaktion des Professionellen mit seinem Klienten, in der besondere gesellschaftliche Funktionen und Werte erfüllt werden, zum Beispiel Wahrheits- Konsens- und Identitätsfindung. Professionelles Handeln ist dabei immer bezogen auf lebenspraktische Probleme.

"In den komplexen in der Regel in Face-to-face-Interaktionen stattfindenden Vollzügen, sind Professionelle dabei in konstitutive Handlungsdilemmata involviert, die nicht aufgehoben, sondern nur reflexiv gehandhabt werden können. “'148

Eine professionelle Berufsgruppe zeichnet sich dadurch aus, dass sie für die Bearbeitung von Problemen der Strukturerhaltung, der Strukturänderung und der Identitätserhaltung von Personen zuständig ist. Das professionelle Handeln gerät unter einen praktischen Handlungsdruck und Entscheidungszwang, wenn diese Personen nicht aus eigener Kraft für die Wahrung oder Wiederherstellung leiblicher, psychischer, moralischer und sozialer Anerkennung sorgen können, denn dann treten Professionelle zu ihrer Sicherung in Kraft. Helsper (1996) trifft eine gewichtige Unterscheidung zwischen Professionellen und Experten. Der Professionelle verfüge über theoretische Wissensbestände und wissenschaftliche Deutungsmuster, könne sein Wissen aber nicht schematisch auf die konkrete Handlung bzw. den Einzelfall übertragen. Im Gegensatz dazu würde der Experte über ein technologisch kontrollierbares Regelwissen verfügen. ${ }^{149} \mathrm{Hier}$ grenzt sich Helsper (1996) von Ansätzen ab, die Professionalisierung vornehmlich unter dem Fokus Wissensanreicherung sehen. Pädagogisches Handeln wird in diesem Professionskonzept also als ein professionelles verstanden, das typische Handlungsdilemmata aufweist. Ausgehend von Entwürfen des idealen Lehrerhandelns skizziert er pädagogische Handlungsprobleme und beschreibt folgende „konstitutive Antinomien“ des pädagogischen Handelns:

- Distanz versus Nähe

- Subsumtion versus Rekonstruktion

- Einheit versus Differenz

- Organisation versus Interaktion/Kommunikation

- Heteronomie versus Autonomie $e^{150}$

Helsper (2000) beschreibt und erläutert im Rahmen seines Konzeptes der konstitutiven Antinomien ${ }^{151}$ diese auf fünf Ebenen.

1. Die konstitutiven, nicht aufhebbaren professionellen Antinomien. Hiermit sind Widersprüche gemeint, die dem lebenspraktischen Handeln imma-

\footnotetext{
${ }^{148}$ Helsper 1996, 528.

${ }^{149}$ Vgl. Helsper 1996, 529.

${ }^{150}$ Vgl. Helsper 1996, 530

151 Innerhalb der Professionstheoretischen Diskussion werden die Begriffe Paradoxie, Antinomie, Dilemmata und Widerspruch verwendet. Schütze spricht ausschließlich von Paradoxien, Helsper hingegen greift alle Begriffe auf, gebraucht dann für seine Ausführungen den Begriff Antinomien. Aus meiner Sicht beinhaltet der Begriff Paradoxie stärker das Element der Widersinnigkeit, der Begriff greift die Unvereinbarkeit auf. Der Begriff Antinomie bezeichnet den Widerspruch zweier an sich gültiger Sätze und der Begriff Dilemmata deutet im Sinne einer Zwangslage auf eine Wahl zwischen zwei gleich unangenehmen Dingen hin.
} 
nent sind, wenn Krisen bewältigt werden müssen, dann gerät das professionelle Handeln unter Entscheidungsdruck. Hinzu kommt, dass professionelles Handeln begründbar und legitimiert sein sollte, im aktuellen Handeln lägen aber abgesicherte Begründungen (noch) nicht immer vor. (Begründungsantinomie)

An dieser Ebene setzt nach Helsper die widersprüchliche Einheit der Vermittlung von Theorie und Praxis an. Das professionelle Handeln zeichnet sich dadurch aus, dass es auf Grundlage theoretischer und wissenschaftlicher Erkenntnisse legitimierbar ist. Diese Erkenntnisse seien zwar angeeignet, aber in der Praxis nicht einfach um- oder übersetzbar. Jede Einordnung eines Falles unter allgemeine Kategorien birgt die Gefahr dem Einzelfall nicht gereicht zu werden und auf gewohnte Handlungsmuster zurückzugreifen. Das professionelle Handeln bedarf zwar einerseits der Routine, erfordert aber zugleich eine bewusste und kritische Haltung gegenüber jeder Gewohnheit. ${ }^{152}$ (Subsumtionsantinomie) Hinzu kommt, dass das professionelle Handeln abhängig ist von dem Gelingen der Interaktion unter Mitwirkung des Klienten/ Schülers bzw. Kollegen. Der Erfolg des Handelns lässt sich also im Vorfeld nicht zusichern, daher ist das Professionelle Handeln anfällig für Fehler. (Ungewissheistsantinomie).

Einerseits die asymmetrische Struktur der Beziehung, die sich etwa durch Wissensbestände, Kompetenzen aber auch Ressourcen ausdrücken kann, und andererseits die Notwendigkeit Problemlösungen nicht vorzugeben, sondern gemeinsam auf einer symmetrischen Ebene mit dem Gegenüber zu erreichen, stellen einen weiteren konstitutiven Widerspruch des professionellen Handelns dar. (Symmetrie- bzw. Machtantinomie). Die interaktive Gegenseitigkeit bedarf, um gelingende Handlungsverkettungen zu erreichen, einer wechselseitigen Vertrauensbasis. Je stärker die Abhängigkeiten sind und je weitreichender Handlungen die persönliche Ebene betreffen, desto schwieriger wird es, eine vertrauensvolle Konstellation herzustellen. (Vertrauensantinomie)

2. Konstitutive Antinomien durch widerspruchsvolle Beziehungsstrukturen. Am Beispiel der Lehrer/-in/ Schüler/-in - Beziehung werden Widersprüche des professionellen Handelns verdeutlicht, so muss das Handeln der Lehrkraft einerseits die psychosoziale Integrität der Heranwachsenden fördern und andererseits die Autonomie zulassen. Das professionelle Handeln erfordert strukturell Nähe und gleichzeitig Distanz. (NäheDistanz-Antinomie). Bei der Vermittlung von fachlichen Inhalten muss der lebensgeschichtliche Hintergrund und die aktuelle persönliche Situation der Lernenden berücksichtigt werden, was zu Antinomien von Person und Sache führen kann. (Sachantinomie) Eine grundlegende Antinomie besteht zwischen dem Anspruch alle Adressaten gleich zu behandeln (Einhaltung einer Gerechtigkeitshaltung), dies steht in einem Spannungsverhältnis zur Berücksichtigung eines Einzelfalls, z.B. durch spezielle Zuwendung, Förderung und Unterstützung einzelner SchülerInnen. (Differenzierungsantinomie)

Die organisationsförmige Institutionalisierung (Schulsystem) ermöglicht auf der einen Seite die Gewährleistung personenunabhängiger Standards, die Vorstrukturierung durch gemeinsame Regeln und rollenförmige Hand-

\footnotetext{
${ }^{152}$ Vgl. Helsper 2000, 145.
} 
lungsmuster, steht aber auf der anderen Seite im Spannungsverhältnis zur Offenheit der durch Interaktion erfolgenden professionellen Praxis, d.h. dass ein fest gefügter Organisationsapparat die Berücksichtigung individueller Entwicklungen vernachlässigt. (Organisationsantinomie) Ein pädagogisches Grunddilemma zeichnet sich dadurch aus, dass der Professionelle auf der Grundlage bestehender Ungleichheit den Klienten/ Lernenden zur Autonomie erziehen muss, es besteht die Gefahr, dass durch das professionelle Handeln Abhängigkeit bzw. Unselbstständigkeit erzeugt wird. Hinzu kommt, dass Lehrende selbst in einem organisatorischen Rahmen handeln, d.h. nicht losgelöst von Abhängigkeiten handeln. (Autonomie-Antinomie)

\section{Historische, kulturell ausgeformte Widerspruchsverhältnisse:}

Diese Ebene bezieht sich auf die strukturellen Bedingungen des Bildungssystems, denn es besteht ein Grundwiderspruch zwischen einer zwangsförmigen allgemeinen Schulpflicht und dem Verspechen zur Selbstständigkeit und Mündigkeit zu erziehen.

Durch den Widerspruch zwischen Fördern und Auslesen werden die bisher genannten Antinomien verschärft, weil dadurch die Konstruktion symmetrischer Verhältnisse erschwert wird. Innerhalb dieses soziokulturellen Rahmens des Schulwesens findet jedes Lehrerhandeln statt und unter den konstitutiven Bedingungen dieses Rahmens ist mit den professionellen Antinomien umzugehen. Helsper (2000) verweist an dieser Stelle auf eine klare Trennung zwischen einem soziokulturellen Professionalisierungsbedarf im Handeln der Lehrer und Lehrerinnen auf der Systemebene Schule und einer Professionalisierung der Berufsgruppe der Lehrerinnen und Lehrer auf der Individualebene.

\section{Professionelle Handlungsdilemmata}

Diese Handlungsdilemmata beziehen sich auf die konkrete Ausformung des professionellen Umgangs mit Antinomien. Dabei wird dieses Handeln sowohl auf der Ebene der Einzelschule (unterschiedliche Lehrergruppen) als auch auf der Individualebene Lehrerin/Lehrer betrachtet. Helsper (2000) geht davon aus, dass sich Zuspitzungen des Handelns ergeben, wenn in einer einzelschulspezifisch ausgebildeten Schulkultur widersprüchliche Anforderungen an das professionelle Handeln gestellt werden, z.B. höchste Leistungsansprüche und eine Hervorhebung differenzierungs- und selektionsorientierter Haltung vs. das Bild einer integrierten Schulgemeinde. Hier muss die einzelne Lehrkraft eine Haltung entwickeln und aufgrund dieser Positionierung prägen sich damit zusammenhängende Handlungsdilemmata aus. ${ }^{154}$

\section{Modernisierungsantinomien}

Ausgehend von der These, dass ein enger Zusammenhang zwischen einer umfassenden sozialen und kulturellen Modernisierung und dem Fortschreiten der konstitutiven Antinomien des Lehrerhandelns besteht, zeigt Helsper (2000) vier Modernisierungsantinomien auf: ${ }^{155}$

\footnotetext{
${ }^{153}$ Vgl. Helsper 2000, 154.

${ }^{154}$ Ebd. 155.

${ }^{155}$ Helsper (2000), bezieht sich auf das konzeptionelle Schema der Modernisierungstendenzen von van der Loo und van Reijen (1992). Anhand der vier Dimensionen Struktur,
} 
Rationalisierungsantinomie: Das Spannungsverhältnis der Professionellen zu der sie beschäftigenden Organisation, hier können zweckrationale abstrakte Regeln im Widerspruch stehen - zum Beispiel im Fall der Schulezu den inhaltlichen Zielen. Die Organisation kann einerseits Entlastungen durch geregelte Strukturen bieten, andererseits zu Beschränkungen führen.

Differenzierungsantinomien: Durch eine wachsende Spannung zwischen der Tendenz zur Vereinheitlichung (Globalisierung) einerseits und andererseits durch die voranschreitende Ausdifferenzierung von Lebensformen und Weltdeutungen ergibt sich eine Zunahme von Entscheidungsmöglichkeiten bei gleichzeitiger Zunahme von Entscheidungszwängen.

Zivilisierungsantinomie: Im Zuge der Modernisierung nehmen Anforderungen an die Affekt- und Selbstkontrolle des Individuums zu und gleichzeitig wird ein hohes Maß an Verständigung und emotionaler Nähe gefordert.

Individualisierungsantinomie: Einerseits ist die Individualisierung durch die Aufhebung gesellschaftlicher Zwänge befördert worden, beinhaltet aber andererseits die Last der eigenverantwortlichen Entscheidungsfindung.

Ausgehend von Schützes Darstellung der Profession als Beruf, deren Besonderheit durch professionelle Paradoxien gekennzeichnet ist, sollten die Ausführungen von Helpers Konzept zu einer genaueren Bestimmung dieser Widersprüche führen. Auch bei Helsper werden strukturelle Besonderheiten des professionellen pädagogischen Handelns hervorgehoben, um fundamentale Antinomien zu bestimmen. Da sich die pädagogische Praxis nicht vollkommen geändert hat und es auch nicht zu grundlegenden strukturellen Umbrüchen in der Organisation Schule gekommen ist, kann man davon ausgehen, dass die Antinomien auch in der Gegenwart Bedeutung haben.

Helsper (2000) führt die Probleme des professionellen Handelns im Wesentlichen auf die Institutionalisierung des pädagogischen Handelns und die sich daraus ergebenden Widersprüche zurück, er bezeichnet die strukturtheoretische Ebene als die grundlegende Ebene. Die Antinomie von Organisation versus Interaktion ist bereits im Rahmen der klassischen Professionstheorie beschrieben worden, auch Schütze (1996) geht davon aus, dass ein wesentlicher Teil der professionellen Handlungsparadoxie durch den Kontext der Organisation entstehen würde. Er weist darauf hin, dass es durch das eingegangene Arbeitsbündnis und den sich daraus ergebenden Aktivitätsbereich für die Professionsratio besonders schwierig sei, ihre Umsicht bei der Bearbeitung der unaufhebbaren Paradoxien zu

Person, Kultur und Natur arbeiten sie die Modernisierungstendenzen (Differenzierung, Rationalisierung, Individualisierung, Domestizierung) heraus und ordnen diesen widersprüchliche Begleiterscheinungen (Modernisierungsparadoxien) zu. Helsper ordnet die fünf strukturellen Antinomien dem Schema zu, wobei zum Beispiel die Zuordnung der Antinomie Nähe/Distanz zu der Dimension Natur nicht unhinterfragt bleiben dürfte. Des Weiteren verwendet Helsper die Begriffe Modernisierungstendenz und Paradoxien meist synonym. Im Rahmen dieser Arbeit ist es nicht möglich, auf die sich daraus ergebenden Schwierigkeiten einzugehen. Hierzu vgl. Soertz (2002), 163ff. 
bewahren. Zudem würde die zunehmende Durchorganisiertheit und Technologisierung heutiger Komplexgesellschaften die Organisationsstrukturen immer umfangreicher und anspruchsvoller werden lassen. Dadurch würden sich die Paradoxien des professionellen Handelns fortlaufend verschärfen. ${ }^{156}$

Die Betonung dieses Aspektes birgt die Gefahr, dass alle „NichtProfessionalität" auf das Grundparadoxon der Organisation reduziert werden könnte. Mit dem Hinweis auf die vielfältigen und unterschiedlichen, nicht immer widersprüchlichen Anforderungen des pädagogischen Handelns formuliert Soretz (2003): „Hier wäre allerdings die Beobachtung auszuschließen, dass das inflationäre Insistieren auf paradoxale, widersprüchliche und antinomische Konstellationen auch als Versuch einer Aufwertung des Lehrberufs angesichts seines ungeklärten Professionsstatus interpretiert werden kann." ${ }^{157}$

Positiv gewendet könnte also folgende Professionalisierungsanforderung formuliert werden: Der organisatorische Rahmen der Schule sollte so genutzt werden, dass dem Berufsauftrag möglichst professionell nachzukommen ist, zumal Lehrkräfte und Schulleiterinnen und Schulleiter sich freiwillig diesen organisatorischen Zusammenhang für ihre berufliche Tätigkeit gewählt haben, was auf Schülerinnen und Schüler nicht zutrifft.

\subsubsection{Pädagogisches Professionswissen}

Als ein Kennzeichen der Profession wurde, wie in Kapitel 4.3.1 dargelegt, die Basis eines systematisierten Wissens genannt. Die Anwendung des Professionswissens geschieht mit Rückbezug auf spezielle professionelle Wissensbestände und konkretisiert sich durch den pädagogischen Handlungskontext in der Praxis. Der Nachweis einer exklusiven Fachwissenschaft, die die professionelle Unabhängigkeit begründet, ist das umstrittenste Kriterium im Rahmen der Diskussion über pädagogische Professionalität. Was genau die speziellen Wissensbestände, welches also die wissenschaftliche Basis der Berufsausübung bei Pädagogen sei, lässt sich dabei schwerer beantworten als bei den „klassischen“ Professionen z.B. Ärzten.

Unter einer systemtheoretischen Betrachtungsweise beschäftigt sich Lilian Fried (2002) mit dem pädagogischen Professionswissens. Durch die Reflexion der pädagogischen Praxis entstehe pädagogisches Wissen, welches zur Erfassung, zum Begreifen und gegebenenfalls zur Veränderung der Erziehungspraxis genutzt wird. Je nach Aneignungstyp des Wissens spricht Fried (2002) von Praxiswissen, das durch die Reflexion von subjektiven Erfahrungen, die in und mit der pädagogischen Praxis gewonnen wurden, erworben wird und Theoriewissen, welches durch eine systematische Reflexion über pädagogische Wirk- und Geltungszusammenhänge entstanden ist. ${ }^{158}$ Die gegenseitige Beeinflussung dieser Wissenskategorien wurde bereits bei Schütze $(1996)^{159}$ thematisiert. Die Wissensbasis der Professionellen wird sowohl durch Aspekte des Theoriewissens als

\footnotetext{
${ }^{156}$ Vgl. Schütze 1996, 252.

157 Soretz 2003, 156.

${ }^{158} \mathrm{Vgl}$. Fried 2002, $12 \mathrm{f}$.

159 Vgl. Schütze 1996, 195.
} 
auch durch Erfahrungen des Praxiswissens angereichert, wodurch Wahrnehmungs- und Deutungsmuster entwickelt werden, die die Grundlage für das pädagogische Handeln bilden. Diese Wissensqualität, die die Wissensbereiche Fachwissenschaft, didaktisches Wissen, pädagogisches Wissen, berufsethisches Wissen beinhaltet, bezeichnet Fried (2002) als Professionswissen der Schulpraxisexperten. ${ }^{160}$

Die Vielschichtigkeit der Wissensgebiete, auf die Professionelle im pädagogischen Kontext zurückgreifen, machen deren Besonderheit aus, aber darin liegt zugleich auch ihre Problematik. Denn neben dem aus subjektiven Erfahrungen extrahierten Wissen wird Wissen aus unterschiedlichen Beständen ausgewählt und übernommen, dies können neben im Studium erworbenen wissenschaftlichen Theorien auch bildungspolitische Programmatiken, organisatorische Strategien oder fachlich, pädagogisch und methodisch ausgerichtetes Wissen sein.

Es handelt sich um ein interdisziplinär vernetztes Wissen, welches nicht unbedingt aufeinander abgestimmt ist und in das Professionswissen übernommen wird, dies birgt die Gefahr, dass Professionelle aufgrund ihres in der Praxis bestehenden Handlungsdruckes zu Reflexionsdefiziten neigen. $^{161}$

Die Wissensformen des pädagogischen Professionswissens werden in unterschiedlichen Systemen und den damit gekoppelten Funktions-, Organisations-, Interaktions- und Personalsystemen erzeugt. Neben dem Wissenschaftssystem und dem Erziehungssystem können dies auch das Politik- und Wirtschaftssystem sein, dadurch wird eine differente Perspektive auf die pädagogische Profession möglich.

Das Professionswissen wurde bereits oben in Theorie- und Praxiswissen untergliedert, unter dem Anspruch einer Wissenschaftsorientierung der Lehrerbildung wurde neben das klassische Theoriewissen (Grundlagen und Orientierungswissen) das Technologiewissen (didaktischmethodisches bzw. handlungsanleitendes Wissen) gestellt, welches einerseits wissenschaftlich legitimiert und andererseits berufspraktisch nutzbar ist, Fried (2003) nennt diese Wissensform Kopplungswissen. ${ }^{162}$

Die Berufspraxis vollzieht sich vor allen Dingen in Interaktionssytemen, diese bestehen sowohl aus Kommunikation als auch aus den Wahrnehmungen der jeweiligen Interaktionssituation. Dadurch bilden sich implizite Wissensbestände für den Beruf aus. Diese durch eigene Erfahrungen der Praxis entwickelten Vorstellungen, z.B. über Lernen, Schule, Rollenverständnis usw. können im Widerspruch zu theoretischen oder organisationalem Wissen stehen. Solange dieses Wissen unbewusst bleibt, wird es beibehalten und dient als Wissensstruktur für die weitere Interaktion, insofern ist es prägend und wirkt sich auf den weiteren Wissenserwerb aus. Die berufsrelevanten Erfahrungen werden in Form eines Interaktionswissens habitualisiert.

\footnotetext{
${ }^{160}$ Vgl. Fried 2002, 13.

161 Ebd. 130.

162 Vgl. Fried 2003, 113.
} 
Die pädagogische Praxis ist in Organisationssysteme eingebunden und daher stellt sich die Frage nach einem expliziten Organisationswissen. Im Zusammenhang einer Untersuchung zum Fortbildungsbedarf und der Analyse der Daten nach Rückgriff auf Wissenssysteme kommt Fried 2002 zu dem Ergebnis, dass sich ein Organisationswissen kaum nachweisen lässt. Wobei sie darauf hinweist, dass die Wissensbasis durch implizites Organisationswissen, z.B. in Form von Ordnung, Standards, Konventionen verinnerlicht ist, aber zu wenig reflektiert sei. ${ }^{163}$ Dies deckt sich mit der Einschätzung Helspers (vgl. Kapitel 3.3.2), der von der Verstärkung der Antinomien durch mangelnde Reflexion des Zusammenhanges Interaktion und Organisation ausgeht.

Wenn sich pädagogisch Professionelle ihre unterschiedlichen Wissensbestände (Theorie- und Praxiswissen) nutzbar machen wollen, um ihren Beruf durch eine Verschränkung der Perspektiven bewältigen zu können, benötigen sie Kopplungswissen, also Wissen, wie man sich welcher Wissensbestände bedient. Dabei stehen innen das im Wissenschaftssystem produzierte und in der Ausbildung vermittelte Technologiewissen sowie das in der Praxis durch Erfahrung generierte Wissen, das Interaktionswissen, zur Verfügung. Fried (2003) weist nach, dass die berufspraktische Bedeutung von Technologiewissen, das im Wissenschaftssystem produziert wird, deutlich mehr Bedeutung für pädagogisch Professionelle hat als die berufsbezogene Leistungsfähigkeit von Interaktionswissen. Sie geht davon aus, dass durch Beobachtung und Reflexion des eigenen Blickwinkels der Berufspraxis das Interaktionswissen stärker nutzbar gemacht werden könnte. Bisher hat sich das Professionsverständnis aus der Perspektive des Wissenschaftssystems heraus entwickelt, was dafür spricht, dass eine wissenschaftliche Basis der Berufsausübung besteht. ${ }^{164}$

\subsubsection{Professionalität von Schulleiterinnen und Schulleitern}

Auf die zentrale Frage des Professionsstatus des Lehrberufs und auch des Berufes Schulleiterin/ Schulleiter gibt es keine einheitliche Antwort, überwiegend wird aber davon ausgegangen, dass der Beruf professionalisierbar bzw. professionalisierungsbedürftig ist. Wird das Kriterium der wissenschaftlichen Basis der Berufsausübung in den Mittelpunkt gestellt, so gibt es bisher keine eigenständige Berufsqualifikation für Schulleiterinnen und Schulleiter, die zum Beispiel die wissenschaftlichen Grundkenntnisse im Bereich Führungstheorie und Organisationstheorie vermittelt, obwohl das Wissen dieser Theorien im "neuen“ Berufsbild vorausgesetzt wird. Sicherlich gibt es aber einen bestimmten Grad an Wissen aus den genannten Bereichen, das sich akkumuliert hat, hier wird insbesondere das in der Praxis erworbene Wissen von Bedeutung sein. Die Professionalität der Schulleiterinnen und Schulleiter lässt sich bisher nur über den Vergleich mit der Professionalität des Lehrberufs bestimmen, dabei ist davon auszugehen, dass die Professionalität der Schulleiterinnen und Schulleiter zusätzliche Erfordernisse zu erfüllen hat. In seinem kriterienbezogenen Vergleich der Professionalität von Lehrkräften und Schulleiterinnen und Schulleitern kommt Neulinger (1990) zu dem Ergebnis, dass die Schulleitertätigkeit insbesondere durch ihre fehlende Ausbildung weniger professionell sei als die des Lehrberufes. Dabei geht er davon aus, dass profes-

\footnotetext{
${ }^{163}$ Vgl. Fried 2002, 172.

${ }^{164}$ Vgl. Fried 2003, 124.
} 
sionelles Schulleitungshandeln erst im Verlauf der Berufsausübung durch Erfahrung, Routine und Talent möglich wird. ${ }^{165}$

Im Hinblick auf das Autonomiepostulat muss der Kontext Schule beachtet werden, denn es kann sich nicht um eine freie Profession handeln, sondern höchstens um einen Beruf, in dem das Berufshandeln in einem hierarchisch geordneten Organisationssystem stattfindet, welches schon aus sich heraus eine Entwicklung zur klassischen Profession stark einschränkt. Daher erhält bei der Betrachtung des Berufes Schulleiterin/ Schulleiter das Verhältnis zwischen Individuum und Organisation eine besondere Bedeutung, insbesondere Schütze (1996) verweist darauf, dass es die paradoxalen Strukturen der professionellen Berufe sind, die zu strukturellen Fehlern bei der Arbeit führen können und dass diese auf zwei Ebenen wirksam sind, auf der Ebene der Interaktionsbeziehungen zwischen Professionellen und Klienten oder in der Beziehung des Professionellen zur Organisation. ${ }^{166}$ Die in der Professionstheorie vielfach thematisierte Spannung zwischen Profession und Organisation wird für Schulleiterinnen und Schulleiter, die an der Spitze der Organisation Schule stehen, besonders relevant. Aus meiner Einschätzung heraus ergeben sich folgende Spannungszustände:

- Schulleiterinnen und Schulleiter stehen an der Schnittstelle zwischen Schulbehörde und Kollegium der Schule, sie müssen für die Umsetzung von Erlassen sorgen, auch wenn sie diese selbst eventuell nicht begrüBen.

- Schulleiterinnen und Schulleiter sind in erheblichem Maß von politischen Entscheidungen und Eingriffen abhängig. Die Bereitstellung von materiellen und personellen Ressourcen ist immer motiviert von politischen Relevanzsetzungen. Reformen müssen in kurzer Zeit umgesetzt werden, z.B. Auflösung der Orientierungsstufe, unabhängig davon, ob die personellen, materiellen Voraussetzungen für die Situation der Einzelschule gegeben sind.

- Gerade die einzelschulspezifische Situation stellt den Handlungskontext der Schulleiterinnen und Schulleiter dar und hat erheblichen Einfluss auf die Gestaltungsmöglichkeiten der Schule, z.B. Anmeldezahlen, vorgefundenes Personal, Unterrichtsversorgung, finanzielle Mittel des Schulträgers, Attraktivität des Standortes etc. Je nach Ausgangssituation müssen andere Prioritäten gesetzt werden bzw. entstehen Widersprüche zwischen eigenen Entwicklungsideen und Machbarkeit, die nicht unbedingt allein durch die Schulleiterin/ den Schulleiter aufzulösen sind.

- Die Position der Schulleiterin/ des Schulleiters weist ein grundsätzliches berufsimmanentes Spannungs- und Konfliktpotential im dienstlichen Bereich auf. Der Umgang mit Unterschiedlichkeit, mit Subgruppen und Leistungsschwächen im Kollegium stellt ein großes Feld der Alltagpraxis dar. Demgegenüber steht aber eine strukturelle Schwäche der Leitungsperson.

Diese Aspekte möglicher widersprüchlicher Anforderungen richten den Fokus auf das Verhältnis Schulleiterin/ Schulleiter zur ihren Organisations-

\footnotetext{
${ }^{165}$ Vgl. Neulinger 1990, 51.

${ }^{166} \mathrm{Vgl}$. Schütze 1996, 187.
} 
strukturen. Soretz (2003) gibt einen wichtigen Hinweis darauf, dass im Zusammenhang professioneller Interaktion die Gesichtspunkte Autonomie und Autorität als Bestimmungsmerkmale bisher wenig Beachtung fanden. ${ }^{167}$ Die Einbindung in den organisationalen Zusammenhang, hier der Schule, ermöglicht den Schulleiterinnen und Schulleitern einerseits Gestaltungsräume, verlangt ein hohes Maß an kommunikativen Absprachen und erfordert zugleich eine Unterordnung unter spezifische organisationale Zwänge bis hin zu Verfahrensvorschriften und Sparvorgaben.

Neben der Interaktion zwischen Schulleiterinnen/ Schulleitern und allen an Schule beteiligten Personengruppen, vornehmlich den Kolleginnen und Kollegen, auf deren freiwillige Mitarbeit sie angewiesen sind, ist von Bedeutung für das Handeln der Akteure, wie es innen gelingt, die Organisation Schule zur Erledigung ihrer Aufgabe nutzbar zu machen und in welcher Weise sie mit den vorgefundenen Grenzen umgehen.

Der Anspruch des professionellen Handelns als ein theoriegeleitetes Handeln bedeutet zwar nicht, dass jegliches berufliche Handeln der Ausübenden theoriegeleitet sein muss, wie die Begriffe Routine und Interaktionswissen verdeutlichen, allerdings wird das individuelle Handlungsrepertoire sicherer, wenn es auf verdichteten Wissensbeständen basiert. Im Rahmen der Auseinandersetzung mit der Berufsausübung von Schulleiterinnen und Schulleitern ist von Interesse, welche Wissensformen in der Berufspraxis überhaupt zum Tragen kommen bzw. rekonstruiert werden können. Bauer et al (1999) sprechen in diesem Zusammenhang von der Ausbildung eines professionellen Selbst und gelangen zu folgender Definition der pädagogischen Professionalität: „Pädagogisch professionell handelt eine Person, die gezielt ein berufliches Selbst aufbaut, das sich an berufstypischen Werten orientiert, sich eines umfassenden pädagogischen Handlungsrepertoires zur Bewältigung von Arbeitsaufgaben sicher ist, sich mit sich und anderen Angehörigen der Berufsgruppe Pädagogen in einer nichtalltäglichen Berufssprache verständigt, ihre Handlungen unter Bezug auf eine Berufswissenschaft begründen kann und persönlich die Verantwortung für Handlungsfolgen in ihrem Einflussbereich übernimmt." ${ }^{168}$ Die Sichtweise des Berufes hat große Bedeutung für die Auseinandersetzung mit den beruflichen Anforderungen und nimmt eine handlungsleitende Funktion ein. Die Entwicklung der Berufsauffassung lässt sich in die Beschreibung von Professionalität einfügen.

\subsubsection{Berufsauffassungen}

Ausgehend von der Annahme, dass Schulleiterinnen und Schulleiter sich im Rahmen ihrer Tätigkeit einerseits an den Anforderungen der Berufsaufgaben orientieren und andererseits die Auseinandersetzung mit beruflichen Aufgaben vor dem Hintergrund eines individuellen Berufskonzeptes gestalten wird, ist es im Rahmen dieser Untersuchung von Bedeutung, welche Berufsauffassungen sich rekonstruieren lassen.

${ }^{167}$ Vgl. Soretz 2003, 187.

${ }^{168}$ Bauer et al 1999, 15. 
Des Weiteren spielt das vom Schulleitungsverband erarbeitete Berufsleitbild im Sinne eines äußeren Images eine Rolle, in dem programmatische Vorgaben immer auch orientierende und motivierende Funktion enthalten und damit Einfluss auf die Berufsausübung haben.

Einen Hinweis auf Zusammenhänge zwischen Berufsauffassungen und Tätigkeiten der Schulleiterinnen und Schulleiter geben die Berufsleitbilder (vgl. S. 87 in dieser Arbeit), die im Kontext veränderter Aufgaben formuliert und gefordert werden. In beruflichen Leitbildern werden ideale Orientierungen formuliert, aber die Berufsauffassungen müssen nicht diesen Leitbildern entsprechen, es können Diskrepanzerfahrungen entstehen. Passungen oder Widersprüche zwischen den vorhandenen Berufsauffassungen und den Berufsaufgaben erleichtern bzw. erschweren die Auseinandersetzung mit dem beruflichen Altag. Tätigkeiten, die sich in Auffassungsbereiche einpassen lassen, werden eher integriert als Tätigkeiten, die im individuellen Berufskonzept nicht verankert werden können. ${ }^{169}$ Für das Handlungsfeld der Schulleiterinnen und Schulleiter lassen sich drei Aspekte und Dimensionen beschreiben:

- die Anforderungsdimensionen auch im Hinblick auf neue Aufgaben und Tätigkeitsbereiche

- die aus den theoretischen Bezügen hervorgehenden Dimensionen auf den Ebenen Führungsverhalten, pädagogische Professionskompetenz und Organisationsverständnis

- die subjektiven Wahrnehmungen, Haltungen und Überzeugungen innerhalb der Berufsausübung

Da die vorliegende Untersuchung insbesondere den dritten Bereich, nämlich die individuelle Sichtweise des Berufes in den Mittelpunkt rückt verwende ich den Begriff der Berufsauffassung, der häufig synonym mit dem Bergriff Berufsverständnis verwendet wird. Der Begriff der Berufsauffassung beinhaltet berufsbiografisch erworbene Urteile, die sich auf bestimmte Inhalte- hier den Beruf Schulleiterin/ Schulleiter beziehen. Terhart (1995) spricht von der orientierenden Funktion der Berufauffassung, weil sie durch ihre stabilen Anteile (Berufskonzept) ein Repertoire an Verhaltens- Einstellungs- und Positionsstandards bereitstellt. ${ }^{170}$ Berufsauffassungen umfassen Meinungen, Urteile und Einstellungen und konstruieren, obwohl sie nicht vollständig bewusst sein müssen, einen Teil der beruflichen Identität, als Niederschläge beruflicher Erfahrungen können sie expliziert werden. Die Berufsauffassung beinhaltet kognitive Anteile (z.B. Beurteilen als Berufsaufgabe), affektive Anteile (z.B. Zufriedenheit im Beruf) und handlungsleitende Anteile (z.B. Bereitschaft Konflikte anzugehen, Bereitschaft sich für eine verbindliche Umsetzung von Zielen einzusetzen).

Die Berufsauffassungen enthalten persönliche Anteile, die dem Selbstkonzept zugerechnet werden, fokussieren aber vorwiegend die beruflichen

${ }^{169}$ Vgl. Esslinger 2002, 17.

170 Vgl. Terhart 1995, 227. 
Auffassungen. Während das Selbstkonzept als die Gesamtheit der auf die eigene Person bezogenen, einigermaßen stabilen Kognitionen und Bewertungen verstanden wird, lässt sich mit dem Begriff Berufskonzept die Gesamtheit der auf den Beruf bezogenen Kognitionen und Bewertungen beschreiben. Das Berufskonzept bildet auf diese Weise einen Anteil an den beruflichen Auffassungen. Die Berufsauffassung kann somit als eine integrierende Konzeption persönlicher Berufs- und Selbstwahrnehmung bezeichnet werden. Die individuelle Sichtweise des Berufes beeinflusst dabei die eigenen Relevanzsetzungen, Interpretations- und Bewertungsmuster der beruflichen Alltagspraxis.

Ausgehend davon, dass die Sichtweise des Berufes eine handlungsleitende Funktion einnimmt, gilt nun im Hinblick auf mein Forschungsanliegen zu fragen, ob es eine berufsspezifisch konsensualisierbare Berufsauffassung von Schulleiterinnen und Schulleitern gibt. Terhart (1997) spricht von Berufskultur und gibt folgende Definition: „Er (der Begriff Berufskultur) bezeichnet die für einen bestimmten Beruf bzw. für ein Berufsfeld typischen Wahrnehmungsweisen, Kommunikationsformen und langfristigen Persönlichkeitsprägungen derjenigen Personen, die in diesem Beruf arbeiten."171 Da der „Kulturbegriff“ sehr häufig im schulpädagogischen Kontext Verwendung findet, z.B. Lernkultur, Schulkultur, verwende ich den Begriff der berufsspezifischen Auffassung, gibt es also solche „typischen“ Muster einer Berufsauffassung? Die Beobachtung und Interpretation berufsinterner Reflexionen von Schulleiterinnen und Schulleitern können es ermöglichen Einblicke in die Berufspraxis zu erhalten und damit in die innere „Berufskultur" zu gewähren, der gegenüber ein äußeres Image steht.

Bezüglich einer Neueinschätzung der von Schulleiterinnen und Schulleitern geforderten Aufgaben und unter dem Blickwinkel moderner Führungsansätze wäre eine Berufsauffassung wünschenswert, die eine eigene Berufsdefinition (professionelles Selbst) zulässt und gleichzeitig Anforderungen an ihre Position im Kontext der Organisation Schule gerecht wird.

Auf der Basis der hier dargelegten theoretischen Bezüge lassen sich meines Erachtens im Sinne eines sensibilisierenden Konzeptes folgende Aspekte und Dimensionen einer Berufsauffassung von Schulleiterinnen und Schulleitern beschreiben. Im Gegensatz zu den weit verbreiteten Programmatiken über die „richtige Schulleiterin/ den richtigen Schulleiter" zeichnet sich aus der Theorie ein Bild ab, welches auf Handlungsparadoxien eingeht und davon ausgeht, dass nur ein reflektierter Umgang mit Widersprüchen und die dafür notwendige Distanzierungsfähigkeit seitens der Akteure es ermöglicht, angemessen den Berufsanforderungen zu entsprechen. Der Aufbau einer integrativen Berufsauffassung kann in diesem Sinne als Prozess der Professionalisierung angesehen werden.

${ }^{171}$ Terhart 1997, 452. 
Abb. 8: $\quad$ Modell einer Berufsauffassung bezogen auf den Beruf Schulleiterin/Schulleiter

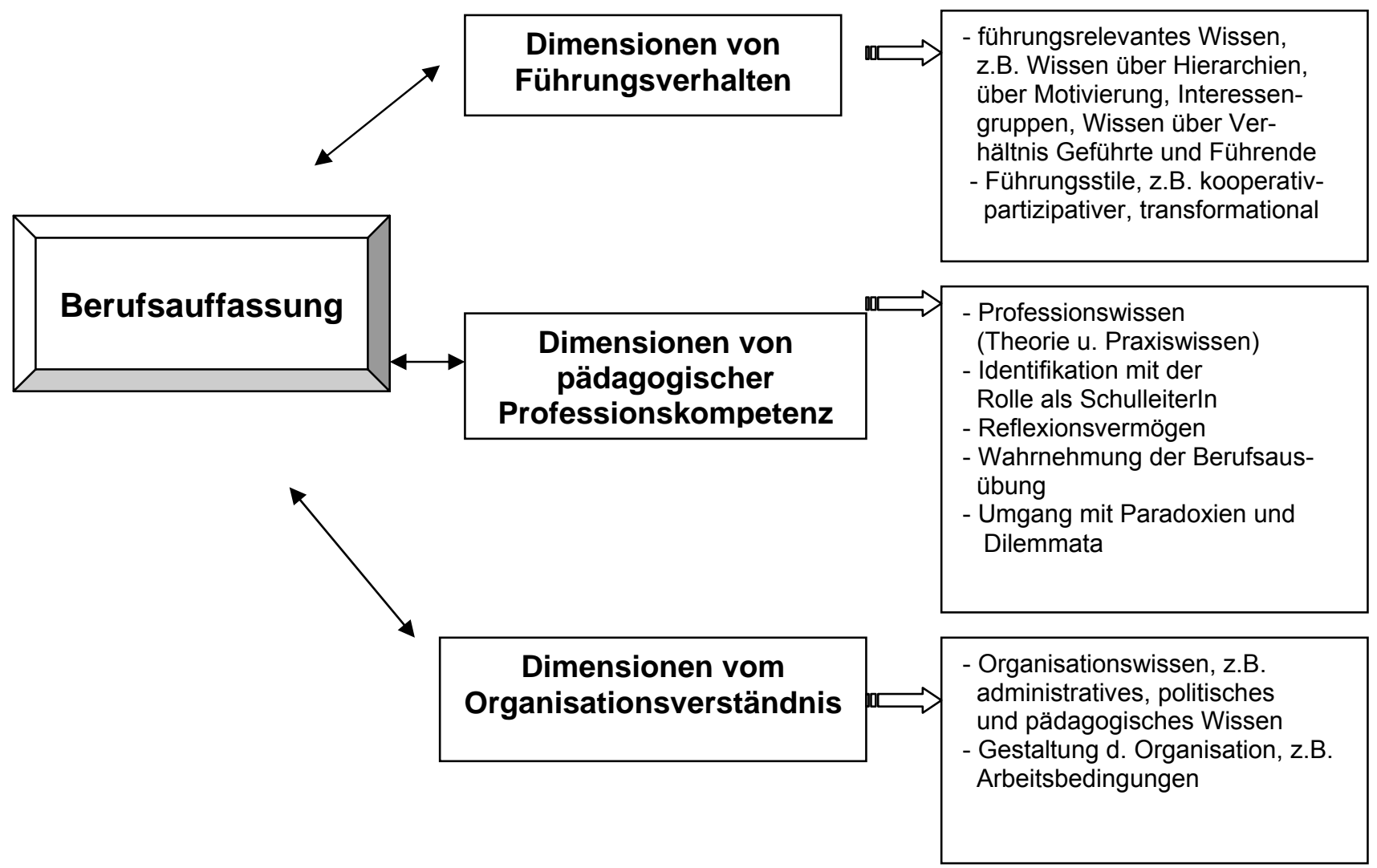

Ziel der qualitativen Interviewuntersuchung ist es, einen Zugang zu den subjektiven Wahrnehmungen der Akteure zu erhalten, also die subjektive Sichtweise und Interpretation der eigenen Berufsrolle rauszufiltern. Durch die Rekonstruktion der Berufsauffassungen der Schulleiterinnen und Schulleiter sollen Aufmerksamkeitsrichtungen im Hinblick auf führungsrelevantes, organisationales und professionelles Wissen analysiert und erforscht werden, ob es verallgemeinerbare Muster der Auseinandersetzung mit den beruflichen Anforderungen gibt.

Dabei dient das implizite Theoriewissen als "Linse“, durch die die empirische Realität wahrgenommen wird. Es gilt einerseits zu prüfen, ob und inwieweit Führung, Organisation und Profession in der sozialen Lebenswelt der Schulleiterinnen und Schulleiter eine Rolle spielen und welche Konzepte die Befragten von diesen Theorien haben bzw. in welcher Weise sie sich zu ihnen stellen, andererseits ist es möglich, ungewöhnliche und überraschende Befunde theoretisch einzuordnen. ${ }^{172}$

\subsubsection{Zusammenfassung}

Der Wissensbasis der Professionellen wird in den verschiedenen Ansätzen eine zentrale Bedeutung beigemessen. Es wird davon ausgegangen, dass Professionelle über ein ausgefeiltes professionelles Wissenssystem verfügen. Dieser Wissenskorpus ist auf Anwendung angelegt, muss also

${ }^{172}$ Vgl. Kelle/ Kluge 1999, 29. 
folglich in der Praxis rekonstruiert werden. Dabei müssen Professionelle flexibel auf unterschiedliche Wissensbestände zurückgreifen, um in unterschiedlichen situativen Kontexten dieses Wissen spezifizieren zu können. Andererseits muss das Wissen stabil sein, um eine verlässliche Handlungsgrundlage bieten zu können. Ein Zusammenspiel von handlungssichernden Gültigkeiten, also berufsrelevantem Fachwissen und berufsbezogenem Erfahrungswissen, entsteht.

In der aktuellen Professionstheorie der Lehrerinnen und Lehrer wird überwiegend davon ausgegangen, dass die Erfüllung der komplexen Berufsausübung an bestimmte antinomische bzw. paradoxale Interaktionsmodi gebunden ist. Schulleiterinnen und Schulleiter müssen sich in noch stärkerem Maße als Lehrkräfte mit ihrem eigenen Verhältnis gegenüber den strukturellen Gegebenheiten der Organisation auseinandersetzen, um in der Berufspraxis organisationale Bedingungen in ihren ermöglichenden Strukturen annehmen und nutzbar für die Erledigung der Arbeitsaufgaben machen zu können. In diesem Kontext ist es bedeutsam, wie die Akteure die Frage der Balance von eigener professioneller Autonomie und Organisationsratio halten.

Die professionstheoretischen Überlegungen zum Verhältnis Individuum und Organisation erscheinen im Hinblick auf die Anforderungen der Schulentwicklung insbesondere auf der Ebene des Schulleitungshandelns wichtig, denn die Selbststeuerung der Schule ist von Seiten der Politik besonders dort gewünscht, wo sie zu Einsparungen führt- Schulleiterinnen und Schulleiter stehen im Spannungsverhältnis zwischen mehr Gestaltungsfreiheit, damit verbunden ist die Übertragung von mehr Aufgaben, unter den Bedingungen knapperer personeller und finanzieller Mittel.

Ferner entspricht der Anspruch auf Weiterentwicklung der eigenen Unterrichtspraxis und der Schule nicht automatisch den Überzeugungen der Lehrerinnen und Lehrer, gerade dort, wo Veränderungen eine Einschränkung ihrer individuellen pädagogischen Autonomie bedeutet oder Routinen in Frage stellt, kann mit Widerstand des Kollegiums gerechnet werden. Die Schulleiterinnen und Schulleiter müssen Wege aushandeln und dabei sowohl Kontinuität als auch Entwicklung im Blick haben.

Im Rahmen der Beschäftigung mit den Berufsauffassungen von Schulleiterinnen und Schulleitern ist von Interesse, auf welche Wissensformen die Akteure durch ihren persönlichen Stil der Auseinandersetzung mit den komplexen Berufsaufgaben Bezug nehmen.

\section{4. $\quad$ Eigene Untersuchung}

\subsection{Zielsetzung und methodisches Vorgehen}

Ziel meiner Untersuchung ist es, durch eine quantitative Befragung zu prüfen, ob sich die durch die Schulleitungsforschung herausgearbeiteten führungsrelevanten Handlungsdimensionen in der Selbsteinschätzung der niedersächsischen Schulleiterinnen und Schulleiter wieder finden lassen. In einem zweiten Untersuchungsteil soll anhand qualitativer Interviews rekonstruiert werden, an welchen Dimensionen die Schulleiterinnen und Schulleiter sich innerhalb ihrer Berufsauffassung orientieren: 
- Welches Führungsverständnis ist für die befragten Schulleiterinnen und Schulleiter bedeutsam? Welche Führungsverständnisse werden im empirischen Material deutlich, gibt es ein dominantes Führungsverständnis?

- Auf welches Wissen über die Organisation von Schule greifen die befragten Schulleiterinnen und Schulleiter bei ihrer Tätigkeit zurück bzw. welches Verständnis von Organisation wird in ihren Berufsauffassungen sichtbar?

- Welches pädagogische Professionswissen ist für sie handlungsleitend bzw. auf welches Professionswissen greifen sie zurück?

Die Untersuchung in Form einer quantitativen Befragung orientiert sich an den bereits gewonnenen Erkenntnissen anderer Untersuchungen, so untersuchte Wissinger (1996) mit einem standardisierten Fragebogen das Selbstverständnis von 196 Schulleiterinnen und Schulleitern aller Schulformen in Bayern unter der Perspektive des Führungshandelns. Wichtigstes Ergebnis war hier, dass Schulleiterinnen und Schulleitern ihr schulisches Führungshandeln auf die Gestaltung der zwischenmenschlichen Beziehungsarbeit fokussieren und damit auf der Individualebene verhaftet bleiben, anstatt ihre Schule auf der Systemebene zu lenken. ${ }^{173}$

Bonsen (2002) untersuchte in Nordrhein-Westfalen die Wirksamkeit von Schulleitung und ermittelte dabei fünf Bedingungsbereiche einer gelungenen Führungskonzeption: Zielgerichtete Führung, Innovationsbereitschaft und Organisationskompetenz sowie Management der sozialen Beziehung und Partizipation an Entscheidungsfindungen. Diese ermittelten Dimensionen, die auch durch die Schulqualitätsforschung bestätigt worden sind, sollen nun zunächst auf ihre Relevanz in der Selbsteinschätzung der Schulleiterinnen und Schulleiter dieser Stichprobe überprüft werden. ${ }^{174}$

Daher wurde in einem ersten Schritt eine standardisierte quantitative Schulleiterinnen/ Schulleiter-Befragung durchgeführt. So konnten Handlungsdimensionen der Schulleiterinnen und Schulleiter mit Hilfe von Korrelationsanalysen exploriert werden. Dadurch konnten erste Hinweise auf die Berufsauffassungen gewonnen werden, die dann durch die qualitative Befragung differenziert herausgearbeitet wurden.

Mit dem skizzierten Vorgehen wird eine Verknüpfung eines quantitativen Verfahrens mit einer qualitativen Analyse des Interviewmaterials möglich. Die Kombination beider Auswertungsstränge sollte vertiefende Erkenntnismöglichkeiten bieten. Das Ziel war die Rekonstruktion subjektiver Deutungsmuster der Schulleiterinnen und Schulleiter. Dagegen sollten nicht die Effekte ihrer Handlungen überprüft werden, wie es sich die Wirksamkeitsforschung zur Aufgabe gemacht hat.

\footnotetext{
${ }^{173}$ Vgl. Wissinger 1996, 169-170.

${ }^{174}$ Vgl. Bonsen 2002, $75 f$
} 


\subsubsection{Fragestellung}

a. Durch den standardisierten Fragebogen soll geklärt werden, ob sich bei niedersächsischen Schulleiterinnen und Schulleitern die führungsrelevanten Dimensionen: Zielgerichtete Führung, Innovationsbereitschaft, Organisationskompetenz, Management der sozialen Beziehung und Partizipation der Entscheidungsfindung abbilden lassen. Wenn dies nachgewiesen werden kann, dann kann das als ein Hinweis dafür angesehen werden, dass einem „effektiven“ Führungsverständnis zumindest zugestimmt wird. Interessant ist in diesem Zusammenhang die Beantwortung der

Items, die nicht nur nach Zielen fragen, sondern auch nach der Bereitschaft, sich für die konkrete Umsetzung dieser Ziele einzusetzen. Des Weiteren wird der Fragekomplex Schulentwicklung nicht nur Aufschluss über die Befürwortung oder Ablehnung von Innovationen geben, sondern auch die Rolle der Schulleiterin/ des Schulleiters in diesem Prozess aufzeigen. Daneben sollen die Berufszufriedenheit und das Belastungsempfinden der Schulleiterinnen und Schulleiter erfragt werden.

b. Die leitfadengestützen qualitativen Interviews sollen einerseits vertiefenden und differenzierten Aufschluss über die in der Handlungspraxis zum Tragen kommenden Handlungsdimensionen, wie sie im Fragebogen erfragt wurden, geben. Andererseits soll aufgrund theoretischer Vorüberlegungen die Berufsauffassung der Schulleiterinnen und Schulleiter ermittelt werden und geprüft werden, ob sich eine verallgemeinerbare berufsspezifische Berufsauffassung beschreiben lässt.

\subsection{Triangulation}

Der Begriff der Triangulation bezeichnet im Kontext der Sozialforschung die Betrachtung eines Forschungsgegenstandes aus zwei unterschiedlichen Perspektiven. Durch die Triangulation (verschiedener Methoden oder verschiedener Datensorten) soll ein Erkenntniszuwachs möglich werden, also beispielsweise Kenntnisse auf unterschiedlichen Ebenen gewonnen werden, die weiter reichen, als es mit einem Zugang möglich wäre. ${ }^{175}$ In der Diskussion um qualitative Forschung hat die Triangulation in den 70er Jahren durch die Konzeptualisierung von Denzin (1970) starke Beachtung gefunden. Denzin (1970) unterscheidet verschiedene Formen der Triangulation: „Data-Triangulation“ bezeichnet die Einbeziehung unterschiedlicher Datenquellen in Abgrenzung zur Verwendung unterschiedlicher Methoden der Hervorbringung von Daten. ${ }^{176}$ Als zweite Form nennt Denzin (1970) die „Investigator Triangulation“, hier werden unterschiedliche Beobachter oder Interviewer eingesetzt, um Verzerrungen durch die Person des Forschers zu vermeiden. Der dritte Typ ist die „TheorienTriangulation“. Die Theorien-Triangulation stellt unterschiedliche theoretische Sichtweisen auf den Forschungsgegenstand nebeneinander.

Die vierte Form, die „methodologische Triangulation“ entweder innerhalb einer Methode (within-method) oder von verschiedenen Methoden (bet-

\footnotetext{
${ }^{175}$ Vgl. Flick 2004,12.

${ }^{176}$ Vgl. Denzin 1970, 301.
} 
ween-method) findet die stärkste Beachtung und soll nachfolgend erläutert werden.

\subsubsection{Methodologische-Triangulation}

Die Methodologische-Triangulation kann sowohl als Triangulation innerhalb einer Methode (within-method), zum Beispiel die Verwendung verschiedener Subskalen in einem Fragebogen, als auch als Triangulation zwischen verschiedenen Methoden (between-method) durchgeführt werden.

Mit der zweiten Form, also der Kombination zweier Methoden, wird nach Denzin (1970) Auffassung die Reliabilität der Ergebnisse einer Untersuchung gesteigert. Gerade an diesem Punkt setzt die Kritik an Denzins Konzeptualisierung an. Silvermann (1985) verweist auf die Reaktivität von Methoden, denn jede Methode konstituiert den Gegenstand, der mit ihr erforscht wird, auf spezifische Weise. ${ }^{177}$ Bei der Kombination zweier Methoden, zum Beispiel qualitativer und quantitativer Verfahren, kann nicht unbedingt davon ausgegangen werden, dass jeweils der eine Ansatz die gleichen Erkenntnisse erbringt wie der andere bzw., dass bei Diskrepanzen der Ergebnisse das eine oder andere Resultat damit widerlegt wird.

Als Konsequenz dieser Diskussion wird eine eher pragmatischere Umgehensweise mit der methodologischen Triangultaion praktiziert, so schlägt Lamnek (1988) vor, die Erwartungen an Triangulation realistischer anzusetzen, da er davon ausgeht, dass übereinstimmende Ergebnisse nicht zu erwarten seien, wenn Methoden von unterschiedlicher Qualität eingesetzt werden. Die Ergebnisse können sich aber gegenseitig ergänzen. ${ }^{178}$

\subsection{Quantitative Erhebung}

Die Kombination quantitativer Forschung und qualitativer Forschung findet in der vorliegenden Arbeit durch die Verknüpfung eines standardisierten Fragebogens mit qualitativen Interviews Anwendung. Es wird eine in die Breite gehende standardisierte Befragung mit einer in die Tiefe gehenden qualitativen Befragung kombiniert.

Ziel dieser methodologischen Triangulation („,between-method“) ist es, durch Einbeziehung verschiedener Erhebungs- und Auswertungsmethoden eine umfassendere und vielschichtigere Erkenntnis zu erhalten, dabei wird die Fragestellung von unterschiedlichen Perspektiven aus betrachtet. Hierbei muss berücksichtigt werden, dass zwei methodische Zugänge möglichst an einem Fall trianguliert werden müssten, um die Ergebnisse der unterschiedlichen Zugänge miteinander vergleichen zu können, d.h. jeder ausgefüllte Fragebogen mit dem jeweiligen Interview. Die Ergebnisse der standardisierten Schulleiter-/Schulleiterinnenbefragung können zwar allgemein mit den Daten der qualitativen Interviews verglichen werden, der Rückschluss eines Interviews auf einen Fragebogen oder umgekehrt ist aber aus Datenschutzgründen zu keinem Zeitpunkt der Untersuchung möglich. Ergebnisse können also nur in ihrer Gesamtheit miteinander verglichen werden. Die Antworten der Fragebögen werden in ihrer Häufigkeit und Verteilung analysiert. Die Interviews werden interpretiert und die Ergebnisse in Beziehung gesetzt. Dabei wird versucht, beiden An-

\footnotetext{
177 Vgl. Silvermann, 1985, 21.

178 Vgl. Lamnek 1988, 236.
} 
sätzen in ihrer Besonderheit Rechnung zu tragen und ebenso die Unterschiede der Ergebnisse kritisch zu thematisieren.

Abb. 9: $\quad$ Triangulation

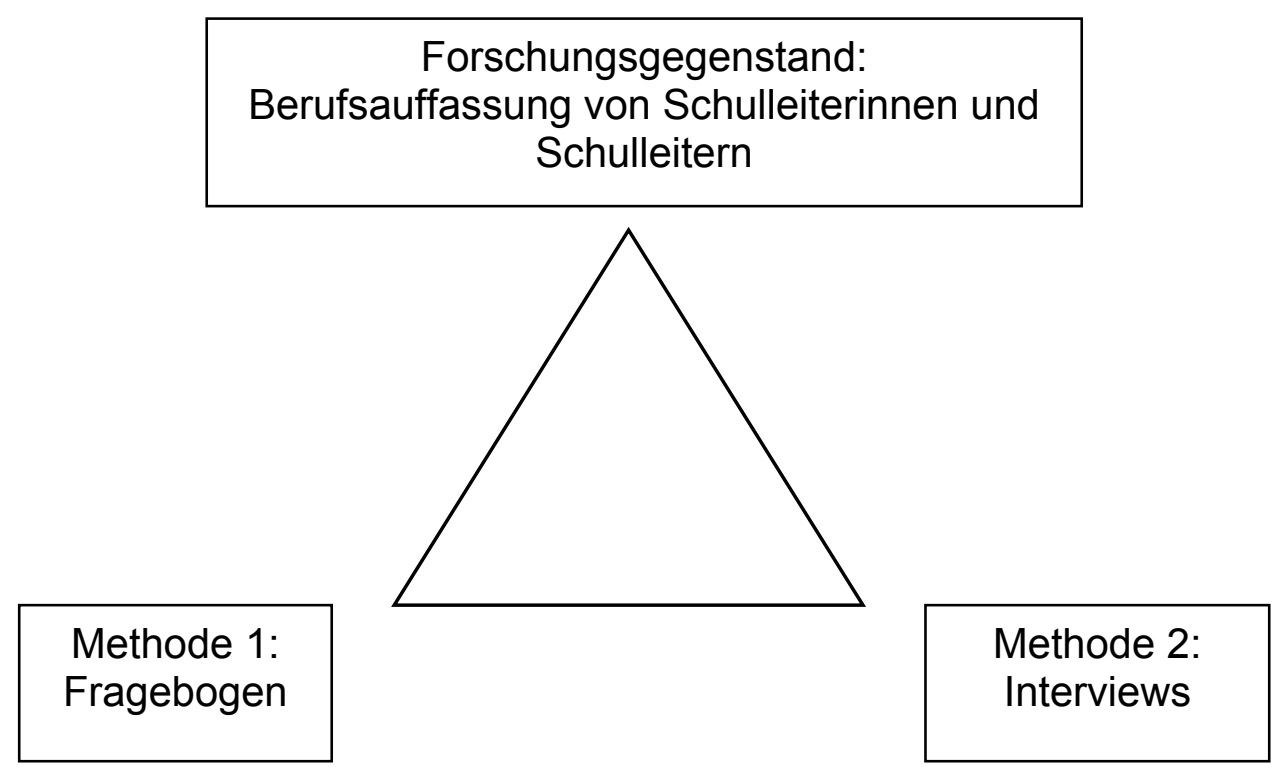

\subsubsection{Fragebogen}

Der von mir entwickelte Fragebogen orientierte sich an den oben genannten führungsrelevanten Dimensionen. Die einzelnen Fragenkomplexe greifen Fragen der Untersuchung von Wissinger (1996) zum Selbstverständnis von Schulleiterinnen und Schulleitern in Bayern auf. ${ }^{179}$

Ein Pre-Test wurde aus forschungsökonomischen Gründen lediglich mit zwei Probanden durchgeführt, mit einem ehemaligen Schulleiter und einer ehemaligen Schulleiterin, die sowohl den Fragebogen ausfüllten als auch ein Interview mit mir führten. Das Ziel dieses Testes war es, einerseits die Verständlichkeit der Items zu kontrollieren und andererseits durch die Befragten auf weitere für die Interviews bedeutsame Inhalte aufmerksam gemacht zu werden. Die Items erschienen den Befragten angemessen, der Bereich Umgang mit Ressourcen, der in der Probe keine große Rolle spielte, wurde im Leitfaden ergänzt.

Der Fragebogen der Hauptuntersuchung enthält insgesamt 62 Fragen/ bzw. Items (vgl. Anlage 1). Zu Beginn des Fragebogens werden persönliche Merkmale wie Geschlecht, Alter, aber auch die bisherige Dienstzeit der Schulleiter/Schulleiterin abgefragt. Außerdem werden Informationen über die jeweiligen Schulen erhoben, z.B. Schultyp und Schülerzahl. Dieser Teil beinhaltet außerdem eine offene Frage zu dem vor der Schulleitertätigkeit ausgeübten Beruf.

Hauptbestandteil sind die Items zu den arbeitsbezogenen Wahrnehmungen und Einstellungen der Schulleiter. Entsprechend dem theoretischen Hintergrund und der allgemeinen Fragestellung wurden diese 62 Fragen den folgenden vier thematischen Bereichen zugeordnet: „Umgang mit den Lehrerinnen und Lehrern“ (Item 1-8), z.B. ich gebe häufig individuelle Be-

\footnotetext{
${ }^{179}$ Wissinger 1996, 83f.
} 
ratung und Hilfe, „Arbeitszufriedenheit/ Berufszufriedenheit" (Item 9-30), z.B. das Leiten der Schule ist für mich mit einem hohen Belastungsdruck verbunden, „Schulentwicklung“ (Item 31-40), z.B. Ich sorge dafür, dass die pädagogischen Ziele verbindlich umgesetzt werden, „Führungsverhalten“ (Item 41-62), z.B. ich diskutiere alle wichtigen Entscheidungen offen im Kollegium.

Durch den Fragenkomplex „Umgang mit Lehrerinnen und Lehrern" sollte einerseits das Verhältnis zwischen Schulleiterin/ Schulleiter und Kollegium ermittelt werden, andererseits Rückschlüsse auf den Führungsstil hinsichtlich Mitarbeiterorientierung möglich werden. Insbesondere im Bereich Arbeitszufriedenheit/ Berufszufriedenheit geht es darum, etwas über das subjektive Belastungserleben zu erfahren. Im Bereich "Schulentwicklung" geht es um die Einstellung zur Weiterentwicklung der Schule und die Bedeutung der eigenen Position in diesem Prozess, also auch um Innovationsbereitschaft. Der letzte Bereich „Führungsverhalten“ umfasst Fragen zum Führungsstil, z.B. wie werden Entscheidungen getroffen, inwieweit wird das Kollegium mit einbezogen?

Bei den 62 Items wurden Aussagen vorgegeben, die von den Befragten bewertet werden sollten. Hierbei gab es vier Antwortmöglichkeiten, die angekreuzt werden konnten. Stimme zu, stimme eher zu, stimme eher nicht zu, stimme überhaupt nicht zu.

Teilweise haben die Befragten Schulleiterinnen und Schulleiter ihr Kreuz genau auf die Grenze zwischen zwei Antwortkategorien gesetzt, zumeist zwischen „stimme zu“ und „stimme eher nicht zu“. Dies könnte als Hinweis darauf verstanden werden, dass eine mittlere, neutrale Antwortmöglichkeit gewünscht gewesen wäre. Darauf wurde aber bewusst verzichtet, um die Befragten zu einer Entscheidung zu zwingen, ihnen damit eine „Zuflucht“ in die neutralen Aussagen zu versagen. Die Antworten auf den Grenzen wurden im Datensatz als fehlend kodiert.

Die Aufbereitung und Auswertung der Daten wurde mit Hilfe einer wissenschaftlichen Hilfskraft der Universität Göttingen durchgeführt. Die Datenauswertung erfolgte in einem ersten Analyseschritt mit dem SPSS- Programm (Statistical Package for the Social Science).

\subsubsection{Auswahl des Samples}

Die Auswahl der Befragten wurde auf die Gruppe der Schulleiterinnen und Schulleiter in Niedersachsen beschränkt. Durch die Eingrenzung auf das allgemein bildende Schulwesen in Niedersachsen wurde damit dem Umstand Rechnung getragen, dass die Organisation der staatlichen Schulaufsicht je nach Bundesland hinsichtlich der Rechts- und Verwaltungsvorschriften differieren, diese bundeslandspezifischen Unterschiede beeinflussen die Handlungsbedingungen und Handlungsanforderungen der Schulleiterinnen und Schulleiter. ${ }^{180}$ Diese Unterschiede betreffen auch die jeweiligen Schulformen, des Weiteren spielt die Größe der Schule bei der Berechnung der Entlastungsstunden des Schulleitungspersonals eine Rolle. Insofern habe ich auch hier eine Einschränkung auf die Schulform des

\footnotetext{
${ }^{180}$ Vgl. Cortina/ Baumert 2003, 180ff.

Hier finden sich ein Überblick sowie eine ausführliche Erläuterung der rechtlichen Bestimmungen je Bundesland.
} 
Gymnasiums, der IGS und KGS vorgenommen, um die äußeren Bedingungen der Schulorganisation vergleichbar zu machen, dies betrifft nicht die inhaltliche Gestaltung, sondern zunächst die Position der Schulleiterin/ des Schulleiters sowie die Aufgabenanforderungen.

Der Fragebogen wurde an alle niedersächsischen Gymnasien, kooperativen und integrierten Gesamtschulen, die eine Sekundarstufe II haben, versandt. Dies sind 235 Schulen. Nach vorheriger Genehmigung der Bezirksregierungen wurde die Erhebung als schriftliche, postalische Befragung von Februar bis April 2004 durchgeführt. In einem Anschreiben wurde das Forschungsvorhaben erläutert und um Unterstützung gebeten (siehe Anlage 2). Die Rücksendung des Fragebogens und die eventuelle Einwilligung zu einem Interview wurden mit getrennter Post an die Universität Göttingen gesandt.

\subsubsection{Status der Schulleiterinnen und Schulleiter in Niedersachsen}

Im Folgenden möchte ich kurz auf die rechtliche Stellung und das niedersächsische Berufsbild eingehen, um die Ausgangssituation der Untersuchung darzulegen.

\subsubsection{Rechtliche Situation}

Die Schule als Institution des öffentlichen Rechts ist eine Behörde, also braucht sie eine Leitung. Die Ausgestaltung der Leitungsaufgaben ist durch schulrechtliche Vorgaben determiniert, d.h. das Handlungsfeld bestimmt sich durch Rechts- und Verwaltungsvorschriften, die zunächst den normativen Rahmen bilden. Die Gesetzgebung der Länder hat die Position des Schulleiters/der Schulleiterin mit unterschiedlichen Aufgaben und Pflichten - und damit Verantwortlichkeiten - ausgestattet. Der Schulleiter/die Schulleiterin trägt im Schulgesetz der Bundesländer und im Verständnis der Öffentlichkeit die gesamte Verantwortung für die Schule, d.h. er/sie ist für die Erfüllung des staatlichen Erziehungs- und Unterrichtsauftrages verantwortlich ${ }^{181}$.

Mit dem gesetzlichen Auftrag sind Anforderungen in den Aufgabenfeldern Sicherung des Bildungsauftrages, Schulprogrammentwicklung, Evaluation, Umgang mit Schülerinnen und Schülern, Lehrerinnen und Lehrern, Erziehungsberechtigten, Schulpersonal, Mitwirkungsgremien, Schulträger, Schulaufsicht und Öffentlichkeit verbunden. ${ }^{182}$

Im niedersächsischen Schulgesetz ist die formalrechtliche Stellung der Schulleiterin/ des Schulleiters im Paragraphen $43 \S$ wie folgt beschrieben:

§ 43 Stellung der Schulleiterin und des Schulleiters

(1) Jede Schule hat eine Schulleiterin oder einen Schulleiter.

(2) Die Schulleiterin oder der Schulleiter

1. trägt die Gesamtverantwortung für die Schule,

2. vertritt die Schule nach außen,

3. führt die laufenden Verwaltungsgeschäfte,

4. führt den Vorsitz in der Gesamtkonferenz sowie in deren Ausschuss nach § 39 Abs. 1 oder 2, bereitet die Sitzungen vor und führt die Beschlüsse aus,

5. sorgt für die Einhaltung der Rechts- und Verwaltungsvorschriften und der Schulordnung,

\footnotetext{
$181 \S 2$ NSchG 2004.

182 Vgl. Habeck 2002, 249.
} 
6. ergreift die notwendigen Maßnahmen in Eilfällen, in denen die vorherige Entscheidung der zuständigen Konferenz oder des zuständigen Ausschusses nicht eingeholt werden kann, und unterrichtet hiervon die Konferenz oder den Ausschuss unverzüglich,

7. besucht die an der Schule tätigen Lehrkräfte im Unterricht und berät sie,

8. sorgt für die Qualitätssicherung und Qualitätsentwicklung der Schule,

9. nimmt die übrigen, nicht den Konferenzen vorbehaltenen Aufgaben wahr.

(3) Die Schulleiterin oder der Schulleiter kann in Erfüllung der Aufgaben nach Absatz 2 allen an der Schule tätigen Personen Weisungen erteilen; $\S 50$ Abs. 1 Satz 1 bleibt unberührt.

(4) 1Die Schulleiterin oder der Schulleiter hat innerhalb von drei Tagen Einspruch einzulegen, wenn nach ihrer oder seiner Überzeugung ein Beschluss einer Konferenz oder eines Ausschusses

1. gegen Rechts- oder Verwaltungsvorschriften,

2. gegen eine behördliche Anordnung,

3. gegen allgemein anerkannte pädagogische Grundsätze oder Bewertungsmaßstäbe verstößt oder

4. von unrichtigen tatsächlichen Voraussetzungen oder von sachfremden Erwägungen ausgeht. Der Einspruch hat aufschiebende Wirkung. Über die Angelegenheit hat die Konferenz oder der Ausschuss in einer Sitzung, die frühestens am Tage nach der Einlegung des Einspruchs stattfinden darf, nochmals zu beschließen. Hält die Konferenz oder der Ausschuss den Beschluss aufrecht, so holt die Schulleiterin oder der Schulleiter die Entscheidung der Schulbehörde ein. In dringenden Fällen kann die Entscheidung sofort eingeholt ${ }^{183}$

Insbesondere Punkt 1 (Gesamtverantwortung) und Punkt 8 (Qualitätssicherung und Qualitätsentwicklung) geben den nötigen Raum für vielfältige Aufgaben und Kompetenzbeschreibungen und haben durch die Schulentwicklung zunehmend an Bedeutung gewonnen.

Diese Tendenz der Erweiterung der Aufgaben und Verantwortlichkeiten geht einher mit dem Bestreben zur verpflichtenden Einführung der Eigenverantwortlichen Schulen zum Schuljahr 2006/2007. Der Gesetzentwurf für das Jahr 2006 sieht vor, dass die Schulleiterinnen und Schulleiter nach $\S 43$ Absatz 4 „Vorgesetzter aller an der Schule tätigen Personen“ sind, dass sie darüber hinaus verpflichtet sind, an den Sitzzungen des zu gründenden Schulbeirates teilzunehmen und diesen Beirat über pädagogische und wirtschaftliche Entwicklung der Schule zu informieren (§ 42a Schulbeirat). Alle an Schule beteiligten Personengruppen sind verpflichtet an Befragungen, Erhebungen und Unterrichtsbeobachtungen im Rahmen der Evaluation teilzunehmen ( $\S 30$ ). Die Koordinierung und Durchführung all dieser Maßnahmen obliegt selbstverständlich der Schulleiterin/ dem Schulleiter. Deutlich wird hier, dass die Stärkung der Position meist zu einem Mehr an Aufgaben führt. ${ }^{184}$

\subsubsection{Berufsbild in Niedersachen}

Die Forderung nach Anerkennung der Schulleiterin/ des Schulleiters als eigenständiger Beruf und nach berufsspezifischer Qualifikation wird geäußert. Im Jahre 1999 erstellte die „Arbeitsgemeinschaft der Schulleiterverbände in Deutschland" ein Positionspapier (ASD) und beschrieb ein Berufsbild von Führungskräften im Schulwesen, dies wurde 2005 aktualisiert. ${ }^{185}$ Folgende Aufgaben werden genannt: Qualitätsentwicklung, Qualitätssicherung, Qualitätskontrolle, Personalführung, Personalauswahl, Be-

\footnotetext{
${ }^{183}$ Niedersächsische Schulgesetz vom August 2004, 15.

${ }^{184} \mathrm{Vgl}$. Gesetzentwurf des Landes Niedersachsen zur Einführung der Eigenverantwortlichen Schule (Stand 20.10.2005).

${ }^{185} \mathrm{Vgl}$. ASD-Bundesvorstand 1999. und ASD Schulleitung in Deutschland 2005, 5.
} 
urteilung von Personal, Mittelbewirtschaftung, Erschließung zusätzlicher Mittel, pädagogische und organisatorische Entwicklungsplanung, Evaluation und Berichterstattung, Vertretung der Schule nach außen.

Der ASD fordert auf Grund der vielfältigen Aufgaben eine fundierte Ausbildung von Schulleiterinnen und Schulleitern. Der ASD äußert Kritik daran, dass die Auswahl bei der Besetzung des Amtes nach wie vor nach einer dienstlichen Beurteilung des Unterrichts getroffen wird. Zu wenig würden Führungsqualitäten, Organisationsfähigkeiten und Fähigkeiten zur Mitarbeiterführung berücksichtigt.

Die damalige Niedersächsische Kultusministerin Renate Jürgens-Pieper beauftrage 2001 eine Projektgruppe „Arbeitsplatz Schulleitung“, die aufgrund vielfältiger Veränderungen der Anforderungen an die Tätigkeit der Schulleiterinnen und Schulleiter eine umfassende Arbeitsplatzbeschreibung erstellte und konzeptionelle Überlegungen für die Qualifizierung zukünftiger Schulleiterinnen und Schulleiter erarbeitete. Dieser Bericht wurde 2002 vorgelegt.

Das folgende Berufsbild ist von der Projektgruppe in Niedersachsen erarbeitet worden: „Schulleiterinnen und Schulleiter brauchen eine hohe Leitungs- und Führungskompetenz. Dazu gehören klare Leitvorstellungen und perspektivisches Denken ebenso wie persönliche Leistungsbereitschaft und die Fähigkeit zu überzeugen, durchzusetzen und Entscheidungen zu treffen -Glaubwürdigkeit und Konsensbereitschaft ebenso wie Fähigkeit zu Planung und Organisation“". ${ }^{186}$

Im Bereich des erstellten Anforderungsprofils gelten als zentrale Kategorien die Leitungs- und Führungskompetenz, darunter werden sechs Kompetenzbereiche mit 64 Qualifikationen und persönlichen Dispositionen aufgezählt. Zu den Kompetenzen gehören:

- soziale Kompetenz (Kommunikationsfähigkeit, kooperatives Arbeitsklima etc.)

- pädagogische Kompetenz (sie sind Lehrkräfte, erfüllen den Bildungsauftrag)

- rechtliche Kompetenz (überblicken den relevanten Rechtsrahmen, wenden Rechts- und Verwaltungsvorschriften situationsgemäß an)

- strategische Management Kompetenz (entwickeln langfristige Konzepte, strategische Ressourcenplanung, sorgen für eine nachhaltige Qualitätssicherung)

- Organisatorische Managementkompetenz (sie schaffen einen reibungslosen, transparenten Ablauf des Schulalltages)

- Personalentwicklungskompetenz (analysieren Personalbedarf, fördern Leistungsbereitschaft und berufliche Entwicklung $)^{187}$

Aus den umfangreichen Ausführungen wird deutlich, dass das Aufgabenspektrum der Schulleiterinnen und Schulleiter in differenzierter Weise erweitert wurde. Die zunehmende Selbstständigkeit und Eigenverantwortung

\footnotetext{
${ }^{186}$ Niedersächsisches Kultusministerium 2002, 13.

${ }^{187}$.Vgl. Projektbericht 2002, 13.
} 
der Schulen hat Einfluss auf die Funktion und Rolle von Schulleiterinnen und Schulleitern. Die Vorstellung, dass Schulleiterinnen und Schulleiter gute Lehrkräfte mit Verwaltungskompetenz seien oder Schulleiter/Schulleiterinnen sich als „primus inter pares“ begreifen, gelte nicht mehr, so der Schulleitungsverband. ${ }^{188}$ Klar und deutlich wird im niedersächsischen Curriculum gefordert, dass ein Rollenwechsel von der Lehrkraft zur Führungskraft stattfinden soll. Die Schulleitungsfunktion unterliege dem Wandel von einer Verwaltungs- und Kontrollfunktion hin zu einer Führungs- und Managementfunktion (vgl. Kapitel 2.1 in dieser Arbeit). ${ }^{189}$

\subsubsection{Auswahl- und Qualifizierung}

Die Auswahlverfahren zur Besetzung von Schulleitungsstellen in Niedersachsen werden in der Regel nach wie vor über das Verfahren der dienstlichen Beurteilung vollzogen. Die Kandidatin/ der Kandidat wird im Unterricht hospitiert und es wird ein ausführliches Gespräch zum Amt geführt. Anwesend sind der zuständige Dezernent, ein Dezernent aus dem Kultusministerium sowie die Fachmoderatoren.

Über beides erhält die Bewerberin/ der Bewerber eine schriftliche Rückmeldung mit einer Note. Nach Ablauf des Verfahrens aller Kandidaten findet ein Auswahlgespräch im Kultusministerium statt.

Die Schulbehörde der Stadt und das Kollegium der Schule müssen Voten abgeben, die aber keine verbindliche Richtlinie für die Entscheidung darstellen.

Die in Niedersachsen erarbeiteten ersten Vorschläge einer dreiwöchigen Qualifizierungsphase, die als Voraussetzung für eine Bewerbung angesehen werden sollte, ist nicht verpflichtend eingeführt worden.

Die daraufhin vorgeschlagene berufsqualifizierende Begleitung neuer Schulleiterinnen und Schulleiter ist ebenfalls nicht bindend, sondern stellt ein freiwilliges Angebot dar.

Sicherlich spielen Zusatzqualifizierungen eine Rolle bei der Beurteilung der Kandidatinnen und Kandidaten, aber im Prinzip kann sich in Niedersachsen jeder ohne jegliche Zusatzausbildung für das Amt der Schulleiterin/ des Schulleiters bewerben. Es erfolgt nach wie vor eine dienstliche Beurteilung durch das Amt für Schule (Stunden und Gespräch zum Amt), sowie eine Prüfung im Kultusministerium, durch die schulrechtliche Kenntnisse überprüft werden - letztendlich entscheidet der Kultusminister über die Besetzung der Stellen.

\subsubsection{Beschreibung der untersuchten Stichprobe}

Im Folgenden wird nun konkret die Stichprobe dieser Untersuchung beschrieben. Insgesamt haben von 235 angeschriebenen Schulleiterinnen und Schulleitern 145 den ausgefüllten Fragebogen zurückgeschickt. Das entspricht einer Beteiligungsquote von 61,7\%. Diese hohe Rücklaufquote spricht sicherlich für die Aktualität des Themas und die Bereitschaft der Schulleiterinnen und Schulleiter, sich intensiv mit ihrer eigenen Position auseinander zu setzen. Ähnlich hohe Rücklaufquoten hatte auch Wissinger (1996) in seiner Untersuchung. Über die Beteiligung an der Fragebogenuntersuchung hinaus waren 60 der von mir angeschriebenen Schullei-

\footnotetext{
${ }^{188} \mathrm{Vgl}$. ASD 2005, 12.

${ }^{189}$ Vgl. Wissinger 2000.
} 
terinnen und Schulleiter zu einem Interview bereit, also 41,4 \% der teilnehmenden Schulleiterinnen und Schulleiter.

Von den befragten Schulleiterinnen und Schulleitern arbeiten 103 der 145 an einem Gymnasium, 21 an einer Kooperativen Gesamtschulen und 21 an einer Integrierten Gesamtschule. Von den 145 Schulleitern, die den Fragebogen beantwortet zurückgesandt haben, sind 28 weiblich und 117 männlich. (19,3 bzw. 80,7 \%) Der jüngste Schulleiter/ die jüngste Schulleiterin ist 43 Jahre alt, die/ der älteste 66. Das durchschnittliche Alter beträgt 54,7 Jahre, die Standardabweichung liegt bei 4,3 Jahren. Nur 7,7 \% der Schulleiter sind unter 50 Jahre alt.

$75,7 \%$ der Schulen (109) haben mehr als 600 Schüler, 24,3 \% (35) weniger als 600 Schüler, bei einer Schule fehlt die entsprechende Angabe. An $52,4 \%$ der Schulen liegt der Frauenanteil im Kollegium unter 50 \%. Differenziert nach Schulformen zeigt sich vor allem an den Gymnasien ein vergleichsweise geringer Frauenanteil. An knapp $70 \%$ der Gymnasien ist weniger als die Hälfte des Kollegiums weiblich, bei immerhin gut $27 \%$ dieser Schulen liegt der entsprechende Anteil sogar unter $40 \%$. Dagegen liegt der Frauenanteil in allen kooperativen Gesamtschulen mindestens bei $50 \%$. Auch an den integrierten Gesamtschulen liegt der Frauenanteil überwiegend (81,0 \% der Schulen) bei $50 \%$ und mehr (vgl. Tab. 1)

\begin{tabular}{|c|c|c|c|c|c|}
\hline $\begin{array}{c}\text { Stichprobe in } \\
\text { Niedersachsen } \\
\text { N } 145\end{array}$ & & Gymnasium & KGS & IGS & Summe \\
\hline $\begin{array}{l}\text { Geschlecht der } \\
\text { Schulleiterinnen und } \\
\text { Schulleiter }\end{array}$ & w & $\begin{array}{c}18 \\
(17,5 \%) \\
85 \\
(82,5 \%)\end{array}$ & $\begin{array}{c}7 \\
(33,3 \%) \\
14 \\
(66,7 \%)\end{array}$ & $\begin{array}{c}3 \\
(14,3 \%) \\
18 \\
(85,7 \%)\end{array}$ & $\begin{array}{c}28 \\
(19,3 \%) \\
117 \\
(80,7 \%)\end{array}$ \\
\hline Größe der Schule & $\begin{array}{l}>600 \\
<600\end{array}$ & $\begin{array}{c}72 \\
(70,6 \%) \\
30 \\
(29,4 \%) \\
\end{array}$ & $\begin{array}{c}19 \\
(90,5 \%) \\
2 \\
(9,5 \%) \\
\end{array}$ & $\begin{array}{c}18 \\
(85,7 \%) \\
3 \\
(14,3 \%) \\
\end{array}$ & $\begin{array}{c}109 \\
(75,7 \%) \\
35 \\
(24,3 \%) \\
\end{array}$ \\
\hline $\begin{array}{l}\text { Frauenanteil im } \\
\text { Kollegium }\end{array}$ & $\begin{array}{l}\geq 50 \% \\
<50 \%\end{array}$ & $\begin{array}{c}31 \\
(30,1 \%) \\
72 \\
(69,9 \%)\end{array}$ & $\begin{array}{c}21 \\
(100,0 \%) \\
0 \\
(0,0 \%)\end{array}$ & $\begin{array}{c}17 \\
(81,0 \%) \\
4 \\
(19,0 \%)\end{array}$ & $\begin{array}{c}69 \\
(47,6 \%) \\
76 \\
(52,4 \%)\end{array}$ \\
\hline
\end{tabular}

Tabelle 1: Schulformbezogene Darstellung ausgewählter Statistiken

\subsubsection{Antworthäufigkeiten zu einzelnen Items}

Zunächst werden Ergebnisse der einzelnen Items aus den vier Fragekomplexen betrachtet (1. Umgang mit Lehrerinnen und Lehrern; 2. Arbeitszufriedenheit, hier ist das Belastungsempfinden ein Bestandteil; 3. Schulentwicklung; 4. Führungsverhalten). Bei der Interpretation dieser Ergebnisse ist immer auch zu berücksichtigen, dass eine standardisierte Befragung in Form eines Fragebogens ebenso wie offene Verfahren dazu verleiten, dass die untersuchte Person aufgrund ihrer Definition der Rolle der 
Forscherin ihre Antworten so geben, wie es den vermeintlichen Erwartungen der Forscherin an sie gerecht wird. Sie reagieren sozial erwünscht. ${ }^{190}$

\subsubsection{Umgang mit Lehrerinnen und Lehrern}

Im Bereich der Fragen zum Umgang mit Lehrerinnen und Lehrern stimmen die Schulleiterinnen und Schulleiter klaren Zielvereinbarungen und auch Unterrichtsbesuchen mehrheitlich uneingeschränkt zu. Die dienstliche Beurteilung der Lehrkräfte bei der Bewährungsfeststellung von Beamten auf Probe zu unbefristeten Beamten liegt erst seit Ende der 90er Jahre in der Hand der Schulleiterinnen/Schulleiter. Dienstliche Beurteilungen dieser Art durch die Schulleiterinnen und Schulleiter befürworten 65,7 \% der Befragten, ähnlich fällt die Beurteilung von Unterrichtsbesuchen aus. $97,2 \%$ sind der Meinung, dass Unterrichtsbesuche ein wichtiger Baustein für die Personalentwicklung seien. Demnach hat sich ein Bewusstsein in die Richtung des Vorgesetzten durchgesetzt, der die Aufgabe des Beurteilens für seine Position annehmen kann.

Abb. 10: Umgang von Schulleiterinnen und Schulleitern mit den Lehrerinnen und Lehrern

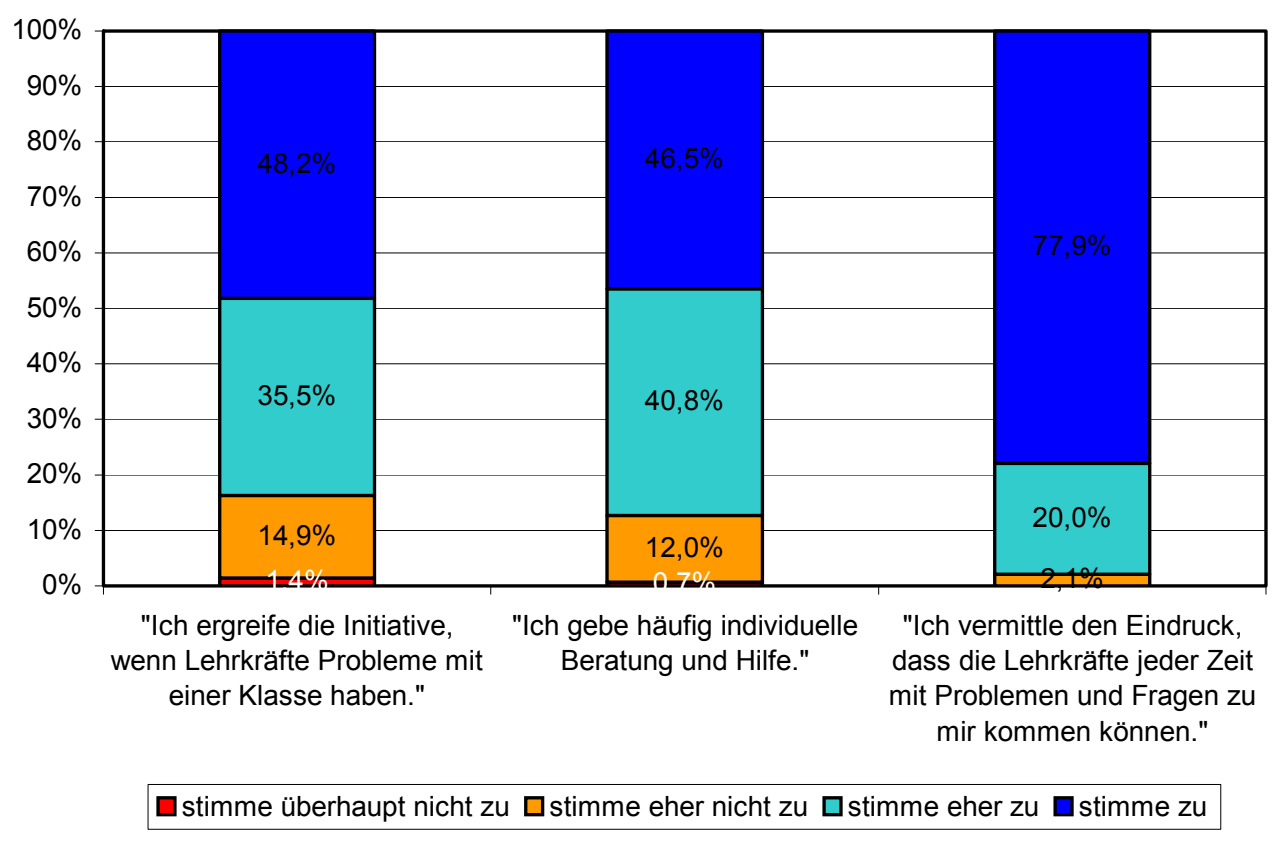

Besonders auffällig ist im Rahmen des Fragekomplexes die gleichzeitig hohe Priorität eines guten sozialen Klimas für Schulleiterinnen und Schulleiter. Die Befragten nehmen bewusst die Rolle der Beraterin/ des Beraters an und sind ansprechbar für die Probleme ihrer Kolleginnen und Kollegen (vgl. Abb. 10)

${ }^{190}$ Vgl. Friedrichs 1990, 150 
Abb. 11: Führungsverhalten von

Schulleiterinnen und Schulleitern

(ausgewählte Items)

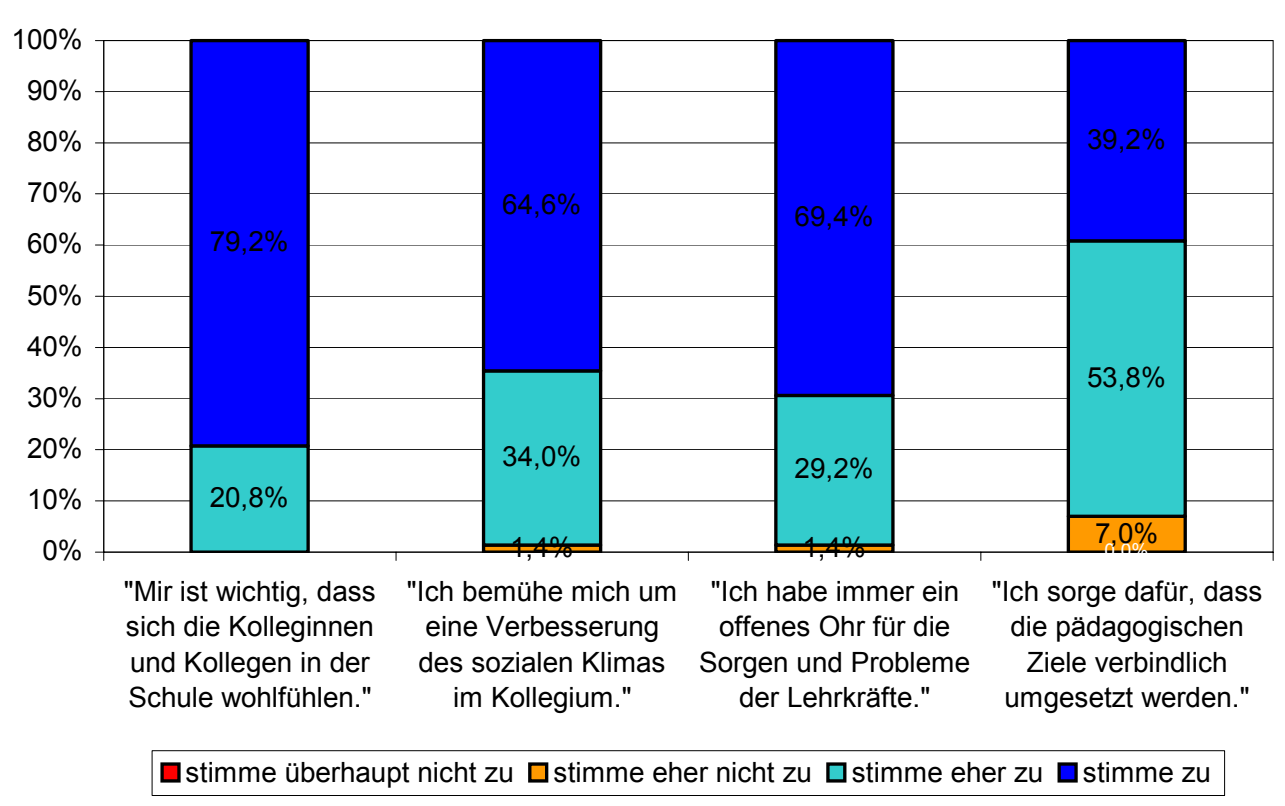

Die Mehrheit der Schulleiterinnen und Schulleiter bemüht sich um eine Klimaverbesserung (64,3\%). 79,2\% ist es wichtig, dass sich Kolleginnen und Kollegen in der Schule wohl fühlen, die übrigen 20,8\% halten die entsprechende Aussage zumindest für eher wichtig. 70,7\% der Befragten erachten Fortbildungen für das Kollegium als wichtig

Das Management der sozialen Beziehungen hat für das Führungsverhalten der Schulleiterinnen und Schulleiter offenbar einen großen Stellenwert. Die entsprechenden Aussagen erfahren eine sehr große, überwiegend uneingeschränkte Zustimmung. Es ist insgesamt eine starke Mitarbeiterorientierung festzustellen.

In den Aussagen zum Umgang mit Lehrerinnen und Lehrern wird zwar einerseits eine führungsbetonte Rolle sichtbar, denn Zielvereinbarungen, Mitarbeitergespräche und das Beurteilen von Lehrkräften sind Aufgaben, die die Rolle der Schulleiterin/ des Schulleiters neu definieren und auf eine Steuerungsfunktion im Bereich Personal hinweisen. So stimmen der Aussage „Unterrichtsbesuche sind ein wichtiger Baustein für Personalentwicklung" $75,0 \%$ voll zu und Zielvereinbarungsgespräche halten $73,6 \%$ für wichtig. Andererseits lassen sich Widersprüche dazu aufzeigen, denn nur $30,9 \%$ der Befragten stimmen der Frage zu, dass sie auch für die Übereinstimmung der Weiterbildung mit den pädagogischen Zielen sorgen (vgl. Abb. 18). 


\subsubsection{Berufszufriedenheit und Belastungsempfinden}

Abb. 12: Berufszufriedenheit von Schulleiterinnen und Schulleitern (ausgewählte Items)

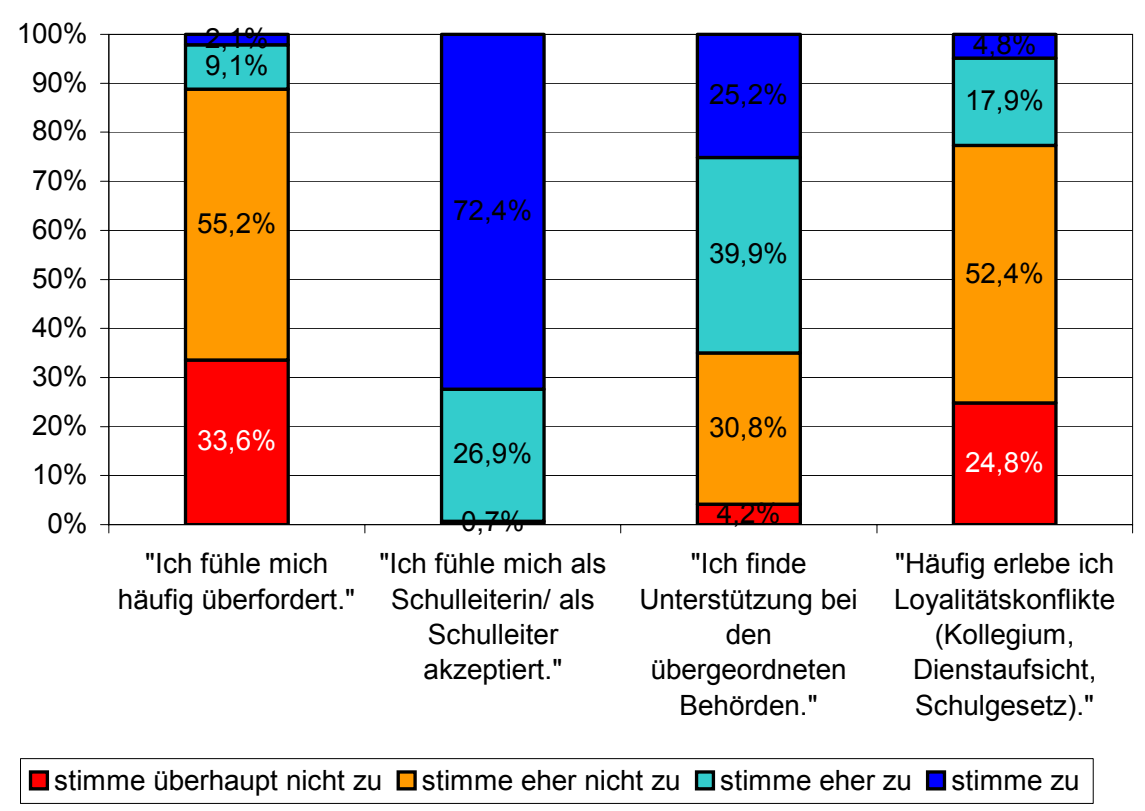

Die hier befragten Schulleiterinnen und Schulleiter sind in einem erstaunlich hohen Maß mit ihrer beruflichen Position zufrieden und fühlen sich als Schulleiterin/als Schulleiter akzeptiert.

$72,4 \%$ stimmen der Frage "Ich fühle mich akzeptiert" voll und $26,9 \%$ eher zu. Etwa gleich hohe Zustimmung erhält die Frage nach der Zufriedenheit mit der beruflichen Position. 78,5\% der Befragten stimmen „Ich bin zufrieden mit meiner beruflichen Situation" uneingeschränkt zu.

Lediglich $11,2 \%$ der Befragten halten dagegen die Aussage „Ich fühle mich überfordert" für eher oder vollkommen zutreffend. Vor dem Hintergrund der allgemein hohen Zustimmungen bezüglich der eigenen beruflichen Zufriedenheit fällt auf, dass mehr als ein Drittel der befragten Schulleiterinnen und Schulleiter (35\%) angeben, keine oder eher wenig Unterstützung bei den übergeordneten Behörden zu finden. Die Mehrheit der Befragten erlebt allerdings keine Loyalitätskonflikte (vgl. Abb. 12).

Diese Ergebnisse, die auf eine insgesamt hohe Berufszufriedenheit schließen lassen, werden durch die folgenden Items zum Belastungsempfinden relativiert und differenziert. 
Abb. 13: Berufszufriedenheit und Belastungsempfinden von

Schulleiterinnen und Schulleitern

(ausgewählte Items)

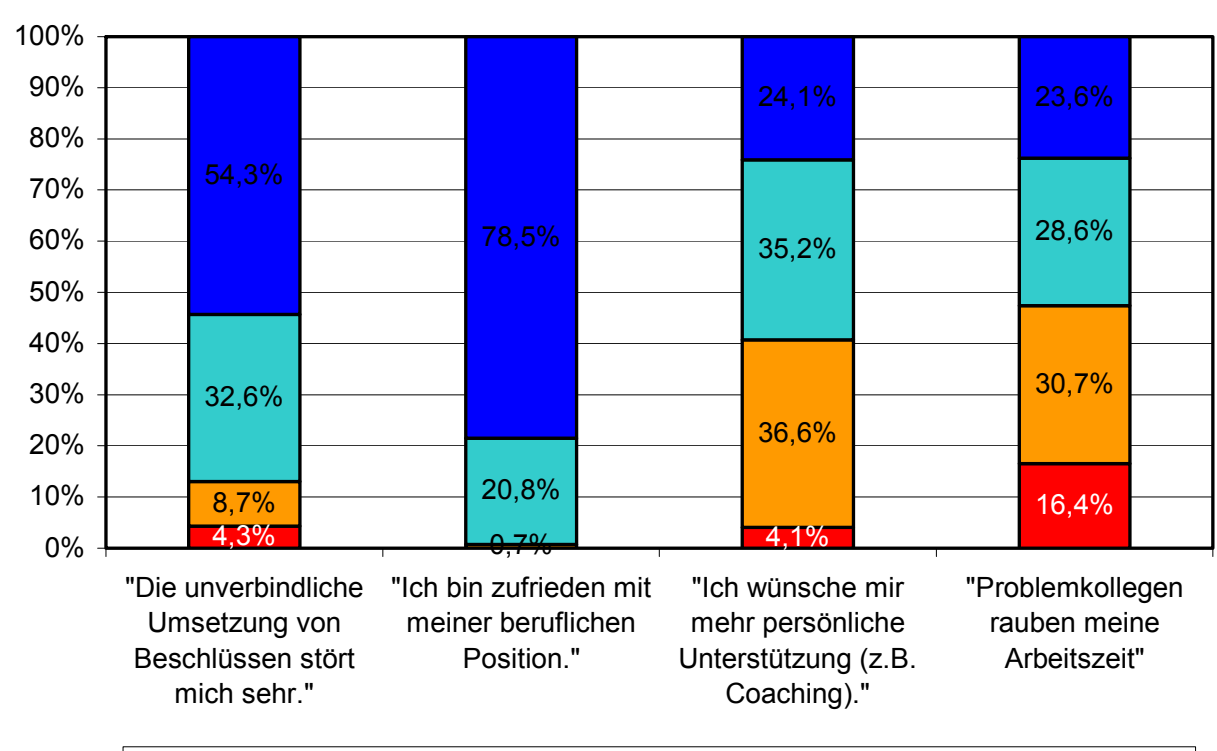

$\square$ stimme überhaupt nicht zu $\square$ stimme eher nicht zu $\square$ stimme eher zu $\square$ stimme zu

Es besteht eine Unzufriedenheit über die unverbindliche Umsetzung von Beschlüssen, dieser Aussage stimmen 86,9 \% zu (54,3 \% voll zu und 32,6 $\%$ eher zu), beides kann als ein Anzeichen dafür gesehen werden, dass der Wunsch nach Steuerung zwar da ist, deren konsequente Umsetzung in der Praxis aber nicht immer gelingt. Als einen auch zeitlich belastenden Faktor erleben die Befragten „Problemkollegen“, 23,6\% stimmen dieser Aussage voll zu und $28,6 \%$ stimmen eher zu.

Was genau „Problemkollegen sind kann das Item nicht aufklären. Es können unterschiedliche Anlässe sein, aus denen heraus Schulleiterinnen und Schulleiter Kollegen als Problemkollegen bezeichnen, so können es Kollegen sein, die ihrer Dienstpflicht nicht ordnungsgemäß nachkommen oder über die Beschwerden seitens der Schülerinnen und Schüler oder Eltern vorliegen, aber auch Kollegen, die mit eigenen Problemen zu der Schulleiterin /dem Schulleiter kommen.

Die Zustimmung zu klaren Zielvereinbarungen muss im Zusammenhang mit der Unzufriedenheit über die unverbindliche Umsetzung von Beschlüssen (Abb. 13) gesehen werden, 54,0\% der Befragten geben an, dass sie mit der Unverbindlichkeit unzufrieden sind, aber lediglich 39,2 \% sorgen für die Umsetzung von Beschlüssen. 
Abb. 14: Berufszufriedenheit und Belastungsempfinden von Schulleiterinnen und Schulleitern

(ausgewählte Items)

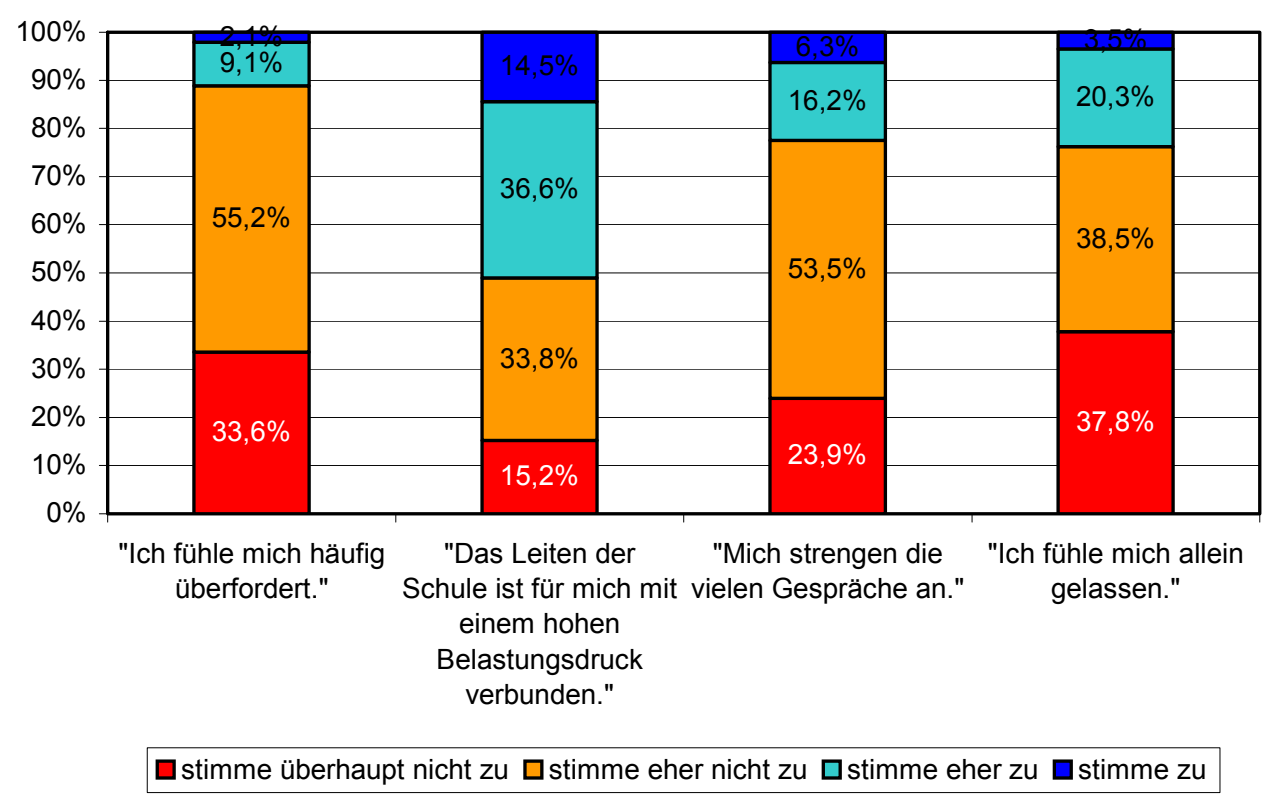

Wie Abb.14 zeigt geben zwar 88,8 \% (stimme überhaupt nicht zu + stimme eher nicht zu) der befragten Schulleiterinnen und Schulleiter an, dass sie sich nicht überfordert fühlen. Gleichzeitig trifft aber für $14,5 \%$ der Befragten uneingeschränkt und für weitere 36,6 \% der Schulleiter eher zu, dass das Leiten der Schule mit einem hohen Belastungsdruck verbunden ist. Dies könnte ein Hinweis darauf sein, dass das Belastungserleben nicht an einer persönlichen Überforderung festzumachen ist, sondern dass vielmehr Belastungen durch Faktoren außerhalb des eigenen Kompetenzerlebens erfahren werden. $50,7 \%$ der Befragten äußern, dass ihnen „Problemkollegen Arbeitszeit rauben“ (22,9 \% stimme zu + 27,8\% stimme eher zu), ebenso stören sich $86,4 \%$ an der unverbindlichen Umsetzung von Beschlüssen (54\% stimme zu $+32,4 \%$ stimme eher zu). Darüber hinaus wünschen sich $24,1 \%$ der Befragten mehr persönliche Unterstützung, z.B. in Form von Coaching. In diesem Zusammenhang muss auch beachtet werden, dass $3.5 \%$ der Aussage „Ich fühle mich allein gelassen“ uneingeschränkt zustimmen und immerhin 20,3\% der Schulleiterinnen und Schulleiter dieser Aussage eher zustimmen.

\subsubsection{Ergebnisse Schulentwicklung}

Im Bereich der Schulentwicklung zeichnet sich ein deutliches Bild der Befürwortung von Schulprogrammen und Weiterentwicklung der Schule ab. $74,1 \%$ der Befragten halten eine Schulprogrammarbeit für wichtig, 44,3\% sehen sich dabei als Initiator der Schulentwicklung. 78,5\% der Befragten halten Veränderungen im Bereich des Unterrichts für notwendig und $53,1 \%$ arbeiten in Netzwerken oder Gremien mit. Lediglich 26,4 \% stimmen dagegen der Aussage „Es gelingt mir, die Schule im Hinblick auf ein gemeinsames Ziel zu lenken"vollkommen zu, 69,3 \% der Befragten tun dies nur eingeschränkt (vgl. Abb. 15). 
Abb. 15: Schulentwicklung

durch Schulleiterinnen und Schulleiter

(ausgewählte Items)

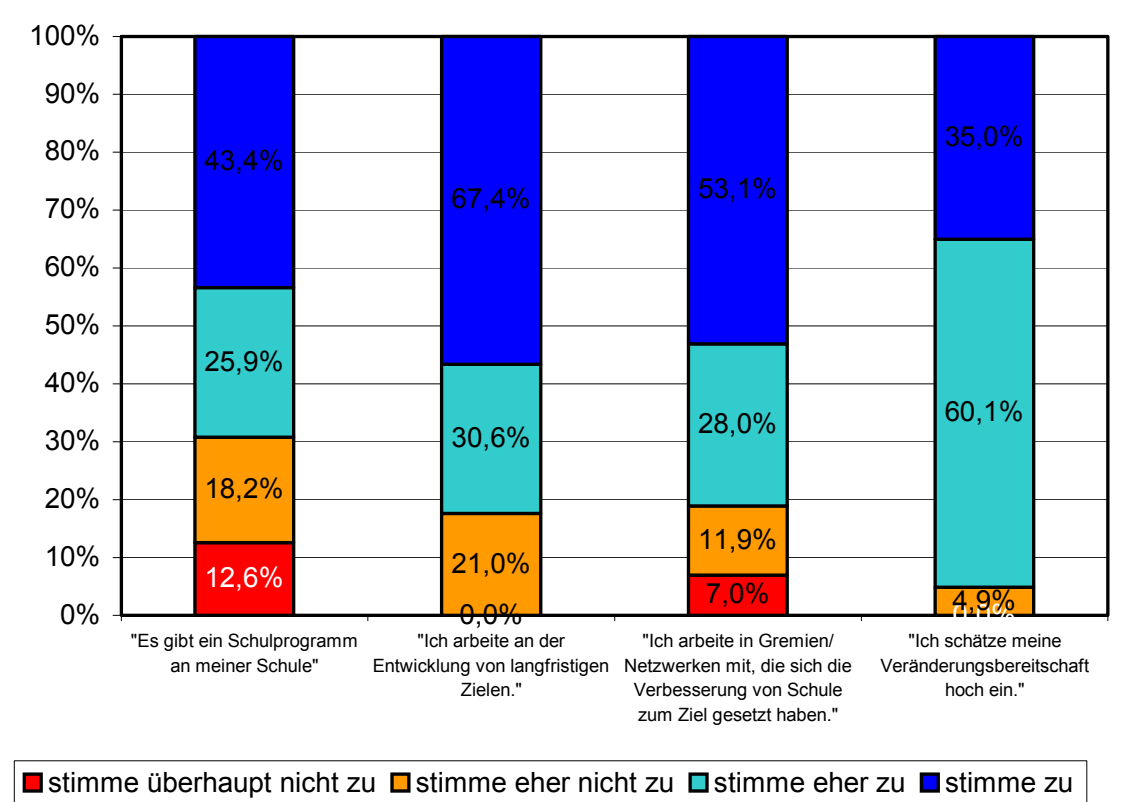

An der Entwicklung von langfristigen Zielen arbeiten zwar insgesamt $67,4 \%$ der Schulleiterinnen und Schulleiter, dort, wo es um die verbindliche Umsetzung dieser Ziele geht, sinkt jedoch die Zustimmung zu den entsprechenden Aussagen. So sorgen 39,2 \% für die verbindliche Umsetzung der Ziele und lediglich 34,3\% überprüfen die Umsetzung von Beschlüsse. Es wird ein Widerspruch deutlich zwischen der Absicht, die Schule im Hinblick auf gemeinsame Ziele zu leiten und dem Gelingen der Zielumsetzung.

Dies könnte ein Anzeichen für institutionell bedingte Probleme sein, denn in der Schule fehlen Sanktionsmöglichkeiten bei Nichteinhaltung von Beschlüssen bzw. bei nicht ordnungsgemäßer Erledigung von Aufgaben. Die Schulleiterin/ der Schulleiter benötigt alle bzw. den größeren Teil des Kollegiums, um Schulentwicklungsprozesse umzusetzen. Dabei sind Schulleiterinnen und Schulleiter auf die Freiwilligkeit zur Mitarbeit angewiesen.

Auffällig ist bei der allgemein hohen Zustimmung zur Schulentwicklung und des Rollenverständnisses als Initiatorin/Initiator für diese Prozesse (vgl. Abb. 16), dass die hier befragten Schulleiterinnen und Schulleiter ihre eigene Veränderungsbereitschaft eher niedrig einschätzen. Während $35 \%$ der Befragten ihre Veränderungsbereitschaft hoch bzw. eher hoch einschätzen, hält sich der bedeutend größere Teil der Befragten (65\%) nicht für besonders veränderungsbereit. Dies kann als ein "selbstkritisches" Ergebnis angesehen werden, welches hinsichtlich der hohen Anforderungen insbesondere des Aufgabengebietes Schulentwicklung eine Erklärung geben kann und erneut auf die Diskrepanz zwischen Wunschdenken, z.B. der hohen Befürwortung der Unterrichtsentwicklung (vgl. Abb. 16), und den eigenen Einstellungen hinweist. 
Abb. 16: Schulentwicklung durch Schulleiterinnen und Schulleiter (ausgewählte Items)

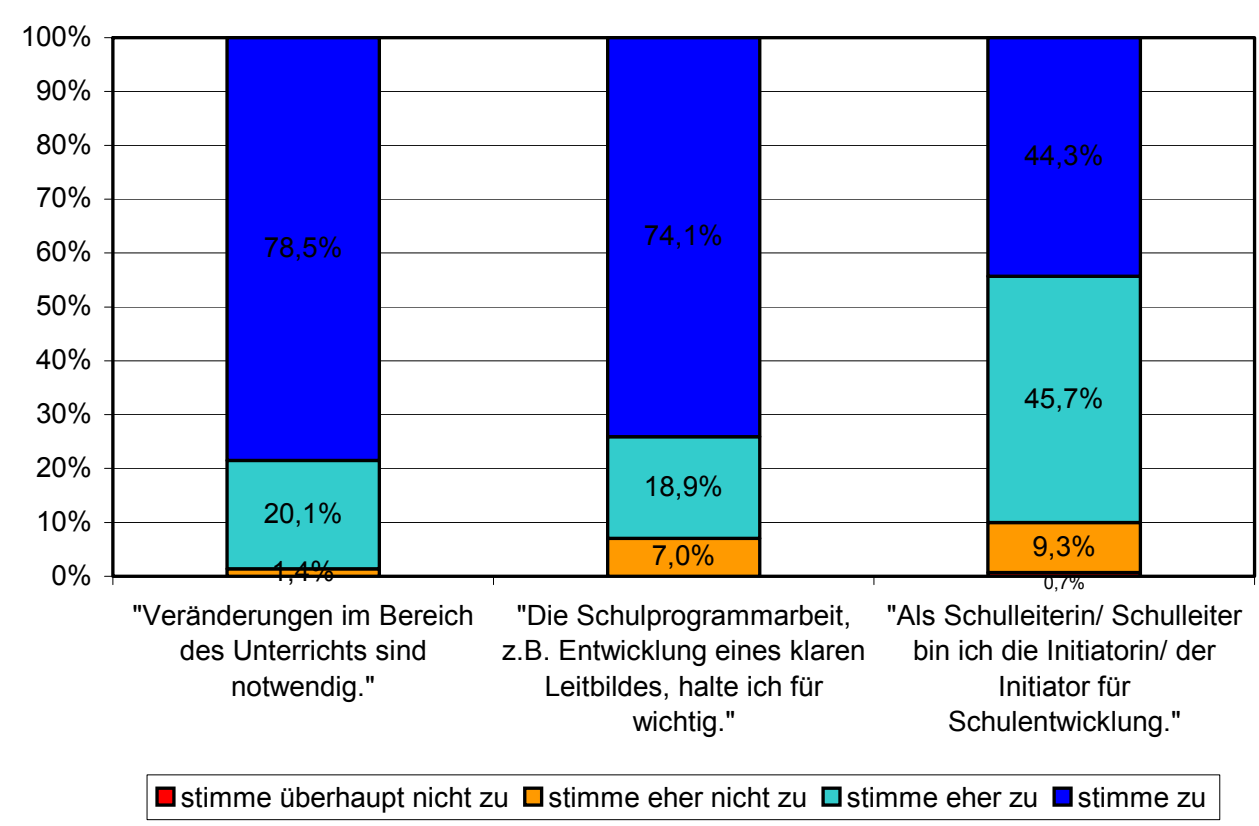

Wird davon ausgegangen, dass die Gruppe der hier befragten Schulleiterinnen und Schulleiter schon als diejenige eingeschätzt wird, die die Schule gestalten und voranbringen will, dann ist die geringe Zustimmung zu einer veränderungsfreudigen Haltung bedenklich. Denn im Zuge der Erweiterung schulischer Autonomie, in dem Entscheidungs- und Handlungsspielräume der Einzelschule erhöht werden, wird die durch die Schulleiterinnen und Schulleiter geförderte Schulentwicklung nur dann wirksam werden können, wenn diese innere Überzeugungen zu Veränderungen transportieren und dadurch Veränderungsprozesse überzeugend im Kollegium initiieren können.

\subsubsection{Führungsverhalten}

Im Bereich Führungsverhalten lassen sich Fragen zum eigenen Führungsstil und zu den eigenen Berufsauffassungen von Fragen bezüglich des Umganges mit Personal trennen (Führungsverhalten/ Mitarbeiterorientierung). Die eigene Auffassung über den Beruf ist von klaren Vorstellungen geprägt, die sich vor allen Dingen im Bereich der Schulentwicklung bereits zeigten. 97,9\% der Befragten haben klare Vorstellungen über ihre Arbeit (69,9\% stimmen dieser Aussage voll und $28,0 \%$ eher zu).

Der Aussage „ich delegiere viele Aufgaben“ stimmen 27, $5 \%$ voll und $60,6 \%$ eher zu (vgl. Abb. 17). Gerade die Beantwortung dieser Frage ist wahrscheinlich nicht nur vom persönlichen Führungsstil abhängig, sondern auch von den personalen Gegebenheiten vor Ort, inwieweit ist die jeweilige Schule mit ausreichend Koordinatoren versorgt und in welchem Maße ist das Kollegium bereit selbstständig Entwicklung und Entscheidungen zu bestimmen. 
Abb. 17: Führungsverhalten von Schulleiterinnen und Schulleitern (ausgewählte Items)

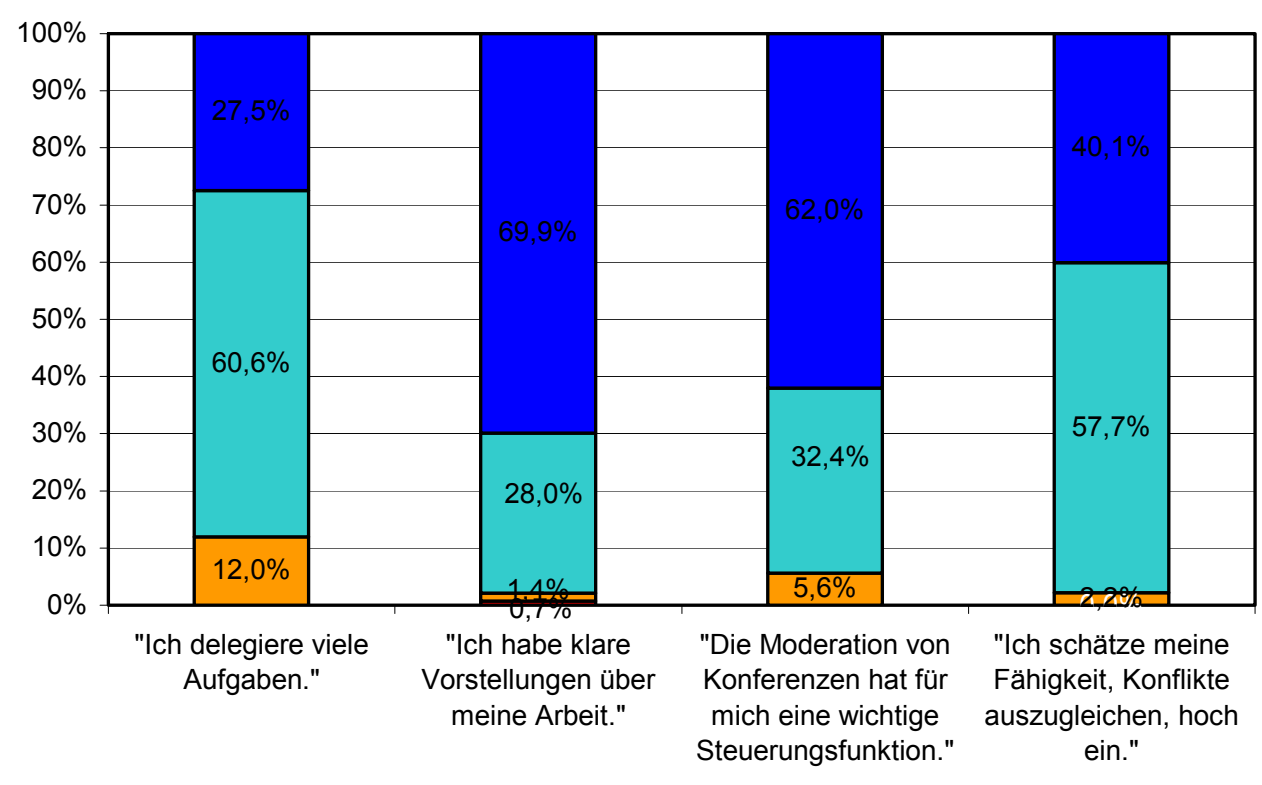

$\square$ stimme überhaupt nicht zu $\square$ stimme eher nicht zu $\square$ stimme eher zu $\square$ stimme zu

Die Gestaltung und Durchführung von Konferenzen hat für die Mehrheit der Befragten eine wichtige Steuerungsfunktion. Die eigene Konfliktfähigkeit schätzen die Befragten überwiegend hoch ein.

Abb. 18: Führungsverhalten

von Schulleiterinnen und Schulleitern

(ausgewählte Items)

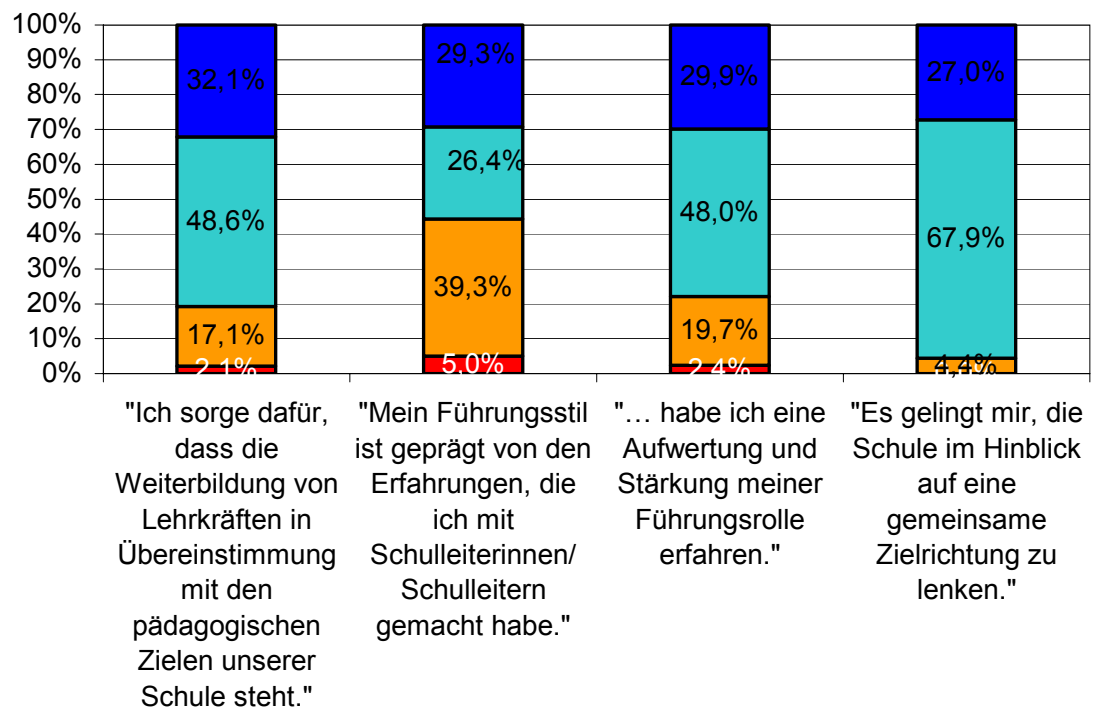

$\square$ stimme überhaupt nicht zu $\square$ stimme eher nicht zu $\square$ stimme eher zu $\square$ stimme zu

Für die Befürwortungen von klaren Vorstellungen spricht ebenso die Zustimmung zu einem Fortbildungskonzept, welches die Weiterentwicklung 
der eigenen Schule gezielt steuert. Die überwiegende Mehrheit der Befragten ist der Überzeugung, dass es innen gelingt, die „Schule im Hinblick auf eine gemeinsame Zielrichtung zu lenken", 27,0 \% stimmen dieser Aussage voll zu, 67,9 \% stimmen eher zu. Dennoch stimmen 0,7 \% der Befragten überhaupt nicht zu und $4,4 \%$ eher nicht.

Die hier befragten Schulleiterinnen und Schulleiter orientieren sich zu einem Teil an dem selbst erlebten Führungsstil, dabei kann an dieser Stelle nicht deutlich werden, welche Art von Führung sie erlebt haben. Einerseits kann eine Orientierung an Vorbildern vorliegen oder aber auch der Wunsch, es besser machen zu wollen als derjenige/diejenige, die man selbst als Schulleiter/ Schulleiterin erlebt hat.

\subsubsection{Entscheidungsfindung}

Interessant sind die Ergebnisse bezüglich des Bereiches Entscheidungsfindung, denn $42,9 \%$ der Schulleiterinnen und Schulleiter geben an, dass sie Entscheidungen allein treffen ( $\mathrm{vgl}$. Abb. 19). Hier spiegelt sich eine Tendenz wider, die ein führungsbetontes Bild von Schulleitung gerade im Bereich der Entscheidungsfindung nachzeichnet. Bei aller Wichtigkeit der Beteiligung von Kollegien an Entscheidungen wünschen sich gerade Lehrerinnen und Lehrer zunehmend klarere Richtungsvorgaben der Schulleitung, damit auf Kollegiumsebene die oft aufreibenden und langwierigen Aushandlungsprozesse reduziert werden. ${ }^{191}$

Abb. 19: Führungsverhalten von Schulleiterinnen und Schulleitern (ausgewählte Items)

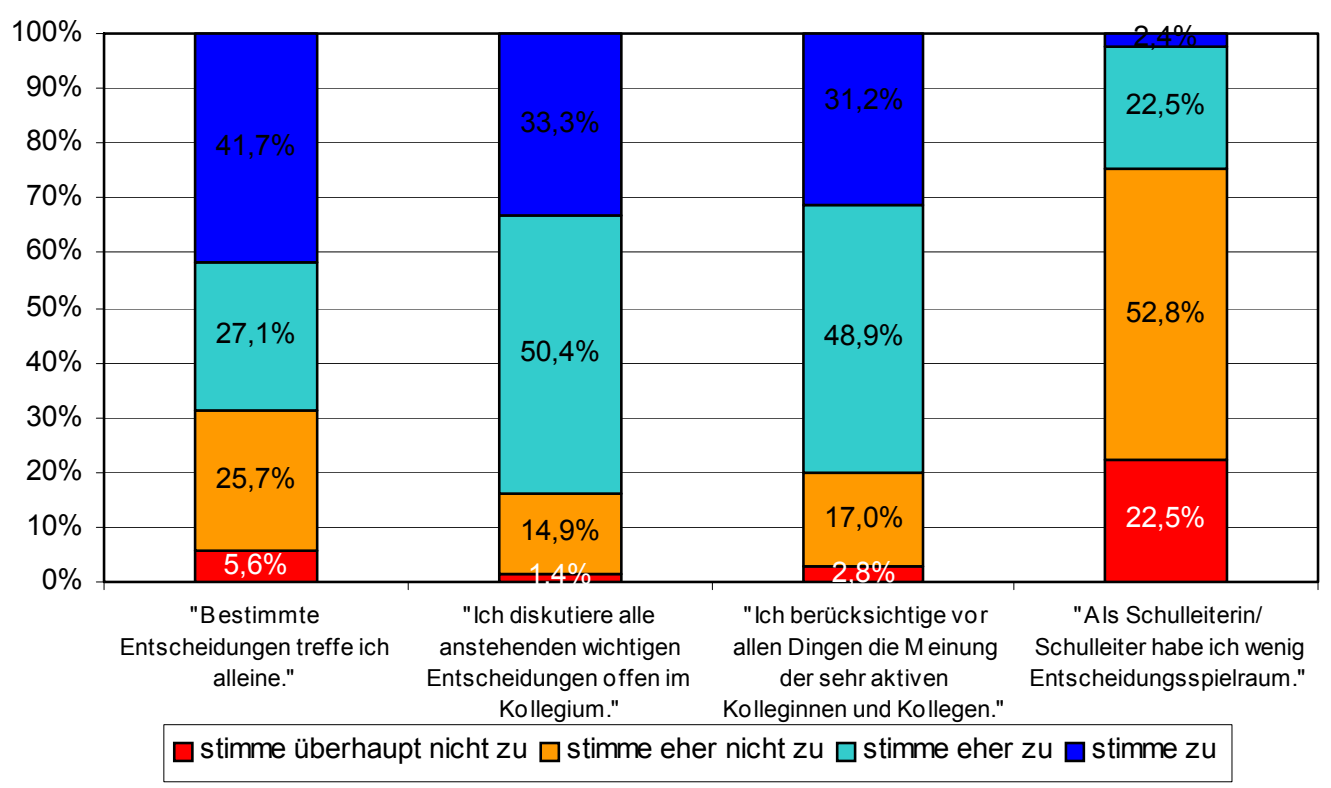

Immerhin diskutieren 16,3\% der Befragten wichtige Entscheidungen nicht oder eher nicht im Kollegium, dahingegen wird einer Beteiligung bei Entscheidungen mehrheitlich zugestimmt. Diese Ergebnisse können als An-

${ }^{191}$ Vgl. Bonsen 2002, 75. 
deutung einer inneren Ambivalenz gedeutet werden, der Wunsch nach einem kooperativen-partizipativen Führungsstil ist sicherlich vorhanden, aber insbesondere Personalentscheidungen und auch organisatorische Entscheidungen müssen allein getroffen werden, dies auch, weil vieles ad hoc zu entscheiden ist.

Nach der Analyse der Einzelitems wird durch eine konfirmatorische Faktorenanalyse überprüft, ob sich die zuvor dargestellten Handlungsdimensionen anhand der erhobenen Daten empirisch bestätigen lassen, ob also Faktoren als latente Konstrukte - in diesem Fall bestimmte grundlegende Einstellungen und Eigenschaften der Schulleiterinnen und Schulleiter das Antwortverhalten erklären können. ${ }^{192}$

\subsubsection{Dimensionen schulischen Führungshandelns}

Ursprünglich ist davon ausgegangen worden, dass sich Schulleitungshandeln in unterschiedlichen Handlungsdimensionen nachweisen lässt. Auf dieser theoretischen Grundlage ist der verwendete Fragebogen entwickelt worden. Bei der Auswertung der ausgefüllten Fragebögen sollte erforscht werden, ob sich die theoretisch definierten Handlungs- bzw. Einstellungsdimensionen empirisch bestätigen lassen. Es wurde untersucht, inwiefern sich die Ergebnisse der einzelnen Items auf dahinter stehende Faktoren zurückführen lassen bzw. ob sie zu reliablen Skalen zusammengefasst werden können. ${ }^{193}$

Zunächst wurde die angenommene Struktur schulischen Führungshandelns mittels konfirmatorischer Faktorenanalysen überprüft, wofür die Software AMOS genutzt wurde. Da die entsprechenden statistischen Tests des "model-fit" eine vergleichsweise hohe Fallzahl voraussetzen, die in der vorliegenden Untersuchung nicht erreicht wird, können ihre Ergebnisse nur einen ersten Eindruck der Gültigkeit der ursprünglichen strukturbezogenen Annahmen und der Eignung der verwendeten Items geben. Die Resultate der konfirmatorischen Faktoranalysen finden sich im Anhang (vgl. Anlage 3). Im Anschluss an die konfirmatorischen Faktorenanalysen wurden die theoretisch zueinander passenden Items zu Skalen zusammengefasst und eine umfassende Itemanalyse nach dem Konzept der klassischen Testtheorie durchgeführt. Jede der gebildeten Skalen repräsentiert gewissermaßen eine Dimension schulischen Führungshandelns, hinter der grundlegende Eigenschaften und Einstellungen der Befragten stehen. Es konnten in weitgehender Übereinstimmung mit den Ergebnissen der konfirmatorischen Faktorenanalysen vier konsistente Skalen schulischen Führungshandelns bestätigt werden.

Die Skala „Mitarbeiterorientierung“ fasst insgesamt sieben Items zusammen, wozu unter anderem „ich gebe häufig individuelle Beratung und Hilfe" und "mir ist wichtig, dass sich Kolleginnen und Kollegen in der Schule wohlfühlen" gehören. Cronbachs Alpha beträgt für diese Skala 0,74, die Trennschärfekoeffizienten der Items liegen zwischen 0,38 und 0,57.

Zum Bereich „Zielorientiertes Führungsverständnis" sind zehn Items des verwendeten Fragebogens zu zählen. Beispiele dafür sind die Items „ich arbeite an der Entwicklung von langfristigen Zielen für die Schule“ und „es gelingt mir, die Schule im Hinblick auf eine gemeinsame Zielrichtung zu

\footnotetext{
${ }^{192}$ Konfirmatorische Faktorenanalyse siehe Anlage 3.

${ }^{193}$ Backhaus 2000, $189 \mathrm{ff}$.
} 
lenken“. Die Reliabilität der Skala misst 0,74 (Cronbachs Alpha), die Trennschärfekoeffizienten der Items betragen zwischen 0,31 und 0,58.

Als dritte Dimension schulischen Führungshandelns konnte die „Zufriedenheit mit der eigenen Stellung und dem beruflichen Umfeld" empirisch bestätigt werden. Beispielitems dafür sind „ich bin zufrieden mit meinem Verhältnis zu meinem Kollegium“ und „ich bin zufrieden mit der Organisation unseres Schulbetriebes“. Insgesamt gehören sechs Items zu dieser Skala mit einer Reliabilität von 0,73 (Cronbachs Alpha). Die Trennschärfekoeffizienten der Items belaufen sich auf Werte von 0,35 bis 0,59.

Die „empfundene Belastung“ wird ebenfalls mittels sechs Items gemessen, zu denen unter anderem „Ich fühle mich häufig überfordert“ und „Problemkollegen rauben meine Arbeitszeit“ gehören. Cronbachs Alpha liegt für diese Skala bei 0,71, die Trennschärfekoeffizienten der Items zwischen 0,30 und 0,59. Für alle getesteten Skalen und ihre Items gilt, dass Cronbachs Alpha wie auch Trennschärfekoeffizienten akzeptable Werte annehmen. ${ }^{194}$ Eine einfache Mittelwertbildung der jeweiligen Antworten auf den vier Skalen - z.B. für die zehn Antworten zu den Items im Bereich „Zielorientiertes Führungsverständnis“ - gibt eine Anschauung des absoluten Niveaus der gemessenen Konstrukte, wie hoch z.B. die Zielorientierung der Schulleiterinnen und Schulleiter in ihrer Führungsrolle ist oder wie stark ihre Belastung eingeschätzt wird. ${ }^{195}$

Abbildung 20 zeigt, dass insbesondere die „Mitarbeiterorientierung“ der befragten Schulleiterinnen und Schulleiter hoch ist.

Abb. 20: Führungsverhalten und Arbeitserleben von Schulleiterinnen und Schulleitern

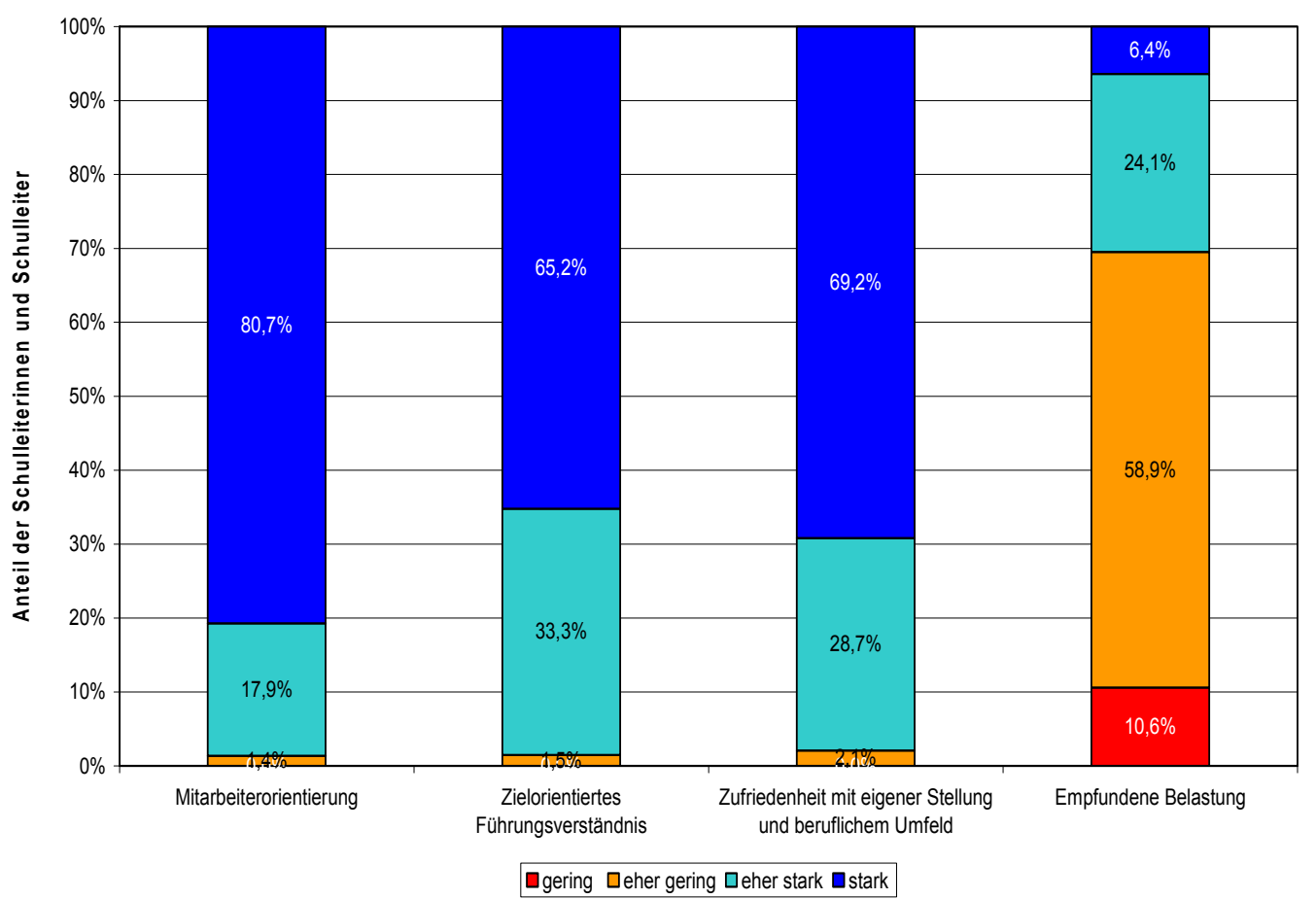

\footnotetext{
${ }^{194}$ Für eine detaillierte Darstellung der Item- und Skalenkennwerte siehe Anhang.

195 Die individuellen Skalenwerte der Schulleiterinnen und Schulleiter liegen hier zwischen 1 und 4. Wird theoretisch mögliche Spannweite von 3 durch 4 gewünschte Kategorien dividiert, ergibt sich eine Kategorienbreite von 0,75. Dementsprechend umfasst die Bezeichnung "gering“ Werte zwischen 1,00 und 1,75, „eher gering" die Werte 1,75 bis 2,50 usw..
} 
Bei $80,7 \%$ von ihnen liegen die Antworten zu den entsprechenden Items im Durchschnitt zwischen 3.25 und 4,00. Weitere 17,9\% der Befragten weisen eine „eher starke" Mitarbeiterorientierung auf, mit durchschnittlichen Antwortwerten von 2,50 bis 3,25. Eine eher geringe Mitarbeiterorientierung weisen offenbar nur wenige Schulleiterinnen und Schulleiter auf.

Die Schulleiterinnen und Schulleiter verfügen in der Regel neben einer meist starken Mitarbeiterorientierung auch über ein ausgeprägtes zielorientiertes Führungsverständnis. 65,2 \% der Befragten erreichen hierbei einen durchschnittlichen Antwortwert über 3,25, weitere 33,3\% einen Wert zwischen 2,5 und 3,25. Nur 1,5\% der Fälle sind der Gruppe mit einer eher geringen Zielorientierung des Führungsverständnisses zuzuordnen.

Die „Zufriedenheit mit der eigenen Stellung und dem beruflichen Umfeld“ ist bei fast $70 \%$ der Schulleiterinnen und Schulleiter sehr hoch. 28,7\% der Befragten zeigen eine geringere, jedoch immer noch überwiegende Berufszufriedenheit. Nur ein kleiner Teil der Stichprobe $-2,1 \%$ der Befragten - ist der Gruppe der eher mit der beruflichen Situation unzufriedenen Schulleiterinnen und Schulleiter zuzuordnen.

Trotzdem fühlen sich einige Schulleiterinnen und Schulleiter durch ihren Beruf belastet - offenbar ohne dass ihre Berufszufriedenheit unbedingt stark herabgesetzt werden muss. Immerhin 6,4\% der Befragten empfinden eine starke Belastung im Schullalltag. Weitere $24,1 \%$ fühlen sich zwar etwas weniger, doch immer noch überwiegend durch ihre Arbeit belastet. 58,9 \% zeigen eine eher geringe Belastung, 10,6\% nahezu gar keine mit durchschnittlichen Antwortwerten zwischen 1,00 und 1,75.

Wie bereits beschrieben, vermitteln diese Ergebnisse einen Eindruck von dem absoluten Niveau der gemessenen Konstrukte. Streng genommen sind aber bei den vorliegenden Daten die Bildung arithmetischer Mittelwerte sowie andere Analysen, die von dem gleichwertigen Abstand der Antwortalternativen ausgehen, nicht zugelassen. Schließlich sind die hier berücksichtigten Variablen ordinalskaliert. ${ }^{196}$ Eine notwendige Voraussetzung für die Berechnung der Skalenwerte und daran anschließende Berechnungen sind Daten auf Intervallskalenniveau. Mittels des PartialCredit-Modells lassen sich ordinalskalierte Daten auf dieses Niveau bringen und für Skalenbildungen, Mittelwertberechnungen, Korrelationsanalysen usw. nutzen.

\subsubsection{Korrelationen zwischen individuellen Merkmalen und den vier Dimensionen}

Nach der Errechnung der Werte, also der Summierung der Werte für die Items der gebildeten Skalen, wurden mittels Korrelationsanalyse unterschiedliche Zusammenhänge überprüft. ${ }^{197}$

Hierbei konnte kein signifikanter Zusammenhang zwischen dem Alter und den Ausprägungen auf den vier Dimensionen schulischen Führungshandelns festgestellt werden. Gleiches gilt für das Verhältnis von Dienstjahren

\footnotetext{
${ }^{196}$ Vgl. Husfeldt 2003, 402.

197 Es wurden also Wertepaare gebildet (bivariate Korrelation), die Stärke des Zusammenhangs wird mit einer Maßzahl angegeben, den Korrelationskoeffizienten. Dieser Koeffizient der stets mit $r$ symbolisiert wird, liegt zwischen -1 und +1 , eine Zahl nahe bei 1 bedeutet einen starken und ein Betrag nahe 0 einen schwachen Zusammenhang. Man spricht ab 0,7 von einer mittleren und ab 0,9 von einer hohen Korrelation.
} 
als Schulleiterin/Schulleiter und schulischem Führungshandeln. Die Annahme, dass Alter bzw. Dienstjahre insbesondere auf das Engagement im Bereich der Schulentwicklung oder auf die Berufszufrieden allgemein einen deutlichen Einfluss ausüben, konnte folglich nicht bestätigt werden.

Betrachtet man die Kategorie Geschlecht, so besteht kein signifikanter Zusammenhang zwischen dem Geschlecht und der Mitarbeiterorientierung oder dem Geschlecht und der Zielorientierung des Führungsverständnisses. Der diesbezügliche Korrelationskoeffizient beträgt lediglich -0,114. Es besteht jedoch ein (auf dem Niveau von 0,05) signifikanter Zusammenhang zwischen dem Geschlecht und der Zufriedenheit mit der eigenen Stellung und dem Arbeitsumfeld. Der Korrelationskoeffizient hierfür beträgt 0,191, was bedeutet, dass Männer im Durchschnitt mit ihrer beruflichen Situation zufriedener als Frauen sind. Des Weiteren besteht ein signifikanter Zusammenhang (auf dem Niveau von 0,05) zwischen dem Geschlecht und der empfundenen Belastung durch den Beruf. Dabei beläuft sich der Korrelationskoeffizient auf $-0,176$. Frauen fühlen sich eher belastet als Männer.

\subsubsection{Zusammenhänge zwischen den vier Dimensionen}

Abb. 21: Zusammenhänge zwischen den vier Dimensionen schulischen Führungshandelns (nur signifikante (*, 0,05-Niveau) und hochsignifikante (**, 0,01-Niveau) Korrelationen)

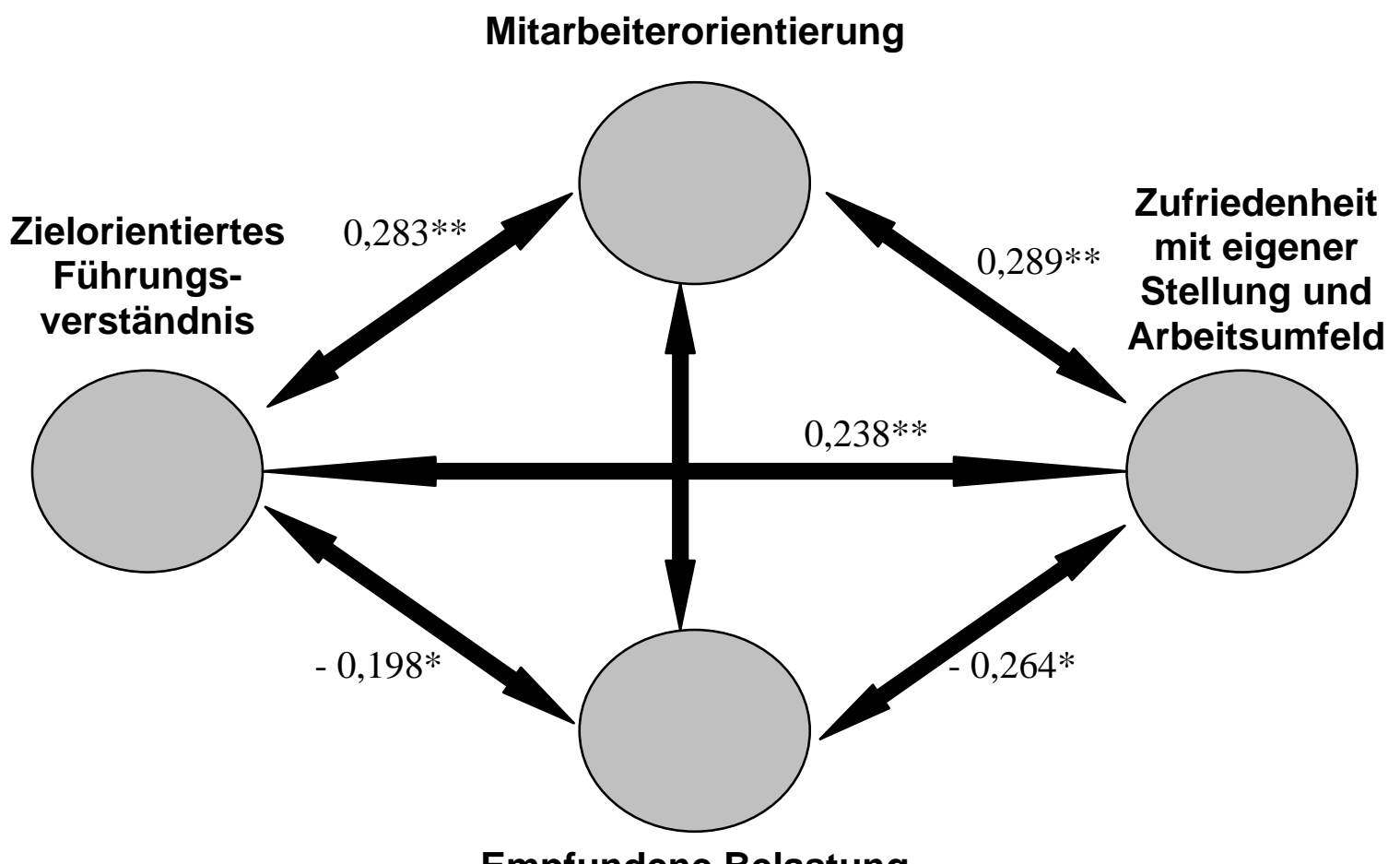

Empfundene Belastung

Es besteht ein hoch signifikanter positiver Zusammenhang (auf einem Niveau von 0,01) zwischen der „Mitarbeiterorientierung“ und der „Zufriedenheit mit der eigenen Stellung und dem Arbeitsumfeld" (Korrelationskoeffizient $=0,289$ ). Wer eine höhere Mitarbeiterorientierung aufweist, ist im 
Schnitt auch mit seiner Arbeit zufriedener, wobei hier wie bei den übrigen Korrelationen nicht von kausalen Richtungen gesprochen werden kann. Dennoch ist dieser Zusammenhang ohne weiteres nachvollziehbar, denn wer sich stärker akzeptiert fühlt oder in der eigenen Schule wohl fühlt, der wird insgesamt zufriedener sein. Darüber hinaus besteht ein signifikanter Zusammenhang zwischen den pro-sozialen Einstellungen und der Zielorientierung des Führungsverständnisses. (Korrelationskoeffizient $=0,283$ ). Dies kann wieder als ein Hinweis darauf gedeutet werden, dass die Ziele der Schule maßgeblich von dem gesamten Kollegium beeinflusst werden und dass die Zielerreichung davon abhängig ist, wie sich das Kollegium einer Schule für die Weiterentwicklung und Veränderung von Schule einsetzt. Wer eine stärkere Mitarbeiterorientierung hat, besitzt im Schnitt auch ein ausgeprägteres zielorientiertes Führungsverständnis. Die Zielorientierung des Führungsverständnisses korreliert außerdem hoch signifikant mit der Arbeitszufriedenheit. Der entsprechende Koeffizient misst 0,238. Ein signifikanter (auf dem Niveau von 0,05) negativer Zusammenhang besteht zwischen einem zielorientiertem Führungsverständnis und Belastungsempfinden. Der Korrelationskoeffizient beläuft sich hierbei auf 0,198. Es wurde also festgestellt, dass Schulleiterinnen und Schulleiter mit einem höheren Maß an zielorientiertem Führungsverständnis im Schnitt eine geringere Belastung durch ihre Arbeit empfinden.

Ein interessantes Ergebnis der Korrelationsanalysen ist der hochsignifikante (0,01-Niveau), aber relativ geringe negative Zusammenhang zwischen der „Zufriedenheit mit der eigenen Stellung und dem Arbeitsumfeld“ und der empfundenen Belastung. Der Korrelationskoeffizient liegt bei 0,264 , das bedeutet, dass Schulleiterinnen und Schulleiter, die eine höhere Arbeitszufriedenheit zeigen, im Schnitt eine geringere Belastung empfinden.

\subsubsection{Zusammenfassung}

Insgesamt deuten die Aussagen der Schulleiterinnen und Schulleiter an, dass sie selbst der Überzeugung sind, ihre Schule ziel- und ergebnisorientiert zu führen. Dabei haben sie sowohl die Weiterentwicklung und Gestaltung der eigenen Schule im Blick als auch fördernde Arbeits- und Beziehungsstrukturen für Kolleginnen und Kollegen.

Die Beantwortung der Fragen zum Führungsverhalten geben Hinweise darauf, dass sich die Führungskonzeptionen heutiger Schulleiterinnen und Schulleiter in Niedersachsen stark an den Forderungen nach Schulentwicklung orientieren. Des Weiteren konnte ein Verständnis der eigenen Rolle nachgewiesen werden, das Führungsaufgaben aktiv wahrnimmt, z.B. das Beurteilen von Kolleginnen und Kollegen.

Hinsichtlich der Dimension „Zielorientierte Führung" wie auch im Bereich der Schulentwicklung zeichneten sich dabei aber Widersprüche zwischen den Vorstellungen bzw. Wünschen und den tatsächlichen Handlungen, die zu einer Verwirklichungen dieser Ansprüche führen müssten, ab. Die Ergebnisse machen darauf aufmerksam, dass es im Rahmen der Schulentwicklung nicht nur um die Erarbeitung und Verabschiedung gemeinsamer Programme und Ziele gehen kann, sondern dass es eine bedeutende 
Führungsaufgabe ist, die Umsetzung eben dieser Ziele immer wieder einzufordern und dafür Sorge zu tragen, dass Ergebnisse erreicht werden.

Bei der gelungenen Bewältigung des Berufes spielt die empfundene Berufzufriedenheit eine große Rolle, dies haben die Ergebnisse gezeigt. Eine zielgerichtete Führung, die sich vor allem durch eine Führung des Kollegiums hinsichtlich gemeinsamer innovativer Entwicklungsbemühungen ausdrückt, hat einen positiven Einfluss auf die eigene Berufszufriedenheit.

Das Leiten einer Schule ist mit hohen Anforderungen verbunden und doch fühlen sich die meisten Schulleiterinnen und Schulleiter akzeptiert in ihrer Rolle als Führungsperson, gerade diese Zufriedenheit reduziert das subjektive Belastungserleben. Dies lässt den Umkehrschluss zu, dass diejenigen, die sich stark belastet fühlen, weniger meinen, in der Lage zu sein, die Schule zielorientiert zu führen.

Alle Ergebnisse müssen vor dem Hintergrund der Organisation Schule betrachtet werden, neben einem individuellen Handlungsrepertoire spielen die rechtlichen und organisatorischen Gegebenheiten der Schule selbst eine Rolle. Wie Schulleiterinnen und Schulleiter konkret mit den Alltagsanforderungen und den erlebten Widersprüchen umgehen, darüber sollen die nun folgenden vertiefenden qualitativen Interviews Auskunft geben.

\subsection{Leitfadengestützte qualitative Interviews mit Schulleiterinnen und Schulleitern- methodisches Vorgehen bei der Datenerhe- bung}

\subsubsection{Erhebungsverfahren}

In der hier vorgelegten Untersuchung wurde zuvor bereits eine standardisierte quantitative Befragung von Schulleiterinnen und Schulleitern durchgeführt, die als eine Form des Vorwissens in den nun folgenden qualitativen Forschungsteil integriert werden muss. Beide Untersuchungsteile haben das Ziel, Auskunft über die Berufsauffassung von Schulleiterinnen und Schulleitern zu geben. Diese Konzeption und das methodische Vorgehen sind insofern theoriegeleitet, als die Ergebnisse der Schulleitungsforschung bei der quantitativen und qualitativen Erhebung eine Rolle spielen. Des Weiteren wurde aus den Theorien (Führungstheorie, Organisationstheorie und Professionstheorie) im Sinne eines sensibilisierenden Konzeptes ein Konstrukt der Berufsauffassung entworfen (vgl. S. 75 in dieser Arbeit).

Die qualitativen Interviews mit Schulleiterinnen und Schulleitern verfolgen das Ziel, die subjektiven Berufsauffassungen von Schulpraxisexperten zu erheben. Es soll untersucht werden, ob sich die Berufsauffassung von Schulleiterinnen und Schulleitern an Wissensbereichen aus den genannten Theorien orientiert. In diesem Kontext sollten die Interviews Auskunft über Berufsorientierungen und die Entwicklung eines professionellen Selbst im Spannungsverhältnis programmatischer Zielvorgaben und alltäglicher Praxis geben. Methodisch muss dabei ein Weg beschritten werden, der sowohl theoretisches Vorwissen als auch bereits gewonnene Ergebnisse berücksichtigt und zugleich der geforderten Offenheit gerecht werden kann. 
Das qualitative Interview bietet dem Befragten die Möglichkeit, seine Wirklichkeitsdefinition mitzuteilen. Die Datenerhebung erfolgt daher durch leitfadengestützte qualitative Interviews mit Experten, denn anders als bei biographischen Interviews interessieren die Befragten weniger als ganze Person, sondern in ihrer Eigenschaft als Experten für ein bestimmtes Handlungsfeld - hier als Schulleiterinnen und Schulleiter im Kontext Schule. Der Leitfaden dient der thematischen Vorstrukturierung und erhält dadurch eine inhaltliche Steuerungsfunktion, um dem spezifischen Erkenntnisinteresse Rechnung zu tragen. Er bietet damit zwar ein Gerüst für die Datenerhebung, eine offene Gesprächsführung lässt aber dem Befragten genügend Raum für die Entfaltung seiner Sichtweise. ${ }^{198}$

Inhaltlich wurde der Leitfaden aus bereits vorliegenden Untersuchungen, aus Theorien und den Ergebnissen des quantitativen Untersuchungsteils sowie aus der eigenen Kenntnis des Feldes abgeleitet. Der eingesetzte Interviewleitfaden wird durch allgemeine Fragen zur Tätigkeit und zum Arbeitsalltag strukturiert, der Schwerpunkt liegt auf den Aufmerksamkeitsschwerpunkten Führungshandeln, Organisationsverständnis und persönliche Berufsauffassung.

Die strikte Trennung zwischen der Gesamtperson und einer Expertin/ eines Experten, wie sie Meuser/Nagel (1997) vorschlagen, wird in der Methodendiskussion der Experteninterviews als eigenständiges, verallgemeinerbares Interviewverfahren kontrovers diskutiert. ${ }^{199}$ Denn die Inhalte des Expertenwissens, die analysiert werden sollen, beinhalten bereits immer das subjektive Deutungsmuster der interviewten Person, daher sprechen Kassner/ Wassermann (2005) von „der Rekonstruktion sozialer Deutungsmuster von ExpertInnen mittels leitfadengestützter qualitativer Interviews“. ${ }^{200}$ In der hier vorliegenden Studie geht es um Interviews mit Experten im Handlungskontext Schule, es geht nicht um die Erfassung biografischer oder privater Themen. Dennoch ist davon auszugehen, dass die Interviewten als Person präsent sind; insbesondere Interviewpassagen, in denen subjektive Deutungsmuster des Handlungskontextes offenbart werden, sollen im Sinne des Erkenntnisinteresses nutzbar gemacht werden. Im Folgenden werde ich kurz auf die Definitionen des Expertenbegriffes eingehen.

\subsubsection{Expertenstatus}

Für das Feld Schule muss davon ausgegangen werden, dass die Befragten sich selbst als „Experten“ in Fragen der Leitung und Organisation der Schule betrachten, dabei werden sie sich selbst Kompetenzen zuschreiben in Bezug auf Führungsfähigkeiten und sich als Sachverständige der Organisation Schule erleben. Fried (2002) spricht in diesem Zusammenhang von schulrelevantem Professionswissen von Schulpraxisexperten; Schulleiterinnen und Schulleiter planen, evaluieren oder revidieren ihr Handeln mit Hilfe von Wissen. ${ }^{201}$

\footnotetext{
${ }^{198}$ Vgl. Friebertshäuser 1997, 375.

199 Vgl. Meuser/Nagel 1997, 486f.

200 Kassner/ Wassermann 2005,107.

${ }^{201}$ Vgl. Fried 2002, 175.
} 
In der Literatur werden die Begriffe Experte, Spezialist und Professioneller unterschieden. Die Auffassung über Experten entspricht häufig dem Bild des Professionellen, der seine Kompetenzen über die Erfüllung formaler Ausbildungsanforderungen erlangt. Kennzeichen der Professionellen ist, dass sie sich einen kanonisierten Sonderwissensbestand über eine institutionelle und formalisierte Ausbildung erworben haben. ${ }^{202}$ Dies trifft auf Schulleiterinnen und Schulleiter, wie oben bereits erörtert, nicht zu, dennoch verfügen sie über ein Sonderwissen bezüglich der Schulpraxis und der darin zum Tragen kommenden Wissensbestände (vgl. Kap. 3.3 in dieser Arbeit). Der Begriff 'Schulpraxisexperte' eignet sich aus meiner Einschätzung heraus daher für die Bezeichnung der Berufsgruppe Schulleiterinnen und Schulleiter.

Allgemein wird dem Expertenstatus die exklusive Verfügbarkeit von Wissen zu einem bestimmten Thema in einem beruflichen Kontext zugewiesen und den Experten wird die Kompetenz zugesprochen, dass sie reflektorisch mit diesem Wissen umgehen.

Als Experte wird angesprochen,

- $\quad$ wer Verantwortung trägt für einen Entwurf, die Umsetzung oder die Kontrolle einer Problemlösung

- wer über einen besonderen Zugang zu Informationen über Personengruppen oder Entscheidungsprozesse verfügt. ${ }^{203}$

Schulleiterinnen und Schulleiter tragen die Gesamtverantwortung für die einzelne Schule, dies beinhaltet allgemein formuliert den Bildungs- und Erziehungsauftrag des staatlich institutionalisierten Schulsystems. Darüber hinaus sind sie verantwortlich für einen möglichst reibungslosen Gesamtablauf der Organisation. Vor allen Dingen haben sie gegenüber den Lehrerinnen und Lehrern eine personale Fürsorgepflicht und verfügen über informelle und formale Informationen (Personalakten).

\subsubsection{Wissenssoziologische Definition des Expertenbegriffs}

Nach Alfred Schütz (1972) handelt es sich beim Experten um einen Wissenschaftler, der über ein sicheres Wissen verfügt, welches jeder Zeit verfügbar und kommunizierbar ist, Sprondel (1979) grenzt den Expertenbegriff im Kontrast zum Begriff des Laien ab, er bezeichnet den Experten als Verwalter von Sonderwissen, wobei nicht jedes Sonderwissen auch gleich Expertenwissen sein muss. Das Expertenwissen ist an einen Beruf geknüpft, daher spricht Sprondel (1979) auch vom Expertenwissen als „sozial institutionalisierter Expertise".204

Hitzler (1994) grenzt den Expertenbegriff von dem Spezialisten ab. Der Experte zeichnet sich durch weitestgehende Autonomie aus, während das Arbeitsgebiet des Spezialisten klar vorgegeben und begrenzt ist. Der Definition von Schütz (1972) entsprechen Schulleiterinnen und Schulleiter nicht, sie verfügen zwar über ein durch ein Studium angeeignetes wissenschaftliches Wissen bezüglich ihres Primärberufes Lehrerin/Lehrer, ein Großteil ihres Wissensbestandes wird aber durch die Praxis ihres Berufes

\footnotetext{
202 Vgl. Pfadenhauer 2005, 123ff.

${ }^{203}$ Vgl. Bogner/ Menz 2002, 7-31.

${ }^{204}$ Vgl. Sprondel 1979, 148.
} 
angeeignet und evtl. durch zusätzliche Ausbildungsangebote angereichert. Insofern kann Sprondels (1979) Definition Rechnung getragen werden. In täglichen Entscheidungen, in längerfristigen inhaltlichen Schwerpunktsetzungen der Schule und in der Organisation des Schulalltages sind Schulleiterinnen und Schulleiter relativ autonom, aber bezüglich des Personals und des Budgets sind sie von der übergeordneten Schulbehörde abhängig.

Abb. 22: Expertenstatus

\begin{tabular}{|c|c|c|}
\hline Experte & Spezialist & Professioneller \\
\hline $\begin{array}{ll}\text { - } & \text { Überblick über } \\
\text { exklusiven Wis- } & \text { sensbestand } \\
\text { - } & \text { berufliches Son- } \\
\text { derwissen } \\
\text { - } \quad \text { verantwortliche } \\
\text { Zuständigkeit } \\
\text { - } \quad \text { eigene Relevan- } \\
\text { zen, subjektive } \\
\text { Bedeutungszu- } \\
\text { schreibungen }\end{array}$ & 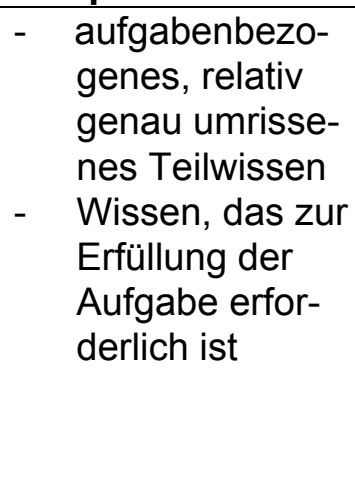 & $\begin{array}{ll}\text { - } & \text { kanonisierter } \\
\text { Wissensbestand } \\
\text { - } \quad \text { in der Berufsrolle } \\
\text { kristallisierte Ex- } \\
\text { pertise }\end{array}$ \\
\hline
\end{tabular}

Zusammenfassend schließe ich mich bezogen auf die Schulleiterinnen und Schulleiter der Definition von Bogner/Menz (2001) an:

Schulleiterinnen und Schulleiter können als Experten angesehen werden, denn sie verfügen über ein spezifisches Prozess- und Deutungswissen, das sich auf ihr berufliches Handlungsfeld bezieht. Ihr Wissen besteht nicht nur aus Fach- bzw. Sonderwissen, sondern weist zum großen Teil den Charakter von Praxis- und Handlungswissen auf. Ihr Handeln kann von unterschiedlichen Handlungsgrundsätzen sowie von individuellen Entscheidungsregeln, kollektiven Orientierungen und sozialen Deutungsmustern beeinflusst sein. Das Wissen der Schulleiterinnen und Schulleiter, ihre Handlungsorientierung, d.h. auch ihre unterschiedlichen Aufmerksamkeitsschwerpunkte hinsichtlich der relevanten Wissensbestände, wird in inrem Funktionskontext Schule praxiswirksam und beeinflusst die Handlungsbedingungen anderer Akteure. ${ }^{205}$

\subsubsection{Erhebung leitfadengestützter problemzentrierter Interviews mit Experten}

Im Rahmen meines Forschungsinteresses habe ich Schulleiterinnen und Schulleiter als Experten im Handlungsfeld Schule bestimmt und ausgewählt. In der vorliegenden Studie bestand nun die methodische Schwierigkeit darin, dass die Akteure als Experten betrachtet werden und so zunächst von einem leitfadengestützten Experteninterview ausgegangen wurde. Bereits die Interviews im Pre-Test, aber auch erste Interviews im Feld, verdeutlichten Probleme, denn das Interview brachte viel stärker als zunächst angenommen die Person des Interviewten in den Vordergrund, und die starke Orientierung an den Themen des Leitfadens erschwerte eine größtmögliche Offenheit und Flexibilität, die aber gerade qualitative

${ }^{205}$ Vgl. Bogner/ Menz 2002, 486. 
Interviews ausmachen. Die Auswertungstechnik von Experteninterviews richtet sich jedoch vor allem auf die Analyse und den Vergleich der Inhalte des Expertenwissens, nicht auf die subjektive Problemsicht. ${ }^{206}$ Aus den dargelegten Gründen wurde von dem ursprünglichen Ansatz der leitfadengestützten Experteninterviews tendenziell abgewichen, stattdessen fand eine Annäherung an problemzentrierte Interviews statt.

Da in der Untersuchung die Berufsauffassung von Schulleiterinnen und Schulleitern, ihre Handlungsorientierung bei der Berufsausübung sowie die Verarbeitung von Erfahrungen im Berufskontext untersucht werden soll, wählte ich einen methodischen Weg, der der subjektiven Problemsicht Raum gibt. Das Erkenntnisziel, nämlich die Erfassung einer subjektiven - Problemsicht - einer Berufsauffassung, stimmen mit der Absicht der „Problemzentrierten Interviews“ überein. Des Weiteren ergeben sich auch Parallelen in der Gesprächshaltung, wie sie von Witzel (2000) für das problemzentrierte Interview beschrieben wurden, denn es geht im Interview darum, möglichst viel „hervorzulocken“ an konkreten Erfahrungen, an Sichtweisen, an Wirklichkeitsausschnitten der Akteure. ${ }^{207}$ Im Rahmen der hier geführten Interviews wird der in einen Funktionskontext eingebundene Akteur betrachtet, die Berufsrolle und das berufsbezogene Handeln stehen im Vordergrund. Es liegt ein enger Expertenbegriff zugrunde, denn das Interview zielt auf den Wissensvorsprung, der aus einer besonderen beruflichen Position resultiert. ${ }^{208}$ Der Experte wird als Repräsentant einer Organisation angesprochen, der Interviewbereich bezieht sich auf diesen Wirklichkeitsausschnitt. Das Expertenwissen ist dabei nicht einfach abfragbar, sondern muss aus den Äußerungen der Experten rekonstruiert und interpretiert werden. ${ }^{209}$

Die Interviews fokussieren kontextbezogen die berufliche Orientierung der Schulleiterinnen und Schulleiter, das Forschungsinteresse richtet sich auf die Berufsauffassung, die sich von den Akteuren bei der Bewältigung der zunehmend komplexer werdenden Anforderungen herausbildet. Im Rahmen dieser Forschung ist von besonderem Interesse, wie Schulleiterinnen und Schulleiter aus ihrer Position heraus Führung im Kontext Schule beurteilen, wie sie mit konstitutiven Widersprüchen der Organisation umgehen und welche Bedeutung die veränderten Berufsaufgaben für die eigene Berufsrolle haben. Drei Grundpositionen bestimmten das problemzentrierte Interview: ${ }^{210}$

- $\quad$ Problemzentrierung: Orientierung an gesellschaftlich relevanten Problemstellungen. Die Interviewer nutzen die objektiven Rahmenbedingungen der untersuchten Orientierungen und Handlungen, um die Explikation der Interviewten verstehend nachzuvollziehen und am Problem orientiert Fragen zu stellen.

- $\quad$ Gegenstandsorientierung: Dem Gegenstand der Untersuchung soll flexibel gegenüber getreten werden, d.h., das problemzentrierte Interview stellt das wichtigste Element der Untersuchung dar, es

\footnotetext{
${ }^{206}$ Vgl. Flick 2002,141.

${ }^{207}$ Vgl. Witzel 2000, 2.

${ }^{208}$ Vgl. Bohnsack 2003, 57.

${ }^{209}$ Ebd. 58.

${ }^{210}$ Vgl. Witzel 2000
} 
wird aber darauf verwiesen, dass es sinnvoll sein kann, der Forschungsthematik mit einer Methodenkombination zu begegnen. So lässt sich das Interview auch mit einem standardisierten Fragebogen zur Lösung von Stichprobenproblemen und zur Kombination der mit unterschiedlichen Verfahren gewonnenen Ergebnisse verbinden.

- $\quad$ Prozessorientierung: Dies bezieht sich auf den Forschungsablauf und auf die Vorinterpretation. Ziel der Interviews ist es, dass der Befragte vertrauensvoll und offen über eigene Orientierungen und Handlungen spricht, dazu ist es notwendig, sensibel und akzeptierend den Kommunikationsprozess während des Interviews zu steuern. Die Befragten sollen sich ernst genommen und akzeptiert fühlen, um zu einer Selbstreflexion motiviert zu werden. Nur so können widersprüchliche Handlungsanforderungen, Entscheidungsdilemmata und Interessenwidersprüche zum Ausdruck gebracht werden. ${ }^{211}$

Im Sinne der Offenheit der Fragestellung wird im Interview das Zuhören mit Nachfragen ergänzt, die interviewende Person nimmt eine aktivstimulierende Rolle ein. Das Interview kann gelingen, wenn die Untersuchung bei der Expertin/ dem Experten Neugierde erzeugt und sich die Befragten als Akteure des Interviews verstehen, die für wissenschaftliche Zwecke Auskunft über ihren Berufsalltag geben. Das Interview soll eine Reflexion des eigenen Berufes initiieren, dabei können insbesondere Erzählpassagen, in denen sich die befragte Person ausführlich über die subjektive Problemsicht äußert, von Bedeutung sein und sich als Schlüsselstellen für die Rekonstruktion einer Berufsauffassung erweisen.

Als Erhebungsinstrument hat sich ein leitfadengestütztes Interview bewährt, dies wird einem thematisch begrenzten Erkenntnisinteresse gerecht. Der Leitfaden sollte aber lediglich als Gesprächsstruktur genutzt werden und dem Experten ausreichend Möglichkeit geben, ausführlich und offen zu sprechen und Erläuterungen abzugeben.

In dem hier dargelegten Sinne kann das Problemzentrierte Interview einerseits der befragten Person ein großes Maß an Selbstgestaltung einräumen und damit dem Anspruch der Offenheit gerecht werden, andererseits eine Orientierung an - aus der Sicht der Forscherin - relevanten Themen gewährleisten. Witzel (2000) bezeichnet diese Ausgewogenheit als ein induktiv-deduktives Wechselverhältnis, um das sich der Erkenntnisgewinn im Forschungsprozess organisiert. ${ }^{212}$ Dies beinhaltet auch, dass das unvermeidbare theoretische Vorwissen offen zu legen und als heuristisch-analytischer Rahmen für Frageideen zwischen Interviewer und Befragten anzuerkennen ist. Die Erkenntnisse des bisherigen Forschungsprozesses (standardisierte Befragung, Auseinandersetzung mit Theorien) finden als sensibilisierendes Konzept Eingang sowohl in die Erhebung als auch in die Auswertung. Gleichzeitig wird das Prinzip der Offenheit realisiert, indem die eigenen Relevanzsetzungen des untersuchten Subjekts insbesondere durch Erzählphasen angeregt und zugelassen werden. ${ }^{213}$

${ }^{211}$ Ebd., 3

${ }^{212}$ Vgl. Witzel 2000

${ }^{213}$ Vgl. Witzel 2000, 2. 


\subsubsection{Leitfaden und Durchführung der Interviews}

Die Eingangsfrage des Leitfadens soll einerseits das Thema Führungsperson im Kontext Schule fokussieren, andererseits Raum für freies Erzählen lassen, um eigene Schwerpunktsetzungen zuzulassen. Das Interesse gilt eher den Prozessabläufen, also wie die Befragten ihre berufliche Situation einschätzen, wie sie ihr eigenes Handeln erläutern und welche Berufauffassung sie entwickelt haben. Darüber hinaus sind Frageideen zu den einzelnen Themenbereichen vorformuliert. Wichtig ist aber, dass dem Interviewten zu jeder Zeit die größtmögliche Offenheit eingeräumt wird, ohne dabei die Orientierung am Erkenntnisinteresse zu verlieren. ${ }^{214}$

Der Gesprächsrahmen wurde bereits durch die Teilnahme an der vorhergegangenen standardisierten Befragung vorgegeben und durch den Leitfaden strukturiert, dabei wurde den Befragten ermöglicht, unerwartete Themen zu jeder Zeit zu äußern. So konnten das Wissen und die Erfahrung der Schulleiterinnen und Schulleiter umfassend in das Interview einfließen.

Der Einstieg in die Interviews wurde jeweils über eine Frage zu Situationen im Berufsalltag, in denen Fähigkeiten als Führungsperson gefordert sind, gegeben. „Erzählen Sie Situationen aus Ihrem Alltagsgeschäft, in denen Sie sich besonders als Führungsperson gefordert fühlen“. Die relativ allgemeine Frage lässt eine eigene Richtung der Schulleiterinnen und Schulleiter zu und erlaubt den Befragten ausführlich über ihre Tätigkeit zu sprechen. Dennoch wird ein Fokus auf die eigene Berufsrolle als „Führungsperson" gesetzt, um eine allgemeine Beschreibung der alltäglichen Aufgaben zu vermeiden, sondern vielmehr die Reflexion der eigenen Position in den Mittelpunkt zu rücken.

Den Abschluss eines jeden Interviews bildete die Frage: „Haben wir nach Ihrer Einschätzung etwas Wichtiges vergessen?"

Der Leitfaden gibt zwar Themenaspekte mit vorgeschlagenen Fragen vor, ob die Fragen allerdings alle in der vorgegebenen Reihenfolge gestellt werden, hängt von der Interviewsituation und den Antworten der Befragten ab. Es kann sein, dass die Befragten bereits auf Aspekte einer weiteren Frage eingehen, in diesen Fällen wurde dem offenen Gespräch der Vorrang gelassen. Die inhaltlichen Bereiche und Fragen des Leitfadens sind im Folgenden aufgeführt:

\section{Erfolg und Misserfolg}

Gibt es Situationen, von denen Sie sagen, das hat auf die Leitung bezogen gut geklappt oder nicht gut geklappt?

Praxis der Entscheidungsfindung

Wie und von wem werden an ihrer Schule Entscheidungen getroffen?

Bewertung der eigenen Position

Welche Initiativen von Ihrer Seite waren in der Rückschau erfolgreich? Welche selbst gesetzten Ziele haben Sie als Führungsperson erreicht? Wie würden Sie Ihre Stellung im Gesamtkollegium beschreiben?

${ }^{214}$ Vgl. Witzel 2000, 5 . 
Belastungserleben

Könnten Sie Situationen schildern, von denen Sie meinen, dass es sich für Schulleiterinnen und Schulleiter um besonders anstrengende Situationen handelt?

Wie gehen Sie als Schulleiter/ Schulleiterin mit den immer knapper werdenden Ressourcen um?

Bedeutung der Schulentwicklung und des veränderten Aufgabenspektrums

Haben Schulentwicklungsprozesse in Niedersachsen zu einer Veränderung ihres eigenen Rollenverständnisses geführt? (Schulentwicklung; Unterrichtsentwicklung)

Wie beurteilen Sie den Trend, den Schulleitungen mehr Aufgaben zu übertragen? (Personalkostenbudgetierung).

\section{Zukunftspläne}

Was haben Sie sich für die Zukunft Ihrer Schule vorgenommen?

Eine spezifische Sondierung findet dort statt, wo sich aufgrund des vorher erworbenen Wissens (theoriegeleitet) oder des aus dem Interview selbst erworbenen 'Wissens' Frageideen ergeben haben. Ebenso werden Äußerungen der Befragten zurückgespiegelt, um innen die Möglichkeit zu geben ihre eigene Sichtweise zu behaupten und vertiefend zu reflektieren, dabei dürfen aber keine Rechtfertigungen provoziert werden.

Im Vordergrund der Kommunikationsstrategie steht das Ziel, eine Vertrauensbasis herzustellen, die es den Interviewten erlaubt, sich zu öffnen und möglichst authentisch ihre Sichtweise und ihr Erleben im Beruf zu artikulieren, sozusagen „Preiszugeben“. Die Gesprächshaltung innerhalb der Interviewführung orientiert sich an den Grundsätzen der von Witzel (2000) für das problemzentrierte Interview vorgeschlagenen Vorgehensweise. ${ }^{215}$

In der Interviewsituation selbst werden weder die Gesamtkonzeption noch die theoretischen Überlegungen einer Berufsauffassung thematisiert, so dass die Befragten in ihrer Aufmerksamkeit nicht gelenkt wurden. Allerdings wussten sie natürlich, worum es thematisch geht, dies allein schon deshalb, weil alle Interviewten auch den standardisierten Fragebogen beantwortet hatten. Mit dem Einsatz des Leitfadens wurde versucht, eine vergleichbare Struktur für das Gespräch zu geben.

Bereits durch die Erhebung der ersten Interviews wurde deutlich, dass die Person der Schulleiterin/ des Schulleiters sehr viel stärker als zunächst angenommen den thematischen Verlauf der Interviews bestimmte. In den ersten Interviews versuchte ich dennoch, mich streng am Interviewleitfaden zu orientieren, was zu einer Dialogstruktur der Gespräche führte. Erst mit zunehmender Bewusstheit über den Gewinn gerade der offenen Passagen ließ ich diese zu, so dass es sich um problemzentrierte Interviews handelte. Aus diesem Sample der später geführten Interviews habe ich das auszuwertende Interviewmaterial ausgewählt.

${ }^{215}$ Vgl. Witzel 2000. 


\subsubsection{Die qualitative Stichprobe}

Der Fragebogen wurde gemeinsam mit einem Anschreiben, in dem um die Bereitschaft zu einem Interview gebeten wurde, versandt. Die Bereitschaftserklärung wurde mit gesonderter Post an die Universität gesandt (vgl. Anlage 2). Insgesamt haben sich 56 Schulleiterinnen und Schulleiter (40 Gym/ 6 KGS/10 IGS) bereit erklärt, mit mir ein Interview zu führen.

Eine Auswahl der Interviewten aus diesen 56 Personen habe ich entsprechend nach Verhältnis zu den Beteiligten der Fragebogenuntersuchung vorgenommen, d.h. anteilsmäßige gleiche Verteilung der Schulformen, Berücksichtigung von Frauen und Männern, sowohl ländliche als auch städtische Schule wurden ausgewählt und sowohl größere als auch kleinere Schulstandorte berücksichtigt.

Die Stichprobengröße wurde aus pragmatischen Überlegungen auf die Durchführung von 20 Interviews beschränkt.

\begin{tabular}{|c|c|c|c|c|c|}
\hline $\begin{array}{c}\text { Stichprobe in } \\
\text { Niedersachsen } \\
20\end{array}$ & & Gymnasium & KGS & IGS & Summe \\
\hline weiblich & $\begin{array}{l}\text { städtisch } \\
\text { ländlich }\end{array}$ & $\begin{array}{l}2 \\
1\end{array}$ & 2 & 1 & $\begin{array}{l}5 \\
1\end{array}$ \\
\hline männlich & $\begin{array}{l}\text { städtisch } \\
\text { ländlich }\end{array}$ & $\begin{array}{l}6 \\
5\end{array}$ & & $\begin{array}{l}2 \\
1\end{array}$ & $\begin{array}{l}8 \\
6\end{array}$ \\
\hline Summe & & 14 & 2 & 4 & 20 \\
\hline
\end{tabular}

Tabelle 2: Realisierte qualitative Interviews

Die 20 Interviews wurden im Frühjahr 2004 durchgeführt, alle Interviews wurden in gesamter Länge aufgenommen. Ort der Befragung war das Dienstzimmer der Schulleiterin bzw. des Schulleiters. Ein Zeitlimit wurde nicht gesetzt; im Durchschnitt dauerten die Gespräche ca. 60-100 Minuten. Alle Interviews wurden von mir selbst als Einzelinterviews durchgeführt. Im Anschluss an die Erhebung wurden die Interviews wortgetreu transkribiert und für weitere computerunterstützte Analyseschritte aufbereitet. Aufgrund der dargelegten methodischen Probleme Experten- bzw. Problemzentrierte Interviews wurden lediglich die Interviews aus dem Sample zu weiteren Auswertung herangezogen, die dem Anspruch eines problemzentrierten Interviews gerecht werden konnten. Des Weiteren konnte ein Interview aufgrund von technischen Mängeln nicht transkribiert werden und ein weiteres fiel heraus, da ich den Befragten persönlich kannte, was sich erst vor Ort herausstellte. Insgesamt lag so ein Sample von 15 Interviews vor. 


\subsubsection{Auswertung der problemzentrierten Interviews}

Die Auswertungsmethode der problemzentrierten Interviews von Witzel (2000) lehnt sich an das theoriegenerierende Verfahren der "Grounded Theory" nach Glaser/ Strauss (1967) an. Von Vertretern der "GroundedTheory" wird eine grundsätzliche theoretische Offenheit eingefordert, die zur Generierung von Hypothesen und Theorien führen soll. Das von Glaser/Strauss (1967) entwickelten Analyseverfahren des theoretischen Kodierens folgt dem Anspruch einer gegenstandsbegründeten Theorieentwicklung. Die Auswertungstechnik arbeitet eng am vorgefundenen Material bzw. ist im Material selbst verankert („grounded“). Die Analyse des Datenmaterials ist ein interpretativer Prozess, in diesem induktiven und offenen Verfahren führt über die Herausarbeitung von Kern- und Schlüsselkategorien zur Bildung von Theorien mittlerer Reichweite. ${ }^{216}$ In ihren Grundannahmen gehen Glaser/Strauss davon aus, dass eine 'theoretische Sensibilität' dem Forscher eine Perspektive für relevante Daten ermöglicht, um über das empirische Material in theoretischen Begriffen reflektieren zu können. ${ }^{217}$

Der Prozess der Auseinandersetzung mit dem Material bildet den Mittelpunkt qualitativer Forschung. Dabei wird das Interviewmaterial sorgfältig codiert, d.h., es erfolgt die Zuordnung von Codes zu bestimmten Phänomenen im Datenmaterial. Die Bildung von Kategorien stellt den Prozess der Datenanalyse dar und bildet die Grundlage für die Interpretation der Daten. Die theoretischen Kategorien, die auf der Grundlage erster Datenauswertung entwickelt werden, bestimmen den Prozess der Auswahl der Einzelfälle. Es wird dann eine Interpretation in Form einer vergleichenden Interpretation von Einzelfällen durchgeführt. ${ }^{218}$

Den Kern der Auswertungsarbeit nach der 'Grounded Theory' bildet der Prozess der Kodierens; Glaser/ Strauss (1967) schlagen drei Kodierschritte vor: 1. das offene Codieren; 2 . das axiale Codieren und 3. das selektive Codieren.

1. Das offene Kodieren eröffnet die Forschungsarbeit und zielt darauf ab, Daten und Phänomene in Begriffe zu fassen, Aussagen werden mit Anmerkungen und mit „Begriffen“ (Kodes) versehen. Das Datenmaterial wird sorgfältig Zeile-für-Zeile bearbeitet und es entstehen erste vorläufige

Kodes. Diese können sich auf einzelne Wörter beziehen, auf Sätze oder Abschnitte oder das ganze Dokument. Die Analyse besteht aus der Interpretation des Gesagten, vor dem Hintergrund von Wissen (Alltagswissen, Forscherwissen, wissenschaftlichem Wissen) und von Kenntnis des übrigen Interviewtextes. Im nächsten Schritt werden für die jeweilige Fragestellung besonders relevante Phänomene, die in den Daten entdeckt wurden, gruppiert und dadurch kategorisiert. Die vergebenen Kodes sollen den Inhalt einer Kategorie treffend wiedergeben.

\footnotetext{
${ }^{216}$ Vgl. Bohnsack 2003, 71.

${ }^{217}$ Vgl. Glaser/ Strauss 1998, 47.

${ }^{218} \mathrm{Vgl}$. Kelle/Kluge 1999.
} 
„Mögliche Quellen der Kodes sind Begriffe, die der (sozialwissenschaftlichen) Literatur entlehnt (konstruierte Kodes) oder aus Aussagen der Interviewpartner übernommen (In-vivo-Kodes) sind, wobei letztere wegen ihrer großen Nähe zum untersuchten Material vorzuziehen sind."219 So können auffällige Formulierungen der Befragten zu Kodes werden. Es können einer Textpassage mehrere Kodes zugewiesen werden. Die Entwicklung von Kategorien beinhaltet den Vorgang des Dimensionalisierens; d.h., Kategorien besitzen Attribute und diese lassen sich auf einem Kontinuum beschreiben, z.B. die Kategorie „professionelle Gelassenheit“ kann gering oder groß sein. Das Ziel dieses ersten Kodierungsschrittes ist es, den Text aufzulösen, zu verstehen und dabei Kategorien zu vergeben, zu entwickeln und in eine Ordnung zu bringen. Es werden vorläufige Konzepte und Dimensionen entwickelt, dabei werden dem Datenmaterial zunächst zahlreiche konzeptuelle Kategorien zugewiesen. Als Ergänzung zu den Kodes und Kategorien können Kodenotizen und Memos angelegt werden, die Auffälligkeiten im Material und wichtige Gedanken zu den relevanten Fragestellungen enthalten. ${ }^{220}$

In ähnlicher Weise beschreibt Witzel (2000) die ersten Schritte der Kodierung. Der erste Auswertungsschritt bezieht sich auf die Einzelfallanalyse, das transkribierte Interview wird Satz für Satz deutend nachvollzogen. Der Text wird mit Stichworten aus dem Leitfaden (theoriegeleitet) und mit Begrifflichkeiten, die neue thematische Aspekte aus den Darstellungen der Interviewpartner (induktiv) kennzeichnen, markiert.

Im zweiten Schritt erfolgt eine analytische Zuordnung thematischer Auffälligkeiten zu „In-vivo-codes“, d.h. alltagsnahen Begriffen, aus denen sich Notizen oder kleine Ausarbeitungen ergeben, diese werden auch Memos genannt. Die Textpassagen werden mit Schlagwörtern (Kodes) verknüpft. Dieses Vorgehen computergestützt vorzunehmen, ermöglicht eine komplexe Zugriffsmöglichkeit, denn es lassen sich im weiteren Verlauf der Auswertung Einzelfälle unter verschiedenartigen Aspekten vergleichen. Das Auffinden von Originalstellen und Querverbindungen zwischen unterschiedlichen Textstellen ist möglich. ${ }^{221}$

2. Während das offene Kodieren eher einen breiten und noch relativ ungeordneten Zugang zum Datenmaterial schafft und eine Vielzahl von Kategorien erarbeitet, werden im Prozess des axialen Kodierens Kategorien differenziert und ausgewählt. Es werden in diesem Auswertungsschritt Relevanzentscheidungen getroffen, denn nicht alle im Material identifizierten Phänomene werden systematisch vergleichend auf ihre Ursache, Umstände und Konsequenzen befragt, sondern nur diejenigen, die nach dem

\footnotetext{
${ }^{219}$ Vgl. Flick 2002, 263.

${ }^{220}$ Vgl. Kuckartz 2005, 78. Es wurde das qualitative Datenanalyse - Programm MAXqda Version 2001- benutzt. Kuckartz (1999,75 f.) verweist ausdrücklich darauf, dass auch während einer computergestützten Textanalyse nicht der Computer, der ist, der denkt, interpretiert und codiert, dies ist ausschließlich eine menschliche Interpretationsleistung. Das MAXqda-Programm lässt sich als Ordnungshilfe verstehen und in diesem Bereich ist es auch sinnvoll und hilfreich.

${ }^{221} \mathrm{Vgl}$. Witzel 2000. Witzel bezieht sich in seinen Ausführungen auf Gerhardt. Die um fangreiche Diskussion um das methodische Vorgehen bei der "Typenbildung" kann im Rahmen dieser Arbeit nicht geführt werden, eine übersichtliche Darstellung der Prozessstrukturanalsyse nach Uta Gerhardt findet sich bei Kluge 1999.
} 
vorläufigen Stand der Analyse besonders ertragreich für die Klärung der Forschungsfrage sein können. Diese "Schlüssel- oder Kernkategorien“ werden mit möglichst vielen Textstellen angereichert. Es werden Beziehungen zwischen Kategorien verdeutlicht bzw. Hergestellt: Strauss/Corbin (1990) schlagen den Einsatz eines „Kodierparadigmas“ vor, um solche Beziehungen darzustellen. Die Kategorien werden durch diesen Analyseschritt weiterentwickelt und erlangen so ein höheres Abstraktionsniveau, sodass fallübergreifende Bezüge möglich werden.

Witzel (2000) schlägt vor, durch einen kontrastierenden Fallvergleich „Kernkategorien“ in Form eines Typologiekonzeptes zu entwickeln, die dann in der nächsten Auswertungsstufe als Deutungshypothesen genutzt werden können. ${ }^{222}$

3. Das sich nun anschließende selektive Kodieren setzt den Prozess des axialen Kodierens fort. Ziel ist es, die entwickelten Kernkategorien in einen abschließenden Analyseprozess zu integrieren; Ergebnis dieses Prozesses ist die Entwicklung zentraler Kernkategorien und darauf bezogener Unterkategorien, die das untersuchte Phänomen möglichst genau abbilden. In diese Phase wird die gesamte interpretative Arbeit integriert, die Daten werden gruppiert und eine Theorie über das typische Handeln typischer Akteure im Untersuchungsfeld verdichtet.

Der Analyseprozess nach der Grounded Theory unterliegt keinem festen Ablaufschema, sondern er gibt Leitlinien und Orientierungshilfen. Das Kodieren hat die Funktion der Analyse und ist gleichbedeutend mit der Interpretation von Daten. Diese Kodierung und Überprüfung der Kategorien am Material findet während des gesamten Forschungsprozesses immer wieder statt. Die drei verschiedenen Formen des Kodierens stellen dabei grobe Phasen des Forschungsprozesses dar. ${ }^{223}$

Die theoretischen Kategorien, die auf der Basis der ersten Untersuchungseinheiten entwickelt werden, bestimmen die Auswahl weiterer Fälle. Dabei können die Auswahlkriterien aufgrund der theoretischen Relevanzen verändert werden. Es werden Fälle verglichen, die interessierende Kategorien gemeinsam haben und bezüglich der bedeutsamen Kategorien entweder relevante Unterschiede oder große Ähnlichkeiten aufweisen. Glaser/ Strauss (1998) ${ }^{224}$ sprechen von den Methoden Minimierung und Maximierung. Der Interpretationsvorgang und die Einbeziehung zusätzlichen Materials sind dann abgeschlossen, wenn weitere Kodierung und Anreicherung von Kategorien keine neuen Erkenntnisse mehr liefern, die theoretische Sättigung ist dann erreicht. Theoretische Sättigung erfordert, dass die maximale Variation der Untersuchungsgruppe abgebildet wird, d.h. eine genügende Anzahl unterschiedlicher Gruppen innerhalb des Gegenstandsbereiches untersucht worden ist. ${ }^{225}$

\footnotetext{
${ }^{223}$ Durch die Einführung EDV-gestützter Verfahren der Kodierung qualitativen Daten-materials in den 80er Jahren wurde dieser Kodierungsprozess erheblich erleichtert. ${ }^{224}$ Vgl. Glaser/Strauss 1998, 53.

${ }^{225}$ Vgl. Kelle/ Kluge 1999, 46. Zur Problematisierung der theoretischen Sättigung vgl. Flick 2002, 270ff, Kuckartz 2005, 80f.
} 
Die sich nun anschließende Zeile-für-Zeile-Auswertung der Einzelfälle erfolgt nach dem Prinzip der „Kernstellen“, die aus den Interviews ausgewählt werden, sodass eine Reduzierung des Materials und eine Konzentration auf die Fragestellung der Untersuchung möglich werden. Als Kernstellen können nach Ricker (2000) die Textstellen angesehen werden, „die repräsentativ für die erzählende Person, ihre Erfahrungsaufsicht und die Rekonstruktion ihrer Lebensgeschichte sind." ${ }^{226}$ Bei der Auswahl der Textstellen sind die Fragestellung der Untersuchung und das Thema der Forschungsarbeit von Bedeutung. In der hier vorliegenden Studie wurden zudem die durch den Auswertungsprozess entstanden Kernkategorien mit einbezogen. Dabei fließen in den Auswertungsprozess latent auch diejenigen Interviewpassagen mit ein, die nicht als Kernstellen ausgewählt wurden. $^{227}$

Die Aufgabe dieser abschließenden Interpretationsphase ist es, das jeweils Charakteristische des Einzelfalls unter Bezugnahme der entwickelten Kategorien zu erarbeiten. Nachdem das Charakteristische des ersten Falls erarbeitet wurde, wurden nach dem Prinzip des theoretischen Samplings weitere Fälle ausgewählt und gleichermaßen ausgewertet.

Wie bei vielen qualitativen Untersuchungen stellt sich auch im Rahmen dieser Untersuchung das Problem der Datenfülle. Von jeder interviewten Person liegen ca. 20-30 Seiten transkribierter Text vor. Das gesamte Material auszuwerten war im Rahmen einer Einzelforschung nicht möglich. So habe ich 15 Interviews nach dem oben beschriebenen Analyseverfahren codiert und Kernkategorien für das Material entwickelt, bei der ausführlichen Fallanalyse beschränke ich mich auf fünf Einzelfälle, die eine breite Variation des vorliegenden Materials abbilden.

\section{Berufsauffassungen von Schulleiterinnen und Schulleitern- das empirische Material}

Das empirische Material wurde auf der Basis vollständig transkribierter Interviews ausgewertet. Über die Kodierung der Interviews wurden Kategorien differenziert und die von den Befragten zusätzlich angesprochenen Themen berücksichtigt.

Das für den ersten Fall entwickelte Kategorieschema wird anhand eines systematischen Fallvergleichs erweitert. Die fallvergleichende Kontrastierung dient der Ermittlung von Vergleichsdimensionen, die eine Verallgemeinerung der jeweiligen Fallbesonderheiten ermöglicht.

Für das in dieser Untersuchung vorliegende Datenmaterial konnten nach der Durchführung der Kodierschritte und dem kontrastierenden Fallvergleich folgende fallübergreifenden Vergleichskategorien ermittelt werden.

Diese Kategorien wurden dimensionalisiert und im Rückgriff auf das sensibilisierende Konzept als tragbares Analyseinstrument für den weiteren Forschungsprozess eingesetzt.

\footnotetext{
${ }^{226}$ Ricker 2000, 87.

${ }^{227}$ Die besondere Bedeutung der Eingangserzählung konnte im vorliegenden Material nur bedingt festgestellt werden, dies liegt meines Erachtens daran, dass die Schulleiterinnen und Schulleiter immer in ihrer Schule und in ihrem Dienstzimmer befragt wurden und in den meisten Fällen brauchten sie ein wenig Zeit, um sich thematisch einzulassen.
} 
Abb. 23: Fallübergreifende Vergleichskategorien

\section{Handlungsrepertoire}

(Handlungs- und Interaktionsstrategien)

- Aufgabenspektrum

- Erwartungen an den Handlungsausgang

- Einschätzung von Problemen

- Umgang mit Konflikten

- Ausgleich widersprüchlicher Beziehungsstrukturen

Stabilisierung durch Arbeitsbündnisse

- Verbundenheit mit Schulleitung, Kollegium, Sch., Eltern

- Perspektivenübernahme

- Arbeitsformen

\section{Berufsrelevantes Selbstkonzept}

- Selbsteinschätzung

- Bewältigung von Stress/ Belastung

- Identifikation mit Rolle als SL

- Berufszufriedenheit vs. Berufsbelastung

Reflexionsvermögen/ Distanzierungsfähigkeit

- Diagnosekompetenz

- Einschätzung sich selbst gegenüber

- Reflexion von Widersprüchen

- Distanz

Nachdem das Charakteristische des ersten Falls erarbeitet wurde, wurden nach dem Prinzip des theoretischen Samplings weitere Fälle - minimal und maximal kontrastiert- ausgewählt und ebenso ausgewertet. Am Ende erfolgte im Vergleich der Einzelfälle die Herausarbeitung der spezifischen Berufsauffassungen. Es ließen sich folgende in sich kohärente Fallbeispiele kontrastieren, diese werden im Kap. 5.1 ausführlich dargestellt.

Abb. 24 : Fallkontrastierung

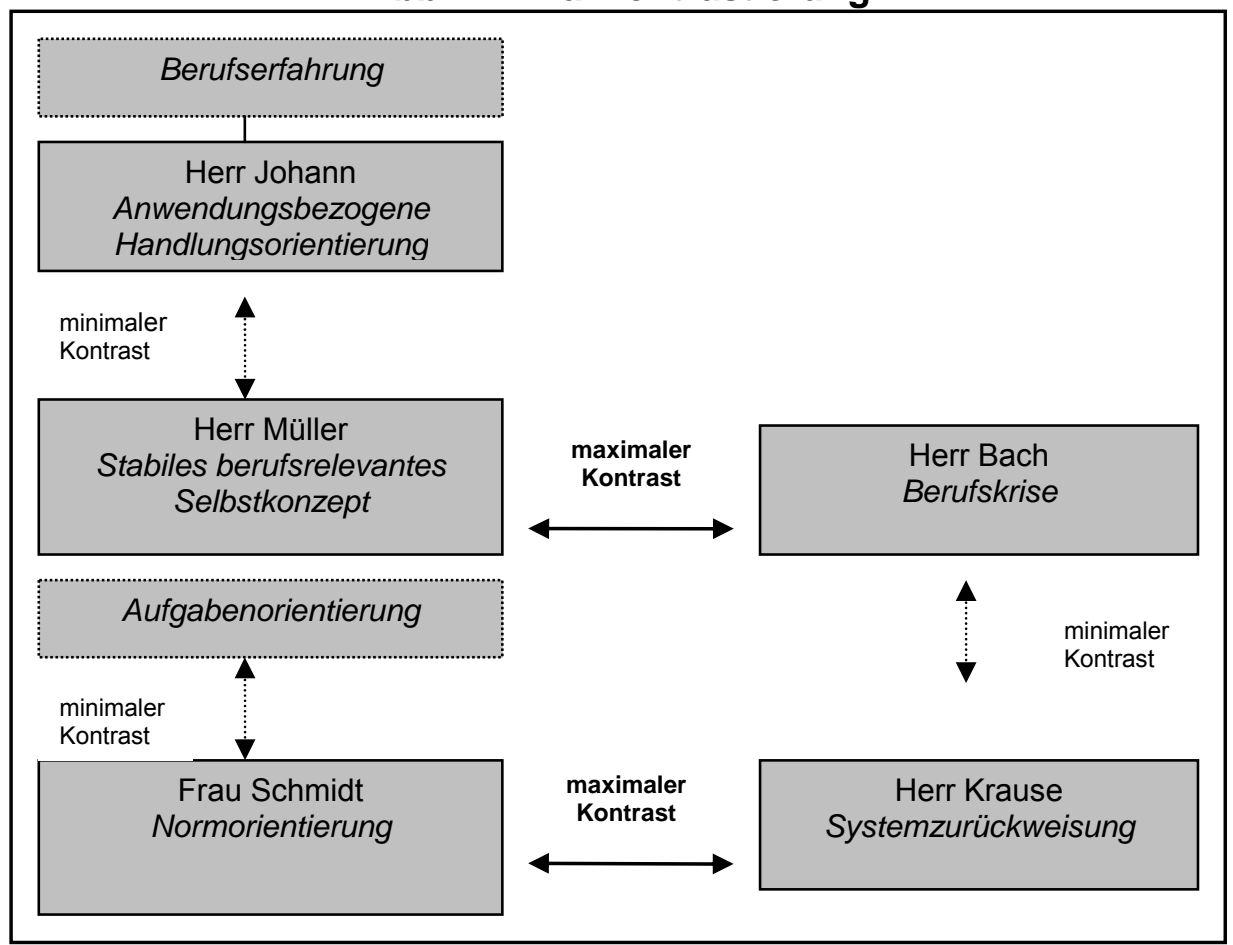




\subsection{Darstellung der Ergebnisse anhand ausgewählter Fallbeispiele} Die vergleichende Auswertung des vorliegenden Materials erfolgt durch dichte Fallbeschreibungen und hat das Ziel, die Berufsauffassungen der interviewten Schulleiterinnen und Schulleitern in den Dimensionen Führungsverhalten, Organisationsverständnis und der Professionskompetenz herauszuarbeiten und zu vergleichen.

Insgesamt lassen sich aus dem vorliegenden Datenmaterial auf der Basis eines minimalen und maximalen Vergleichs 5 unterschiedliche "Typen“228 von Berufsauffassungen als unterschiedliche Kombinationen von Dimensionen herausarbeiten. Die einzelnen „Typen“ sagen nichts aus über die Häufigkeit des Auftretens der einzelnen Typen, sondern letztendlich handelt es sich um unterscheidbare Auffassungen und damit mit großer Wahrscheinlichkeit verbundene Verhaltensmuster.

Im Folgenden wird die Berufsauffassung von 4 Schulleitern und 1 Schulleiterin vorgestellt. Die Darstellung der Einzelfälle beginnt mit einer kurzen Beschreibung der Ausgangssituation; diese Beschreibung wird aus Gründen der Anonymisierung sehr kurz gefasst.

\subsection{1 „Konstruktiver Jongleur“ Herr Müller (SL4) 229}

Herr Müller ist über 20 Jahre im Schuldienst, er war zunächst Lehrer, dann stellvertretender Schulleiter an einer Auslandsschule und leitet seit zwei Jahren die jetzige Schule. Es handelt sich um ein größeres (>600 Sch.) Gymnasium in einer Kleinstadt.

Die Schulleitung besteht aus dem Schulleiter, einem Stellvertreter und drei Koordinatoren. In der Schule gibt es eine Steuergruppe, die Schulenwicklungsaufgaben initiiert. Der Schulleiter hat einem Interview zugestimmt und erscheint im Telefongespräch sehr aufgeschlossen. Das Gespräch findet an einem Freitag nach Unterrichtsschluss im Dienstzimmer des Schulleiters statt.

Auf die Eingangsfrage, welche Kompetenzen von einem Schulleiter jeden Tag gefordert werden, grenzt Herr Müller die Arbeit eines Schulleiters deutlich von der eines Lehrers ab. Als Unterscheidungsmerkmal führt er vor allem den Umgang mit Erwachsenen unterschiedlicher Konstellationen an. Er erlebt die ihm gestellten Aufgaben als „Bereicherung“.

\footnotetext{
228 Eine Typologie ist das Ergebnis eines Gruppierungsprozesses, bei dem das vorliegende Datenmaterial anhand von Merkmalen in Gruppen bzw. Typen eingeteilt wird, so dass sich Elemente eines Typus möglichst ähnlich sind. Der Typus bezeichnet die Teilgruppe, die gemeinsame Eigenschaften aufweist. Auf der Ebene des Typus wird vorrangig nach Gemeinsamkeiten gesucht, auf der Ebene der Typologie wird eine Abgrenzung zwischen einzelnen Typen vorgenommen. Da sich alle Typen auf eine Untersuchungsgruppe beziehen, weisen sie trotz der Unterschiede auch Gemeinsamkeiten auf, indem es sich z.B. bei allen Befragten um Schulleiterinnen und Schulleiter handelt, die zu einem bestimmten Zeitpunkt in einer bestimmten Region eine Schule leiten. Dies macht deutlich, dass die in meiner Untersuchung konstruierten Typen nicht ohne weitere Untersuchung auf andere Gruppen übertragen werden können. (Vgl. Kluge 1999.)

${ }^{229}$ Die Namen sind geändert worden. Alle wörtlichen Zitate sind in den Fallbeschreibungen kursiv gedruckt und entstammen den Interviews mit der jeweiligen Schulleiterin/ dem jeweiligen Schulleiter, die Kennzeichnung erfolgt mit Nummern weiblich SLi/ männlich SL. In Klammern sind jeweils die genauen Zeilenangaben der Interviewtranskripte angegeben. Informationen, die Rückschlüsse auf die Person oder die Schule zulassen würden, sind aus den Texten entfernt worden, ohne die inhaltliche Aussage zu verändern.
} 
„ICh bin noch nicht so lange im Amt, ich bin jetzt im zweiten Jahr Schulleiter und war vorher im Ausland und war dort stellvertretender Schulleiter an einer allerdings großen und auch komplizierten Auslandsschule. Und ich glaub', dass ich da 'ne Menge gelernt hab' für dieses Geschäft, und es ist für mich erst mal, wenn ich's mal so abgrenze von der normalen Lehrertätigkeit, die ich ja auch über 20 Jahre gemacht habe, doch 'ne - ja Horizonterweiterung oder Bereicherung, einfach weil ich viel mehr mit erwachsenen Menschen und Personen und Gruppen, unterschiedlichen Konstellationen zu tun habe, also das ist für mich sozusagen immer so 'n bisschen wie ein Tanz auf 'm Vulkan. Also äh das ist teilweise kompliziert und stressig, aber teilweise macht's mir auch Spaß, so zwischen verschiedenen Kräften zu jonglieren und zu schauen, wie man aber dann für die eigene Schule dann das Beste dabei herausholt. Also das sind ja sehr unterschiedliche Gruppierungen, mit denen ich's zu tun habe - so mit Leuten aus der Verwaltung oder auch aus der Politik, mit Elterninteressen, mit Schülern, mit Kollegen, mit NachbarSchulleitern, mit den nicht-pädagogischen Angestellten, die an der Schule auch wichtig sind, usw. Und das ist eigentlich ein sehr buntes Panorama von Tätigkeitsfeldern, und allein das macht mir Spaß, und ich glaube, dass ich mit diesen unterschiedlichen Personen und Personengruppen im Prinzip ganz gut umgehen kann, dass ich die Leute auch wahrnehme, wie sie so aus ihrer Interessenposition heraus auf mich zukommen und dass ich aber dabei doch so mit innen umgehen kann, dass auch für die Schule etwas Gutes dabei herauskommt. "(8-25) 230

Herr Müller verweist zu Beginn des Interviews darauf, dass er noch nicht lange im Amt ist, also nur über begrenze Erfahrungen als Schulleiter verfügt, aber über seine Tätigkeit als stellvertretender Schulleiter an einer großen und komplizierten Auslandsschule von Anfang an gut auf seine Tätigkeit vorbereitet war. Herr Müller greift in seiner Einschätzung der beruflichen Vorbereitung auf ein in der Praxis erworbenes Wissen zurück und fühlt sich durch ähnliche berufliche Erfahrungen auf seines Amt vorbreitet. Den Wechsel der Tätigkeit klassifiziert er als eine „Horizonterweiterung“ und „Bereicherung“, Herr Müller begründet das damit, dass er es im Vergleich mit der Tätigkeit als Lehrer nicht mehr in erster Linie mit Kindern, sondern mit erwachsenen Menschen, mit Personen zu tun hat und nicht so sehr mit einzelnen- wie z.B. beim Lehrer - sondern mit Gruppen, die - worauf er wenig später verweist- unterschiedliche Interessen vertreten und von unterschiedlichen Interessenspositionen auf ihn zu kommen. Die Interessensbezogenheit der Gruppen stellt ein wesentliches Element von Organisationen und damit ein Element seines Organisationswissens dar. Das Wissen um die unterschiedlichen Gruppierungen und deren Interessensbezogenheit wird in der entsprechenden Literatur als wesentliche Voraussetzung für die Bewältigung von Führungsaufgaben genannt. Diese Voraussetzung als Gelingensbedingung für professionelles Führungshandeln scheint bei Herrn Müller vorhanden zu sein. Dies ermöglicht es berufliche Anforderungen sowohl als belastend zu erkennen- d.h. nicht zu verkleinern- als auch als produktive Herausforderung und als "Spaß“ es macht Herrn Müller Spaß zwischen den Kräften zu jonglieren- zu erleben. In seinen Äußerungen spiegelt sich eine hohe Berufszufriedenheit und ein persönliches Engagement.

Verbunden sind dieses Wissen und die Wahrnehmung der Aufgaben als Herausforderung mit einer positiven Selbsteinschätzung hinsichtlich der

Praxiswissen

Organisation swissen

\footnotetext{
${ }^{230}$ Vgl. Kuckartz 1999. Er verweist darauf, dass Textsegmente so zu skizzieren sind,
} dass die Textstellen im Kontext verständlich bleiben und daher eher größer sein sollten.

Positive Selbsteinschätzung 
eigenen Kompetenzen, konkret schätzt er seine Kompetenz zum Umgang mit unterschiedlichen Gruppen und zur Wahrnehmung der ungleichen Interessenspositionen positiv ein. Der Ausgleich zwischen zum Teil widersprüchlichen Beziehungsstrukturen wird innerhalb der Professionstheorie als eine wichtige Voraussetzung im Umgang mit beruflichen Anforderungen beschrieben (vgl. Kapitel 3.3 in dieser Arbeit).

Ein drittes zeigt sich bereits in diesem ersten Interviewauszug: Herr Müller verfolgt bei seinen Handlungen ein klares Ziel, er betont zwei Mal, dass sich sein Handeln am Wohl der Schule orientiert, dass er für die Schule als Ganzes das Beste herausholen will.

In seinen Äußerungen spiegelt sich ein Organisationsverständnis wider, welches von der Individualebene des Handelns abrückt und den Systemzusammenhang der Gesamtorganisation anerkennt.

Diesen Anforderungen bzw. Herausforderungen - d.h. der Komplexität und tendenziellen Widersprüchlichkeit der Aufgaben und Zielsetzungen versucht Herr Müller mit einem Handlungskonzept des Ausbalancierens, des Jonglierens zu begegnen. Metaphorisch beschreibt er seine berufliche Situation und seine Tätigkeit als Schulleiter als „Tanz auf dem Vulkan“.

Zusammenfassend lässt sich anhand dieses ersten Zitats als ein vorläufiges Ergebnis herausarbeiten, dass das Handeln von Herrn Müller orientiert ist an der in der einschlägigen Literatur immer wieder betonten Komplexität und tendenziellen Widersprüchlichkeit pädagogischen Führungshandelns und daran, dass er als Schulleiter - und dies lässt sich als ein Element seiner Berufsauffassung herausarbeiten - derjenige sein muss, der durch geschicktes Jonglieren das Beste für die Schule erreichen muss. Dieses Jonglieren versteht er als Herauforderung, ohne die damit verbundenen Belastungen zu verdrängen. Betrachte ich diese vier, sich bereits in den ersten Aussagen des Interviewten zeigenden Dimensionen, so sind darin bereits wesentliche Elemente enthalten, die z.B. Wunderer für ein erfolgreiches Führungshandeln benennt (vgl. Kap 3.1 in dieser Arbeit).

Im zweiten Interviewausschnitt illustriert und konkretisiert Herr Müller seine Arbeit als Schulleiter an einem Beispiel.

\footnotetext{
„Heute morgen z.B. bin an einer der beiden künftigen Außenstellen zum Besuch gewesen und habe mit 'ner Kollegin gesprochen, die mir noch vor vier Wochen gesagt hat, sie will die Leitung der Außenstelle übernehmen, und ich hab' die besagte Personalplanung darauf abgestellt und in der Rücksprache mit der Bezirksregierung, und die Kollegin sagte dann: „Ach, ich hab' mir das anders überlegt. Ich möchte mich doch lieber also auf den Unterricht in der Klasse konzentrieren und mich mehr den Kindern wieder zuwenden in den acht Dienstjahren, die noch vor mir stehen, und die Außenstellen-Leitung kommt für mich nicht mehr in Frage. "Und das ist eigentlich - für mich ist es fast ein Wortbruch, aber ich muss also diese Realität akzeptieren. Stress ist auch, dass Leute ihre Arbeit schlecht machen. Lehrer machen ihre Arbeit schlecht. Man müsste sie eigentlich rausschmeißen, und das Beamtenrecht lässt es nicht zu. Das Beamtenrecht ist so - das gibt eine solche Sicherheit den Kollegen, dass sie also sozusagen schon silberne Löffel klauen müssten, um irgendwie aus dem Dienst entfernt werden zu können. "(29-40)
}

Der Schulleiter beurteilt das Verhalten der Kollegin kritisch, sie hat sich aus seiner Sicht nicht an Abmachungen gehalten, was ihn unter Druck für 
die weitere Planung setzt. Die Personalplanung ist von subjektiven persönlichen Gegebenheiten der Kollegin abhängig, anders als im privatwirtschaftlichen Bereich kann der Schulleiter nicht die Übernahme eines verantwortungsvolleren Amtes anordnen, im öffentlichen Bereich und in der Schule als Non-Profit Organisation muss der Schulleiter sowohl Bezirkregierung als auch die Personen vor Ort im Blick haben. Die Kollegin kann ablehnen und begründet ihre Entscheidung mit dem Wunsch in der Rolle als „Lehrerin“ zu verbleiben.

Der Schulleiter muss es als „Realität“ anerkennen, dass die Kollegin aus persönlichen Gründen davon Abstand nimmt. Aus dem Erzählkontext heraus erscheint es glaubhaft, dass er dies nicht als persönlichen Affront das zeigt seine Professionalität- er reflektiert das Problem auf der Metaebene. Herr Müller schätzt diese exemplarische Situation insgesamt als Strukturproblem der Organisationsform Schule ein und äußert Kritik an dem Beamtenstatus, die er später erneut aufgreift. Herr Müller orientiert sich in seiner Berufsauffassung an seinen berufstypischen Werten, nämlich dass alle an Schule Beteiligten verbindlich und zuverlässig arbeiten und Absprachen einhalten- wer dies nicht tut, müsste seiner Ansicht nach mit Sanktionen rechnen- Herr Müller orientiert sich in seiner Berufsauffassung an einem transaktionalen Führungsverständnis, welches auf der Grundlage von Leistung und Gegenleistung beruht, diese Auffassung steht aber im Widerspruch zu den strukturellen Gegebenheiten der Schule (vgl. Kap. 3.1 in dieser Arbeit).

Die Fremdbestimmung durch den Beamtenstatus kann als Dysstress für den Schulleiter gedeutet werden; dass er trotzdem in der Lage ist, Stress problembezogen zu bewältigen, wird auch durch die sich anschließende Erläuterung eines weiteren Beispiels deutlich.

„Und ich hab' also 'ne Situation beispielsweise erlebt, dass 'n (...) Lehrer, der an unsere Schule versetzt wurde, noch vor meiner Zeit, der ist sozusagen gleichzeitig mit mir hier angefangen, aber schon nach wenigen Wochen gab's also schon massive Kritik von Eltern-Seite - sowohl was das Fachliche angeht, als auch was das Persönliche angeht im Umgang mit den Kindern (...). Und ich hab' dann aufgrund dieser Kritik der Eltern im Unterricht dieses Kollegen selbst hospitiert, hab' mich überzeugt davon, dass diese Kritik berechtigt ist, hab' das schriftlich angemahnt bei der Bezirksregierung, dass das eine unhaltbare Situation ist, dass die Bezirksregierung sofort eingreifen möge, und äh diese und je nachdem eben die Maßgabe hätte sein können, ihn aus den Unterricht raus zu nehmen und ihn dann zum Fortbildungsinstitut zu schicken, so dass er dort sozusagen fortgebildet worden wäre, um zu schauen, ob er sich soweit entwickelt - fachlich und persönlich -, dass er für den Unterricht wieder fähig gewesen wäre. Die Bezirksregierung hat über Wochen daraufhin überhaupt nicht geantwortet auf diesen Brief, und nach Wochen kam dann: „Wir haben nicht die personelle Kapazität", dass etwa der Fachberaterin seinen Unterricht geht (...) also da fühlte ich mich von der Behörde im Stich gelassen. Und der Kollege hat dann diese Konfrontation hier weiter ertragen müssen, und am Ende ist der Kollege dann an Krebs erkrankt. Also es ist echt 'ne tragische Komponente auch noch mit drin. Und da fühle ich mich eigentlich auch von der Behörde total im Stich gelassen.(41-66)

Herr Müller agiert auf der Ebene seiner Schule konsequent, er hospitiert den Unterricht und führt Gespräche mit dem Kollegen. Er strebt eine Lösung im Sinne aller Beteiligten an, indem er für den Kollegen nach Unter- 
stützungsmöglichkeiten sucht. Herr Müller versucht, die organisationellen und individuellen Bedürfnisse in Einklang zu bringen, er geht hier zielorientiert vor und ist von einem positiven Handlungserfolg überzeugt. Erneut wird durch diese Äußerungen deutlich, dass Herr Müller auf unterschiedlichen Handlungsebenen ,jonglieren“ kann und somit ein Balance zwischen seinem eigenen beruflichen Möglichkeiten und den strukturell gegebenen Organisationslogiken, die er zwar kritisiert, die ihn aber nicht handlungsunfähig machen. Es wird eine in der klassischen Professionstheorie als professionelle Handlungsparadoxie beschriebene Situation dargelegt, denn es liegt eine Antinomie von Organisation versus Interaktion vor. Die Fähigkeit zwischen der eigenen Berufsratio und der Organisationsratio zu balancieren wird in der Literatur als ein entscheidendes Moment professionellen Handelns beschrieben (vgl. Kapitel 3.3 in dieser Arbeit). Herr Müller belässt es aber nicht dabei, sein Handeln aufgrund dieser Paradoxie zu reduzieren, sondern auf seiner Handlungsebene verhält er sich weiterhin konsequent, ihm bleibt das Gespräch vor Ort, welches er auch angeht.

(..) Und ich habe ihm gesagt. „Herr Sowieso , ich hab' mir jetzt durch die Hospitation in Ihrem Unterricht selbst ein Bild gemacht und bin in der Tat davon überzeugt, dass also hier dringend Änderungen herbeigeführt werden müssen", hab' ihm also in Aussicht gestellt, dass der Fachberater massiv seinen Unterricht hospitieren wird, und dass es auch nötig ist - dies - und eben auch die anderen Schritte, die ich jetzt auch genannt habe. In aller menschlicher Akzeptanz diesem Kollegen gegenüber $\langle h m>$. Ich will ihm ja nichts Böses. Absolut nicht. Und dann ist aber auch das Beamtenrecht zu unflexibel, (...). Das ist ja ein armer Mensch, der psychisch auch unter diesem Druck leidet, und im Grunde genommen müsste das Beamtenrecht flexibilisiert werden dahingehend, dass so jemand in ein anderes System des öffentlichen Diensts überführt wird, wo er mehr Verwaltungstätigkeit machen kann oder Dinge machen kann, die seiner Kompetenz entsprechen."(6980)

Dieser Ausschnitt verdeutlicht das Abwägen unterschiedlicher Ebenen im Rahmen der Organisation Schule, Herr Müller ist bereit, beide Seiten des Konfliktes zu sehen, er zeigt Empathie mit dem Kollegen und vollzieht eine Perspektivenübernahme. Dieser Umgang mit dem Kollegen und das Wissen um seine schwierige Situation spiegelt ein berufsethisches Wissen wider. Dennoch sieht Herr Müller seine Hauptaufgabe aber darin, die Qualität des Unterrichts für Schülerinnen und Schüler zu sichern. Der Schulleiter ist trotz seiner Enttäuschung über die mangelnde Unterstützung der Behörde bereit, den Konflikt, so weit es auf der Ebene seiner Schule möglich ist, anzugehen und er übernimmt eine klare Führungsrolle, indem er den beteiligten Kollegen im Unterricht hospitiert und ihm offen seine Kritik mitteilt. An dieser Interviewpassage wird deutlich, dass Herr Müller die nötige Distanzierungsfähigkeit besitzt, er agiert neben dem personalen Rahmen ebenfalls auf der Ebene des politischen und strukturellen Rahmens, dies illustriert folgendes Beispiel. Der hier beschriebene Interessenskonflikt verdeutlicht das Dilemma des Schulleiters, denn die Organisationsform von Schule mit Lehrkräften im Beamtenstatus, die sich - so seine Sichtweise- nicht an der Qualität der Arbeit orientiert und der Hierarchie der Behörde, die ebenfalls nicht an Qualität interessiert ist, stehen im Widerspruch zu seinen Auffassungen der Qualität von Schule, in dem einen Fall ist er Vorgesetzter im anderen Fall ist er Untergebener. Herr Müller kritisiert das Führungsverhalten der Schulbehörde. 
„Oder ein anderes Beispiel war, dass wir ein neues Fach einführen wollten, und das Fach Spanisch, was vom Schulelternrat seit langem gefordert ist, für das es ein sehr großes Interesse gibt, äh, es gab auch Lehrkräfte, die sich für unsere Schule interessierten, und man hat dann seitens der Bezirksregierung dann gesagt: „Na ja, ihr könnt das Fach Spanisch nicht einführen, weil ihr ja nicht zwei Spanisch-Lehrer schon habt. Dann habe ich gesagt: „Gut, dann lasst uns 'n zweiten Spanischlehrer kommen“. Dann hieß es: „Nein, ihr kriegt keinen zweiten Spanischlehrer, weil ihr ja das Fach Spanisch nicht habt. Und es musste dann politisch Druck gemacht werden bis hin in die Regierung, bis hin in das Ministerium hinein und über bestimmte parteipolitische Kanäle hinein, bis die Bezirksregierung dann eingeknickt ist, und das eigentlich, das spricht eigentlich Hohn dem Credo, das man auf der anderen Seite postuliert, dass die Schulen ihr Profil entwickeln sollen, dass sie ihre Schwerpunkte setzten sollen, wenn man mit solchen bürokratischen Hürden zu tun hat, die also wiederum damit zusammenhängen, dass dann bei der Bezirksregierung, der also da Verantwortung trägt, 'ne besondere Affinität hat zum Nachbargymnasium, was Spanisch schon hat und was er schützen will. Ne? Und das sind solche Dinge, und die verursachen natürlich massiv Stress auch, und da kann man dann rätseln, ob das ' $n$ positiver oder negativer Stress ist - positiv insofern jetzt, als wir uns durchgesetzt haben $\langle j a>$, aber negativ eigentlich als etwas, was von der Sache her eigentlich offensichtlich sinnvoll ist, also sozusagen mit solchen Barrieren versehen wird."(102-119)

Herr Müller ist bereit, für die Interessen der Schule Konflikte mit der übergeordneten Behörde einzugehen und sich Unterstützung im Außenbereich zu suchen, um seine Ziel durchzusetzen. Dabei analysiert er die Interessenlagen, deckt die Widersprüchlichkeit von Programm (Profilbildung) und konkreter Umsetzung (Verteilung von Ressourcen) auf. Die ihm gesetzten „Barrieren“ erlebt der Schulleiter als „Stress“. Hier spricht Herr Müller das ambivalente Phänomen Stress an. Er selbst hat durch Eigeninitiative und durch den letztendlichen Erfolg seines Agierens den Stress positiv erlebt. Er hatte ein klares Ziel, welches er in Form eines strategischen Managements erreicht. Die paradoxe und kontraproduktive Argumentation der Schulbehörde hat er als zu vermeidende Barriere erfahren und dies als extern verursachten Stress wahrgenommen. Herr Müller beweist aber in der Situation Überblick, Weitsicht und klare Analyse der Handlungsebenen. Diese Situation zeigt, dass der Schulleiter in der Lage ist, das wirklich Machbare in einer schwierigen Situation herauszuarbeiten und durchzusetzen. Die Berufsauffassung orientiert sich in diesem Beispiel an einer zielbezogenen Führung bei gleichzeitiger Berücksichtigung unterschiedlicher Handlungsebenen: Einzelschule, Schulbehörde, politische Einflussmöglichkeiten.

„Weil ich jedes Projekt, was ich vorhabe, (...), jeweils vorbespreche, also wir haben eine Steuergruppe in der Schule, in der acht Personen mitarbeiten und dazu gehören in der Hauptsache Lehrkräfte, aber auch ein Eltern- und Schüler-Vertreter ist dabei. <hm> Dort werden solche Ideen ausgebrütet im Hinblick auf die Weiterentwicklung der Schule. Wir werden jetzt im nächsten Jahr 'ne Bläserklasse z.B. auch haben. Das wird dann besprochen mit der Schulleitungsrunde, aber eben auch mit dem Personalrat. Also mir persönlich ist der Bezug zum Personalrat sehr, sehr wichtig. Ich bin eigentlich als Schulleiter ein zahnloser Tiger, und ich kann eigentlich auch Maßnahmen, die unpopulär sind, meinen meisten Kollegen gegenüber nur durchsetzen im Schulterschluss mit dem Personalrat. Also ich hab' z.B. eine Dienstaufsichtsbeschwerde gegen einen Kollegen auf den Weg gebracht, der seit Jahrzehnten, wie ich gehört habe, zu spät in den Unterricht geht. Und da war mir der Schulterschluss mit dem Personalrat sehr wichtig, damit das Kollegium 
weiß, ich meine nicht das Kollegium. Überhaupt nicht. Sondern ich meine diesen einzelnen Kollegen." (123-135)

Neben einer starken Betonung der eigenen Verantwortung ist es für Herrn Müller wichtig, die unterschiedlichen Gremien innerhalb der Schule einzubeziehen, wenn es z.B. um die Einführung und Umsetzung neuer Projekte bzw. Unterrichtsfächer geht.

Durch die Formulierung „was ich vorhabe“, wird deutlich, dass der Schulleiter sehr klare Vorstellungen darüber hat, was die Schule aus seiner Sicht voranbringt, er hat klare Werthaltungen, lässt aber eine breite Beteiligung dennoch zu. Dies kann als eine notwendige Vorstrukturierung und Entlastung des Kollegiums anerkannt werden, er hat „Visionen“ für die Schule und steht für seine Ideen ein. Herr Müller transformiert Werthaltungen, denn Werte wie Pünktlichkeit sind inm im Sinne aller Kolleginnen und Kollegen wichtig, er wünscht sich ein Verantwortungsbewusstsein jedes Einzelnen für einen reibungslosen Ablauf des Schulbetriebes.

Die Formulierung „zahnloser Tiger“ erklärt die Bedeutung eines notwendigen Zusammenhalts mit dem Personalrat gerade in Bezug auf unangenehme Entscheidungen, die der Akzeptanz des Kollegiums bedürfen, um die Kolleginnen und Kollegen nicht zu verunsichern. Der Schulleiter differenziert zwischen dem Kollegium und einigen „schwarzen Schafen“, er will durch die Konfrontation mit Fehlverhalten letztendlich auch diejenigen unterstützen, die sich regelkonform verhalten. Oder um im Bild zu bleiben, er zeigt dort Zähne, wo es notwendig ist. Herr Müller nutzt dieses Vorgehen auch im Sinne seiner Stabilisierung durch Arbeitsbündnisse.

Die Berufsauffassung ist von einem kooperativ-partizipativen Führungsstil geprägt, des Weiteren fließen Elemente einer transformationalen Führung mit ein (vgl. Kap. 3.1 in dieser Arbeit). Ebenso verweist die Aussage „zahnlose Tiger" aber auch auf eine selbstreflexive Kompetenz. Herr Müller sieht darin nicht Machtgefälle oder mangelnde Entscheidungsmöglichkeiten begründet. Er weiß vielmehr, wie er seine Position situationsangemessen nutzen kann, um problembezogene Lösungen herbeizuführen.

„Also die Dinge müssen mit den maßgebenden Gremien - auch mit dem Elternrat, auch mit dem Schülerrat und mit dem Personalrat - in der Schulleitung - in der Steuergruppe alle soweit vorgesprochen sein, dass das, was nachher in der Gesamtkonferenz als dem entscheidenden Gremium läuft, im Grunde genommen 'n glatter Durchlauf ist. (...)ich hab' jetzt acht Gesamtkonferenzen gehabt, und die sind immer genau in $21 / 2$ Zeitstunden zu Ende gewesen, weil sie soweit vorstrukturiert waren, dass wir das durchhalten konnten. Das machen wir dann auch, und da bin ich dann auch froh drüber, da bin ich dann auch mit meiner Arbeit zufrieden, weil das dann so läuft."(141-148)

(Auf Nachfrage, ob es auch Entscheidungen gegen seine Überzeugung gegeben hätte, antwortet der Schulleiter)

„Bis jetzt noch nicht. Aber das könnte natürlich u.U. auch passieren. Da muss man dann auch aufpassen, dass man sich nicht emotional so hineinsteigert <ja>, dass man das als Katastrophe oder als Niederlage oder als Abwertung für sich definiert." (151-153)

Organisationsverständnis

Führungsverhalten 
Herr Müller ist sich bewusst, dass Kooperation im Zusammenhang mit Schulentwicklung notwendig und wichtig ist, denn ohne Gesamtkonferenzbeschlüsse kann der Schulleiter keine Projekte verankern.

Die Bedeutung der Gesamtkonferenz wird akzeptiert und eine notwendige Vorstrukturierung durch alle Gremien gewährleistet, der Schulleiter ist sich seiner Rolle als Leiter dieser Konferenz bewusst und erkennt einerseits den Erfolg einer gelungenen Konferenzleitung an, andererseits beweist er Distanzierungsfähigkeit, wenn er Beschlüsse, die nicht seinen Vorstellungen entsprechen, nicht als emotionale Niederlage bewerten würde. An dieser Stelle wird deutlich, dass er eine emotionale Beteiligung von vornherein antizipiert und reflektiert damit umgeht. Partizipation und das Nutzen organisationaler Strukturen ergänzen sich in diesem Beispiel. Herr Müller greift auf Professionswissen zurück, denn Distanzierungsfähigkeit wird als ein hilfreiches Element im Umgang mit Widersprüchen definiert, des Weiteren orientiert er sein Führungsverhalten an machbaren Konzepten- hier kooperativer Führungsstil- obwohl und dies ist bedeutsam für seine Berufsauffassung sein eigenes Führungsverständnis also von der Organisation gelöstes Verständnis von Führung eher einem transaktionalen Führungsstil entsprechen würde (vgl. Kap. 3.1 in dieser Arbeit).

Die Partizipation des Kollegiums bezieht der Schulleiter nicht nur auf die Beteiligung an Entscheidungen, sondern auch auf die Konfliktlösung im Alltag, einen Konflikt der Unterrichtsverteilung bezeichnet er selbst als „klassischen Konflikt".

„Da hab ich dann also den Englisch-Fachobmann gebeten, dass er sich drum (um Unterrichtseinsatz , Anmerkung d. Verf.) kümmert und mir einen Vorschlag machen möge, wer jetzt diesen Leistungskurs - es war einer - sowieso, dann vertreten könnte. Und der Kollege ist nicht fündig geworden. [P] Ehrlich gesagt, ich hab' niemanden. Ich weiß niemanden. Niemand ist bereit, das zu machen. Und ich kann's auch nicht. Also ich selbst konnte es wirklich nicht, das wusste ich. Und ich habe dann eben die Englisch-Fachschaft hier an den Tisch geholt, habe alle versammelt und hab auch insistiert, dass alle kommen und zwar inklusive der Referendare, inklusive der Fachberater, inklusive auch der Leute, die also offensichtlich gar nicht in Frage dafür kamen, hab" 'nen Runden Tisch gemacht, hab" alle 14 versammelt, und jeder Einzelne musste erklären, wie er persönlich dazu steht, dass dieser - also inwieweit er bereit sei oder aus welchen Gründen er nicht bereit sei, diesen Kurs zu übernehmen. Und dann haben sich in diesem Gespräch alle 14 geäußert, und dann war klar, wer am nächsten dran war, diesen Kurs zu übernehmen. Der hat aber sich nicht bereit erklärt, das zu tun. Ich hab“ dann die Runde beendet. Hab“ gesagt: „Dankeschön“ - jeder möge das sacken lassen. Es war aber klar, wer es machen müsste, und dann ist dieser Kollege, noch bevor er die Order von mir bekommen hat, ist er dann selbst zu mir gekommen und hat gesagt, er sei bereit, das zu tun. [P]“ (261-275)

Der Schulleiter versucht, über das Instrument des Runden Tisches alle zu Beteiligten des Problems zu machen und Lösungen transparent herbei zu führen, er erwartet von seinem Kollegium Lösungskompetenz und macht sie zu Beteiligten. Andererseits kann dies auch als ein Beispiel dafür angesehen werden, dass der Schulleiter Gruppendruck initiiert, um zu Lösungen zu gelangen, die das Durchsetzen von Schulinteressen zur Folge haben. Dies gelingt inm nicht sofort, er ist daher aber auch bereit, seine Professionswissen Position als Schulleiter einzusetzen und dem Kollegen gegebenenfalls „Order“ zu geben. Der Konflikt lässt sich hier lösen, beide Seiten werden
Distanzierungsfähigkeit 
dennoch veranschaulicht: Auf der einen Seite Partizipation und auf der anderen Seite, die Notwendigkeit, unangenehme Entscheidungen allein treffen zu müssen. Der Schulleiter besitzt die notwendige Ruhe und Ausdauer, um auf eine Lösung zu warten, was für eine professionelle Gelassenheit spricht. Es handelt sich um eine konstitutive Antinomie zwischen Nähe und Distanz und Gesamtverantwortung vs. Einzelverantwortung, die Herr Müller ausbalanciert und so zu einer akzeptierten Lösung kommt (vgl. Kap. 3.3 in dieser Arbeit).

„Aber das ist ein hochsensibler Punkt, und ich glaube, dass ich da permanent dranbleiben muss an diesem Verhältnis zum Kollegium. Also dass man immer Gefahr läuft, - äh ja - abzuheben und zu denken, es sei alles in Butter, und in Wirklichkeit gibt es da massive Probleme, die sich irgendwo auftürmen und negative Emotionen gegenüber der Schulleitung. Und von daher ist mir das wichtig, über den Personalrat immer auch zu erfassen, was da an Stimmungen da ist und darauf zu reagieren so konstruktiv, wie's geht."(185-190)

Seine eigene Stellung im Kollegium beschreibt Herr Müller als „gut akzeptiert", trotz dieser Einschätzung ist er sich darüber bewusst, dass dies kein gegebener Status quo ist, sondern das Verhältnis zum Kollegium sowohl positiv wie negativ wandelbar ist und er selbst die Nähe zum Kollegium herstellen muss. In seinem Rollenverständnis ist er aber nicht Gleicher unter Gleichen (,inter pares“), sondern vorgesetzte Führungsperson. Der Schulleiter will bewusst Interaktionsprozesse wahrnehmen, dies deutet daraufhin, dass Herr Müller weiß, dass in seiner Berufspraxis Kommunikation und Interaktionssituation von Bedeutung sind.

Im Verlauf des Interviews wird deutlich, dass Herr Müller durchaus Innovationsvorhaben angeregt und durchgesetzt hat, um seine Schule in der Stadt zu positionieren. Interessanterweise schätzt sich der Schulleiter selbst als "nicht ganz so innovativer Schulleiter" ein und zieht personelle Konsequenzen daraus.

„Auf der anderen Seite bin ich nicht so 'n ganz innovativer Schulleiter, und hab dann auch bei der Neubesetzung einer Stelle in der Koordinatorenrunde drauf geachtet, dass so ein ganz dynamischer Kollege dann auch mit reingekommen ist, der mir dann auch noch mal wieder so einen Anschub gibt.

$[P]$ Zum Beispiel was neue Unterrichtsmethoden angeht, Stationen lernen, offenen Unterricht, äh Projektarbeit, da bin ich - äh - da gibt es sicher Leute, die viel weiter sind als ich. Und, und äh, da brauche ich in der Leitungsrunde dann auch jemanden, der von der Seite her mir - mir und der Schulleitung oder der Schule Impulse gibt. <hm> Und so jemand hab ich auch gefunden, der also jetzt mit am Tisch sitzt, und der ist dann auch zusätzlich mit mir in der Steuergruppe und arbeitet dort mit."( 203-213)

Dieses Zitat zeigt, dass Herr Müller seine eigenen Kompetenzen, aber eben auch Defizite reflektiert und im Sinne der Schulentwicklung, hier konkret Unterrichtsentwicklung, andere kompetente Leute in der erweiterten Schulleitung mitarbeiten lässt, um Ausgleich zu schaffen. Dieses Vorgehen von Herrn Müller verweist erneut auf die Notwendigkeit, unterschiedliche Orientierungen des eigenen Berufes zu reflektieren und zu akzeptieren, dass auch im Leitungsteam nicht alle Orientierungen von einer Person vertreten oder gelebt werden können; daher ist es unter die-
Führungsverständnis

Kritische Einschätzung sich selbst gegenüber 
sem Aspekt wichtig, die ergänzende oder ausgleichende Besetzung von Teams zu gestalten. ${ }^{231}$

Aufgrund einer kritischen Reflexion und einer sachlichen Analyse dieser Selbsteinschätzung ist Herr Müller in der Lage, wichtige Führungsaufgaben zu delegieren und ernst gemeinte Kooperation zu praktizieren. Die Berufsauffassung ist von Authentizität geprägt, dies spricht für ein stabiles berufliches Selbstkonzept (vgl. Bauer, S. 76 und Schütze, S. 69 in dieser Arbeit).

Im weiteren Verlauf des Interviews wird eine differenzierte Haltung gegenüber Innovationen deutlich, die Herr Müller hinsichtlich seiner zukünftigen Zielsetzungen formuliert.

\begin{abstract}
„Ja, ich würd' mir wünschen, dass dieser Innovationsdruck, den wir uns jetzt selbst auch gesetzt haben und der sich jetzt noch überlappt mit der Strukturreform, die aus Hannover kommt, und beides zusammen also bringt 'ne Menge von enormen Stress eigentlich, von enormen Arbeitsbelastungen weit über die 40-Stunden-Woche hinaus. Ich würde mir wünschen, dass sich die Schulstruktur stabilisiert und infolgedessen etwas mehr Ruhe eintritt und wir dann den Innovationsdruck dann auch so 'n bisschen rausnehmen können, äh, so dass ich meine Schule mit etwas mehr Ruhe führen kann dann. Dabei muss ich sicherlich auch noch mehr delegieren lernen, ähm, aber ansonsten habe ich kein weiteres Ziel. Nee. Nee, wenn es denn in so ein stabileres Fahrwasser geriete und ich dann auch etwas mehr Freizeit hätte, dann wäre ich schon zufrieden."(232-241)
\end{abstract}

Das Problem einer sowohl personellen Überforderung als auch den Konflikt zwischen Bewahren und Erneuern spricht Herr Müller hier an. Auch die Literatur zur Schulentwicklung verweist inzwischen auf einen kaum zu bewältigenden Innovationsdruck an Schulen. „Sättigungsgrenzen“ sind vor allen Dingen dann auszumachen, wenn viele aufwändige Initiativen gleichzeitig oder in kurzem Rhythmus aufeinander folgen. Wenn von außen Reformmaßnahmen festgesetzt werden und gleichzeitig Ressourcen gekürzt werden, sind Zielsetzungen und Zielerreichung nicht mehr unbedingt einsichtig dem Kollegium zu vermitteln. Herr Müller verknüpft für die Analyse dieser Situation Theoriewissen der Organisation mit Praxiswissen, welches er durch Erfahrung mit Schulentwicklungsprozessen gesammelt hat, d.h., er weiß, welche Aufgaben Schulen anzugehen haben und er will auch Veränderungen herbeiführen und dennoch hat sich durch seine Berufserfahrung eine Einschätzung herausgebildet, die die Grenzen von Innovationen und die Notwendigkeit zur Stabilität berücksichtigt (vgl. Kap. 3.3 in dieser Arbeit).

Von Bedeutung erscheint der Hinweis auf die eigene Rolle. Dass Herr Müller derjenige sein muss, der mehr Ruhe und Stabilität in die Schule bringt, deutet auf eine führungsbetonte bzw. führungsbewusste Haltung hin, denn er muss den Überblick beweisen und Abwägen zwischen Erneuerung und Bewahrung, was als ein Führungsdilemmata angesehen wird.

Herr Müller äußert sich selbstkritisch zu seiner Führungskompetenz und schätzt das eigene Belastungsempfinden ein. Er betrachtet Delegation als Entlastungsmoment seiner eigenen Berufsausübung und sieht, dass er
Organisationswissen

Problembezogene Bewältigung von Belastung

${ }^{231}$ Vgl. Wunderer 2003, 541. 
bisher zu wenig delegiert hat, dies will er ändern, er strebt eine problembezogene Bewältigung von Stress an.

Diese selbstreflexive Haltung des Schulleiters wird im Zusammenhang mit Qualifikationswünschen erneut deutlich.

„Es würde mir gut tun, wenn ich so eine regelmäßige Gesprächsrunde hätte mit anderen Schulleitern. Das könnten dann schlecht die aus derselben Stadt sein, äh, weil man zu denen ja doch in 'ner gewissen Konkurrenz steht (...) Und insofern wäre es gut, mal einen Kreis zu haben, wo man einmal im Monat oder vielleicht sogar 14- tägig sich trifft, um so Erfahrungen auszutauschen sowohl auf der praktischen Ebene, als auch auf der Ebene der Führungsarbeit. Und äh so eine Beziehung eigentlich - so eine Gruppe habe ich nicht, so 'ne Gruppe würde mir sicher Freude bereiten und würde mir auch gut tun. Ich würde mich auch sofort so 'ner Gruppe anschließen." (334-343) (Auf Nachfrage, was er unter Führungsarbeit verstehen würde, ergänzt der Schulleiter) „Ja, es gibt teilweise sehr, sehr stressige Konflikte. Ich hab' Ihnen jetzt welche beschrieben $\langle$ hm>, wo ich dann vielleicht auch so 'n bisschen - äh - was man dann gerne erzählen möchte, wo ich dann glaubte, die Dinge dann gut auf den Weg gebracht zu haben. <ja> Aber es gibt wirklich Konflikte, die so hart sind, dass es gut ist, jemanden Kompetenten zu haben, mit dem man sich unmittelbar austauschen kann." (346-350)

An dieser Stelle des Interviews wird offenkundig, dass sich der Befragte zu jeder Zeit der Interviewsituation bewusst ist und dass er auf einer Metaebene darüber reflektiert, was er erzählen bzw. preisgeben darf, ohne dem Ansehen der Schule oder seiner eigenen Person zu schaden. Es gibt darüber hinaus Begebenheiten und Probleme, die den Schulleiter beschäftigen und evtl. auch belasten, für diese Ebene sucht er kompetente Unterstützung, ist also auch hier bereit, Hilfe und Unterstützung in einem geschützten Rahmen anzunehmen, bewusst ist ihm die Konkurrenzsituation der Schulen untereinander. Die Berufsauffassung ist, wie oben herausgearbeitet worden ist, von einem hohen Verantwortungsgefühl geprägt, es liegt eine hohe Identifizierung mit berufsrelevanten Werten vor, dennoch beweist Herr Müller die notwendige Distanzierungsfähigkeit, die als Element des Professionswissens gilt, diese schützt davor, dass der Beruf zur Belastung wird (vgl. Kap. 3.3)

Auf die Nachfrage, ob er dafür ein Beispiel nennen möchte, erzählt der Schulleiter von seiner starken Belastung durch den oben bereits erwähnten Konflikt.

„Ähm, - doch - [P] Ja, z.B. hat ein - was war das denn neulich? - äh ein Kollege, das war im Französisch-Fachbereich -, ich hab' den Konflikt verdrängt, so dass ich ihn gar nicht mehr erinnere. Na ja, also ich kann aber auch diese Spanisch-Sache nennen noch mal. Vielleicht fällt mir da der andere Konflikt dann auch noch ein.

In diesem Spanisch-Konflikt da war ich teilweise wirklich verzweifelt, weil ich das Gefühl hatte, also ich werde hier vorgeführt. Ich hatte Ihnen das ja beschrieben mit diesem äh...mit diesem Widerspruch: „Sie haben nicht die Lehrkräfte, also kriegen Sie das Fach nicht. Und Sie kriegen die Lehrkräfte nicht, weil Sie das Fach nicht haben", das war so was, da hab' ich Beratungsbedarf auch gebraucht und hab" den auch eingeholt. Ich hab" mich dann mit einem Freund beraten, der ganz woanders in Niedersachsen arbeitet, mehrfach am Telefon, wie ich taktisch vorgehe, welche Politiker ich einschalte, ob ich die Politiker einschalte oder ob ich andere Leute anspreche, die dann in ihrem Namen denn ich kann ja auch schlecht gegen meinen Dezernenten in der Bezirksregierung ope-
Identifikation mit der Rolle als Schulleiter 
rieren, aber andere können das tun. Der Schulelternratsvorsitzende kann das tun usw., also da ist es wirklich sehr gut und wichtig, sich mit anderen Leuten austauschen und beratschlagen zu können - mit kompetenten Leuten. Also es ist nicht so, dass ich in solchen Konflikten immer schon wüsste, wie der nächste Schritt aussieht. Und von daher suche ich mir auch fortlaufend Rat. Also es gibt auch noch in meiner Leitungsrunde jemanden, der ist erfahrener als ich, und deshalb spreche ich den oft an, wenn ich nicht weiter weiß, um Anregungen mir zu holen und das dann wieder zu reflektieren. Und - jawenn man nun so 'ne Gruppe hätte, könnte man das eigentlich selbstverständlicher und systematischer machen. (352-377)

Die unterschiedlichen Handlungsebenen des Schulleiters werden erneut benannt. Das Hierarchieverhältnis zwischen ihm als Schulleiter und der Schulbehörde ist linear, d.h., Herr Müller selbst ist weisungsgebunden, er muss Entscheidungen der Zuweisung von Lehrkräften, der Genehmigung von Fächern akzeptieren und umsetzen, es sei denn, es gelingt ihm, sich in einem komplizierten Aushandlungsprozess durchzusetzen. Dieses Widerspruchsverhältnis wird besonders dort deutlich, wo es zur Verknappung der Ressourcen kommt.

Auf der Ebene Schulleiter und Kollegium ist die Hierarchiestruktur komplexer, er ist zwar Vorgesetzter der Lehrkräfte, er ist aber nur sehr bedingt weisungsberechtigt. Hinzu kommt, dass die Kolleginnen und Kollegen die gleiche akademische Qualifikation haben. Die Entscheidungs- und Führungsstruktur in der Schule lässt wenig Eindeutigkeit zu und macht ein geschicktes Ausbalancieren nötig.

Dass Herr Müller ebenso auf ein Team angewiesen ist wie andere Kolleginnen und Kollegen, wird durch die Art deutlich, wie er seine Position und das Schulleitungsteam insgesamt einschätzt.

„ICh habe einen Stellvertreter und vier Koordinatoren, das ist ein angenehmes Arbeiten miteinander. Also wir lachen auch miteinander und äh - ja - karikieren unsere eigene Situation manchmal miteinander, und ich hab das Gefühl, dass es so von der menschlichen Seite her sehr angenehm ist miteinander, und äh auch dass die unterschiedlichen Rollen und so ganz gut verteilt sind. Das heißt nicht, dass es da nicht auch mal irgendwelche Konflikte gäbe, aber wenn ich diese Runde in der Konstellation nicht hätte, dann wäre die Arbeit für mich sehr viel schwieriger. Also das ist auch so ein psychischer Stabilitätsanker diese Leitungsrunde für mich. - Ja. Gut."(378-385)

Für Herrn Müller sind das eigene Wohlfühlen und die Akzeptanz im Leitungsteam von Bedeutung, dies bezieht er nicht nur auf das Gelingen von Leitungsaufgaben, sondern ganz besonders deutlich auf die psychische Ebene „psychischer Stabilitätsfaktor“. Es zeigt sich, dass der Schulleiter seine eigene Rolle nicht überschätzt und auf das gegenseitige Angewiesen- Sein eingeht, für inn stellt eine gelungene Zusammenarbeit in einem auch menschlich angenehmen Klima eine Bedingung für die eigene Stabilität dar. Die Berufsauffassung orientiert sich an kooperativen Arbeitsformen und stabilen sozialen Kontakten, die Identifikation mit der Rolle als Schulleiter stellt für Herrn Müller kein Hindernis gegenüber einer Beziehungsorientierung dar.

An einer Stelle im Interview fasst Herr Müller seine Hauptaufgaben als Schulleiter wie folgt zusammen:
Reflexionsvermögen

Problembezogene Bewältigung von Belastung

Stabilisierung durch Arbeitsbündnisse 
„Die Hauptaufgaben als Schulleiter- also ich muss da Motivation schaffen bei allen Beteiligten, denke ich, das ist 'ne ganz - und Identifikation mit dieser Schule, mit diesem Arbeitsplatz, und ich muss entscheiden, ich muss strukturieren, dass das System funktioniert, ich muss also mithelfen, dass das gesamte organisatorische, technische Gefüge funktioniert. Und ich muss sicherlich in meiner ganzen Haltung im Umgang mit den Menschen auch Vorbild sein.(246-251)

In diesem Zitat sind mehrere Ebenen benannt, die durch Beispiele im Interview untermauert werden. Zunächst verweist Herr Müller auf seine Rolle als motivierende Identifikationsfigur, die bei seinem Kollegium die Identifikation mit der Schule als Arbeitsplatz fördern will, eine starke Mitarbeiterorientierung wird erkennbar.

Die subjektive Bedeutsamkeit der Arbeit als Schulleiter wird durch den letzten Satz verständlich, er selbst will „Vorbild“ sein. Vorbild kann im Sinne der allgemeinen Definition verstanden werden, d.h. Vorbild als Modellperson, die in ihrem konkreten Lebensvollzug so sehr zu überzeugen mag, dass andere sich an ihr orientieren. Dieser Akt der Identifikation wird aus psychologischer Sicht als Vorgang definiert, „durch den das Subjekt einen Aspekt, eine Eigenschaft, ein Attribut eines anderen assimiliert und sich vollständig oder teilweise nach dem Vorbild des anderen umwandelt". 232

Seine Haltung im Umgang mit Menschen wird durch unterschiedliche Interviewaussagen explizierbar, er möchte seine Schule voran bringen und dabei die verschiedenen Akteure im Blick haben. Herr Müller ist sich bewusst, dass es in der Organisation Schule immer Konflikte auf unterschiedlichen Ebenen geben wird, er ist aber bereit, diese Konflikte offensiv anzugehen, ohne einen Teil des Gefüges aus den Augen zu verlieren. Der Schulleiter wirkt hier als positivistisch-humanistischer Führungstyp. Sein Führungsstil ist auch an dieser Stelle an einem kooperativ-partizipativen Führungsverständnis orientiert, zudem wird ein wichtiger Aspekt der transformationalen Führung angesprochen, Herr Müller möchte als Identifikationsperson für Leistung und Erfolg angesehen werden (vgl. S. 44 in dieser Arbeit).

In den Äußerungen von Herrn Müller spiegelt sich ein Führungsverständnis, welches von einer Verantwortlichkeit im Bereich „Entscheidungen treffen" geprägt ist, das System seiner Einzelschule soll so strukturiert werden, dass es bestmöglich funktioniert. Herr Müller greift auf einen mehrperspektivischen Handlungsrahmen zurück.

Die zu Beginn des Interviews geäußerte metaphorische Beschreibung seines Berufes „Tanz auf dem Vulkan“(12) lässt sich abschließend so erklären, dass der Schulleiter den gefährlichen Stress durchaus positiv nutzen kann, er hat die Kompetenz des Tänzers und kennt die Eigenheiten des Vulkans. „Auf dem Vulkan“, der Schulleiter beweist Überblick und verfügt über unterschiedliche problembezogene Bewältigungsstrategien.

Anhand dieser Fallbeschreibung lassen sich folgende wichtige Elemente der Berufsauffassung von Herrn Müller zeigen. Herr Müller identifiziert sich positiv mit der Rolle des Schulleiters. Dies zeigt sich in einer realistischen Einschätzung des aus seiner Sicht Machbaren und einer gleichzeitig indi-
Kooperative

Arbeitsformen

Identifikation mit Rolle als Schulleiter

\footnotetext{
${ }^{232}$ Vgl. Laplanche/ Pontalis 1972, 219.
} 
viduell hohen Verausgabungsbereitschaft. Herr Müller kann eigene Kompetenzen und persönlichen Schwächen reflektieren und im Gespräch formulieren.

In seiner Berufsauffassung greift Herr Müller auf ein Organisationsverständnis zurück, welches die interne Strukturlogik der Organisation Schule einschätzt und zugleich die Grenzen des Agierens auf der Ebene der Schulbehörde wahrnimmt. Dadurch gelingt es ihm, die Gestaltungsmöglichkeiten innerhalb der Organisation zu nutzen, z.B. fördernde Arbeitsstrukturen aufzubauen. Herr Müller orientiert sich innerhalb seines Agierens in der Schule an einer mehrperspektivischen Sichtweise der Organisation, denn er reflektiert unterschiedliche Handlungsebenen und begegnet widersprüchlichen Anforderungen bzw. paradoxen Situationen mit einem Ausbalancieren auf unterschiedlichen Organisationsebenen, z.B. initiiert er politischen Druck durch Öffentlichkeitsarbeit.

Herr Müller greift auf führungsrelevantes Wissen in unterschiedlichen Formen zurück, so praktiziert er einen kooperativ-partizipativen Führungsstil dort, wo es um die Entwicklung neuer Schulkonzepte geht, z.B. Methodencurriculum. Herr Müller orientiert sich in seiner Berufsauffassung in seinen kognitiven Anteilen an einem transaktionalen Führungsverständnis, er weiß aber - und dies reflektiert er- dass diese Führungskonzeption im Rahmen der Organisation Schule nicht durchzusetzen ist und zeigt in seinen handlungsleitenden Anteilen Bereitschaft Konflikte so weit anzugehen, wie es in seinem Handlungsrahmen möglich ist. Er praktiziert eine zielorientierte Führung und bezieht sich in seiner Handlungskonzeption auf die Schule als Gesamtkomplex, so kann Herr Müller zwischen Individualebene und Systemebene unterscheiden.

Zusammenfassend kann Herr Müller in seiner Berufsauffassung als konstruktiver Jongleur umschrieben werden, weil er die Bedingungen der Schule nutzt und aktiv gestaltend in die Prozesse der Schule eingreift. Dabei balanciert er geschickt zwischen unterschiedlichen Interessengruppen und verfolgt dennoch seine Zielvorstellungen im Sinne der Schule als Ganzes.

\subsection{2 „Resignierter Einsamer" Herr Bach (SL 11)}

Herr Bach war 17 Jahre Lehrer, dann 4 Jahre als Dezernent tätig und leitet seit 5 Jahren das kleine (> 600 Schülerinnen und Schüler) Gymnasium in einer niedersächsischen Großstadt. $\mathrm{Er}$ hat die Schule in einer sehr schwierigen Situation übernommen, die Anmeldezahlen für das neue Schuljahr waren auf 36 zurückgegangen, inzwischen hat er 162 Anmeldungen. Der Schulleiter fand ein „überaltertes“ Kollegium vor. Die Schulleitung besteht neben dem Schulleiter aus einem Stellvertreter und 4 Koordinatoren. Das Gespräch fand an einem Freitag nach Schulschluss im Dienstzimmer des Schulleiters statt.

Angesichts dieser Ausgangssituation verwundert es nicht, dass Herr Bach gleich zu Beginn des Interviews nicht auf die Eingangsfrage, sondern zunächst auf die für sein Schulleitungshandeln entscheidende Situation der Schule zu Beginn seiner Tätigkeit eingeht. 
„Ja, das ist einmal der ganze Bereich „Bildungsangebote der Schule“ eben in der Bildungslandschaft, wie positionieren wir uns im Wettbewerb mit den anderen Gymnasien in (der Stadt Anm. d. Verf.) Hier war speziell die Situation: 98 bin ich Schulleiter geworden, 98 war die Schule auf einem Tiefpunkt, was die Anmeldezahl anbetraf: 39. Und jetzt hatten wir 162 (...) Also ich musste die Schule neu positionieren in der Bildungslandschaft. Ich musste Schüler besorgen, Schüler werben, habe eben mit einem attraktiven Angebot (...) (geworben Anm. Verf.). Und das hat sozusagen guten Anklang gefunden bei der Elternschaft und bei der Schülerschaft zu den genannten Zahlen. [P] Ja, da bin ich sicherlich gefordert bei der Frage: Welche Lehrkräfte kommen? Durch die neuen Schüler haben wir viele neue Lehrkräfte bekommen können, also Personalauswahl ist also ganz klar meine Aufgabe. Wie war Ihre Frage noch?" (5 19)

Herr Bach verweist zu Beginn des Interviews auf die besonders schwierige Lage der Schule, die „Positionierung“ und die Sicherung des Schulstandortes sieht er als seine Haupttätigkeit an. Ein „attraktives“ Bildungsangebot für Schülerinnen und Schüler und deren Eltern zu schaffen, war ein Hauptziel bei Dienstantritt und ein Kriterium für Erfolg sind die gestiegenen Schülerzahlen. Die Auseinandersetzung mit der schulspezifischen Situation macht es notwendig, strategische Maßnahmen sowohl hinsichtlich personeller Ressourcen als auch insbesondere im Bereich pädagogischer Schulentwicklung zu ergreifen. Herr Bach übernimmt Verantwortung für die pädagogische Profilierung der Schule - dies ist bedeutsam für seine Berufsauffassung als Schulleiter - denn der Aspekt der pädagogischen Schulentwicklung und die Außenorientierung im Sinne der Schülerrekrutierung treten in den Vordergrund seiner Orientierung, dabei erhält der Zuspruch der Interessengruppen Schüler/ Schülerinnen und Eltern besondere Bedeutung.

„Durch die neuen Schüler haben wir viele neue Lehrkräfte bekommen können, also Personalauswahl ist also ganz klar meine Aufgabe. Ja, Ja. Dann das Führen im Kollegium. Da war auch viel zu tun, ist viel zu tun, also da haben wir die pädagogische Qualität der Schule entwickelt, und das ist meine Hauptaufgabe. Das ist ja meine eigentliche Hauptaufgabe. Das andere ist jetzt, in der Bildungslandschaft uns zu positionieren und gute Lehrer zu gewinnen. Und die pädagogische Qualität der Schule zu entwickeln, darin sehe ich meine eigentliche Hauptaufgabe, und das ist auch also auch - ohne wenn und aberdie größte Schwierigkeit. Was meine ich damit? Ja, was meine damit? Ich meine damit, [überlegt] kann ich so reden, wie ich denke oder (...) wie allgemeine Begriffe so sind.

Ich gehe da immer davon aus, ja, jetzt komme ich ganz gut in Schwung, wenn ich das jetzt, ohne da Begriffe zu benutzen, die jetzt in der Öffentlichkeit so in sind (P) Ich meine, ich hab' da auch meine eigene Terminologie. Ne? Also mein Hauptanliegen wäre jetzt da, die - ja, ich kann's nicht anders ausdrücken, die Selbstreflexionsfähigkeit von Lehrkräften zu erhöhen. Damit meine ich die Wahrnehmungsfähigkeit erhöhen: Augen und Ohren auf. Damit meine ich die Empathie: hinversetzen in die Schüler, die Perspektive der Schüler wahrnehmen, Perspektivenwechsel - und zwar wirklich, nicht nur im Unterricht, im Unterricht auch, aber auch zu Hause: „Wie nehmen Schüler meinen Unterricht wahr? Wie nehmen Sie mich wahr? Was möchten Schüler im Unterricht? Warum verhalten sie sich daneben oder warum verhalten sie sich gut?" Also Wahrnehmung, Hineinversetzen und dann eben -möglichst gelassen - schauen: „Wie ist der Prozess? Wie kann ich den Prozess fördern? Also den kognitiven Lernprozess aber auch den sozialen Lernprozess fördern. Interaktion, Intergruppe, Zusammenhalt in der Gruppe, Gruppenkonflikt, Klärung in der Gruppe, also all diese ganzen Aspekte, die auf der Beziehungsebene angesiedelt sind, halte ich auch für mindestens so wichtig wie die inhaltlichen, kognitiven Aspekte des Lernprozesses. (21-42) 
Herr Bach nennt drei Aufgabenbereiche seines Handelns: die „Personalauswahl“, das „Führen im Kollegium“ und die Entwicklung der „pädagogische Qualität der Schule“, die pädagogische Qualitätsentwicklung sondiert er als seine Hauptaufgabe, dies wiederholt er drei Mal, gleichzeitig erlebt er diese Aufgabe als größte „Schwierigkeit“.

Der erste Teil seiner Äußerungen zeigt durch die Wortwahl einen Bezug zu den allgemein gängigen Schlagwörtern, die im Kontext der Beschreibung von Schulleitungskompetenz benutzt werden. Von dieser „Terminologie" möchte sich Herr Bach abgrenzen und seine eigenen Begrifflichkeiten einbringen. Die Zwischenbemerkung „kann ich so reden wie ich denke“ könnte ein Hinweis darauf sein, dass er zuvor sehr kontrolliert gesprochen hat und nun, wie er ja auch selbst beschreibt, "ganz gut in Fahrt kommt".

Die nachfolgende Interviewpassage verweist einerseits auf seine Ziele als Schulleiter, denn er will aus seiner Funktion heraus die Selbstreflexionsfähigkeit der Lehrkräfte fördern, um mehr Schülerorientierung im Bewusstsein des Kollegiums zu initiieren - andererseits und dies ist bedeutsam für die Berufsauffassung von Herrn Bach- beziehen sich seine Ausführungen auf Schülerkategorien, Herr Bach orientiert sich in seiner Handlungskonzeption auf der Ebene Schüler-Lehrerinteraktion und betont dabei die Beziehungsebene der Lernprozesse. Diese Zielrichtung gibt einen Hinweis auf seinen eigenen hohen pädagogischen Berufsethos. Neben der Rettung der Schule geht es inm vornehmlich um Lernprozesse auf der Ebene Lehrkraft -Schüler.

„Also die Isolation der Lehrkraft halte ich für ein zentrales Problem auch, und das ist auch die Ursache für manche Berufsmacken - déformation professionelle. Die Isolation am Arbeitsplatz generell und hier in der -Schule speziell - das Kollegium war unheimlich überaltert und war, ist, weiß ich auch nicht genau, wo wir da stehen, sehr individualistisch ausgerichtet. Und es für mich, wie ich denn praktisch vor einer Klasse stehe und diese ganzen Punkte, die ich jetzt genannt habe, dann auf den Unterricht bezogen jetzt - diese Punkte und: wie kann's weitergehen? Was sind so sinnvolle Anknüpfungspunkte? Wo will ich hin? Genau die Probleme stellen sich mir natürlich in der Führung des Kollegiums, und das ist eine ungeheure Herausforderung, ist auch 'ne Schwierigkeit, zu wissen, (...)wo stehen die? Was beschäftigt sie? Wo kann ich sie abholen? Also bei den verbindlichen Arbeitstagen habe ich im Grunde an dem Thema wochenlang herumassoziiert, richtig rumgedacht am Schreibtisch und dann mir einzelne Leute vor Augen geführt und einzelne Gruppe vor Augen geführt. Wo stehen die, und was könnte die interessieren? Wie kann ich sie mitnehmen?" (50-60)

Herr Bach analysiert Gründe für das Scheitern der Unterrichtsentwicklung und sieht die fehlende Teamorientierung der Lehrkräfte als ein „zentrales Problem“ an. Die „Führung des Kollegiums“ stellt für Herrn Bach eine „ungeheure Herausforderung“ dar. Hierdurch wird erkennbar, dass der Schulleiter nicht auf gewohnte Handlungsmuster, z.B. auf die des Lehrers, zurückgreifen kann und es ihm schwer fällt, die Perspektive des Kollegiums aus der Sicht des Schulleiters einzunehmen, die Äußerung „wie kann ich sie mitnehmen? "- kann ein Hinweis darauf sein, dass er das Kollegium als Schülergruppe betrachtet. Die Aneinanderreihung von unbeantworteten Fragen gibt Anhaltspunkte dafür, dass er genau diesen Punkt, wo er nämlich ansetzen soll mit seinen pädagogischen Verbesserungsprozessen, nicht kennt. Herrn Bach fehlen Handlungsstrategien, die es ihm ermögli-

Einschätzung von Problemen 
chen, gemeinsam mit dem Kollegium die Schule weiter zu entwickeln. Führung deutet Herr Bach als Ausübung von Einfluss auf die praktizierte Unterrichtsqualität des Kollegiums. Wie der folgende Interviewauszug zeigt, klassifiziert Herr Bach diesen Schulentwicklungsprozess innerhalb der Schule als gescheitert, z.B. fehlende Teamstrukturen, es gelingt inm aber nicht, sich selbst innerhalb dieser Prozesse als Initiator der Veränderung zu erleben.

„Unterrichtsentwicklung, Kollegiumsentwicklung. Ja, Unterrichtsentwicklung selber, muss ich sagen, ist eigentlich auch natürlich meine Aufgabe, aber ich versuche im Grunde über Kollegiumsentwicklung in dem angesprochenen Sinne auch, dass das dann ins Kollegium ausstrahlt. Das Kollegium - ist nach meiner Wahrnehmung vormodern gewählt. Also die sind irgendwo - entweder schon von Anfang an sehr unbeweglich gewesen, [P] eine Gruppe, glaube ich, war von Anfang an sehr unbeweglich, also schon mit Berufseintritt. Eine andere Gruppe war - ist auf diesem irgendwie nach-antiautoritärem Status, ich weiß nicht, 82 stehen geblieben oder so, also sind irgendwie in diesem antiautoritärem Affekt stehen geblieben. Nur wenige sind im Grunde weitergegangen. Ich finde, da - also ich gehöre zur antiautoritären Zeit ohne wenn und aber, aber was ich so mit Grenzsetzung anspreche oder mit Führung von Unterricht: Ich mache den Unterricht. Ich gestalte den Unterricht. Ich setze die Grenzen - oder auch inhaltliche Ansprüche stellen, habe ich immer gemacht, aber da sind manche auch irgendwie so in so 'ner Beliebigkeit stecken geblieben. Also da - das finde ich eben schade, dass da also auch - ja, das meine ich mit „vormodern“. Also es gab immer 'ne ganz alte Pädagogik, mehr so 'ne KasernenPädagogik einerseits, andererseits ganz große Beliebigkeit und auch viel Gleichgültigkeit. Na gut. Ich hab also wirklich gewartet, gewartet, gewartet, immer mal wieder geschubst oder gestoßen und gestupst und auch mal ein paar verbindliche Arbeitstage auch, aber es auch heute - bis heute - nicht gelungen, das Kollegium wirklich zu gewinnen für Unterrichtsentwicklung, Schulentwicklung. Da muss man sagen. Also wenn ich so für mich hier alleine sitze oder am Schreibtisch, das ist nicht gelungen. "(91-110)

Herr Bach sieht die Verknüpfung zwischen Unterrichtsentwicklung und Kollegiumsentwicklung, schätzt seinen Erfolg als Schulleiter im Bereich Kollegiumsentwicklung aber als gering ein. Der Wunsch, dass das Kollegium guten Unterricht macht, ist stark ausgeprägt, auch hier wird der pädagogische Anspruch des Schulleiters deutlich. Die geringe Selbstwirksamkeitsüberzeugung könnte das Resultat bereits gemachter negativer Erfahrungen im Kollegium sein. Seine Berufsauffassung, die sich stark auf die pädagogische Schüler-Lehrer-Interaktion bezieht, scheint nicht vereinbar zu sein mit der Haltung des vorgefundenen Kollegiums. Herr Bach beschreibt unterschiedliche Kollegiumsgruppen, eine Gruppe erscheint inm als „unbeweglich“, eine andere sei „stehen geblieben“. Herr Bach nennt "ganze alte Pädagogik, so' ne Kasernen-Pädagogik“ und „große Beliebigkeit" als gegensätzliche Orientierungspole. Die Bewertungen bzw. Einschätzungen gegenüber seinem Kollegium sind kritisch und von Misserfolg geprägt. Erneut wird an diesem Interviewausschnitt deutlich, dass Herr Bach seine Zielvorstellungen bisher nicht verwirklichen konnte. Ein Erklärungsansatz bietet der nächste Interviewausschnitt.

„ICh bin ein engagierter, das merken Sie, ich bin ein ganz engagierter Lehrer immer schon gewesen. Ich habe immer schon Schulentwicklung betrieben - auch als Lehrer. Aber es war für mich immer schon ein Herzensanliegen, guten Unterricht zu machen und Schule so zu gestalten, dass Schüler lernen und sich wohl fühlen. Und das ist vielleicht allen nicht recht - allen. Und das habe ich schmerzhaft erfahren müssen. Das ist mir 
überhaupt, - dass man anders sein kann, das fällt mir unheimlich schwer, also da habe ich schon damit meine Empathieprobleme. Also und die kriege ich auch irgendwie nicht. Das weiß ich auch nicht." (192-200)

In den Äußerungen von Herrn Bach zu seiner Rolle als „engagierten“ Lehrers für den es ein „Herzensanliegen“ ist guten Unterricht zu machen, spiegelt sich eine starke Identifikation mit dem Beruf des Lehrers, was ein Hinweis darauf sein kann, dass Herr Bach den Berufswechsel zum Schulleiter nicht vollzogen hat. Des Weiteren geht Herr Bach davon aus, dass diese pädagogische Haltung nicht von allen Lehrerinnen und Lehrern seines Kollegiums anerkannt wird und dies hat er schon „schmerzhaft erfahren" - seine emotionale Verarbeitung negativer Berufserfahrungen sind ein bedeutsames Element seiner Berufsauffassung und kann als ein Hinweis auf sein starkes Belastungsempfinden gedeutet werden.

Die Wortwahl "Empathieprobleme“ in diesem und insgesamt auch in den in folgenden Interviewpassagen ist aus dem psychologisch- fast therapeutischen- Bereich entnommen. Je stärker Herr Bach auf Konflikte im Alltagserleben eingeht, desto deutlicher spiegelt sich diese psychologische Verarbeitungsform in seiner sprachlichen Ausrucksweise (vgl. besonders S. 143).

Es fällt Herrn Bach schwer, Empathie gegenüber Kolleginnen und Kollegen zu entwickeln, die die diese Unterrichtsauffassung aus seinem Erleben heraus nicht teilen. In seiner Selbsteinschätzung hinsichtlich seiner eigenen Kompetenz als Lehrer orientiert sich Herr Bach an einem idealen Bild der pädagogischen Praxis und erlebt eine Diskrepanz zu der real vorgefundenen Situation. Die hohe biographische Identifikation des Schulleiters mit seiner Identität als Lehrer und eine nur zu geringe Identifizierung in der zweiten Rolle als Schulleiter, die die erste nicht ablöst, sondern ergänzen müsste, stehen sich gegenüber.

Die fehlende Übernahme der Rolle als Schulleiter und die mangelnde wechselseitige Vertrauensbasis blockieren eine gelingende Interaktion, die eine notwendige Voraussetzung für eine Problemlösung wäre.

Herrn Bach gelingt es nicht, Kolleginnen und Kollegen als einzelne Personen mit je individuellen Arbeitsstilen und Interessen zu akzeptieren. Seine eigene Festlegung auf konkrete pädagogische Ziele auf der Ebene des Unterrichts scheinen ein Teil seines Problems zu sein, denn Herr Bach müsste sich konkret in den Handlungsspielraum der Lehrkräfte einmischen, wenn er sein Ziel ernsthaft verfolgen würde. Hier stößt aber seine Berufsaufassung auf die Grenzen der Organisation Schule, denn es wäre für Herrn Bach einerseits zeitökonomisch nicht möglich, alle Lehrkräfte regelmäßig im Unterricht zu hospitieren, andererseits hätte er auch wenig Handlungsmöglichkeiten, wenn er Unterricht sehen würde, der seinen Ansprüchen nicht genügen würde, wie auch die Beispiele aus dem Interview mit Herrn Müller zeigen. Herr Bach steht vor der Frage der Balance von eigenen Überzeugungen und Gegebenheiten der Organisation Schule, dies wird in der Professionsdebatte als bedeutsames Belastungsmoment in pädagogischen Führungspositionen angesehen - Herr Bach leidet unter diesem zentralen Grundkonflikt seiner Berufsauffassung.
Fehlende Identifikation mit Rolle als Schulleiter

Fehlender Ausgleich widersprüchlicher Beziehungsstrukturen 
Herr Bach schildert im weiteren Verlauf ein Konfliktbeispiel, in dem ein Lehrer sich weigert Klassenlehrer zu werden.

„Nun auch gerade gestern z.B. eine idiotischste Stelle, wo, jetzt will der ((Kollege) Anm. d. Verf.) auf einmal kein Klassenlehrer werden, keine Klassenfahrt mehr machen. Einer, der 48 ist und kinderlieb. Ist mir unvorstellbar, wie einer mit 48, der ist aber schon so, der weiß: „Wenn ich Klassenlehrer werde, da hängt auch die Klassenfahrt dran. Und auf einmal kriege ich Gäste, und jetzt sind die Ferien aber bald da", das ist in diesem Jahr äuBerst kompliziert, weil wir drei Klassen kriegen. Da muss quasi jeder, der irgendwie zur Verfügung steht, Klassenlehrer werden.

(auf Nachfrage, ob er diesen Konflikt anspricht, entgegnet Herr Bach)

"Ich spreche ihn nicht offen an, weil ich nicht weiß, wie ich's machen soll. Ich meine, ich versuche das dann, wenn äh konkrete Themen sind meinetwegen wie dies jetzt mit diesem Klassenlehrer, ich meine, dann setze ich mich natürlich schon mit ihm zusammen, warum und wieso. Aber meine Vermutung ist, dann kriege ich irgendwie so 'ne Antwort, wo ich dann machtlos dastehe: „Ich bin krank“. Oder: "Meine Frau hat Geburtstag.“ Ja? Irgend so 'ne Antwort kommt, und da bin ja hilflos. Was soll ich denn dazu sagen? Der wird ja nicht sagen, ja, also, ich glaub' nicht, dass er die Wahrheit sagen würde." (120141)

Herr Bach weiß, dass er jeden Kollegen für eine Klassenleitung benötigt und trotzdem scheut er das Gespräch mit dem Kollegen, weil er nicht an eine Art positiver Überzeugung seinerseits glaubt. Herr Bach orientiert sich an einem Führungsverständnis, was als „machtlos“ eingeschätzt wird. In seinen Äußerungen über den Kollegen wird zudem deutlich, dass Herr Bach eine negative und misstrauische Haltung ausgebildet hat. Für Herrn Bach ergibt sich das Handlungsdilemma: Berücksichtigung der Individualebene des Kollegen oder Handeln auf der Systemebene Schule als Ganzes. Von noch größerer Bedeutung sind aber das Vermeiden des Gespräches und das fehlende Vertrauen in seine Mitarbeiter. Deutlich wird hier, dass Herr Bach keine Verhaltensstrategie hat - im Unterschied zu Herrn Müller, der einen ähnlichen Konflikt beschreibt (vgl. S. 131), gelingt es Herrn Bach nicht, sich aktiv einzuschalten. Herr Bach ordnet den Konflikt von vornherein auf der persönlichen Ebene ein, je stärker die Handlungen, hier des Kollegen, auf dieser Ebene verarbeitet werden, desto schwieriger wird es, Lösungen für Konflikte zu entwickeln. Die asymmetrische Struktur der Beziehung kann als Vertrauensantinomie eingeordnet werden (vgl. Helsper, S. 69 in dieser Arbeit).

„Also ich hab am Anfang viel Widerstand gehabt - war wirklich schon auch arger Widerstand auch - unbegreiflich letztendlich. Also so, dass der Personalrat hier war und mit Zahlen kam, die nicht stimmten. So Belastung, Belastung, Belastung. Und die Kursrate sei hochgegangen, also so von 16 nach 18, so krass war es nicht. Aber das Gegenteil war der Fall. Und das finde ich schweinisch. Das finde ich schweinisch, mir mit so einer Geschichte zu kommen, was nicht stimmt. Ich bin Deutsch-Politik-Lehrer, ich bin im Grunde kein Zahlenmensch, aber das war ein Mathematiker, der mir da kam, aber das ist im Grunde jetzt Beiwerk. Aber das, das nehme ich im Grunde, obwohl das der einer ist, der schon weg ist, das nehme ich dem immer noch übel, mir da mit falschen Zahlen zu kommen, also das finde ich wirklich übel." (145-153)

Herr Bach hat vornehmlich zu Beginn seiner Dienstzeit „Widerstand“ erlebt und fühlte sich persönlich vom Verhalten des Personalrates angegriffen. Die Aussage „das nehme ich ihm immer noch übel“ verweist darauf, dass
Keine Erwartung an einen positiven Handlungsausgang

Umgang mit Konflikten 
für Herrn Bach das Gefühl der Enttäuschung über so ein Verhalten immer noch sehr präsent ist, was erneut auf eine emotionsbezogene Bewältigung von Konflikten deutet. Herr Bach kann in dieser geschilderten Situation nicht die Vielschichtigkeit des Handlungskontextes analysieren und das Verhalten des Personalrates auf einer Metaebene betrachten, er ist zu sehr verstrickt. Eine distanzierte Haltung wäre aber notwendig, um förderliche Arbeitsstrukturen aufzubauen. Das Führungsverständnis und verhalten von Herrn Bach sind persönlich und emotional ausgelegt, ihm gelingt kein Perspektivenwechsel als Schulleiter, der aufgrund der Gesamtverantwortung für die Schule systembezogen handeln müsste (vgl. Wissinger, S. 27 in dieser Arbeit). Etwas später kommt Herr Bach erneut auf den erlebten Widerstand im Kollegium zurück.

„Dann war das (ein fachliches Profil, Anm. d. Verf.) hier in dem Bildungsgebiet hier in der Bildungsanstalt unbesetzt -(...) und dann haben wir's gemacht. Also es war: wir mussten was machen, es war die Tradition der Schule, es war unbesetzt, und dann haben wir Gott sei Dank - das besetzt als Erste in (der Stadt, Anm. d. Verf.) und profitieren da auch bis heute von. „Also der Ruf der Schule - ob das die innere Qualität des (...) Unterrichts wirklich so ist, dass das gerechtfertigt ist, da habe ich ja ehrliche Zweifel. Aber gut. Der Ruf ist erst mal da, und wir kriegen die Schüler. Aber, ja, also wir mussten was tun. Eltern standen hinter mir. Ja, die Eltern stehen ja hinter mir - nach wie vor. Und das pflege ich natürlich auch, das ist klar. Eltern, wenn Eltern was wollen, das hat immer Vorrang. Tja. Jedenfalls habe ich sie mitgenommen. Also da habe ich keine großen Probleme. Und in der Schulleitung sind wir uns einig. Also es ist ja leider Gottes so, das ist auch noch nicht ganz zufrieden stellend, dass ich dann doch sehr stark mit 'nem Stellvertreter zusammenarbeite, ne? wir beide. Und der Stellvertreter hat ein äußerst hohes Ansehen, der geht jetzt in den Ruhestand. Ja, hat ein sehr hohes Ansehen, und äh, ja, Gott sei Dank war der von vornherein 100 \% loyal, so dass - äh -, also in der damaligen Situation, wenn er gegen mich intrigiert hätte, also dann wäre das wahnsinnig schwer gewesen. Also wir beide [standen zusammen. Und die anderen Fünf auch, und dann, ich weiß nicht, (...), ich meine, ich bin glücklich, aber das müsste sich doch im Kollegium auch irgendwie, dass sie sagen: "Mensch, wir sind wieder wer". Oder so in diesem Sinne. Oder dass man sagt: „Also wir kriegen auch gute, wir kriegen auch wirklich viel, viel bessere Kinder", dass das keiner Mal sagt, das verstehe ich z.B. überhaupt nicht. Und da bin auch enttäuscht, (....)- ja- sie sagen's nicht. Es kommt keiner und sagt zu mir: "Sie machen's gut". Und das finde ich schon schlimm. Das finde ich schon schlimm. Und das ist, also das mit dem Kollegium, das ist mir nicht gelungen. Also, wenn wir hier nicht zusammenhalten würden hier - da ist enger Zusammenhalt hier in der Schulleitung und auch hier so Sekretärinnen, Hausmeister, aber vom Kollegium selber gibt's nicht viele positive Rückmeldungen. (191-236) ${ }^{233}$

Herr Bach hat durch gezielte Initiativen der Schule ein eigenes Profil ge- Zweifel an Erfolg geben und dadurch höhere Anmeldezahlen erreicht. Damit scheint er zufrieden zu sein, gleichzeitig zweifelt er aber an der „inneren Qualität“. Dies ist auf seine bereits erläuterte hohe Anspruchshaltung und der erlebten Frustration über ungleiche Vorstellungen zu erklären. Dennoch wird das Zweifeln an der inneren Schulqualität insofern handlungsleitend für Herrn Bach, als dass er dem Kollegium generell misstraut. Diese Haltung steht in klarem Widerspruch zu einem Führungsverständnis, welches auf Kooperation und Partizipation ausgerichtet ist (vgl. S. 42 in dieser Arbeit).

\footnotetext{
${ }^{233}$ Passagen, die evtl. Rückschlüsse auf einzelne Personen oder Schulen zulassen, sind gekürzt worden, ohne die inhaltlichen Aussagen zu verändern.
} 
Herr Bach sieht lediglich die Eltern als seine Verbündeten an, „Eltern, wenn Eltern was wollen, das hat immer Vorrang“. Diese besondere Bedeutung des Zuspruchs der Eltern lässt sich einerseits aus der erlebten Anfangssituation an der Schule heraus erklären, denn Herr Bach musste dringend Schüler rekrutieren und Eltern für die Wahl seiner Schule gewinnen. Von Beginn an nahm damit die Interessengruppe Eltern eine wichtige Rolle im Arbeitskontext von Herrn Bach ein. Andererseits kann die Fixierung der Interessengruppe Eltern auch ein Anzeichen dafür sein, dass Herr Bach "hier keine Probleme“ hat, weil Hierarchieebenen geklärt sind und er sich in keinem Rollenkonflikt befindet. Innerhalb der Schule erlebt Herr Bach einen starken Rollenkonflikt, denn es kollidieren die Rollenerwartungen an die Position als Schulleiter mit seinem Selbstverständnis als Pädagoge.

Herr Bach verfügt nur über wenige stabile Arbeitsbündnisse, dies wird aus der Situationsbeschreibung in der Schulleitung erkennbar. Herr Bach erlebt die Konstellation der erweiterten Schulleitung als nicht zufriedenstellend, obwohl inm die Schulleitungsmitglieder Loyalität entgegen bringen. Herr Bach hebt insbesondere das hohe Ansehen des Stellvertreters zweimal hervor- was als Hinweis darauf gedeutet werden kann, dass er selbst auch in der Gunst dieses Ansehens stehen möchte, der Pensionierung des Stellvertreters wird Herr Bach wahrscheinlich als Verlust ansehen.

Am Ende dieses Interviewauszuges wird erneut ein Kernproblem seiner Berufsauffassung deutlich. Herr Bach wartet auf offene Anerkennung des Kollegiums, „es kommt keiner und sagt zu mir: „Sie machen's gut“. In diesem Zitat wird seine Enttäuschung fühlbar, er wünscht sich ganz persönlichen Zuspruch und Lob. Es gelingt ihm nicht, eine Verbindung zwischen seinem eigenen Bedürfnis nach Anerkennung und seiner ausbleibenden Anerkennung gegenüber dem Kollegium zu erkennen, es fehlt ihm das nötige Reflexionsvermögen. Das Ausbalancieren einer für inn widersprüchlich erlebten Handlungslogik, denn er hat etwas erreicht und wird dafür nicht gelobt, gelingt ihm nicht. Eine zu starke persönliche Identifizierung mit dem schulischen Geschehen verhindert ein problemorientiertes Handeln (vgl. Schütze, S.65 in dieser Arbeit).

Auf die Nachfrage, wie er denn mit dieser Enttäuschung umgehen würde, erzählt Herr Bach von seinen „Fluchtphantasien“.

„Ja. Also das habe ich überlegt. Ich hatte auch wirklich keinen in der Bezirksregierung. Keiner war mal da. Na gut. Das ist wieder ein anderes Kapitel, was nun da nicht so gut war. Ich war 17 Jahre an der Basis (...), 23 Stunden gemacht, dann bin ich vier Jahre in der Bezirksregierung, hier in der Schulaufsicht, (...) Das habe ich vier Jahre gemachtUnd danach bin ich dann hier Schulleiter geworden. Also es war auch so ein bisschen 'ne Tradition, wenn man da war, dann sah man zu, nach 'ner bestimmten Zeit Schulleiter zu werden. Inzwischen ist das auch nicht mehr so. Aber halt - ich hab noch von diesem Weg profitiert in Anführungsstrichen. Ich will nur sagen, ich hab also diese Fluchtphantasien habe ich zigmal gehabt." (241-256)

Herr Bach verweist darauf, dass er die Unterstützung von der Bezirksregierung vermisste, was sich wahrscheinlich insbesondere auf die Anfangsjahre an der Schule bezieht. Herr Bach hat dann Basisarbeit in der Schule
Verbundenheit mit Eltern

Ausbleibende Anerkennung

Belastungserleben 
ausgeübt und dies ist für inn Unterrichten. Die Abfolge der Tätigkeiten Dezernent und dann Schulleiter zu werden, scheint aus der Sicht von Herrn Bach eine gewisse „Tradition“ gehabt zu haben. Herr Bach betont durch die Formulierung „in Anführungszeichen“, dass er von dem Dienstweg wohl profitiert hat. Aber - und das bestimmt seine Berufsauffassung wesentlich - er hat „Fluchtphantasien“, d.h., die Position Schulleiter schätzt er nicht als persönlichen Gewinn ein, sondern erlebt sie als Bedrohung, aus der er manchmal fliehen möchte.

„Also es gibt, ich versuche schon, das ein neuer Anlauf jetzt seit Ostern so 'nen kollegialen Supervisionskreis aufzubauen, da bin (ich) aber sozusagen erst durch Ansprache rein gekommen. Sieht ganz gut aus, dass das so klappen kann, wir sind zu Sechst. Wir haben uns erst zweimal getroffen, aber ich glaub', das wird was. Also mir gefällt das - zu Sechst, das sind so mehr so konkrete Fallbesprechungen, dass man so ein konkretes Muster hat, das hat ein Kollege mitgebracht, der sich da besser auskennt als ich." (262267)

Auf Nachfrage, wie Herr Bach mit seiner Frustration umgeht, äußert der Schulleiter, dass er durch seine Familie Rückhalt erfährt und nennt einen kollegialen Supervisionskreis. Herr Bach sucht vor allen Dingen Hilfen dafür, wie er konkrete Situationen in der Schule lösen kann. Ein klares Handlungsmuster erscheint ihm dabei hilfreich sein zu können.

„Das hat also durchaus geholfen, so bei schwierigen Kollegen-Gespräche oder, ja, gut, das ist oft Thema so ein bisschen von mir, aber es ist auch so für die Psychohygiene so, und wenn man merkt, dass andere es auch schwer haben oder auch ähnliche Konfliktsituationen, ist das im Sinne so: „Also ich bin nicht da alleine“, das ist schon ganz gut. Weil im Kollegium ist man wirklich alleine. Also die Einsamkeit im Kollegium ist erheblich meine Einsamkeit, also andere Kollegen sehen das sicher besser als ich." (271-276)

Herr Bach erfährt Hilfe in dem Bereich "schwierige Kollegen-Gespräche“, dies deutet darauf hin, dass Herr Bach das Führen von Mitarbeitergesprächen als ein Problem seines Handlungsrahmens einschätzt. Der Hinweis, dass es sich um „schwierige“ Gespräche handelt, ist ein Anzeichen dafür, dass Herr Bach Kommunikationssituationen eher als asymmetrisch erlebt. Herr Bach nutzt das Gremium aber auch für seine „Psychohygiene“, d.h., psychische Belastungen finden ein Ventil durch das Mitteilen in der Gruppe. Herr Bach verweist darauf, dass es ihn erleichtert zu erfahren, dass es andere auch "schwer" haben. Die soziale Verbundenheit gewinnt an Bedeutung. Herr Bach spricht in diesem Zitat direkt seine eigene Isolation in der Schule an: „meine Einsamkeit“. Diese stark emotionale Belastung erklärt die widerspruchsvolle Beziehungsstruktur seines Handelns, denn in der Position des Schulleiters müsste Herr Bach einerseits Nähe zu seinem Kollegium aufbauen, um kooperative Arbeitsprozesse zu fördern, andererseits müsste er die nötige Distanz bewahren, um als Vorgesetzter agieren zu können.

„Das ( Personalauswahl, Anm. d. Verf.) ist im Grunde genommen Sozialpsychologie letztendlich, aber ich hab da ganz gut gelegen mit. Das muss man sagen. Also ich hab auch Fehler gemacht, ist klar, aber die allermeisten, da würde ich sagen, also die, wo ich wirklich Ruhe hatte mit der Auswahl, dass ich das lange anbahnen konnte, da würde ich sagen, habe ich eigentlich fast nichts falsch gemacht. Dieses Jahr ist es wieder, das ist 
ja, ich mag das grobe Wort nicht sagen, aber es ist wirklich ganz blöd, weil so viele Leute kommen, dass man das nicht mehr steuern kann. Und das ist für die Schule das A und $O$. Und das weiß man, also ich weiß das jedenfalls, also ich gehe davon aus, also die Qualität von Schule - dabei ist die Personalauswahl mit die entscheidende Größe. Gut. Wenn sie jetzt mit 30 kommen, dann muss man hoffen, dass sie dann wirklich auch 'n guten Weg gehen, dass sie dann 50 auch noch einigermaßen - das ist natürlich ein Thema. $\mathrm{Ne}$ ? Aber - nun muss ich sagen, durch meine Kenntnisse aus der Bezirksregierung hat das für mich gar nicht so 'nen großen Unterschied gemacht. Das -weil ich dahinter eben die Abläufe (kenne, Anm. d. Verf.) Na ja, letztes Jahr - zwei Lehrkräfte [lacht] - unsicher bin ich, ob das wirklich richtig war.(...)

(Auf Nachfrage, wie er zu dieser Einschätzung komme, ob er sie schon im Unterricht hospitiert habe, antwortet Herr Bach:)

„Nee, die beiden noch nicht. Aber bei dem einen war schon 'ne Elternbeschwerde, das ist schon so 'n Signal. So viele Beschwerden kommen hier nicht an. Erstaunlicherweise. Ich glaube das liegt an den Eltern, weil sie sich nicht trauen- also bislang.(...)Tja, dann merk ich, gut, vielleicht liege ich auch falsch, aber ich hab' bisschen so Sorge, dass - ja, ich werde nicht vermieden, wenn ich gemieden werde, sagen wir mal ganz banal, wenn der Kontakt nicht gesucht wird. Und ich denke: „Wieso gehen die an mir vorbei?" oder so. Ich stehe oft im Lehrerzimmer, und die anderen sagen "Hallo" und fragen mal was oder so, oder wollen irgendwas wissen, auch irgendwie, was weiß ich: "Wie ist es mit dem Zensuren-Eintragen, und andere fragen mich gar nicht. Das finde ich schon seltsam, entweder sie suchen den Kontakt oder sie vermeiden den Kontakt. Aber das kann sich auch alles noch als Irrtum herausstellen. Also bis jetzt - die Zensuren waren gut, und ich hab eigentlich auch ganz gute Hoffnung, Klassenlehrer sind sie ja sozusagen, die sie jetzt werden im neuen Schuljahr, da habe ich auch keine Beschwerden, nein, aber keinen Widerstand gehabt so: „Ich will nicht", oder „Ich will die Klasse“. So - das lief eigentlich alles ziemlich geschmeidig ab. Aber trotzdem - also... ,(331-352)

Herr Bach erläutert, wie er sein Personal, sofern er Mitspracherecht hat, auswählt. Seine Einschätzung, dass Personalauswahl „Sozialpsychologie“ sei, ist ein Hinweis darauf, dass Herr Bach den persönlichen Eindruck einer Person ebenso ernst nimmt wie die fachlichen Aspekte.

Die Personalauswahl über schulscharfe Stellen bezeichnet Herr Bach als "entscheidende Größe“ für die Qualität der Schule. Obwohl er insgesamt seine Personalauswahl positiv bewertet, räumt er hier auch ein, Fehler gemacht zu haben. Herr Bach erklärt, dass er bei zwei Einstellungen unsicher war. Er deutet die Elternbeschwerde als Indiz für seinen personellen Fehler, wodurch erneut deutlich wird, dass er das Urteil der Eltern als Bezugsgruppe seines Arbeitskontextes für ausgesprochen wichtig hält. Herr Bach findet es „erstaunlich“, dass so wenig Elternbeschwerden kommen würden, dies beleuchtet seine eher negativ geprägte Einstellung gegenüber seinem Kollegium. In dem Führungsstil von Herrn Bach fehlt der Aspekt Mitarbeiterloyalität, was den Schulleiter darin hindert, stabile Arbeitsbündnisse innerhalb der Schule aufzubauen (vgl. S.48 in dieser Arbeit).

Im weiteren Verlauf des Interviewzitates veranschaulicht Herr Bach seine Einschätzung hinsichtlich der Kontaktaufnahme, er räumt zwar ein, dass sich alles „noch als Irrtum“ herausstellen kann, der von inm wahrgenommene Umstand, dass "der Kontakt nicht gesucht" wird, fließt aber in sein Urteil über den neuen Kollegen ein. Deutlich wird hier, dass Herr Bach darauf angewiesen zu sein scheint, dass auf inn zugegangen wird. Ihm ist der persönliche Kontakt einerseits wichtig, andererseits fällt es ihm aus seiner Position heraus schwer, diese Arbeitsbeziehungen selbst aktiv zu gestalten. Herr Bach vollzieht keine Perspektivenübernahme und versetzt

Fehlende Perspektivenübernahme 
sich nicht in die Situation neuer, junger und unerfahrener Kollegen, die auf seine Fürsorgepflicht angewiesen wären. Herrn Bachs Interpretation dieser Alltagsituation im Lehrerzimmer lassen vermuten, dass ihm aufgrund seiner emotionalen Verarbeitung die notwendige Distanz fehlt. Die Berufsauffassung von Herrn Bach orientiert sich an persönlichen und emotionalen Verarbeitungsmechanismen.

„Also ich führe schon ein Gespräch mit dem Kollegen dann, und dann hole ich alle Leute an den Tisch. Also die Eltern, den Kollegen und ich. Oder wenn's Schüler sind: Schüler, Kollegin und ich. Und meine Hauptaufgabe ist dann halt zu moderieren. Also meistens ist es so, na ja so viel Fälle waren's nicht, aber meistens, also ich hatte das Gefühl, Klärung der Beziehungssituation, Metakommunikation - da war eigentlich schon 50 \% Lösung erreicht, nicht wirkliche Lösung jetzt so, aber doch, dass das erst mal - auch die Vorbehalte und die Bilder übereinander, die Sichtweisen ausgetauscht wurden in etwas Ruhe, da bin wirklich so, das versuche ich wirklich so, ein bisschen Geburtshelfer zu sein, äh auch die Strukturierung dann der Gespräche, und das fällt mir dann auch gar nicht so schwer. Da kommt mir auch die Amtsautorität dann zu Hilfe (...).

In dem Sinne gibt's keine Lösung, aber dass eben die Eltern oder die Schüler eben mal Gelegenheit hatten, mal ihre Sichtweisen in Ruhe auszusprechen, und dass der Kollege dann dort antworten muss, sich verteidigen muss, ich nehme ihn nicht in Schutz, also nur dann, wenn ich wirklich überzeugt bin von der Sache her. Das ist aber eher nicht der Fall. Also ich könnte mir vorstellen, dass mir das vom Kollegium auch mal vorgehalten wird. Das ist bislang nicht an mich herangetragen worden. Das ist nicht so, überhaupt nicht so, das ist für mich nicht mein Leitbild, jetzt von vornherein Lehrer in den Schutz nehmen." Ich hab jetzt auch 'ne schlimme Sache am Hals, konkret will ich das jetzt nicht sagen, also da bin ich eher der Moderator, [P] na, Richter bin ich ja nicht, mehr so eben-ich schlag mich nicht auf die Seite des Lehrers. Auf keinen Fall. (361-385)

Besonders an diesem Interviewausschnitt wird der psychologisierte Sprachgebrauch von Herrn Bach erneut deutlich, welcher Ausdruck seiner psychologischen Betrachtungsweise der Schule insgesamt ist.

Herr Bach sieht eine weitere Hauptaufgabe seiner Tätigkeit darin, in Konfliktsituationen zwischen unterschiedlichen Interessengruppen das Gespräch zu „moderieren“. Herrn Bach ist bewusst, dass sich Konflikte auf den Ebenen Beziehungs- und Sachaspekt bewegen, er hat Wissen darüber, bezieht sich aber in seiner Rolle auf „die Amtsautorität“, was eine Hierarchieebene hindeutet. Die Kommunikation verläuft asymmetrisch. Herr Bach hält es zwar für wichtig, dass Schüler und Eltern Gelegenheit haben, ihre Sichtweise zu schildern, die Sichtweise des Kollegen ist inm nicht wichtig, denn dieser „muss antworten, sich verteidigen“"- es geht also nicht um Klärung. Herr Bach nimmt keine unparteiische Gesprächshaltung ein, obwohl er betont, dass er "Moderator“ sei. Herr Bach betont zweimal, dass er sich nicht auf die Seite des Lehrers stellt. Herr Bach antizipiert, dass ihm das Kollegium dies einmal vorhalten könnte, er problematisiert dennoch nicht die Gründe dafür und reflektiert nicht die möglichen Erwartungen des Kollegiums. Die Rolle des Moderators kann Herr Bach nicht einnehmen, denn ihm fehlen die nötige Distanz und Akzeptanz, um eine situationsangemessene Konfliktbehandlung zu handhaben. Die formulierte Position in der Kommunikation - die vornehmlich durch den Status als Vorgesetzter legitimiert wird - stellt in dieser Situation ein Problem der Rolle dar, Herr Bach kann hier weder in der Führungsrolle kommunizieren, noch die Aufgaben des Moderators übernehmen. Herr Bach kann Konflikten

nicht beide Seiten des Konfliktfalles berücksichtigen und daher keinen 
Ausgleich zwischen unterschiedlichen Interessengruppen schaffen. (vgl. S. 73 in dieser Arbeit).

\begin{abstract}
„Also wie gesagt, es fällt schwer, jetzt Schulleiter und Kollegen da in eine, ja, möglichst offene Kommunikation über Unterricht zu machen, weil ja immer noch dieser Dienstvorgesetzten-Status ja dazwischen steht. Also wenn ich denn, ich beurteile ja nicht, aber sie fühlen sich beurteilt. Also das stimmt nicht. Man müsste den, also ich könnte mir das besser vorstellen. Ich würde es besser finden, wenn Hospitationsregeln stünden Da würde ich auch Zeit für zur Verfügung stellen. Wenn vier Leute sagen: „Wir wollen zusammen in diesem halben Jahr uns gegenseitig hospitieren" und einfach den Unterricht besprechen, auswerten und wollen schauen, was man besser machen kann“, das würde viel mehr bringen, als wenn ich da rumliefe. Das hat immer diesen Beiklang von: „Vorgesetzter kommt. Prüft." Das ist nicht äh, also ich mache es nicht - erst mal aus zeitlichen Gründen, dann auch wegen des Tabus, das würde sicherlich Aufruhr geben ,bei den Bewährungsleuten kann man das machen, aber auch das würde garantiert auch empfunden wie Prüfung und äh zeitlich, dieses Tabu, und dann natürlich auch: Wie kriegt man ein offenes Gespräch - ein weiterführendes Gespräch. Ich finde das wirklich - ich hab ja nun einige Gespräche geführt, wie gesagt, ich habe da wirklich bislang eigentlich Glück gehabt, also es war wirklich 'n ziemlich, ziemlich guter Unterricht, würde ich sagen - oder sehr guter Unterricht, den ich gesehen habe bei den Neuen. (434-448)
\end{abstract}

An dieser Stelle analysiert Herr Bach selbst das Problem einer offenen Kommunikation. Die eben noch als Legitimation herangezogene Rolle als Vorgesetzter erscheint dem Schulleiter nun als Hindernis für eine gelungene Kommunikation über Unterricht. Herr Bach verweist darauf, dass er nicht beurteilt und Unterrichtsbesuche deshalb nicht durchführt, weil er kein Tabu brechen will. Die Beurteilung des Unterrichts als Führungsperson gesteht er sich nicht zu, Herr Bach möchte die Unterrichtshospitation Anspruch an koopeauf die Kollegiumsebene verschieben, um zu teamorientierten Unterrichts- rative Arbeitsformen verbesserungen zu gelangen. Die Berufsauffassung von Herrn Bach orientiert sich also einerseits an kooperativen Arbeitsformen, er selbst- und das ist bedeutsam - erlebt sich hingegen nicht als Teil dieses Arbeitsbündnisses.

„Also das ist das Problem der Gesprächsstrukturierung, (...) so Personalentwicklungsgespräche also wie Leute das gut hinkriegen in 'ner Schule, das wäre, da habe ich wirklich Fortbildungsbedarf. Ich bin skeptisch, also ich da nichts gegen, also nicht, dass ich also von der Sache selber irgendwie denke: „Das ist blöd, das also irgendwie so 'n moderner Kram" oder so was. Im Gegenteil. Ich halte das ganz bestimmt für 'ne Sache, die in Unternehmen angebracht und auch funktioniert, aber die haben auch einen anderen Gegenstandsbereich. Wir haben natürlich hier Hierarchien. Die können Leute entlassen. Die Rahmenbedingungen sind anders. Und, was auch noch ganz schlicht und ergreifend dazu kommt, ist: Was ist denn unser Ziel? Also unser Ziel ist ja nicht konkret. Im Unternehmen ist es die Effizienz. Kostensenkung. Gute Produkte bringen. Das ist in der Schule (...) viel zu diffus. Leider. (...) Es gibt zu wenig wirkliche Gütekriterien, die so Konsens sind, wo auch die Leute das Gleiche drunter verstehen. Das ist in der Schule ungeheuer schwer." (465-484)

Herr Bach verweist auf seinen Fortbildungsbedarf im Bereich „Personalentwicklungsgespräche". Herr Bach differenziert zwischen den Organisationen Unternehmen und Schule, er schätzt die Rahmenbedingungen der Schule für ungünstig. Die in der Schule vorhandenen Hierarchien führen eben nicht dazu, dass im Unterschied zum Unternehmen „Leute entlas- 
sen“ werden können. Herr Bach empfindet die Ziele der Schule als zu „diffus“, auch die zuvor geäußerte Zielorientierung auf Unterrichtsentwicklung und Kollegiumsentwicklung ist aus seiner Einschätzung heraus nicht konkret genug zu messen, es fehlen „Gütekriterien“. Der zweite Aspekt, den Herr Bach anspricht bezieht sich auf gemeinsame Zielsetzungen, die ja ein wichtiges Element im Rahmen der Schulentwicklung darstellen. Eine Übereinstimmung vermisst Herr Bach. Herr Bach- und das wurde an unterschiedlichen Interviewauszügen deutlich- verfügt über Theoriewissen von gutem Unterricht, er kann dieses aber nicht handlungsleitend nutzen. Der Schulleiter geht davon aus, dass er nicht die Rolle des Unterrichtsentwicklers einnehmen kann und möchte diese Aufgabe auf Kollegiumsebene verlegen.

„Was ist guter Unterricht?", bevor ich mich da mit Kollegen verständige. Also notwendig ist, dass, also ich würde es lieber auf kollegialer Ebene, also wenn ich da träumen dürften, ganz klar, ein guter Schulpsychologe, nicht alle sind gut, aber es müsste, na gut, ich will jetzt nicht so von oben herab reden, aber es müsste einer sein, der Feeling hat, der auch gut mit Leuten umgehen kann und dann so ein Hospitationsteam von 6 Leuten mit Außenleitung, das wäre spitzenmäßig. Da würde was bei rauskommen. Mit Außenleitung, das ist nicht unwichtig, dass einer also auch die Fäden in der Hand hält und die Gruppe zusammenhält und auch Ziele erreichen will." (490-494)

Dieses Zitat von Herrn Bach verdeutlicht, dass er sich von einem Außenstehenden Unterstützung in dem Bereich wünscht, in dem es ihm selbst nicht gelingt, die Aufgaben umzusetzen. Es sollte jemand sein, der das nötige „Feeling“ hat und dennoch die „Fäden in der Hand hält". Der Schulleiter expliziert unbewusst durch diese Analyse seine eigenen Probleme, inm fehlt die nötige Nähe - die Vertrauensbasis- zum Kollegium, um gemeinsam eine Verständigung auf Ziele zu initiieren. Einerseits ist er zwar bereit sich externe Hilfe zu holen, andererseits sind die internen Prozesse der Schule sicherlich nicht langfristig von außen zu lösen.

"Ich habe auch schon Erfahrung. Ich hab TZI gemacht, ich kenn' die Psychoanalyse recht gut. Und ich hab also Persönlichkeitserfahrung, Selbsterfahrung viel, viel gemacht. Supervisionen auch gemacht. Ich hab also in meinem eigenen Gymnasium da selber 'ne Supervisionsgruppe installiert mit Anleiter von außen gegen Bezahlung. Also das - ich hab 'ne kleine Erfahrung da. Zwei Jahre lang haben wir das gemacht. Also auf jeden Fall war es gut. Dass es nun gerade bombig war, will ich nicht sagen, aber es war bestimmt gut, dass wir uns da so balintmäßig uns da ausgetauscht haben - auch wirklich mit einem Leiter, der auch gut war. Also ich hab da viel Erfahrung. Und insofern habe ich natürlich auch Ansprüche (...), dann ist das auch für mich leicht vertane Zeit." (510-518) ${ }^{234}$

Herr Bach hat unterschiedliche Erfahrungen im Bereich der themenzentrierten Interaktion und in Balintgruppen gesammelt, er schätzt seine Kompetenzen in diesem Bereich daher als hoch ein und schlussfolgert daraus Ansprüche an Fortbildungsangebote. Die positive Erfahrung „auf jeden Fall war es gut", die Herr Bach in seiner Rolle als Lehrer gemacht hat, kann er nicht in seiner Rolle als Schulleiter als Stärke einsetzen. Interes-

\footnotetext{
${ }^{234}$ Themenzentrierte Interaktion nach Ruth Cohn (Ich/Wir/Es =Thema sollen in einer dynamischen Ausgewogenheit stehen). Balint-Gruppen sind von dem Psychoanalytiker Michael Balint entwickelte berufsbezogene Gruppenarbeiten, die mithilfe von Rollenspielen das berufliche Verhalten insbesondere in Konfliktsituationen stabilisieren sollen.
} 
sant an dieser Stelle ist, dass Herr Bach das Gymnasium, an dem er Lehrer war, auch heute noch als sein „eigenes Gymnasium“ bezeichnet, sich also nicht mit seiner jetzigen Schule identifiziert. Auffällig ist die wiederholte Betonung von unterschiedlichen Verfahren, die das Ziel der Selbsterfahrung haben, im Kontrast dazu steht, dass es Herrn Bach kaum gelingt Kommunikationsprozesse innerhalb der Schule situationsangemessen zu analysieren.

Im folgenden Auszug bezieht Herr Bach sich auf die Ebene der Organisationsentwicklung.

„Dann diese Organisationsentwicklung, so, da, Sie wissen wahrscheinlich letztendlich sicherlich genau, was ich meine, aber so diese Kollegiumsentwicklung so, wo ich ja nun wirklich mein Haupt-, also für die innere Qualität, denke ich, ist das eigentlich der Hauptansatzpunkt, die Kollegen miteinander ins Gespräch zu bringen, miteinander über Unterrichtsstrukturierungsprozesse, das ist in diesem Fall auch „soziales Lernen“, darüber die Leute ins Gespräch zu bringen, dass sie da 'ne gemeinsame Sprache finden und ' $n$ Stück aus ihrer Isolation da rauskommen. Das wäre eigentlich - aber wie man das in Gang bringen könnte, also da hab ich auf jeden Fall Bedarf. Ich hab letztes Jahr, vor einem Jahr 'ne Fortbildung beendet. „Führung von Schule“ -das war dieser systemische Ansatz, das war auch ganz gut. Nee, „das war auch ganz gut", hört sich blöd an, das war wirklich gut. Das war dann immer so Freitag, Sonnabend, alle vier Wochen, das war meine Hauptfortbildung als Schulleiter. Da war ich froh, dass ich da reingerutscht bin. Und das war so 'ne Mischung, so 'n bisschen so erfahrungsorientiert und theorieorientiert, aber ich würde mir das noch viel intensiver wünschen. Aber das war ganz gut. Und diese beiden Bereiche - so Organisationsentwicklung, so Geld sammeln, ich hab dann so Dinge wie Geld besorgen, natürlich da bin (ich, Anm. d. Verf.) ein Analphabet. Das äh - da so Sponsoren gewinnen - also da ist viel Bedarf. Also Schule - dieses ganze GeldBesorgen-Thema, da hab ich viel Bedarf muss ich sagen. Oder Öffentlichkeitsarbeit -wie man das besser hinkriegt. Da hätte ich auch Bedarf, und da wäre ich auch interessiert." (542-560)

Herr Bach kommt erneut auf die zu Beginn geäußerte Kollegiumsentwicklung zurück und äußert klar, dass er nicht weiß, wie er solche Gespräche und Auseinandersetzungen „in Gang bringen könnte“. Herr Bach schätzt die absolvierte Fortbildung „Führung und Schule“ als gut ein, hierbei betont er, dass beide Aspekte der Auseinandersetzung theorie- und erfahrungsorientiert einbezogen wurden. Herr Bach wünscht sich eine noch intensivere Auseinandersetzung, vor allen Dingen mit dem Thema Organisationsentwicklung. Herr Bach bezeichnet sich selbst als „Analphabeten“ im Bereich Sicherung und Beschaffung von finanziellen Ressourcen, des Weiteren hat er Bedarf im Bereich „Öffentlichkeitsarbeit“. Gerade unter der Berücksichtigung der Ausgangssituation seiner Schule, hätten Kenntnisse dieser Bereiche zu einer Handlungssicherheit beigetragen und Herrn Bach eventuell den Einstieg in den Beruf Schulleiter, erleichtert.

Herr Bach sagt offen, wo er eigene „Defizite“- dies muss im Zusammenhang der bereits geschilderten Konflikte aber auch als ein Hinweis darauf gedeutet werden, dass Herr Bach in seinem Handlungskontext die Organisation Schule, d.h. zum Beispiel die Möglichkeiten der Ressourcensicherung und Außenrepräsentation der Schule zugunsten einer pädagogischen Unterrichtsentwicklung vernachlässigt hat. (vgl. S. 60 in dieser Arbeit).

Kritische Einschätzung sich selbst gegenüber 
„Ja, dieses Jahr war ja nun wirklich geprägt durch die Behauptung am Markt mit den drei Jahrgängen. (...) Das Bildungsangebot musste neu akzentuiert werden, äh, die Eltern, also diese ganze Strukturierung der Entscheidungsprozesse damals. Dann mit den Leuten, was diesmal also wirklich so unübersichtlich war, Mist war das, wirklich Mist. Wirklich Mist! Also die Schule wird schlechter, weil schlechte Leute kommen. Und ich konnte es nicht verhindern. Ne? Das liegt aufgeschlagen, wir haben mit unserem Dezernenten telefoniert da die ganze Zeit, weil ich ihm sagen muss: „Also wenn's irgend geht, bitte, bitte ,mach' es anders. Das geht, das fährt vor die Wand mit bestimmten Leuten." (597-604)

Herr Bach geht am Ende des Interviews erneut auf die Bedeutung des Bildungsangebotes und die „Behauptung am Markt" ein. Dabei fokussiert er den Erfolg des Bildungsangebotes auf die Personalsituation, die er durch die Auflösung der Orientierungsstufe nicht in der Form beeinflussen konnte, wie er sich das gewünscht hätte. Herr Bach äußert große Skepsis gegenüber den neuen Kolleginnen und Kollegen, die durch Versetzungen an seine Schule kommen. Gutes Personal ist für Herrn Bach eine Voraussetzung für das Gelingen einer guten Schule. Herr Bach- und das ist von Bedeutung für seine Berufsauffassung nimmt eine Personalisierung von Problemen vor- dies stellt einen Grundkonflikt innerhalb seiner Tätigkeit dar. Die Formulierung "das fährt vor die Wand mit bestimmten Leuten" kann auch als ein Hinweis gesehen werden, dass er selbst sich wenig zutraut in der Personalpflege und Personalentwicklung.

„Also die Kollegiumsentwicklung ist für mich wichtig, weil das Kollegium ist für mich der Motor einer guten Schule, und da haben wir noch viel zu tun." (661-662)

Herr Bach misst dem Kollegium eine hohe Bedeutung im Rahmen „einer guten Schule" bei und verweist gleichzeitig darauf, dass die jetzige Situation nicht seinen Vorstellungen entspricht. Herr Bach orientiert sich in seinem Führungsverständnis an einem Führungskonzept, welches Kooperation in den Mittelpunkt stellt, in seinen Äußerungen spiegelt sich ein Widerspruch zwischen der hohen Bedeutung, die der Schulleiter dem Kollegium beimisst und seinen eigenen Handlungsstrategien wider.

Zusammenfassend ist festzuhalten, dass sich bei Herrn Bach eine Berufsauffassung spiegelt, die von einer nicht vollzogenen Identifikation mit der Berufsrolle Schulleiter geprägt ist. In der strukturell problematischen Doppelrolle Schulleiter/Lehrer greift Herr Bach auf Denk- und Verhaltensmuster zurück, die er als Lehrer erworben hat.

Die eigenen beruflichen Erfahrungen als „engagiertet Lehrer“ und auch pädagogisches Wissen, z.B. über TZI und Supervision, können kaum für die konkrete Gestaltung der jetzigen Arbeitsaufgaben genutzt werden. Konflikte wie die Erkenntnis, dass seine beruflichen Interessen und Ziele nicht in gleicher Weise von seinem Kollegium getragen werden, werden emotional verarbeitet. Herr Bach fühlt sich „einsam“ und erfährt die Auseinandersetzung mit den Arbeitsaufgaben als hohe Belastung. Die individuelle Umgehensweise mit den Problemen der Alltagpraxis ist von einer psychologischen Betrachtungsweise der Organisation Schule geprägt. Diese Sichtweise verstellt den Blick für die Wahrnehmung struktureller Konflikte und führt, wie einige Beispiele seiner Äußerungen zeigen, zu einer Personalisierung von Problemen.
Widerspruch zwischen Wunsch nach Kooperation und Handlungsstrategien 
In seiner Berufsauffassung nimmt Herr Bach die Organisation Schule in ihren begrenzenden Strukturen wahr, daher gelingt es ihm nicht, die Gestaltungsmöglichkeiten innerhalb der Organisation zu nutzen. Herr Bach denkt und handelt eher auf der Individualebene als auf der Systemebene. Das Führungsverhalten von Herrn Bach ist auf persönliche, emotionale Zuwendung ausgelegt - das Ausbleiben von Anerkennung wird als Affront gewertet und führt zu einer negativen Einschätzung des eigenen Handlungserfolges. Herr Bach vertritt eine Berufsauffassung, die auch aus der Rolle des Schulleiters die Pädagogik ins Zentrum stellt, einen Teilbereich des Aufgabenspektrums, nämlich die qualitative Verbesserung des Erziehungsprozesses, rückt Herr Bach in den Mittelpunkt seiner Tätigkeit. Eine solche Berufsauffassung stößt an Grenzen, weil einerseits die strukturellen Bedingungen der Schule ein Eingreifen in die „pädagogische Freiheit der Lehrer" nur bedingt zu lassen. Andererseits kann Erziehung nicht mehr die vorrangige Berufsaufgabe von Herrn Bach sein. Die Fokussierung auf eine Dimension der Schule gerät in Konflikt mit dem komplexen Handlungskontext und berücksichtigt nicht die Veränderungsmöglichkeiten, die zum Beispiel durch die Initiierung anderer Arbeitsstrukturen möglich wären. Herr Bach nimmt auf dieser Handlungsebene (Unterrichtsentwicklung) die begrenzenden Strukturen der Organisation Schule wahr und reagiert mit Rückzug.

Herr Bach kann in seiner Berufsauffassung als resignierter Einsamer beschrieben werden, denn es ist ihm nicht gelungen, Kontakt zum Kollegium aufzubauen und auf der Grundlage einer gegenseitigen Vertrauensbasis gestaltend in die Prozesse der Schule einzugreifen. Insgesamt richtet sich Herr Bach an sozi-emotionalen Handlungsorientierungen aus - wie problematisch dieser persönliche Stil der Auseinandersetzung mit den Arbeitsaufgaben ist, macht die hohe emotionale Belastung, die Herr Bach empfindet, deutlich. Die fehlende Distanz gegenüber schulspezifischen Konflikten und die Vernachlässigung der Interessengruppe Lehrerinnen und Lehrer führen zu einer resignativen Einschätzung des beruflichen Erfolges.

Im Unterschied zu dem vorher geschilderten Fall haben Erfahrungen des Scheiterns die Berufsauffassung von Herrn Bach geprägt, die persönliche und emotionale Verarbeitung von Konflikten wird zu einem zentralen Belastungsmoment in der Berufspraxis und verhindern eine Selbsteinschätzung, die an den eigenen Erfolg glaubt. Insbesondere die berufsbiographische Orientierung an den positiv besetzten Erfahrungen als Lehrer erschwert es Herrn Bach, strukturelle Konflikte der Organisation Schule wahrzunehmen und ihnen mit einer nötigen Distanz zu begegnen. Im Gegensatz zu Herrn Müller gelingt es Herrn Bach nicht, zwischen unterschiedlichen Interessengruppen der Schule zu vermitteln.

\subsection{3 „Formelle Führungskraft“ Frau Schmidt (SLi 2)}

Die Schulleiterin ist seit 17 Jahren im Schuldienst und war bereits als Koordinatorin tätig. Sie leitet seit drei Jahren die sehr große Gesamtschule (> 1000 Schülerinnen und Schüler) in einer Großstadt. Die Schulleitung besteht aus fünf Schulleitungsmitgliedern und dem erweiterte Schulleitungsteam aus fünf Jahrgangsleitungen. Frau Schmidt hat vor der Übernahme 
ihres Amtes an einer dreiwöchigen Qualifizierungsmaßnahme für künftige Schulleiterinnen und Schulleiter teilgenommen. Das Interview wurde an einem Freitagnachmittag im Dienstzimmer der Schulleiterin geführt.

Auf die Eingangsfrage, in welchen Altagssituationen besondere Fähigkeiten als Schulleiterin gefordert seien, nennt Frau Schmidt den „Gesprächsbereich“.

„Hm, das betrifft alle Gesprächsbereiche, alle Kommunikationssituationen - da ist Führung erforderlich. Das heißt, sowohl bei Auftritten in Gesamtkonferenzen, bei öffentlichen Auftritten, da sind Statements erforderlich, da muss das Credo der Schule vorgetragen werden, aber auch in Einzelgesprächen, wenn Lehrer das machen sollen, was sie sollen, wenn ich mit denen Kritikgespräche habe, oder aber auch wenn ich Zielvereinbarungsgespräche mache, wenn wir in die Zukunft sehen, wie die Arbeit weitergehen soll, dann ist das erforderlich. In allen Konferenzen ist das erforderlich. Ich kann mir nicht vorstellen außer der Schreibtischarbeit, wo ich also direkt Aktendreikampf mache [lacht], den die Sekretärin dann nicht macht, wenn sie ihn nicht macht, dann ist immer Führungsverhalten erforderlich aus meiner Sicht. "(6-14)

Frau Schmidt differenziert hier zwischen Kommunikation und Gesprächen. Zu dem Bereich der Kommunikationssituationen zählt sie Anlässe, in denen schulspezifische Rahmenbedingungen neben sprachlichem Austausch auch ein nonverbales Verhalten erfordern, z.B. „Auftritte auf der Gesamtkonferenz", „Statements“. Frau Schmidt betont, dass sie in der Öffentlichkeit Verlautbarungen und das "Credo“ der Schule vortragen müsse.

Zu den Gesprächsbereichen zählt Frau Schmidt Einzelgespräche, die sie führt, wenn sie Lehrkräften sagt, was sie „machen sollen“. Die Begriffe Kritikgespräch und Zielvereinbarungsgespräche" sind fachlich ausgerichtete Termini und zählen im schulischen Arbeitsfeld zu dem Kompetenzbereich Personalentwicklung. Frau Schmidt greift auf ein situationsorientiertes Führungsverhalten zurück, denn unterschiedliche Gespräche im Alltag fordern ein bestimmtes Führungsverhalten, welches auch autoritäre Aspekte aufweisen kann.

Die Schreibtischarbeit also die Erledigung administrativer Tätigkeiten klammert Frau Schmidt als Teil des Führungsverhaltens aus- dies und auch der Verweis darauf, dass die Sekretärin die Aufgaben nicht erledigtkönnen als Hinweis darauf gedeutet werden, dass sich Frau Schmidt nicht unbedingt als Vorgesetzte des Schulpersonals ansieht und ihr eventuell in diesem Bereich eine Delegation von Aufgaben nicht gelungen ist.

„ICh beziehe immer Position als Führungskraft. Es gibt, also es gibt ja die Unterschiede: Entweder ich bin in den Konflikt involviert und Konfliktpartei, das tritt ja immer dann auf, wenn ein dienstliches Fehlverhalten von mir gerügt wird und die Kollegin das nicht einsehen kann oder der Kollege, dass das ein Fehlverhalten ist, dann sind wir Konfliktpartei. Aber es kommt auch vor, und da würde ich das mit dem Führungsverhalten 'nen bisschen zurücknehmen wollen: Ich trete in die Stelle des Mediators, d.h. zwei kommen zu mir und brauchen Schlichtung. Dann hängt's natürlich ganz davon ab, inwieweit dienstliche Gegebenheiten übertreten werden, dann ist die Sache klar. Wenn es sich aber um einen Konflikt handelt z.B. in der Beziehungsebene, der geklärt werden muss, um die Weiterarbeit zu ermöglichen, dann bin ich natürlich als Führungsperson nicht gefragt, sondern dann als Beratung bzw. als Schlichter." (17-26)
Bedeutung von Kommunikationsprozessen 
Frau Schmidt erläutert in dieser Passage die unterschiedlichen Rollen, die sie innerhalb einer Konfliktbearbeitung einnimmt. Als Teil des Konfliktes erlebt sie sich, wenn "dienstliches Fehlverhalten von ihr gerügt wird“, in diesem Fall definiert sie ihre Rolle als Vorgesetzte. Frau Schmidt bezieht sich hier auf die Grundstruktur der hierarchischen Ordnung der Organisation Schule, die sich durch den Amtscharakter definiert. Wenn Frau Schmidt nicht selbst in den Konflikt mit einbezogen ist, dann nimmt sie ihr Führungsverhalten zurück und tritt an die Stelle der Mediatorin, also der Vermittlerin in einem Kommunikationsprozess. Frau Schmidt betont, dass sie nur als Vermittlerin auftreten kann, wenn keine dienstlichen Gegebenheiten mit einbezogen werden müssen. In Konflikten, die die Beziehungsebene zweier Kollegen/Kolleginnen betreffen, trennt sie die Rolle der Führungsperson von der Rolle der Beraterin. Als Beratung wird im Allgemeinen ein Gespräch in helfender Absicht bezeichnet, in diesem Sinne wird es von Frau Schmidt gedeutet, d.h. andere suchen eine Lösung für ein benanntes Problem, sie kommen als Ratsuchende zu ihr. Die betonte Verwendung des Begriffes „Führungskraft“ kann als Hinweis darauf gedeutet werden, dass Frau Schmidt die eigene Positionierung in jedem der hier genannten Konfliktbereiche geklärt zu haben scheint. Die Schulleiterin bezieht klar Stellung zu ihrer übergeordneten Führungsposition, dies vornehmlich im Rückgriff auf ihren rechtlichen Status (vgl. S. 85 in dieser Arbeit).

Der diese Interviewpassage einleitende Satz „Ich beziehe immer Position als Führungskraft" kann als eine apodiktische Aussage verstanden werden, die tendenziell auf Distanz zwischen Frau Schmidt und den geführten Kolleginnen und Kollegen hinweist.

Auf Nachfrage, wie Frau Schmidt Konfliktfälle z.B. mit dem hypothetischen Fall eines alkoholkranken Kollegen lösen würde, erläutert die Schulleiterin ihre Umgehensweise.

\footnotetext{
„Aber der Fall ist ganz leicht zu lösen, weil da sind konkrete Suchtvereinbarungen geschlossen, und das habe ich auch aus eigener Erfahrung auch schon erlebt hier, das ist leicht möglich. Also diese Sachen laufen sehr einfach, weil alles geregelt ist und man einfach nur als Führungskraft den Mut aufbringen muss [lacht], das ist vielleicht dann auch die Führungsleistung, hinzusehen, den Fall anzusprechen, den ersten Schritt zu tun und die Maßnahmen konsequent durchzuführen. Also da ist ja Führung ganz eng in dem Fall." (32-37)
}

Frau Schmidt bezieht sich hier auf formell vorgezeichnete Kommunikationslinien, nämlich den Dienstweg, die eindeutigen gesetzlichen Vorgaben zum Umgang mit Suchtkranken an Schulen geben der Schulleiterin Handlungssicherheit und vereinfachen aus ihrer Sicht den Umgang mit dem Problem (vgl. S. 56 in dieser Arbeit). Frau Schmidt verweist darauf, dass „Mut“ aufgebracht werden müsse und dass die eigentliche „Führungsleistung" darin bestehe, Fälle dieser Art wahrzunehmen und offen anzusprechen. Die Aussage „da ist Führung ganz eng“ meint wahrscheinlich, dass die klaren Vorgaben ein genaues und damit eng umrissenes Handlungsrepertoire vorgeben.

„Ich hätte mich auf die Stelle nicht beworben, wenn ich das nicht gewusst hätte, dass ich das muss. Ich versteh" mich nicht als prima inter pares - - gar nicht."(40-41) 
Frau Schmidt distanziert sich von der Vorstellung „Erste unter Gleichen“ zu sein, sowie es auch der Deutsche Schulleitungsverband fordert, dass nämlich eine Abkehr von der Vorstellung vom „primus inter pares“ selbstverständlich sein müsse und eine Konzentration auf Führungsaufgaben nötig sei (vgl. S. 26 in dieser Arbeit). Frau Schmidt war sich dessen anscheinend bereits vor Amtsantritt bewusst, dass sie eine andere Rolle, nämlich eine eindeutig geregelte Führungsrolle als Dienstvorgesetzte, einnehmen werden würde. Als ein erstes Ergebnis kann herausgearbeitet werden, dass Frau Schmidt sich an einem Führungsverständnis orientiert, welches die Rolle als Schulleiterin hierarchisch einordnet und auf die Distanz zwischen Führungsperson und Geführten verweist, diese Einschätzung ist dem autokratischen Führungsstil zuzuordnen (vgl. Staehle, S. 37 in dieser Arbeit).

„Also äh es gibt Situationen, wo ich gedacht hab, dass es einfacher ist, d.h. äh das Leitbild und den Konsens über die Leitung der Schule, die wir als kollegiale Schulleitung machen, das ist ja noch 'ne Besonderheit, also das habe ich mir einfacher vorgestellt. Also den Konsens innerhalb der Schulleitung herzustellen, der ist deswegen schwierig, weil das Verständnis von Leitung bei den unterschiedlichen Leitern nicht meins ist. Also sie sind zum Teil noch verhaftet in diesen 70er Jahren, und das trifft auf die Fachbereichsleiter und auf die Jahrgangsleiter eben auch zu. Also dieses neue Führungsverständnis, was wir haben, ist da noch nicht angekommen, und dieser Wechsel ist ja auch mit der Veränderung der Persönlichkeit verbunden, ist noch nicht an allen Stellen gelungen, und das habe ich mir leichter vorgestellt. Mit den einzelnen Kollegen äh, die akzeptieren die Führungsrolle leichter, obwohl ich viel jünger bin als die meisten, und meine Signale, sage ich mal, sind wohl so deutlich, dass sie das auch können, und da geht das ganz einfach. Erstaunlicherweise. Das habe ich mir schwieriger vorgestellt, weil ich eben jünger bin, dass ich gedacht hab: „Oh, oh, da musst du aufpassen, dass sie deine Rolle akzeptieren und dass du die gut durchführst". Das geht gut. Aber im Rahmen des gesamten Leitungsteams äh ist das schwieriger." (47-61)

Frau Schmidt geht im weiteren Interviewverlauf auf die Zusammenarbeit in der kollegialen Schulleitung und ihren Stand im Kollegium ein. Frau Schmidt hätte es sich „leichter vorgestellt“, Übereinstimmung in den Überzeugungen über die "Leitung der Schule“ zu erreichen. Sie sieht den Grund dafür, dass dies nicht gelungen sei, darin, dass ihr Verständnis von Führung ein anderes sei als bei den anderen Mitgliedern der Schulleitung. Frau Schmidt erklärt, dass "dieses neue Führungsverständnis“ noch nicht angekommen sei. Mit der sprachlichen Wendung vom Singular zum adhortativ gebrauchten wir „was wir haben“ möchte Frau Schmidt eventuell zum Ausdruck bringen, dass die neuen Schulleiterinnen und Schulleiter generell ein neues Führungsverständnis in ihrem Sinne aufweisen. Die eigene Führungsrolle wird von Frau Schmidt in Bezug gesetzt zu der Bedeutung ihres Alters. Dies gibt einen Hinweis darauf, dass das Führungsverständnis eingebettet ist in den geschichtlichen Kontext- mit den 70er Jahren bezieht sie sich wahrscheinlich auf ein Führungsverständnis der $68 e r-$ das viel stärker antiautoritär und auf Konsens ausgerichtet war. Das Leitungsverständnis der Schulleitungsmitglieder steht aufgrund dieser unterschiedlichen Führungsverständnisse im Gegensatz zu ihrem.
Führungsverhalten

Widersprüchliche Führungsverständnisse 
Frau Schmidt erfährt durch die vorgefundene Situation in der kollegialen Schulleitung ein Handlungsdilemma, denn ihre Vorstellung über Führung kollidiert mit der einzelschulspezifischen ausgebildeten Schulkultur. Da Frau Schmidt ihr "Leitbild“ von Führung aber für das richtigere hält, gelingt es ihr nicht, die Prozesse im Schulleitungsteam distanzierter zu analysieren und evtl. auszugleichen.

Frau Schmidt betont, dass der Wechsel von der Lehrkraft zur Schulleiterin bzw. zur Schulleitungsperson auch mit einer „Veränderung der Persönlichkeit" einhergehe. Dieser Anspruch, dass die Persönlichkeit auf die Berufssituation reagieren müsse, veranschaulicht die hohe biographische Identifikation der Schulleiterin mit ihrer Aufgabe als Führungsperson. Ein wichtiges Element der Berufsauffassung von Frau Schmidt ist die Überzeugung, dass der personale Einflussfaktor, also die Persönlichkeit der Führungskraft, von entscheidender Bedeutung für das Gelingen von Führung ist. Einerseits erfährt die Rolle der Führungskraft dadurch eine Aufwertung auch im eigenen Erleben, andererseits werden hohe Erwartungsnormen aufgestellt. Frau Schmidt orientiert sich an einem Führungsverständnis, welches den eigenschaftstheoretischen Ansätzen zuzuordnen ist, wobei sie sich vornehmlich auf Eigenschaftskategorien bezieht, was als Prädisposition der Einflussbefähigung bezeichnet wird (vgl. Wunderer S. 33 in dieser Arbeit).

Frau Schmidt fühlt sich im Kollegium ihrer Schule in ihrer Führungsrolle akzeptiert, dort hatte sie mehr Schwierigkeiten erwartet, vor allen Dingen, weil sie den Altersunterschied als eventuelles Hindernis der Akzeptanz vermutet hatte. Nochmals wird durch die Formulierung „dass sie deine Rolle akzeptieren und dass du die gut ausführst" der eigene Anspruch von Frau Schmidt deutlich.

„Ich spreche das an, ich geh' da offensiv mit um, ich benenne das deutlich, ich benenne die Differenzen, die da sind, und versuche - also es ist ja so, wir sind ja als kollegiale Schulleitung haben wir ja bestimmte Dinge, die müssen wir einfach mit Mehrheit tragen und ich versuche, einen Kompromiss zu finden auf der geringsten - also den möglichst besten Kompromiss zu finden und muss auch - ja - Entscheidungen gegen meine Überzeugung tragen - so, und das tue ich auch und auch durchsetzen. Das ist sehr schwer für mich, weil ich im Grunde es anders möchte." (63-69)

Auf die Frage, wie Frau Schmidt mit den Differenzen im Leitungsteam umgeht, erzählt sie, dass sie „offensiv“ und „deutlich“ Unterschiede im Leitungsverständnis benennen würde. Frau Schmidt zeigt hierdurch, dass sie bereit ist, die Meinungsverschiedenheiten zu thematisieren, andererseits wird in ihren Äußerungen sichtbar, dass ihr die Akzeptanz von Mehrheitsentscheidungen schwer fällt, wenn diese gegen ihre inneren Überzeugungen getroffen werden. Frau Schmidt definiert Schulleitung als Prozess des Aushandelns und ist sich bewusst, dass das Gremium der kollegialen Schulleitung einen Mehrheitsbeschluss verlangt. Frau Schmidt ordnet sich aus ihrer Handlungslogik heraus unter.

„Das hatte ich. Ich hatte mir eigentlich im Rahmen der kollegialen Schulleitung das Arbeiten mit mehr Unterstützung erhofft, also insofern, dass es eigentlich leichter ist. Und ich finde es aber schwer, diese Kompromisse zu machen und auszuhalten, also die zu ma- 
chen sind. Also ich glaube, es wäre einfacher für mich, in einer Schulleitung zu arbeiten, wo ich ganz einfach meine Sachen entscheiden kann und dann machen kann. Also diese Kompromisse und diese Schwierigkeiten, die da immer mit verbunden sind in der Kommunikation und in den Missverständnissen, die belasten mich eher. Also das finde ich eher schwierig. Äh, und das ist schon ein Balanceakt." (199-206)

Frau Schmidt vermisst „Unterstützung“ und erachtet es als schwer, sich in der kollegialen Schulleitung über ein gemeinsames Vorgehen zu verständigen. Die Formulierung -,Kompromisse auszuhalten“ - , deutet auf eine emotionale Betroffenheit. Insbesondere die durch Aushandlungsprozesse entstehenden „Missverständnisse" erlebt sie als belastend und wünscht sich autonomere Entscheidungsmöglichkeiten. Frau Schmidt erlebt ein Spannungsverhältnis zwischen Fremdbestimmung und Selbstbestimmung, dies stellt ein konstitutives Führungsdilemma zwischen Dependenz und Autonomie dar (vgl. Neuberger, S. 38 in dieser Arbeit). Zwei unterschiedliche Führungsverständnisse stoßen aufeinander, ein kollegiales und auf Kooperation bzw. Konsens angelegtes Verständnis der Schulleitungsmitglieder und das eher autokratische Verständnis der Schulleiterin: „Es wäre einfacher für mich, in einer Schulleitung zu arbeiten, wo ich ganz einfach meine Sachen entscheiden kann und dann machen kann“.

„Ja, also in der Situation versuche ich das auf der Metaebene. Ich versuche immer, nicht emotional da rein zu gehen. Wenn ich merke, dass ich emotional da reingehe, dann also weil ich auch ein impulsiver Typ bin - ne? -, dann „Puuuuh!“, dann merke ich, wenn da -, und dann merke ich das aber inzwischen, also ich kontrollier' mich dann, fahr' das dann zurück und atme noch mal tief durch und versuche als Erstes, das ist Technik, habe ich mir angeeignet: „Warum macht der das jetzt? Welche Gründe sind das?" Und zwar nicht das vorwurfsvolle "Warum?", sondern das echt fragende „Warum?". Ne? Und dann versuche ich, das auch so zu lösen: „Was genau sind jetzt die Beweggründe, die dich dazu treiben, das so jetzt vorzutragen?" Das versuche ich zu verstehen, damit es mir leichter fällt und ich nicht 'ne emotionale, sondern 'ne sachliche Front fechten kann." (222-231)

Auf die Nachfrage, wie Frau Schmidt mit solchen belastenden Situationen umgehen würde, erläutert Frau Schmidt, dass sie versuchen würde, „nicht emotional" an Problemsituationen heranzugehen. Die Schulleiterin hat sich als Bewältigungsstrategie eine Technik des problembezogenen Umgangs angeeignet und versucht, ihre emotionale Beteiligung aus den Konflikten herauszuhalten. Frau Schmidt greift auf Professionswissen zurück, welches unterschiedliche Interessengruppen wahrnimmt und durch gezielte Perspektivenübernahme zu einem besseren Verstehen der anderen Sichtweise gelangen will. Die Umschreibung „sachliche Front“ gibt dennoch einen Hinweis darauf, dass Frau Schmidt starke Gegensätze erlebt und diese Bewältigung mit Anstrengung verbunden ist.

Bezüglich des Themas „Entscheidungen treffen“ erzählt Frau Schmidt von einer Gesamtkonferenzentscheidung.

„Ja. Wir haben uns dazu entschieden, sechs Schwerpunktklassen anzubieten im nächsten Schuljahr, der Jahrgang lehnt das ab, das ist keine Entscheidung, die die Leitung alleine trifft, weil die Kollegen es ja auch tragen müssen und wir haben dann in der Gesamtkonferenz sozusagen verloren und müssen jetzt die Entscheidung des Jahrgangs
Belastungen

Widersprüchliche Führungsverständnisse

Umgang mit Belastungen 
umsetzen, obwohl wir als Leitungsgremium der Auffassung sind, dass das eigentlich zwar der Weg weiter gegangen wird, aber langsamer als er müsste (...) Ja, das ist so 'ne basisdemokratische Aktion, das ist ganz klar. Also die Schulleitung hat so 'ne Vorstellung, hat 'ne Leitidee rausgeben, und jetzt muss man gucken: Wo haben wir schlecht kommuniziert, dass unsere Ideen nicht durchgegangen sind? Und der Jahrgang hatte sich auch Gedanken gemacht, und er fand sich nicht in der Lage, diesen hohen Anspruch umzusetzen. Und uns ist es wohl nicht gelungen in dieser Phase der Vorbereitung, diese Ängste zu nehmen z.B., also da muss man dann überlegen, wo die Fehlerquelle gelegen hat."(71-88)

Frau Schmidt berichtet, dass der inhaltliche Innovationsvorschlag der Leitung nicht vom Gesamtkollegium getragen wurde. In diesem Punkt "Schwerpunktklassen“ war sich die Schulleitung offensichtlich als Team einig. Frau Schmidt sieht in dem Abstimmungsergebnis keine grundsätzliche Neuorientierung in eine andere Richtung, aber sie schätzt, dass Erneuerungsprozesse "langsamer" vorangehen, als sie es sich wünschen würde. Frau Schmidt ordnet das Abstimmungsergebnis als „basisdemokratische Aktion“ ein. Die Bemerkung, „die Schulleitung hat eine Leitidee herausgegeben“, deutet daraufhin, dass die Partizipation an Entscheidungsprozessen im Vorfeld kaum stattgefunden hat (vgl. S. 14 in dieser Arbeit). Frau Schmidt analysiert die Gründe und trägt die Verantwortung dafür, dass es ihr nicht in ausreichendem Maße gelungen ist, „Ängste“ zu nehmen. Frau Schmidt erlebt eine asymmetrische Beziehungsstruktur gegenüber dem Kollegium, denn sie verfügt aus ihrer Sicht über andere Wissensbestände bzw. in diesem Fall bessere Konzeptideen, eine gemeinsame Problemlösung mit dem Kollegium auf symmetrischer Ebene wird dadurch erschwert. In ihren Äußerungen werden erlebte Widersprüche deutlich, die in der Professionstheorie als konstitutiver Widerspruch des professionellen Handelns (Symmetrie- bzw. Machtantinomie) bezeichnet werden (vgl. Helsper, S. 70 in dieser Arbeit).

„Ja, ich treffe jeden Tag Entscheidungen alleine. Und äh - ja - wenn Sie mich jetzt so direkt fragen [lacht] und mir fällt keine ein, ist es ja schwierig. Könnten wir mal eben von heute eine [überlegt]. Ja! Ich entscheide, wann und mit welcher Tagesordnung sich der Arbeits- und Gesundheitsausschuss mit dem Personalrat trifft und ob er sich trifft. Also das meinen Sie aber nicht. Sie meinen 'ne Personalentscheidung. Ne? Ja, aber das (Versetzungen, Anm. d. Verf.) entscheidet der Dezernent, das entscheide ich nicht. Also ich kann dem 'ne Vorschlagsliste machen, aber das würde ich auch nicht allein entscheiden, weil das mach' ich hier in so 'ner demokratischen Schule auch gemeinsam mit dem Personalrat. [P] Wir machen's in diesem Fall nicht. [P] Wir - äh - wir sitzen das aus." (138-144)

Frau Schmidt verweist darauf, dass sie täglich „alleine Entscheidungen treffe" und nennt ein Beispiel, hier agiert sie auf der strukturellen Ebene ihrer Schule, indem sie formale Arbeitsstrukturen der Organisation schafft. Frau Schmidt greift auf Organisationswissen zurück, das dem strukturellen Führungsrahmen zuzuordnen ist (vgl. Bolmann/Deal, S. 20 in dieser Arbeit). In dem Bereich Personalentscheidungen, z.B. bei eventuellen Versetzungen bzw. Abordnungen, erkennt Frau Schmidt die lineare Hierarchieebene der Schulverwaltung ohne Einschränkungen an: „entscheidet der Dezernent“. Dass hier dennoch zwei Organisationsverständnisse aufeinander treffen, nämlich ein zielorientiert-rationales auf der Ebene der 
Schulbehörde und ein kommunikativ-interaktionales der Einzelschule, an deren Schnittstelle Frau Schmidt steht, wird dadurch erkennbar, dass die Schulleiterin Gebrauch vom Recht einer Vorschlagsliste machen könnte, was sie „an einer so demokratischen Schule gemeinsam mit dem Personalrat" machen würde. ${ }^{235}$ Hierzu wäre ein Aushandlungsprozess innerhalb der Schule notwendig, den Frau Schmidt nicht initiiert- und dies ist von Bedeutung für ihre Berufsauffassung, denn sie erkennt die Aufsichtrechte der bürokratischen Autorität an. Die abwartende Haltung von Frau Schmidt „wir sitzen das aus“ verweist auf die Diskrepanz der zwei Handlungsrationalitäten, denn in allen anderen Bereichen greift sie lenkend in die Prozesse ihrer Schule ein.

„Hm. Ja. In diesem Fall ist es aber anders. Also das ist anders. Also das ist auch in meinem Fall anders, also finde ich nicht in jedem Fall richtig, äh, wenn ich eine IGS im Aufbau, die jetzt von uns 'ne Person braucht, da würde ich natürlich unbedingt jemanden hinschicken, also wenn ich in der schickenden Position wäre, der da auch diesen Aufbau positiv begleitet, und da würde ich auf gar keine Fall versuchen, jemanden loszuwerden, der eigentlich keine Lust mehr hat zu arbeiten. Da wäre das für die Gesamtschulidee ganz kontraproduktiv. Das mach“ich gar nicht. Wenn ich aber finde, dass da jemand dringend an ein Gymnasium möchte, und da bin ich auch froh, weil der keine Lust mehr hat zur Gesamtschule, dann würde ich dem natürlich auch keine Steine in den Weg legen. Und so handele ich auch."( 153-159)

Auf die Nachfrage, ob Frau Schmidt in einer Vorschlagsliste nicht die Möglichkeit sehen würde, bestimmte Kollegen an andere Schule abgeben zu können, antwortet sie, dass der Fall hier anders liegen würde.

Frau Schmidt erläutert ihre personellen Überlegungen, dadurch wird ihre hohe Identifikation mit der Gesamtschule deutlich. Die Schulleiterin würde die Gesamtschulidee insofern ohne Einschränkungen unterstützen, indem sie bereit wäre, Lehrerinnen und Lehrer ihrer eigenen Schule an Schulen im Aufbau abzugeben, die ihre Arbeit gut machen und eine solche Aufgabe bewältigen können. Dieses Beispiel zeigt, dass die Schulleiterin über den Rahmen ihrer eigenen Schule hinausblickt und das Schulsystem insgesamt in den Blick nimmt (vgl. Wissinger, S.25 in dieser Arbeit).

Im weiteren Verlauf des Interviews geht Frau Schmidt auf konkrete Veränderungen der Schule ein, die durch Ressourceneinsparungen seitens der Schulbehörde nötig werden.

„Ja. Wir haben uns zunächst erst mal alle Erlasse genau angeschaut, haben mit den Kollegen dazu Beratungen durchgeführt, haben sie in Kenntnis gesetzt über die Erlasse und haben dann Ziele formuliert: "Was wollen wir erhalten? Was ist uns das Wichtigste? Und was können wir eher lassen?" Diese Beratungen sind auf den unterschiedlichen Ebenen gelaufen, und wir kommen jetzt in die Entscheidungsphase. Äh, es gibt dann ja immer verschiedene Möglichkeiten, diese Wege zu gehen, und wir kommen jetzt auf den nächsten Gesamtkonferenzen dann in die Entscheidungsphase, also wie wir's dann machen werden. Also, ja, ich mache das im Sinne des klassischen Projektmanagements, Veränderungsmanagements. Äh, was natürlich besonders schwierig ist, ist so diese emotionale Betroffenheit, die man hat, wenn man als Gesamtschulmensch mit Herzblut an einer Gesamtschule hängt und merkt, dass so eine Erlassvorlage ja ein Tritt ist, die die Arbeit der gesamten Jahre eben mit Füßen tritt und auch destruktiv behandelt, nicht wür-
Berufsration vs. Organisationsratio

Schulsystem als Orientierungsrahmen

${ }^{235}$ Vgl. Rosenbusch 2005, 68. 
digt und die Kollegen dafür bestraft, dass sie's gemacht haben. Und das ist für alle, die hier mit Herzblut an der Gesamtschule hängen, ist das ein Schlag ins Gesicht und trägt nicht dazu bei, gerne zu arbeiten. "Die (die Stimmung) wird sehr belastet. Und wir versuchen natürlich gegenseitig - äh - also es ist ja oft so, wenn ein äußerer Feind da ist, dann schließt man sich im Innern enger zusammen, aber dieser Feind macht ja dividi et imperia, das heißt, wir haben dann die Abordnungen, dann geht das so los. Ne? Das ist ganz klar. Und das haben wir auch - wir hatten jetzt gerade vorgestern 'ne Dienstversammlung, wo wir versucht haben, da auch noch mal beruhigend einzuwirken durch Information und Zielsetzung und Klarheit eben. " (244-270)

Frau Schmidt verweist sie auf die rechtliche Sachkenntnis und eine transparente Informationspolitik im Umgang mit knapperen Ressourcen. Die Schulleiterin nutzt die organisationalen Gremien der Schule und bereitet in diesem Fall Entscheidungen langfristig vor, sie selbst bezeichnet dies als „Projektmanagement“. Frau Schmidt erlebt die Erlassvorgaben als Missachtung gegenüber der engagierten Arbeit an der Gesamtschule. Durch dieses Zitat wird die Verbundenheit mit der Gesamtschule, vor allen Dingen aber auch mit dem Kollegium, veranschaulicht. Sie versucht dieser schlechteren Stimmung im Kollegium mit Information und klaren Zielsetzungen entgegenzuwirken.

Von Bedeutung für ihre Berufauffassung ist, dass sie als Schulleiterin einer Gesamtschule politische Divergenzen aushalten und aushandeln muss, in dieser Situation erlebt sie die übergeordnete Behörde „als äußeren Feind“. Frau Schmidt orientiert sich aus ihrer verinnerlichten beruflichen Identität als Schulleiterin einer Gesamtschule an anderen politischen Überzeugungen als die übergeordnete Schulverwaltung. Die Schulleiterin muss sich gegen Einschränkungen und Übergriffe der übergeordneten Schulbehörde wehren, also eine autonome Problembearbeitung vollziehen, und dennoch den Weisungen der Behörde Folge leisten.

„Damit meine ich das Führungsverständnis, was das Niedersächsische Kultusministerium im Bericht „Arbeitsplatz Schulleitung" deutlich gemacht hat, und was die Trainer und Trainerinnen, die in der Schulleiterqualifizierung arbeiten, den neuen Schulleitern, mit denen sie arbeiten, versuchen deutlich zu machen. (...)Ja, da kann ich 'ne Stunde jetzt zu erzählen, [lacht], aber - also es entspricht dem, was in dem Bericht steht, Also da steht natürlich ganz oben an die Managementfähigkeiten, die Führungsfähigkeiten, das Organisationstalent, na, Sie kennen ja den ganzen Kreis, also diese Abbildung, die das Ministerium da verlangt. Und das ist 'ne große Herausforderung, weil auch personelle, also persönliche Weiterentwicklung erforderlich ist, und weil man eben diese Rolle annehmen muss - eben auch dieses Leitbild sich verinnerlichen muss."(180-197)

Auf Nachfrage nach dem von der Schulleiterin betonten „neuen Führungsverständnis“, erläutert Frau Schmidt, dass sie sich auf das Leitbild des niedersächsischen Kultusministeriums beziehen würde (vgl. 27 in dieser Arbeit). ${ }^{236}$ Zwei Ebenen spricht Frau Schmidt in dieser Interviewpassage an, einerseits die Übernahme normativer Erwartungen „Managementfähigkeiten“, „Führungsfähigkeiten“ und „Organisationstalent“, die „das Ministerium verlangt" und andererseits die eigene Identifizierung mit diesem Rollenverständnis. Insgesamt wird hier erkennbar, dass sich die Berufsauffassung von Frau Schmidt stark an vorgegebenen und normierten Kategorien orientiert. Die Anwendung dieses Professionswissens durch
Widersprüche aushalten

Führungsverhalten

${ }^{236}$ Siehe Anlage 4. 
konkrete Handlungen im Berufskontext der Schulleiterin stoßen dabei auf Widerstände, so zum Beispiel in der erweiterten Schulleitung, die Rahmenbedingungen müssten zu einer Umformulierung abstrakter Kategorien führen.

„Was insgesamt wichtig ist, denke ich, ist, dass die Leitungspersonen an Schule - auch die, die schon länger Leiter einer Schule sind, - äh, dass die dringend Qualifizierungsmaßnahmen brauchen. Also ich persönlich bin in einer Qualifizierungsmaßnahme, die mir sehr nützt, die ich aber schon lange mache, und denke, dass das erforderlich ist. Also man kann nicht einfach von Lehrer auf Schulleiter gehen. Es ist erforderlich, dass man Qualifizierungsmaßnahmen durchführt. Das Land Niedersachsen hat ja jetzt die „Begleitende Schulleiter-Qualifizierung“ ins Leben gerufen, und das ist für die neuen Schulleiter eine gute Sache. Die machen das und sind froh darum. Und ich denke, die alten Schulleiter, die da auch noch Interesse haben, die sollten das dringend auch aufnehmen und sich da weiterentwickeln. (...),Also ich hab“ daran teilgenommen an dieser Qualifizierungsmaßnahme und muss sagen, dass ich das erstens sehr genossen habe, das zu machen, dass es Klarheit darüber gegeben hat, was das bedeutet, eine Schule zu leiten - auch gerade so 'ne große Schule. Äh, ich hätte mir das noch 'n bisschen intensiver gewünscht, etwas - das liegt aber dann auch am Team, also ich denke, dass das im Grunde 'ne gute Sache gewesen ist, dass viel angesprochen worden, - manches hätte gestrafft werden können. Ich fand das richtig, das zu machen. Ich weiß aber, dass aus meinem Kurs, da waren irgendwie 30 drin, und da sind nachher nur 10 Schulleiter geworden. Insofern ist das natürlich 'ne Ressourcenverschwendung. Deswegen, denke ich, ist es richtig, die Schulleiter jetzt mit dieser Begleitmaßnahme zu schulen, - da werden ja nur die, die auch wirklich ernannt sind, dann geschult, und das ist richtig. Trotzdem ist 'ne Ausbildung im Vorfeld - man bewirbt sich auf 'ne Leitungsstelle und weiß nicht, was auf einen zukommt. Es ist ein Berufswechsel, das muss man ganz klar sehen. Und wenn man einen neuen Beruf anfängt, dann fängt man nicht eigentlich 'n Beruf an, indem man einfach da sitzt und es macht, sondern man macht 'ne kleine Ausbildung im Vorfeld, um überhaupt zu sehen, wie der Beruf geht. “(287-318) ${ }^{237}$

Einerseits befürwortet Frau Schmidt begleitende Qualifizierungsmaßnahmen für Leute, die schon Schulleiterin/Schulleiter sind, andererseits will sie Qualifizierungen vor der möglichen Ernennung, auch für die, die es am Ende nicht werden. In diesem Rahmen kann die Qualifizierung im Vorfeld Klarheit über die Berufsanforderungen schaffen und ebenso erscheint es erforderlich, Hilfestellungen zum Zeitpunkt des Berufseintrittes zu geben. Frau Schmidt unterscheidet deutlich den Beruf der Lehrkraft von dem Beruf der Schulleiterin und greift auf ein Professionswissen zurück, welches ein spezifisches Rollenverständnis als Gelingensbedingung für Schulleitungstätigkeit fordert (vgl. Wissinger S. 28 in dieser Arbeit).

Zusammenfassend ist festzustellen, dass die hier dargestellte Schulleiterin eine Berufsauffassung entwickelt hat, die von einer spezifischen Rollenübernahme als Schulleiterin gekennzeichnet ist. In den Äußerungen von Frau Schmidt spiegelt sich insgesamt eine Übernahme normativer Berufsvorstellungen wider, welchen sie versucht zu entsprechen. Die Schulleiterin orientiert sich in ihrer Handlungskonzeption an einer positiven Selbsteinschätzung hinsichtlich ihrer eigenen Kompetenzen. Frau Schmidt äu-

${ }^{237}$ Hervorzuheben ist an dieser Stelle, dass weder die Qualifizierung vor Amtsantritt noch die Teilnahme an der Begleitfortbildung verbindlich vorgeschrieben sind, sondern vom persönlichen Engagement der jeweiligen Einzelperson abhängen.

Berufwechsel Lehrerin Schulleiterin 
ßert in ihren Ausführungen klare Berufsanforderungen und reflektiert ihren persönlichen Stil der Auseinandersetzungen mit den Arbeitsaufgaben. In ihrer Berufsauffassung greift Frau Schmidt auf ein Organisationsverständnis zurück, welches sowohl die gestaltbaren als auch die begrenzenden Strukturen der Organisation im Blick hat. Aufgrund veränderter politischer Vorgaben seitens der Schulbehörde gerät sie als Schulleiterin einer Gesamtschule in Handlungskonflikte, weil sie Kürzungen umsetzten muss, die politisch motiviert sind und ihren inneren Überzeugungen widersprechen.

Die Gestaltung fördernder Arbeitsstrukturen insbesondere im Leitungsteam gelingt ihr nicht, weil Frau Schmidt einerseits auf ein Führungsverständnis zurückgreift, welches sich an „modernen“ Konzeptionen, z.B. organisatorischen und strategischen Managementkompetenzen orientiert, und sie sich andererseits gleichzeitig an Handlungsorientierungen ausrichtet, die auf formellen Weisungsbefugnissen basieren. Die zwei unterschiedlichen Führungsverständnisse - Schulleiterin versus Leitungsteam welche auch historisch begründbar sind, erlebt Frau Schmidt als starke Differenz und auch als Belastung, die sie in ihrem Arbeiten für die Schule bremsen. Die starke und positiv besetzte Abgrenzung ihrer eigenen Führungsvorstellungen gegenüber dem des Leitungsteams verhindert einen gleichwertigen und offenen Aushandlungsprozess. Eine distanziertere Reflexion dieses Widerspruches und mehr Sensibilität für die vorgefundene Berufspraxis könnten hier die nötige Nähe zur kollegialen Schulleitung und das Erleben unterstützender Arbeitsbündnisse ermöglichen.

Frau Schmidt kann in ihrer Berufsauffassung als formelle Führungskraft umschrieben werden, weil führungsrelevantes Wissen einen hohen Stellenwert in ihrer Berufsauffassung einnimmt und sie sich häufig auf formelle Regelungen in ihrer Berufspraxis bezieht.

Im Unterschied zu den vorher dargestellten Fallbeschreibungen hat Frau Schmidt ähnlich wie Herr Müller eine positive Selbsteinschätzung ausgebildet, ebenso analysiert sie Prozesse der Schule und kann eigene Handlungsorientierungen benennen. Ihre eigenen Berufsvorstellungen, die sich an einem „neuen Führungsverständnis“ orientieren, stoßen in der erlebten Berufspraxis auf Widerstände, die Äußerungen von Frau Schmidt geben Hinweise darauf, dass es ihr nicht gelingt, vermittelnd unterschiedliche Interessenpositionen und vor allen Dingen unterschiedliche Vorstellungen über die Leitung einer Schule auszuhandeln. Ähnlich wie bei Herrn Bach fehlt Frau Schmidt hier die Distanz, um Entwicklungsprozesse der Schule im Vorfeld sensibler anzugehen. Frau Schmidt vermisst im Gegensatz zu Herrn Müller unterstützende Arbeitsbündnisse vornehmlich innerhalb der kollegialen Schulleitung.

\subsection{4 „Resoluter Systemkritiker“ Herr Krause (SL 5)}

Die Ausgangssituation des nachfolgenden Falls ist eine gänzlich andere, denn Herr Krause ist lediglich kommissarischer Schulleiter und zieht ein Resümee über ein Schuljahr in dieser Funktion. Unter dieser Prämisse muss das folgende Material gelesen werden, da es dennoch oder gerade deswegen interessante Deutungen der Berufspraxis enthält, habe ich diesen Fall ausgewählt. 
Herr Krause ist seit 25 Jahren im Schuldienst, er hat eine Ausbildung als Beratungslehrer absolviert, dann war er zunächst Oberstufenkoordinator, dann stellvertretender Schulleiter und seit einem Jahr leitet er das große Gymnasium (>600 Schülerinnen und Schüler) in einer Großstadt. Die Schulleitung ist durch das Fehlen des Schulleiters/ der Schulleiterin um eine Person reduziert, besteht also aus dem stellvertretenden Schulleiter Herrn Krause und drei Koordinatoren. Das Gespräch wurde an einem Freitagvormittag im Dienstzimmer geführt.

Diese besondere Situation thematisiert Herr Krause gleich zu Beginn des Interviews, bevor die Eingangsfrage gestellt werden konnte.

„Ich weiß nicht, inwiefern das standardisiert oder nicht standardisiert ist. Ich bin ja nur kommissarischer Schulleiter für ein Jahr.

Ich bin also nur Stellvertreter $\langle h m>$, und da die Stelle aus wer weiß was für Gründen eben nicht richtig und rechtzeitig besetzt werden konnte, habe ich nun also das zweifelhafte Vergnügen oder das zweischneidige, sagen wir besser, Vergnügen, für ein Jahr Schulleiter zu spielen." (1-5)

Die besondere Rolle wird von Herrn Krause hervorgehoben und bereits in dieser ersten Aussage definiert er die Schulleitertätigkeit allgemein als ambivalentes Aufgabenfeld. Herr Krause erzählt im Folgenden, dass er sich nicht bewerben wollte. Auf Nachfrage nach den Gründen führt er dann Folgendes aus.

„Die Arbeit macht mir Spaß, und ich fühl' mich der Sache durchaus gewachsen und am richtigen Platz - aber - jetzt, was die Schulleitung angeht -, Koordinatorenaufgaben oder Stellvertreter oder meinetwegen auch dies hier. Das ist nicht die Aufgabe, die mich da irgendwie schreckt, oder dass ich das Gefühl hab: „Ich kann das nicht“. Aber ich halte das Prinzip, das System für derart ineffektiv und innovationsfeindlich und Scheuklappen fördernd, das ist also schon, schon grotesk, wie sich das hält. Als Prinzip "(55-60)

In seinen Äußerungen spiegelt sich eine selbstbewusste Einschätzung eigener Kompetenzen wider, denn er fühlt sich unterschiedlichen Tätigkeitsbereichen innerhalb des Aufgabenspektrums Schulleitung gewachsen. Die eigene Kompetenz setzt der Schulleiter in Bezug zu dem "System“, welches Herr Krause als "ineffektiv und innovationsfeindlich" einschätzt. Herr Krause kritisiert deutlich die momentane Struktur der Schulen.

„Also solange die, wie soll ich's nennen? - die Rechtsformen, die organisatorischen, aber vor allen Dingen auch rechtlichen Bedingungen oder der rechtliche Stand des Schulleiters so ist, wie er ist, ist er für mich unattraktiv. Ich sag (es) ganz offen, es wäre sozusagen, ich würde es mit in Kauf nehmen, wenn ich dafür 'ne interessante Schule in der richtigen Gegend finde. Dann würde ich, wenn es da 'ne freie Schulleiterstelle gibt, um dahin zu kommen, auch auf 'ne Schulleiterstelle bewerben. Ansonsten äh kann ich- ehrlich gesagt - keine bestimmte Richtung angeben, die ich ganz konkret anvisiere. Ich würde wieder ganz gern, wie gesagt, an einer [P] sich in einer gewissen Entwicklung befindlichen Schule Stellvertreter spielen, oder 'n Koordinator, mich interessiert, weil ich's noch lange nicht gemacht habe, jetzt der Umbruch Oberstufe. Ich bin aber auch in dem, was wir hier "neue Technologie" nennen - sowohl auf Verwaltungsebene als auch auf pädagogisch-didaktischer Ebene - ganz gut zu Fuß, da was in Gang zu bringen oder auch mit aufzubauen oder weiterzuentwickeln, das könnte mich reizen. Aber genauso gut auch

Eigenes Handlungsrepertoire vs. Schulsystem 
administrative Aufgaben für 'ne gewisse Zeit. Also ich möchte nicht ganz aus Schule raus. Das macht so viel Spaß." (64-76)

Herr Krause thematisiert zunächst die rechtlichen und organisatorischen Rahmenbedingungen des Amtes allgemein, aus seinen Äußerungen wird sichtbar, dass er vornehmlich den begrenzenden Charakter dieser Strukturen wahrnimmt. Dennoch wäre Herr Krause bereit, sich auf eine Schulleiterstelle zu bewerben, wenn er eine „interessante Schule“ finden würde. Herr Krause schildert für inn mögliche Alternativen, die er selbst für sich als real ansieht- und dies kann er vielleicht, weil er kommissarischer Leiter ist. Offensichtlich wird in dieser Interviewpassage der Widerspruch zwischen eigenem persönliche Engagement für die Schule - Herr Krause kann sich vielfältige Aufgabenbereiche vorstellen - und der systemimmanenten Kritik an der beruflichen Position des Schulleiters. Die berufliche Identität, die aus der eigenen Einschätzung heraus unterschiedliche Aufgaben in der Schule bewältigen kann, kollidiert mit den wahrgenommenen Einschränkungen der Organisation - dies wird in der Professionstheorie als eine typische professionelle Paradoxie beschrieben. (vgl. Schütze, S. 65 in dieser Arbeit).

„Nüchtern betrachtet, ist der Schulleiter [P] im Grunde genommen ein rein Abhängiger. $[P]$ In dem Sinne, dass er eine Schule leitet, ist der Name „Euphemismus“ - oder sogar 'ne echte Betrügerei. Er leitet die Schule nicht. Er kann bestimmte Dinge verwalten innerhalb eines sehr eng gestrickten Rechtsrahmens. Und es gibt im Grunde genommen rundherum lauter Gremien, die die eigentliche Entscheidung mit Gewalt von ihm fernhalten oder sie ihm im Falle eines Konflikts sofort abnehmen. [P] Damit will ich jetzt nicht irgendwie 'ner diktatorischen Führung das Wort reden, aber - angefangen von Personalia, über organisatorische Grundprinzipien ist ein Gremium zuständig, das meines Erachtens erstens keine echte Legitimation und vor allen Dingen keine Verantwortung hat: die Gesamtkonferenz zum Beispiel. Sie wird - platt gesagt - dominiert von einer Gruppe, die für das, was sie dort beschließt, eigentlich keinerlei Konsequenzen tragen muss. Der Schulleiter hat das Recht, die Pflicht bei Beschlüssen, die gegen Rechts- und Verwaltungsvorschriften, Gesetz und sonst was verstoßen, Einspruch zu erheben und das eben nicht zu vollziehen. Aber äh [lacht] - ein bisschen locker formuliert - ansonsten kann jeder Unsinn beschlossen werden, ob er machbar ist, mit welchem Aufwand er machbar ist, ob er einem System zuzuordnen ist oder aber ein willkürliches Hin- und Her-gespringe ist, - und das halte ich z.B. in einem doch - unter einem permanenten RechtfertigungsLeistungs-Druck stehenden Gebilde wie Schule - schon für 'ne gut gemeinte, aber in der Praxis doch sehr bedenkliche Konstruktion." (85-101)

Herr Krause beschreibt die zu geringen Einflussmöglichkeiten des Schulleiters und greift dabei auf ein Organisationsverständnis zurück, welches den Handlungsradius auf das Verwalten in einem „engen Rechtsrahmen“ beschränkt. Ein Schulleiter ",eitet die Schule nicht", der Begriff Leitung bezieht sich auf die formal-amtsautoritäre Ebene des Schulleitungshandelns, diese hebt Herr Krause hervor, während der Begriff Führung sich auf die Beeinflussung der Einstellung und des Verhaltens von Einzelpersonen mit Kritik an beruflicher Position des dem Zweck bestimmte Ziele zu erreichen bezieht (vgl. S. 35 in dieser Arbeit). Die emotionale Beteiligung und der Ärger über den so wahrgenommen Zustand werden durch die verwendeten Formulierungen „Euphemismus“, „Betrügerei“ deutlich. Herr Krause nennt dabei zwei Ebenen, die inn in seinem Entscheidungsrecht einschränken würden, die innere Strukturlogik der Schule, z.B. Gremien, und die äußere Strukturlogik der Schulbe- 
hörde, die ihm Entscheidungen im Falle eines Konfliktes abnehmen würde.

Der Schulleiter betont, dass er „diktatorische“ Führungsvorstellungen nicht übernehmen wolle. Das Gremium der Gesamtkonferenz hält er dennoch für „eine bedenkliche Konstruktion“, dies begründet er damit, dass hier eine Gruppe innerhalb der Gesamtkonferenz entscheiden würde, die nicht die Verantwortung für die Konsequenzen zu tragen hat. Herr Krause beurteilt die formale Rechtsgrundlage der Stellung des Schulleiters als zu gering. Durch die Betonung der Amtsautorität und somit der Vorgesetztenrolle wird erkennbar, dass Herr Krause sich in der Rolle des Schulleiters mehr Macht wünscht. Zusammenfassend lässt sich anhand dieser ersten Zitate als ein vorläufiges Ergebnis herausarbeiten, dass sich Herr Krause in seinem Handeln einer starken Diskrepanz der Handlungsrationalitäten ausgesetzt fühlt, wie sie auch in der organisationspädagogischen Literatur beschrieben wird. Er nimmt als kommissarischer Schulleiter- und dies ist für seine Berufsauffassung von Bedeutung- Konflikte und eine einschränkende Strukturlogik der Organisation Schule wahr, die Herr Krause als Hindernis im Rahmen seines Handlungsrepertoires erlebt. Die Systembrüche zwischen eigener Profession, dem Beruf als Schulleiter und der Organisation Schule und deren kritische Reflexion führen bei Herrn Krause zu einer eher verneinenden Haltung gegenüber dem Beruf Schulleiter.

„Und da ist meines Erachtens. jede Fördermöglichkeit, wenn wir bei dem Beispiel bleiben des Schulleiters, eingeengt über Stundenentlastung, dergleichen beschließt die Gesamtkonferenz in den Grundzügen. Geldliche Mittel stehen dem herkömmlichen Schulleiter zur Förderung von besonderen Aktivitäten, besonderem Engagement zumindest im allgemein bildenden Schulbereich nicht oder nicht in irgendwie nennenswertem Umfang zur Verfügung. Das heißt, das Mittelmaß muss regieren. Und der, der sich über das Mittelmaß hinaus engagiert, macht das entweder, bis er verbrannt ist oder er verfügt über eine unerschöpfliche intrinsische Motivation, aber derjenige, der entweder als bewusster Entschluss oder von seinem Naturell her den Durchschnitt oder den Unterdurchschnitt lebt, hat keinerlei Nachteile. Und das kann ein System niemals effektiv machen, sondern wir müssen es in Gang halten - mehr nicht." (112-121)

Herr Krause nennt konkrete Beispiele, in denen das Mitspracherecht der Gesamtkonferenz mit seinen Interessen als Schulleiter kollidiert. Mit dem Beispiel der Stundenentlastung meint Herr Krause, dass die Verteilung von Entlastungsstunden, die jeder Schule in einem bestimmten Rahmen zur Verfügung stehen, über einen Gesamtkonferenzbeschluss vorgenommen wird. Schulleiterinnen und Schulleiter können nicht allein bestimmen, ob er Lehrerinnen oder Lehrern eine Anrechnungsstunde zur Verfügung gestellt wird oder nicht bzw. diese entgeltlich belohnt. Herr Krause orientiert sich an einem transaktionalen Führungsverständnis, welches auf Leistung und Gegenleistung basiert und kritisiert „Mittelmaß" als Folge eines ausbleibenden Anreiz- und Belohnungssystems in Schulen. Das Deutungsmuster von Herr Krause ist das „Mittelmaß“, diese Einschätzung wird sich wahrscheinlich durch berufliche Erfahrungen gefestigt haben.

„Es müsste echte, und zwar punktuell: „Hier und jetzt wird es gebraucht" - Fördermöglichkeiten zumindest geben und ich will nicht zu weit greifen, meines Erachtens geht das nur über ein reduziertes Grundgehalt, das jedem zusteht und ein Prämiensystem, in dem der Schulleiter 'ne sehr starke Rolle spielt - oder Schulleitung von mir aus - , aber jeden-
Führungsstil

Strukturelle Bedingungen der Schule

Führungsverständnis 
falls ein kleineres Gremium, das in irgendeine Weise auch verpflichtet ist, das kann von mir aus auch 'n gemeinsamer Ausschuss - aber nicht dann einfach, dass ich zu diesem Betrieb gehöre, stimme ich im Grunde genommen über mein Wohl und Wehe hundertprozentig selber ab. Das hat sich in keinem anderen Bereich bewährt und kann sich hier auch nicht bewähren, das ist meine tiefste Überzeugung. Noch mal: ich will nicht die EinMann-Diktatur, aber nach 25 Jahren Schuldienst glaube ich sagen zu können: Das kann nicht effektiv funktionieren. Das ist innovationsfeindlich. Das ist Engagement tötend."(128-139)

Herr Krause verfügt über konkrete Ideen, wie er als eventueller Schulleiter das Engagement einzelner fördern könnte. Er wünscht sich, dass der Schulleiter bei den Entscheidungen bezüglich des Grundgehaltes bzw. der Prämienzahlung „eine starke Rolle spielt“. Aus seiner beruflichen Erfahrung heraus kann Schule nicht effektiv wirken, wenn das Gehalt nicht nach der Leistung bemessen wird, sondern standesgemäß und eventuell nach der Dauer der Dienstzeit bemessen wird. Die Berufsauffassung von Herrn Krause steht im Konflikt zu den organisationalen Bedingungen.

„Ein Beispiel: Personalsituation. Wir hatten eine ganz andere Veranstaltung hier in der Nähe, es ging da um den sog. „Schultüv“, und die ersten drei Fragen von Schulleitern hier der Region - unterschiedlichen Schultyps - zielten darauf: „Wie kann ich Qualität von Schule wirklich nachhaltig verbessern, wenn die Kollegen erleben müssen - Tag für Tag -, dass auch der, ich sag mal, faulste und unfähigste Lehrer von seinem Schulunterricht in keinster Weise angreifbar ist. Das ist die Praxis. Ob es andere Rechtsvorschriften gibt - hin und her - und, wenn ich von meiner vorgesetzten Behörde schriftlich auf Nachfrage angewiesen werde, einen Kollegen einzusetzen, der sich selber als todkrank bezeichnet, als schüttelgelähmt und sonst was, der aber ausschließlich Sport unterrichtet, und ich sage: „Diesen Kollegen setze ich nicht ein“. Aber er hat Anspruch auf volles Gehalt. Die Bezirksregierung rief mich dann an und sagte: „Rechtlich gibt es keine Handhabe. Also du setzt ihn gefälligst ein.", und der Kollege dann in $11 / 2$ Jahren vielleicht in der Summe vier Wochen unterrichtet, dann ist das für mich auch 'ne Sache, wo ich einfach weiß: Hier ist systematisch etwas krank. [P] Ich kann diese Schule nicht leiten, wenn mir so etwas einfach aufgezwungen werden kann." (149-164)

Mit dieser pauschal negativen Einschätzung, die Herr Krause im Rückgriff auf andere Schulleiter zu rechtfertigen sucht, definiert die Probleme der Schule aus der personalen Dimension heraus. Auch wenn in der geschilderten Situation eine Kritik am Verhalten der Schulbehörde nachvollziehbar ist, so zeigt sich hier doch auch, dass Herr Krause einerseits die ausbleibende Qualität von Schule auf Personalprobleme zurückführt, dass in seinen Ausführungen aber ebenso sichtbar wird, dass Herr Krause die personale Fürsorgepflicht gegenüber seinem Personal nicht bereit ist zu übernehmen. Seine Berufauffassung orientiert sich an einer Rollenvorstellung, in der das Amt des Schulleiters das Einstellen und Entlassen von Personal beinhaltet, dabei wird „Effizienz“ als entscheidende Normgröße angesehen und soziale Bedürfnisse vernachlässigt.

„Und da stehe ich als Schulleiter, als kommissarischer, und kann sagen: „Hier ist der Ordner. Hier sind die - ich müsste jetzt nachzählen -, aber es sind sicherlich jetzt schon über 50 Faxe, Briefe und sonst was, in denen dienstliche Verfehlungen [P], Handlungen gegen Rechtsvorschriften - und nichts passiert. Gut. Dann [P] ich werde weitermachen, ich werde auch das 60ste Fax schicken, aber die Erkenntnis ist eben durch diesen Einzelfall belegt oder bestärkt, dass das System zu reglementiert, zu justiziabel ist, um auf

Personalhoheit für Schulleiter u. Schulleiterinnen 
die Herausforderung vor Ort - und Schule ist dazu da, um hier und jetzt mit Schülern zu arbeiten und nicht um in drei Jahren Verwaltungsgerichtsurteile entweder zu stützen oder nicht zu stützen. Das ist nicht Aufgabe von Schule. Das kann ein Schulleiter vor Ort schon gar nicht leisten. Da wäre also selbständige Schule 'ne absolute Augenwischerei.

Aber andersrum: er muss Möglichkeiten haben - sei es alleine, oder, was ja viel besser wäre, in einem Team, das auch echt Verantwortung trägt und sogar zur Verantwortung gezogen werden könnte, sei es finanziell durch Einbußen wiederum von Prämien oder Privilegien, welcher Art auch immer, dass da Entscheidungen getroffen werden können, die z.B. jemanden schlichtweg: „Aus Unterricht. Nein! Verlust der Unterrichtsprämie - hin in den Verwaltungsdienst." Das muss möglich sein. Das - natürlich muss 'ne belegbare Verfehlung da sein, und das ist nicht, ich rede nicht davon, dass irgend jemand mal seine Pausenaufsicht nicht wahrgenommen hat, aber wenn es über einen bestimmten Level hinausgeht, kann es nicht sein, dass erst Juristen über offensichtlich Dutzende von Jahren hin sammeln muss, durch mehrere Gerichtsverfahren gehen müssen, nun einfach vor Ort wieder normaler Unterricht gemacht werden kann." (156-180)

Herr Krause verweist in dieser Interviewpassage auf die Rechts- und Verwaltungsvorschriften der Schulaufsicht, die inm als Schulleiter ein schnelles Handeln vor Ort unmöglich machen würden. Herr Krause beleuchtet hier den Konflikt, dass er als Schulleiter unmittelbar mit konkreten Ereignissen, Problemen und Vorfällen in seiner Schule konfrontiert wird und seine Alltagspraxis sich als ein alles andere als gesetzmäßig ablaufendes Geschehen darstellt. Herr Krause bezieht sich in seiner Argumentation auf das Konzept der „Selbstständigen Schule“, dessen Anliegen es ist, Schulleiterinnen und Schulleiter zu Dienstvorgesetzten zu machen und die Gestaltungsfreiheit der Einzelschulen durch die Reduzierung von Verwaltungsvorschriften zu erhöhen. Dies ist nach Ansicht von Herrn Krause nicht gelungen, solange nicht ein Team, welches in der Schule Verantwortung trägt, konsequenter auch personelle Angelegenheiten selbst bestimmen kann.

„Ja .Ich muss so 'nen Job annehmen mit dem Gefühl, nicht von jedermann geliebt werden zu können, sondern ich will respektiert werden, weil man mir abnimmt, und ich glaube, das kann ich auch in Anspruch nehmen nach diesem Jahr, dass ich klarlegen kann, warum wir oder ich so und nicht anders entschieden haben. Warum wir diese Möglichkeiten wahrnehmen und andere nicht. Warum wir nach längerer Diskussion dieses vorschlagen oder jenes. Aber dann muss man dazu auch stehen, selbst wenn ein Teil auch langjähriger Wegbegleiter oder sonst was vielleicht sagt: „Das haben wir aber immer anders gemacht." Man kann es nicht jedem recht machen. Noch mal: ich glaube, was verloren gegangen ist, ist, dass wir tatsächlich in erster Linie dazu da sind, ein Bildungsprodukt, sag ich's jetzt mal salopp, äh zu ermöglichen. Dazu braucht es nun mal 'ner bestimmten Einrichtung, aber auch meines Erachtens in der Schule vor allen Dingen geeignetes Personal und Personal- oder Menschenführung. Da ist Schule falsch konstruiert."(184-194)

Herr Krause erklärt, dass er als Schulleiter in dem „Job“ aufgrund nachvollziehbar getroffener Entscheidungen respektiert werden - auch wenn es nicht möglich sein wird, es allen recht zu machen. Herr Krause orientiert sich in seinen Äußerungen an einem Führungsverständnis, welches personelle Komponenten in den Mittelpunkt stellt, die Struktur - hier die Einrichtung stellt den Organisationsrahmen, aber für wichtiger hält Herr Krause gutes Personal, und wie er bereits mehrfach ausgeführt hat, liegt

Personalhoheit für Schulleiter u. Schulleiterinnen

Führungsverständnis 
aus seiner Sicht eine falsche Struktur vor, wenn Schulleiter nicht mehr Personalhoheiten erhalten.

Auf Nachfrage, woran genau er denn diese falsche Konstruktion festmacht, geht Herr Krause erneut auf personelle Voraussetzungen ein.

„Organisatorische - Personal-Konflikte, halboffizielle Regelungen wieder zurückzufahren auf das, was zulässig ist oder was überhaupt machbar ist. Personen einzubinden oder wieder zurückzuführen in die Bereiche, für die sie auch wirklich zuständig sind, und aus denen sie sich formal mal rausgestohlen haben. Das wäre so das Technischorganisatorische. Und das andere, worauf Sie, glaube ich, eher abzielten, ist, wir haben 'ne Schulstruktur-Reform, die - vor allen Dingen mit der Integration der 5., 6.-Klassen aufs Gymnasium ganz bisher geleugnete oder aber umgangene pädagogische Herausforderung bringt, und da nun anerkennenswerterweise - zumindest an dieser Schule - in den letzten zwei Jahren wirklich eine personelle Auffrischung stattgefunden hat, nicht nur durch junge Kollegen, sondern auch durch Seiteneinsteiger, auch die im Lebensalter schon älter sind, aber ganz andere Erfahrungen mitbringen auch aus außerschulischen Bereichen, diese Gruppe zu motivieren - zusammen mit den noch motivierten, ich sprach ja vorhin von dem unerschöpflichen Vorrat intrinsischer Motivation bei einigen, das ist ja toll, dass es solche Leute auch noch gibt, diese beiden Gruppen: Eingesessene, die noch wollen, Neue, die erst recht wollen oder erstaunt sind, wie bestimmte Zustände in den öffentlichen Schulen sind, die zusammenbringen und zu motivieren, tatsächlich im Team bestimmte Dinge zu durchdenken, vorzubereiten, Vorschläge zu machen und die dann auch durchzusetzen, das haben wir, glaube ich, mit einer sehr gut funktionierenden Schulleitungsmannschaft zumindest befördert". (224-240)

Herr Krause benennt im Folgenden ein Spektrum der Aufgaben des Schulleiters. Neben den bereits mehrfach erwähnten Reglementierungsversuchen gegenüber dem Personal beschreibt er in dieser Interviewpassage seine Aufgabe als motivierender Unterstützer unterschiedlicher Gruppen im Sinne der „pädagogischen Herausforderungen“. Dabei erlebt er Teile des Kollegiums als überaus motiviert und engagiert. Der Schulleiter ist der Überzeugung, dass es inm gemeinsam mit Kolleginnen und Kollegen und dem Schulleitungsteam gelungen ist, die Schule auf inhaltliche und organisatorische Anforderungen, hier die Klassen 5 und 6, vorzubereiten.

„ICh bin also nun mal so 'n Nestbeschmutzer und sage: "Wir verzichten auf zwei Koordinatoren und stellen dafür eine gelernte Verwaltungskraft mehr ein. Dann wäre Schule effektiver“. Aber es wäre eine - in Anführungsstrichen - „klassische Beförderungsmöglichkeit" - weg. So. Warum nicht dann ein Grundgehalt, das von mir aus um 10, 15 oder 20 Prozent niedriger liegt als der [unverständlich] - ein Prämiensystem, das Schulleitungsaufgaben mit so und soviel belohnt oder einen Topf an der Schule schafft, wo Initiativen aus dem Kollegium, Tätigkeiten in der Schulleitung oder sonst was honoriert werden - im wahrsten Sinne des Wortes. Das würde meines Erachtens. nicht unbedingt mehr kosten, es würde vor Ort vielleicht, wenn es so weitergeht, wird ja auch das dann vor Ort nur machbar sein, siehe Stichwort „Budgetierung“ und das Umgehen mit Geldern. Die Schulen wissen ja, die so was machen, dass das sehr leicht zu einer eigenen Finanzund Lohnbuchhaltung führt mit einem irren Aufwand, wenn da Schulen allein gelassen werden, wird auch das 'ne Totgeburt werden. Aber wenn da bestimmte Möglichkeiten da wären, über - so wie das meinetwegen die Ärzte machen mit ihren Abrechnungszentren, dass es dafür Institutionen gibt, die solche Aufgaben der Schule abnehmen, dann würde das ohne, meines Erachtens, materiell höheren Aufwand einen explosionsartigen Effektivitätsschub bringen - sowohl in der Verwaltung als auch in der Förderung von Engagement -sei es bei Standardaufgaben und erst recht bei außergewöhnlichen Aufgaben. (280-296)

Handlungsrepertoire 
Durch die Ausführungen von Herrn Krause wird sichtbar, dass er zwar viel über Möglichkeiten einer Umgestaltung spricht, dass er aber wenig über die real erlebte Praxis redet. Im Bereich der Verwaltung wünscht Herr Krause sich externe Unterstützung, da er der Meinung ist, dass diese Aufgaben von z.B. „Abrechnungszentren“ effektiver erledigt werden könnten. Herr Krause orientiert sich in seinen Führungsverhalten und in seinem Organisationsverständnis an unternehmerischen Prinzipien, so ist das Effektivitätskriterium (Wirksamkeit, Leistungsfähigkeit) eine entscheidende Maßgröße sowohl für die Aufgabenerfüllung als auch in seinen strategischen Überlegungen hinsichtlich der Veränderung der Organisation (vgl. S. 56 in dieser Arbeit). In den Ausführungen von Herrn Krause fehlen auch im Sinne des Umgangs mit Schülerinnen und Schülern pädagogische Überlegungen und Lösungsansätze, die ohne Belohnungssystem greifen könnten.

Auf die Frage nach der Stellung im Kollegium antwortet Herr Krause, dass er sich respektiert fühlt.

„Ich glaube, ich kann sagen, das mag vielleicht 'n bisschen eitel klingen oder so was, respektiert. Also ich sehe grob nach diesem Schuljahr die Entscheidung als gut und richtig an - für mein Selbstgefühl und für die Schule, bestimmte Dinge angegangen zu sein, offen und dann manchmal vielleicht auch, dann im Moment verletzend gewesen zu sein, weil ich gesagt habe: „Also das, und das, und das habe ich jetzt über Jahre erlebt“. Ich konnte und wollte da nicht an anderen vorbei, Dinge aufs Tablett bringen, aber bin ich, ob ich's wollte oder nicht, zuständig und verantwortlich dafür, und jetzt gehe ich die und die Sache an." Und das hat zuerst - in Teilgruppen zu 'ner Mischung aus Irritation und Abwehr oder Gegenwehr natürlich geführt, jetzt, wie gesagt, fast am Ende dieses Schuljahres behaupte ich, und ich hoffe nicht, und ich glaube auch nicht, dass ich mich da täusche, dass sogar in diesen Gruppen und auch von Personen, das kann ich in diesem Falle sogar einmal sagen, die betroffen waren von Maßnahmen und Entscheidungen, die ich nun mal treffen konnte und meinte zu müssen, dass ich da durchaus Respekt verspüre und die Bereitschaft, wieder jetzt auf neuem, auf neu ausgehandelten oder bestimmten Terrain wieder durchaus sinnvoll und, soweit ich das beurteilen kann, offen zusammenzuarbeiten. Also das hat sich gelohnt. Ich fühle, ich bin nicht unzufrieden - also mit diesem Jahr." (310-324)

In seinem eigenen Resümee über sein erstes Jahr als kommissarischer Leiter äußert Herr Krause, dass er respektiert sei und er schätzt die Situation in der Schule so ein, dass das Kollegium seine Handlungsweisen, auch wenn sie unangenehm waren, letztendlich akzeptiert und auch angenommen hat. Herr Krause erzählt davon, dass er als stellvertretender Schulleiter bestimmte Dinge erlebt hat und er diese Dinge aus der Position des Schulleiters angeht, dies kann so gedeutet werden, dass Herr Krause seine Amtsautorität nutzt, um Angelegenheiten - meist Konflikte - anzugehen und auszuhalten, dies konnte er als stellvertretender Schulleiter nicht aktiv forcieren. Trotz „Irritationen“ und „Abwehr" erlebt Herr Krause Akzeptanz gegenüber seinem Vorgehen und es ist ihm gelungen, Bereitschaft für eine offene Zusammenarbeit hervorzurufen. Die Aussage „für mein Selbstgefühl und für die Schule" verweist darauf, dass sich Herr Krause darüber bewusst ist, dass der Erfolg des eigenen Handelns Einfluss auf das Selbstkonzept hat und dass die nötige Akzeptanz seitens des Kollegi-
Strategisches OrganisationsManagement

Berufszufriedenheit 
ums eine wichtige Gelingensbedingung für das eigene Handeln darstellt. Die erfolgreiche Interaktion unter Mitwirkung des Kollegiums führte zum Erfolg des Handelns (Vgl. Helsper, S. 69 in dieser Arbeit).

„Also zur Zeit, dadurch dass eben eine - der Schulleiter eben nicht besetzt ist, haben wir also jetzt noch vier Koordinatoren - und ich als eigentlicher Stellvertreter - und, wie gesagt, wir versuchen das in 'ner zeitweiligen oder befristeten Umverteilung von gewissen Aufgaben, ja, soweit das geht, als Team zu managen. Ich hab das Gefühl, dass mehr, als ich eigentlich wollte, ich tatsächlich dann doch so 'ne Art Schulleiter-Rolle bekommen habe, und da auch von meinen Mitarbeitern [lacht] , meinen Mit-Koordinatoren,-ja, wenn ich jetzt sage, so, so „beschützt“ werde, dann klingt das ganz falsch. Aber mir fällt im Moment kein passendes Wort ein. Ich habe das Gefühl, dass die mir versuchen, für bestimmte, und zwar eben diese Konfliktdinge den Rücken so frei zu halten, wie es irgendwie geht, weil ich glaube, damit einen Bedarf auch in der Runde der Koordinatoren getroffen zu haben, dass bestimmte Dinge angegangen werden. Und da das Ergebnis ja irgendwie mehr als akzeptabel, sondern ganz ermutigend ist, hab ich das Gefühl, manchmal hab ich schon ein schlechtes Gewissen, weil ich weiß, wie viel Stunden die auch noch alle machen, was man da noch hier abgenommen wird, oder gesagt wird: „Kümmere Du Dich jetzt mal drum. Das kriegen wir schon irgendwie hin“, so dass man also nicht von einem ganz fest organisierten Arbeitsplan oder Arbeitsaufteilung, wie wir es eigentlich vorhatten am Anfang des Schuljahres, mehr sprechen kann, sondern dass da schon mehr Kooperation einerseits stattfindet [lacht], die fließend das Organisatorische immer wieder neu anpasst und regelt, auf der anderen Seite doch 'ne eher hierarchische - na - hierarchisch trifft's nicht, aber eine Rollenverteilung, wie ich sie am Anfang des Schuljahres nicht erwartet und nicht angestrebt habe. Also es ist tatsächlich offensichtlich 'n Bedarf nach einem dann doch in Person auch greifbaren Leiter da, der sich um bestimmte Dinge kümmert, und dafür hält man ihm dann auch offensichtlich gern in wiederum Bereichen, die nicht unbedingt die ureigensten sind, den Rücken oder den Schreibtisch oder was auch immer frei. Und Sie fragten ja nach Treffen, das ist auch auf persönlicher Ebene eine vielleicht außergewöhnlich gute Zusammenarbeit." (351-369)

Auch an dieser Interviewpassage wird deutlich, dass Herr Krause oft allgemeine Aussagen macht und er kaum konkrete Alltagspraxis beschreibt. Herr Krause veranschaulicht die Zusammenarbeit im Schulleitungsteam und betont, dass Aufgaben „im Team“ gemanagt werden. Dabei verweist er darauf, dass er mehr und mehr in die originäre Rolle des Schulleiters gerückt sei. Insbesondere für den oben mehrfach betonten Bereich „Konfliktdinge" verschiebt sich der Verantwortungsbereich auch von Seiten der Koordinatoren. Der Schulleiter erklärt, dass er eine enge Zusammenarbeit erlebt, aber dass sich gleichzeitig durch seinen Umgang mit Konflikten die Rollenzuschreibung in Richtung "hierarchische Rollenverteilung“ verändert habe. Herr Krause orientiert sich an einem autoritären Führungsverständnis und aus seiner Einschätzung heraus, wünschen sich auch die anderen Koordinatoren eine gestärkte Leitungsposition. Herr Krause verweist in dieser Interviewpassage darauf, dass sich durch die Wechselwirkung der normativen Erwartungen an die Position des Schulleiters und seine aktivitätsorientierte eigene Rollenbeschreibung sowohl Dominanz als auch Verbundenheit entwickeln konnten.

In diesem Fall stimmen die Erwartungen von zentralen Bezugspositionen und -personen (z.B. Mitarbeiter, Kollegium) an die Führungsrolle mit der eigenen Definition aus der Einschätzung des Schulleiters heraus überein. Unter anderen Umständen könnte dieses zu strukturellen Rollenkonflikten führen, wie sie zum Beispiel von Frau Schmidt beschrieben wurden. 
„Also es ist einfach eine Häufung, die dieses Schuljahr besonders spannend gemacht hat, also ich bin, ich sag Ihnen noch mal, müde, kaput,t aber nicht unglücklich und unzufrieden, das ist es nicht. Aber es hat dann so sehr geballt mir auch noch mal vor Augen geführt, dass mein Gefühl, mein Gefühl, das ist nicht die Objektivität, sondern mein Gefühl, dass an diesem System sehr vieles so unbeweglich und meines Erachtens auch so falsch ist, dass ich mich nicht gezielt auf eine Schulleiterstelle bewerben möchte, weil ich nicht mit Inbrunst sagen kann: „Ja, diese Aufgabe reizt mich“. Denn dazu müsste sie bestimmte andere Voraussetzungen haben. Ich kann sie, sage ich einfach mit 'nem gewissen Selbstbewusstsein. Ich kann das. Aber ich dränge mich nicht danach." (408-416)

Herr Krause verweist erneut auf die zwei Ebenen seines Handelns: Die persönliche Ebene, auf der er sich durch viel Arbeitsbelastung zwar erschöpft fühlt, aber „nicht unglücklich“, und die Systemebene, die ihm vor „Augen geführt" hat, dass vieles falsch läuft. In seiner persönlichen Konsequenz nimmt er davon Abstand, sich auf eine Schulleiterstelle zu bewerben. Wichtig für sein Selbstbild ist dabei, dass er mit einem "gewissen“ Selbstbewusstsein" aus der Situation heraustreten kann. Die Betonung „mein Gefühl“ macht deutlich, dass Herr Krause sein direktes Konfliktverhalten in Verbindung bringt mit Systemfragen und er antizipiert, dass andere nicht in ähnlicher Weise das System als „falsch“ einschätzen müssen.

„ICh hatte das Privileg, 'ne Beratungslehrer,- meines Erachtens gute BeratungslehrerAusbildung zu genießen und in dem Bereich auch zu arbeiten, bis die äußeren Bedingungen da verschlechtert wurden, da hab ich dann diese Aufgabe abgegeben und habe auch mal einen Brief, der mir viel Ärger bereitet hat, an die Bezirksregierung geschrieben. Diese Ausbildung ist mir sehr zugute gekommen, denn ich meine, ein Schulleiter muss konfliktfähig sein. Das heißt für mich praktisch: Erstens, er muss Konfliktstoff erkennen oder zumindest, wenn er ihm dargeboten wird, erkennen oder begreifen. Er muss willens sein, Konflikte im Hause mit Leuten, die er jeden Tag wieder sieht, anzunehmen und auszuhalten, das betrifft Schüler, ihre Eltern, aber auch Kollegen. Das betrifft die Behörde, das betrifft den Schulträger. Er muss also zu einem sachlichen Konflikt willens und fähig sein, und er muss dann über das Instrumentarium verfügen, es gelernt haben oder von Natur aus mitbringen, diesen Konflikt dann so praktisch auszutragen oder auszuleben, dass praktikable Ergebnisse dabei herauskommen, die möglichst eine weitere konstruktive Zusammenarbeit nicht unnötig erschweren oder sogar unmöglich machen. Er muss also auch über ein gewisses diplomatisches Geschick verfügen. Aber er muss Klarheit anstreben. Das ist Konflikt und ohne zeitweise Gegensätze nicht möglich. Das ist 'ne ganz, ganz wichtige Sache - diese Personalebene betreffend. Das gilt auch für die Zusammenarbeit - sei es in Schulleitung mit anderen Gremien oder natürlich dann mit anderen Gruppen wie Eltern-Schülern und dergleichen. Ich meine, der Schulleiter muss willens sein, zu erkennen auf Vorschlag oder selbst, was mit den vorhandenen Ressourcen machbar ist und Widerstandskraft entwickeln gegen einen immer wieder gewünschtes Gießkannenprinzip, das letztlich nichts wirklich fördert und nichts wirklich zu Ende bringt, sondern auch da den Mut haben, damit zu leben, dass er sich unbeliebt macht bei einem Teil, weil er sich entscheidet, begründbar entscheidet, auch offen dann begründet, bestimmte Dinge massiv konsequent zu fördern und andere Dinge auf Kosten dieser als wichtigste oder dringend notwendigste oder unverzichtbarste Aktivitäten andere Dinge, auch wenn es den anderen 13 Kollegen - oder wem auch immer - in dem Moment weh tut, aufzugeben oder aufzuschieben. (420-448)

Herr Krause beschreibt ausführlich das breite Spektrum an Aufgaben, die ein Schulleiter zu erfüllen hat und bezieht dabei unterschiedliche Interes-
Kritische Einschätzung sich selbst gegenüber 
senpositionen mit ein. Dabei verweist er ausdrücklich auf die besondere Bedeutung von einer gelingenden Konfliktbearbeitung, seine Ausbildung zum Beratungslehrer hat Herr Krause dabei als hilfreich erlebt. In der Professionsdebatte wird diese Auseinandersetzung mit unterschiedlichen Gruppierungen als ein besonderes Belastungsmoment pädagogischen Führungshandelns aufgezeigt (vgl. S. 69 in dieser Arbeit). Herr Krause beschreibt vor allen Dingen persönliche Stärken des Schulleiters; und dies ist von Bedeutung für seine Berufsauffassung, denn er hebt hervor, dass es von Bedeutung ist, „Mut“ zu haben und „Widerstandskraft“ zu besitzen, um konsequent auch auf Kosten eigener Unbeliebtheit Prioritäten zu setzen und Ziele klar zu verfolgen. Der Schulleiter orientiert sich an einer zielorientierten Führung, in der die Beziehungsebene zugunsten der Entscheidungsebene zurückgestellt wird, dabei ist er bereit, Verantwortung für die Konsequenzen zu übernehmen. Andererseits könnte sein Vorgehen aber auch als sehr dominant gedeutet werden, denn er scheint auf eine gemeinsame Entwicklung von Zielen weitgehend zu verzichten. Anstatt eines kooperativ- partizipativen Führungsstils praktiziert Herr Krause einen eher dominanten - transaktionalen Führungsstil, was unter anderen Umständen sowohl zu Widerständen im Kollegium als auch im Schulleitungsgremium führen könnte.

„Und drittens: er muss organisieren können. Also ich hab hier, das stinkt, glaube ich, nach Eigenlob, aber ich hoffe, dass es so rüberkommt, äh Zufriedenheit heißt, glaube ich, nicht unbedingt Selbstüberschätzung. Ich hab 'ne gute Lehre gehabt hier, was Organisation angeht, weil ich das Stundenplan-Machen noch mit einem System kennen gelernt habe von jemandem, der wirklich, glaub ich, durch und durch Pädagoge und begeisterter Organisator war, und diese handwerkliche Methode, die dann nun bedeutete, dass man so zwischen den Ferien und an den Wochenenden zwischen 15 und 4 Uhr morgens stundenlang schweigend nebeneinander stand, um dann an einer Sache mit vier oder fünf Sätzen gezielt auszutauschen: „Warum so und nicht anders?“ Äh, das hat den Blick für das Wesentliche geschärft, das hat 'ne gewisse Demut [lacht] erzeugt, dass man nicht ohne große Mühen zu einem wirklich zufrieden stellenden Ergebnis kommt, zu dem man dann auch stehen kann, selbst wenn es bestimmte Dinge eben nicht mehr möglich macht. Dass das mir sehr geholfen hat, [P] - ja - hinter den ganzen brodelnden Einzelaktivitäten eben doch immer wieder so ein gewisses System zu erkennen, das es einem möglich macht, überhaupt zu handeln und sich nicht in Einzelaktivitäten völlig zu verlieren“. (449-466)

Herr Krause beurteilt seine eigene „Lehre“ im Organisieren des Stundenplans als hilfreiche Voraussetzung für eine realistische Einschätzung des Arbeitseinsatzes, denn die handwerklichen Fähigkeiten seien nötig, um Schule zu organisieren. Für seine Berufsauffassung ist von Bedeutung, dass Herr Krause von sich verlangt, pädagogische Qualitäten und organisatorische Kompetenzen gleichermaßen zu besitzen, um den Tagesablauf einer Schule sichern zu können - er greift auf Praxiswissen zurück, welches Fried 2003 als ein wichtiges Element des Professionswissens beschreibt (vgl. S. 73 in dieser Arbeit).

\footnotetext{
„Aber ich glaube, hier in Schule, wo es natürlich auch um Strukturen geht, 5. Klassen noch im Sinne von Grundschule - oder 5. Klasse an weiterführenden Schulen, da kann man einen Systemstreit drum führen, genau wie über integrierte Systeme oder gegliedertes Schulwesen. Aus der Praxis behaupte ich mal: in erster Linie hängt es von den Menschen vor Ort ab. Und wenn das System [lacht], das über der integrierten Gesamtschule
} 
und über dem gegliederten Schulsystem drübergestülpt ist, nicht allein in dieser Gesellschaft, aber eben auch, wenn dieses System ein ganz klares Mittelmaß und Passivität fördert, - sie werden ja belohnt dafür, dass sie eben nichts tun [lacht], dann kann in keinem System wirklich Bewegung drin sein. Und wie diese Bewegung aussieht - ich könnte Ihnen jetzt ein Modell an die Wand malen, das würde auf den ersten Blick wahrscheinlich nur Heiterkeit auslösen bei vielen, aber es ist durchaus ernst gemeint: Schule wäre gedient im Sinne von Erziehung, von Lebensnähe, von Zukunftsorientiertheit, wenn Lehrer gezwungenermaßen irgendwie zwischen fünf und zehn Jahren spätestens aus der Schule raus müssten, nach eins, zwei, drei Jahren, oder sagen wir mindestens ein Jahr aus Schule raus in irgendwelche anderen - mit Lohnzuschüssen oder wie auch immer, andere Welten kennen lernen müssten. Wenn Beförderungen nur in absoluten erklärbaren Ausnahmefällen innerhalb, und zwar rede ich auch schon über die Oberstudienräte zum Beispiel an Gymnasien, nur bei Schulwechsel, wie bei jedem Sparkassenleiter - äh Filialwechsel oder sonst was, bis er dann seine eigne Filiale kriegt zum Lebensweg, äh, wenn mit diesem $[P]$ - ja - voluntaristischen Prinzip in Fortbildung und dergleichen, wo jeder mal was macht, und wenn die Person weg ist, ist dieser Zweig weg, äh, in Schule wenn Kollege $X$ da ist, wird irgend 'nen teures Gerät angeschafft, wenn er dann weggeht, steht das rum. Das sind alles so Ad-hoc-Entscheidungen, so kurzfristige Entscheidungen, die mich, also ehrlich gesagt, immer wieder verblüffen. Immer noch äh - muss ich 'ne Mischung aus Grinsen und Gallengeschmack da erleben, wenn ich sehe, wie [P] - ja wie dieser monströse Apparat Bildungssystem so vor sich hin trabt-oder - was tut er eigentlich? , was macht er eigentlich? Schlendert - so. [P] Also, noch mal: ich meine schon, der Schlüssel liegt beim Personal, nicht bei der Struktur. (524-549)

Der kommissarische Schulleiter geht am Ende des Interviews erneut auf die Bedeutung des Personals ein und grenzt strukturelle Systemfragen, z.B. Gesamtschule contra Gymnasium, davon ab. "Bewegung" im Schulsystem könnte aus seiner Einschätzung heraus nur entstehen, wenn Lehrkräfte einerseits gezwungen würden, andere Berufswelten, und zwar lohnabhängige, kennen zu lernen und den Ort ihrer Berufsausübung als Lehrkraft öfter, spätestens bei Beförderungen zu wechseln und Fortbildungen nicht nach freiem Willen, sondern nach pädagogischen Profilierungen der Schule festgesetzt werden würden. Von Bedeutung für die Berufsauffassung von Herrn Krause ist, dass er das Ziel all dieser Umstrukturierungsvorschläge darin sieht, dass Schule der Erziehung von Schülerinnen und Schülern dienen muss, und zwar so, dass den Lernenden Kenntnisse und Fähigkeiten vermittelt werden müssten, die es ihnen ermöglichen, in Zukunft am Erwerbsleben teilzunehmen. Der Kernpunkt seiner Kritik richtet sich also vornehmlich auf die Unflexibilität des Bildungssystems, welches seiner Ansicht nach zu langsam auf Veränderungsprozesse in der Gesellschaft reagieren würde.

Am Ende der Interviewpassage kommt Herr Krause zu dem Kernpunkt seiner Einschätzung zurück, er stellt strukturelle und personelle Gegebenheit gegenüber- vorher hat er jedoch sehr viele strukturelle VerändeStrukturelle Konflikte rungsmöglichkeiten beschrieben, die die Verzahnung beider Aspekte deutlich zum Vorschein brachten. Auch in dieser Interviewpassage fällt der Sprachgebrauch auf, seine Äußerungen spiegeln insgesamt eine ambivalente Haltung gegenüber der Schule wider, einerseits wird seine starke Kritik deutlich- die sich in dem Credo „dieses System fördert ein ganz klares Mittelmaß und Passivität" ausdrückt - und andererseits ein hohes persönliches Engagement für Veränderungen im Bereich des Personal- und Organisationsweiterentwicklung. 
Die Berufsauffassung des hier dargestellten kommissarischen Schulleiters ist von einer positiven Selbsteinschätzung geprägt. Diese Einschätzung eigener Kompetenzen ermöglicht es, unterschiedliche berufliche Anforderungen wahrzunehmen. In der alltäglichen Praxis greift Herr Krause auf Praxiswissen zurück, welches er durch unterschiedliche Berufserfahrungen erworben hat, z.B. Stundenplanerstellen als stellvertretender Schulleiter, aber ebenso eine Ausbildung als Beratungslehrer. Herr Krause macht in der täglichen Praxis Erfahrungen mit unterstützenden Arbeitsbündnissen, vor allen Dingen in dem Schulleitungsteam, er erfährt Anerkennung und kann ein berufsrelevantes positives Selbstkonzept aufbauen. Dabei muss seine besondere Situation als Schulleiter auf Zeit berücksichtigt werden, denn insbesondere sein Mut, Konflikte anzugehen, selbst wenn die Beziehungsebene dadurch verletzt und gestört wird, kann natürlich auch mit dem befristeten „Aushalten“ dieser Situation zu tun haben.

In seiner Berufsauffassung kollidieren eigene Vorstellungen der Organisation Schule mit vorgefundenen Bedingungen. Herr Krause erlebt einen Widerspruch zwischen seinem eigenen Rollenverständnis der Position des Schulleiters und der vorgefundenen internen Strukturlogik der Schule, dabei nimmt er den Handlungskontext Schule ausschließlich in seinen begrenzenden Strukturen wahr und es gelingt ihm nicht, gestaltbare Freiräume zu nutzen. Die große Kritik an der personellen Situation der Schule geht einher mit seiner Kritik an der Aufgabenzuweisung durch die Schulbehörde. Insofern steht Herr Krause im Spannungsverhältnis zwischen Autonomie und Dependenz.

Herr Krause orientiert sich an Führungsprinzipien, die eher auf der Durchsetzung von Willen basieren und somit einer auf Kooperation und innerer Überzeugung ausgerichteten Führung entgegenstehen. Herr Krause greift auf Wissensbestände aus der Führungstheorie zurück, die sich stark an betriebswirtschaftlichen Konzepten orientieren. Seine Berufsratio steht im Widerspruch zu der Organisationsratio, als Folge dessen hat Herrn Krause sich gegen die Übernahme des Amtes des Schulleiters zumindest an seiner jetzigen Schule entschieden. Die massive und durchgängige Kritik am Bildungssystem liegt wahrscheinlich auch darin begründet, dass er kein Schulleiter auf Dauer ist, sondern nur kommissarisch tätig wird. Herr Krause beschreibt auf der Ebene des Schulsystems interessante Lösungsmöglichkeiten, die aus seiner Einschätzung heraus der Organisation Schule eine echte Weiterentwicklung bringen würden. Insgesamt redet Herr Krause viel über Möglichkeiten, nicht aber über die reale Praxis, an einigen Interviewpassagen spiegeln seine Äußerungen ein starkes Bedürfnis nach einer Artikulation seiner erfahrenen Frustrationen mit Konflikten in der Schule wider - aber auf der Ebene allgemeiner Kritik am bestehenden Schulsystem. Vieles von seinen Ausführungen wirkt daher fast stereotyp, so z.B. die immer wiederkehrende Orientierung am „Mittelmaß der Schule", die er insgesamt als Interpretationsfolie seines Berufserlebens nutzt. Herr Krause geht kaum auf inhaltliche Bereiche seiner Schule ein, sondern beschreibt auf der Ebene der Arbeitsstrukturen von Lehrerinnen und Lehrern Bedingungen, die seiner Einschätzung nach die Weiterentwicklung und Verbesserung von Schulen verhindern. Im Gegensatz zu dem vorherigen Beispiel verfügt Herr Krause über stabile Arbeitsbündnisse, die es inm ermöglichen, dennoch eine Berufszufriedenheit und ein eigenes positives Selbstwertgefühl aufzubauen. 
In seiner Berufsfassung kann er als resoluter Systemkritiker beschrieben werden, weil er energisch Kritik an bestehen Strukturen übt. Für seine eigene berufliche Position zieht er die Konsequenz, das Amt des Schulleiters nicht für längere Zeit zu übernehmen.

Die Besonderheit des Falls liegt im Unterschied zu den drei ersten darin, dass Herr Krause- aus der Position des kommissarischen Schulleiters heraus- massive Kritik am Bildungssystem übt. Ähnlich wie Herr Müller verfügt Herr Krause über unterstützende Arbeitsbeziehungen und schätzt seine eigenen Kompetenzen hoch ein, für ihn stellen nicht eigene mangelnde Fähigkeiten, sondern das Organisationsgefüge (dieser) Schule ein Hindernis für die Erfüllung der Arbeitsaufgaben dar.

\subsection{5 „Bodenständiger Seelsorger“ Herr Johann (SL 12)}

Herr Johann ist seit über 30 Jahren im Schuldienst tätig. Er war zunächst Koordinator, er hat sich von außen auf die Stelle des Schulleiters an seiner jetzigen Schule beworben. Er leitet seit 19 Jahren das große Gymnasium einer Großstadt. Die Schulleitung besteht aus dem Schulleiter, einem Stellvertreter und vier weiteren Koordinatoren. Das Gespräch wurde an einem Schultag vormittags im Dienstzimmer des Schulleiters geführt.

Herr Johann geht bereits sehr ausführlich auf die Eingangsfrage - erzählen Sie Situationen aus ihrem Berufsalltag, in denen Sie sich besonders als Führungsperson gefordert fühlen- ein.

„Ja, werde ich gerne machen. Ein Schulleiter eines großen Gymnasiums hat ja vielfältigste Funktionen wahrzunehmen, und die Frage ist immer, wo man Prioritäten setzt. Also momentan - zu Zeiten der knappen Unterrichtsversorgung - spielt natürlich die Auseinandersetzung, ich sag mal, das Feilschen um einzelne Stunden, die Unterrichtszuweisung von Lehrerstunden eine wichtige Rolle - aber bei weitem natürlich nicht die wichtigste. Die wichtigste Rolle in erster Linie ist das Motivieren von Lehrern, der Umgang mit Lehrern, Konfliktlösungen. Und wir haben ein Kollegium im nächsten Jahr von 95 Lehrern, und ich pflege es oft zu sagen: „Im nächsten Leben werde ich Seelsorger“. Es geht hier weniger um Verwaltung als vielmehr um Führung und Beratung von Kollegen. Also ich hab' 'ne ganz wichtige Funktion. Ich selbst bemühe mich, sagen wir mal, um einen Führungsstil der offenen Tür. Ich kenne keine Sprechstunde, wo die Schule so groß ist, jeder kann jederzeit zu mir kommen. Und das ist für mich auch das Allerwichtigste. Bei einer Unterrichtsverpflichtung von 8,5 Stunden - im Augenblick ist das für mich eine hohe, relativ hohe Zahl, obwohl ich auf dieses Unterrichten nicht verzichten möchte. So. Vorgeworfen wird mir ja manchmal - und der Schulleitung - wir würden uns zu sehr um die Schüler kümmern und weniger um die Lehrer. Das ist ein Vorwurf, der zwar nicht offen geäußert wird, den kann ich eigentlich nicht als Vorwurf akzeptieren, ich empfinde es eigentlich als Kompliment, dass diese Schule - dieser Konkurrenzkampf unter den (...)Gymnasien, der sehr hart ist, ausgezeichnete Anmeldezahlen haben, meistens mehr, als wir aufnehmen können, hat den Ruf, dass sich die Lehrer sehr um die Schüler kümmern. Und dieses also versuche ich zu verstärken. Weil der Ruf nicht vom Schulleiter abhängt, das ist der unwichtigste Teil, sondern die einzelnen Lehrer, und da gibt's an dieser Schule welche, die sich äußerst intensiv um die Schüler kümmern. Gut, alle anderen Aufgaben müssen gemacht werden: Verwaltung, äh, es ist gut, dass eine Schule geordnet verwaltet wird, das halte ich aber für nicht wichtig. Also jetzt momentan ist ein großer Teil der Arbeit, die Zusammenarbeit mit dem Schulträger, weil diese Schule gene- 
ral saniert wird. Und das ist zwar sehr erfreulich, es werden 10 Million in etwa hier investiert, aber es ist elendig, mit deutschen Behörden zu arbeiten. Ja, vielleicht erst mal so weit." (8-33)

Bereits die ersten Ausführungen von Herrn Johann spiegeln eine problemorientierte und sachliche Betrachtung seiner Aufgaben. Er redet sofort über aktuelle konkrete Probleme seiner Schule, z.B. Konkurrenzkampf unter den Gymnasien. Herr Johann greift auf Professionswissen zurück, welches unterschiedliche Interessenpositionen im Blick hat, so den Anspruch des Kollegiums und auf der anderen Seite die Orientierung an der Schülerzufriedenheit. In diesem Zusammenhang reflektiert Herr Johann den versteckten Vorwurf der zu starken Schülerorientierung von seitens des Kollegiums. Herr Johann setzt hier deutlich Prioritäten und schätzt den Umgang mit Schülerinnen und Schülern als eines der wichtigsten Kriterien für den Ruf der Schule ein. Einerseits betont Herr Johann den Unterricht als einen Teil seiner Tätigkeit und er hebt hervor, dass er auf sein eigenes Handeln als Lehrer nicht verzichten möchte, und aus der Rolle des Schulleiters betont er, dass der Ruf der Schule von allen Lehrkräften abhängt. „Führung und Beratung" des Kollegiums sind für Herrn Johann wichtige Elemente innerhalb seiner Berufsauffassung, dabei orientiert er sich in seinem Führungsstil an einem auf Kooperation und Kommunikation ausgerichtetes Führungsverhalten.

Herr Johann nimmt seine Aufgaben außerhalb der Schule ebenso wahr, um materielle Ressourcen, in diesem Fall bauliche Ausstattung, zu sichern, er beurteilt diesen Aufgabenbereich aber als sekundäre Tätigkeit und verweist darauf, dass es mühsam sei, mit Behörden zusammenzuarbeiten. In seinem Organisationswissen greift er auf unterschiedliche Handlungsebenen zurück, setzt selbst die Priorität im Inneren der Schule.

Herr Johann betont zwei Mal - und dies ist von Bedeutung für seine Berufsauffassung- die beratenden Anteile seiner Tätigkeit. Die Aussage „Im nächsten Leben werde ich Seelsorger" veranschaulicht, dass Beziehungsarbeit und Personalpflege einen Hauptteil seiner Arbeit ausmachen. Zusammenfassend lässt sich anhand dieses ersten Zitates als ein vorläufiges Ergebnis herausarbeiten, dass Herr Johann sich an einem beratendkooperativen Führungsstil orientiert und seine kommunikative Kompetenz und Kontaktfähigkeit als wichtige Elemente seiner Berufsauffassung einschätzt (vgl. S. 46 in dieser Arbeit).

„Es sind kleinere Lehrer-Konflikte. Befindlichkeiten der Lehrer. Beispiel: Im Augenblick entstand 'ne ziemliche Unruhe im Kollegium und zwar über das zukünftige Sprachenprofil. (...) Es waren auch häufig Kommunikationsprobleme. Wir haben so viel zu tun im Augenblick, dass wir nicht ständig informieren können. Kurzum: wir haben uns dann alle zusammengesetzt: alle Fachkonferenzleiter und aus der Schulleitung noch jemand und haben klargestellt, dass es hier kein Konzept gibt, das auf kaltem Wege eingeführt werden soll. Wir haben dann gleichzeitig einen Ausschuss eingesetzt, der sich Gedanken macht über das zukünftige Sprachenprofil. Also im Grunde keine ernsthaften Konflikte. Natürlich gibt es ernsthafte Konflikte mit einzelnen Lehrern, die auch dazu führen, dass der eine oder andere Lehrer, es kommt kaum vor, aber das gibt's natürlich, auch die Schule verlässt. Beispiel: ich musste im vorletzten Jahr einen (Lehrer Anm. $d$ Verf.) sechs Wochen vor dem Abitur aus seinem Kurs nehmen, weil er nicht in der Lage war, diesen Kurs vorzubereiten. Ich hab' das erst spät mitbekommen, diese Sache hat dann natürlich zu einem großen Konflikt geführt, (...) der dann auch zu einen Personalgespräch zu mir
Identifikation mit Rolle als Lehrer und Schulleiter

Führungsverständnis 
kommen musste. Das meine ich auch mit Konflikten, und sie sind allerdings ganz, ganz selten."(37-57)

Herr Johann berichtet von Konfliktfällen innerhalb der Schule, seine Äußerungen spiegeln aber eine gelassene Einstellung wider, denn Konflikte auf der Ebene der Schulentwicklung schätzt er als lösbar und weniger ernsthaft ein, als Auseinandersetzungen auf der Unterrichtsebene des einzelnen Kollegen. Die Konfliktlösung bestand in der Entfernung des Kollegen aus dem Unterricht und wohl letztlich auch aus der Schule-, wie der weitere Interviewverlauf verdeutlicht.

„Ja, auf diese Weise: da sind die Schüler zu mir gekommen; dann musste ich allerdings ganz schnell handeln, und dann habe ich auch nichts weiter gemacht, als den - meinen Mitarbeiter, das mache ich immer, wir haben dienstags hier Schulleitungsbesprechung, also (ich) treffe also keine wichtigen Entscheidungen, ohne dass ich das hier mit meinen guten Mitarbeitern (kollegiale Schulleitungsmitglieder, Anm. d. Verf.) besprochen habe ich habe ausgezeichnete. Dann allerdings habe ich ihn (den Kollegen, Anm. d. Verf.) ganz schnell kommen lassen und ihm das mitgeteilt, und dann ist die Maßnahme schon am nächsten Tag vollzogen worden. (61-70)

Herr Johann beschreibt seine Intervention aufgrund der Schülerbeschwerden, da der zeitliche Druck (Abitur) gegeben gewesen sei, habe er schnell gehandelt, jedoch nicht ohne vorher sein Schulleitungsteam zu informieren. Herr Johann verweist darauf, dass er wichtige Entscheidungen immer gemeinsam mit seinen Mitarbeitern aus der Schulleitung trifft. Deutlich wird durch dieses Zitat, dass der Schulleiter mit einem akuten Problem in der Schule konfrontiert wurde, das zügig durch eine Entscheidung seinerseits gelöst werden musste. Sein Vorgehen deutet auf ein pragmatisches Verhältnis zu Vorschriften hin, denn er setzt sie ins Verhältnis zur vorgefundenen Wirklichkeit und er will die Situation für Schülerinnen und Schüler, die kurz vor dem Abitur stehen, klären (vgl. Rosenbusch, S. 55 in dieser Arbeit). Andererseits verweist diese Handlung des Schuleiters auch auf den Widerspruch von Entscheidungsdruck auf der einen Seite und einer abgesicherten Begründung auf der anderen Seite (Begründungsantinomie), aus der Perspektive des Kollegen bzw. des Kollegiums könnte sein Vorgehen ebenso als autoritär und unzureichend begründet gedeutet werden. Wichtig ist für Herrn Johann - und dies ist wichtig für seine Berufauffassung - die Rückversicherung in seinem Arbeitsteam, was auf eine reflexive Distanz auch unter Handlungsdruck hindeutet und seine Teamorientierung veranschaulicht.

Auf die Nachfrage, ob er sich denn selbst zuvor ein Bild vom Unterricht gemacht hätte, berichtet Herr Johann.

„Ich, - der Konflikt war ja nicht nur der. Ich hatte ihn ja im Unterricht schon besucht, weil ich in dem Unterricht nicht vom Fach bin, hatte ich meinen Stellvertreter, der auch Mathematiklehrer ist, dann gebeten, in den Unterricht zu gehen. Der hat Beratungsgespräche geführt, dies ist alles gelaufen, nur wie brisant sich der Konflikt dann auswirkte, das war nicht abzusehen. Sonst hätte der nie den Leistungskurs bekommen. Ja, er wird jetzt versetzt, er wird jetzt auf eigenen Wunsch zum nächsten Schuljahr versetzt. Das war auch nicht sein erster Konflikt, den er hatte, er hatte auch schon ähnliche Konflikte in der vorigen Schule, so ist das dann ja meistens, das wird ja immer weitergegeben. Ne? Wir haben ihm sogar geraten, an dieser Schule zu bleiben. Er hat natürlich Angst vor Gesichtsverlust. Er besteht aber auf eine Versetzung, und dann wird er versetzt. Ja, wie ich 
eben auch dem Dezernenten sagte: da halte ich es wirklich, muss ich mal brutal sagen, mit Sankt Florian. Ich bin für diese Schule verantwortlich. Das andere - die Lösung wäre, dass dieser Kollege aus dem Schuldienst ausscheidet. Er ist nicht geeignet für den Beruf! Er ist ein sehr netter Mensch, er ist fachlich gut, aber er kann sich in keiner Weise bei den Schülern durchsetzen. Und dies habe ich in meiner langen Schulleitertätigkeit, ich bin ja jetzt im 19. Jahre Schulleiter, das habe ich natürlich einige Male ähnlich gehabt. Das heißt, eigentlich müsste er eine Verwaltungstätigkeit oder 'ne Hochschultätigkeit haben. (...). Und auch der Junge wie gesagt - er ist fachlich sehr gut, aber er ist nicht für den Lehrerberuf geeignet."(76-94)

Die Interviewpassage lässt das zielorientierte und konsequente Vorgehen von Herrn Johann erkennen, er hat unterschiedliche Maßnahmen eingeleitet und die Unterrichtshospitation mit anschließender Beratung an den stellvertretenden Schulleiter delegiert, weil er diesen als fachkompetenter einschätzt. Der Schulleiter beleuchtet zwei Seiten dieses Konfliktes und ist in der Lage, sich in die Situation des Kollegen hineinzuversetzen: „Er hat natürlich Angst vor Gesichtsverlust" und rät dem Kollegen an der Schule zu bleiben, dennoch beurteilt Herr Johann die pädagogischen Qualitäten des Kollegen als unzureichend. Er differenziert also zwischen fachlichem Wissen und berufspraktischem Können. In der Diskussion über pädagogisches Professionswissen wird zwischen Theorie-, Praxiswissen und dem nötigen Kopplungswissen unterschieden, um das fachliche Wissen berufspraktisch nutzbar machen zu können (vgl. Fried, S. 73 in dieser Arbeit).

Herr Johann bezieht an dieser Stelle aber ebenso klar die Position des Schulleiters, der für seine eigene Schule verantwortlich ist. Er analysiert zwar die Lage des Kollegen und auch die Situation des Schulsystems mit den mangelnden Möglichkeiten anderer Karrierewege; der Kollege, der als Lehrer ungeeignet scheint, müsste eigentlich in eine "Verwaltungstätigkeit“ oder eine „Hochschultätigkeit“. Dessen ungeachtet - und dies ist bedeutungsvoll für die Berufsauffassung von Herrn Johann- distanziert er sich von dem Geschehen auf der Ebene der Schulbehörde, indem er seinen Handlungskontext unabhängig durch das Selbstverständnis des Schulleiters einordnet. In der Rolle als Schulleiter nimmt er die widersprüchliche Wirkung der staatlichen bzw. organisationalen Steuerungsmechanismen wahr, sie belasten inn aber nicht. Der Schulleiter umschreibt mit dem Bild des heiligen Sankt Florians treffend seine Situation, in dem Sinne von "Verschont mein Haus, zündet andere an“.

Herr Johann greift auf Praxiswissen als Teil des Professionswissens zurück - auf Grund von Erfahrungen des wiederholten Erlebens solcher Si- Berufserfahrung tuationen kann Herr Johann gelassener mit dieser Art von Konflikten umgehen. Es gelingt ihm in diesem Konflikt, die Verschränkung der Perspektiven so zu vollziehen, dass er unterschiedliche Wissensbestände für die Bewältigung des Berufes nutzbar machen kann.

„Also einerseits halte ich das für richtig, denn nur die Schule kann ja entscheiden, ob eine junge Frau oder ein junger Kollege hier zu diesem Kollegium und zu der Schule passt. Auf der anderen Seite ist die Terminierung unmöglich, denn die Einstellungsgespräche laufen während der mündlichen Prüfung immer mit zusammen. Wir haben dies Mal drei Stellen bekommen. Ich hatte während des Abiturs 150 Bewerbungen zu bearbeiten. Erst mal nur das. Äh, die Einstellungsgespräche in einer Zeit, wo ich überhaupt keine Zeit hatte, wir haben jeden Nachmittag hier Einstellungsgespräche geführt, jeden Nachmittag. 
Also die zeitliche Terminierung müsste anders sein. Das ist von den Schulen nicht zu schaffen. So. Das zweite ist die Konkurrenz der Gymnasien untereinander. Und zwar um die Mangelfächer. Wir hier - in einem Zentrum - haben es noch viel leichter. Aber welches Gymnasium in der Fläche kriegt noch einen Mathematik-Physik-Lehrer? Wir haben einen! Oder einen Musiklehrer? Das heißt, ich meine, es müsste in einer Zeit, wo in einzelnen Fächern nicht genügend Bewerber da sind, müsste es zentral koordiniert werden durch die Bezirksregierung unter Beteiligung der Schule. Sind mehr Bewerber da als Stellen, dann, meine ich, kann man es den Schulen überlassen. Aber dieses elendige Windhundrennen. Teilweise haben Schulen die schon eingeladen, als die Bewerbungsfrist überhaupt noch nicht abgeschlossen war. Also völlig illegitim, illegal sogar. Ja. Aber insgesamt halte ich das für sehr wichtig und sehr gut. Ob nun wieder alle Gremien beteiligt werden müssen: Gesamtkonferenz setzt einen Ausschuss ein, und dann muss ein Behindertenbeauftragter dazu und 'ne Frauenbeauftragte und der Personalrat und einer muss 'ne Frau sein - und das halte ich für etwas umständlich alles." (99-118)

Der Schulleiter geht in dieser Interviewpassage auf die Personaleinstellungen in der Schule ein. Herr Johann hält ein Mitspracherecht der Schule für richtig, dabei verweist er auf die Arbeitsbelastung durch Bewerbungsgespräche und die ungünstige zeitliche Terminierung seitens der Schulbehörde. Er spricht sich dort für eine „zentrale“ Koordinierung aus, wo es um die Besetzung von so genannten Mangelfächern geht, insbesondere um die Brisanz der Konkurrenz zwischen den Schulen herauszunehmen. Das Verfahren insgesamt könnte vereinfacht werden. Herr Johann verweist in dieser Interviewpassage auf die Notwendigkeit der Trennung Schulbehörde und Einzelschule und bezieht sich auf die Gesamtverantwortung der Schulbehörde für mehrere Schulen. Damit erkennt er die lineare Hierarchie des Schulsystems an.

„Wir haben einmal, glaube ich, so an die 60 Aufgaben übertragen bekommen. Das sind alles Sachbearbeitertätigkeiten. Also sagen wir mal, die Stellung hat sich überhaupt nicht geändert. Ob ich nun Stillzeiten ausrechnen darf oder Schwangerschaftsbeurlaubungszeiten etc. - das sind Sachbearbeitertätigkeiten, die kann einer mit A6, ein Sachbearbeiter viel schneller als ich. Das, also ich bin mit A16 völlig überbezahlt. Also gar nicht. Es ist geplant, die Schulleiter sollen Dienstvorgesetzte werden. Ja. Dienststellenleiter. Also ich hab eher den Eindruck, dass die Stellung des Schulleiters vielleicht formal gestärkt, aber faktisch geschwächt wird, weil wichtige Entscheidungen in die Konferenzen und in die Elterngremien verlagert werden. Beispiel: es ist zum Glück jetzt noch nicht so gekommen, - wir haben die Entscheidung, uns hier eine Stundentafel zu gestalten. Stundentafel 1 oder 2. Stundentafel. Stundentafel 1 ist die Profilierung in den Klassen 7, 8, 9. Wenn die Konferenz, die Gesamtkonferenz das beschließen, sie brauchen aber, so war es in dem letzten Entwurf, sie brauchen aber die Zustimmung des Schulelternrates. Und das finde ich 'ne Farce, wenn einer Gruppe, noch dazu den Nicht-Fachleuten, ein so großes Gewicht eingeräumt wird. Wobei wir hier eine sehr gute Zusammenarbeit hier an dieser Schule zur Elternschaft haben. Das ist also keine Klage über die Eltern, nur die Tendenz geht dahin, den Eltern als möglichen Wählern eben sehr viele Rechte einzuräumen. Aber die Katastrophe ist - die Frage des Elternrechts. Das habe ich jetzt wieder gemerkt." (144-162)

Herr Johann schätzt die Übertragung von vornehmlich Verwaltungsaufgaben auf den Schulleiter kritisch ein und erlebt dadurch in keiner Weise eine Aufwertung des Amtes. Im Gegenteil er geht davon aus, dass viele Aufgaben von Sachbearbeitern schneller und kostengünstiger erledigt werden könnten. Die begriffliche Präzisierung zwischen „Dienstvorgesetz-

Zunehmende Aufgabenvielfalt des Schulleiters 
ter" und "Dienststellenleiter" lässt erkennen, dass Herr Johann die formaladministrativen Aufgaben als ein Mehr an Arbeit empfindet, aber sein Status als Vorgesetzter dabei nicht erhöht wurde. Im Bereich der Entscheidungsfindung erlebt er eher eine Schwächung seiner Position. Die Beteiligung des Elternrates an der Abstimmung einer Stundentafel für die Schule hält Herr Johann für nicht gerechtfertigt, weil die Gruppe der Eltern keine Fachleute für die inhaltliche Gestaltung der Schule seien. Er betont zwar die gute Zusammenarbeit mit dem Schulelternrat, schätzt die Tendenz, den Eltern mehr Rechte einzuräumen, aber skeptisch ein, dies bezieht er vornehmlich auf das Mitspracherecht bei der Schulwahl. Seine Einschätzung erscheint zunächst plausibel, es ließe sich aber der Punkt „Eltern als mögliche Wähler" einer Schule in die Richtung verstärken, dass Angebot und Nachfrage den Bestand einer Schule sichern und eine inhaltliche ausdifferenzierte Positionierung der Schule nicht ohne Elternmitsprache vollzogen werden sollte.

„Ja. Ja. Ich nehme normal keine Hauptschüler auf. Ich kann das einfach pädagogisch nicht verantworten. Erstmalig hat das jetzt ein Russe durchgesetzt. Ich hab' den dann wieder weggeschickt. Er wollte von mir einen rechtsmittelfähigen Bescheid haben, den konnte ich ihm natürlich nicht geben. Der hat nach einem halben Jahr nur Fünfen und Sechsen. Da bin ich von überzeugt. Obwohl wir die Gutachten unter Verschluss halten. Die kennt keiner. Also da sind keine Vorurteile drin. Es wird auch keiner ausgegrenzt. Ja das, und, und - also, wie gesagt, seit der Schulreform bekommt die Schule viele Aufgaben - eigentlich hoheitliche sehe ich im Augenblick noch nicht. Und das Haupt-, ich unterstütze viele dieser Reformen, ich finde das gut mit den fünften und sechsten Klassen und auch andere Dinge. Aber wir bekommen keinerlei Unterstützungspersonal zusätzlich. Der Schritt z.B. jetzt, dass wir also die Lehrmittel ausleihen müssen - die Schule ist völlig überfordert. Denn die Stadt hat uns gleich erklärt: „Die Sekretärinnen dürfen aber nicht mitarbeiten. Das ist Sache des Staates. Und die Sekretärinnen sind Sache der Kommune." Also praktisch müssen wir - und stellen Sie sich mal vor, bei 1000 Schülern müssen wir ja 10.000 von Büchern wälzen, ohne dass das Sekretariat hier rangezogen werden darf. Ich meine, die Damen machen das ja, weil sie freundlich sind und gut, aber eigentlich dürften sie's nicht. Ne? Das heißt kurzum: jede Reform, die nur dazu dient, Geld zu sparen, ist verjubelt, sage ich mal." (164-180)

Der freie Elternwille, der dem Schüler den Besuch der Schule seiner Wahl zusichert, lässt sich aus Sicht von Herrn Johann nicht mit pädagogischen Grundsätzen vereinbaren. Die rechtliche Situation bindet den Schulleiter und verhindert ein Vorgehen in seinem Sinne, da er den Schulerfolg des Schülers für nicht erreichbar einschätzt. Der Schulleiter betont zwar, dass kein Schüler ausgegrenzt würde, dennoch lässt sich andersherum argumentieren, dass eben durch die Reaktion des Schulleiters doch eine Einordnung nach Gutachten stattfinden würde, wenn die Rechtsvorschrift nicht den Elternwillen verankern würde.

Herr Johann kommt in diesem Zusammenhang erneut auf die Aufgabenverschiebung von der Schulbehörde auf die Einzelschule zurück. Den Schulreformen steht er zunächst positiv gegenüber: „Ich finde das gut mit den fünften und sechsten Klassen“, er vermisst aber Unterstützung in den Aufgabenbereichen der Verwaltung und Organisation, z.B. Bücherausleihe, hier verweist er auf den Widerspruch der Aufgabenverteilung, denn Sekretärinnen dürfen laut Erlass ihre Arbeitszeit nicht damit verbringen. Herr Johann zweifelt an der Praktikabilität mancher Reformvorhaben, vornehmlich, wenn es um Sparen und nicht um eine 
nehmlich, wenn es um Sparen und nicht um eine sinnvolle Umgestaltung gehe.

Auf die Frage, ob es Gesamtkonferenzbeschlüsse gegeben hat, die gegen die Überzeugung von Herr Johann getroffen wurden, antwortet der Schulleiter, dass es solche Entscheidungen nicht gegeben hätte.

„Also, ich bin nun schon so lange Schulleiter, nein, gab's nicht, die gab es nicht. Es gibt natürlich, sagen wir mal, eine natürliche Schranke zwischen Schulleiter und Konferenz, also in dem Augenblick. Ich kann mich mit einzelnen hier sehr gut verstehen, aber in dem Augenblick, wo sie in einer Konferenz sind - also eine Konferenz entwickelt eine Eigendynamik. Im Augenblick der Konferenz waren sie der Meinung, die Schulleitung wollte irgendwas durchdringen, dann gehen sie natürlich auf Opposition. Das ist auch in Ordnung so. Nur wenn man lange genug Schulleiter ist, dann bekommt man seine Entscheidungen durch, im Stundenplan z.B. Spanisch einzuführen. Haben wir auch gemacht. (185-192)

Herr Johann geht auf seine Rolle in Konferenzen ein und verweist auf seine Position des Vorsitzenden. Der Schulleiter erlebt eine widerspruchsvolle Beziehungsstruktur, denn Kolleginnen und Kollegen, die ihm nah stehen, entwickeln durch die Eigendynamik der Konferenz ihm gegenüber als Leiter dieser Konferenz Distanz (vgl. Helsper, S. 69 in dieser Arbeit). Die „Eigendynamik“ und die „Opposition“ seines Kollegiums schätzt Herr Johann als normalen Prozess einer Konferenzkultur ein. Herr Johann bringt erneut seine lange Amtszeit als Schulleiter mit dem Gelingen der Entscheidungsdurchsetzung in Zusammenhang. Das in der Praxis durch Erfahrung generierte Wissen, welches in der Professionsdebatte als Interaktionswissen bezeichnet wird, gewinnt berufspraktische Bedeutung und dient Herrn Johann als Handlungssicherheit, denn er greift auf gewohnte Handlungsmuster zurück. Dennoch birgt diese Routine auch die Gefahr, dass eine kritische Haltung gegenüber der Gewohnheit ausbleibt.

Im weiteren Interviewverlauf bezieht sich Herr Johann auf die schwierige Stellung der Gesamtkonferenz, die über Grundsätze entscheidet. Seine Position erlebt Herr Johann als Gratwanderung.

„Es ist immer eine Gratwanderung. Die Konferenz entscheidet über alle grundlegenden wichtigen Fragen. So. Und über die laufenden entscheidet der Schulleiter. Und jetzt ist die Frage: „Was ist grundlegend?". Ist z.B. eine Zeitstruktur - ist das eine grundlegende Entscheidung? Wann fangen wir demnächst an? Wie groß sind die Mittagspausen? Ganz wichtige Fragen kommen da auf uns zu demnächst, weil wir auch auf zwei Schulen verteilt sind. Oder: "Ist das 'ne Sache einfach der Verwaltung und der Organisation?" Gut. Das muss man einfach auch mit einem bisschen Gespür mitbekommen, was hier möglich ist. Auf der anderen Seite gibt's Entscheidungen, wo ich gar nicht beteiligt bin, immer ausgenommen hier meine Mitarbeiter, mit denen wir alles entscheiden. Außenvertretung der Schule. Dann Aufnahme der Personalpolitik - welche Lehrer holen wir? usw., wobei da die Grenzen schon wieder fließend sind, wenn die neuen Stellen ausgeschrieben werden, das äh - letztendlich Beförderungsstellen. Also ich mache der Bezirksregierung den Vorschlag, wie die Stelle ausgeschrieben wird. Dieses allerdings mache ich unter Hinzuziehung des Personalrates. Aber die Entscheidung treffe ich dann. Aufnahme von Schülern - auch 'ne Entscheidung, die ich treffe. Und dann vor allen Dingen die Unterrichtsverteilung. Die ist ja dem Schulleiter per Schulgesetz zugewiesen, und da wird auch der Personalrat nicht beteiligt. Wird nicht beteiligt! Nein! Welcher Lehrer bekommt welche Klasse? Wobei, ich meine, natürlich, das ist eigentlich - äh die Fachkonferenzen sind
Situationsangemessene Einschätzung von Problemen 
beteiligt, die machen mir auf einer Liste Vorschläge, wer welche Klasse bekommen soll. Aber die letzte Entscheidung - das muss immer ja wieder - ich weiß ja heut noch nicht, was ich bekomme. Die letzte Entscheidung, die Entscheidung treffe ich dann. Da muss ich auch massiv eingreifen. Die Fachkonferenzen geben mir eine Liste, und da sind drei Kurse frei, weil sie keinen gefunden haben. Klar. Muss ich besetzen. Ob durch Kürzungen oder durch Mehrarbeit, das ist ja auch möglich. Also es gibt schon 'ne Reihe von Entscheidungen." (216-242)

Einzelne Bereiche hat der Schulleiter an sein Schulleitungsgremium delegiert, dennoch werden wichtige Entscheidungen von ihm allein getroffen, z.B. Beförderungsstellen unter Einbezug des Personalrates, Schüleraufnahme, und was aus seiner Sicht besonders wichtig ist, alle Entscheidungen der Unterrichtsverteilung. In diesem Beispiel bezieht er sich auf seine Amtsautorität, die es inm z.B. ermöglicht, Mehrarbeit anzuordnen. Herr Johann übernimmt insbesondere auf der internen Schulebene des allgemeinen Schulbetriebes seine Führungsrolle durch eine strategische Leitung im organisatorischen 'Kerngeschäft' der Unterrichtsverteilung, er legitimiert diese durch seine formale Amtsautorität. Wichtige Führungs- und Entscheidungsfunktionen delegiert er nicht, sondern er orientiert sich in seinem Handeln an einem führungsbetonten Bild des Schulleiters. Einem "typischen Konflikt" nämlich mit dem Problem der mangelnden Unterrichtsversorgung umzugehen, begegnen Herr Johann schöpft den Rahmen seiner Dienstbefugnisse aus, z.B. Mehrarbeit anzuordnen.

Auf die Frage wie er sein Verhältnis Kollegium einschätzt, antwortet Herr Johann, dass er die Zusammenarbeit als „partnerschaftlich“ erlebt.

\begin{abstract}
„Also ich äh - insgesamt meine ich, besteht zwischen Schulleitung und mir in besonderem Maße, ich würde eher sagen, ein partnerschaftliches, entkrampftes Verhältnis. [P] Ich versuche, viel ins Kollegium zu gehen Persönliche Kontakte habe ich zwar zu einigen, ich duze mich aber mit keinem. Also,, die Schulleitung duzt sich aber auch untereinander nicht. Das ist eher untypisch heute. Weiß ich nicht. An kleinern Schule, glaube ich, duzen sich die Schulleiter auch. [P] Aber ich denke, das könnte schon Problem machen, wenn ich mich, sagen wir mal, mit 10 Leuten duze und mit den anderen nicht. Also, ich halte das nicht für gut. Ich halte es auch nicht für gut, wenn Schulleiter aus dem eigenen Kollegium kommen. Ich bin von außen gekommen. Nein. Also insgesamt, ich werde jetzt 62 und hab auch vor, noch ein paar Jährchen zu machen, vielleicht ist das auch 'ne Antwort. Denn ich fühle mich hier nach wie vor sehr wohl. Und insgesamt, denke ich, insgesamt ist die - es sind nicht alles meine Freunde im Kollegium - natürlich, aber die Akzeptanz ist schon da und die gegenseitige Respektierung. Also ich gehe jeden Morgen gerne in die Schule. Ist vielleicht ein - aber ist wirklich wahr. Jeden Morgen." (244-263)
\end{abstract}

Am Bespiel „duzen“ veranschaulicht er die Nähe und Distanz, die er zu seinem Kollegium verspürt. Dadurch wird deutlich, dass er für sich eine Balance von Nähe und Distanz gefunden hat, so dass die Arbeitsbeziehung Schulleiter und Kollegium gewährleistet ist. Die Betonung der Partnerschaftlichkeit verweist auf ein wechselseitiges Vertrauensverhältnis, in seiner Stellung als Schulleiter erfährt er "Akzeptanz“ und "gegenseitige Respektierung“. Dies deutet darauf hin, dass sich die Zusammenarbeit und Führung sowohl im fachlichen als auch im persönlichen Dialog vollzieht und Herr Johann sich als Person angenommen und in seiner Autorität als Schulleiter akzeptiert erlebt. Er orientiert sich an einem koopertativdelegativen Führungsstil (vgl. S. 46 in dieser Arbeit). Dass Herr Johann
Treffen von Entscheidungen 
gern in die Schule geht, kann als Anzeichen für eine hohe Berufszufriedenheit und eine vorhandene Identifikation mit der Schule angesehen werden.

Auf die Nachfrage, was aus der Einschätzung des Schulleiters noch nicht gut klappt antwortet Herr Johann zunächst ausweichend.

„Ja, was nach wie vor nicht gut funktioniert, also erst Mal: die Schulleitung besteht ja insgesamt zur Zeit aus sechs Personen. Es sind noch fünf Studiendirektoren da. Vorher waren es sogar sechs Studiendirektoren. Auch die, die nachgekommen sind, wir sind eine sehr harmonische Gruppe. Eine kollegiale Schulleitung. Hier wird auch nicht abgestimmt, und wir haben auch keine Tagesordnung. Jeden Dienstag sitzen wir zwei Stunden zusammen - für uns sicher ein ganz wichtiges Datum. Wir frühstücken schön miteinander und besprechen die entscheidenden Dinge. So. Was sicher Probleme in jeder groBen Schule sind Kommunikationsprobleme. Wir haben jetzt zwar 'ne sehr schöne räumliche Schule, immer aktuell, aber aus meiner Sicht, was nicht klappt, das ist z.B., die Lehrer dazu zu bewegen, manche ihrer lästigen Pflichten auf sich zu nehmen. Aufsicht. Das sind immer dieselben die fehlen. Aufsicht ist erst mal wichtig. Da ist ein Schüler, der lässt da was liegen und 'ne Flasche fallen, und der Lehrer geht vorbei. Es gibt auch andere Lehrer, aber, aber - viele sind es, und die sagen kein Wort. So. Aufsicht übernehmen wird auch sehr großzügig von einigen gesehen. Pünktliches Beginnen des Unterrichts und Schließen. Das heißt, ich würde mir von vielen Lehrern auch einfach mehr Zivilcourage wünschen. Also das ist so die Hauptkritik. Trifft aber auf viele Schulen zu. Und das ist eben; die Belastung, die ist jetzt so groß. Wir haben jetzt innerhalb von sechs Tagen jetzt fast 400 Schüler aufnehmen müssen. Und Abitur hatten wir Montag, Dienstag auch noch usw. Das heißt, ich komme in diesen Phasen kaum ins Lehrerzimmer. Ich überprüfe dann schon und sage mit freundlichen Worten: "Sie haben doch Aufsicht. Haben Sie die vergessen?" Aber das geht meinen Kollegen und Kolleginnen genauso, es ist eigentlich nicht die Zeit dafür, die Lehrer auch noch zu kontrollieren." (268-288)

Herr Johann will zwar auf die Frage antworten, betont aber zunächst, dass Arbeit der kollegialen Schulleitung funktioniert. Seine Berufsauffassung orientiert sich an kooperativen Arbeitsformen und er schätzt die stabilen sozialen Kontakte.

Mit dem Verweis, dass die nachfolgend beschriebenen Probleme auf jede große Schule zutreffen, berichtet Herr Johann erst einmal allgemein von Kommunikationsproblemen, wobei hier wohl Aspekte der Informationsvermittlung gemeint sind. Die Größe der Schule spielt aus der Sicht von Herrn Johann eine Rolle, denn aufgrund der vielfältigen Aufgaben und der sich daraus ergebenden zeitlichen Belastung des Schulleiters und seiner Koordinatoren, würden der notwendige Kontakt und die regelmäßige Kontrolle des Alltagsgeschäftes versäumt werden. Herr Johann appelliert in dieser Interviewpassage an die Vorbildfunktion der Lehrkräfte- in dem Bereich Arbeitsaufgaben der Lehrkräfte, die einen verantwortlichen Umgang in der Schule sichern, hat Herr Johann klare Ordnungsvorstellungen und ist bereit, Versäumnisse zu benennen. Herr Johann nennt Zeitmangel als Belastung seines Arbeitsalltages, was er im weiteren Verlauf ausführt.

„Also es ist hauptsächlich die zeitliche Belastung. Ich habe jetzt, glaube ich, zum ersten $\mathrm{Mal}$ in meinem Leben den Eindruck gehabt, wo ich dachte: "Das schaffst du nicht." Erst mal die Einstellungen, und ich hatte jeden Nachmittag 'ne Veranstaltung. Das Abitur, und dann hatte ich 19 Leistungskurs-Arbeiten innerhalb kürzester Zeit dieses Jahr. Und jetzt mal ganz ehrlich, dass ich dachte: „Wann sollst du die 19 Leistungskursarbeiten korrigieren?" Und hab mir einen Plan gemacht, Wochenende: Sonntag früh drei, nachmittags
Stabile

Arbeitsbündnisse

Führungsverständnis 
drei, Sonnabend dann und so - d.h. ich hatte nie Wochenende und so jung ist man ja nun auch nicht mehr. Ja? Also so, das meine ich, dass es Situationen gibt, wo man wirklich, sagen wir mal, an die eigene Kraftgrenze kommt. Andererseits gibt es so viele schöne Situationen, und da schöpft man dann auch immer wieder Kraft. Auch gerade an dieser Schule. Das ist eine sehr, sehr lebendige Schule." (291-300).

Herr Johann empfindet gerade in diesem Schuljahr seine zeitliche Beanspruchung als zu hoch, da er kaum Regenerationszeiten erlebt. Obwohl er an seine „Kraftgrenze“ gekommen ist, überwiegen für Herrn Johann die vielen positiven Elemente seiner Arbeit. In der Berufsauffassung von Herrn Johann hat die Arbeit große Bedeutung und die hohe Identifikation mit seiner" Schule führen zu einer generellen Berufszufriedenheit, auf die Herrn Johann in einer sehr anstrengenden Arbeitssituation zurück greifen kann und inm die nötige Motivation zur Weiterarbeit gibt.

Auf die Frage, welchen zeitlichen Stellenwert die Schulentwicklungsarbeit in seinem Berufsalltag einnimmt und wie er seine Rolle darin wahrnimmt, antwortet er:

„ICh bemühe mich, sagen wir mal, die Stärken der Lehrerinnen und Lehrer zu fördern, zu aktivieren und versuche, viel zu delegieren. Schulprogramm hat bei uns ein ausgesprochen - auch ein richtiger programmatischer Kopf, Oberstudienrat, der hat das vorangetrieben. Der macht auch das Jahrbuch. Das ist auch gerade erschienen, was auch sehr schön ist. Ein sehr schönes Jahrbuch haben wir jedes Jahr. Nein, ich versuche, Lehrer zu gewinnen, die sich dann aktiv und möglichst auch zunächst eigenverantwortlich fühlen. Ich hätte das nicht gekonnt. Aber gerade Schulprogramm - und dann sogar sind wir drei Tage über ein Wochenende, nach Loccum, kennen Sie doch sicherlich nach Loccum gegangen und haben dann die Grundlagen für unser Schulprogramm gelegt. Ja, ja eine Gruppe von Eltern, Lehrern und Schülern. Das war ein faszinierendes Wochenende gerade weil wir gemeinsam etwas - also das hat so 'ne richtige Aufbruchstimmung erzeugt. Wir sprechen nur immer vom "Geist von Loccum" jetzt. Ja, natürlich, ich bemühe mich jetzt, das umzusetzen. Aber sagen wir mal an der Entstehung habe ich mich nur, habe ich mich hauptsächlich, äh, ich habe natürlich alle Entwürfe gelesen oder Vorschläge, aber ich sehe meine Rolle in erster Linie darin, den Lehrern, sagen wir mal, die Möglichkeiten zu geben, was zu bewegen Und den organisatorischen Rahmen, die Organisation - ich kann nicht alles anstoßen, und ich bin auch nicht der Oberlehrer hier der Institution, sondern es gibt viele Leute, viele Lehrer in vielen Bereichen, die sind in diesen Bereichen einfach besser als ich. Da haben wir, das ist ein Kapital, mit dem die Schule wuchern kann." (305-324)

Im Bereich der Schulentwicklung nimmt Herr Johann seine sonst eher führungsbetonte Rolle zurück und delegiert die Schulprogrammentwicklung sowohl an einen Oberstudienrat als auch an aktive Kolleginnen und Kollegen (vgl. S. 57 in dieser Arbeit). Der Aspekt der Partizipation tritt in den Vordergrund, als Träger der Entwicklungsprozesse sieht Herr Johann das Kollegium selbst an, er -und dies ist bedeutsam für seine Berufsauffassung - ist zwar beteiligt, aber vornehmlich Initiator des eigenverantwortlichen Handelns der Lehrerinnen und Lehrer. In seinem Organisationsverständnis greift Herr Johann auf Konzepte der Organisationsentwicklung zurück. In seinem Führungsverständnis orientiert sich in diesem Bereich an einem kooperativen und partizipativen Führungsstil, den Part der Schulprogrammentwicklung delegiert er und kann andere für ihre Leistungen anerkennen. Für Herrn Johann und dies ist ein wichtiges Element sei- 
ner Berufsauffassung steht der Erfolg der Schule als Ganzes im Mittelpunkt.

Auf die Frage nach den zukünftigen Zielen für seine Schule entgegnet Herr Johann, dass zunächst die Integration der 5. Jahrgänge von Bedeutung ist.

„Ja. Also das Wichtigste ist erst mal die Integration der neuen 5. und 6. Klassen voranzubringen. $<h m>$ Und das eben an zwei Schulen. Wir bekommen noch eine zweite Schule dazu, die liegt $500 \mathrm{~m}$ entfernt und ist aber eine sehr große Schule. Und jetzt das Problem: erstens diese Jahrgänge, ich habe die noch als junger Lehrer erlebt, für mich ist das nicht so neu, aber für die meisten. Viele unserer Lehrer müssen umdenken. Die müssen einfach umdenken. Es ist nicht mehr nur lehrerzentrierter Unterricht 'ne Stunde lang. Vielleicht an der Tafel stehen. Dafür bin ich nicht. Das geht nicht. Das kann ich mit meinem 13. Jahrgang machen. Die kennen das auch so. Gut. Also ganz wichtiges Ziel: Integration der Schülerinnen und Schüler, mindestens aber genauso wichtig ist die Integration der neuen Kolleginnen und Kollegen, die werden kommen. Was wir bekommen, weiß ich nicht. Und die werden offensichtlich uns wieder einige abziehen. Also insgesamt so an die 18 neue Lehrer werden kommen. Und zwar eben aus einer anderen Schulform. Und das wird einfach schwierig. Es wird eben so, dass ich auch eine Zahl von 95 Lehrern anstelle, verteilt auf zwei Schulen, also die Lehrer sehen sich nicht teilweise. Also das ist die Hauptaufgabe, die ich im nächsten Jahr hinkriegen muss, sonst halte ich das für eine schlimme Entwicklung. Also so für's nächste Jahr - was ich mir vorgenommen habe schon seit einem Jahr plane ich das, ich will auf eine Tagung in Jena - „Lehrerberatung“, also um die einzelnen Lehrer besser zu beraten. Ich möchte das anfangen mit den Fachkonferenzleitern. Also so 'ne Art von Personalgespräch, Personalrat kann zugezogen werden - muss aber nicht. Die Tagesordnung wird dann festgelegt, der Zeitpunkt, und es gibt hinterher ein Protokoll. Es wird Unterstützungsbedarf festgehalten, also so was wie 'ne Zielvereinbarung, ohne dass man das nun so technokratisch macht. Also das wären sozusagen wichtige Ziele für mich." (344-363)

Die Hauptaufgaben für das nächste Schuljahr sieht Herr Johann darin, die Schulstrukturreform gewinnbringend umzusetzen. Dies bedeutet für inn neben der Integration der jüngeren Schülerinnen und Schüler vornehmlich die Einbindung neuer Kolleginnen und Kollegen in die Schule. Herr Johann ist sich bewusst, dass dies für seine Schule und für inn eine besondere Herausforderung darstellt, die er aktiv lenken möchte.

Für sich persönlich möchte er das Thema „Zielvereinbarungsgespräche“ angehen, dazu will er an einer Fortbildung teilnehmen. Unterschiedliche Aspekte werden durch dieses Zitat deutlich, zunächst beweist Herr Johann Überblick und setzt Prioritäten, wie er es ja auch zu Beginn des Interviews als wichtige Aufgabe umschrieben hat. Er erlebt eine von außen gesetzte Umgestaltung der Schule als persönlichen Auftrag und ist bereit, Verantwortung für das Gelingen bzw. Misslingen zu tragen. Des Weiteren nimmt er trotz seiner vielen Dienstjahre neue Themen wie „Zielvereinbarungsgespräche" auf und will sich selbst weiter entwickeln. Sein Hinweis darauf, deutet an, dass diese Gespräche nicht „technokratisch“ abgehandelt werden sollen, sondern für inn Lehrerberatung und Unterstützungsbedarf im Vordergrund stehen. Herr Johann sieht seine Aufgabe in einer zielorientierten Führung vornehmlich im Bereich der Personalpflege und Personalentwicklung (vgl. S. 45 in dieser Arbeit). 
„Also die Lehrerversorgung ist die entscheidende Schulreform. Wenn wir gut gebildete, gut ausgebildete Lehrkräfte haben, das ist das Wichtigste - und nicht irgendwelche Richtlinien oder zentralen Prüfungen. Das ist alles unwichtig. Auch nicht die renovierten Räume, die wir jetzt kriegen. Die gut ausgebildeten Lehrer in überschaubaren Klassen, das ist das Entscheidende. Mehr brauchen wir gar nicht“.(404-409)

In den Ausführungen von Herrn Johann wird sichtbar, dass seine Berufsauffassung stark auf das innere Geschehen in der Schule ausgerichtet ist, seine Zielsetzungen orientieren sich an dem Gelingen des pädagogischen Alltags und darin sieht er auch - wie bereits herausgearbeitet - eine seiner Hauptaufgaben. Implizit wird hier seine Kritik an anderen Entscheidungen der Schulreform deutlich.

„Also ich bemühe mich, ich kontrolliere auch Aufsicht. Natürlich wenn ich feststelle, ein Lehrer hat die Aufsicht nicht gemacht, dann stell'ich den nicht öffentlich zur Rede, sondern dann bitte ich ihn hier zu einem Gespräch und versuche ihm das, sagen wir mal ruhig, in entspannter Atmosphäre klarzumachen. So dass er auch, dass er auch bereit ist, gern weiter in der Schule mitzuarbeiten. Das heißt, manchmal gibt es so Sachen, ich sage das nach der Arbeit auch schon zu dem Lehrer: „Oh, da müssen wir aber mal ein bisschen dafür sorgen, dass die Aufsichten gemacht werden, denn die einen machen sie, Sie machen sie nicht." Und dann mache ich ihm das auch klar. Und das muss das Kollegium überhaupt nicht wissen, mit wem ich hier Gespräche führe. Wenn ich merke, ein Lehrer kommt viel zu spät zum Unterricht, dann werde ich, ich versuche, Konflikte möglichst unter vier Augen zu klären, keinen hinzuziehen. Allerdings bei Konflikten, wie damals mit dem Mathe-Lehrer, da bitte ich dann schon meinen Stellvertreter dazu. Also möglichst, und möglichst alle Konflikte im Haus zu lösen. Also die Dezernenten grundsätzlich nicht einzuschalten. Das können wir alles allein. Wir haben einen Beratungslehrer, mit dem spreche ich dann das eine oder andere Mal auch, wenn es um Konflikte zwischen Lehrern geht. Die gibt es ja dann auch. Am schlimmsten sind eigentlich Konflikte, sagen wir mal, zwischen Sekretärinnen und Hausmeister, die Sekretärinnen haben manchmal Schwierigkeiten mit dem Hausmeister, der übrigens ein sehr guter ist, aber es gibt durchaus Lehrer, denen gegenüber er sich im Ton vergreift. Und die Lehrer dann kommen: „Jetzt müssen Sie aber mal von Ihrem Weisungsrecht Gebrauch machen." Weisung. Den Begriff Weisung benutze ich auch nicht. Ich sage dann zu jemanden: „Bitte, ich habe die Weisung, das ist 'ne Dienstanweisung, also ich versuche, es möglichst freundlich zu machen, ist $n$ bisschen auch anstrengend manchmal." (451-471)

Herr Johann geht am Ende des Interviews erneut auf den Umgang mit Konflikten ein. Bei all seinen Interventionen ist inm der persönliche Umgang miteinander wichtig, dabei beruft er sich stärker auf persönliche Autorität als auf formale Weisungsrechte.

Herr Johann reflektiert Unterstützungsbedarf und wägt ab, welche Dinge sich informell klären lassen. Wichtig ist ihm als Schulleiter möglichst viele interne Konflikte auch intern zu lösen, den Dezernenten will er nicht dabei haben. Selbstständige Konfliktbearbeitung betrachtet er als Zeichen von Autonomie und Kompetenz. Die lineare Hierarchieebene wird von Herrn Johann zwar jeder Zeit anerkannt, er betrachtet seine Rolle als Schulleiter der Schule aber als weitgehend autonom innerhalb des Organisationsgefüges. In der Professionsdiskussion wird die Einbindung in eine Organisationsratio und das gleichzeitige Streben nach professioneller Autonomie als Belastungsmoment beschrieben. Herrn Johann scheint es gelungen zu sein, eine professionelle Unabhängigkeit in den gegebenen Organisationsstrukturen zu entwickeln (vgl. S. 67 u. 74 in dieser Arbeit). In seiner 
beruflichen Identität als Schulleiter versucht Herr Johann neben zeitweise notwendigen führungsbetonten Vorgehensweisen vor allen Dingen eine kooperative und mitmenschliche Umgangsweise anzustreben. Dass dies auch ein Belastungsmoment für inn darstellen kann, wird aus der letzten Äußerung deutlich: „,ist auch anstrengend manchmal“.

Bei Herrn Johann lässt sich eine Berufsauffassung beschreiben, die von einer positiven Identifikation mit der Rolle des Schulleiters und mit „seiner eigenen Schule" geprägt ist. In den Auseinandersetzungen mit den alltäglichen Berufsaufgaben beruft sich Herr Johann mehrfach auf Praxiswissen. Insbesondere die langjährige Berufserfahrung des Schulleiters haben zu einer gewissen Gelassenheit in Konfliktsituationen geführt hat.

In seinem beruflichen Selbst erlebt Herr Johann Anerkennung und Zuspruch, er hat unterstützende Arbeitsbeziehungen aufgebaut und ist sich bewusst über die Problematik Nähe und Distanz. Die Erfolge der Schule kann er einerseits dem Kollegium zusprechen und andererseits für sich selbst als Motivation zur Weiterarbeit nutzen.

In seiner Berufsauffassung greift Herr Johann auf ein Organisationsverständnis zurück, welches sich vornehmlich an der inneren Gestaltung der Schule orientiert. Herr Johann delegiert Schulentwicklungsaufgaben und sieht seine Aufgabe darin, förderliche Arbeitstrukturen zu organisieren. Herr Johann reflektiert und analysiert unterschiedliche Prozesse in der Schule und kann situationsangemessen reagieren, dabei nutzt er den Gestaltungsraum, z.B. Schulprogramm, ebenso, wie er begrenzende Strukturen akzeptiert und sich mit innen arrangiert, z.B. Unterrichtsversorgung. Der Schulleiter beweist Überblick und ist in der Lage, Prioritäten im Sinne der Schule als Ganzes zu setzen.

In seinem Führungsverhalten unterscheidet Herr Johann situationsangemessen, wann ein eher führungsbetontes Vorgehen wichtig ist, z.B. innerhalb der Unterrichtsverteilung, und wann ein stärker kooperativpartizipativer Führungsstil förderlich ist. Für Herrn Johann sind Personalpflege und Personalentwicklung wichtige Führungsaufgaben, die er wahrnimmt. Die Betonung der Führungsaspekte Fördern und Beraten auf der Ebene des Kollegiums verweisen auf die prosoziale Dimension eines kooperativen Führungsstils, die Aussage „im nächsten Leben werde ich Seelsorger", lässt sich abschließend so erklären, dass der Schulleiter rückblickend das Hauptgewicht seiner Tätigkeit deutlich im Bereich Mitarbeiterführung sieht und in vielen Bereichen der väterliche- partnerschaftliche Vorgesetzte ist.

In seiner Berufsauffassung kann Herr Johann als bodenständiger Seelsorger umschrieben werden, weil er seinen Aufgabenbereich vornehmlich in der Betreuung von Kolleginnen /Kollegen und Schülerinnen/ Schülern sieht. Er richtet sich in seiner Handlungsorientierung eher an inneren Prozessen der Schule aus und betrachtet äußere Einflüsse gelassener, hier greift er auf den Boden seiner Erfahrung zurück und dadurch gelingt es inm, bestimme Entwicklungen distanzierter zu analysieren.

Im Unterschied zu anderen Fallbeispielen kommt hier dem Aspekt Berufserfahrung eine bedeutende Rolle zu, denn Herr Johann beruft sich sowohl 
im Rahmen von Konfliktbewältigungen als auch im Treffen von Entscheidungen auf seine langjährige Erfahrung als Schulleiter. Dies zeigt sich in einem pragmatischeren Umgang mit Erlassvorgaben, einer klaren und unaufgeregten Durchsetzung von Entscheidungen. Auch für Herrn Johann spielen Arbeitsbündnisse vor allen Dingen innerhalb der kollegialen Schulleitung eine wichtige Rolle, er erlebt diese als stabil und unterstützend. Im Unterschied zu Herrn Müller und Frau Schmidt scheint Herr Johann viel gelassener mit Anforderungen der Neuerung oder Ressourcenverknappung umzugehen, er sucht praktikable Lösungen und beruft sich auf routinierte Handlungserfahrungen. Im Gegensatz zu Herrn Bach und auch Herrn Krause erlebt Herr Johann die Bewältigung der Alltagspraxis als überwiegend positiv besetzt und „machbar“. Herr Johann hat sich stark mit „seiner" Schule identifiziert und betont ausdrücklich, dass er gern Schulleiter dieser Schule ist.

\section{Schule leiten in der beruflichen Praxis}

Qualitative Forschung gewinnt ihre Gültigkeit durch die Untersuchung von subjektiven Deutungsmustern des alltäglichen Erlebens und Handelns. Anstatt allein von Konzepten oder Theorien auszugehen, wird durch ein induktives Verfahren versucht, aus dem empirischen Material selbst neue Erkenntnisse über den Untersuchungsgegenstand zu gewinnen. Die Auswertung der Einzelfälle hat die Aufgabe, einerseits der Besonderheit des Einzelfalls Rechnung zu tragen und andererseits dem Anspruch an Verallgemeinerbarkeit gerecht zu werden.

Die im empirischen Material durch die Einzelfallanalyse herausgearbeiteten Berufsauffassungen skizzieren Unterschiede in der Art und Weise, wie die Akteure und Akteurinnen bei ihren Auseinandersetzungen in dem beruflichen Kontext und bei den beruflichen Anforderungen inhaltlich Bezug nehmen auf führungsrelevantes Wissen, unterschiedliche Organisationsverständnisse und Formen der pädagogischen Professionskompetenz.

Aus dem vorliegenden empirischen Material konnten aufgrund der Ergebnisse auf der Einzelfallebene und der vergleichenden Auswertung unterschiedliche Variationen von Berufsauffassungen rekonstruiert werden. ${ }^{238}$ Es gibt Anhaltspunkte für fünf Variationen von beruflichen Auffassungen im Hinblick auf die Kombination der drei Dimensionen Führungsverhalten, Organisationsverständnis und Professionskompetenz beschreiben. Im Rahmen der letztgenannten Dimension und in der abschließenden Verdichtung wird die subjektive Bewertung dargelegt. Zunächst werden diese Variationen von Haupttypen skizziert und auf der Basis der gewonnenen Erkenntnisse wird dann die Berufsauffassung von Schulleiterinnen/Schulleitern und deren Bedeutung für die Schulentwicklung eingeschätzt und mit programmatischen Zielvorstellungen verglichen.

\subsection{Muster von Berufsauffassungen}

Unter beruflichen Auffassungen verstehe ich im Kontext dieser Untersuchung die berufliche Selbsteinschätzung, die Urteile und Einstellungen bezüglich der Berufsaufgaben und die Handlungsorientierungen, die die Akteurinnen und Akteure in der Auseinandersetzung mit ihrem beruflichen

${ }^{238}$ Vgl. Flick 2002, 338 
Kontext entwickelt haben. Vor dem Hintergrund dieser Berufsauffassung begegnen die Akteure ihren beruflichen Anforderungen in der Praxis, dass dies sehr unterschiedlich geschieht, konnte die Einzelfallanalyse zeigen. Insgesamt lassen sich fünf unterscheidbare Haupttypen skizzieren, die ich jetzt noch einmal auf einer abstrakteren Ebene darstellen werde. Es lassen sich im empirischen Material auf den Ebenen des Führungsverhaltens, des Organisationsverständnisses und der pädagogischen Professionskompetenz bestimmte Muster der Selbsteinschätzung und Handlungsorientierung aufzeigen. Das Charakteristische dieser "Muster von berufsbezogenen Auffassungen" besteht in einer Kombination von Merkmalen, die kennzeichnend sind für die unterschiedliche Art der Auseinandersetzung mit beruflich vorgefundenen Bedingungen, den beruflichen Anforderungen und der Verarbeitung beruflicher Erfahrungen.

\section{Typ 1: Professionelle Berufsauffassung}

Berufsauffassungen bezogen auf

\section{die Dimensionen des Führungsverhaltens}

- die Akteure verfügen über ein breites und flexibles Repertoire an Führungsverhalten, sie sind in der Lage sich entsprechend der situativen Anforderungen verhalten zu können. Die Handlungsorientierung ist darauf ausgerichtet, der Komplexität der Aufgaben gerecht zu werden und gleichzeitig die wahrgenommenen Widersprüchlichkeiten der Aufgaben auszubalancieren;

- die Akteure können unterschiedliche Führungsstrategien einsetzen, weil sie ein breites Wissen und vielfältige Führungskompetenz besitzen;

- das Führungsverständnis orientiert sich langfristig an der Sicherung eines "guten" Unterrichts und die Akteure richten ihre Handlungen danach aus;

- die Akteure reflektieren die Besetzung von Teams, delegieren Aufgaben und übertragen aktiv Kolleginnen und Kollegen Verantwortung.

die Dimensionen des Organisationsverständnisses

- die Akteure verfügen über ein umfassendes Wissen über die strukturellen Bedingungen der Organisation Schule. Unterschiedliche Interessengruppen in der Organisation Schule werden angemessen eingeschätzt und in Beziehung gesetzt zu den organisationellen Bedürfnissen;

- die Akteure agieren auf der administrativen, politischen und pädagogischen Ebene und haben die Schule als Ganzes im Blick.

- strukturelle und normative Vorgaben werden so weit wie möglich im Sinne einer Ressourcensicherung genutzt;

- die Akteure reflektieren vorhandene Strukturen kritisch und können den Handlungskontext- weil sie inn als widersprüchlich erkennennutzen, ihr Handeln ist an Veränderung ausgerichtet;

- Schulentwicklung hat Bedeutung im Organisationsverständnis und wird durch eine aktive Gestaltung der Organisationsstrukturen begleitet. 
die Dimensionen der Professionskompetenz

- im Zentrum der beruflichen Auffassungen steht ein positives berufliches Selbstkonzept, das von einer hohen Identifikation mit der Berufsrolle Schulleiterinnen und Schulleiter geprägt ist;

- die Handlungsorientierungen richten sich an einer Wahrung der professionellen Autonomie gegenüber den Erfordernissen der Organisation aus, d.h., strukturelle Bedingungen werden reflektiert und im Rahmen des Handlungskontextes wird nach individuellen Handlungsmöglichkeiten gesucht;

- die Akteure zeigen reflexive Distanz sowohl gegenüber Prozessen der Schule und den als widersprüchlich eingeordneten Organisationsstrukturen als auch gegenüber ihrem eigenen Handeln.

Bei diesem Typ der Berufsauffassung kommt es zu einer konstruktiven Handlungsorientierung, die sowohl eigene berufliche Ziele als auch unterschiedliche Interessenpositionen innerhalb der Schule berücksichtigt. Dabei gelingt es den Akteuren, Widersprüche auf der Ebene der Organisation Schule zu analysieren und auf der Grundlage eines positiven beruflichen Selbstkonzeptes Prozesse innerhalb der Schule aktiv gestaltend zu beeinflussen.

\section{Typ 2: Resignative Berufsauffassung}

Berufsauffassungen bezogen auf

\section{die Dimensionen des Führungsverhaltens}

- die Akteure erleben ihre eigene Führungsrolle als wenig einflussreich, das eigene Rollenverständnis orientiert sich an der berufsbiographisch erworbenen Position des Lehrers/ der Lehrerin;

- die Akteure können kaum Führungsstrategien einsetzen, da sie von einem geringen Erfolgserleben bei sich selbst und anderen ausgehen;

- die Handlungsorientierung ist darauf ausgerichtet, sozialemotionale Anerkennung zu erhalten;

- das Führungsverständnis orientiert sich ausschließlich an dem Ziel "guter Unterricht“, den Akteuren fehlen die nötigen Führungskompetenzen, um eine langfristige Zielerreichung zu ermöglichen;

- den Akteuren gelingt es nicht, unterschiedliche und widersprüchliche Anforderungen anzuerkennen und ihre Handlungsorientierung situativ daran auszurichten.

die Dimensionen des Organisationsverständnisses

- die Akteure agieren hauptsächlich auf der pädagogischen Ebene und haben die Organisation Schule mit ihren strukturellen Bedingungen nicht im Blick;

- Hindernisse und Konflikte des beruflichen Altags werden als subjektiver Misserfolg gedeutet und können nicht in Bezug zum Handlungskontext der Organisation gesetzt werden;

- die Rahmenbedingungen der Organisation Schule werden als wenig gestaltbar wahrgenommen und eigene Handlungsräume können nur bedingt für die Veränderung nutzbar gemacht werden; 
- Schulentwicklung- hier speziell die pädagogische Schulentwicklunghat Bedeutung im Organisationsverständnis, die Akteure sind überzeugt von der Notwendigkeit einer Verbesserung des Unterrichts gleichzeitig zweifeln sie aber an dem Gelingen von Umsetzungsprozessen.

die Dimensionen der Professionskompetenz

- die berufliche Auffassung ist durch ein negatives Selbstkonzept geprägt, in der Selbsteinschätzung der Akteure spiegelt sich eine geringe Selbstwirksamkeitsüberzeugung wider, gleichzeitig wird eine ausbleibende Anerkennung in der Fremdbewertung wahrgenommen, dies führt zu einer emotionalen Belastung;

- die Akteure verfügen über ein kognitives Interaktionswissen, sie können Prozesse der Schule analysieren, aber in ihrer Handlungsorientierung dieses Wissen und ihre Kompetenzen nicht einbringen;

- die Akteure zeigen keine Distanz gegenüber ihrem eigenen Handeln und reflektieren Prozesse der Schule sowie Organisationsstrukturen ausschließlich vor dem Hintergrund der individuellen Handlungsebene.

Bei diesem Typ der Berufsauffassung besteht der Unterschied zu dem vorhergehenden Typ vor allem darin, dass die Akteure sich nicht an einem positiven beruflichen Selbstkonzept orientieren. Die vorwiegend emotionale Verarbeitung von Konflikten und die Diskrepanz zwischen eigenen Wünschen und dem vorgefundenen Handlungskontext werden zum belastenden Moment der Berufsauffassung. Eine subjektiv hohe Bedeutsamkeit der Arbeit und ein dem gegenüberstehendes niedriges Erfolgserleben führen zu einer Resignationstendenz.

\section{Typ 3: Programmatische Berufsauffassung}

Berufsauffassungen bezogen auf

\section{die Dimensionen des Führungsverhaltens}

- die Akteure verfügen über ein führungsrelevantes Wissen, welches einen hohen Stellenwert innerhalb ihrer Berufsauffassung einnimmt, sie definieren sich in erster Linie als Führungsperson;

- in ihrem Führungsverhalten orientieren sich die Akteure einerseits an einem kooperativen Führungsstil und andererseits berufen sie sich auf ihre hierarchisch zugewiesene Position, die Akteure finden aber keine Balance zwischen diesen Verhaltensweisen und können diese nicht situationsangemessen nutzen;

- den Akteuren fällt es schwer, unterschiedliche Auffassungen über Führung anzuerkennen, da sie selbst ihr Führungsverständnis für das richtige halten und die notwendigen Aushandlungsprozesse innerhalb der Schule als belastend erleben;

- die Akteure orientieren sich in ihrem Führungsverhalten an einem normativen Leitbild.

\section{die Dimensionen des Organisationsverständnisses}

- die Akteure verfügen über Wissen über die strukturellen Bedingungen der Organisation Schule und nutzen diese Strukturen so weit 
wie möglich für die Erfüllung ihrer Aufgaben, die begrenzenden Strukturen der Organisation werden auf der politischen Ebene festgestellt;

- Schulentwicklung wird als Möglichkeit einer eigenen Profilierung der Einzelschule wahrgenommen und aktiv selbst initiiert;

- die Akteure können unterschiedliche Interessenpositionen einschätzen, es gelingt innen aber nicht, vermittelnd zwischen diesen Positionen zu agieren.

die Dimensionen der Professionskompetenz

- eine hohe Identifikation mit der Berufsrolle als Schulleiterin/ Schulleiter prägt das Selbstkonzept der Akteure, die eigenen Kompetenzen werden positiv eingeschätzt;

- die eigene Berufsauffassung und die vorgefundenen Handlungsbedingungen können teilweise nicht in Einklang gebracht werden, es fehlt eine distanzierte Reflexion dieser Widersprüche;

- den Akteuren fehlt eine kritische Distanz gegenüber ihren eigenen Handlungsorientierungen, das übernommene berufliche Leitbild und die in der Berufspraxis wahrgenommenen ungleichen Berufsauffassungen werden als Diskrepanzerfahrung verarbeitet und als belastend erlebt.

Bei diesem Typ der Berufsauffassung kollidieren ein positives Selbstkonzept und eine hohe Einschätzung eigener Kompetenzen mit den vorgefundenen Bedingungen der Praxis. Vor allen Dingen die wahrgenommene Berufsauffassung anderer Akteure des Handlungskontextes wird als widersprüchlich zu den eigenen Auffassungen erlebt und als „defizitär" bewertet, was ein Aushandeln unterschiedlicher Umgangsweisen mit Prozessen der Schule verhindert. Es gelingt den Akteuren zu wenig, unterschiedliche Interessenpositionen zu akzeptieren und diese integrativ zu nutzen.

\section{Typ 4: Skeptische Berufsauffassung}

Berufsauffassungen bezogen auf

die Dimensionen des Führungsverhaltens

- die Akteure orientieren sich in ihrem Führungsverhalten an betriebswirtschaftlichen Führungskonzepten, die anderen Bedingungen des vorgefundenen Handlungskontextes stehen im Widerspruch dazu;

- die Akteure versuchen den unterschiedlichen Anforderungen ihres Tätigkeitsfeldes gerecht zu werden, nehmen dabei die widersprüchlichen Aufgaben wahr und führen diese auf die begrenzenden Strukturen zurück;

- die Führungsrolle als Schulleiterin/Schulleiter wird in ihrer formalen Verankerung im Schulsystem als zu schwach eingeschätzt, insbesondere der rechtliche Rahmen der Kontrollfunktion wird als Einschränkung des eigenen Handlungsfreiraums bewertet.

die Dimensionen des Organisationsverständnisses

- $\quad$ die Akteure verfügen über Wissen bezüglich der strukturellen 
Bedingungen der Organisation Schule und üben Kritik an den organisatorischen Restriktionen;

- die Balance zwischen der formalen Struktur der Schule und dem eigenen Handlungsspielraum kann nicht zufriedenstellend gehalten werden, es entsteht ein belastendes Spannungsverhältnis zwischen Autonomie und Dependenz

- die Realisierungsmöglichkeiten von Schulentwicklungsprozessen durch den Schulleiter/ die Schulleiterin werden unter den gegebenen Bedingungen von den Akteuren als nicht ausreichend angesehen;

- der organisatorische Rahmen der Schule wird als paradox interpretiert und die eigenen Gestaltungsmöglichkeiten werden gering eingestuft

die Dimensionen der Professionskompetenz

- die berufliche Auffassung ist von einem positiven Selbstkonzept geprägt, dies zeigt sich durch eine selbstsichere Einschätzung der eigenen Kompetenzen, eine Identifikation mit der Rolle Schulleiter/ Schulleiterin wird abgelehnt

- die Akteure erleben eine Unvereinbarkeit zwischen der eigenen Berufsauffassung und der Fremdsteuerung durch die gegebene Organisationsstruktur der Schule

- die Akteure reflektieren kritisch Prozesse der Schule und Systemzusammenhänge der Organisation, eine distanzierte Betrachtung des eigenen Handelns im Kontext dieser Organisation gelingt den Akteuren nicht, dies führt zu einer Verschiebung von Verantwortlichkeit nach außen und verhindert eigene Einflussmöglichkeiten.

Bei dieser Berufsauffassung stehen sich berufliche Überzeugungen und die Handlungsrationalität der Organisation unvereinbar gegenüber, die eigenen beruflichen Ziele werden als konträr zu den Organisationszwängen erlebt. Trotz eines positiven Selbstkonzeptes gelingt es den Akteuren nicht, die Widersprüche zwischen den eigenen Berufszielen und den strukturellen Bedingungen des Handlungskontextes Schule auszubalancieren.

\section{Typ 5: Pragmatische Berufsauffassung}

Berufsauffassungen bezogen auf

\section{die Dimensionen des Führungsverhaltens}

- die Akteure können flexibel und situationsangemessen ihr Führungsverhalten einsetzen, dabei hat die zugewiesene Amtsmacht Bedeutung;

- das Führungsverständnis orientiert sich an der Gestaltung eines schülerorientierten Unterrichts und einer schülerfreundlichen Schulkultur, die Interessenposition der Kolleginnen und Kollegen wird unter diesem Blickwinkel eingeschätzt;

- die Handlungsorientierung ist an den inneren Prozesse der Schule ausgerichtet, die Komplexität der Aufgaben wird akzeptiert und in der eigenen Auseinandersetzung mit den Berufsanforderungen reduziert durch klare Prioritätensetzungen und einem gelassen Umgang mit Konflikten in der Alltagspraxis; 
- die Akteure delegieren Aufgaben aus dem Bereich der Schulentwicklung.

die Dimensionen des Organisationverständnisses

- die Handlungsorientierung der Akteure ist darauf ausgerichtet, die innere Ablauforganisation der Schule optimal zu gestalten;

- die Akteure agieren vornehmlich auf der pädagogischen und administrativen Handlungsebene, politische Dimensionen werden als gegeben akzeptiert;

- Schulentwicklung erscheint den Akteuren dort wichtig, wo sie zur Profilierung des eigenen Schulstandortes dient.

die Dimensionen der Professionskompetenz

- im Mittelpunkt der beruflichen Auffassung steht ein positives berufliches Selbstkonzept, das von einer hohen Identifikation mit der Berufsrolle Schulleiterin/ Schulleiter geprägt ist. Ein wichtiges Element der Berufsauffassung ist die bejahende Einstellung gegenüber der eigenen Schule. Beides führt zu einer hohen Berufszufriedenheit;

- Die Akteure zeigen reflexive Distanz sowohl gegenüber den Prozessen der Schule als auch gegenüber den Organisationsstrukturen und wahrgenommen Einschränkungen;

- Die Akteure beziehen sich innerhalb ihrer Handlungsorientierung auf eine langjährige Berufserfahrung, die einen routinierten Umgang mit widersprüchlichen Anforderungen ermöglicht.

Bei dieser Berufsauffassung kommt es zu einer routinierten und pragmatischen Handlungsorientierung, die Akteure suchen im Rahmen ihres Handlungskontextes nach praktikablen Lösungen, dabei begegnen sie Widersprüchen und Veränderungsanforderungen mit einer professionellen Gelassenheit. Wie beim Typ I steht im Zentrum der Berufsauffassung ein positives Selbstkonzept und die Identifikation mit der Rolle Schulleiterin ISchulleiter, aber dieser Typ unterscheidet sich von Typ I vor allem dadurch, dass Berufserfahrung einen pragmatischeren und ruhigeren Umgang mit widersprüchlichen Anforderungen ermöglicht.

Auf der Basis der Einzelfallauswertung und der dort gefundenen Berufsauffassungen habe ich fünf Typen von Berufsauffassungen gebildet. Die Abbildung 26 zeigt die Zuordnung der Einzelfälle zu den fünf Typen von Berufsauffassungen, die sich aus den in Kapitel 5 präsentierten Einzelfallauswertungen ergibt.

Die Nähe von Typ 1 und Typ 5 ist gegeben, dennoch habe ich mich entschieden, einen eigenen Typus zu bilden, weil bei Typ I die Reflektion der strukturellen Widersprüche stärker ausgeprägt ist und die Handlungsorientierungen vielfältiger und zielbezogener sind, was sich im Folgenden noch in der Bedeutung für die Schulentwicklung zeigen lassen wird. 
Abb. 25 Typen von Berufsauffassungen

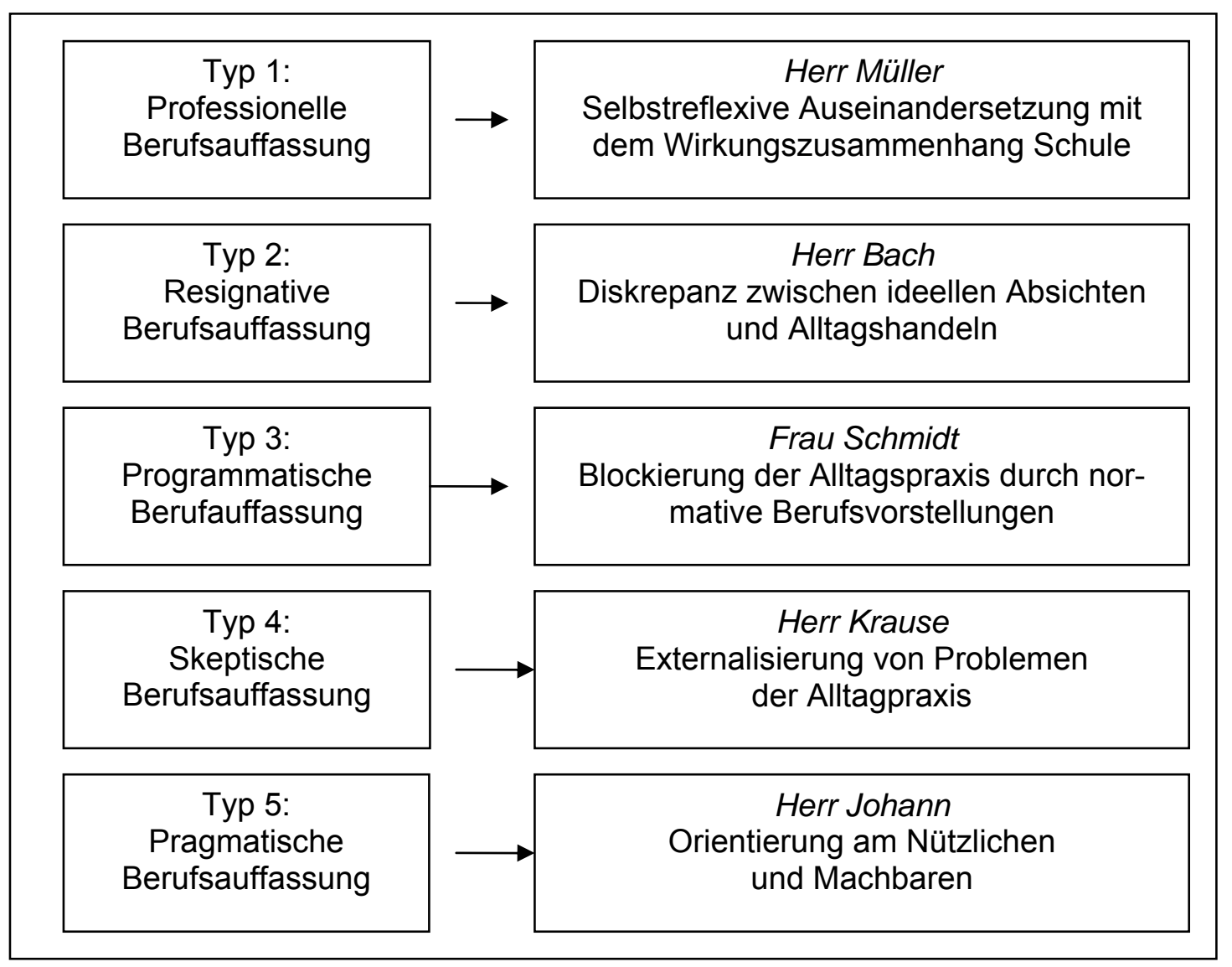

\subsection{Schulleitungshandeln- Aushandlungsprozess zwischen Stabilität und Innovation}

Forderungen, dass sich Schule entwickeln müsse, begleiten diese Organisation von Anfang an. Es wurde und wird immer wieder auf die Notwendigkeit von Reformen, Innovationen, Lernen von Schule und Schulentwicklung hingewiesen. Seit den 80er Jahren wird der Begriff „Schulentwicklung" hierfür verwendet, weil verstärkt von der Ebene der Einzelschule als Ausgangspunkt jeglicher Veränderung ausgegangen wird. Es fand ein Wechsel statt von Gesamtlösungsstrategien hin zu innerschulischen kleineren befristeten Vorhaben in den Schulen selbst. Dabei ist der Begriff Schulentwicklung offen, es geht um angestrebte Veränderungen, die den Schulen eine Vielfalt von Entwicklungen unter vorgegebenen äußeren Rahmenbedingungen frei stellt. Im Zusammenhang mit dieser Verschiebung gerieten die Schulleitungen bzw. die Schulleiterinnen und Schulleiter in den Fokus der Schulforschung. Es wird konstatiert, dass Schulleitung bei allen Entwicklungsbemühungen eine Schlüsselrolle zukommt, die Schulleiterinnen und Schulleiter gelten als zentrale Instanz für Schulreformprozesse, ja, dass es gar gerechtfertigt sei, von einer Reformgröße zu sprechen. ${ }^{239}$

${ }^{239}$ Vgl. Holtappels 2005, 85. 
Die Ergebnisse dieser Studie legen dies nicht nahe, hierfür können unterschiedliche Gründe genannt werden. Zum einen bestehen nach Auffassung der hier befragten niedersächsischen Schulleiterinnen und Schulleiter sich widersprechende Anforderungen, nämlich die Aufforderung zur Einzelschulentwicklung und die gleichzeitige geforderte äußere Strukturreform. Beides zieht eine Zunahme der Arbeitsaufgaben nach sich, wobei die Arbeitsbedingungen und Handlungsspielräume teilweise ungeklärt bleiben. Den Schulen und damit den Schulleiterinnen und Schulleitern wurde durch eine äußere Strukturreform die Auflösung der Orientierungsstufe in Niedersachsen zum Schuljahr 2004/2005, also die Verantwortung für eine gravierende Umstrukturierung ihrer bestehenden Schulen, übertragen.

Die Ergebnisse dieser Studie müssen auch vor dem Hintergrund dieser Herausforderung gedeutet werden. Organisationale Einflussfaktoren, die die Folge von politischen Entscheidungen waren, erforderten eine komplette Umstrukturierung der Einzelschule. Dies zog veränderte Arbeitsbedingungen und die Neuzusammensetzung des Personals nach sich. Vielleicht ist dies ein Grund dafür, dass die befragten Schulleiterinnen und Schulleiter in ihren Aussagen relativ wenig Bezug nehmen auf Schulentwicklungsaufgaben, da die äußere Strukturveränderungen zunächst umgesetzt werden müssen und dann einer inneren Stabilisierung bedürfen.

Ein weiterer Grund dafür, dass Schulleiterinnen und Schulleiter die Schlüsselrolle innerhalb von Schulentwicklungsprozessen nicht einnehmen können, liegt darin, dass ein Merkmal der Berufsauffassung aller hier Befragten ist, dass innen die Aufgabe zukommt, Konflikte mit Kolleginnen und Kollegen, den anderen Schulleitungsmitgliedern und der Schulbehörde auszutragen und sie daher vielfach nicht in Harmonie und Übereinstimmung mit den Mitgliedern ihrer Organisation stehen können. Gerade aber die Schulentwicklungsprogrammatiken zielen auf Konsens, Harmonie, Bewältigung gemeinsamer Aufgaben als Leitideen des pädagogischen Handelns. Visionen und Ziele allein können den Weg nicht aufzeigen, denn schulischer Wandel erfordert eine permanente Neuausrichtung und durchaus differente Entwicklungsrichtungen. Die einzelschulspezifische Organisationsstruktur ist dabei von exogenen Faktoren abhängig, wie z.B. gewandelten Bildungsorientierungen oder auch ganz konkret der Lehrerzuweisung, der Schulausstattung und den unterschiedlichen Zielvorstellungen von Lehrkräften. Vieles davon entzieht sich der Kontrolle und Lenkung der Schulleiterinnen und Schulleiter.

Nicht nur die zeitliche Arbeitsbelastung, das so genannte Alltagsgeschäft, schränkt die Schulleiterinnen und Schulleiter in ihrer Teilnahme an konkreten Entwicklungsprozessen und konzeptioneller Arbeit ein, es scheint ebenso von Bedeutung bei der Ausübung ihrer Tätigkeit zu sein, gerade den Anspruch an Schulentwicklung und die Selbstansprüche an ihre eigenen Berufsaufgaben stärker an Wirkrealitäten als an Programmzielen auszurichten. Die Zielsetzungen müssen ausgehandelt werden mit den unterschiedlichen Interessengruppen der Schule, unter Berücksichtigung der vorhandenen Ressourcen und den schulübergreifenden Bildungsorientierungen, dabei gilt es abzuwägen zwischen Innovation und Stabilität. 
So hemmt der Berufsauffassungstyp 2 - resignative Berufsauffassung die Prozesse der Schulentwicklung, denn individuelle Orientierungen stehen im Gegensatz zu Erfordernissen des Handlungskontextes, in einem solchen Fall wird Schulentwicklung blockiert. Dies konnte das Beispiel von Herrn Bach zeigen. Eine zu starke Fokussierung auf eine Zielvorstellunghier die konkrete Unterrichtsentwicklung- verkennt die Komplexität der gegebenen Arbeitssituation. In dem Kollegium von Herrn Bach wäre zunächst ein diskursiver Verständigungsprozess notwendig, den er als Schulleiter aufgrund einer Verhaltensblockierung aber nicht initiiert. Herr Bach gerät mit seiner Berufsauffassung in einen selbstwidersprüchlichen Konflikt, auf der einen Seite er als Pädagoge und auf der anderen Seite er als Schulleiter. Dieses Beispiel zeigt eindrücklich, dass Schulentwicklung auch davon abhängig ist, in welcher „Entwicklungsphase“ sich die jeweilige Schule befindet.

Im Unterschied zum Typ 2 führt beim Berufsauffassungstyp 3 - programmatische Berufsauffassung - die zu starke Orientierung an normativen Vorgaben und Überzeugungen zu kontraproduktiven Handlungsorientierungen der Akteure, die eher zur Konsolidierung des bestehenden Handlungskontextes führen als zur Veränderung. Am Beispiel von Frau Schmidt ließ sich zeigen, dass sie sich zwar an Veränderungen orientiert und sich dabei in ihrer Berufsauffassung auf ein Leitbild des Berufes bezieht, welches die Aufgabe der Qualitätsentwicklung hervorhebt. Weil sie aber den vorgefundenen Gesamthandlungskontext in ihrer Handlungsorientierung nicht berücksichtigen kann, gelingt es ihr dennoch nicht, sich von ideellen Anforderungen zu lösen und konkrete Folgerungen für die vorgefundene Praxis daraus abzuleiten. Sie möchte vielmehr als eine Art „Einzelkämpferin" Schulentwicklungsprozesse vorgeben und nicht dazu anregen. Frau Schmidt fehlt die nötige Unterstützung im Leitungsteam und damit letztendlich im Kollegium, um Veränderungen im Sinne der Schule zu implementieren.

Im Berufsauffassungstyp 4 - skeptische Berufsauffassung - ergibt sich aus der Berufserfahrung eine Diskrepanz zwischen eigener Vorstellung und beruflich vorgefundener Realität, was zu einer kritischen und abwehrenden Haltung gegenüber Veränderungsprozessen von Schule führt.

Am Beispiel von Herrn Krause lässt sich zeigen, dass die Hervorhebung der Übermacht der hierarchischen Organisation, die Betonung unzulänglicher Rahmenbedingungen und das Beklagen mangelnder Ressourcen dazu führen, dass in der eigenen Berufsauffassung die Wirksamkeit von Gestaltungsprozessen der Schule radikal in Frage gestellt wird.

Im Gegensatz zum Typ 4 steht der Berufsauffassungstyp 5- pragmatische Berufsauffassung - Veränderungsprozessen gelassen gegenüber und die Akteure schätzen ihren eigenen Gestaltungsspielraum als hinreichend groß ein. Die positive Einstellung gegenüber der eigenen Schule führt da$\mathrm{zu}$, dass eher stabilisierende als verändernde Handlungsorientierungen vorliegen. Das Beispiel von Herrn Johann zeigt, dass er seine Berufsauffassung entlang langjähriger Erfahrungen ausgeformt hat und es ihm zu gelingen scheint, pragmatisch abzuwägen, was für seine Schule notwendig und gleichzeitig machbar ist. In seiner Berufsauffassung spielt die Be- 
ratungsfunktion eine wichtige Rolle, da er erkannt hat, dass Kolleginnen und Kollegen, die die Schule voranbringen wollen, vor allem nach Beratung und Begleitung verlangen. Dabei betrachtet Herr Johann „Schulentwicklung" aus der Innensicht seiner Schule und begegnet äußeren Einflüssen routiniert. Herr Johann ist zunächst darum bemüht, den „guten Stand“ seiner Schule zu stabilisieren und innerschulische Prozesse zu regulieren.

Bei dem Berufsauffassungstyp 5 bestehen auf den ersten Blick große Ähnlichkeiten mit dem Berufsauffassungstyp 1- professionelle Berufsauffassung - , gerade aber im Hinblick auf die Bedeutung der Schulentwicklung, die im Rahmen der Analyse dem Organisationsverständnis zugeordnet worden ist, zeigen sich Unterschiede hinsichtlich der Handlungsorientierung. Die Berufsauffassung des Typs I ist durch eine reflektierende konstruktive Aushandlung von Schulentwicklungsprozessen gekennzeichnet. Wenn auch die Handlungsorientierung an Veränderung ausgerichtet ist, so werden ebenso Widersprüche und Hindernisse dieser Veränderung wahrgenommen und kritisch analysiert, es wird zwischen Innovation und Stabilität im Sinne der Schule als Ganzes ausbalanciert. Am Beispiel von Herrn Müller konnte dieser reflektierende Umgang mit den vorhandenen Strukturen gezeigt werden. Herr Müller nutzt sein flexibles Handlungsrepertoire und agiert auf allen Ebenen des Handlungskontextes Schule. Zudem hat er unterschiedliche Interessengruppen ebenso im Blick wie strukturelle Bedingungen und Bedürfnisse der Organisation Schule.

Herr Müller unterstützt Kolleginnen und Kollegen, die sich um die Gestaltung und Veränderung der Schule bemühen, er schafft und sichert fördernde Arbeits- und Beziehungsstrukturen und überträgt Verantwortung für Weiterentwicklung.

Gerade auf die Veränderung und Weiterentwicklung von Schule - dies legen die Ergebnisse dieser Studie nahe - hat Einfluss, wie die Akteure/ Akteurinnen mit den strukturellen Bedingungen der Organisation Schule umgehen. Insgesamt muss für die hier untersuchte Gruppe von Schulleiterinnen und Schulleitern konstatiert werden, dass die Alltagspraxis vornehmlich von Aufgaben bestimmt ist, die außerhalb von Maßnahmen der Schulentwicklung liegen. Die Sicherung von personellen und materiellen Ressourcen, der Umgang mit täglich auftretenden Konflikten sowie die Erledigung administrativer Aufgaben nehmen einen zunehmend großen Raum im alltäglichen Handeln ein. Dennoch beurteilen die hier befragten Schulleiterinnen und Schulleiter Schulentwicklung positiv, sie schätzen aber aus genannten Gründen ihre eigene Wirksamkeit als eher gering ein. Die Schulleiterinnen und Schulleiter können Kolleginnen und Kollegen anregen, sich auf Prozesse der Schulentwicklung einzulassen. So weit wie es ihr Handlungsspielraum zulässt, können sie flexible Organisationsbedingungen schaffen, die es Kollegien ermöglichen, Neues auszuprobieren und gegebenenfalls schulintern einzusetzen, insofern steuern und initiieren Schulleiterinnen und Schulleiter Schulentwicklung, aber sie erscheinen im Kontext dieser Untersuchung nicht als der Motor dieser Entwicklungen. 


\subsection{Das Spannungsverhältnis zwischen Programmatiken und Alltagshandeln}

Ein Beispiel für die Kluft zwischen Praxiswissen, also Wissen, welches durch die Reflexion subjektiver Erfahrungen in der Praxis erworben wurde, und Theoriewissen konnte eindringlich am Beispiel des Führungsverhaltens, insbesondere der alltäglichen Konfliktbewältigung, gezeigt werden. Alle interviewten Schulleiterinnen und Schulleiter nennen den Umgang mit Kolleginnen und Kollegen als die stärkste Belastung. Dieser Umgang, so zeigt es die Rekonstruktion in den Interviews, ist deshalb so schwierig, weil anders als z.B. in Privatunternehmen im Rahmen der Organisation Schule ein Widerspruch besteht zwischen der Funktion des Leitens und der tatsächlichen Weisungsbefugnis. Ein transaktionaler Führungsstil, wie er für die Privatwirtschaft als effektiv beschrieben wird und bei dem Leistungen belohnt und fehlende Leistungsbereitschaft sanktioniert werden, ist in Schule unter gegebenen Bedingungen nicht zu verwirklichen. Die theoretischen Erkenntnisse aus der Führungstheorie, wonach transaktionale Führung zu einer hohen Zielerreichung führt, ist in der schulischen Praxis nicht anwendbar. Für die Schulpraxisexpertinnen und - experten entstehen konfligierende Rationalitäten. So wendet sich Typ 4 -skeptische Berufsauffassung- wie am Beispiel von Herr Krause gezeigt wurde, nicht aufgrund des Führungsverständnisses gegen die Schule als Organisation, sondern aufgrund der Tatsache, dass Kolleginnen und Kollegen, die unzureichende Leistung erbringen, nicht ausgetauscht werden können, wie es im Unternehmen möglich wäre.

Das Leiden am Beruf bei Typ 2- resignative Berufsauffassung- resultiert daraus, dass die eigenen Überzeugungen von einer hohen Verausgabungsbereitschaft getragen sind und gleichzeitig an dem Arbeitsengagement vieler Kolleginnen und Kollegen gezweifelt wird. Wie an dem Beispiel von Herrn Bach gezeigt wurde, führt die subjektive Erfahrung sich widersprechender Handlungsorientierungen zu einer Vermeidungshaltung, Herr Bach hat aus seiner Sicht heraus keinerlei Handhabe und kann nur auf die Möglichkeiten der Motivation setzen.

Ähnlichen Problemen im Schulalltag wird unterschiedlich begegnet, gerade im Bereich Konflikte erwies sich ein selbstsicheres Verhalten, welches Ausdruck in einem positiven Selbstkonzept findet, als handlungsentlastend. Denn wie am Typ 1- professionelle Berufsauffassung- gezeigt werden konnte, ist es von entscheidender Bedeutung, ob sich Schulleiterinnen und Schulleiter ihrer eigenen Rolle und dem schulischen Spielraum gemäß verhalten. Dies zeigte sich an dem Beispiel von Herrn Müller, der situationsangemessen auf den Einzelkonflikt reagiert und dennoch die Schule als Ganzes im Blick hat. Hingegen kann und will Herr Krause die Balance zwischen eigenen Berufsüberzeugungen, die sich an unternehmerischen Handlungsrationalitäten orientieren, und interner Struktur der Schule nicht aushalten, er kann nicht zwischen Handeln und Struktur vermitteln.

Das programmatische Berufsbild des Landes Niedersachsen fordert eine hohe Leitungs- und Führungskompetenz von Schulleiterinnen und Schulleitern (soziale, pädagogische, rechtliche, strategische, organisatorische und Personalentwicklungskompetenz). Die Dimensionen der pädagogi- 
schen Professionskompetenz spielen im Rahmen des Berufsbildes keine Rolle, zeichnen sich aber auf Grund der Forschungsergebnisse dieser Studie als wesentliches Element der Berufsausübung aus. Denn dort, wo eine Reflexion tendenziell widersprüchlicher struktureller Bedingungen erfolgt und daraus dennoch konstruktive Konsequenzen für das eigene Handeln gezogen werden, gelingt es, sich positiv mit dem Beruf Schulleiterin/ Schulleiter zu identifizieren und Prozesse der Schule aktiv zu gestalten. Ein positives berufliches Selbstkonzept, das durch Selbstreflexionsvermögen und Distanzierungsfähigkeit gekennzeichnet ist, macht es möglich, mit auftretenden Widersprüchen umzugehen und die Differenz zwischen Theorie- und Praxiswissen anzuerkennen. Das Fehlen eines positiven Selbstkonzeptes und ein emotionaler Umgang mit täglichen Problemen verhindern situationsange-messene Problemlösungen in der Praxis.

Am Typ 3 - programmatische Berufsauffassung - konnte gezeigt werden, dass eine starke Orientierung an idealen Zielvorstellungen und die Übernahme eines normativen Berufsbildes in der Praxis einerseits als Stabilisator für Handlungsstrategien fungiert, sich aber insbesondere im Umgang mit innerschulischen Arbeitspartnern negativ auswirken kann. Denn Programm und die vorgefundene Alltagspraxis stehen im Widerspruch, die Negierung von Paradoxien und eine fehlende Perspektivenübernahme erschweren die gestaltende Arbeit. Ähnlich wie bei Herrn Bach zeigt sich auch bei Frau Schmidt, dass neben einem hohen Arbeitsengagement der soziale Rückhalt am Arbeitsplatz von Bedeutung im eigenen Belastungsempfinden ist. Ansätze einer Professionalisierung des Schulleitungshandelns müssten Schulleiterinnen und Schulleiter darin unterstützen, eine reflexive Haltung auszubilden. Insbesondere reflexive Distanz gegenüber widersprüchlichen Altagserfahrungen und Handlungslogiken kann dort hilfreich sein, wo Misserfolge zu verzeichnen sind, um nicht den eigenen Handlungsraum einzuengen.

Schulleiterinnen und Schulleiter müssen Unvereinbarkeiten aushalten und Differenzen zwischen Alltagspraxis und Programmatiken anerkennen, um sich selbst und andere nicht zu überfordern. Zunehmend wird in der Führungs- und Organisationsforschung mehr Sensibilität für Paradoxien gefordert. Für die Schulpraxisexpertinnen und -experten bedeutet das, dass sie mit Widersprüchen, wie z.B. Vertrauen und Misstrauen, Rationalität und Emotionalität, Konkurrenz und Kooperation, Flexibilisierung und Stabilität umgehen können müssen, um situationsgerecht auf diese gegensätzlichen Anforderungen reagieren zu können.

Wenn durch die Ergebnisse dieser Untersuchung konstatiert werden muss, dass sich die Berufsauffassung vornehmlich durch Erfahrungen in der Praxis formt, so muss gleichzeitig gefordert werden, dass sie durch wissenschaftliche Kenntnisse modifiziert werden muss. Ausgehend davon, dass das Praxiswissen von Schulleiterinnen und Schulleitern mittels Theoriewissen ausdifferenziert werden kann, wäre es aufgrund der Ergebnisse dieser Arbeit wünschenswert, dass Schulleiterinnen und Schulleiter sich stärker mit der Professionstheorie auseinandersetzen und diese als Deutungsangebot ihrer Berufspraxis nutzen. Wissenschaftliche Erkenntnisse könnten so verknüpft werden mit individuellen Berufsauffassungen und zu einem berufsrelevanten Fachwissen der Schulleiterinnen und Schulleiter 
entwickelt werden. Gerade die in der Professionstheorie thematisierte Spannung zwischen eigener Profession und Organisation ermöglicht die Akzeptanz der konstitutiven Paradoxien und kann den Weg öffnen für einen konstruktiven Umgang mit Problemen und Barrieren der Schulpraxis.

\section{7.}

Resümee

7.1 Zusammenfassende Diskussion der Ergebnisse

Ziel der vorliegenden Arbeit war es, die Berufsauffassung von Schulleiterinnen und Schulleitern anhand theoretischer Überlegungen der FührungsOrganisations- und Professionstheorie zu rekonstruieren. Ausgehend von der Annahme, dass die Wahrnehmung und die Gestaltung der Organisationsrealität durch Schulleiterinnen und Schulleiter von den jeweils individuellen Ausprägungen der Berufsauffassung beeinflusst werden, konnten auf der Grundlage empirisch abgebildeter Kategorien die vorausgehend beschriebenen fünf Typen von Berufsauffassungen ermittelt werden.

Zwei Untersuchungsteile wurden durchgeführt, zunächst eine standardisierte Fragebogenerhebung, in der 145 Fragebögen ausgewertet werden konnten, vertiefend wurden dann 20 qualitative Interviews mit Schulleiterinnen und Schulleitern durchgeführt.

Im Rahmen der Untersuchung wurde dem qualitativen Teil größeres Gewicht eingeräumt, weil es im quantitativen Teil vornehmlich darum ging, erste Einschätzungen über das Berufs- bzw. Führungsverständnis nachzuweisen, relevante Handlungsdimensionen von Schulleiterinnen und Schulleitern zu ermitteln und einen Zugang zum Feld zu erhalten.

1. Die Ergebnisse der quantitativen Befragung mit einer Rücklaufquote von $61,7 \%$ zeigen, dass die hier befragten niedersächsischen Schulleiterinnen und Schulleiter einem Führungsverhalten zustimmen, welches von prosozialen Einstellungen gegenüber dem Kollegium geprägt und gleichzeitig zielorientiert ausgerichtet ist (vgl. in dieser Arbeit S. 100). Die Befragten zeigen eine hohe Berufszufriedenheit und fühlen sich nur gering belastet.

Im Bereich der führungsrelevanten Dimensionen zeichnet sich ein eher führungsbetontes Bild $a b$, eine zielorientierte Führung wird überwiegend befürwortet. In diesem Bereich sind zustimmende Aussagen zu Zielvereinbarungsgesprächen, dienstlichen Beurteilungen ebenso enthalten wie das Durchsetzen von Entscheidungen. Dass zwischen Zuspruch und Umsetzung allerdings Missverhältnisse auftreten, wird gerade in diesem Komplex deutlich. Zielerreichung, was ja die Umsetzung von konkreten Arbeitszielen bedeuten würde, wird nur eingeschränkt bestätigt. Hier stören sich zwar Schulleiterinnen und Schulleiter an der fehlenden Konsequenz bei der Umsetzung von Beschlüssen (54,5\%), aber sie selbst sorgen nur zu einem verhältnismäßig kleinen Teil für die Umsetzung von Beschlüssen $(39,2 \%)$ und lediglich etwa ein Drittel $(34,3)$ überprüft die Wirkungen.

Diese Ergebnisse legen nahe, dass Diskrepanzen zwischen Absichten und konkreten Umsetzungen bestehen. Die Bereitschaft, für die eingeforderte Verbindlichkeit die entsprechenden Handlungen zu vollziehen, ist 
nur bedingt vorhanden. Der Wunsch nach mehr Entscheidungs- und Handlungsspielräumen auf Seiten der Schulleiterinnen und Schulleiter müsste aber einhergehen mit einem konsequenten Umdenken und einem Handeln, welches sich entsprechend der eigenen Berufsdefinition auswirkt.

Den Befragten ist ein soziales und auf Kooperation ausgerichtetes Klima wichtig und es ließ sich eine insgesamt starke Mitarbeiterorientierung nachweisen. Ebenso hohen und uneingeschränkten Zuspruch erhielt die Bedeutung der Schulentwicklung. Sowohl die Schulprogrammarbeit als auch die gemeinsame Weiterentwicklung und Verbesserung des Unterrichts halten die Befragten für bedeutungsvoll. Ihre eigene Rolle in diesem Prozess schätzen die Befragten überwiegend als aktiv, gestaltend und initiierend ein.

Entgegen meiner Annahme stufen die Schulleiterinnen und Schulleiter ihre Belastung weniger hoch ein als vermutet. Die befragten Schulleiterinnen und Schulleiter fühlen sich mehrheitlich in ihrer Position $(72,4 \%)$ akzeptiert und weisen eine große Berufszufriedenheit auf $(78,5 \%)$, dennoch schätzen sich $(11,2 \%)$ als überfordert und $(3 \%)$ als allein gelassen in ihrem Beruf ein. Von besonderer Bedeutung sind diese Ergebnisse auch insofern, als dass Korrelationen zwischen den Dimensionen zielorientierte Führung und Berufszufriedenheit festgestellt werden konnten. Dieser Zusammenhang zwischen beruflicher Zufriedenheit und einer auf die Weiterentwicklung der Schule ausgerichteten Führungshaltung konnte durch die qualitativen Interviews ebenfalls bestätigt werden.

2. Die Rekonstruktion der Berufauffassung durch die qualitativen Interviews konnte zeigen, dass Schulleiterinnen und Schulleiter über ein flexibles und breites Handlungsrepertoire verfügen müssen, wenn sie den unterschiedlichen und tendenziell widersprüchlichen beruflichen Anforderungen begegnen. Dabei müssen sie die Prozesse der Schule aus vielfältigen Blickwinkeln analysieren können, um Spannungsverhältnisse in der Praxis auszuhalten und gleichzeitig konstruktiv Lösungen für auftretende Probleme entwickeln zu können.

Die analysierten Interviewdaten deuten darauf hin, dass ein berufsbezogenes positives Selbstkonzepts großen Einfluss sowohl auf die Erfüllung des Aufgabenspektrums als auch auf die Berufszufriedenheit hat.

Die Auseinandersetzung mit den an Schule beteiligten Akteuren, das Handeln auf den unterschiedlichen Ebenen und die Kommunikation mit den verschiedenartigen Interessengruppen, ob in Arbeitsteams, im Gesamtkollegium, auf Schulebene oder darüber hinaus, kann als die große Herausforderung der Alltagspraxis von Schulleiterinnen und Schulleitern angesehen werden. Insbesondere der Umgang mit Störungen und Konflikten stellt eine Schlüsselstelle im Alltagshandeln dar. In den Interviews zeigt sich, dass den schwierigsten und sensibelsten Bereich des Schulleitungshandelns der Umgang mit Kolleginnen und Kollegen der Schule darstellt, dabei wird das Verhalten einiger „Problemkolleginnen und kollegen" durchgehend als stärkste Belastung im Berufsalltag angesehen. Die befragten Schulleiterinnen und Schulleiter erleben häufig einen Spannungszustand zwischen zur Zusammenarbeit verpflichteten und führungsorganisatorisch gleichrangigen Organisationsmitgliedern, die nicht mit Mit- 
teln der direkten Weisung, sondern über Konsensfindung gelöst werden muss. Laterale Kooperationskonflikte entstehen dort, wo das Prinzip individueller Ergebnisverantwortung nicht greift und Zielkonflikte mit einzelnen Lehrkräften oder im Kollegium entstehen. Hier wünschen sich die Befragten klarere Befugnisse und Möglichkeiten der Belohnung und Sanktion.

Greifen Schulleiterinnen und Schulleiter ausschließlich auf Verhaltensmuster zurück, die sie in der Berufsrolle der Lehrerin/ des Lehrers erworben haben, so kann dies dem komplexen Aufgabenfeld Schulleitung nicht gerecht werden. Am Typus 2 -resignative Berufsauffassung- konnte gezeigt werden, dass eine zu starke Orientierung am Berufsbild des guten Lehrers/ der guten Lehrerin zu einer Verkennung der Realität des Berufes als Schulleiterin/ als Schulleiter führen kann. Eine fehlende reflexive Distanz zu den Aufgaben und Erfordernissen des Berufes Schulleiterin/ Schulleiter korreliert mit einem Leiden am Beruf, da die subjektiven Ansprüche an das Gelingen der Berufsaufgabe nicht übereinstimmen mit den Erfordernissen der Organisation und da organisationale Strukturen nicht genutzt werden können für ein „professionelles“ Handeln.

Eine zu stark an normativen Vorgaben orientierte Berufsauffassung kann ein flexibles Reagieren auf innerschulische Prozesse verhindern und zum Beharren auf der eigenen Position führen. Beides schränkt das eigene Handlungsrepertoire im Sinne einer situationsangemessenen Einschätzung von Problemen und einem förderlichen Umgang mit Konflikten ein. Programmatiken dienen zwar einer allgemeinen Beschreibung und können Orientierungspunkte bieten, sie können aber nicht unbedingt handlungsleitend wirksam werden, da sie von dem durch die Praxis erfahrenen Wissen oftmals abweichen. Dies konnte am Typus 3 - programmatische Berufsauffassung gezeigt werden.

Eine skeptische Berufsauffassung bildet sich dort heraus, wo eine hohe Einschätzung der eigenen Fähigkeiten und persönlichen Führungskompetenzen sich paart mit einer Abwehr von organisatorisch gegebenen strukturellen Bedingungen. Dies kann organisationale Konfliktpotenziale erhöhen und zu Verhärtungen führen. Wenn der als hierarchisch erlebte Organisationskontext Übermacht über die eigenen professionellen Gesichtspunkte erhält, wird die Handlungsfähigkeit im Kontext innerschulischer Gestaltung eingeschränkt. Spannungen zwischen der eigenen Berufsratio und der Organisationsratio sind unvermeidlich und führen in diesem Berufsauffassungstyp aber dazu, dass Veränderungschancen nicht erkannt werden. Wenn es nicht gelingt, sich entsprechend der eigenen Berufsdefinition zu verhalten, wird dies oft mit fehlenden Rahmenbedingungen erklärt. Dass ein beständig als lähmend erlebter Widerspruch dieser Handlungsrationalitäten letztendlich zur Ablehnung der Berufsaufgabe führt, zeigte der Typ 4 -skeptische Berufsauffassung.

Insbesondere in diesem Wechselspiel eigener Berufsauffassung und vorgegebener Strukturen der Organisation Schule können organisationale Abhängigkeiten und Ressourcenknappheit als Störgrößen angesehen werden. Professionstheoretische Überlegungen könnten eine wichtige Hilfestellung sein bei der Entwicklung von mehr Sensibilität für Paradoxien, die in Führung und Kooperation unweigerlich bestehen. Die eigene Fähig- 
keit zur Reflexion dieser Widersprüche im Altagshandeln kann das Schulleitungshandeln positiv beeinflussen, dies konnte durch den Typ 1 - professionelle Berufsauffassung- gezeigt werden.

Betrachte ich die fünf gebildeten Typen in ihrer Bedeutung für die Schulentwicklung, so gilt für alle Typen, dass das berufsrelevante Selbstkonzept einen wesentlichen Faktor bei der Ausübung des Berufes darstellt. Eine eindeutige Identifikation mit der Rolle der Schulleiterin/ des Schulleiters und eine positive Einschätzung der eigenen Kompetenzen tragen dazu bei, fördernde Arbeits- und Beziehungsstrukturen aufzubauen und zwischen unterschiedlichen Interessengruppen in der Schule zu vermitteln. Dennoch muss gerade im Rahmen der Schulentwicklung berücksichtigt werden, in welcher Schulentwicklungsphase sich die Schule befindet.

Wie die Darstellung der Typen von Berufsauffassungen gezeigt hat, ließen sich in den Berufsauffassungen von Schulleiterinnen und Schulleitern große Unterschiede skizzieren. Der individuelle Stil der Auseinandersetzung mit den Berufsaufgaben, eigene Kompetenzen und die unterschiedlichen Aufmerksamkeitsrichtungen beeinflussen maßgeblich die Ausübung des Berufes. Von Bedeutung innerhalb der Berufsauffassungen ist es, dass es den Schulleiterinnen und Schulleiter gelingt, ein positives Selbstkonzept auszubilden und berufliche Erfahrungen als machbare Beanspruchung zu verarbeiten. Eine reflexive Distanz gegenüber Prozessen der Schule, gegenüber den als widersprüchlich eingeordneten Organisationsstrukturen und gegenüber dem eigenen Handeln hat großen Einfluss auf die Entwicklung eines positiven Selbstkonzeptes.

Eine professionelle Berufsauffassung von Schulleiterinnen und Schulleiter - und dies kann zusammenfassend festgehalten werden - zeichnet sich durch ein flexibles Repertoire an Führungsverhalten aus. Die Akteure können sich entsprechend situativer Anforderungen verhalten. Die Komplexität der Aufgaben wird auch in ihrer Widersprüchlichkeit wahrgenommen und im Rahmen des Handlungskontextes nach individuellen Handlungsmöglichkeiten gesucht. Die Organisation Schule wird aus unterschiedlichen Blickwinkeln betrachtet und den Akteuren gelingt es, auf der administrativen, politischen und pädagogischen Ebene zu agieren. Ein umfassendes Wissen über die strukturellen Bedingungen der Organisation Schule ermöglicht es, zwischen unterschiedlichen Interessengruppen der Schule und den organisationalen Bedürfnissen der Schule auszubalancieren. Gerade unter den Bedingungen erweiterter Autonomie erhält das Handeln der Schulleiterinnen und Schulleiter eine besondere Bedeutung für die Qualität der Einzelschule. Da das eigene Handeln kontextuell in der Struktur des Schulsystems eingebettet ist, unterliegt es damit einer Fremdsteuerung durch schulexterne Instanzen. Insbesondere die von der Politik initiierte Selbststeuerung der Schulen geschieht auch unter den Gesichtpunkten des Einsparens personeller und ökonomischer Ressourcen. Auf der anderen Seite haben die Ergebnisse der Vergleichsstudien (PISA) dazu geführt, dass die Bildungspolitik die Vereinheitlichung (Vergleichsarbeiten, Zentralabitur) zugunsten individueller pädagogischer Autonomie anstrebt. Daher besteht eine wichtige Aufgabe für Schulleiterinnen und Schulleiter darin, Balance zu halten zwischen Veränderungen der kontinuierlichen Schulentwicklungen und einer stabilisierenden Funktiona- 
lisierung des Schulbetriebes. Gerade in diesem Bereich der regelmäßigen Evaluationen aller an Schule beteiligten Personengruppen wird der Druck der Schulleiterinnen und Schulleiter zwischen Rechenschaft und Weiterentwicklung auszugleichen größer werden. ${ }^{240}$

Dies zeigt, dass die Akteure in der Schule ihre Einstellungen und Handlungsrepertoires verändern und erweitern müssen, um den komplexen und widersprüchlichen Berufsanforderungen gerecht werden zu können. Der Kontext Schule birgt in sich selbst das Problem, dass manche unbewussten oder bewussten Berufsrollenvorstellungen nicht unbedingt in Einklang zu bringen sind mit den einerseits vorgegebenen Funktionen, z.B. Dienst-, Aufsichts- oder Beratungsfunktion, den eigenen berufsrelevanten Werten und andererseits der vorgefundenen Realität der Praxis. Die Fähigkeit zwischen widersprüchlichen Anforderungen, unterschiedlichen Interessengruppen und dem eigenen Anspruch die Balance zu halten, gilt sowohl in der Führungstheorie als auch in der Professionstheorie als die Voraussetzung für ein situationsgerechtes Verhalten, welches die Gestaltung fördernder Arbeits- und Beziehungsstrukturen sichert und Weiterentwicklung ermöglicht. Wenn Schulleiterinnen und Schulleiter sich selbst bewusst machen, nach welchen Mustern sie agieren und dass ihre Deutungsmuster andere sein können als die des Kollegiums, dann kann diese Reflexion des eigenen Handelns dazu dienen, strukturelle Gegebenheiten zu erkennen, adäquater darauf zu reagieren und Schule professioneller zu gestalten. Das Bewusstsein von sich selbst erhöht die eigene Handlungsfähigkeit und kann als eine Bedingungsvariable innerschulischer Veränderung angesehen werden.

\title{
7.2 Ausblick
}

\author{
„Man müsste schon drei Jahre Schullei- \\ ter gewesen sein, um Schulleiter werden \\ zu können. ${ }^{241}$
}

Mit dieser Arbeit wurde der Versuch unternommen, Einblicke in die Berufsauffassung von Schulleiterinnen und Schulleitern zu erhalten, Muster dieser Auffassungen abzubilden und in Beziehung zu setzen zu den Forderungen nach Schulentwicklung. Die Handlungsmöglichkeiten von Schulleiterinnen und Schulleitern sind entgegen manchem Wunschdenken weit weniger geprägt von dem Konstrukt eines beruflichen Leitbildes - wie es für die niedersächsischen Schulleiterinnen und Schulleiter entworfen wurde-, sondern das Handeln wird maßgeblich von individuellen Überzeugungen und Vorstellungen bestimmt. Innerhalb dieser Berufsauffassungen konnte das berufsrelevante Selbstkonzept als Bedingungsvariable herausgearbeitet werden. Veränderungsprozesse erfordern neben beruflichen Kompetenzen und individuellen Ressourcen eine Berufsauffassung, die die Berufserfordernisse im Blick hat und sich durch ein großes Handlungsrepertoire auszeichnet. Als wichtiges Ergebnis kann festgehalten werden, dass eine Berufsauffassung, die sich sowohl an eigenen Wertvorstellungen als auch an berufsrelevanten Werten orientiert - also eine pro-

\footnotetext{
${ }^{240}$ Vgl. Gesetzentwurf zur Eigenverantwortlichen Schule, 2005.

${ }^{241}$ Zitat eines amtierenden Schulleiters.
} 
fessionelle Berufsauffassung - dazu verhilft, den komplexen Anforderungen des Berufsalltages gewachsen zu sein.

Die vielfältigen Konzepte aus dem Bereich der Führungs- und Organisationstheorie sowie die zahlreichen Empfehlungen für das Berufsbild Schulleiterinnen/Schulleiter können nicht reduziert werden auf einen Anforderungskatalog, sondern es bedarf einer ernsthaften Auseinandersetzung mit den Schulpraxisexperten vor Ort. Denn Schulentwicklung braucht neben der Professionalität der Schulleiterinnen und Schulleiter auch einen institutionellen Rahmen, der dem Anspruch nach Weiterentwicklung selbst gerecht wird. Die Veränderungs- und Entwicklungsempfehlungen der hier befragten Schulleiterinnen und Schulleiter könnten für die Weiterentwicklung des beruflichen Kontextes Schule genutzt werden. Dies wäre allerdings auch die Aufgabe derer, die Verantwortung tragen für die Gestaltung des Gesamtsystems Schule, also die Beamten der Kultusbehörden und die Bildungspolitik.

Genannt werden sollen nur einige Anregungen, die sich aus den Ergebnissen dieser Untersuchung ableiten lassen:

\section{Auf der Ebene der Qualifikation...}

- Eine systematische und verbindliche Ausbildung vor Berufseintritt muss Pflicht werden. Diese Ausbildung muss neben handlungsorientierten Kompetenzen auch wissenschaftlich begründetes theoretisches Wissen vermitteln, welches als Deutungsrahmen für die Berufpraxis genutzt werden kann. Diese Ausbildungszeit sollte separat von der eigenen Tätigkeit als Lehrerin/ Lehrer absolviert werden. In diese Ausbildungszeit müssen Hospitationen amtierender Schulleiterinnen und Schulleiter integriert werden.

Auf der Ebene des Berufsalltages...

- $\quad$ Eine berufsbegleitende Supervision wäre eine Unterstützung. Dabei wäre es unabdingbar, dass diese Supervision von Externen durchgeführt wird, die gleichzeitig ausreichende Kenntnisse des Feldes Schule haben

- Des Weiteren wären überregionale Treffen von Schulleiterinnen und Schulleitern sinnvoll, die auch Raum für einen geschützten Austausch untereinander bieten, die also außerhalb von Tagungen mit der Schulbehörde stattfinden.

\section{Auf der institutionellen Ebene...}

- Einführung einer leistungsbezogenen Besoldung für Beamte.

- Kurzfristig handhabbare Sanktionsmöglichkeiten bei vorliegenden Dienstvergehen, z.B. Gehaltskürzungen, durch die Schulleitung, evtl. den Schulbeirat.

- Abschaffung der A 14 Stellen. Das eingesparte Geld sollte als Budget an den Schulen verbleiben, die damit besondere Leistungen von Lehrerinnen und Lehrern zeitgebunden belohnen

- Des Weiteren erachte ich es als sinnvolle Maßnahme, alle A 15 und A16 Stellen auf Zeit zu besetzen, um auf der Führungsebene einerseits Evaluation zu ermöglichen und andererseits auch Schulleitungspersonal die Gelegenheit zu geben, einen souveränen und 
selbst gewünschten Ausstieg aus ihrem Leitungsamt zu ermöglichen.

Durch die vorliegende Untersuchung konnte das breite Spektrum der Berufsauffassungen amtierender Schulleiterinnen und Schulleiter aufgezeigt werden. Offenkundig wurde, dass bestimmte Berufsauffassungen weniger dem Anspruch an Schulentwicklung und den Anforderungen des Berufes entsprechen als andere. Hier wird nicht nur Schule nicht weiter entwickelt, sondern es kommt auch zu persönlichen Niederlagen. Auf der anderen Seite gibt es Schulleiterinnen und Schulleiter, die mit hohem persönlichen Engagement und großem Sachverstand die Weiterentwicklung ihrer Schulen vorantreiben und dabei die Differenz zwischen Visionen und Realität anerkennen können.

Gerade denjenigen könnte der Berufsalltag erleichtert werden, wenn sich Bildungspolitikerinnen und Bildungspolitiker dazu durchringen könnten, grundlegende Veränderungen im Bildungssystem durchzusetzen. Es geht dabei nicht um große Zukunftsvisionen, um ideelle Programmatiken, sondern eher um adaptionsfähige Handlungspläne, die den Möglichkeiten der Praxis entsprechen. Schule will und muss sich auf Veränderungen einlassen, der Handlungskontext selbst birgt jedoch eine Vielzahl unsicherer Variablen, die berücksichtigt werden müssen.

Nicht idealisierte Leitsterne am Lehrerhimmel werden in der Praxis benötigt, sondern klarsichtige Balancierer, die Spannungen und Widersprüche aushalten und konstruktiv mit Barrieren umgehen. 


\section{Literatur}

Ackermann, Helga (2003): Erwartungen von Schulleitungen an die neue Bildungspolitik. In: Schulleitungsverband Niedersachsen e.V., 2003, 81, 1-5.

Ackermann, Helga / Wissinger, Jochen (Hg.) (1998). Schulqualität managen. Von der Verwaltung der Schule zur Entwicklung von Schulqualität. Neuwied: Luchterhand.

Altrichter, Herbert/ Schley, Wilfried./ Schratz, Michael (Hg.) (1998): Handbuch zur Schulentwicklung. Innsbruck: Studienverlag.

Altrichter, Herbert (2000): Qualitätsforderungen, Schulevaluation und die Rolle der Schulleitung. In: Schulleiter-Handbuch 93, 85-97.

Arbeitsstabteilung Forum Bildung (1999): Bildungs- und Qualifikationsziele von morgen. Bonn: Langer Design.

Arbeitsstabteilung Forum Bildung (2001): Qualitätsentwicklung und Qualitätssicherung im internationalen Wettbewerb. Bonn: Langer Design

Arbeitsstabteilung Forum Bildung (2001): Lernen - ein Leben lang. Bonn: Langer Design

Arbeitsstabteilung Forum Bildung (2001): Neue Lern- und Lehrkultur, Bonn: Langer Design.

Arnold, Rolf/ Faber, Klaus (2001): Qualität entwickeln - aber wie? Qualitätssysteme und ihre Relevanz für Schule. Seelze: Kallmeyer.

Arnold, Rolf / Griese, Christiane (2004): Schulleitung und Schulentwicklung. Hohengehren. Schneider Verlag.

ASD (allgemeiner Schulleitungsverband Deutschland) (1999). Schulleitung in Deutschland. Stuttgart: Raabe.

ASD (allgemeiner Schulleitungsverband Deutschland) (2005). Schulleitung in Deutschland 2005. Ein Berufsbild in der Weiterentwicklung. Stuttgart: Raabe.

Auering, Rupert (1986): Die Funktion des Schulleiters bei der Durchsetzung schulischer Innovationen. Frankfurt am Main: Lang.

Backhaus, Klaus (2000): Multivariate Analysemethoden. Berlin: Springer.

Bartz, Adolf (2004): Personalmanagement in Schule. Landesinstitut für Schule. Soest: Kettler. 
Bass, Bernhard M./ Steyrer, Johannes $\left(1995^{2}\right)$ : Transaktionale und transformationale Führung. In: Kieser, Alfred/ Reber, Gerhard Wunderer, Rolf (Hg.) (19952): Handwörterbuch der Führung.

Stuttgart. Schäffer-Poeschel, 2053-2062.

Bastian, Johannes (1997): Pädagogische Schulentwicklung. Von Unterrichtsreform zur Entwicklung der Einzelschule. In: Pädagogik, Heft 2, 1997, 6-11.

Bastian, Johannes (2000): Schulentwicklung in der Region. In: Pädagogik, H. 7-8. Weinheim: Beltz.

Bastian, Johannes / Helsper, Werner / Reh, Sabine/Schelle, Carla (Hg.)(2000): Professionalisierung im Lehrerberuf. Von der Kritik der Lehrerolle zur pädagogischen Professionalität. Opladen: Leske+Budrich.

Baumert, Jürgen/ Leschinsky, Achim (1986): Berufliches Selbstver ständnis und Einflussmöglichkeiten von Schulleitern. In: Zeitschrift für Pädagogik 1986/ 32 Jg./2, 247-263.

Baumert, Jürgen(1989): Forschungsergebnisse zur Schulleiterproblematik. Schulleitung in der empirischen Forschung. In: Rosenbusch, Heinz/ Wissinger, Jochen (1989): Schulleiter zwischen Administration und Innovation. Braunschweig: Schulleiter-Handbuch, 52-63.

Baumgartner, Irene/ Häfele, Walter/ Schwarz, Manfred/ Sohm, Kuno $\left(1998^{2}\right)$ : OE-Prozesse. Die Prinzipien systemischer Organisationsentwicklung. Ein Handbuch für Beratende, Gestaltende, Betroffene, Neugierige und OE- Entdeckende. Bern: Haupt.

Bauer, Karl-Oswald/ Kopka, Andreas /Brindt, Stefan (1999): Pädagogische Professionalität und Lehrerarbeit. Weinheim: Juventa.

Bennis, Warren (1998): Menschen führen ist wie Flöhe hüten.

Frankfurt/Main: Campus,

Berthel, Jürgen (2000): Personalmanagement. Stuttgart: SchäferPoeschel, 55-108.

Bessoth, Richard (1996): Organisationsklima und Organisationskultur von Schulen. Synergien und Potential an Schulen. In: Pädagogische Führung, 7. Jg.; Heft 4, 1996, 175-177.

Bildungskommission NRW (1995): Zukunft der Bildung - Schule der Zukunft. Neuwied: Luchterhand,

Bogner, Alexander/ Menz, Wolfgang (2002): Expertenwissen und Forschungspraxis: die modernisierungstheoretische und die methodische Debatte um die Experten. Zur Einführung in ein unübersichtliches Problemfeld. In: Bogner, Alexander/ Littig, Beate /Menz, Wolf- 
gang (Hg.): Das Experteninterview, Theorie, Methode, Anwendung. Opladen: Leske\&Budrich, 7-31.

Bogner, Alexander/Littig, Beate /Menz, Wolfgang (Hg.) $\left(2005^{2}\right)$ : Das Experteninterview. Wiesbaden: VS-Verlag.

Bohnsack, Ralf (1991): Rekonstruktive Sozialforschung. Einführung in die Methodologie und Praxis qualitativer Forschung. Opladen: Leske\&Budrich.

Bohnsack, Ralf/Marotzki, Winfried/ Meuser, Michael (Hg) (2003): Hauptbegriffe qualitativer Sozialforschung. Opladen: Leske \& Budrich.

Bolmann, Lee G. /Deal, Terrence E. $\left(1997^{2}\right)$ : Reframing OrganizationsArtistry, Choise and Leadership. San Francisco: Sage Publishing.

Bonsen, Martin /lglhaut, Claus/Pfeiffer, Hermann (1999): Schulleitungshandeln aus Schulleitungssicht. Dortmund: IFS-Verlag.

Bonsen, Martin (2002): Schulleitungshandeln aus Lehrersicht- Einschätzungen zu schulentwicklungsbezogenen Handlungsdimensionen. In: Wissinger, Jochen/ Huber, Stephan Gerhard (Hg.)(2002):

Schulleitung- Forschung und Qualifizierung. Opladen: Leske\& Budrich.

Bonsen, Martin/ von der Gathen, Jan/ Iglhaut, Claus /Pfeiffer, Hermann (2002a): Die Wirksamkeit von Schulleitung. München: Juventa.

Bonsen, Martin/ Pfeiffer, Hermann (2002b): Erkundungen zur Wirksam keit von Schulleitungshandeln. Ergebnisse einer empirischen Explorationsstudie. In: Journal für Schulentwicklung 1/2002, 23-31.

Bonsen, Martin/ von der Gathen, Jan/ Iglhaut, Claus /Pfeiffer, Hermann (2002c): Wie wirkt Schulleitung? Schulleitungshandeln als Faktor der Schulqualität. In: Rolff, Hans-Günter u.a. (Hg.): Jahrbuch der Schulentwicklung, Bd. 12, Weinheim.

Bonsen, Martin (2003): Schule, Führung, Organisation, Münster: Waxmann.

Boomgaarden, Hero / Lehmann, Jochen (2003): Schule leiten will gelernt sein. Niedersächsischer Bildungsserver. (Online)Abrufbar: http://www.nibis.de/slq/1. (Zugriff 04.08.2003.)

Bormann, Inka (2002): Organisationseinheit und organisationales Lernen von Schulen. Opladen: Leske \& Budrich,

Brinkmann, Ralf (1999): Techniken der Personalentwicklung. Heidelberg. Sauer-Verlag

Brockhaus (2006): Enzyklopädie digital. Mannheim. 
Brockmeyer, Rainer (1997): Was sollen wir für morgen lernen? Perspektiven für die Weiterentwicklung des Bildungswesens. In: BLK (HG.) (1997): Schulleitung und Schulaufsicht: neue Rollen und Aufgaben im Schulwesen einer dynamischen und offenen Gesellschaft. Innsbruck, Wien: Studienverlag, 17-48.

Brockmeyer, Rainer (2000): Die Bedeutung „der großen Konzeption“. Anmerkungen zu Entwicklungsverläufen und Wirkungen.

In: Frommelt, Bernd/Klemm, Klaus/Rösner, Ernst/Tillmann, KlausJürgen (2000): Schule am Ausgang des 20. Jahrhunderts. München: Juventa, 197-209.

Brosziewski, Achim (1994): Expertenschaft in Führungskritik. In: Hitzler, Ronald/ Honer, Anne/ Maeder, Christian (Hrsg.), Expertenwissen. Die institutionalisierte Kompetenz zur Konstruktion von Wirklichkeit, Opladen, 104-121.

Buchen, Herbert /Horster, Leonard /Rolff, Hans-Günter (1999): Schulleitung und Schulentwicklung. Berlin: Raabe.

Buchen, Herbert/ Rolff, Hans-Günter (Hg.) (2006): Professionswissen Schulleitung. Weinheim. Beltz.

Buhren, Claus (2001): Leistungslohn für Lehrer. In: Journal für Schulentwicklung, 3/2001, 5, 35-42.

Buhren, Claus/ Rolff, Hans-Günter (2002): Personalentwicklung in Schulen. Weinheim: Beltz.

Bühl, Achim/ Zöfel, Peter (2002):, München: Pearson Studium.

Bulla, Hans-Georg (1982): Probleme einer Organisationseinheit in der Schule, Frankfurt am Main: Lang.

Bund Länder Kommission für Bildungsplanung und Forschungsforderung (Hg.) (1998): Schulleitung und Schulaufsicht. Studienverlag, Wien.

Burns, James (1978): Leadership. New York: Harper\&Row.

Brügelmann, Hans (1999): Was leisten unsere Schulen. Seelze: Kallmeyer:

Byrne, Brian (2001): Factorial Validity of a theoretical Construct (First Or der CFA-Model). In: Bryne, Brian: Structural equation modelling with Amos basic concepts, applications and programming, New Jersey. S. 57-97. 
Cloer, Ernst /Klika, Dorle/Kunert, Hubertus (Hg) (2000): Welche Lehrer braucht das Land? Notwendige und mögliche Reformen der Lehrerbildung. München: Juventa.

Combe, Arno/Helsper, Werner (Hg) (1996) Pädagogische Professionalität. Untersuchung zum Typus pädagogischen Handelns. Frankfurt a. M.: Suhrkamp.

Cortina, Kai/ Baumert, Jürgen/ Leschinsky, Achim/ Mayer, Karl Ulrich/ Trommer, Luitgard (Hg.) (2003): Das Bildungswesen in der Bundesrepublik Deutschland. Reinbek: Rowohlt.

Dalin, Per/ Rolff, Hans-Günter/ Buchen, Herbert (1995): Institutioneller Schulentwicklungsprozess. Bönen: Kettler.

Davies, Brent (2002): Rethinking the educational Context - A reengineering approach. In: Davies, Bronwyn/ Ellison, Larry (Hg.): School Leadership for the $21^{\text {st }}$ Century. London, MCB, 1-22.

Denzin, Norman (1970): The Research Act in Sociology. London: Butterworth.

Dewe, Bernd/ Ferchhoff, Wilfried/ Radtke, Frank-Olaf (1992): Auf dem Weg zu einer aufgabenzentrierten Professionstheorie pädagogischen Handelns. In: dies (Hg.): Erziehen als Profession. Zur Logik professionellen Handelns in pädagogischen Feldern. Opladen: Leske \& Budrich, 7-20.

Dewe, Bernd (1998): Zur Relevanz der Professionstheorie für pädagogische Handlungsfelder. In: Schulz, Wolfgang K.: Expertenwissen. Soziologische und pädagogische Perspektiven. Opladen: Leske\& Budrich, 67-86.

Deutscher Bildungsrat, Empfehlungen der Kommission (1970): Strukturplan für das Bildungswesen. Bonn.

Deutsches PISA-Konsortium (Hg) (2000): PISA 2000. Leske \& Budrich.

Diehl, Jörg/ Kohr, Heinz (1994 ${ }^{11}$ ): Deskriptive Statistik. Eschborn: Klotz, 338-375.

Diekmann, Andreas (2002): Empirische Sozialforschung. Reinbek. Rororo.

Dirks, Una /Hansmann, Wilfried (Hg.) (2002).Forschendes Lernen in der Lehrerbildung. Bad Heilbrunn. Klinkhardt.

Driftmann, Hans/ Erdsiek-Rave, Ute/ Riecke-Baulecke, Thomas (2004):

Eigenverantwortung annehmen und Schule führen. Schulmanagement-Handbuch 112. Oldenburg. 
Dubs, Rolf (1994): Die Führung einer Schule. Leadership und Management Stuttgart: SKV.

Dubs, Rolf (1997): Teilautonome Schule- ein Thema für die berufsbildende Schule? In: Euler, Dieter/ Sloane, Peter F.E. (Hg.): Duales System im Umbruch. Eine Bestandsaufnahme der Modernisierungsdebatte. Pfaffenweiler, Bd. 2, 105-120.

Dubs, Rolf (1999): Qualitätsmanagement an Schulen. Bestandsaufnahme und Perspektiven. In: Grogger, Günther/ Specht, Werner (Hg.) (1999): Evaluation und Qualität im Bildungswesen. Wien: Bundesministerium für Unterricht und kulturelle Angelegenheiten, 80-94.

Edelstein, Wolfgang (2002): Selbstwirksamkeit, Innovation und Schulreform. In: Zeitschrift für Pädagogik 2002/ Beiheft 44, 13-27.

Ellrich, Hans / Hoos, Klaus (1998): Vorbild gefragt? Über beispielhaftes Verhalten in Erziehung und Führung. Schulleiter-Handbuch, 88. München: Oldenbourg.

Ender, Bianca / Strittmatter, Anton (2001): Personalentwicklung als Schulleitungsaufgabe. Innsbruck. Studienverlag.

Ehinger, Wolfgang/ Hennig, Claudius $\left(1997^{2}\right)$ : Praxis der Lehrersupervision. Weinheim und Basel: Beltz.

Eikenbusch, Gerhard (1998): Praxishandbuch Schulentwicklung. Frank furt am Main: Cornelsen.

Esslinger, llona (2002): Berufsverständnis und Schulentwicklung: ein Passungsverhältnis?. Bad Heilbrunn: Klinkhardt,

Fend, Helmut (1981): Theorie der Schule. München: Uban\&Schwarzenbeck.

Fend, Helmut (1998): Qualität im Bildungswesen, München: Juventa.

Fiedler, Frank (1967):A Theory of Leadership Effectiveness. New York: McGraw-Hill.

Fiedler, Frank (1987): Führungstheorien-Kontingenztheorie. In: Kieser, Alfred/ Reber, Gerhard/ Wunderer, Rolf (1987): Handwörterbuch der Führung. Stuttgart: Poeschel, 809-823.

Fischer, Walther A../ Schratz, Michael (1999): Schule leiten und gestalten. München. Studienverlag.

Flaake, Karin (1989): Berufliche Orientierungen von Lehrerinnen und Lehrern. Frankfurt a. M., New York: Campus Verlag. 
Flick, Uwe $\left(2002^{6}\right)$ : Qualitative Sozialforschung. Rowohlt. Hamburg.

Flick, Uwe (2002): Triangulation. In: Flick, Uwe/ von Kardorff, Ernst: Handbuch qualitativer Sozialforschung. Hamburg Rowohlt.

Flick, Uwe (2004): Triangulation. Verlag für Sozialwissenschaften, Wies baden.

Forberg, Andrea (1997): Rollen- und Führungsverständnis von Schulleiterinnen Beruflicher Schulen. Eine berufsbiographisch-orientierte Untersuchung. Weinheim 1997.

Frey, Dieter (2003): Schlechte Führung. Ein Interview mit dem Psychologen in der Zeit. Die Zeit, 36/2003. Hamburg.

Frick , Roman (1990): Pädagogische Führung in der guten Schule. In: Pädagogische Führung, 1. Jg., Heft 1, 1990,12-15.

Frick, Roman (1994): Betriebsleitung und Vermögensverwaltung in der teilautonomen Schule. In: Buchen, Herbert/ Horster, Leonard / Rolff, Hans-Günter (1994): Schulleitung und Schulentwicklung. Berlin: Raabe.

Friebertshäuser, Barbara (1997): Interviewtechnik - ein Überblick. In: Friebertshäuser, Barbara ( $\mathrm{Hg}$.):Handbuch qualitative Forschungsmethoden in der Erziehungswissenschaft, Weinheim/München: Juventa.

Fried, Lilian(2002): Pädagogisches Professionswissen und Schulentwicklung. München: Juventa.

Fried, Lilian (2003a): Dimensionen pädagogischer Professionalität. In: Lemmermöhle, Doris/Jahreis, Dirk: Professionalisierung der Lehrerbildung. Die deutsche Schule 7/2003, 7-25.

Fried, Lilian (2003b): Pädagogisches Professionswissen als Form und Medium der Lehrerbildungskommunikation- empirische Suchbewegung. In: Zeitschrift für Pädagogik 2003/49. Jg./1, 112-124.

Friedrichs, Jürgen $\left(1990^{14}\right)$ : Methoden empirischer Sozialforschung. Opladen: Westdeutscher Verlag, 148-153.

Friedrich, Ernst Christian (2000): Soziale Arbeit - Berufsethos - SozialManagement. München: Deutscher Studienverlag.

Frommelt, Bernd/Klemm, Klaus/Rösner, Ernst/Tillmann, Klaus-Jürgen (2000): Schule am Ausgang des 20. Jahrhunderts. München: Juventa.

Fullan, Michael (1999): Change Forces: Probing the Depths of Educati onal Reform. London. 
Fullan, Michael (2000): Schulentwicklung im Jahr 2000. In: Journal für Schulentwicklung,4, 4, 9-16.

Fürstenau, Peter/ Dallmann, Gerhard (1972): Zur Theorie der Schule. Weinheim: Beltz.

Glaser, Barney/ Strauss, Anselm (1967): The Discovery of Grounded Theory. Strategies for Qualitative Research. Chicago: Aldine Atherton.

Glaser, Barney/ Strauss, Anselm (1998): Grounded Theorey. Strategien qualitativer Forschung.Bern, Göttingen, Toronto, Seattle. (Erstveröffentlichung 1967 unter dem Titel: The Discovery of Grounded Theory: Strategies for Qualitative Research. New York.)

Glasl, Friedrich ( $2001^{6}$ ): Konfliktmanagement: Ein Handbuch für Führungskräfte, Beraterinnen und Berater. Bern: Haupt.

Gläser, Jochen/ Landel, Grit (2004) Experteninterviews und qualitative Inhaltsanalyse. Wiesbaden: Verlag für Sozialwissenschaften.

Goleman, Daniel (2002): Emotionale Führung. München: Econ.

Green, Norm (2001): Schule bedeutet Wandel: Wege zu neue Quali tätsgemeinschaften, Partnerschaften und Schulen. In: Blickpunkt Schulleitung (2002): Impulse. Der Beitrag des Schulleitungsverbandes Niedersachsen zur Entwicklung von Schulleitung und Schule. 20 Jahre Schulleitungsverband Niedersachsen. Hannover: Freimann \& Fuchs, 67-76.

Grimm, Matthias Alexander (1993) Kognitive Landschaften von LehrernBerufszufriedenheit und Ursachenzuschreibung angenehmer und belastender Unterrichtssituationen. Frankfurt am Main: Lang.

Habeck, Heinfried (2002): Berufsbild und Rollenverständnis Schulleiter. In: Rolff, Hans-Günter/ Schmidt, Hans-Joachim (2002): Brennpunkt Schulleitung und Schulaufsicht. Neuwied: Luchterhand, 245-250.

Haberleitner, Elisabeth/ Deistler, Elisabeth/ Ungvari, Robert (2003): Führen, Fördern, Coachen. München: Piper.

Häder, Michael (2002): Delphi-Befragungen. Wiesbaden: Westdeutscher Verlag.

Haller, Ingrid/ Wolf, Hartmut (2004):Dialogische Führung. Soest: Verlag für Schule und Weiterbildung.

Hallinger, Philip /Heck, Ronald H. (1998): Exploring the Principial's Contribution to School Effectiveness: 1980-1995. In: School Effectiveness and School Improvement., Vol. 9, No 2, 157-191. 
Hasenbank, Thomas (2000): Führung und Leitung einer Schule als Dimension und Rahmenbedingung Berufsschulischer Entwicklung. München: Diss.

Hauschildt, Jürgen/ Gemünden, Hans Georg (Hg.) (1999): Promotoren. Wiesbaden: Gabler.

Heinze von, Thomas (2001): Qualitative Sozialforschung. München: Oldenbourg,12-165.

Heitmann, Hartmut (2000): Schulmanagement Handbuch, 101: Grund lagen der Personalentwicklung, München.

Helsper, Werner (1996): Antinomien des Lehrerhandelns in modernisierten pädagogischen Kulturen. Paradoxe Verwendungsweisen von Autonomie und Selbstverantwortlichkeit. In: Combe, Arno/ Helsper, Werner (Hg) (1996) Pädagogische Professionalität. Untersuchung zum Typus pädagogischen Handelns. Suhrkamp, Frankfurt a. M, 521-612.

Helsper, Werner (2000): Antinomien des Lehrerhandelns und die Bedeutung der Fallrekonstruktion - Überlegungen zu einer Professionalisierung im Rahmen der universitären Lehrerausbildung: In: Cloer, Erns/ Klika, Dorle/ Kunert, Hubertus (2000): Welche Lehrer braucht das Land? Notwendige und mögliche Reformen der Lehrerbildung. Weinheim: Juventa, 142-177.

Hentze, Joachim/ Kammel, Andreas/ Lindert, Klaus (1990): Personalführungslehre. Stuttgart: UTB.

Herrlitz, Hans-Georg/ Hopf, Wulf/ Titze, Hartmut/ Cloer, Ernst $\left(2005^{4}\right)$ :

Deutsche Schulgeschichte von 1800 bis zur Gegenwart. Weinheim: Juventa.

Hierdeis, Helmwart/ Hug, Theo. (1997): Pädagogische Alltagstheorien und erziehungswissenschaftliche Theorien, Bad Heilbrunn: Klinkhardt.

Hitzler, Ronald (1994): Wissen und Wesen der Experten. Ein Annähe rungsversuch -zur Einleitung, In: Hitzler, Ronald/ Honer, Anne/ Maeder, Christian (Hrsg.), Expertenwissen. Die institutionalisierte Kompetenz zur Konstruktion von Wirklichkeit.

Opladen: Westdeutscher Verlag, 13-30.

Hobeck, Dorothea (2005): Die Unterrepräsentanz von Frauen in Schulleitung. Erlangen-Nürnberg. Dissertation. 
Hoffmann, Frank-Peter/ Mattusch, Norbert/ Heitmann, Hartmut (2002): Motivation, Zielvereinbarungen, Entgeltregelungen. Schulmanagement-Handbuch 21, 2002. München:Oldenbourg.

Holtappels, Heinz-Günter (1995): Innere Schulentwicklung; Innovationsprozesse und Organisationsentwicklung. In: Rolff, Hans-Günter (Hg.): Zukunftsfelder in der Schulforschung. Weinheim: Deutscher Studienverlag.

Holtappels, Heinz-Günter/ Höhmann, Katrin (Hg.) (2005): Schulentwicklung und Schulwirksamkeit. Weinheim/ München: Juventa.

Hopf, Christel (1978): Die Pseudo-Exploration. Überlegungen zur Technik qualifizierten Interviews in der Sozialforschung, In: Zeitschrift für Soziologie, Jg. 7, S. 97-115.

Hopf, Christel/ Weingarten, Elmar (1979): Qualitative Sozialforschung, Wiesbaden: Klett-Cotta, 11-35.

Huber, Stephan Gerhard (1999a): School Effectiveness: Was macht die Schule wirksam? Internationale Schulentwicklungsforschung. In: schul-management, 2, 10-17.

Huber, Stephan Gerhard (2002): Machbarkeits- und Konzeptionsstudie zur Gründung einer Länderakademie für pädagogische Führungskräfte: Bericht für die Cornelsen Stiftung Lehren und Lernen. In der Reihe „Länderakademie für pädagogische Führungskräfte“ (Band 2) von Rolff, Hans-Günter/ Rosenbusch, Heinz S./ Huber, Stephan Gerhard $(\mathrm{Hg})$. Bamberg: Länderakademie für pädagogische Führungskräfte.

Huber, Stephan Gerhard (2002): Schule im internationalen Trend erweiterte und neue Aufgaben. In: Journal für Schulentwicklung, 1/2002, 7-19.

Huber, Stephan Gerhard (2003): Qualifizierung von Schulleiterinnen und Schulleitern im internationalen Vergleich. Eine Untersuchung in 15 Ländern zur Professionalisierung von pädagogischen Führungskräften für Schule. Kronach: Wolters Kluwer.

Husfeldt, Vera / Nikolova, Roumiana (2003): Students' Concept of Democracy. In: European Educational Research Journal, Volume 2, Number 3, 396-409.

Ilien, Albert (2000a): Entwurf eines professionstheoretisch fundierten Konzepts zur systemischen Kollegiumsberatung. Hannover: MS.

Jetter, Wolfgang (2000): Performance Management. Zielvereinbarungen, Mitarbeitergespräche, leistungsabhängige Entlohnungssysteme. Stuttgart: Schäffer-Poeschel. 
Kansteiner-Schänzlin, Katja (2002) Personalführung in der Schule. Rieden: Klinkhardt.

Kassner, Karsten /Wassermann, Petra (2005): Nicht überall wo Methode draufsteht, ist auch Methode drin. Zur Problematik der Fundierung von Experteninterviews. In: Bogner, Alexander/ Littig, Beate (Hg.): Das Experteninterview. Wiesbaden: VS Verlag, 95-130.

Kelle, Udo/ Kluge, Susann (1999): Vom Einzelfall zum Typus. Opladen: Leske \& Budrich.

Kempfert, Guy/Rolff, Hans-Günter (1999): Pädagogische Qualitätsentwicklung. Weinheim: Beltz.

Klippert, Heinz (1997): Schule entwickeln - Unterricht neu gestalten. Weinheim: Beltz.

Kieser, Alfred/ Reber, Gerhard/ Wunderer, Rolf (1987): Handwörterbuch der Führung. Stuttgart: Schäffer-Poeschel.

Kieser, Alfred/ Reber, Gerhard/ Wunderer, Rolf (1995²): Handwörter buch der Führung, Stuttgart: Schäffer-Poeschel.

Kieser, Alfred/ Reber, Gerhard/ Wunderer, Rolf (20014 ): Handwörter buch der Führung, Stuttgart: Schäffer-Poeschel.

Klafki, Wolfgang (2002): Schultheorie, Schulforschung und Schulent wicklung.Weinheim. Beltz.

Klippert, Heinz (2000): Pädagogische Schulentwicklung. Weinheim und Basel: Beltz.

Kluge, Susann (1999): Empirisch begründete Typenbildung. Opladen: Leske-Budrich.

Kluge, Susann (2000): Empirisch begründete Typenbildung in der quali tativen Sozialforschung (20 Absätze). Forum Qualitative Sozialforschung/ Forum: Qualitative Social Research (Online Journal), 1 (1). Abrufbar über: http://qualitative-research.net/fqs (Zugriff: 05.04.2001).

König Eckard/ Volmer, Gerda $\left(2000^{7}\right)$ : Systemische Organisationsberatung. Grundlagen und Methoden. Weinheim: Deutscher Studienverlag.

König Eckard (19997): Reformen der Schule: ein organisations- pädagogisches Thema? In: Wissinger, Jochen (Hg) (1997): Schulleitung als pädagogisches Handeln. München: Oldenbourg.

Kuckartz, Udo (1999): Computergestützte Analyse Qualitativer Daten, Westdeutscher Verlag, Wiesbaden. 
Kuckartz, Udo (2005): Einführung in die Computergestützte Analyse qualitativer Daten. Wiesbaden: Verlag für Sozialwissenschaften.

Kunz-Heim, Doris (2002): Qualität durch Qualifizierung. München: Juventa.

Lamnek, Siegfried (1988): Qualitative Sozialforschung, Band I: Methodologie. München: Psychologie Verlags Union.

Lamnek, Siegfried (1989): Qualitative Sozialforschung Bd. II. Methoden und Techniken. München: Psychologie Verlags Union, 247-264.

Lamnek, Siegfried (1995): Qualitative Sozialforschung, Band 1: Methodologie. Weinheim: Klett-Cotta.

Lamnek, Siegrfied $\left(2005^{4}\right)$ : Qualitative Sozialforschung. Weinheim: Beltz.

Laplanche, Jean/ Pontalis, Jean- Bernhard (1972) Das Vokabular der Psychoanalyse. Bd. 1. Frankfurt am Main: Suhrkamp.

Larisch, Marianne /Joksimovic, Ljijana / von Knesebeck, Olaf/ Starke, Dagmar/ Siegrist, Johannes (2003):Berufliche Gratifikationskrisen und depressive Symptome. In: Zeitschrift für Psychotherapeutische Psychologische Medizin; 53. Stuttgart, New York: Thieme, 223-228.

Lehmann, Jochen/ Boomgaarden, Hero (2002): Schule leiten will gelernt sein. Niedersächsisches Landesinstitut für Schulentwicklung. Hannover.

Lemmermöhle, Doris / Große, Stefanie/ Schellack, Antje / Putschbach, Renate (2005): Passagen und Passantinnen - biographisches Lernen junger Frauen in den Statuspassagen zur Erwerbsarbeit. Münster: Waxmann.

Lenzen, Dieter (1996): Handlung und Reflexion, Weinheim, Basel.

Leppin; Jörg/ Sauer, Karl-Volker (2001): Rückmeldungen an Schulleitungen. In: Journal für Schulentwicklung, 3/2001, 5, 43-49.

Liebel, Hermann (1991): Motivieren durch Kooperation, In: Wissinger, Jochen/Rosenbusch, Heinz S. (Hg.) (1991), Motivation durch Kooperation. Schulleiter Handbuch 58, SL Verlag, Braunschweig, 4056.

Liebel, Hermann/ Oechsler, Walter (1994): Handbuch Human Ressource Management. Wiesbaden: Gabler.

Lohmann, Armin/ Hajek, Manfred/ Döbrich, Peter (1997): Identität und Schulprogramm. München: Lexika-Verlag. 
Lohmann, Armin (1999): Führungsverantwortung der Schulleitung. Neuwied: Luchterhand.

Lohmann, Armin/ Minderop, Dorothea (2004): Führungsverantwortung der Schulleitung. München: Luchterhand.

Lüde von, Rolf/ Philipp. Elmar (2004): Schlüsselrolle der Schulleitung.

In: Driftmann, Hans/ Erdsiek-Rave, Ute/ Riecke-Baulecke, Thomas (2004): Eigenverantwortung annehmen und Schule führen. Schulmanagement-Handbuch 112. München: Oldenbourg, 69-77.

Lüders; Martin(1998): Die Bedeutung der Professionalisierung des Lehrers für die Qualitätssicherung von Schule. In: Ackermann, Helga/ Wissinger, Jochen: (1998): Schulqualität managen, Neuwied: Luchterhand, $77-87$.

Luhmann, Niklas (1984): Soziale Systeme. Grundriss einer allgemeinen Theorie. Frankfurt am Main: Suhrkamp.

Malik, Fredmund (2000): Führen, Leisten, Leben. München: Heyne.

Malik, Fredmund (2001): Personalauswahl und Stellenbesetzung. In: Buchen, Heinz/Horsten, L./ Rolff, Hans-Günter (Hg.) (2001), Schulleitung und Schulentwicklung. 35. Berlin. Raabe.

Malik, Fredmund (2002): Was echte Führer wirklich tun. Wider dem Glauben an angeborene, charismatische Führerpersönlichkeiten. In: Buchen, Herbert/ Horster, Leonard/ Rolff, Hans-Günter (Hg.) (2002), Schulleitung und Schulentwicklung. 36. Raabe, Berlin.

Mauch, Siegfried (1999): Zielorientiertes Führen - ein Umsetzungsmodell für die öffentliche Verwaltung. Düsseldorf: Raabe.

Mayring, Philipp (2002): Einführung in die Qualitative Sozialforschung. Weinheim. Beltz.

MAXqda (2001): Handbuch. Berlin.

Meuser, Michael /Nagel, Ulrike (1991): ExpertInneninterviews - vielfach erprobt, wenig bedacht. In: Garz, Detlef / Kraimer, Klaus (Hg.): Qualitativ-empirische Sozialforschung. Opladen: Westdeutscher Verlag, 441-471.

Meuser, Michael/ Nagel, Ulrike (1997): Das Experteninterview Wissenssoziologische Voraussetzungen und methodische Durchführung. In: Friebertshäuser, Barbara / Prengel, Annedore (Hg.): Handbuch Qualitativer Forschungsmethoden in der Erziehungswissenschaft. Weinheim: Juventa, 481-491. 
Meyer, Rita (2000): Qualifizierung für moderne Beruflichkeit. Soziale Organisation der Arbeit von Facharbeiterberufen bis zu Managertätigkeit. Münster, New York, München, Berlin: Waxmann.

Miller, Reinhold (2003): Selbst-Coaching für Schulleitung. Weinheim: Beltz.

Miller, Susanne (2001): Schulleiterinnen und Schulleiter. Hohengehren: Schneider Verlag,

Morgan, Gareth $\left(2000^{2}\right)$ : Bilder der Organisation. Stuttgart. Klett.

Müller, Alfred/ Gampe, Harald/ Rieger, G. / Risse, Erika (Hg.)(1997): Leitung und Verwaltung einer Schule. Kapitel 6. Neuwied: Luchterhand.

Münch, Elke (1999): Neue Führungsperspektiven in der Schulleitung Kooperation zwischen Schulleiter und Stellvertreter. Luchterhand, Münster.

Neubauer, Walter / Gampe, Harald/ Knapp, Rudolf/ Wichterich, Heiner (1999): Konflikte in der Schule. Aggression, Kooperation, Schulentwicklung. München: Luchterhand.

Neubauer, Walter (2003): Organisationskultur. Stuttgart: Kohlhammer.

Neubauer, Walter (2004): Führung und Personalmanagement. In:

Driftmann, Hans/ Erdsiek-Rave, Ute/ Riecke-Baulecke, Thomas (2004): Eigenverantwortung annehmen und Schule führen. Schulmanagement-Handbuch 112. München: Oldenburg 35-51.

Neuberger, Oswald (1984): Führung - Ideologie - Struktur - Verhalten, Stuttgart. Enke.

Neuberger, Oswald $\left(1994^{2}\right)$ : Personalentwicklung. Stuttgart: Enke, 59-72.

Neuberger, Oswald $\left(1995 \mathrm{a}^{2}\right)$ : Führungstheorien - Machttheorie. In: Hitzler, Ronald/ Honer, Anne/ Maeder, Christian (Hg.):Handwörterbuch der Führung. Stuttgart. 831-843.

Neuberger, Oswald $\left(2002^{6}\right)$ : Führen und Führen lassen. Stuttgart: Luci us \& Lucius.

Neulinger, Klaus (1990): Schulleiter in Baden-Württemberg - Lehrerelite zwischen Job und Profession. Herkunft, Motive und Einstellungen einer Berufsgruppe. Frankfurt am Main. Dissertation.

Nevermann, Knut (1982): Der Schulleiter. Juristische und pädagogische Aspekte zum Verhältnis von Bürokratie und Pädagogik, Stuttgart: Klett-Cotta. 
Niederhuber, Susanne (2002): Schulleiterhandeln aus der Lehrerperspektive. Universität Bamberg.

Niedersächsisches Kultusministerium (1996): Schulentwicklung, Beratung und Fortbildung in Niedersachsen. Stand-Perspektiven- Empfehlungen. Bericht der Kommission „Schulentwicklung, Beratung und Fortbildung" vom 29.11.1996. Hannover.

Niedersächsisches Kultusministerium (1997):

Schul(verwaltungs)reform. Schulprogramme in Niedersachsen Perspektiven und Empfehlungen. Bericht der Arbeitsgruppe „Schulprogramme" im Niedersächsischen Kultusministerium vom 06.10.1997. Hannover.

Niedersächsisches Kultusministerium (1998): Schulprogrammentwick lung und Evaluation. Stand, Perspektiven und Empfehlungen. Niedersachsen macht Schule. Hannover.

Niedersächsisches Kultusministerium (2004): Niedersächsisches Schulgesetz. Hannover.

Niedersächsisches Kultusministerium (2002): Die Schulleiterinnen und Schulleiter in Niedersachsen. Projektgruppe „Arbeitsplatz Schulleitung" vom 04.11.2002. Hannover.

Niedersächsisches Kultusministerium (2002): Niedersachsen macht Schule mit der selbstständigen Schule. Hannover.

Niedersächsisches Kultusministerium (2003): Orientierungsrahmen Schulqualität in Niedersachsen. Hannover.

Niedersächsisches Kultusministerium (2005): Entwurf zum Gesetz der Einführung der Eigenverantwortlichen Schule (Stand 20.10.2005). Hannover.

Obermeyer, Klaus (2005): Die Schulleitung als Coach. In: Schulmanagement Handbuch 36/2005. Oldenbourg, 33-35.

Oelkers, Jürgen (1995): Wie lernt ein Bildungssystem? In: Die Deutsche Schule, Jg. 87, H 1, 4-20.

Oevermann, Ulrich $\left(1996^{2}\right)$ : Theoretische Skizze einer revidierten Theorie professionellen Handelns. In: Combe, Arno/ Helsper, Werner $(\mathrm{Hg})$ (1996) Pädagogische Professionalität. Untersuchung zum Typus pädagogischen Handelns. Frankfurt am Main : Suhrkamp, 70-182.

Orth, Gerhard (2001): Gestaltungsspielräume als Innovations- und Leistungsanreiz. In: Journal für Schulentwicklung 4/2001, 5, 20-26. 
Pallasch, Waldemar $\left(1997^{3}\right)$ : Supervision: Neue Formen beruflicher Praxisbegleitung. In pädagogischen Arbeitsfeldern. Weinheim, München: Juventa.

Pallasch, Waldemar/ Kölln, Detlev $\left(2002^{5}\right)$ : Pädagogisches Gesprächstraining. Weinheim und München: Juventa.

Pallasch, Waldemar/ Simon, Rainer (2003): Professionelles Coaching im Schulbereich. In: Journal für Schulentwicklung. Innsbruck Wien, 17-26.

Pallasch, Waldemar /Petersen, Ralf (2005): Coaching. Weinheim, München. Juventa.

Peters, Tom (1998): Der Innovationskreis. Düsseldorf, München: Econ.

Philipp, Elmar (1996 $\left.{ }^{4}\right)$ : Gute Schule verwirklichen. Weinheim und Basel: Beltz.

Philipp, Elmar/ Rolff, Hans-Günter (1998): Schulprogramm und Leitbilder entwickeln. Weinheim und Basel: Beltz.

Pfadenhauer, Michaela ( 2005):Das Experteninterview - ein Gespräch Zwischen Experte und Quasi-Experte. In: Bogner, Alexander/ Littig, Beate/ Menzel, Wolfgang (Hg.): Das Experteninterview. VS Verlag, Wiesbaden, , 113130.

Pfiffner, Martin/ Stadelmann, Peter (1994): Expertenwissen von

Wissensexperten. In: Hitzler, Ronald/ Honer, Anne/ Maeder, Christian(Hrsg.): Expertenwissen. Die institutionalisierte Kompetenz zurKonstruktion von Wirklichkeit, Opladen: Westdeutscher Verlag, 146153.

Pollack, Walter/ Pirk, Dieter (2001): Personalentwicklung in lernenden Organisationen. Wiesbaden: Gabler.

Probst, Gilbert $\left(1992^{2}\right)$ : Regeln des systemischen Denkens, In: Probst, Gilbert Siegwart, Hans (1992): Integriertes Management, Bausteine des systemischen Managements, Bern/ Stuttgart: Verlag Haupt,181-205.

Prondczynskiy von, A. (2001): Evaluation der Lehrerausbildung in den USA: Geschichte, Methode, Befunde. In: Keiner, Edwin (Hg): Evaluationen der Erziehungswissenschaft, Weinheim, Berlin.

Ratzki, Anne (2002): Schule und Schulaufsicht - ein mehrdeutiges Verhältnis. In: Rolff, Hans-Günter/ Schmidt, Hans-Joachim (Hg.) (2002): Brennpunkt Schulleitung und Schulaufsicht Neuwied, Kriftel: Luchterhand. 60-72. 
Rahm, Sibylle (2002): Schulentwicklung als Aufgabe und Handlungsfeld der Schulleitung - Die Perspektive der schottischen Qualitätsinitiative. In: Wissinger, Jochen/ Huber, Stephan Gerhard (2002): Schulleitung- Forschung und Qualifizierung, Opladen: Leske \& Budrich, 79-96.

Rahm, Sibylle (2005): Einführung in die Theorie der Schulentwicklung. Weinheim/ Basel: Beltz.

Rahm, Sibylle/ Schley, Wilfried (2005): Von der Kraft der Paradoxien. In: Journal für Schulentwicklung, 9. Jg., 2/2005, 9-21.

Rauscher, Heinz (2001): Verändertes Lehrerimage als Herausforderung. Schulleiter- Handbuch; 97. München: Oldenbourg.

Rauscher, Heinz (1995): Der Umgang mit Belastungen im Schulleiter beruf, Schulleiter-Handbuch, 77, SL-Verlag.

Regenthal, Gerhard (1999): Corporate Identity in Schulen. Neuwied: Luchterhand. Richter, Jens (1995): Theorien der Schulentwicklung, In: Daschner, Peter/ Richter, Thomas/ Doppke, Michael (2002): Grundlagen der Personalentwicklung 1; Personalführung, Jahresgespräche, Führungsfeedback. Schulmanagement- Handbuch 21, 2002. München: Oldenbourg.

Ricke-Baulecke (2001): Schulprogramme und wirksames Management: Grundsätze, Verfahren, Instrumente. Schulleiter-Handbuch, 100. München. Oldenbourg.

Ricker, Kirsten (2000): Migration, Sprache und Identität. Eine biographie-analytische Studie zu Migrationsprozessen von Französinnen in Deutschland,. Bremen.

Riegel, Enja (2006):Starke Schulleitung und innere Schulreform. In: Die Deutsche Schule, 98. JG, 2006/2, 139-141.

Rinne, Karin (2003): SPSS - Statistical Package for the Social. Frankfurt am Main: Franzis Verlag.

Risse, Erika (Hg.) (1998): Schulprogramm - Entwicklung und Evaluation. Neuwied: Luchterhand.

Risse, Erika./ Schmidt, Hans-Joachim (Hg.) (1999): Von der Bildungsplanung zur Schulentwicklung. Neuwied: Luchterhand.

Rolff, Hans-Günter (1986): Daten, Beispiele und Perspektiven. In: Jahrbuch der Schulentwicklung. Band 4, Weinheim: Beltz.

Rolff, Hans-Günter (1991): Schulentwicklung als Entwicklung von Ein zelschulen? Zeitschrift für Pädagogik, 37, S. 865-886. 
Rolff, Hans-Günter(1992): Die Schule als besondere soziale Organisation- Eine komparative Analyse. Zeitschrift für Sozialisationsforschung und Erziehungssoziologie (ZSE), 12. Jahrgang. 4, S. 304323.

Rolff, Hans-Günter (1993): Wandel durch Selbstorganisation. Theoretische Grundlagen und praktische Hinweise für eine bessere Schule. Weinheim/ München: Juventa.

Rolff, Hans-Günter, Stryck, Tom (1995): Schulautonomie - Chancen und Grenzen. Weinheim: Juventa.

Rolff, Hans-Günter (1998): Schulaufsichtsentwicklung in Deutschland.

Ein vergleichender Bericht aus Sicht des nordrhein-westfälischen QUESS-Projektes. In: Ministerium für Schule und Weiterbildung und Landesinstitut für Schule und Weiterbildung (Hg.) (1998), Schulentwicklung und Schulaufsicht - Qualitätsentwicklung und Qualitätssicherung von Schule. Soest :Verlag für Schule und Weiterbildung, $95-120$.

Rolff, Hans-Günter /Buhren, Claus G./ Lindau-Bank, Detlev/ Müller, Sabine (1998): Manual Schulentwicklung. Weinheim: Beltz.

Rolff, Hans-Günter /Schmidt, Hans-Joachim (Hg) (2002): Brennpunkt Schulleitung und Schulaufsicht. Neuwied, Kriftel: Luchterhand.

Rolff, Hans-Günter /Buhren, Claus G. (2002): Personalentwicklung in Schulen. Weinheim: Beltz.

Rolff, Hans-Günter /Philip, Elmar (2004): Schulprogramme und Leitbil der entwickeln. Weinheim: Beltz.

Rosenbusch, Christa (2000). Schulpsychologen - Unterstützung oder Belastung für Schulleiter. schul-management, I, S. 37-42.

Rosenbusch, Heinz S. (1989): Der Schulleiter - ein notwendiger Ge genstand organisationspädagogischer Reflexion. In: Rosenbusch, Heinz S./ Wissinger, Jochen (Hg) (1989), Schulleiter zwischen Administration und Innovation. SL-Verlag. Braunschweig.

Rosenbusch, Heinz S. (1995): Reform der Schulverwaltung aus organisationspädagogischer Sicht. Schulleitung und Schulaufsicht als erzieherisch bedeutsame Wirklichkeit. schul-management, 3642.

Rosenbusch, Heinz S./Wissinger, Jochen (Hg.) (1989): Schulleiter zwischen Administration und Innovation. Schulleiter - Handbuch 50. Braunschweig: SL-Verlag. 
Rosenbusch, Heinz (1997): Organisationspädagogische Perspektiven einer Reform der Schulorganisation. In: SchulVerwaltung, 10, 329334.

Rosenbuch, Heinz S. (1999): Schulleitung und Schulaufsicht. (Studien Brief der Fernuniversität Hagen). Weiterbildendes Studium „Vorbereitung auf Leitungsaufgaben in Schulen" (Vorl. Auf). Modul 7/3. Hagen.

Rosenbusch, Heinz S. (2000): Schulleitung - Reformgröße im gesellschaftlichen Umbruch. In: Scheunpflug. Annette $(\mathrm{Hg})$ (2000),Schulleitung im gesellschaftlichen Umbruch, Schulleiter Handbuch 93, München: Oldenbourg, 7-20.

Rosenbusch, Heinz S./Huber, Stephan Gerhard (2001): Qualifizierungsmaßnahmen von Schulleiterinnen und Schulleitern in den Ländern der Bundesrepublik Deutschland. schul-management, 4, S. 8-16.

Rosenbusch, Heinz S./Huber, Stephan Günter (2002): Organisation und Merkmale der Qualifizierung von Schulleiterinnen und Schulleitern in den deutschen Bundesländern. In: Wissinger, Jochen/ Huber, Stephan Gerhard (2002): Schulleitung - Forschung und Qualifizierung. Opladen: Leske \&Budrich, 111-128.

Rosenbusch, Heinz (2003): Schulleitung-Reformgröße im gesellschaftlichen Umbruch. In: Schulleiter-Handbuch 93. SL-Verlag,7-21.

Rosenbusch, Heinz (2005): Organisationspädagogik der Schule. München: Luchterhand.

Rosenholtz, Susan (1991): Teachers' Workplace. The Social Organization Of Schools. New York: College Press.

Rosenstiel, Lutz von (2003): Kommunikation und Führung in Arbeitsgruppen. In: Schuler, Heinz (Hg.): Lehrbuch Organisationspsychologie. Bern: Verlag Hans Huber, 321-351.

Rosenstiel, Lutz von/ Regnet, Erika/ Domsch, Michel E. (1993): Führung von Mitarbeitern: Handbuch für erfolgreiches Personalmanagement. Stuttgart: Schäffer-Verlag.

Scala, Klaus /Grossmann, Ralph (19972): Supervision in Organisationen. Weinheim und München: Juventa.

Schaarschmidt, Uwe/ Arold, Helga/ Kieschke, Ulf (2003): Die Bewältigung psychischer Anforderungen durch Lehrkräfte. In: SchulleiterHandbuch 93.München: Oldenbourg, 62-73. 
Schauenberg, Bernd (1995): Wissenschaftstheoretische Grundfragen der Führungsforschung-Phänomenologie und Konstruktivismus. In: Kieser, Arno/ Reber, Gerhard/ Wunderer, Rolf (Hg.) $\left(1995^{2}\right)$ : Handwörterbuch der Führung. Stuttgart: Schäfer-Poeschel, 22062214.

Schettgen, Peter (1991): Führungspsychologie im Wandel. Neue Ansätze in der Organisations-, Interaktions- und Attributionsforschung. Wiesbaden. Deutscher Universitätsverlag.

Schlee, Jörg (2002): Ressourcen für Personen mit Leitungs- und Führungsaufgaben- Zur Notwendigkeit eines Unterstützungssystems für Schulleitungen. In: Schule als Chance. Dokumentation Schulmanagement-Tagungen. Oldenburg, 57-62.

Schley, Wilfried (1998). Konfliktmanagement- Zum Verhältnis von Schulleitung, Lehrern, Eltern und Schülern. In: Ackermann, Helga/ Wissinger, Jochen (Hg.) (1998), Schulqualität managen. Von der Verwaltung der Schule zur Entwicklung von Schulqualität Neuwied: Luchterhand, 178-199.

Schley, Wilfried (2001): Sozialpsychologie der Schulentwicklung. In: Journal für Schulentwicklung 4/2001, 5, 8-14.

Schnell, Rainer/ Hill, Paul B./ Esser, Elke $\left(1998^{15}\right)$ : Methoden der empirischen Sozialforschung. Band 28, Opladen: Westdeutscher Verlag, 13-37

Schütz, Alfred (1971-1972):Gesammelte Schriften (Bd. 1-2). Den Haag.

Schütze, Fritz (1987): Das narrative Interview in Interaktionsfeldstudien: erzähltheoretische Grundlagen. 1. Studienbrief der Fern Universität Hagen. Nr. 3757.Hagen.

Schütze, Fritz (1996): Organisationszwänge und hoheitsstaatliche Rahmenbedingungen im Sozialwesen: Ihre Auswirkungen auf Paradoxien des professionellen Handelns.

In: Combe, Arno/Helsper, Werner (Hg) (1996) Pädagogische Professionalität. Untersuchung zum Typus pädagogischen Handelns. Frankfurt a. M.: Suhrkamp, 183-275.

Schütze, Fritz/ Bräu, Karin/ Liermann, Hildegard/ Prokop, Karl/ Speth, Martin/ Wiesemann, Jutta (1996): Überlegungen zu Paradoxien des professionellen Lehrerhandelns in Dimensionen der Schulorganisation. In. Helsper, Werner/ Krüger, Heinz-Hermann/ Wenzel, Hartmut (Hg.) (1996): Schule und Gesellschaft im Umbruch. Deutscher Studienverlag, 333-375.

Scholl, Armin (2003): Die Befragung. Das Leitfaden- und Experteninterview. Konstanz. UVK 
Schratz, Michael (1996): Die Rolle der Schulaufsicht in der autonomen Schulentwicklung. Innsbruck: Studienverlag.

Schratz, Michael (1996): Gemeinsam Schule lebendig gestalten. Weinheim und Basel: Beltz.

Schratz, Michael (1997): Qualität sichern: Schulprogramme entwickeln. Weinheim: Beltz.

Schratz, Michael (2002): Die Schulaufsicht und die teilautonome Schule. In: Rolff, Hans-Günter/Schmidt, Hans-Joachim (Hg.) (2002), Brennpunkt Schulleitung und Schulaufsicht (S. 27-42). Neuwied, Kriftel: Luchterhand.

Schreyögg, Georg (1995): Führungstheorie - Situationstheorie. In: Kieser, Alfred /Reber, Gerhard/ Wunderer Rolf (Hg.) Handwörterbuch der Führung.2. Stuttgart, 993-1005.

Schulleitungsverband Niedersachsen e.V. (2003): Berufsbild Schulleiterin/ Schulleiter. Hannover: SLNV 2003, H. 77.

Schwarz, Ralf/ Jerusalem, Matthias (2002): Das Konzept der Selbstwirksamkeit, In: Zeitschrift für Pädagogik, 44, 2002, 29-38.

Seidel, Eberhard/ Jung, Rüdiger/ Redel, Wolfgang (1988): Führungsstil und Führungsorganisation. Bd. 1. Darmstadt: Wissenschaftliche Buchgesellschaft.

Senge, Peter $\left(1999^{7}\right)$. Die fünfte Disziplin. Stuttgart: Klett-Cotta. (amerikanische Originalausgabe (1990) The Fifth Discipline: The art und practice of de learning organization. New York: Doubleday).

Senge, Peter/ Kleiner, Art/ Roberts, Charlotte/ Ross, Richard/ Bryan, Smith (2000): The Dance of Chance: 10 Herausforderungen tiefgreifender Veränderungen in Organisationen. Wien, Hamburg: Signum Verlag.

Siedenbiedel, Georg (2001): Organisationslehre. Stuttgart., Berlin, Köln: Kohlhammer.

Siegrist, Johannes (1995): Medizinische Soziologie. München, Wien, Baltimore: Urban \& Schwarzenberg, 180-190.

Silvermann, David (1985): Qualitative Methodology and Sociology. Aldershot:Gower.

Simon, Rainer (2001): Personalmanagement als Gesamtaufgabedas Beispiel Schleswig Holstein. In: Journal für Schulentwicklung, 3/2001, 5, 49-62. 
Smolka, Dieter (Hg.) (2000): Motivation und Mitarbeiterführung in der Schule: Empfehlungen für die Schulpraxis. Neuwied, Kriftel: Luchterhand.

Soretz, Friedrich (2003): Pädagogische Professionalität und schulische Organisationsentwicklung, Verlag Dr. Kovac.

Söll, Florian (2002): Was denken Lehrer/innen über Schulentwicklung? Weinheim und Basel: Beltz.

Spreemann, Sandra (2000): Geschlechtsstereotype Wahrnehmung von Führung, Verlag Kovac

Spöhring, Walter (1989): Qualitative Sozialforschung. Stuttgart: Teubner.

Sprenger, Reinhard K. $\left(2001^{2}\right)$ : Aufstand des Individuums. Frank furt/Main: Campus.

Sprenger, Reinhard K. (2003): Warum Vertrauen? ... weil es Führung erfolgreich macht. Pädagogische Führung, 3,116-118.

Sprondel, Walter (1979): Zur Entwicklung von Typenbegriffen in der Wissenssoziologie, In: Sprondel, Walter/ Grathoeff, Richard (Hg.): Alfred Schütz und die Idee des Alltags in den Sozialwissenschaften, Stuttgart,140-154.

Staehle, Wolfgang (1999): Management. München: Valden.

Steffens, Ulrich/ Bargel, Tino (Hg.) (1993): Erkundungen zur Qualität von Schule. Neuwied: Luchterhand.

Steffens, Ulrich (1991): Kooperation in Kollegien und Schulqualität Empirische Befunde aus der Konstanzer Schulforschung. In: Wissinger, Jochen/ Rosenbusch, Heinz S. (Hg.) (1991), Motivation durch Kooperation. Schulleiter-Handbuch 58. SL-Verlag. Braunschweig, 30-40.

Steyrer, Johannes (1996): Prozesse der Führung. In: Kasper, Helmut/ Mayrhofer, Wolfgang (Hg.) (1996): Personalmanagement, Führung, Organisation. Wien: Linde Verlag,153-224.

Stichweh, Rudolf (1987): Professionen und Disziplinen - Formen der Differenzierung zweier Systeme beruflichen Handelns in modernen Gesellschaften. In: Harney, Klaus/ Jütting, Dieter/ Koring, Bernhard (1987): Professionalisierung der Erwachsenenbildung. Frankfurt am Main, Bern, New York: Peter Lang, 210-275.

Stichweh, Rudolf (1996): Profession in einer funktional differenzierten 
Gesellschaft. In: Combe, Arno/Helsper, Werner (Hg) (1996) Pädagogische Professionalität. Untersuchung zum Typus pädagogischen Handelns. Frankfurt am Main: Suhrkamp, 49-69.

Stogdill, Ralph Melvin (1974): Stogdill's Handbook of Leadership: A survey of the literature. New York: Berrett.

Storath, Roland (1995): Praxisschock bei Schulleitern? Neuwied, Kriftel: Luchterhand.

Strasser, Franz (1998): Probleme und Lösungen im Alltag der Schulleitung. Schulleiter-Handbuch 87, 1998. München: Oldenbourg.

Strasser, Franz (1997): Herausforderungen für die Schulleitung- Probleme und Lösungen. Schulleiter-Handbuch 85, 1997. München: Oldenbourg.

Strauss, Anselm/ Corbin, Juliet (1996): Grounded Theory: Grundlagen Qualitativer Sozialforschung. Weinheim: Beltz/ Psychologie Verlags Union.

Stroebe, Rainer W. (2002): Grundlagen der Führung. Heidelberg. Sauer-Verlag.

Struck, Peter/ Würtl, Ingo (1999): Vom Pauker zum Coach, München: Hanser-Verlag.

Strübing, Jörg (2004): Grounded Theory. Wiesbaden: Verlag Sozialwissenschaften

Tannenbaum, Robert/ Schmidt, Warren H. (1958): How to Chose a Leadership Pattern. In: Harvard Business Review 36, (2), 95-101.

Tenorth, Heinz-Elmar (1989) Professionstheorie für die Pädagogen, In: Zeitschrift für Pädagogik, 35, 809-823

Terhart, Ewald (1986): Organisation und Erziehung. Zeitschrift für Pädagogik, 32 (2), 205-223.

Terhart, Ewald (1995): Lehrerprofessionalität, In: Rolff, Hans-Günter (1995): Zukunftsfelder in der Schulforschung, Weinheim: Beltz, 225266,

Terhart, Ewald (1996): Berufskultur und professionelles Handeln bei Lehrern. In: Combe, Arno/ Helsper, Werner (Hg) (1996) Pädagogische Professionalität. Untersuchung zum Typus pädagogischen Handelns. Suhrkamp, Frankfurt am Main 448-471. 
Terhart, Ewald (1997): Schulleitungshandeln zwischen Organisation und Erziehung. In: Wissinger, Jochen/Rosenbuch, Heinz (Hg) (1997), Schulleitung als pädagogisches Handeln. SchulleiterHandbuch 83 München: Oldenbourg, 7-20.

Tillmann, Klaus-Jürgen (1995): Schulentwicklung und Lehrerarbeit. Nicht auf bessere Zeiten warten. Bergen: Bergmann und Helbig.

Titscher, Stefan/ Wodak, Ruth/ Meyer, Michael/ Vetter, Eva (1988): Methoden der Textanalyse. Wiesbaden. Westdeutscher Verlag.

Türk, Klaus (1989): Neuere Entwicklungen in der Organisationsforschung. Stuttgart: Enke.

Ulrich, Peter/ Probst, Gilbert $\left(1991^{3}\right)$ : Anleitung zum ganzheitlichen Denken und Handeln: ein Brevier für Führungskräfte. Bern, Stuttgart: Haupt.

Universitäts-Rechenzentrum Trier (Hg.) (2001): Analyse von Strukturgleichungsmodellen mit AMOS 4.0; 4-8.

Wahl, Diethelm (1991): Handeln unter Druck. Strukturierter Dialog als Verbalisationsmethode. Weinheim: Deutscher Studien Verlag, 70-84.

Weick, Karl E. (1976): Educational Organizations as Loosely Coupled Systems. Administrative Science Quarterly, 21, 15-19.

Weik, Elke (1998): Zeit. Wandel und Transformation. Mehring.

Weinert, Ansfried (2004 ${ }^{4}$ ): Menschenbilder und Führung. In: Weinert, Ansfried (2004): Menschenbilder in Organisationen. Arbeits- und Organisations- psychologie 5. Weinheim: Beltz Psychologie Verlags Union, 777-790.

Weinert, Ansfried (1981²): Lehrbuch für Organisationspsychologie. München: Weinheim: Urban \& Schwarzenberg.

Weitzel, Ulrike (2002): Berufsbild und Rollenverständnis der Schulaufsicht In: Rolff, Hans-Günter./Schmidt, Hans-Joachim (Hg) (2002), Brennpunkt Schulleitung und Schulaufsicht Neuwied, Kriftel: Luchterhand, 323-335.

Wildt, Johannes (1999): Lehrerprofessionalität und Schulentwicklung. In: Rösner, Ernst (Hg.) (1999): Schulentwicklung und Schulqualität. Dortmund. IFS-Verlag, 121-142.

Winkel, Rainer (1998): Theorie und Praxis der Schule: oder: Schulreform konkret- im Haus des Lebens und Lernens. Baltmannsweiler: Schneider-Verlag. 
Willms, Johannes (2004): Führungsmodelle in der Organisationsberatung unter integraler Perspektive. Göttinger Beiträge 27. Göttingen.

Winkler, Rainer(1989): Was ist eine gute Schulleitung. Zusammenfassende Vorstellung und Kommentar zu den Arbeitsgruppenergebnissen. In: Ermert, Karl (Hg.): Schulleitung zwischen Verwalten und Gestalten. Loccum: Evangelische-Akademie.

Winkler, Rainer, (1997): Theorie und Praxis der Schule. Hohengehren: Schneider Verlag, 351-389.

Winterhagen-Schmidt, Luise (1997): Berufsziel Schulleiterin. Weinheim: Juventa.

Wirries, Ingeborg (1993): Führen in der Schule. Schulleiter-Handbuch 67,1993. Braunschweig: Schulleitungs-Verlag.

Wirries, Ingeborg (2001): Erfolgsfaktor: Mitarbeiter. SchulleiterHandbuch, 98. München: Oldenbourg.

Wissinger, Jochen (1993): Schulleiterinnen und Schulleiter, die Rollenwahrnehmung in ihrer Bedeutung für die schulische Entwicklung. In: Wissinger, Jochen/ Rosenbusch, Heinz S. (Hg.) (1993), Schule von innen verändern. Schulleiter-Handbuch 66 (S. 68-80). Braunschweig: SL-Verlag.

Wissinger, Jochen (1996): Perspektiven schulischen Führungshandelns. Eine Untersuchung über das Selbstverständnis von Schulleiterlnnen. Weinheim, München: Juventa-Verlag.

Wissinger, Jochen (Hg). (1997): Schulleitung als pädagogisches Handeln. Schulleiter-Handbuch 83. München: Oldenbourg.

Wissinger, Jochen (2000): Rolle und Aufgaben der Schulleitung bei der Qualitätssicherung und -entwicklung von Schulen. Zeitschrift für Pädagogik, 6, 851-865.

Wissinger, Jochen/ Huber, Stephan Gerhard (Hg.) (2002): Schulleitung -Forschung und Qualifizierung. Leske \& Budrich, Opladen

Wiswede, Günter/ Wiendieck, Gerd (Hg.) (1990): Führung im Wandel, Neue Perspektiven der Führungsforschung und Führungspraxis. Stuttgart: Ferdinand Enke Verlag.

Wiswede, Günter (1995): Führungsrollen. In: Kieser, Arno/ Reber, Ger hard Wunderer, Rolf (Hg.) (1995²):Handwörterbuch der Führung. Stuttgart. 826-839.

Witzel, Andreas (1985): Das problemzentrierte Interview. In: Jüttemann, Gerd (Hg.): Qualitative Sozialforschung in der Psychologie. 
Weinheim: Beltz, 227-255.

Witzel, Andreas (2000): Das problemzentrierte Interview (26 Absätze). Forum Qualitative Sozialforschung, Forum: Qualitative Social Research. (Online Journal), 1 (1). Abrufbar über: http://qualitativeresearch.net/fsq. (Zugriff 01.01.2001)

Wunderer, Rolf / Grunwald, Wolfgang (1980): Führungslehre. Band 1: Grundlagen der Führung. Berlin/ New York: Walter de Gruyter.

Wunderer, Rolf (1995): Mitarbeiterführung- Entwicklungstendenzen. In: Kieser, Alfred/ Reber, Gerhard/ Wunderer, Rolf $\left(1995^{2}\right)$ : Handwörterbuch der Führung, Stuttgart: Schäffer-Poeschel.

Wunderer, Rolf/Kuhn, Thomas, (2000): Führungsrelevante Grundfragen der Philosophie und der Sozialwissenschaften, Stuttgart: SchäfferPoeschel.

Wunderer, Rolf $\left(2003^{5}\right)$ Führung und Zusammenarbeit. München: Luchterhand.

Zöfel. Peter (2002): Statistik verstehen. Ein Begleitbuch zur computergestützten Anwendung. München: Pearson Studium.

Zündorf, Lutz (1986): Macht, Einfluss, Vertrauen und Verständigung. Zum Problem der Handlungskoordinierung in Arbeitsorganisationen. In: Seltz, Rüdiger/ Mill, Ulrich/ Hildebrandt, Eckart (Hg.) (1986): Organisation als soziales System. Berlin: Sigma, 33-48.

Zymnek, Bernd /Sikorski, Sandra (2005): Der Beitrag der empirischen Schulforschung zu einer dezentralen Schulpolitik. In: Journal für Schulentwicklung 1/2005, 9, 10-20. 
9. Übersicht über Abbildungen und Tabellen

\begin{tabular}{|c|c|c|c|}
\hline Abbildungen & & Titel & Seite \\
\hline Abbildung & 1 & Aufbau der Arbeit & 10 \\
\hline Abbildung & 2 & Vier-Rahmen-Modell Bolman/Deal & 21 \\
\hline Abbildung & 3 & Theoretische Bezüge & 34 \\
\hline Abbildung & 4 & $\begin{array}{l}\text { Führungsstilkontinuum nach Tannen- } \\
\text { baum/Schmidt }\end{array}$ & 37 \\
\hline Abbildung & 5 & Führungsstiltypologie & 38 \\
\hline Abbildung & 6 & Entwicklung der Organisationstheorie & 49 \\
\hline Abbildung & 7 & Schulentwicklungsstationen & 54 \\
\hline Abbildung & 8 & $\begin{array}{l}\text { Modell einer Berufsauffassung für Schulleite- } \\
\text { rinnen und Schulleiter }\end{array}$ & 76 \\
\hline Abbildung & 9 & Triangulation & 81 \\
\hline Abbildung & 10 & $\begin{array}{l}\text { Umgang von Schulleiterinnen und Schulleiter } \\
\text { mit Lehrerinnen und Lehrern }\end{array}$ & 88 \\
\hline Abbildung & 11 & $\begin{array}{l}\text { Führungsverhalten von Schulleiterinnen und } \\
\text { Schulleitern }\end{array}$ & 89 \\
\hline Abbildung & 12 & $\begin{array}{l}\text { Berufszufriedenheit von Schulleiterinnen und } \\
\text { Schulleitern }\end{array}$ & 90 \\
\hline Abbildung & 13 & $\begin{array}{l}\text { Berufszufriedenheit und Belastungsempfinden } \\
\text { von Schulleiterinnen und Schulleitern (1) }\end{array}$ & 91 \\
\hline Abbildung & 14 & $\begin{array}{l}\text { Berufszufriedenheit und Belastungsempfinden } \\
\text { von Schulleiterinnen und Schulleitern (2) }\end{array}$ & 92 \\
\hline Abbildung & 15 & $\begin{array}{l}\text { Schulentwicklung durch Schulleiterinnen und } \\
\text { Schulleiter (1) }\end{array}$ & 93 \\
\hline Abbildung & 16 & $\begin{array}{l}\text { Schulentwicklung durch Schulleiterinnen und } \\
\text { Schulleiter (2) }\end{array}$ & 94 \\
\hline Abbildung & 17 & $\begin{array}{l}\text { Führungsverhalten von Schulleiterinnen und } \\
\text { Schulleitern (1) }\end{array}$ & 95 \\
\hline Abbildung & 18 & $\begin{array}{l}\text { Führungsverhalten von Schulleiterinnen und } \\
\text { Schulleitern (2) }\end{array}$ & 95 \\
\hline Abbildung & 19 & $\begin{array}{l}\text { Führungsverhalten von Schulleiterinnen und } \\
\text { Schulleitern (3) }\end{array}$ & 96 \\
\hline Abbildung & 20 & $\begin{array}{l}\text { Führungsverhalten und Arbeitserleben von } \\
\text { Schulleiterinnen und Schulleitern }\end{array}$ & 98 \\
\hline Abbildung & 21 & $\begin{array}{l}\text { Zusammenhänge zwischen den vier Dimensio- } \\
\text { nen schulischen Führungshandelns }\end{array}$ & 100 \\
\hline Abbildung & 22 & Expertenstatus & 105 \\
\hline Abbildung & 23 & Fallübergreifende Vergleichskategorien & 115 \\
\hline Abbildung & 24 & Fallkontrastierung & 115 \\
\hline Abbildung & 25 & Typen von Berufsauffassungen & 187 \\
\hline Tabelle & & Titel & Seite \\
\hline Tabelle & 1 & $\begin{array}{l}\text { Schulformbezogene Darstellung ausgewählter } \\
\text { Statistiken }\end{array}$ & 87 \\
\hline Tabelle & 2 & Realisierte qualitative Interviews & 110 \\
\hline
\end{tabular}


Anlagen 


\section{Anlage 1}

I. Umgang mit den Lehrerinnen und Lehrern

\begin{tabular}{|c|l|}
\hline & \\
\hline 1 & $\begin{array}{l}\text { Das Beurteilen der Lehrkräfte ist für mich } \\
\text { eine unangenehme Dienstpflicht }\end{array}$ \\
\hline 2 & $\begin{array}{l}\text { Unterrichtsbesuche sind ein Baustein für } \\
\text { die Personalentwicklung der Schule }\end{array}$ \\
\hline 3 & $\begin{array}{l}\text { Ich finde es wichtig, Zielvereinbarungen mit } \\
\text { Fachkonferenzen zu treffen }\end{array}$ \\
\hline 4 & $\begin{array}{l}\text { Zielvereinbarungs-/ Mitarbeitergespräche } \\
\text { sind für die Unterrichtsentwicklung wichtig }\end{array}$ \\
\hline 5 & $\begin{array}{l}\text { Ich ergreife die Initiative, wenn Lehrkräfte } \\
\text { Probleme mit einer Klasse haben. }\end{array}$ \\
\hline 6 & $\begin{array}{l}\text { Ich gebe häufig individuelle Beratung und } \\
\text { Hilfe }\end{array}$ \\
\hline 7 & $\begin{array}{l}\text { Probleme, die Lehrkräfte mit einer Klasse } \\
\text { haben, löse ich gemeinsam mit den Betrof- } \\
\text { fenen }\end{array}$ \\
\hline 8 & $\begin{array}{l}\text { Ich vermittle den Eindruck, dass die Lehr- } \\
\text { kräfte jeder Zeit mit Problemen und Fragen } \\
\text { zu mir kommen können }\end{array}$ \\
\hline
\end{tabular}

\begin{tabular}{|c|c|c|c|}
\hline stimme zu & $\begin{array}{l}\text { stimme } \\
\text { eher zu }\end{array}$ & $\begin{array}{c}\text { stimme } \\
\text { eher nicht } \\
\text { zu }\end{array}$ & $\begin{array}{l}\text { stimme } \\
\text { überhaupt } \\
\text { nicht zu }\end{array}$ \\
\hline & & & \\
\hline & & & \\
\hline & & & \\
\hline & & & \\
\hline & & & \\
\hline & & & \\
\hline & & & \\
\hline & & & \\
\hline & & & \\
\hline & & & \\
\hline & & & \\
\hline & & & \\
\hline
\end{tabular}

II. Arbeitszufriedenheit/ Berufszufriedenheit:

\begin{tabular}{|c|c|c|c|c|c|}
\hline & & $\begin{array}{l}\text { stimme } \\
\mathrm{zu}\end{array}$ & $\begin{array}{l}\text { stimme } \\
\text { eher zu }\end{array}$ & $\begin{array}{c}\text { stimme } \\
\text { eher nicht } \\
\text { zu }\end{array}$ & $\begin{array}{c}\text { stimme } \\
\text { überhaupt } \\
\text { nicht zu }\end{array}$ \\
\hline & Ich fühle mich häufig überfordert & & & & \\
\hline 10 & $\begin{array}{l}\text { Ich schätze meine Veränderungsbereit- } \\
\text { schaft hoch ein }\end{array}$ & & & & \\
\hline 11 & $\begin{array}{l}\text { Oft wird meine Arbeit durch unvorhergese- } \\
\text { hene Situationen unterbrochen }\end{array}$ & & & & \\
\hline 12 & $\begin{array}{l}\text { Ich fühle mich als Schulleiterin/ als Schullei- } \\
\text { ter akzeptiert }\end{array}$ & & & & \\
\hline 13 & $\begin{array}{l}\text { Kolleginnen und Kollegen nehmen mein } \\
\text { Beratungsangebot häufig wahr }\end{array}$ & & & & \\
\hline 14 & $\begin{array}{l}\text { Das Leiten der Schule ist für mich mit ei- } \\
\text { nem hohen Belastungsdruck verbunden }\end{array}$ & & & & \\
\hline
\end{tabular}




\begin{tabular}{|c|c|c|c|c|c|}
\hline & & $\begin{array}{c}\text { stimme } \\
\mathrm{zu}\end{array}$ & $\begin{array}{l}\text { stimme } \\
\text { eher zu }\end{array}$ & $\begin{array}{c}\text { stimme } \\
\text { eher nicht } \\
\text { zu }\end{array}$ & $\begin{array}{c}\text { stimme } \\
\text { überhaupt } \\
\text { nicht zu }\end{array}$ \\
\hline & Mich strengen die vielen Gesprächen an & & & & \\
\hline & Problemkollegen rauben meine Arbeitszeit & & & & \\
\hline 10 & & & & & \\
\hline 17 & $\begin{array}{l}\text { Ich finde Unterstützung bei den übergeord- } \\
\text { neten Behörden }\end{array}$ & & & & \\
\hline 18 & $\begin{array}{l}\text { Die unverbindliche Umsetzung von Be- } \\
\text { schlüssen stört mich sehr }\end{array}$ & & & & \\
\hline & Ich bin zufrieden mit... & & & & \\
\hline 19 & - meiner beruflichen Position & & & & \\
\hline 20 & $\begin{array}{l}\text { - meiner persönlichen Arbeitsorgani- } \\
\text { sation }\end{array}$ & & & & \\
\hline 21 & - dem Klima an meiner Schule & & & & \\
\hline 22 & $\begin{array}{l}\text { - der Organisation unseres Schulbe- } \\
\text { triebes }\end{array}$ & & & & \\
\hline 23 & $\begin{array}{l}\text { - den Kommunikationsstrukturen in } \\
\text { unserer Schule }\end{array}$ & & & & \\
\hline 24 & $\begin{array}{l}\text { - meinem Verhältnis zu meinem Kol- } \\
\text { legium }\end{array}$ & & & & \\
\hline 25 & $\begin{array}{l}\text { - meinem Kontakt zu Schülerinnen } \\
\text { und Schülern }\end{array}$ & & & & \\
\hline 26 & - $\quad$ meinem Verhältnis zu den Eltern & & & & \\
\hline 27 & $\begin{array}{l}\text { - meiner Zusammenarbeit mit den } \\
\text { Sekretärinnen }\end{array}$ & & & & \\
\hline 28 & $\begin{array}{l}\text { Ich wünsche mir mehr persönliche Unter- } \\
\text { stützung (z.B. Coaching) }\end{array}$ & & & & \\
\hline 29 & $\begin{array}{l}\text { Häufig erlebe ich Loyalitätskonflikte } \\
\text { (Kollegium, Dienstaufsicht, Schulgesetz) }\end{array}$ & & & & \\
\hline 30 & $\begin{array}{l}\text { In meinem Berufsalltag fühle ich mich allein } \\
\text { gelassen }\end{array}$ & & & & \\
\hline
\end{tabular}


III. Schulentwicklung

\begin{tabular}{|c|c|c|c|c|c|}
\hline & & stimme zu & $\begin{array}{l}\text { stimme } \\
\text { eher zu }\end{array}$ & $\begin{array}{l}\text { stimme } \\
\text { eher nicht } \\
\text { zu }\end{array}$ & $\begin{array}{c}\text { stimme } \\
\text { überhaupt } \\
\text { nicht zu }\end{array}$ \\
\hline 31 & $\begin{array}{l}\text { Es gibt ein Schulprogramm an meiner } \\
\text { Schule. }\end{array}$ & & & & \\
\hline 32 & $\begin{array}{l}\text { Ich arbeite an der Entwicklung von langfris- } \\
\text { tigen Zielen für die Schule }\end{array}$ & & & & \\
\hline 33 & $\begin{array}{l}\text { Ich sorge dafür, dass die pädagogischen } \\
\text { Ziele verbindlich umgesetzt werden }\end{array}$ & & & & \\
\hline 34 & $\begin{array}{l}\text { Ich überprüfe die wirkliche Umsetzung von } \\
\text { Beschlüssen }\end{array}$ & & & & \\
\hline 35 & $\begin{array}{l}\text { Ich arbeite in Gremien/ Netzwerken mit, die } \\
\text { sich die Verbesserung von Schule zum Ziel } \\
\text { gesetzt haben }\end{array}$ & & & & \\
\hline 36 & $\begin{array}{l}\text { Veränderungen im Bereich des Unterrichts } \\
\text { sind notwendig }\end{array}$ & & & & \\
\hline 37 & $\begin{array}{l}\text { Die Schulprogrammarbeit, z.B. Entwicklung } \\
\text { eines klaren Leitbildes, halte ich für wichtig }\end{array}$ & & & & \\
\hline 38 & $\begin{array}{l}\text { Als Schulleiterin/Schulleiter bin ich die Initi- } \\
\text { atorin/der Initiator für Schulentwicklung }\end{array}$ & & & & \\
\hline 39 & $\begin{array}{l}\text { Ich bin häufig bei außerunterrichtlichen } \\
\text { Aktivitäten der Schülerinnen anwesend }\end{array}$ & & & & \\
\hline 40 & $\begin{array}{l}\text { Ich nehme mir Zeit dafür, in den Pausen } \\
\text { mit Schülerinnen u. Schülern u. Lehrkräften } \\
\text { zu sprechen }\end{array}$ & & & & \\
\hline
\end{tabular}

IV. Führungsverhalten

\begin{tabular}{|c|c|c|c|c|c|}
\hline & & stimme zu & $\begin{array}{l}\text { stimme } \\
\text { eher zu }\end{array}$ & $\begin{array}{c}\text { stimme } \\
\text { eher nicht } \\
\text { zu }\end{array}$ & $\begin{array}{c}\text { stimme } \\
\text { überhaupt } \\
\text { nicht zu }\end{array}$ \\
\hline 41 & $\begin{array}{l}\text { Mir ist wichtig, dass sich die Kolleginnen } \\
\text { und Kollegen in der Schule wohl fühlen }\end{array}$ & & & & \\
\hline 42 & $\begin{array}{l}\text { Ich bemühe mich um die Verbesserung des } \\
\text { sozialen Klimas im Kollegium }\end{array}$ & & & & \\
\hline 43 & $\begin{array}{l}\text { Ich schätze meine Fähigkeit, Konflikte aus- } \\
\text { zugleichen, hoch ein }\end{array}$ & & & & \\
\hline 44 & $\begin{array}{l}\text { Ich habe immer ein offenes Ohr für die } \\
\text { Sorgen und Probleme der Lehrkräfte }\end{array}$ & & & & \\
\hline 45 & Bestimmte Entscheidungen treffe ich allein & & & & \\
\hline
\end{tabular}




\begin{tabular}{|c|c|c|c|c|c|}
\hline & & stimme zu & $\begin{array}{l}\text { stimme } \\
\text { eher zu }\end{array}$ & $\begin{array}{l}\text { stimme } \\
\text { eher nicht } \\
\text { zu }\end{array}$ & $\begin{array}{c}\text { stimme } \\
\text { überhaupt } \\
\text { nicht zu }\end{array}$ \\
\hline 46 & $\begin{array}{l}\text { Ich diskutiere alle anstehenden wichtigen } \\
\text { Entscheidungen offen im Kollegium }\end{array}$ & & & & \\
\hline 47 & $\begin{array}{l}\text { Ich berücksichtige vor allen Dingen die } \\
\text { Meinung der sehr aktiven Kolleginnen und } \\
\text { Kollegen }\end{array}$ & & & & \\
\hline 48 & $\begin{array}{l}\text { Ich kann Gesamtkonferenzentscheidungen } \\
\text { gut annehmen, auch wenn meine eigenen } \\
\text { Vorschläge abgelehnt werden }\end{array}$ & & & & \\
\hline 49 & $\begin{array}{l}\text { Als Schulleiterin/Schulleiter habe ich wenig } \\
\text { Entscheidungsspielraum }\end{array}$ & & & & \\
\hline 50 & $\begin{array}{l}\text { Außerordentliche Leistungen von Leh- } \\
\text { rern/Lehrerinnen werden im Kollegium be- } \\
\text { kannt gegeben }\end{array}$ & & & & \\
\hline 51 & Ich delegiere viele Aufgaben & & & & \\
\hline 52 & $\begin{array}{l}\text { Ich versuche das Kollegium bzw. betroffene } \\
\text { Lehrer bei Entscheidungen zu beteiligen }\end{array}$ & & & & \\
\hline 53 & $\begin{array}{l}\text { Ich habe klare Vorstellungen über meine } \\
\text { Arbeit }\end{array}$ & & & & \\
\hline 54 & $\begin{array}{l}\text { Es gelingt mir die Schule im Hinblick auf } \\
\text { eine gemeinsame Zielrichtung zu lenken }\end{array}$ & & & & \\
\hline 55 & $\begin{array}{l}\text { In den letzten Jahren habe ich eine Aufwer- } \\
\text { tung und Stärkung meiner Führungsrolle } \\
\text { erfahren }\end{array}$ & & & & \\
\hline 56 & $\begin{array}{l}\text { Auf Konferenzen werden inhaltliche The- } \\
\text { men diskutiert, z.B. Ergebnisse aktueller } \\
\text { Projekte, Schulentwicklung }\end{array}$ & & & & \\
\hline 57 & $\begin{array}{l}\text { Die Moderation von Konferenzen hat für } \\
\text { mich eine wichtige Steuerungsfunktion }\end{array}$ & & & & \\
\hline 58 & $\begin{array}{l}\text { Ich bereite jede Sitzung/ Konferenz aus- } \\
\text { führlich vor und nach }\end{array}$ & & & & \\
\hline 59 & $\begin{array}{l}\text { Mir ist die Fortbildung des Kollegiums ein } \\
\text { großes Anliegen }\end{array}$ & & & & \\
\hline 60 & $\begin{array}{l}\text { Lehrerinnen u. Lehrer berichten über Er- } \\
\text { gebnisse von Fortbildungen im Rahmen } \\
\text { von Fachkonferenzen }\end{array}$ & & & & \\
\hline 61 & $\begin{array}{l}\text { Ich sorge dafür, dass die Weiterbildung von } \\
\text { Lehrkräften in Übereinstimmung mit den } \\
\text { pädagogischen Zielen unserer Schule steht }\end{array}$ & & & & \\
\hline 62 & $\begin{array}{l}\text { Mein Führungsstil ist geprägt von den Er- } \\
\text { fahrungen, die ich mit Schulleiterin- } \\
\text { nen/Schulleitern gemacht habe }\end{array}$ & & & & \\
\hline
\end{tabular}




\section{Anlage 2}

Maike Languth

C/o. Prof. Dr. Lemmermöhle

Pädagogisches Seminar der Universität Göttingen

Baurat Gerber Str. 4/6

37073 Göttingen

Sehr geehrte Frau Schulleiterin, sehr geehrter Herr Schulleiter,

ich bitte Sie um Mithilfe bei der Umsetzung meines Forschungsvorhabens „Schulleitungshandeln unter der besonderen Berücksichtigung des subjektiven Führungsverständnisses“. Das Forschungsvorhaben wird im Zentrum für empirische Unterrichts- und Schulforschung (ZeUS) an der Universität Göttingen unter der Leitung von Frau Prof. Dr. Lemmermöhle durchgeführt.

Durch meine eigene Tätigkeit in der kollegialen Schulleitung beschäftige ich mich intensiv mit dem Thema "Leitung und Führung von Schule“. Dabei ist mir aufgefallen, dass der Beruf Schulleiterin/Schulleiter zunehmend mit Sollvorstellungen und Kompetenzprofilen ausgefüllt wird. Die Ansprüche an die Berufsausübung steigen, aber meines Erachtens kommen die Schulleiterinnen und Schulleiter selbst bisher zu wenig zu Wort. Die von mir recherchierten Untersuchungen zum Thema Schulleitungshandeln beschäftigen sich meistens mit der Wirksamkeit und Effektivität von Schulleitungshandeln.

Ich möchte mit meiner Untersuchung, die eine Kombination aus einem standardisierten Fragebogen und qualitativen Interviews darstellt, die subjektive Wahrnehmung des Alltagshandelns von Schulleiterinnen und Schulleitern in den Blick nehmen. Dabei soll auch nach Ihrer konkreten Belastungen gefragt werden.

Aus rechtlichen Gründen lag der Fragebogen dem Kultusministerium vor. Sowohl die Schulen selbst als auch das Kultusministerium erhalten nach Abschluss der Arbeit eine zusammenfassende Rückmeldung, aus der aber keine Daten von Einzelschulen abzuleiten sind. Die Ergebnisse meiner Arbeit können einen Beitrag zur höheren Wertschätzung für den Beruf Schulleiterin/Schulleiter leisten und einen Anstoß für unterschiedliche Unterstützungssysteme geben. Die Teilnahme ist selbstverständlich freiwillig.

Ich bitte Sie den beigefügten Frageboten auszufüllen und bis zum 15.02.2004 an die obige Adresse der Universität Göttingen zu senden. Ihre Anonymität bleibt gewahrt. Der Frageboten wurde an alle niedersächsischen Gymnasien, kooperativen Gesamtschulen und integrierten Gesamtschulen versendet, die eine Sekundarstufe II haben. Somit lassen sich die inhaltlichen und organisatorischen Bereiche vergleichen. Sollten Sie darüber hinaus die Bereitschaft haben, mit mir persönlich ein vertiefendes Interview zu führen, wäre ich Ihnen sehr dankbar.

Bitte füllen sie dann den unteren Abschnitt dieses Schreibens mit Ihrem Namen und der Schuladresse aus und senden Sie diesen mit getrennter Post ebenfalls bis 15.02.2004 an die obige Adresse der Universität Göttingen. Ich melde mich dann umgehend bei Ihnen.

Ich danke Ihnen sehr herzlich

Mit freundlichen Grüßen

Maike Languth

Ja, ich bin bereit, mit Ihnen ein Interview zu führen.

Name:

Schuladresse: 


\section{Anlage 3}

\section{Handlungsdimensionen der Schulleiterinnen und Schulleiter}

Im folgenden Analyseschritt wird nun mit Hilfe der konfirmatorischen Faktorenanalyse getestet, ob die theoretisch bestimmten Konstrukte (bzw. Faktoren) wie zum Beispiel Zielorientierung, die erfassten Einstellungen der Schulleiterinnen und Schulleiter erklären können. Die Items (Fragen des Fragebogens) waren ja entworfen worden, um Einstellungen und Handlungsdimensionen von Schulleiterinnen und Schulleitern zu erfassen. Können die theoretischen Zusammenhänge nicht bestätigt werden, dann kann dies einerseits daran liegen, dass die ermittelten Handlungsdimensionen nicht ausreichend operationalisiert waren.

Die konfirmatorischen Faktorenanalyse wurde mit der Analysesoftware AMOS 5.0 durchgeführt. ${ }^{242}$

\subsection{Ergebnisse Umgang mit Lehrerinnen und Lehrern}

Die Itembatterie der Fragen zum Umgang mit Lehrerinnen und Lehrern lässt sich die Dimension Mitarbeiterorientierung abbilden. Die Handlungsdimension „Management der sozialen Beziehung“ ließ sich also in dieser Befragung nachweisen.

\section{Abb. 1: Konfirmatorische Faktorenanalyse Umgang mit Lehrerinnen und Lehrern $^{243}$}

Ich gebe häufig individuelle Beratung und Hilfe (recodiert)

Ich vermittele den Eindruck, dass die Lehrkräfte jeder Zeit mit Problemen und Fragen zu mir kommen können. (recodiert)

„Kolleginnen und Kollegen nehmen mein Beratungsangebot häufig wahr" (recodiert)

Mir ist wichtig, dass sich die Kolleginnen und Kollegen in der Schule wohl fühlen (recodiert)

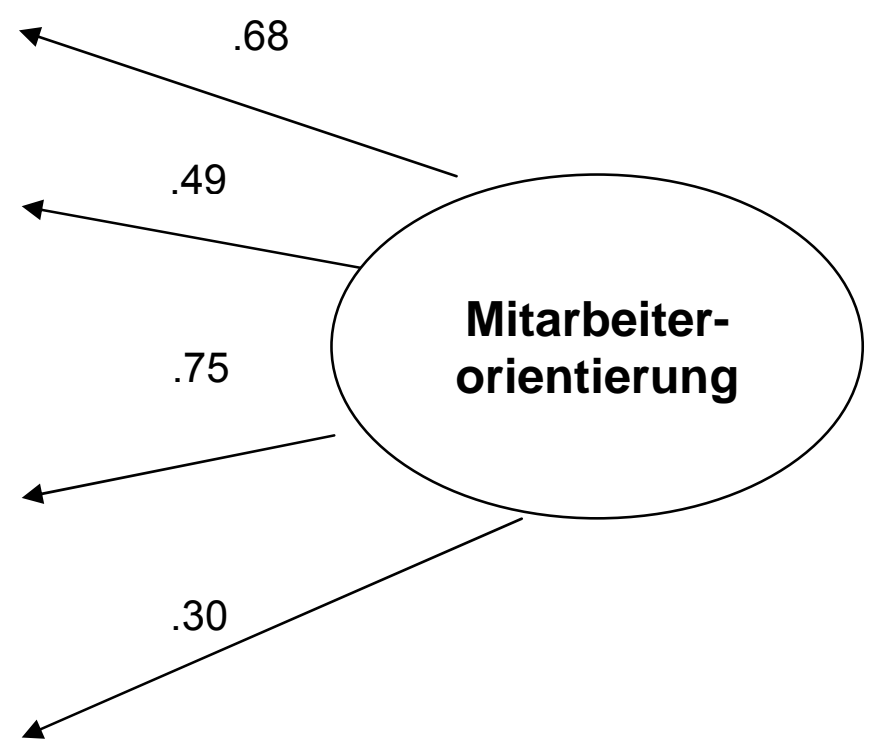

$\mathrm{N}=146 \quad$ Chi-Square $/ \mathrm{df}=2.689 ; \mathrm{p}=0.68 ; \mathrm{CFi}=.956 ; \mathrm{AGFI}=.909 ; \mathrm{RMSEA}=.110$

\footnotetext{
${ }^{242}$ Vgl. Byrne, Barbara M. 2001, 57ff

Anzumerken ist hier, dass der Datensatz von 145 Fragebögen für das Programm Amos sehr klein

ist, normalerweise wird mit Datensätzen über 1000 gerechnet.

${ }^{243}$ Aus Gründen der Übersichtlichkeit und besseren Lesbarkeit werden Angaben der Messfehler weggelassen.
} 
Für die empirische Bestätigung der theoretischen Zusammenhänge ist es zunächst wichtig, dass die Faktorenladung, also die Korrelation zwischen Ausgangsvariablen und Faktor hoch ist. Die standardisierten (Werte zwischen -1 und 1) Faktorenladungen stehen oberhalb der Pfeile zwischen Faktor (hier Mitarbeiterorientierung) und den Variablen (Fragen/ltems). Um empirisch abgesicherte Berechnungen vorzulegen, sollte der ChiSquare/df unter 2,2 und der AGFI über 0,90 liegen. ${ }^{244}$

\subsection{Berufszufriedenheit}

Nach Anpassung des Modells kann eine latente Dimension „Berufszufriedenheit" bestehend aus vier Items ermittelt werden. In den Fragen zur Berufzufriedenheit wird die Trennung zweier Aussagenmodi deutlich, die sich durch das Modell abbilden lässt. Es gilt zu unterscheiden zwischen Items, die die subjektive Zufriedenheit mit der eigenen Position darstellen und denen die Zufriedenheit mit dem beruflichen Umfeld anzeigen. Nur für den zweiten Bereich ließ sich eine konsistente Dimension nachweisen. Dies kann darin begründet sein, dass die Aussagen zum subjektiven Erleben eine zu geringe Streubreite der Aussagen ermöglichte.

\section{Abb. 2: $\quad$ Konfirmatorische Faktorenanalyse Berufszufriedenheit}

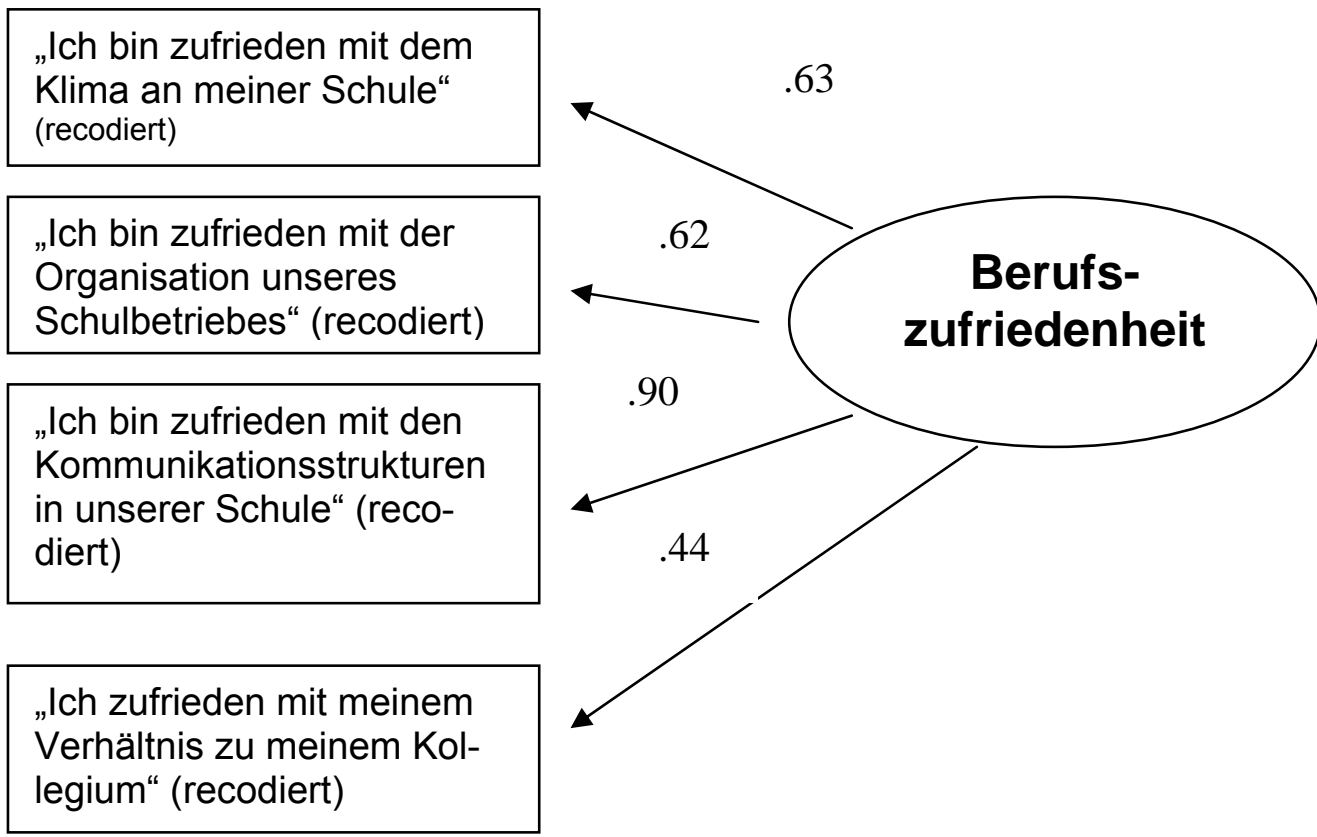

244 Weitere Erläuterung zur Abbildung: für Chi-Square/df (Chi-Quadrat dividiert durch Freiheitsgrade) gilt: je niedriger desto besser, am besten unter 2,2;

$\mathbf{p}$ ist mit Chi-Square verbunden; für $\mathbf{p}$ gilt, dass er umso höher ist, je näher das hypothetisches Modell und empirisches Modell beieinander liegen, also Werte um 0,05 sind gut. AGFI ist der adjusted goodness-of-fit-index, bei dem die Zahl der Freiheitsgrade im spezifizierten Modell berücksichtigt wird; dieser Wert sollte über 0,90 liegen.

CFI (comparative fit index), bei dem die Stichprobengröße berücksichtigt wird; sollte über 0,95 oder zumindest nicht weit davon entfernt liegen;

RMSEA (root mean square error of approximation); Beantwortet die Frage, wie gut das Modell (mit unbekannten aber optimal gewählten Parametern) zur PopulationsKovarianzmatrix passt, wenn sie erhältlich wäre; Werte unter 0,05 zeigen eine gute Passung, bis 0,08 bleiben die Werte in Ordnung; wenn die Stichprobe (wie in meinem Fall klein ist), reagiert der RMSEA über, dann sind möglicherweise Werte bis 0,10 noch vertretbar. Vgl. Byrne, Barbara M. (2001). 
$\mathrm{N}=146 \quad$ Chi-Square $/ \mathrm{df}=.367 ; \mathrm{p}=0.545 ; \mathrm{CFi}=.956 ; \mathrm{AGFI}=.987 ; \mathrm{RMSEA}=.000$ Zu problematisieren ist an dieser Stelle der extrem niedrige RMSEA-Wert. Ein derartig hoher Wert zeigt an, dass viele Personen einheitlich hoch angekreuzt haben und diese mangelnde Polarisierung in den Antworten ist für die Errechnung einer Dimension nicht förderlich.

\subsection{Empfundene Belastung}

Das Gleiche gilt für die Dimension „Belastungsempfinden“, die nach ihrer Berechnung nur aus vier Items besteht, die sehr ähnlich sind und daher den Faktor „Belastung“ noch nicht differenziert genug ausdrückt.

Anzustreben wäre es, dass sich Items finden lassen, die genauer die Belastungen thematisieren, also ein breiteres Spektrum abbilden und die sich trotzdem in einer aussagekräftigen Dimension abbilden lassen

\section{Abb. 3: Konfirmatorische Faktorenanalyse Belastungsempfinden}

Ich fühle mich häufig überfordert (recodiert)

Das Leiten der Schule ist für mich mit hohem Belastungsdruck verbunden (recodiert)

Mich strengen die vielen Gespräche an (recodiert)

In meinem Berufsalltag fühle ich mich allein gelassen (recordiert)

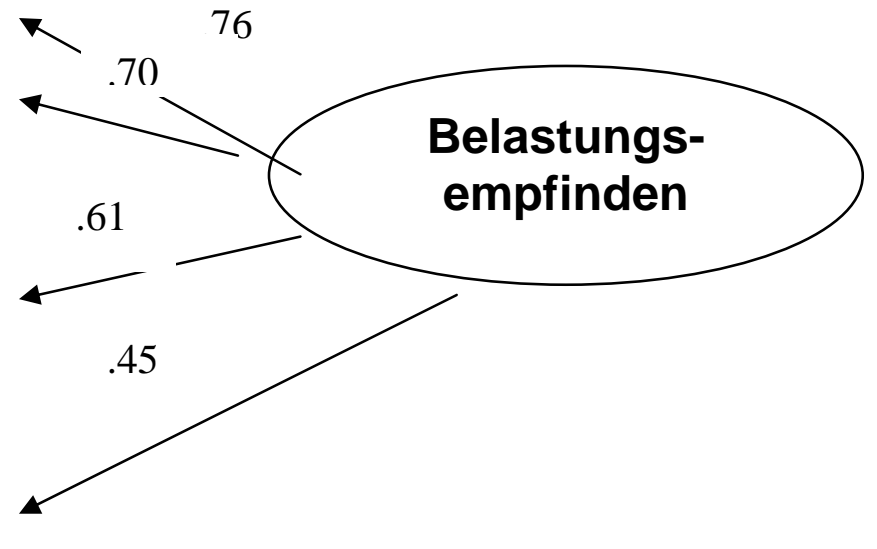

$\mathrm{N}=146 \quad$ Chi-Square $/ \mathrm{df}=.971 ; \mathrm{p}=0.379 ; \mathrm{CFi}=1000 \mathrm{AGFI}=.964$;

RMSEA $=.000$

\subsection{Ergebnisse Zielorientierung}

Da sich in den Ergebnissen zum Fragenkomplex Führungsverhalten ein Schwerpunkt im Bereich „Zielorientierte Führung“ herausgebildet hat, soll anhand dieses Konstruktes die Tragfähigkeit dieser Dimension, die ja in Untersuchungen von Bonsen (2002) als bedeutsamste Dimension von Seiten des Kollegiums herausgestellt worden war, überprüft werden. Interessanter Weise ließ sich keine Dimension abbilden, die empirisch abgesicherte und vertretbare Werte liefert. Der Handlungsdimension „Zielorientierte Führung" kommt statistisch in den Antworten der hier untersuchten 145 Schulleiterinnen und Schulleitern keine Bedeutung zu.

Sowohl die erste Berechnung des Faktors Zielorientierung zeigte, dass die die Werte nicht tolerierbar waren. Auch der Versuch den Bereich Zielorientierung in zwei Teilbereiche, nämlich Zielorientierung und konsequentes Verhalten zu trennen, ergab kein akzeptables Modell. Dies überrascht insofern nicht, als sich hier Widersprüche bereits andeuteten zwischen Zielsetzungen und Zielumsetzungen. Deutlich wird, dass das latente Konstrukt Zielorientierung sich bisher nicht aus den Antworten ableiten lässt. Die 
Dimension Zielorientierung scheint daher einerseits ein zu abstraktes Konstrukt darzustellen, andererseits lässt sich der inhaltliche Anspruch im eigenen Handeln scheinbar nicht verwirklichen. Zielorientierte bezeichnet ja sowohl die Kommunikation von Visionen und Leitvorstellungen als auch die Erarbeitung und Umsetzung von Arbeitszielen. 


\section{Anlage 4}

\section{Das Berufsbild für Schulleiterinnen und Schulleiter Arbeitsplatz Schulleitung ${ }^{245}$}

\section{Soziale Kompetenz}

Sie verfügen über ein hohes $M a ß$ an Kommunikationsvermögen. Durch ihre Menschenkenntnis, Begeisterungsfähigkeit, Fürsorge, und Toleranz erzeugen sie ein kooperatives Arbeitsklima, knüpfen stabiles Beziehungs- und Kommunikationsnetz nach innen und nach außen.

\section{Rechtliche Kompetenz}

Sie überblicken den für Schule relevanten Rechtsrahmen und wenden die entsprechenden Rechts- und Verwaltungsvorschriften situationsgemäß und flexibel an.

\section{Schulleiterinnen und Schulleiter haben eine hohe Leitungs- und Führungs- kompetenz}

Pädagogische Kompetenz Sie sind ausgebildete Lehrkräfte, sie erfüllen den Bildungsauftrag der Schule und fordern die pädagogische Qualität ihrer Schule ein.
Personalentwicklungskompetenz Sie analysieren Personalbedarf an ihren Schulen. Sie kennen die beruflichen Qualitäten der an ihren Schulen arbeitenden Personen und fördern deren Leistungsbereitschaft und berufliche Entwicklung.

\section{Organisatorische Managementkompetenz} Sie sind effiziente Organisationsmanager, sie schaffen reibungslosen, transparenten und akzeptierten Ablauf des Schulalltages, sie gehen verantwortlich mit Ressourcen um.

245 „Arbeitsplatz Schulleitung“ Nds. Kultusminister 2002, 13. 
Tabellarischer Lebenslauf

Name:

Maike Languth

Geburtsdatum/-ort:

10.12.1967 in Kiel

Schulbildung:

1974-1978

Grundschule Kiel

1978-1987

Gymnasium Kiel

Studium:

1987-1994

Studium für das Lehramt an Gymnasien an der Georg-August Universität in Göttingen Hauptfächer Sport/ Deutsch

Studienabschluss:

12/1994

1. Staatsexamen

Referendariat:

05/1995 -05/1997

05/1997

Referendariat an der IGS-Linden Hannover

2. Staatsexamen

Berufstätigkeit:

08/1997-02/1998

Angestellt als teilzeitbeschäftigte Lehrerin an der KGS-Salzhemmendorf

09/1997-09/1998

Dozentin an der Volkshochschule Hannover "Deutsch als Fremdsprache“

02/1998-02/1999

Beamtin auf Probe an der

KGS Salzhemmendorf

02/1999

Ernennung zur Studienrätin

01/1999-07/2002

Didaktische Leiterin der Georg-ChristophLichtenberg Gesamtschule in Göttingen

09/2001-02/2004

Weiterbildungsstudium „Pädagogische Supervision" an der Christian Albrecht Universität Kiel Abschluss: Zertifikat Pädagogische Supervision

$08 / 2004$

Koordinatorin am Max-Planck-Gymnasium in Göttingen

$02 / 2006$

Ernennung zur Studiendirektorin am Max-Planck-Gymnasium in Göttingen

$12 / 2006$

Promotion zur Doktorin der

Sozialwissenschaften (Dr. dis. pol.) 